\title{
SYNTHESIS OF LANTHIONINE-CONTAINING PEPTIDES ON SOLID PHASE VIA AN ORTHOGONAL PROTECTING GROUP STRATEGY
}

Mohd Firouz $\mathcal{M O H D} \mathcal{M U S \mathcal { T A P } A}$

A thesis presented in partial fulfillment of the requirements for the Doctor of Philosophy degree of the University of London

\author{
Department of Chemistry \\ UNIVERSITY COLLEGE LONDON
}

August 2002 
ProQuest Number: U641868

All rights reserved

INFORMATION TO ALL USERS

The quality of this reproduction is dependent upon the quality of the copy submitted.

In the unlikely event that the author did not send a complete manuscript and there are missing pages, these will be noted. Also, if material had to be removed, a note will indicate the deletion.

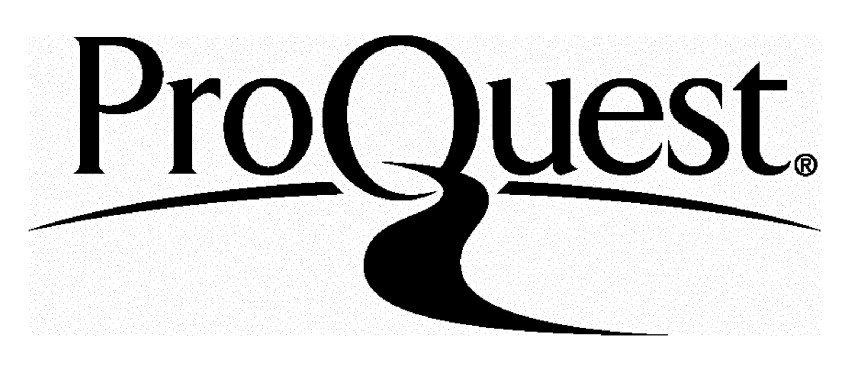

ProQuest U641868

Published by ProQuest LLC(2015). Copyright of the Dissertation is held by the Author.

All rights reserved.

This work is protected against unauthorized copying under Title 17, United States Code. Microform Edition (c) ProQuest LLC.

\author{
ProQuest LLC \\ 789 East Eisenhower Parkway \\ P.O. Box 1346 \\ Ann Arbor, MI 48106-1346
}


"Life is like a box of chocolate-you never know what you're gonna get"

Tom Hanks as "Forest Gump" 


\section{Declaration}

I, Mohd Firouz Mohd Mustapa, hereby state that the following is entirely my own work and has not been for any other degree or examination.

Mohd Firouz MOHD MUSTAPA

August 2002 


\section{Acknowledgement}

First and foremost I would like to thank God Almighty for providing me the strength, courage, perseverance and sanity (at times when sanity was precious indeed) to carry out this project to the very end.

My utmost gratitude goes to Dr. Alethea Tabor for choosing me to embark on this project, for her constant guidance, assistance and support over the past four years, and for her sheer patience during the course of the write-up of this thesis!

My thanks go to Dr. Richard Harris and Dr. Paul Driscoll (Department of Biochemistry) for their extensive NMR work on the controversial "lanthionine" amino acids and peptides.

I must thank Dr. Piers "daddy-to-be" Gaffney for his invaluable input in the construction of my thesis, as well as all the bright ideas over the years! Oh, and thanks for the job! (:)

My thanks also go to my industrial supervisors Dr. Nathan Chubb and Dr. Darren Schulz as well as Dr. Jessica Mould and the entire Veterinary Science team at Pfizer Central Research, Sandwich, for their generosity and hospitality.

I would also like to thank the following: Dr. Marianne Groussier (grandmamma of lanthionine) for pioneering the ABT group's epic adventure into the syntheses of "lanthionine" amino acids and peptides (it ain't over yet sweetie - it's all Mitsunobu now!) and for all her help in the laboratory; Dr. Linda "allylic sister" Alexander McNamara and Chris McNamara, for the countless dinners, barbecues and nights out I (my turn to host dinner next - oh wait, got to find somewhere to live first... oh well, good luck with baby Ethan- he's soooo cute!); Dr. Erwann Guenin for all his help with mass spectrometry and the peptide synthesizer, "Joey" - merci; Dr. Sarah "been around the world you lucky so and so" Sheady; Paul Free and Chris Hurley (life in the lab would be dull without the both of you 
man to fill your shoes...?!?! When are you making curry again?); Dr. Paul "B" Bell (sorry you couldn't keep the snazzy safety glasses for good...); Dr. Paul Mendonça (comrade, coffee break companion, lab-mate, officemate, cat-keeper, what can't you be?!); Suzie "Mitsy" Elliot and Nives Bulic-Subanovic for keeping the hope of one day synthesizing lanthionine peptides alive!

I am very grateful to UCL Graduate School, Overseas Research Students' Award Scheme (ORSAS) and Pfizer UK Ltd. for their generosity in providing solid financial support for this Ph.D. project. 8

My thanks and love go to my family in Malaysia - my parents, my two sisters G and Tasya and brother-in-law Arman including the latest additions to the family Hykel and Erin - for their support, love and encouragement. Thank you Payie, Rizal, Duan, Azneal, as well as Stuart and Mike for their support, encouragement and most importantly their friendships.

Last but not least "tack så myket" Göran. 


\section{Abstract}

Lanthionine (Lan), a monosulfide analogue of cystine, is a naturally occurring amino acid found in a group of polycyclic peptide antibiotics called lantibiotics. Nisin is so far the only lantibiotic that has been produced synthetically. Biological studies of these peptides are of interest, due to their activity against Gram-positive bacteria, especially in the search for new groups of antibiotics. With the difficulty in obtaining lantibiotics in respectable quantities for biological studies it was therefore the aim of this Ph.D. project to establish the fundamentals of the total synthesis of lantibiotics solely on solid phase, by investigating the possibility of a rapid and high yielding method for synthesizing smaller Lan-containing peptides.

Following literature procedures of the formation of Lan residues, the synthesis of Lan derivative 1 was attempted. Using a triply orthogonal protecting group strategy the syntheses of several Lan-containing cyclic peptides were attempted; only the 21 membered nisin ring $\mathrm{C}$ analogue 2 yielded enough material for extensive NMR studies (TOCSY, HSQC, HMQC, HMBC) to be carried out. The two major products were found to be diastereoisomers 6 and contained nor-Lan, a regioisomer of the desired product $\mathbf{1}$. nor-Lan 3 was formed from an enantiomeric mixture of $\alpha$-iodo- $\beta$ alanine derivatives $\mathbf{4}$, originally identified in literature as a locked conformer of the desired $\beta$-iodo- $\alpha$-alanine derivative $\mathbf{5}$.

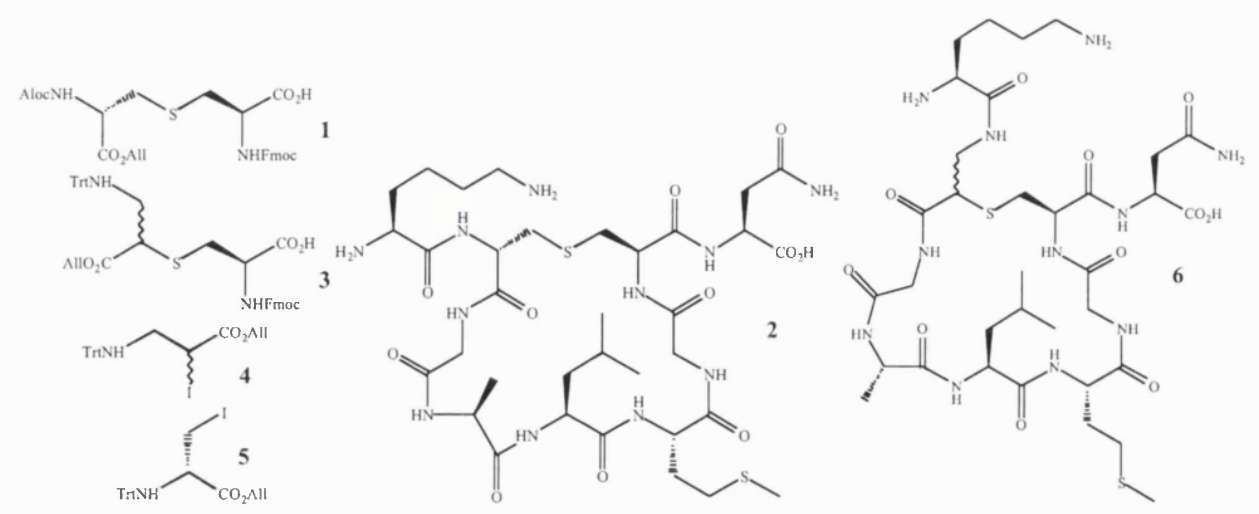

Despite the lack of success in terms of the target peptides and amino acids, it has been shown that the use of a differentially-protected double-headed amino acid monomer could be used in the synthesis of cyclic peptides on solid phase utilizing an orthogonal protecting group strategy. 


\section{CONTIENTS}

Abbreviations $\quad 11$

List of Figures 15

\section{Chapter 1}

Introduction I $\quad 23$

1.1. Lanthionine 23

1.2. Lantibiotics 24

1.3. Nisin 31

1.4. Synthetic lanthionine-containing peptides 32

1.5. Synthesis of lanthionine residues 34

1.5.1. Sulfur extrusion 34

1.5.2. Biomimetic approach $\quad 37$

1.5.3. Serine $\beta$-lactones 38

1.5.4. $\beta$-Haloalanine derivatives $\quad 39$

1.5.5. Ring opening of aziridine-2-carboxylates 41

\section{Chapter 2}

Introduction II $\quad 42$

2.1. Solid phase peptide synthesis $\quad 42$

2.2. Resins for Fmoc solid phase peptide synthesis 45

2.2.1. Cleavage conditions 46

2.2.1.1. Acid labile resins yielding peptide acids 46

2.2.1.2. Acid labile resins yielding peptide amides 47

2.2.1.3. Resins cleaved via nucleophilic displacement and photolysis $\quad 48$

2.3. Coupling reagents and cyclisation reagents (via amide bond formation) $\quad 50$

2.4. Orthogonal protecting groups 55

2.4.1. Allylic protecting groups $\quad 55$

2.4.2. Dimedone-based protecting groups $\quad 60$

2.5. Synthesis of cyclic and polycyclic peptides 67 
2.5.1. Multiply-orthogonal protecting group strategies for SPPS

2.6. Synthesis of lanthionine-containing peptides $\quad 72$

2.6.1. Sulfur extrusion $\quad 72$

$\begin{array}{ll}\text { 2.6.2. Biomimetic approach } & 73\end{array}$

2.6.3. Serine $\beta$-lactones $\quad 74$

2.6.4. $\beta$-Haloalanine derivatives $\quad 76$

\section{Chapter 3}

$\begin{array}{lr}\text { Introduction III } & 80\end{array}$

3.1. Nucleophilic ring opening of aziridine-2-carboxylates 80

3.2. Aims $\quad 92$

\section{Chapter 4}

$\begin{array}{ll}\text { Results and Discussion I } & 97\end{array}$

4.1. Synthesis of protected cysteine 9

4.2. Synthesis of protected $(R)$-iodoalanine $(\boldsymbol{R})$-6a $\quad 101$

4.3. Synthesis of protected lanthionine $(\boldsymbol{R}, \boldsymbol{R})-\mathbf{1 0} \quad 105$

4.4 Synthesis of protected $(S)$-iodoalanine $(S)$-6a 107

4.5. Synthesis of protected lanthionine $(S, R)-10 \quad 109$

4.6. Synthesis of ivDde/Dmab protected $(S, R)$-lanthionine 2

\section{Chapter 5}

$\begin{array}{ll}\text { Results and Discussion II } & 116\end{array}$

5.1. Investigation of the conformational isomer issue 116

5.1.1. HPLC analyses 117

5.1.2. NMR analyses 119

$\begin{array}{ll}\text { 5.1.3. Test reactions } & 128\end{array}$

5.1.4. Synthesis of $(R, R)$ and $(S, R)$-lanthionine derivatives 14 and 15

(via the Mitsunobu reaction) 131 


\section{Chapter 6}

$\begin{array}{ll}\text { Results and Discussion III } & 133\end{array}$

6.1. Synthesis of a lanthionine analogue of an enkephalin opioid peptide 133

6.2. Synthesis of a lanthionine analogue of ring C of nisin 143

6.3. Synthesis of an analogue of rings A and B of nisin 146

$\begin{array}{ll}\text { 6.4. Further NMR analyses of peptide } 25 & 149\end{array}$

Chapter 7

$\begin{array}{lr}\text { Experimental } & 153\end{array}$

$\begin{array}{ll}\text { Appendix A } & 203\end{array}$

$\begin{array}{ll}\text { Appendix B } & 204\end{array}$

$\begin{array}{ll}\text { Appendix C } & 205\end{array}$

$\begin{array}{ll}\text { Appendix D } & 206\end{array}$

$\begin{array}{ll}\text { Appendix E } & 207\end{array}$

$\begin{array}{ll}\text { Appendix F } & 208\end{array}$

$\begin{array}{ll}\text { Appendix G } & 209\end{array}$

$\begin{array}{ll}\text { Appendix H } & 210\end{array}$

$\begin{array}{ll}\text { Appendix I } & 211\end{array}$

$\begin{array}{ll}\text { Appendix J } & 212\end{array}$

$\begin{array}{lr}\text { Appendix K } & 213\end{array}$

$\begin{array}{ll}\text { Appendix L } & 214\end{array}$

$\begin{array}{lr}\text { Appendix M } & 215\end{array}$

$\begin{array}{ll}\text { Appendix N } & 216\end{array}$

$\begin{array}{ll}\text { Appendix O } & 217\end{array}$

$\begin{array}{ll}\text { Appendix P } & 218\end{array}$

$\begin{array}{ll}\text { Appendix Q } & 219\end{array}$

$\begin{array}{ll}\text { Appendix R } & 220\end{array}$

$\begin{array}{ll}\text { Appendix S } & 221\end{array}$

$\begin{array}{ll}\text { Appendix T } & 222\end{array}$

$\begin{array}{ll}\text { Appendix U } & 223\end{array}$ 
Appendix V 224

Appendix W 225

Appendix $X$

226

Appendix X-a

227

Appendix $\mathbf{Y}$

228

Appendix Z

References 


\section{ABBREVIATIONS}

ADDP : 1,1'-(azodicarbonyl)dipiperidine

All : allyl

Ala : alanine

Aloc : allyloxycarbonyl

AOP : (7-azabenzotriazol-1-yloxy)-tris-(dimethylamino)-phosphonium hexafluorophosphate

APCI : atmospheric pressure chemical ionisation

Asn : asparagine

Asp : aspartic acid

Boc : tertiary butoxycarbonyl

BOP : (benzotriazol-1-yloxy)-tris-(dimethylamino)-phosphonium hexafluorophosphate

${ }^{\mathrm{t}} \mathrm{Bu} \quad$ : tertiary butyl

$\mathrm{Bz} \quad$ : benzyl

$\mathrm{Clz}$ : 2-chlorobenzyloxycarbonyl

COSY : correlation spectroscopy

Cys : cysteine

Da : Daltons

Dab : $N$-Dde-4-aminobenzyl

DBU : 1,8-diazabicyclo[5,4,0]-undec-7-ene

DCC : $N, N^{\prime}$-dicyclohexylcarbodiimide

Dcm : N-4,4-dimethyl-2,6-dioxocyclohexylidenemethyl

DCM : dichloromethane

DCU : $N, N^{\prime}$-dicyclohexyl urea

Dde : $N$-1-(4,4-dimethyl-2.6-dioxocyclohexylidene)ethyl

DEPT : distortionless enhancement by polarisation transfer

Dha :2,3-dehydroalanine

Dhb : 2,3-dehydrobutyrine

DIC : $N, N$ '-diisopropylcarbodiimide

DIPEA: $N, N$-diisopropylamine 
Dmab : 4-[N-\{1-(4,4-dimethyl-2,6-dioxocyclohex-1-ylidene)-3-methylbutyl $\}$ amino] benzyl

DMAD: dimethylazodicarboxylate

DMF : $N, N^{\prime}$-dimethylformamide

DMSO : dimethylsulfoxide

DPPA : diphenylphosphoryl azide

EI : electron ionisation

equiv. : (molar) equivalents

ES+ : electrospray positive

FAB : fast atom bombardment

FLEC : 1-(9-fluorenyl)ethyloxycarbonyl

Fm : fluorenylmethyl

Fmoc :9-fluorenylmethoxycarbonyl

Glu : glutamic acid

Gly : glycine

HATU : $N$-[(dimethylamino)-1 $H$-1,2,3-triazolo[4,5-b]-pyridin-1-yl-methylene]- $N$ methylmethanaminium hexafluorophosphate $\mathrm{N}$-oxide

HAPyU: 1-[1-pyrrolidinyl-1H-1,2,3-triazolo[4,5-b]-pyridin-1-yl-methylene]-pyrrolidinium hexafluorophosphate $N$-oxide

HBTU : $N$-[1H-benzotriazol-1-yl]-(dimethylamino)-methylene]- $N$-methylmethanaminium hexafluorophosphate $N$-oxide

His : histidine

HMPA : hexamethylphosphoric triamide

HMPB : 4-(4-hydroxymethyl-3-methoxphenoxy)butyryl

HOAt : 1-hydroxy-7-azabenzotriazole

HOBt : 1-hydroxybenzotriazole

HPLC : high performance liquid chromatography

Ile : isoleucine

I.R. : infra-red

ivDde : $N$-1-(4,4-dimethyl-2,6-dioxocyclohexylidene) isovaleryl

LCMS : liquid chromatography-mass spectrometry

Lan : lanthionine 
Leu : leucine

Lys : lysine

Me : methyl

MeLan: methyl lanthionine

Met : methionine

Ms : methanesulfonyl

NMA : $N$-methylaniline

NMM : $N$-methylmorpholine

NMR : nuclear magnetic resonance

NOESY: nuclear overhauser effect spectroscopy

OPfp : pentafluorophenyl esters

PAL : tris(alkoxy)benzylamide linkage

PCOR : peptide cyclisation on an oxime resin

PEG-PS: poly(ethylene glycol)-polystyrene

Phe : phenylalanine

Pro : proline

PyAOP: (7-azabenzotriazol-1-yl)-tris(pyrrolidino)-phosphonium hexafluorophosphate

PyBOP: (benzotriazol-1-yl)-tris(pyrrolidino)-phosphonium hexafluorophosphate

Ser : serine

SPPS : solid phase peptide synthesis

TBDMS: tertiary butyldimethylsilyl

TBTU : 2-(1H-benzotriazol-1-yl)-1,1,3,3-tetramethyluronium tetrafluoroborate

TEA : triethylamine

TES : triethylsilane

TFA : trifluoroacetic acid

THF : tetrahydrofuran

Thr : threonine

TIPS : triisopropylsilane

TMS : trimethylsilyl

tlc : thin layer chromatography

Trt : triphenylmethyl 
Tyr : tyrosine

U.V. : ultra-violet

Val : valine

VT : variable temperature

$\mathrm{Z}$ : benzyloxycarbonyl 


\section{List of Figures and Tables}

$$
\text { Page }
$$

Figure 1.1. (a) meso-Lanthionine and (b) cystine 24

Figure 1.2. Unusual amino acids in lantibiotics 25

Figure 1.3. Unusual amino acids in lantibiotics $\quad 25$

Figure 1.4. Biosynthesis of lantibiotics $\quad 26$

$\begin{array}{ll}\text { Figure 1.5. Mode of action of mersacidin } & 28\end{array}$

Figure 1.6. Structures of the two-component lantibiotic, Plantaricin W 31

Figure 1.7. Mode of action of nisin $\quad 32$

Figure 1.8. A lanthionine enkephalin opioid analogue 33

Figure 1.9. (a) Lanthionine-somatostatin analogue; (b) proposed bioactive conformation 33

Figure 1.10. Structures of (a) Lan-7; (b) TT-232 33

Figure 1.11. (a) Monosulfide and (b) disulfide analogues of an integrin antagonist 34

Figure 1.12. A thioether cyclic peptide 34

Figure 1.13. Formation of a $(R, R)$-lanthionine derivative via sulfur extrusion method

Figure 1.14. Mechanism of sulfur extrusion from a disulfide; stereochemical inversion of a $\beta$-substituted disulfide $\quad 35$

Figure 1.15. Cross-coupling in sulfur extrusion reaction 36

Figure 1.16. meso-Lanthionine formation via a cyclic cystine derivative 36

Figure 1.17. Retrosynthetic analysis of meso-lanthionine 37

Figure 1.18. Michael addition of cysteine to dehydroalanine 38

Figure 1.19. Competing reactions; (a) thioester formation, (b) thioether formation 38

Figure 1.20. Lanthionine formation using a $\beta$-iodo- $(R)$-alanine derivative

with a cysteine derivative $\quad 39$

Figure 1.21. Determination of chiral integrity of a $(R, R)$-lanthionine derivative $\quad 40$

Figure 2.1. Different degrees of amino acid protection, allowing for repeated amide bond formation $\quad 43$

Figure 2.2. General scheme for Fmoc SPPS 44

Figure 2.3. Cleavage mechanism for Wang and NovaSyn ${ }^{\circledR}$ TGA resins 46 
Figure 2.4. Cleavage mechanism for Rink acid, 2-chlorotrityl, SASRIN and HMPB resins

Figure 2.5. Cleavage mechanism for Rink amide and PAL (5-(4- $N$-fluorenylmethoxycarbonyl-aminomethyl-3,5-dimethoxyphenoxy)valeryl) resin 48

Figure 2.6. Cleavage mechanism for Sieber amide resin 48

Figure 2.7. Cleavage mechanism for HMBA resin 48

Figure 2.8. Cleavage mechanism for 3-nitro-4-hydroxymethyl benzoyl and $\alpha$-methyl-6-nitro-veratrylamine alcohol resins

Figure 2.9. Formation of oxazolone (b) and its enantiomer (d) from an activated amino acid (a) via enolisation of the oxazolone (c) resulting in racemisation: (e) 50

Figure 2.10. Coupling via the azide method using DPPA 51

Figure 2.11. A Pfp-activated amino acid 51

Figure 2.12. Formation of the $O$-acylisourea intermediate (a) followed by nucleophilic attack of an amino group

Figure 2.13. Formation of the symmetrical anhydride (a) followed by nucleophilic attack of the amino group yielding the desired amide bond

Figure 2.14. Formation of an amide bond via in situ activation of the

$\mathrm{O}$-acylisourea intermediate (b) with $\mathrm{HOBt}(\mathrm{a})$

Figure 2.15. Formation of the unreactive $N$-acylisourea (a) 53

Figure 2.16. Formation of the carcinogenic HMPA during BOP activation 53

Figure 2.17. Various coupling and/or cyclisation reagents for SPPS 54

Figure 2.18. Stabilising effect of the pyridinium nitrogen on HOAt 54

Figure 2.19. Allylic protection of various functional groups 56

Figure 2.20. Incorporation of allyl and Aloc onto alcohols, carboxyls and amines 56

Figure 2.21. Mechanism for allylic deprotection $\quad 57$

Figure 2.22. Competing allyl amine formation reaction via routes (a) and (b) 58

Figure 2.23. Scavengers used for palladium-mediated allylic deprotection $\quad 59$

Figure 2.24. Structures of (a) 4-bromocrotonic acid, (b) 4-trityloxybut-(Z)-2enyloxyacetic acid and (c) 4-(4'-methoxytrityloxy)-but-2-enyloxyhexanoic acid 59

Figure 2.25. Synthesis of Dcm and its incorporation into amino acids 60

Figure 2.26. Mechanism for the deprotection of Dcm 61

Figure 2.27. Synthesis of Dde, incorporation onto Fmoc-Lys-OH and deblock 62 
$\begin{array}{ll}\text { Figure 2.28. Structure of ivDde } & 63\end{array}$

Figure 2.29. Synthesis of Dab and its incorporation onto amino acids 64

Figure 2.30. Synthesis of Dmab, its protection of and deblock from amino acids 65

Figure 2.31. Formation of the pyroglutamyl ring on Glu 65

Figure 2.32. Unsuccessful cyclisation due to incomplete removal of Dmab 66

Figure 2.33. Peptide cyclisation mechanism on an oxime resin 67

Figure 2.34. On-resin peptide cyclisation: (a) head-to-tail (b) tail-to-side chain

(c) side chain-to-head and (d) side chain-to-side chain 68

Figure 2.35. On-resin head-to-tail cyclisation via Lys side chain anchoring $\quad 69$

Figure 2.36. On-resin head-to-tail cyclisation via Asp side chain anchoring $\quad 69$

$\begin{array}{ll}\text { Figure 2.37. Solid phase synthesis of a monocyclic peptide } & 70\end{array}$

Figure 2.38. Synthesis of a branched cyclic peptide via a three-dimensional $\begin{array}{ll}\text { orthogonal protecting group strategy } & 71\end{array}$

Figure 2.39. Synthesis of an overlapping bi-cyclic peptide 71

Figure 2.40. Schematics for the synthesis of nisin 72

Figure 2.41. Synthesis of a lanthionine analogue of an enkephalin opioid peptide 73

Figure 2.42. Formation of ring B of nisin via a stereospecific Michael addition $\quad 74$

Figure 2.43. Synthesis of Lan-7 in solution $\quad 75$

Figure 2.44. Synthesis of a lanthionine-somatostatin analogue 76

Figure 2.45. Synthesis of a $\beta, \beta$-dimethyllanthionine analogue of an enkephalin $\begin{array}{ll}\text { opioid peptide } & 77\end{array}$

Figure 2.46. Lanthionine macrocyclisation by in situ activation of serine 78

$\begin{array}{ll}\text { Figure 2.47. Synthesis of a thioether cyclic peptide } & 79\end{array}$

Figure 3.1. Nucleophilic ring opening at $C_{2}$ or $C_{3}$ of aziridine-2-carboxylates 81

Figure 3.2. Reaction of an aziridine-2-carboxylate with ${ }^{\mathrm{n}} \mathrm{BuCu}(\mathrm{CN}) \mathrm{Li}_{2}$

Figure 3.3. Reaction of an aziridine-2-carboxylic acid with organocuprates $\quad 82$

Figure 3.4. Reaction of an aziridine-2-carboxylate with Grignard reagents in the presence of copper(I) bromide-dimethylsulfide $\quad 82$

Figure 3.5. Reaction of an aziridine-2-carboxylates with a Wittig reagent 83

Figure 3.6. Aziridine-2-carboxylate reaction with tetrabutylammonium

$\left[{ }^{11} \mathrm{C}\right]$ cyanide

Figure 3.7. Hydration of aziridine-2-carboxylates $\quad 84$ 
Figure 3.8. Reaction of aziridine-2-carboxylates with various alcohols $\quad 84$

Figure 3.9. Reaction of aziridine-2-carboxylates with methanol 85

Figure 3.10. Reaction of a $C_{3}$-substituted aziridine-2-carboxyl amide with $\mathrm{NH}_{3} \quad 85$

Figure 3.11. Reaction of an aziridine-containing peptide with diethylamine 85

Figure 3.12. Reaction of an aziridine-2-carboxylate with the $\alpha$-amino function of $\begin{array}{ll}\text { L-His } & 86\end{array}$

Figure 3.13. Reaction of an aziridine-2-carboxylate with trimethylsilyl azide $\quad 86$

Figure 3.14. Reaction of an aziridine-2-carboxylate with methylmercaptan $\quad 86$

Figure 3.15. Reaction of aziridine-2-carboxylates with various thiols 87

Figure 3.16. Formation of lanthionine and 3-methyllanthionine derivatives $\quad 88$

Figure 3.17. Reaction of an aziridine-2-carboxylate with a racemic thiol 88

Figure 3.18. Formation of lanthionine and 3-methyllanthionine derivatives $\quad 89$

Figure 3.19. Reaction of a trifluoromethyl $C_{3}$-substituted aziridine-2-carboxylate $\begin{array}{ll}\text { with various thiols } & 89\end{array}$

Figure 3.20. Reaction of an aziridine-2-carboxylate with hydrogen fluoride $\quad 90$

Figure 3.21. Reaction of aziridine-2-carboxylates with metal halides 90

Figure 3.22. Reaction of an aziridine-2-carboxylate with hydrogen chloride 91

Figure 3.23. Reduction of an aziridine-2-carboxylate 91

Figure 3.24. Aziridine-2-carboxylate system 92

Figure 3.25. Proposed mechanism for Lewis acid-mediated $C_{3}$ ring opening of aziridine-2-carboxylates $\quad 92$

Figure 3.26. Target molecule 1 for SPPS 93

Figure 3.27. General scheme for proposed synthesis of lanthionine containing peptides, using a linker yielding peptide amides $\quad 94$

Figure 3.28. Target molecule 2 for SPPS 95

Figure 3.29. General scheme for the proposed synthesis of lanthionine containing peptides with overlapping thioether linkages, using a linker yielding peptide acids 96 Figure 4.1. General scheme for the synthesis of lanthionine derivatives 98 Figure 4.2. Structure of $(R / S)$-2-iodo-3-(triphenylmethylamino)-propionic acid allyl ester, an $\alpha$-iodo- $\beta$-alanine derivative $\quad 99$

Figure 4.3. Trans-esterification of $(R, R)$-cystine with "butyl acetate 99

Figure 4.4. Fmoc protection of $(R, R)$-cystine bis- ${ }^{t}$ butyl ester 100 
Figure 4.5. Disulfide reduction of $N, N^{\prime}$-bis(fluorenylmethoxycarbonyl)- $(R, R)$ cystine-bis-butyl ester 100

Figure 4.6. $N$-Triphenylmethylation of $(S)$-serine 101

Figure 4.7. Allylation of $N$-triphenylmethyl-(S)-serine 102

Figure 4.8. Mesylation of $N$-triphenylmethyl-(S)-serine allyl ester 103

Figure 4.9. Iodination of $N$-triphenylmethyl-( $S)$-serine( $O$-mesyl) allyl ester $\quad 103$

Figure 4.10. (S)-1-Triphenylmethyl-aziridine-2-carboxylic acid allyl ester $\quad 104$

Figure 4.11. Fisher projections displaying three conformations of $N$-trityl-3-iodo-

(R)-alanine 6-nitroveratryl, ${ }^{78}$ benzyl, ${ }^{76}$ methyl ${ }^{76}$ and allyl ester 105

Figure 4.12. Formation of the triphenylmethyl-derived $(R, R)$-lanthionine $\quad 105$

Figure 4.13. Replacement of triphenylmethyl with allyloxycarbonyl on $(R, R)$-lanthionine $(\boldsymbol{R}, \boldsymbol{R})-\mathbf{1 0}$

Figure 4.14. $N$-Triphenylmethylation, allylation and mesylation of $(R)$-serine followed by conversion to $(S)$-iodoalanine derivative $(S)$-6a 108

Figure 4.15. ( $R$ )-1-Triphenylmethyl-aziridine-2-carboxylic acid allyl ester $\quad 108$

Figure 4.16. Formation of the lanthionine derivative $(\boldsymbol{S}, \boldsymbol{R})-\mathbf{1 0} \quad 109$

Figure 4.17. Replacement of triphenylmethyl with allyloxycarbonyl on $\begin{array}{ll}\text { lanthionine }(S, R)-10 & 110\end{array}$

Figure 4.18. Removal of butyl from lanthionine $(S, R)-12 \quad 110$

Figure 4.19. ivDde/Dmab derivative of $(S, R)$-lanthionine

Figure 4.20. Unsuccessful attempts at esterification of $N$-triphenylmethyl- $(R)$-serine (R)-3 with Dmab-OH

Figure 4.21. Scheme for carboxyl protection of $N$-triphenylmethyl- $(R)$-serine $(\boldsymbol{R})-3$ with Dmab-OH via TBDMS $\beta$-OH protection 112

Figure 4.22. Bromination of Dmab-OH 113

Figure 4.23. Replacement of triphenylmethyl protecting group with ivDde on compound $(S, R)-10$

Figure 4.24. Replacement of allyl with Dmab protecting group on ivDde-derived $(S, R)$-lanthionine 16

Figure 4.25. Removal of butyl from ivDde-derived $(S, R)$-lanthionine $17 \quad 115$

Figure 5.1. Iodoalanine and lanthionine targets 117

$\begin{array}{ll}\text { Figure 5.2. Structures of }(R, R)-10 \text { and }(S, R)-10 & 118\end{array}$ 
Figure 5.3. Mechanism for the formation of aziridine $(S)-11$ from $N$ -

triphenylmethyl- $\alpha$-iodo- $(R)$-alanine allyl ester $(\boldsymbol{R})-\mathbf{6 a}$

Figure 5.4. Regioisomeric iodination product, $(R / S)$-2-iodo-3-

(triphenylmethylamino)-propionic acid allyl ester, an $\alpha$-substituted $\beta$-alanine derivative

Figure 5.5. Indication of $\alpha_{1}-\mathrm{C}, \alpha_{2}-\mathrm{C}, \beta_{1}-\mathrm{C}$ and $\beta_{2}-\mathrm{C}$ for $(\boldsymbol{R}, \boldsymbol{R})-10$ and $(\boldsymbol{S}, \boldsymbol{R})-10$

Figure 5.6. Expected $\mathrm{HMBC}^{3} J$ correlations for $(\boldsymbol{S}, \boldsymbol{R})-\mathbf{1 0}$ across the thioether linkage

Figure 5.7. Structure of nor-lanthionine 18, as proposed by NMR

Figure 5.8. Scheme indicating how $(S)-6$ gave $(S, R)-10$ and hence $(S, R)-12$; and $6 \mathrm{~b}$ gave 18 and hence 19. Similarly, $(R)-6$ gave $(R, R)-10$ and hence $(R, R)-12$; and $6 \mathbf{b}$ gave 18 and hence 19. The loss of stereochemical integrity is discussed in Chapter 6

Figure 5.9. Structure of nor-lanthionine 20, a regioisomer of 1

Figure 5.10. Structure of nor-lanthionine 21, a regioisomer of 16

Figure 5.11. Lanthionine derivatives 17 and 2 and their regioisomers 22 and 23, respectively

Figure 5.12. Proposed mechanism for the formation of regioisomers of $N$-trityl- $\alpha$ iodo-(S)-alanine allyl ester $(S)$-6a via the aziridine-2-carboxylate $(\boldsymbol{R})-\mathbf{1 1}$

Figure 5.13. Structures of all nor-lanthionine derivatives synthesised

Figure 5.14. Partial conversion of $N$-triphenylmethyl-( $R)$-serine( $O$-mesyl) allyl ester $(\boldsymbol{R})-5$ into aziridine $(\boldsymbol{R})-\mathbf{1 1}$

Figure 5.15. Ring opening of aziridine $(R)-11$ by sodium iodide

Figure 5.16. Equilibrium between the mesylate serine $(\boldsymbol{R})-\mathbf{5}$, aziridine derivative

$(R)-11$, the correct iodoalanine $(S)-6 \mathbf{a}$ and its regioisomers $6 \mathbf{b}$

Figure 5.17. Unsuccessful ring-opening reaction of aziridine derivative $(\boldsymbol{R})-11$

with cysteine derivative 9

Figure 5.18. Formation of lanthionine via Mitsunobu reaction conditions

Figure 6.1. nor-Lanthionine derivatives 20 and 23 for SPPS

Figure 6.2. Lanthionine opioid enkephalin peptide analogue (a) and the analogue synthesised 24

Figure 6.3. Synthesis of a sulfur-bridged opioid enkephalin peptide analogue 24135 
Figure 6.4. Sieber amide resin

Figure 6.5. NovaSyn ${ }^{\circledR}$ TG Sieber resin 138

Figure 6.6. Structure of the doubly oxidised peptide 24a 140

Figure 6.7. (a) Ring $C$ of nisin and an analogue of ring $C$ of nisin 25

Figure 6.8. NovaSyn $^{\circledR}$ TGT alcohol resin pre-loaded with Asn(Trt) 144

Figure 6.9. Synthesis of a lanthionine analogue of ring C of nisin 25

Figure 6.10. Lanthionine derivative $(\boldsymbol{S}, \boldsymbol{R})-\mathbf{1 0} \quad 146$

Figure 6.11. Rings A and B of nisin 146

Figure 6.12. nor-Lanthionine analogue of rings A and B of nisin 26

Figure 6.13. Synthesis of an analogue of rings A and B of nisin 26

Figure 6.14. Structure of the nor-Lan analogue of ring $C$ of nisin 25 across the thioether linkage as proposed by NMR

Figure 6.15. Structures of $25 \mathrm{a}$ and $\mathbf{2 5 b}$, indicating the stereochemistry at the $\beta$ amino acid moiety

Figure 6.16. Formation of lanthionine via Mitsunobu reaction conditions $\quad 152$

Figure 6.17. Application of Mitsunobu conditions to the synthesis of MeLan 152

Figure 7.1. Major product: ( $R / S$ )-2-iodo-3-(triphenylmethylamino)-propionic acid allyl ester

Figure 7.2. Major product: (R/S)-2-iodo-3-(triphenylmethylamino)-propionic acid allyl ester

Figure 7.3. Major product: $(R / S)-2-[(R)-2$-'butoxycarbonyl-2-(fluoren-9-ylmethoxycarbonylamino)-ethylsulfanyl]-3-(triphenylmethylamino)-propionic acid allyl ester

Figure 7.4. Major product: $(R / S)$-2-[(R)-2-'butoxycarbonyl-2-(fluoren-9-ylmethoxycarbonylamino)-ethylsulfanyl]-3-(triphenylmethylamino)-propionic acid allyl ester

Figure 7.5. Major product: $(R / S)$-2[( $R)-2$-'butoxycarbonyl-2-(fluoren-9-ylmethoxycarbonylamino)-ethylsulfanyl]-3-(allyloxycarbonylamino)-propionic acid allyl ester

Figure 7.6. Major product: $(R / S)$-2-[(R)-2-'butoxycarbonyl-2-(fluoren-9-ylmethoxycarbonylamino)-ethylsulfanyl]-3-(allyloxycarbonylamino)-propionic acid allyl ester 
Figure 7.7. Intended product: 3-[(S)-2-allyloxycarbonyl-2-(allyloxycarbonylamino)ethylsulfanyl]-(R)-2- (fluoren-9-ylmethoxycarbonylamino)-propionic acid

Figure 7.8. Intended product: 3-[(R)-2-'butoxycarbonyl-2-(fluoren-9-ylmethoxycarbonylamino)-ethylsulfanyl]-(S)-2-\{1-(4,4-dimethyl-2,6-dioxocyclohex-1-ylidene) isovalerylamino -propionic acid allyl ester

Figure 7.9. Intended product: 3-[(R)-2-'butoxycarbonyl-2-(fluoren-9-ylmethoxycarbonylamino)-ethylsulfanyl]-(S)-2-\{1-(4,4-dimethyl-2,6-dioxocyclohex-1-ylidene) isovalerylamino\}-propionic acid

Figure 7.10. Intended product: 3-[(R)-2-'butoxycarbonyl-2-(fluoren-9-ylmethoxycarbonylamino)-ethylsulfanyl]-(S)-2-\{1-(4,4-dimethyl-2,6-dioxocyclohex-1-ylidene) isovalerylamino $\}$-propionic acid 4-\{N-[1-(4,4-dimethyl-2,6-dioxocyclohex-1ylidene)-3-methylbutyl]amino benzyl ester

Figure 7.11. Intended product: $3-[(S)-2-\{4-[N-(1-(4,4-d i m e t h y l-2,6-d i o x o c y c l o h e x-1-$ ylidene)-3-methylbutyl)amino]benzyloxycarbonyl $\}-2-\{1-(4,4-$ dimethyl-2,6-dioxocyclohex-1-ylidene)isovalerylamino -ethylsulfanyl]-(R)-2-(fluoren-9-ylmethoxy carbonylamino)-propionic acid

Figure 7.12. Major product: $(R / S)$-2-iodo-3-(triphenylmethylamino) propionic acid allyl ester

Table 1. Type A lantibiotics, structures and applications

Table 2. Type B lantibiotics, structures and applications 30

Table 3. Product of Cys-Ala ((R)-Ala) couplings with cesium carbonate in DMF 39

Table 4. Optical rotation values for $(R)-6$ and $(S)-6$ 108

Table 5. Optical rotation values for $(R, R)-10$ and $(S, R)-10$ 110

Table 6. ${ }^{1} \mathrm{H}$ and ${ }^{13} \mathrm{C}$ chemical shifts for peptide 25a 200

Table 7. ${ }^{1} \mathrm{H}$ and ${ }^{13} \mathrm{C}$ chemical shifts for peptide $25 \mathbf{b}$ 201 


\section{Chapter 1}

\section{Introduction I}

\subsection{Lanthionine}

Lanthionine (Lan) is an unusual amino acid which was first isolated from wool which had been treated with sodium carbonate in $1941^{1}$ and has since been found in human hair, chicken feathers and lactalbumin. ${ }^{2,3}$ It is a double-headed or bifunctional amino acid (Figure 1.1) which is analogous to cystine, having two alanine residues linked by a thioether bridge instead of a disulfide bridge. It occurs in nature as the $(S, R)$-diastereoisomeric form (meso-lanthionine, meso-Lan $)^{4}$ whereas cystine exists naturally as a $(R, R)$-diastereoisomer. 
<smiles>N[C@@H](CSC[C@H](N)C(=O)O)C(=O)O</smiles>

(a)

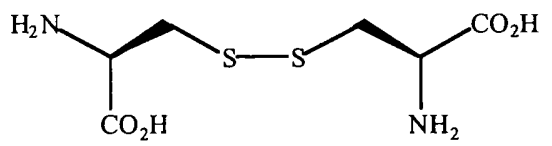

(b)

Figure 1.1. (a) meso-Lanthionine and (b) cystine

The presence of lanthionine in peptides plays a very important role. When two alanine residues on a peptide are connected via a thioether bridge (i.e. lanthionine), the degree of conformational freedom of the now cyclic peptide is considerably reduced. The peptide becomes more rigid ${ }^{5}$ and behaves differently compared to its linear parent. The presence of more cyclic units (i.e. more lanthionine) would reduce the degrees of conformational freedom even more. Polycyclic lanthionine-containing peptides have been shown to have high specificity against Gram-positive bacteria. Studies have shown that peptides containing thioether bridges have greater stability towards enzymatic degradation compared to their disulfide analogues. ${ }^{5}{ }^{6}$ The synthesis of these naturally-occurring lanthionine-containing peptides would therefore lead to further studies of conformationally restrained peptides, their applications in the food and pharmaceutical industry and the complete understanding on their extensive biological activities, as well as the development of analogues to improve solubility and bioactivity.

\subsection{Lantibiotics}

Polycyclic lanthionine-containing peptides are known as lantibiotics. They have been shown to exhibit antibiotic properties. To date over thirty lantibiotics have been identified and characterised including nisin, ${ }^{7}$ subtilin, ${ }^{8}$ epidermin, $,{ }^{9}, 10,11$ gallidermin, ${ }^{12}$ ancovenin, ${ }^{13}$ duramycin, ${ }^{14}$ duramycin $\mathrm{B},{ }^{15}$ duramycin $\mathrm{C},{ }^{15}$ cinnamycin, ${ }^{16}$ Pep $5,{ }^{17}$ actagardine,${ }^{18}$ mersacidin, ${ }^{19}$ streptococcin A-FF22, ${ }^{20}$ epilancin $\mathrm{K} 7,{ }^{21}$ lactocin $\mathrm{S},{ }^{22}$ salivaricin $\mathrm{A},{ }^{23}$ variacin, ${ }^{24}$ lacticin $481,{ }^{25}$ bacteriocin ISK-1, ${ }^{26}$ plantaricin $\mathrm{C},{ }^{27}$ staphylococcin $\mathrm{T},{ }^{28}$ along with other lantibiotics whose structures have not been completely assigned such as mutacin $\mathrm{II},{ }^{29}$ sublancin $168,{ }^{30}$ carnocin UI $49^{31}$ and nukacin ISK-1. ${ }^{32}$ 
These lantibiotics consist of $19-34$ amino acid residues with up to five thioether bridges. Lanthionine is present in all lantibiotics, and is only one of four such thioether-containing residues. Other unusual amino acids also contribute to the polycyclic structure of these lantibiotics such as 3-methyllanthionine (threo-MeLan), $S$-aminovinyl-D-cysteine, $S$-aminovinyl-D-methylcysteine and lysinoalanine (Figure 1.2).<smiles>C[C@@H](SC[C@H](N)C(=O)O)[C@H](N)C(=O)O</smiles>

(a)<smiles>C[C@H](S/C=C\N)[C@H](N)C(=O)O</smiles>

(b)

(c)<smiles>N/C=C\SC[C@H](N)C(=O)O</smiles>

(d)<smiles>N[C@@H](CCCCNC[C@H](N)C(=O)O)C(=O)O</smiles>

(e)

Figure 1.2. Unusual amino acids in lantibiotics; (a) $(2 S, 6 R)$-Lanthionine (mesoLan), (b) $(2 S, 3 S, 6 R)-3-M e t h y l l a n t h i o n i n e ~(t h r e o-M e L a n)$, (c) $(2 S, 3 S)-[(Z)-2-$ aminovinyl]-D-methylcysteine (MeCys(Avi)), (d) $S$-[(Z)-2-aminovinyl]-D-cysteine (Cys(Avi)), (e) $(2 S, 9 S)$-Lysinoalanine (Lys- $N^{\prime}$-Ala)

Lantibiotics also contain other unusual amino acids, which include 2,3dehydroalanine (Dha), (Z)-2,3-dehydrobutyrine (Dhb) and 3-hydroxyaspartic acid (Figure 1.3).<smiles>C=C(N)C(=O)O</smiles>

(a)<smiles>C/C=C(\N)C(=O)O</smiles>

(b)<smiles>N[C@@H](C(=O)O)C(O)C(=O)O</smiles>

(c)

Figure 1.3. Unusual amino acids in lantibiotics; (a) 2,3-dehydroalanine (Dha), (b) (Z)-2,3-dehydrobutyrine (Dhb), (c) erythro-3-hydroxy-L-aspartic acid (HyAsp) 
The occurrence of meso-Lan, threo-MeLan, Cys(Avi), MeCys(Avi), Lys-N'-Ala, Dha and Dhb can be attributed to the biosynthetic mechanism of the formation of lantibiotics (Figure 1.4). ${ }^{1}$

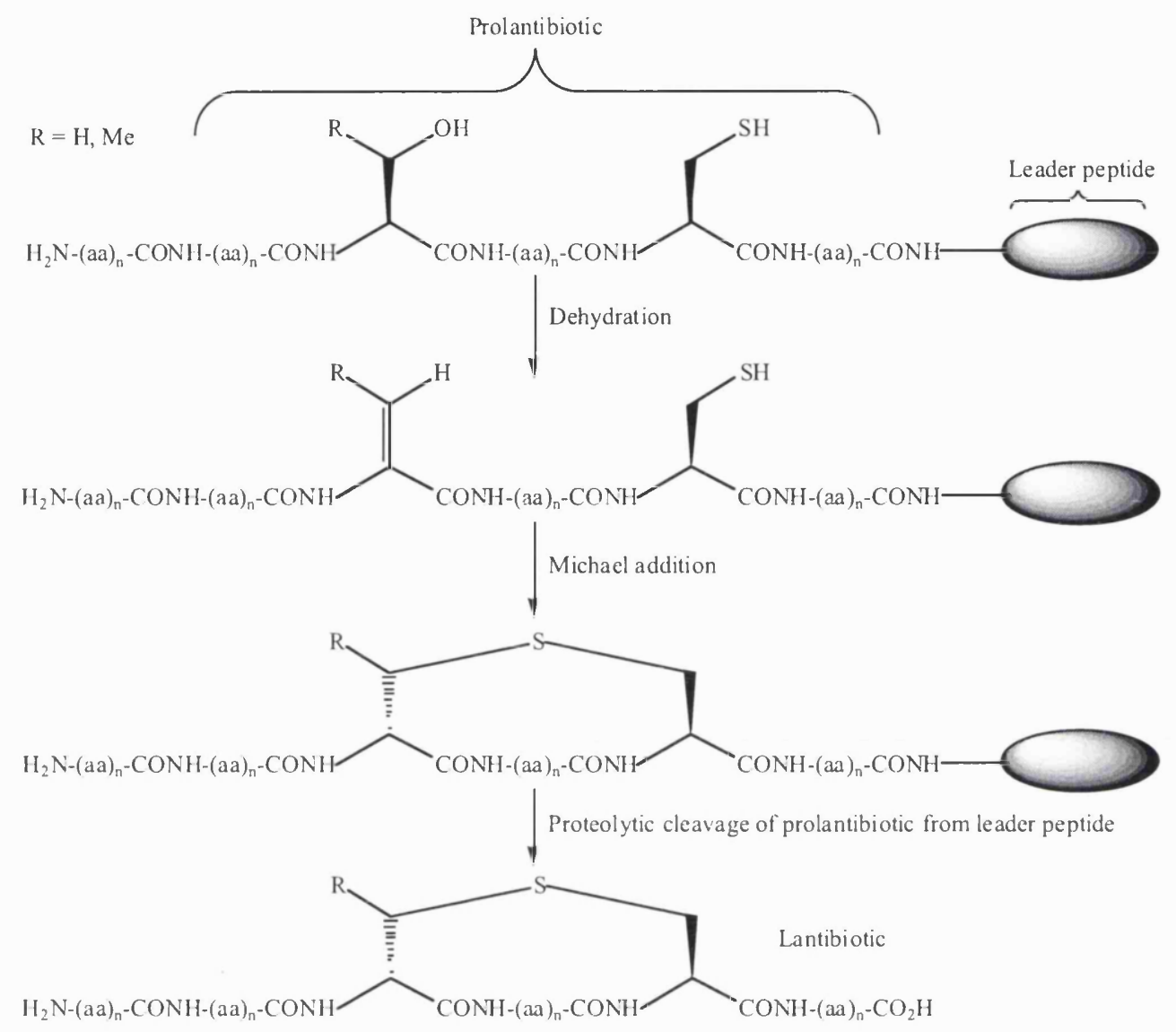

Figure 1.4. Biosynthesis of lantibiotics

A precursor peptide is initially formed in the ribosome through processes of transcription and translation. These "prelantibiotics" are each made up of a leader peptide and a "prolantibiotic". These "prelantibiotics" are then enzymatically modified resulting in dehydration of $\beta$-hydroxy amino acids such as serine and threonine to give Dha and Dhb respectively, allowing regio- and stereospecific Michael addition of cysteine residues forming Lan and MeLan, and lysine residues forming Lys- $N$ '-Ala. ${ }^{11,14,17}$ The number of cysteine residues does not necessarily match the number of dehydroamino acids hence the presence of the latter in lantibiotics. The occurrence of Cys(Avi) and MeCys(Avi) is a result of $C$-terminal cysteine residues in lantibiotics such as epidermin and mersacidin which undergo oxidation and decarboxylation by modifying enzymes before addition to 
dehydroamino acids forming the corresponding thioether linkages. ${ }^{11,14}$ Proteolytic cleavage of the modified "prolantibiotics" from the leader peptides and liberation from the cell yield the antimicrobial lantibiotics. These post-translational side-chain modifications of lantibiotics are unique and not known to arise in any other peptide antibiotics.

Structural elucidation of these lantibiotics proved to be difficult due to the presence of the dehydroamino acids. By Edman degradation, proteinogenic amino acids on a peptide are converted to their phenylthiohydantoin derivatives and analysed by HPLC, as part of an automated Edman sequencer, with comparisons of retention times with standard values. Lan, MeLan, Dha, Dhb and other unusual amino acids in lantibiotics give blanks in the HPLC trace. $N$-terminal Dha or Dhb on the peptides are extremely unstable and "de-aminate" after spontaneous addition of water during the sequencing reaction hence resulting in a halt in the process. ${ }^{33,}{ }^{34}$ Meyer et al. converted these $\alpha, \beta$-unsaturated amino acids into their cysteinyl- $S$-alkyl derivatives by reaction with ethanethiol. ${ }^{33}$ These derivatives do not block the sequencing. Interestingly, Lan and MeLan thioether bridges are cleaved under the reaction condition and form stable, sequenceable cysteine derivatives. Jung et al. have also incorporated the use of electrospray mass spectrometry in series with the automated Edman sequencer to improve amino acid identification in peptides. ${ }^{34}$

Lantibiotics are generally divided into two subtypes; Type A and Type B. ${ }^{14}$ Type A lantibiotics are in excess of $2000 \mathrm{Da}$ in molecular weight, adopt corkscrew-like structures and are positively charged. There have been studies on the structureactivity relationship of nisin, a Type A lantibiotic, where its flexible and elongated structure is utilised for creating transient pores in cytoplasmic membranes of Grampositive bacteria, allowing smaller molecules from the cytoplasm to exit resulting in cell death. ${ }^{35}$ It has been shown that an interaction with lipid II is required for nisin to create these pores, however Pep5 - also a type A lantibiotic - has no affinity for it. ${ }^{36}$ It is not yet known which membrane molecule Pep5 targets for the purpose of pore formation. 
Type B lantibiotics adapt more rigid and globular structures due to their head-to-tail cross-linkages and are either negatively charged or neutral. ${ }^{14}$ Despite the lack of head-to-tail cross-linkages mersacidin and actagardine (Table 2) are regarded as Type B lantibiotics ${ }^{32}$ due to the overall similarity of their precursor peptides although this view was not shared by Jung ${ }^{14}$ who initially suggested the classification of lantibiotics.

Little is known about these lantibiotics except for the fact that some of them are enzyme inhibitors. ${ }^{14}$ Both mersacidin and actagardine potently inhibit the biosynthesis of bacterial cell walls by inhibiting transglycosylation although the biosynthesis of RNA, DNA and proteins are not affected (Figure 1.5). ${ }^{32}$

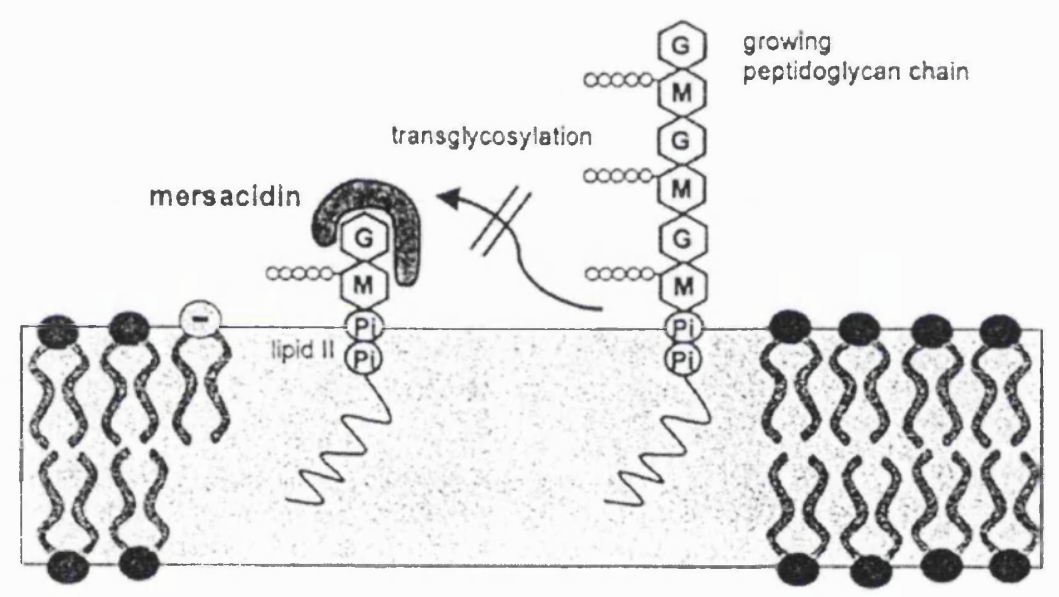

Figure 1.5. Mode of action of mersacidin. Cell wall precursor lipid II is transferred to the outer membrane during peptidoglycan synthesis. This accessibility to mersacidin allows complexation with the disaccharide moiety hence inhibiting its incorporation into the growing peptidoglycan chain. Diagram taken from ref. ${ }^{32}$

Tables 1 and 2 list some of the better known Type A and B lantibiotics, respectively. 


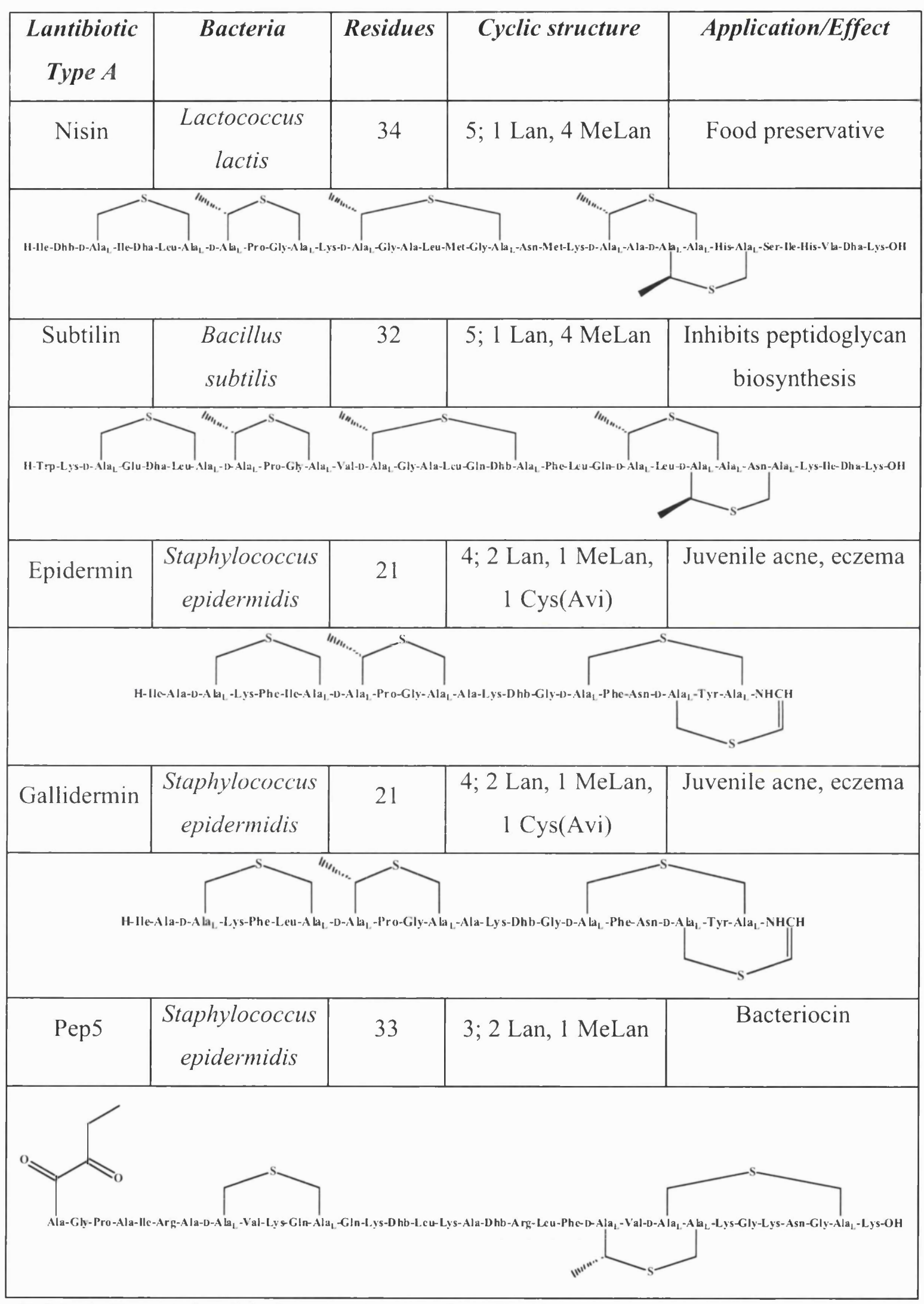

Table 1. Type A lantibiotics, structures and applications 


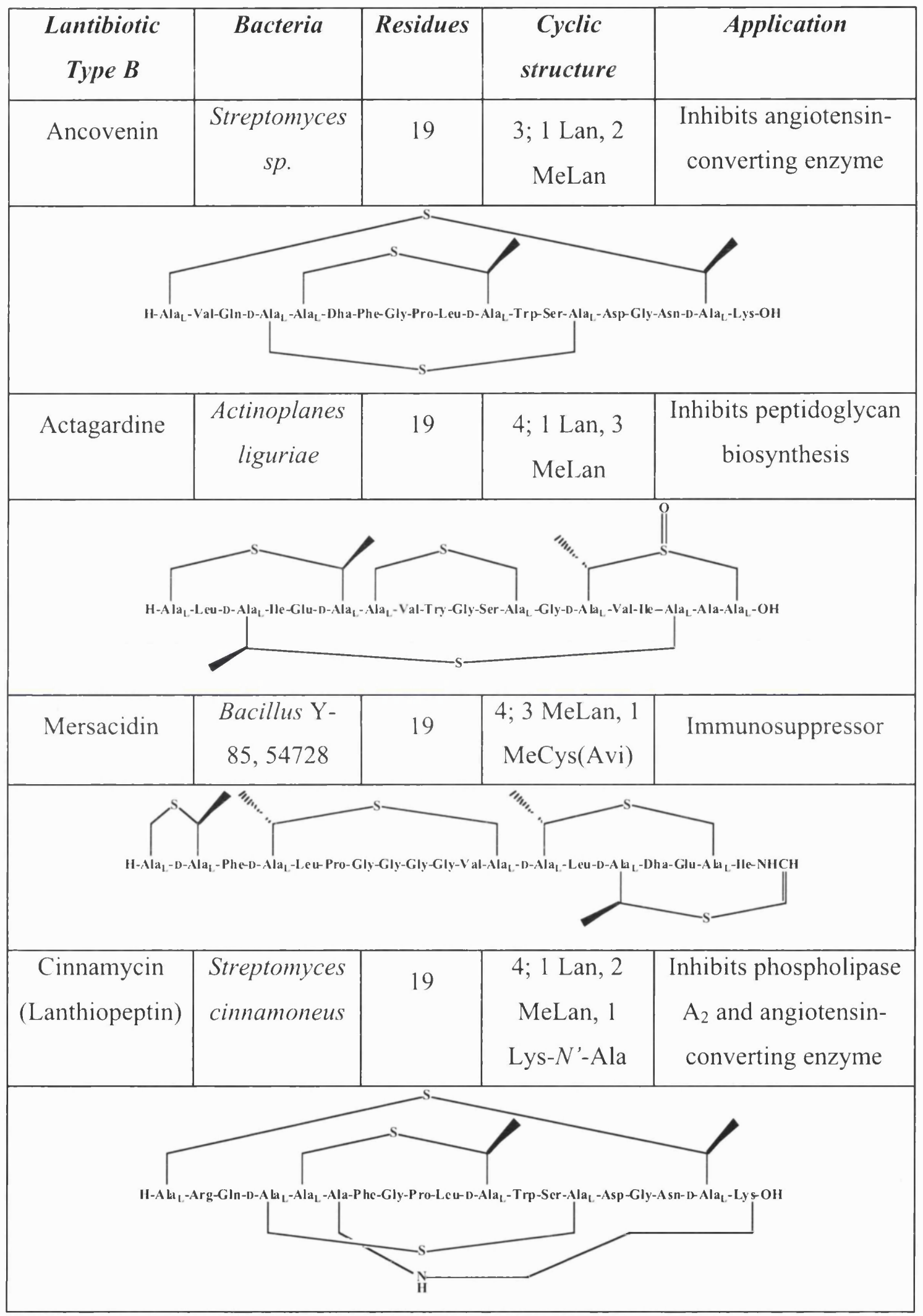

Table 2. Type B lantibiotics, structures and applications 
The classification of lantibiotics into subtypes $\mathrm{A}$ and $\mathrm{B}$ is limited to the classic definition of these peptide antibiotics. Plantaricin $W,{ }^{37}$ lacticin $3147,{ }^{38}$ cytolysin ${ }^{39}$ and staphylococcin $\mathrm{C} 55^{40}$ are all unique two-component lantibiotics which do not possess antimicrobial properties when the two lanthionine-containing peptidic components are separated. Figure 1.6 illustrates the structure for Plantaricin W as an example.

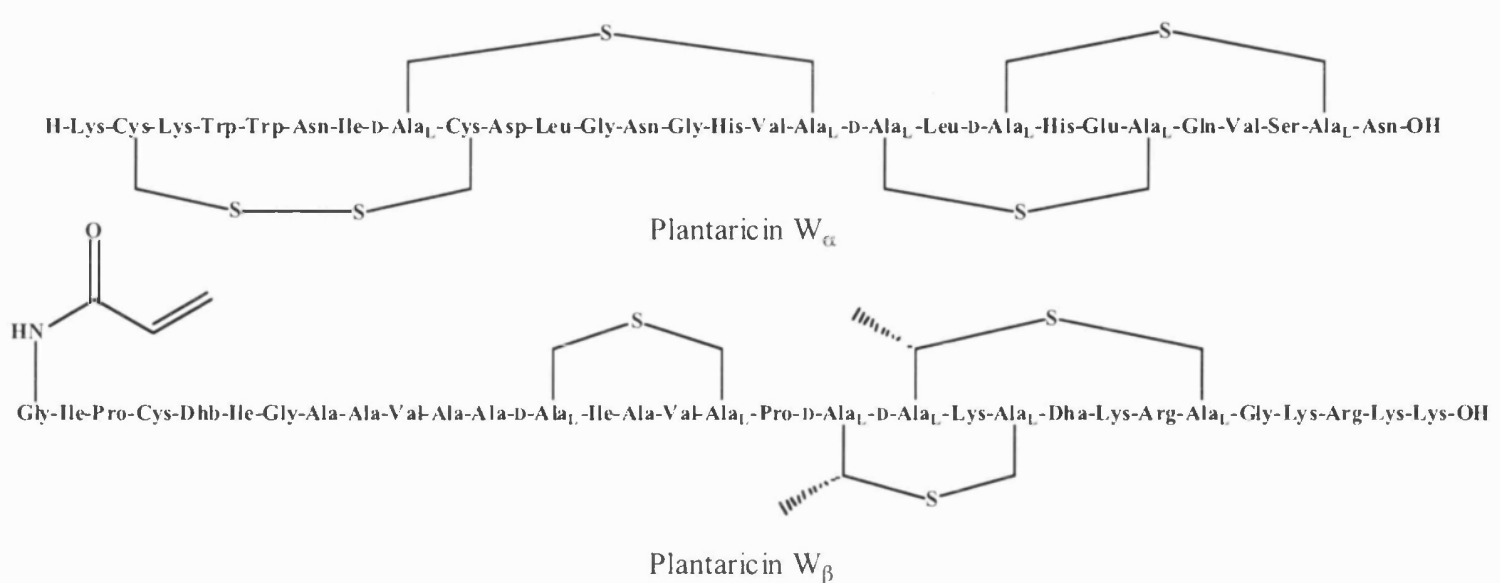

Figure 1.6. Structures of the two-component lantibiotic, Plantaricin W

\subsection{Nisin}

Nisin is perhaps the most well known lantibiotic. It was the first to be discovered in 1928 from a culture broth of Lactococcus lactis. ${ }^{41,42}$ However it was not isolated until 1947 from a culture broth of Streptococcus lactis $^{43}$ and it was only in 1971 that Gross and Morrell proposed the complex structure of this lantibiotic. ${ }^{7}$ It contains 5 cyclic structures made up of one Lan and four MeLan residues together with two Dha and one Dhb residues (Table 1). Since 1951 nisin has been an important preservative $^{44,45}$ in the food industry due to its remarkable anti-growth effect on Gram-positive microorganisms. ${ }^{46}$ It has also been found that it exhibits antimalarial properties, ${ }^{47}$ however, due to its poor solubility in water and low stability at physiological pH nisin has not been developed into a chemotherapeutic drug. ${ }^{48}$ Already wild-type nisin has been modified through site-directed mutagenesis to give nisin $Z$, substituting the histidine at position 27 or 31 with lysine, which showed increased solubility in water without affecting its antimicrobial activity. ${ }^{49}$ 
Classified as a Type A lantibiotic, nisin creates pores in bacterial cell walls resulting in cell death. Its $N$-terminal ionic interaction with lipid II is a vital docking mechanism for targeted pore formation and simultaneously blocks peptidoglycan synthesis, much like mersacidin and actagardine (Figure 1.7). ${ }^{32}$

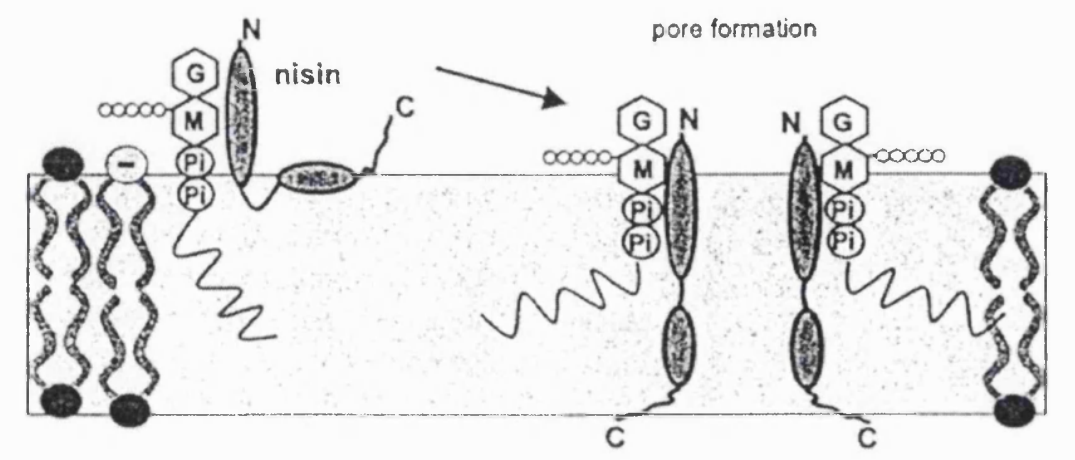

Figure 1.7. Mode of action of nisin. Nisin binds to the cell wall precursor, using it as a docking molecule for pore formation and inhibits peptidoglycan synthesis. Diagram taken from ref. ${ }^{32}$

\subsection{Synthetic lanthionine-containing peptides}

Some naturally occurring opioids show marked receptor selectivity ${ }^{50}$ but their linearity and high flexibility hinders any conformational analyses. Their cyclic analogues, useful in structure-activity studies due to their constrained structures, are studied in the hope of elucidating the critical portions of the molecule for opiate receptor binding. There have been several reports on the incorporation of lanthionine, as analogues, in these natural peptides.

Enkephalins are important in a variety of physiological functions such as the modulation of pain and regulation of the immune system. ${ }^{51}$ Since their discovery, several classes of opioids have been identified and a lanthionine analogue (Figure 1.8) has been synthesised via several different methods. ${ }^{50,52,53,54}$ Although lanthionine analogues of these peptides are more constrained than disulfide analogues, it was shown that this did not result in increased activity. ${ }^{50}$ 


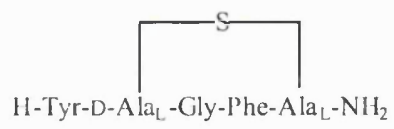

Figure 1.8. A lanthionine enkephalin opioid analogue

Somatostatin is a 14-residue peptide, which plays important physiological roles such as being an inhibitor of the release of several growth hormones. ${ }^{55}$ Several highly bioactive somatostatin analogues have been synthesised. Sandostatin is a cyclic peptide somatostatin analogue with eight residues containing a disulfide bridge. ${ }^{56}$ It was suggested that the disulfide bridge might not be sufficient to constrain the cyclic backbone in the proposed bioactive conformation (Figure 1.9). ${ }^{57}$ Therefore a lanthionine analogue was developed; this showed similar activity to sandostatin but with increased stability against enzymatic degradation. ${ }^{6,58}$

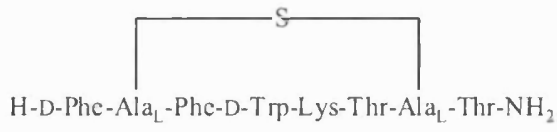

(a)

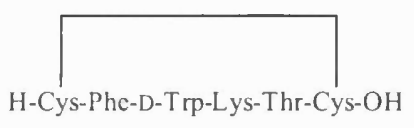

(b)

Figure 1.9. (a) Lanthionine-somatostatin analogue; (b) proposed bioactive conformation

Lan-7 is a monocyclic lanthionine-containing peptide, which has been shown to have a remarkable potential as a cancer chemotherapeutic agent. It is a monosulfide analogue of the less stable TT-232, a sandostatin analogue, which has been shown to exhibit strong anti-tumour effects both in vitro and in vivo by inhibiting growth on a number of human tumour cell lines. ${ }^{59}$ Its potency has been shown to be two to three times that of the already-clinically-used octreotide. ${ }^{59,60}$ The reduction of the ring size, from TT-232 to Lan-7, did not affect its anti-tumour activity but Lan-7 showed no toxicity to normal human cells.

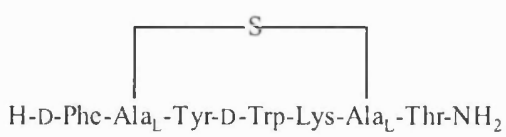

(a)

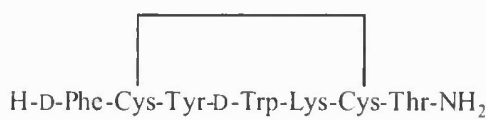

(b)

Figure 1.10. Structures of (a) Lan-7; (b) TT-232 
There have also been reports on the use of lanthionine in peptides as analogues to a cell adhesion modulator. ${ }^{61,62}$ Cell adhesion is important in biological processes such as wound healing and inflammation. ${ }^{63}$ Proteins that mediate the adhesion process are called integrins, and finding integrin antagonists and agonists is of great interest as either could have potential therapeutic value. ${ }^{64}$ The disulfide peptide in Figure 1.11 acts as an antagonist to a specific integrin involved in inflammation. The lanthionine analogue was shown to exhibit the same selectivity as the disulfide analogue.

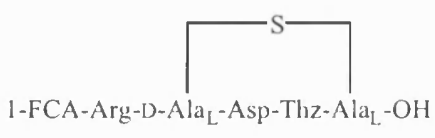

(a)

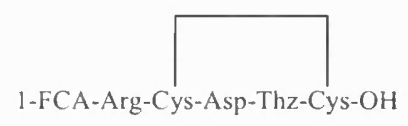

(b)

Figure 1.11. (a) Monosulfide and (b) disulfide analogues of an integrin antagonist

In addition, there has been a report on the synthesis of a lanthionine-type cyclic peptide (Figure 1.12). ${ }^{65}$

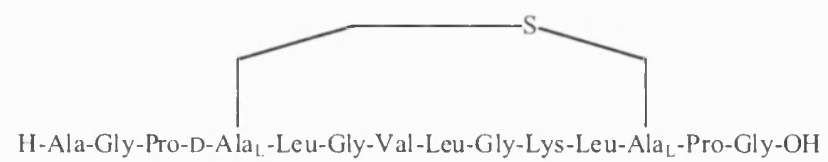

Figure 1.12. A thioether cyclic peptide

\subsection{Synthesis of lanthionine residues}

A number of approaches to the synthesis of lanthionine, both as a monomer and as part of a peptide, have been reported. These are discussed in this section.

\subsubsection{Sulfur extrusion}

The first method reported for the synthesis of lanthionine was via extrusion of one sulfur atom from a protected cystine residue (Figure 1.13). ${ }^{66}$

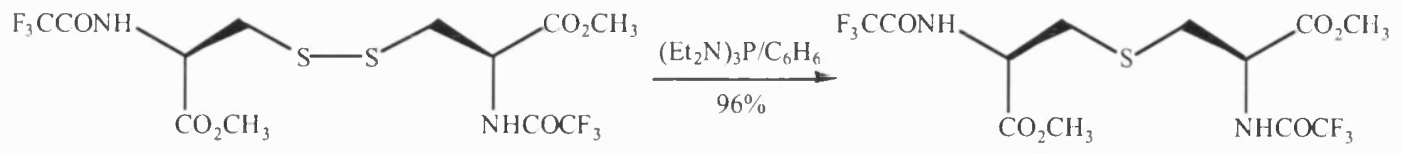

Figure 1.13. Formation of a $(R, R)$-lanthionine derivative via sulfur extrusion method 
Initial studies on small sulfur-containing molecules indicated that tris(diethylamino)phosphine was most effective for selective extrusion of one sulfur atom from a disulfide. ${ }^{67,}{ }^{68}$ Any carboxyl function present required protection, otherwise a competing amide formation occurred instead of the intended sulfur extrusion reaction. ${ }^{67}$

The mechanism of the sulfur extrusion involves a fragmentation of the disulfide, followed by an $\mathrm{S}_{\mathrm{N}} 2$ step. For $C_{\beta}$-substituted cystine, therefore, this results in stereochemical inversion at the $C_{\beta}$-position (Figure 1.14). ${ }^{68}$

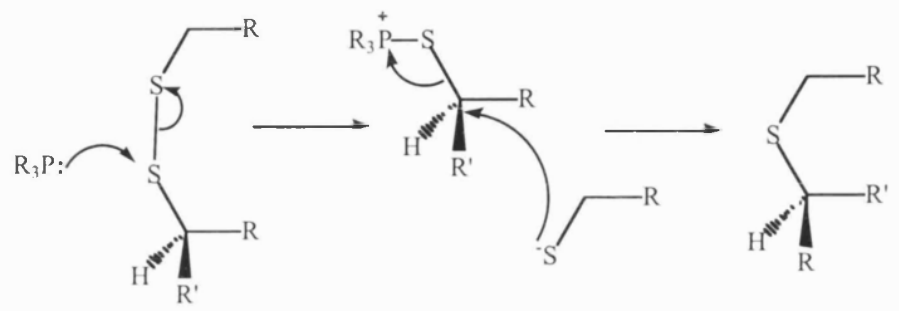

Figure 1.14. Mechanism of sulfur extrusion from a disulfide; stereochemical inversion of a $\beta$-substituted disulfide

Problems, however, occur in asymmetrical or acyclic disulfides where crosscoupling could take place (Figure 1.15) ${ }^{66}$ As a result, meso-lanthionine, which has one $R$ and one $S$ chiral centre, cannot be synthesised via this method. Similarly, it is impossible to produce differentially protected lanthionine derivatives. This problem can be solved by tethering the $R$ and $S$ cysteine precursors together (Figure 1.16) and forming the cyclic disulfide. ${ }^{69}$ Sulfur extrusion can then be successfully carried out, to give the desired lanthionine, at high dilution to minimise the formation of intermolecular thioethers. 


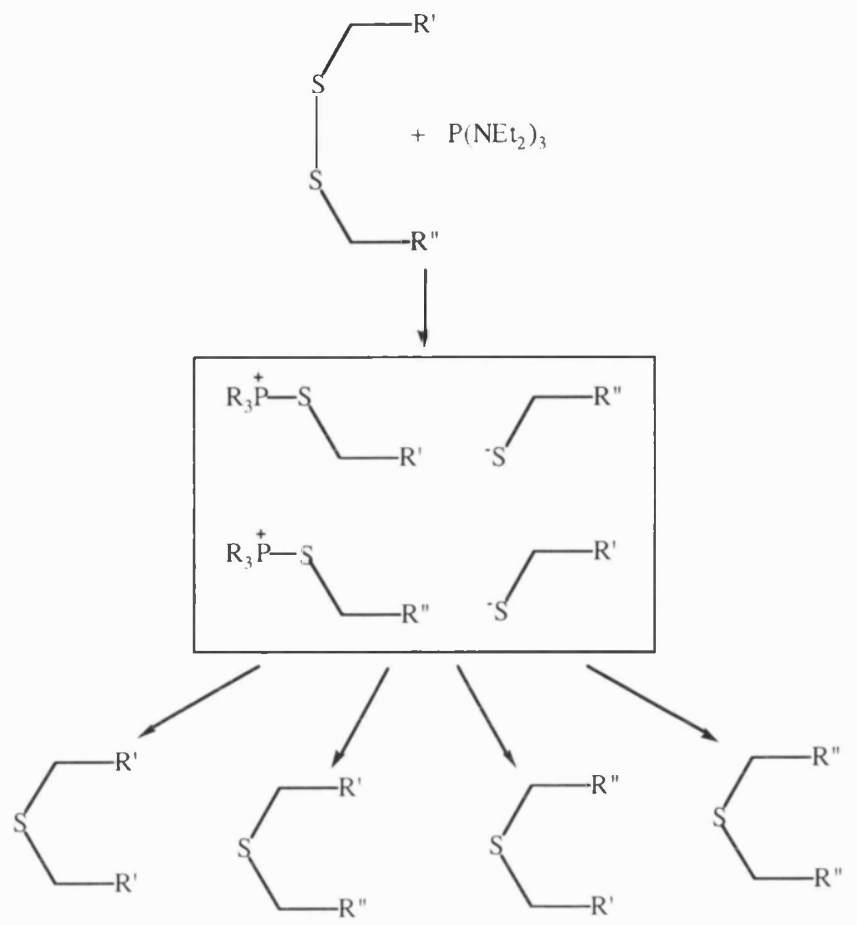

Figure 1.15. Cross-coupling in sulfur extrusion reaction<smiles>O=C(OCCO)[C@H](C[C@@H](C[SnH2])C(=O)OCCO)NCCO</smiles>

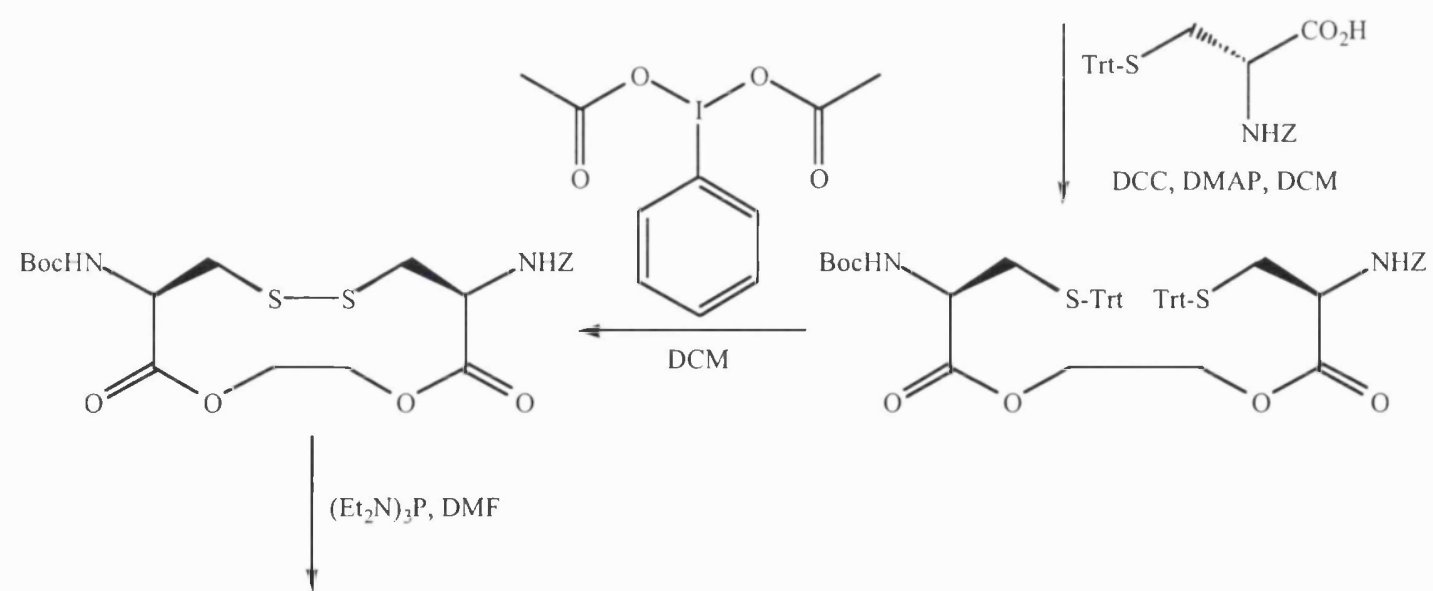<smiles>CC(=O)N[C@@H](CSC[C@@H](N)C(=O)OCCOC(=O)[C@H](N)CSC[C@H](N)C(=O)O)C(=O)O</smiles>

Figure 1.16. meso-Lanthionine formation via a cyclic cystine derivative 
However, in an acyclic structure much like the synthesis of a derivative of a lanthionine monomer - instead of being part of a peptide - this procedure would still be faced with the cross-coupling problem described above. Additionally, the use of tris(diethylamino)phosphine might not be compatible with the proceedings of solid phase peptide synthesis. Besides, the method described in Figure 1.16 is such that the two carboxyl functions of the meso-lanthionine are unprotected. This removes the element of a discriminative peptide coupling as well as the possibility of the use of a triply orthogonal protecting group strategy in solid phase peptide synthesis.

As a result of these limitations other synthetic strategies have been sought. Retrosynthetic analysis of lanthionine (Figure 1.17) produces synthons, which suggest three possible approaches; cysteine and dehydroalanine, cysteine and serine $\beta$-lactone, and cysteine and $\beta$-halo-alanine. All the abovementioned approaches have been reported and are discussed below.

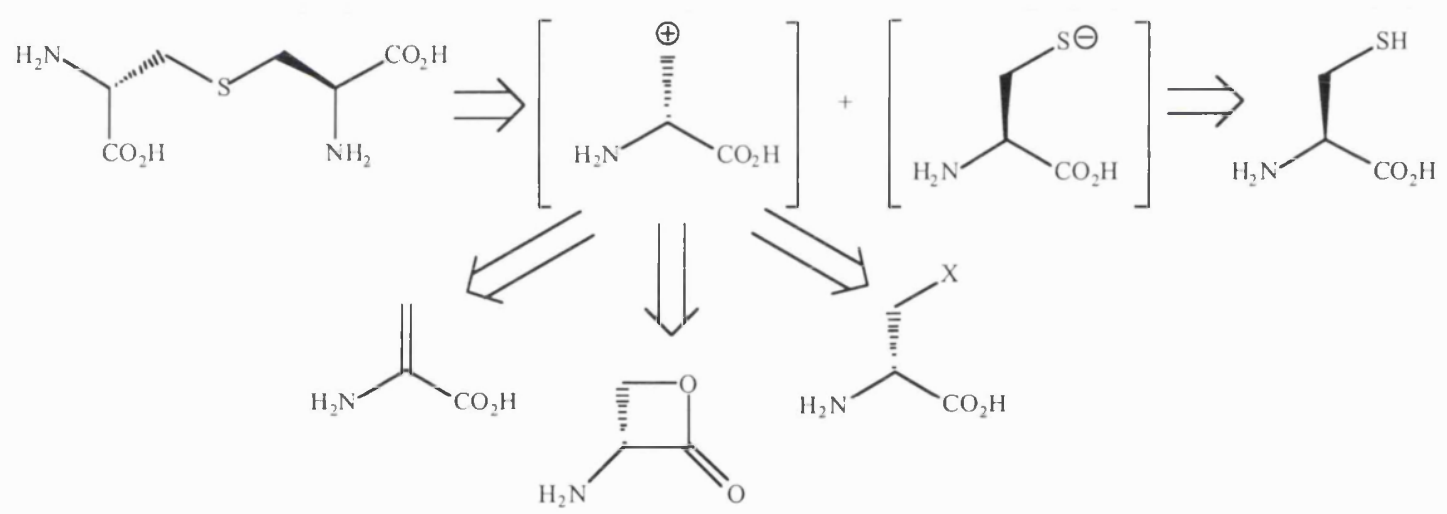

Figure 1.17. Retrosynthetic analysis of meso-lanthionine

\subsubsection{Biomimetic approach}

As described above, serine and threonine residues on prelantibiotic peptides undergo dehydration to give dehydroalanine and dehydrobutyrine respectively. Subsequent Michael addition by cysteine - SH results in cyclic thioether-bridged lantibiotics, in which the regio- and stereospecificity is enzymatically controlled. This biosynthetic mechanism was mimicked by Bradley et al. by reacting a dehydroalanine derivative with a cysteine derivative (Figure 1.18). ${ }^{70}$ 


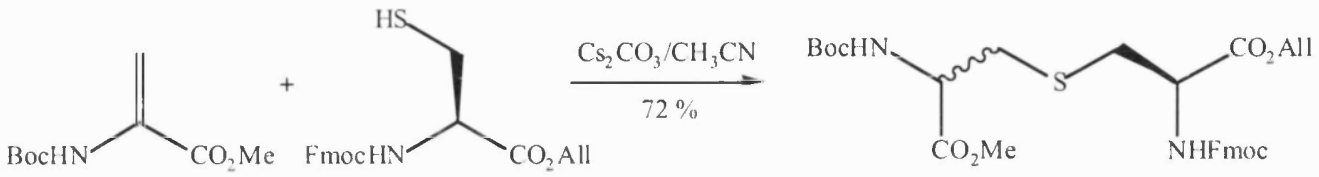

Figure 1.18. Michael addition of cysteine to dehydroalanine

The reaction proceeded to form the lanthionine derivative monomer. Unsurprisingly, the reaction was non-stereospecific and gave both diastereoisomers with thiolate attack of the cysteine taking place on both faces of the dehydroalanine derivative. The two diastereoisomers were separable by HPLC. Monteiro et al. have also reported on the same approach of synthesising lanthionine derivatives. ${ }^{71}$

\subsubsection{Serine $\beta$-lactones}

$(S)$-Serine and $(R)$-serine $\beta$-lactones were synthesised $(76-81 \%)^{72}$ by treating $N$ protected $(Z$ or $B o c)$ serine with triphenylphosphine and dimethyl azodicarboxylate in THF at $-78{ }^{\circ} \mathrm{C} .{ }^{73}$ These lactones were then treated with $(S)$-cysteine and $(R)$ cysteine derivatives in the presence of a base to form lanthionine. ${ }^{5}$ However a competing reaction can also take place forming a thioester instead of the desired lanthionine (Figure 1.19). ${ }^{5}$ Although the reaction was stereospecific, the yields of the desired lanthionine residues were only $50 \%$ due to the competing reaction.

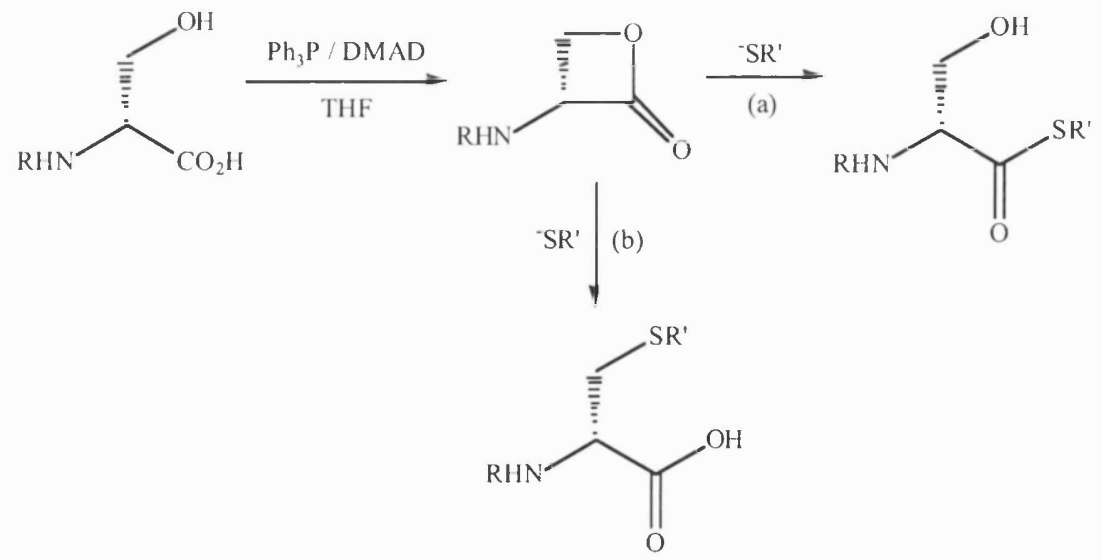

Figure 1.19. Competing reactions; (a) thioester formation, (b) thioether formation

Vederas et al. discovered the ring-opening of serine $\beta$-lactone derivatives by silylamines yields more of the $O$-acyl cleavage product when the reactions were 
carried out in chlorinated solvents, and the regiospecificity is changed when acetonitrile is used as solvent, yielding $95 \%$ of the $O$-alkyl cleaved product. ${ }^{74}$ Shirahama on the other hand reacted $N$-Boc-serine- $\beta$-lactone with sodium phenylselenide in THF and yielded the selenide ether exclusively. ${ }^{75}$ A more reliable method is still needed to produce lanthionine derivatives.

\subsection{4. $\beta$-Haloalanine derivatives}

The use of $\beta$-bromo- and $\beta$-iodoalanine derivatives for the $\beta$-cationic alanine synthon in the formation of lanthionine have been reported. In each case the $\beta$-haloalanines were derived from serine.

Dugave and Menez reported the successful synthesis of the natural $(S, R)$ - and unnatural $(R, R)$-lanthionine derivatives using $N$-triphenylmethylated $(R / S)$ serine benzyl/methyl esters which were mesylated at the $\beta$-hydroxy position, followed by iodination to form the $\beta$-iodoalanine derivatives. ${ }^{76}$ These were then treated with cysteine and $\beta$-substituted cysteine derivatives in the presence of caesium carbonate to yield Lan and MeLan derivatives in high yields (Figure 1.20, Table 3).
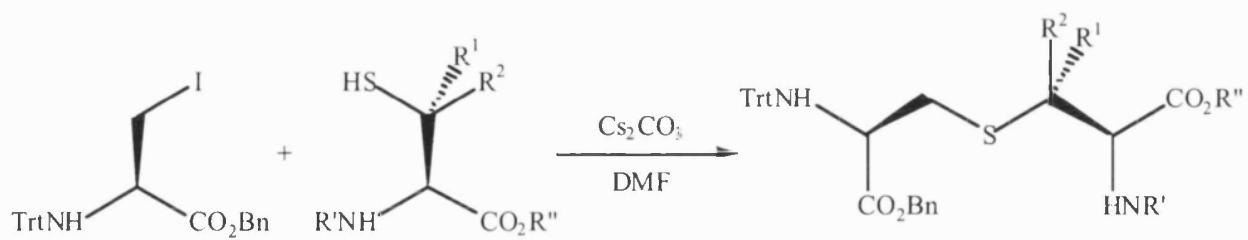

Figure 1.20. Lanthionine formation using a $\beta$-iodo- $(R)$-alanine derivative with a cysteine derivative

\begin{tabular}{|c|c|c|c|c|}
\hline $\mathbf{R}^{\mathbf{1}}$ & $\mathbf{R}^{2}$ & $\mathbf{R}^{\prime}$ & R" & Yield /\% \\
\hline $\mathrm{H}$ & $\mathrm{H}$ & Fmoc & ${ }^{\mathrm{t}} \mathrm{Bu}$ & 88 \\
$\mathrm{H}$ & $\mathrm{H}$ & $\mathrm{Boc}$ & $\mathrm{Me}$ & 79 \\
$\mathrm{H}$ & $\mathrm{Me}$ & $\mathrm{Boc}$ & $\mathrm{Me}$ & 83 \\
$\mathrm{Me}$ & $\mathrm{Me}$ & $\mathrm{Boc}$ & $\mathrm{Me}$ & 81 \\
\hline
\end{tabular}

Table 3. Product of Cys-Ala $((R)$-Ala) couplings with cesium carbonate in DMF 
The iodoalanine derivatives were reported to be present as two conformers, not interconverting due to the bulkiness of the triphenylmethyl group and the size of the iodine atom. Restricted rotation, resulting in two conformers at ambient temperature, has been reported in triphenylmethylated homoserine lactones. ${ }^{77}$

The lanthionine derivatives produced in Table 2 also gave ${ }^{1} \mathrm{H}$ NMR signals with similar patterns to the iodoalanine derivatives, indicating the presence of conformers. The chiral integrity of the first two entries was investigated, to ascertain that the two species observed by NMR were not diastereoisomers. Removal of the triphenylmethyl group and desulfurisation was followed by acidic hydrolysis and $(+)$-FLEC derivatisation of the resulting alanine residues. These were analysed by chiral HPLC, where elution of the (+)-FLEC-derivatised alanine with genuine samples of $(+)$-FLEC-(D,L)-alanine and (+)-FLEC-(L)-alanine indicated that the chiral integrity of the lanthionine derivatives was retained (Figure 1.21). The enantiomeric excess of the alanine derivatives were in excess of $98 \%$, which clearly dismissed the hypothesis of a significant degree of racemisation during the coupling.

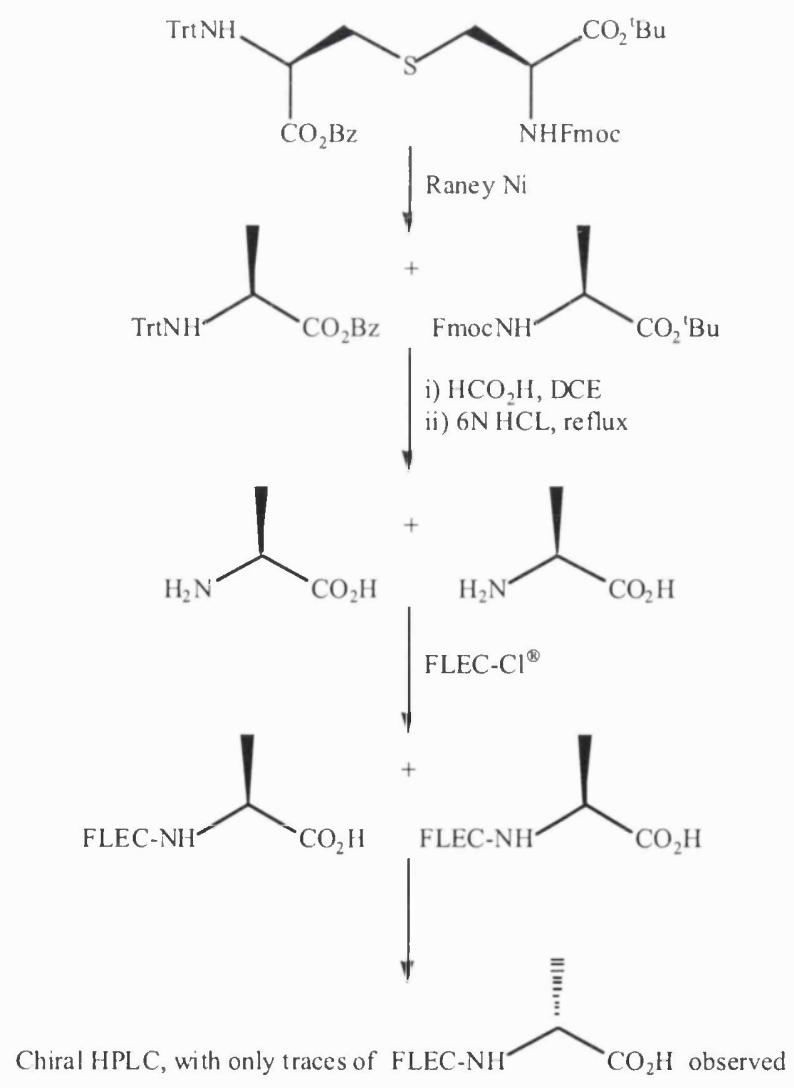

Figure 1.21. Determination of chiral integrity of a $(R, R)$-lanthionine derivative 
Other tests were carried out to confirm the presence of conformers. VT ${ }^{1} \mathrm{H}$ NMR experiments on the iodoalanine derivatives did not confirm the theory, as no coalescence of signals was observed due to the formation of aziridine at temperatures higher than $50^{\circ} \mathrm{C}$. VT experiments of the lanthionine derivatives, however, were not carried out. Since there was no indication of diastereoisomer formation at the lanthionine stage, as determined by the experiments described above which yielded only $(+)$-FLEC-derivatised L-alanine residues for $(R, R)$-lanthionine, Dugave and Menez concluded that the two conformers produced at the iodoalanine stage reacted with the cysteine derivatives to give two lanthionine conformers. Previous work in the Tabor group, in which the nitroveratryl group and its oxycarbonyl derivative were used as photolabile protecting groups, appeared to corroborate these observations: two conformers were present at both the iodoalanine and lanthionine stages. $^{78}$

\subsubsection{Ring opening of aziridine-2-carboxylates}

Lanthionine derivatives can also be formed from nucleophilic ring opening reactions of aziridine-2-carboxylates by cysteine derivatives. ${ }^{79,80}$ This, and other ring opening reactions of aziridine-2-carboxylates are discussed in Chapter 3. 


\section{Chapter 2}

Introduction II

\subsection{Solid phase peptide synthesis}

Merrifield was awarded a Nobel Prize in 1984 for his seminal work on solid phase peptide synthesis (SPPS) ${ }^{81}$ The principal of solid phase synthesis is that an insoluble polymeric matrix in the form of resin beads, appropriately functionalised with a linker or handle, can be used to host reactions forming amide or ester bonds with the linker (Figure 2.1). The functional groups on the amino acids need to be suitably protected. The $\alpha-N$ protection needs to be temporary, being easily removed without affecting any other protecting groups, to allow for the formation of repeated amide bonds to form the peptide. The side chain protecting groups, on the other hand, are 
semi-permanent as they need to be able to withstand the conditions applied throughout the peptide synthesis and are only removed at the end of the synthesis, often concurrently with peptidyl-resin cleavage to yield the fully deprotected peptide.

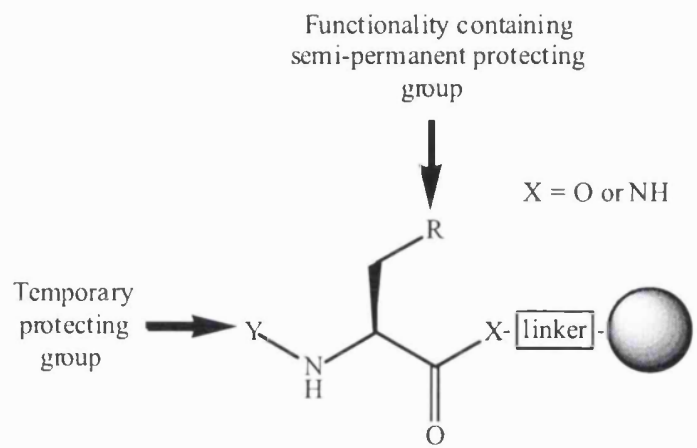

Figure 2.1. Different degrees of amino acid protection, allowing for repeated amide bond formation

Due to the insolubility of the resins, which could be placed on sinters, excess reagents, solvents and by-products for the removal of the temporary protection and amide bond formation could simply be filtered through once the reactions have taken place, avoiding tedious aqueous work-up procedures required after each amino acid coupling carried out in solution.

The Boc methodology employed by Merrifield involves the use of a copolymer of styrene and $2 \%$ divinylbenzene as the insoluble support, with chloromethylation to provide the active site for first residue coupling. The temporary Boc $N$-protection used is cleaved in relatively mild acidic conditions $(\mathrm{HCl} / \mathrm{AcOH})$ whereas the peptidyl-resin cleavage is carried out using hydrogen fluoride, which also cleaves semi-permanent side chain protections such as benzyl or $\mathrm{Z}$. The serious hazards of the use of hydrogen fluoride in the cleavage of peptides from Merrifield-type resins led Sheppard in the 1970 s to develop a different approach to SPPS. ${ }^{82 a, 82 b}$ Instead of the acid-based Boc methodology employed by Merrifield, a new methodology was developed: Fmoc-based $C \rightarrow N$ methodology used in conjunction with acid labile semi-permanent side chain protections and peptide cleavage from the linker-resin system. Figure 2.2 shows the general scheme for an Fmoc SPPS using a resin with an amino-linker yielding a $C$-terminal peptide amide upon cleavage. 


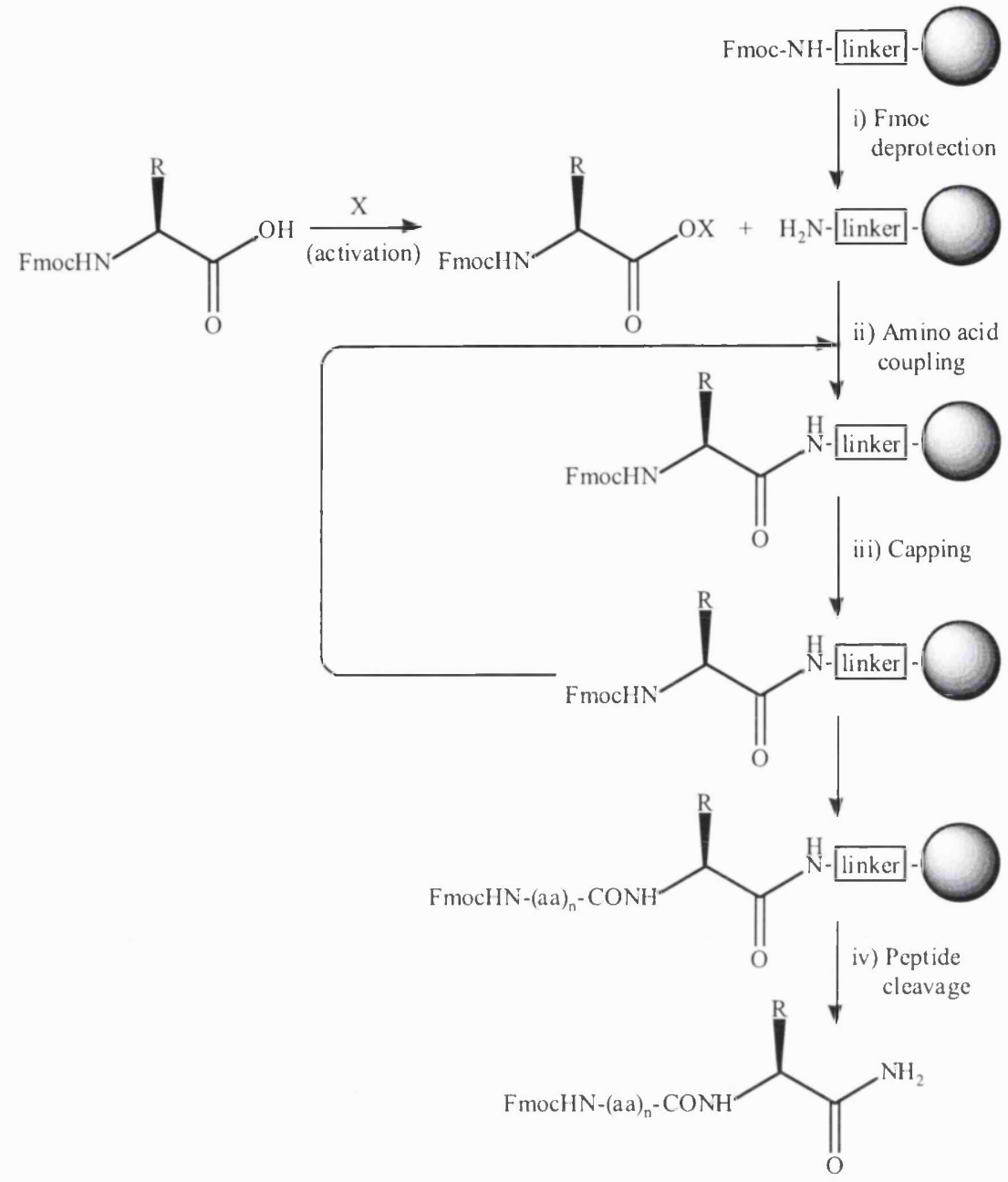

Figure 2.2. General scheme for Fmoc SPPS

In step (i) the temporary Fmoc protecting group is removed with a mild base, typically with $20 \%$ piperidine solution in DMF. In step (ii) the first amino acid, preactivated to form an active ester, is coupled onto the resin. This is followed by the $\mathrm{N}$ acetylation step (iii), carried out to "cap" any unreacted amino group, minimising the amount of by-products - in the form of peptides with varying lengths - formed during the synthesis. This sequence is then repeated as necessary to form the required peptide sequence. The manual repeated cycle of SPPS has been adapted for automation in the development of the solid phase peptide synthesiser.

Following each Fmoc deprotection and amino acid coupling steps in a manual synthesis, a small amount of resin is normally removed, washed and treated with solutions of Kaiser test. ${ }^{83}$ The Kaiser test is a mixture of solutions which essentially 
is a ninhydrin test for free amines, which indicates a dark blue to purple colouration. A positive Kaiser test should be observed after an Fmoc deprotection step and a negative one (colour of resulting solution remains unchanged) after an amino acid coupling step. In automated SPPS - either batch (automated delivery of reagents to sintered column containing resin and agitation during reaction) or continuous flow (automated delivery to column followed by continuous flow of reagents in a loop) each Fmoc deprotection and amino acid coupling is monitored by U.V. instead. Finally, in step (iv) the peptide is cleaved from the linker-resin.

\subsection{Resins for Fmoc solid phase peptide synthesis}

The original resin developed by Merrifield was polystyrene-based. Atherton and Sheppard developed a polyamide resin which overcame the problems of aggregation of the growing peptide observed during SPPS with Merrifield's resin and possessed greater swelling properties in DMF. ${ }^{82}$ Both types of resins were found to be unable to withstand high pressures, and hence were unsuitable for continuous flow peptide synthesis. A new type of resin was then developed in 1985 by Bayer and Rapp where poly(ethylene glycol) was grafted to polystyrene (PEG-PS). ${ }^{84}$ This resin is stable to the increased flow rates and hence increased pressure in continuous flow peptide synthesis. Its swelling properties have also been shown to be superior and have been described as tentacle-like; these resins are known as Tentagel resins. ${ }^{85}$

An important factor in the choice of resins used for SPPS is the linker. The sensitivity of the linker to acids, bases, nucleophiles, metal catalysts and U.V. radiation plays an important role in the success of a synthesis. For Fmoc SPPS, a wide variety of functionalised resins are currently commercially available. Rink, ${ }^{86}$ Sieber, ${ }^{87}$ 2-chlorotrityl, ${ }^{88}$ Wang, ${ }^{89} \mathrm{TGT}^{90,91}$ and $\mathrm{PAL}^{92}$ resins and their derivatives and refinements are widely used. These are chosen based on whether peptide acids or amides are required, and whether fully protected or deprotected peptides are desired. 


\subsubsection{Cleavage conditions}

The linkers in Fmoc SPPS have to be stable to the reaction conditions carried out during the synthesis. Fmoc is removed by base and therefore most of the linkers are base-stable and acid-labile. The resins mentioned above are of varying degrees of sensitivity to acids.

\subsubsection{Acid labile resins yielding peptide acids}

Resins such as the Wang resin ${ }^{89}$ and NovaSyn ${ }^{\circledR}$ TGA resin ${ }^{93}$ are cleaved under highly acidic concentrations, typically with $90-95 \%$ TFA solutions in the presence of scavengers such as triethylsilane or triisopropylsilane for up to $2 \mathrm{hr}$ (Figure 2.3). These conditions allow for the production of fully deprotected peptides by simultaneous deprotection of acid labile side chain protecting groups such as ${ }^{\mathrm{t}} \mathrm{Bu}$ and Boc.

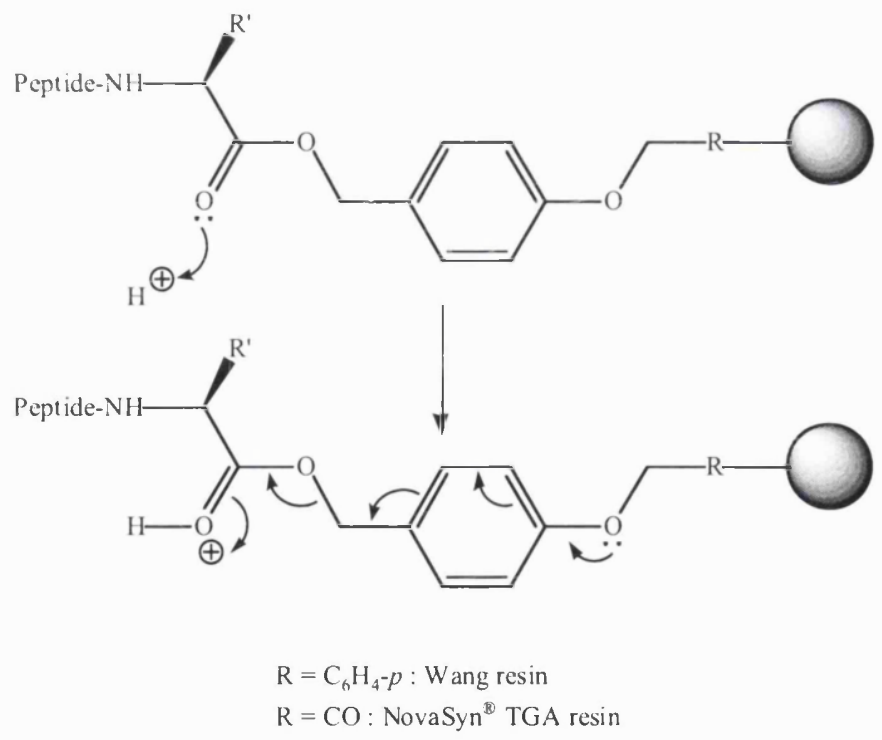

Figure 2.3. Cleavage mechanism for Wang and NovaSyn ${ }^{\circledR}$ TGA resins

Resins such as the Rink acid resin, ${ }^{86} 2$-chlorotrityl resin, ${ }^{88}$ SASRIN resin ${ }^{94}$ and HMPB resin ${ }^{95}$ contain hyperacid labile linkers. Peptide cleavage is carried out with $0.1-5 \%$ TFA solutions in DCM between $1 \mathrm{~min}$ and $1 \mathrm{hr}$ (Figure 2.4). Under these conditions the peptides produced are fully protected. The use of these resins is beneficial for monitoring the synthesis, where small amounts of the peptide could be 
cleaved from the resin at different stages in the synthesis, with all side chain protecting groups intact for LCMS analyses. Another advantage of using such hyperacid labile linkers is the possibility of synthesising protected peptide fragments, which could then be coupled via segment condensation in solution.

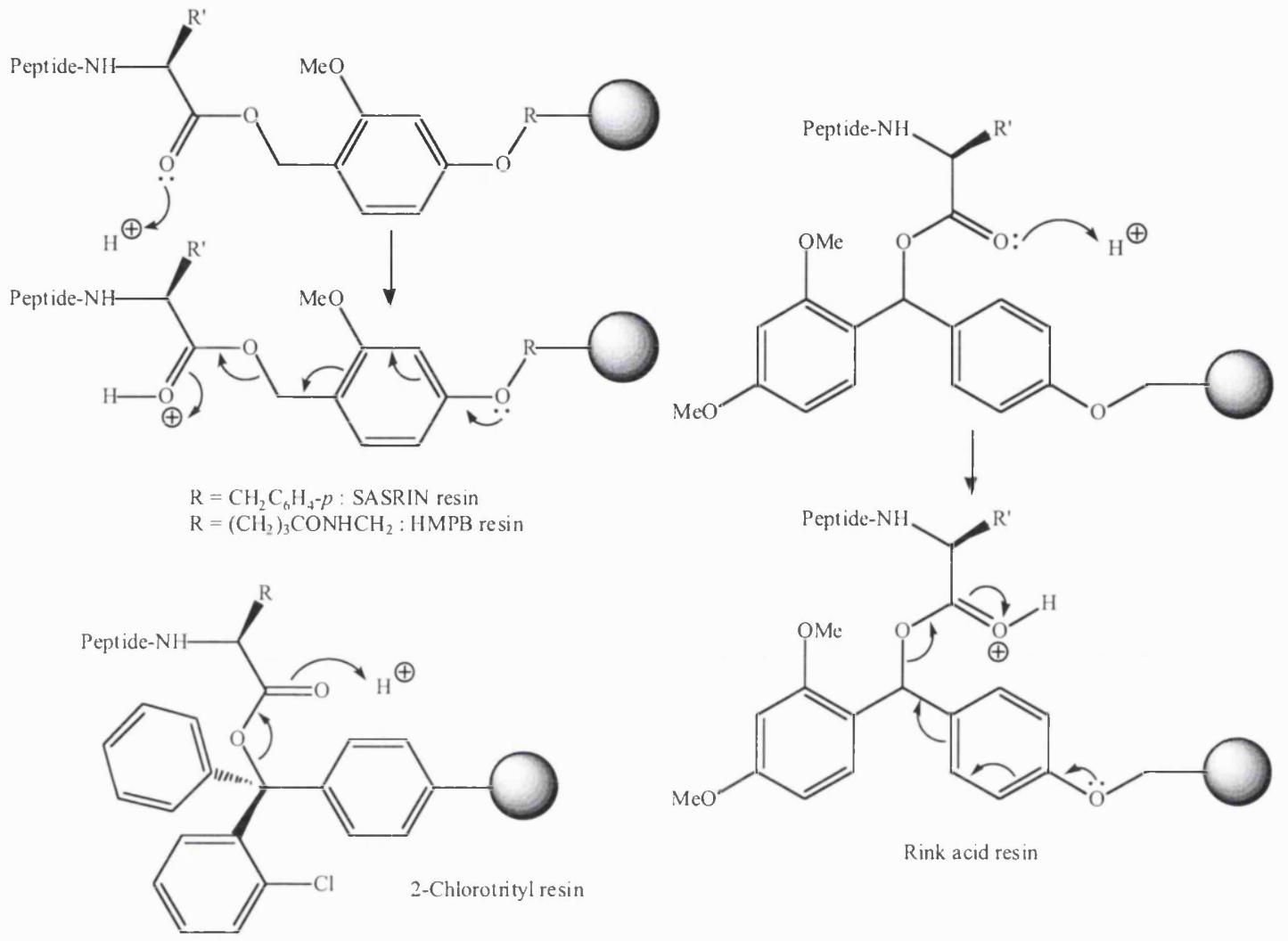

Figure 2.4. Cleavage mechanism for Rink acid, 2-chlorotrityl, SASRIN and HMPB resins

\subsubsection{Acid labile resins yielding peptide amides}

Resins such as the Rink amide ${ }^{86}$ and PAL resins ${ }^{92}$ are cleaved under high acidic concentrations, typically with $50-95 \%$ TFA solutions in the presence of scavengers such as triethylsilane or triisopropylsilane for up to $2 \mathrm{hr}$ (Figure 2.5). These conditions also allow for the production of fully deprotected peptides by simultaneous deprotection of acid labile protecting groups such as ${ }^{\mathrm{t}} \mathrm{Bu}$ and $\mathrm{Boc}$.

Resins such as the Sieber amide $\operatorname{resin}^{87}$ contain hyperacid labile linkers with peptide cleavage carried out with $1-5 \%$ TFA solutions in DCM for up to 15 min (Figure 
2.6). Similar to hyperacid labile linkers yielding peptide acids, the use of this resin is beneficial for monitoring the peptide synthesis at various stages by LCMS.

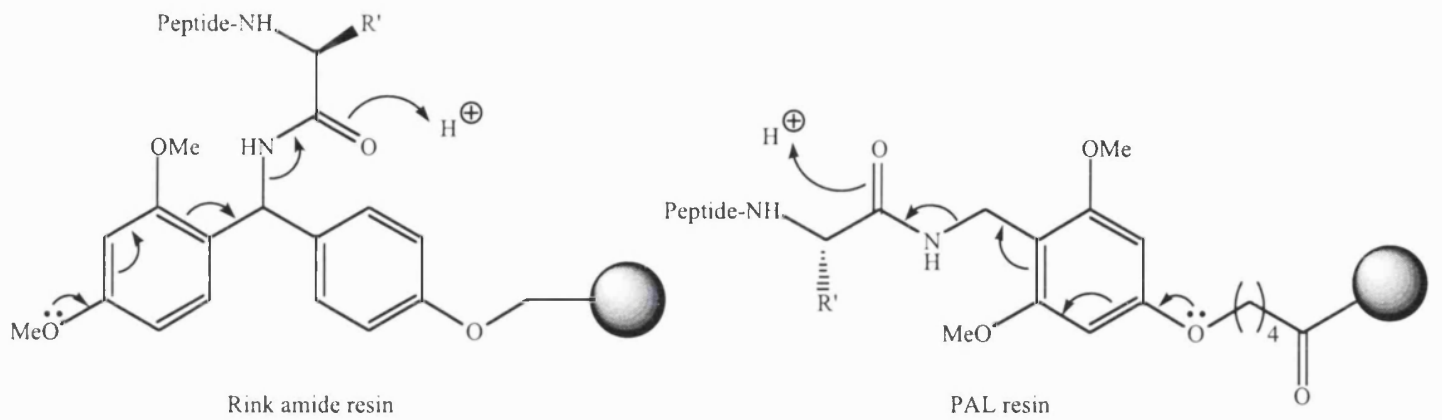

Figure 2.5. Cleavage mechanism for Rink amide and PAL (5-(4-Nfluorenylmethoxycarbonyl-aminomethyl-3,5-dimethoxyphenoxy)valeryl) resin

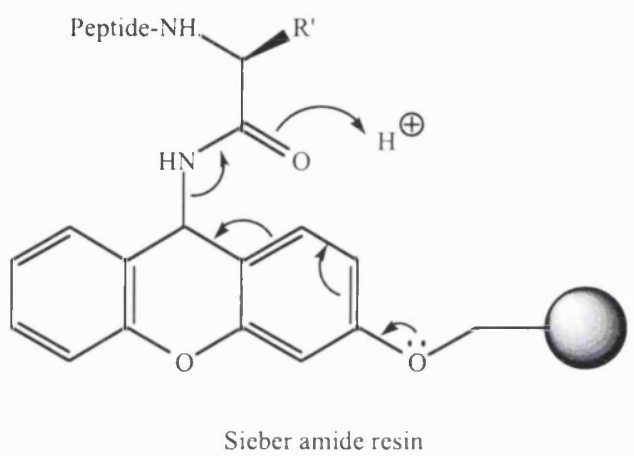

Figure 2.6. Cleavage mechanism for Sieber amide resin

\subsubsection{Resins cleaved via nucleophilic displacement and photolysis}

Ammonia has been used to cleave peptides from HMBA resins yielding peptide amides. ${ }^{96}$ Its use does not interfere with the Fmoc protecting group as cleavage is carried out at the end of the synthesis, once the base labile group has been removed (Figure 2.7).

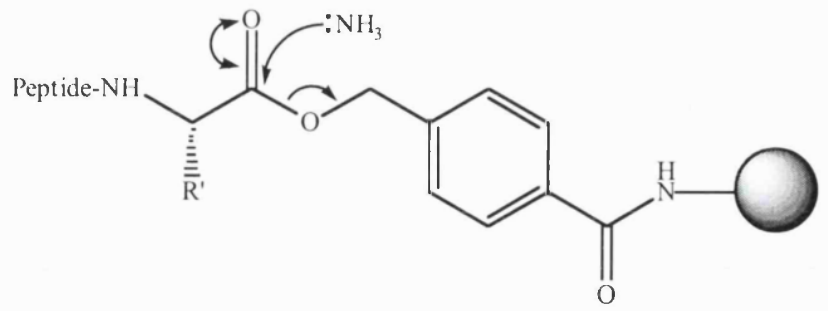

HMBA resin

Figure 2.7. Cleavage mechanism for HMBA resin 


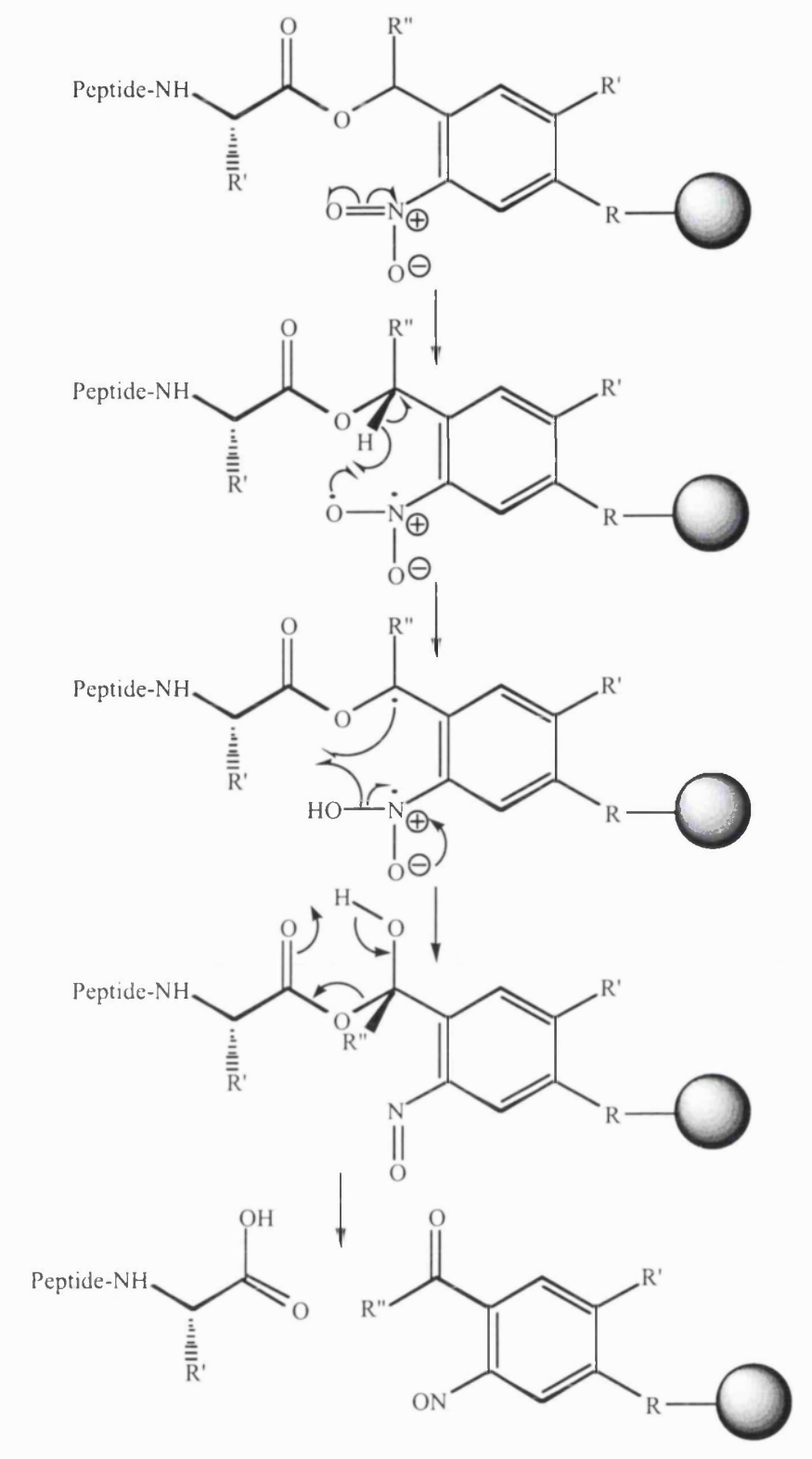

$\mathrm{R}=\mathrm{CO}, \mathrm{R}^{\prime}=\mathrm{H}, \mathrm{R}^{\prime \prime}=\mathrm{H}:$ 3-Nitro-4-hydroxymethyl benzoyl resin

$\mathrm{R}=\mathrm{O}\left(\mathrm{CH}_{2}\right)_{3} \mathrm{CO}, \mathrm{R}^{\prime}=\mathrm{OMe}, \mathrm{R}^{\prime \prime}=\mathrm{Me}: \alpha$-Methyl-6-nitro-veratrylamine resin

Figure 2.8. Cleavage mechanism for 3-nitro-4-hydroxymethyl benzoyl and $\alpha$ methyl-6-nitro-veratrylamine alcohol resins

There have also been reports of linkers that undergo photolytic cleavage, yielding both peptide acids and amides. The $\alpha$-methyl-6-nitro-veratrylamine based $\operatorname{resin}^{97}$ is cleaved by irradiating with U.V. light of a specific wavelength, typically $350 \mathrm{~nm}$, over periods of $1-3 \mathrm{hr}$ (Figure 2.8). 


\subsection{Coupling reagents and cyclisation reagents (via amide bond formation)}

In coupling a carboxylic acid group with an amino group to form an amide bond, the reaction requires harsh conditions of strong acid or base. This less than ideal method for peptide synthesis could be avoided by increasing the electrophilicity of the carboxylic acid group, making it more susceptible to the amino nucleophilic attack.

For peptide bond formation, two factors are important: high yields and retention of stereochemical integrity. Amino acids can undergo racemisation should the carboxyl group be "over-activated". This could take place via the formation of an oxazolone (Figure 2.9).<smiles>[R]c1nc(-c2ccccc2)oc1OC1=N[C@@H]([2H])C(=O)O1</smiles>

(c)<smiles>[R]NC(=O)[C@H]([R])NC(=O)OF</smiles>

Figure 2.9. Formation of an oxazolone (b) and its enantiomer (d) from an activated amino acid (a) via enolisation of the oxazolone (c) which results in racemisation: (e)

In solution peptide synthesis, the azide method is perhaps the earliest means of amide bond formation. ${ }^{98}$ In SPPS, diphenylphosphoryl azide (DPPA) is still used for amide bond formation due to its efficiency at forming activated peptide azides in situ (Figure 2.10). ${ }^{99,100}$ 


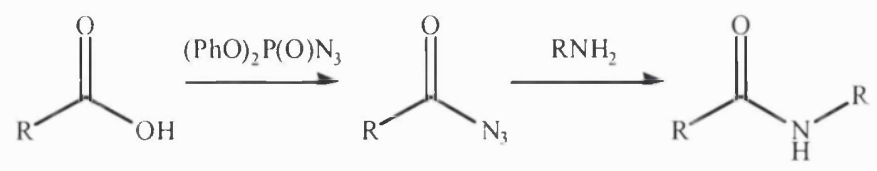

Figure 2.10. Coupling via the azide method using DPPA

Pentafluorophenyl esters (OPfp) of amino acids (Figure 2.11) have found wide application in Fmoc SPPS. These active esters react to form amide bonds rather slowly, but the addition of HOBt increases the rate of reaction. ${ }^{101}$<smiles>[CH][C@@H](NC)C(=O)Oc1c(F)c(F)c(F)c(F)c1F</smiles>

Figure 2.11. A Pfp-activated amino acid

Merrifield originally used dicyclohexylcarbodiimide (DCC) as coupling reagent in SPPS. ${ }^{81}$ The formation of the $O$-acylisourea intermediate leads to nucleophilic attack of the amino group, forming the required amide bond (Figure 2.12).

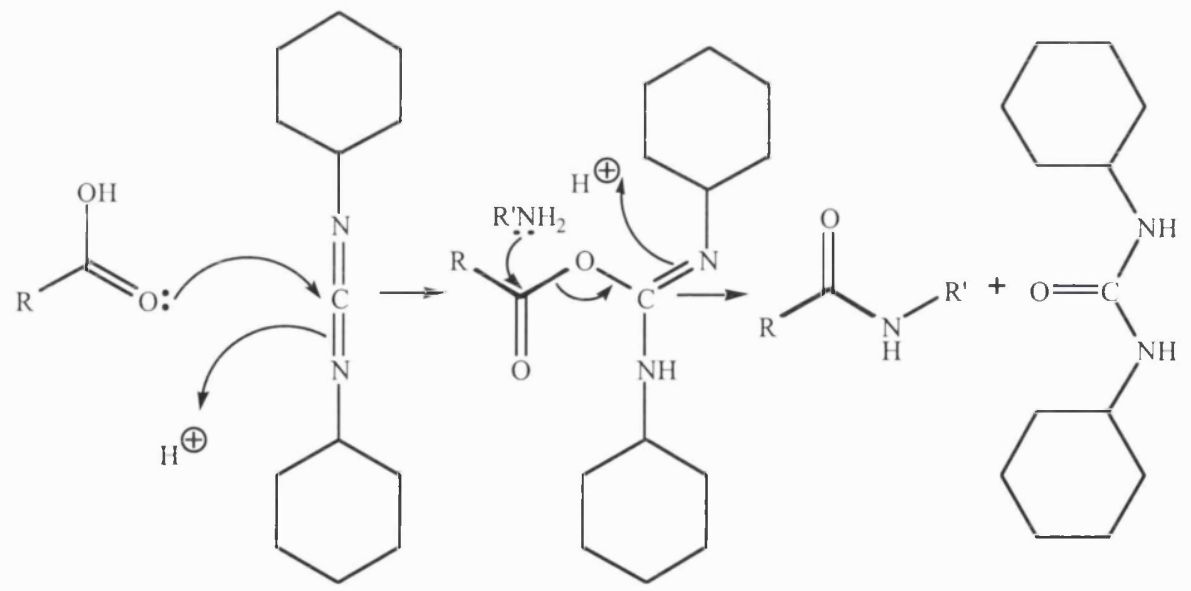

(a)

Figure 2.12. Formation of the $O$-acylisourea intermediate (a) followed by nucleophilic attack of an amino group

The formation of a symmetrical anhydride is possible via reaction of the $O$ acylisourea intermediate with a second, non-activated carboxylic acid (Figure 2.13). This symmetrical anhydride can also react with an amino group to form the desired 
amide bond. Pre-formed symmetrical anhydrides are generated by reaction of the Fmoc amino acid with 0.5 equivalents of carbodiimide. However, this means of amide bond formation is, among other things, wasteful as only one amino acid from the anhydride is used for amide bond formation.

(a)

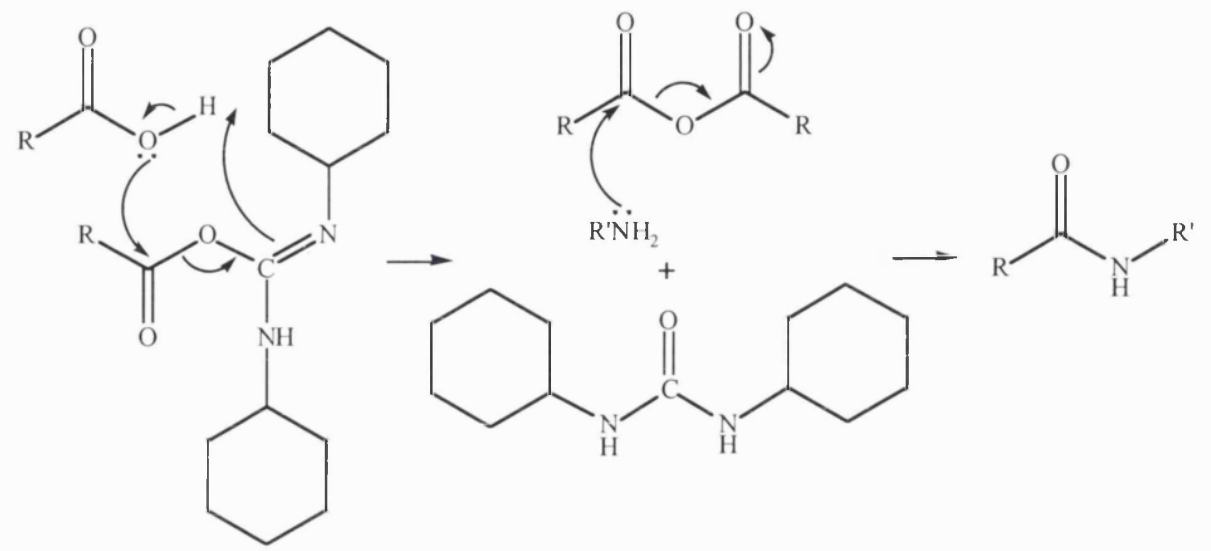

Figure 2.13. Formation of the symmetrical anhydride (a) followed by nucleophilic attack of the amino group yielding the desired amide bond.

In solution peptide chemistry, the urea by-product is simply filtered off due to its sparing solubility in most organic solvents. However, repeated washing is a requirement in SPPS. The introduction of diisopropylcarbodiimide (DIC) eliminates this problem. ${ }^{102}$ It is often used in conjunction with a hydroxylamine such as 1 hydroxybenzotriazole (HOBt), ${ }^{103}$ forming the active ester in situ, to avoid possible racemisation via the symmetrical anhydride formation (Figure 2.14).

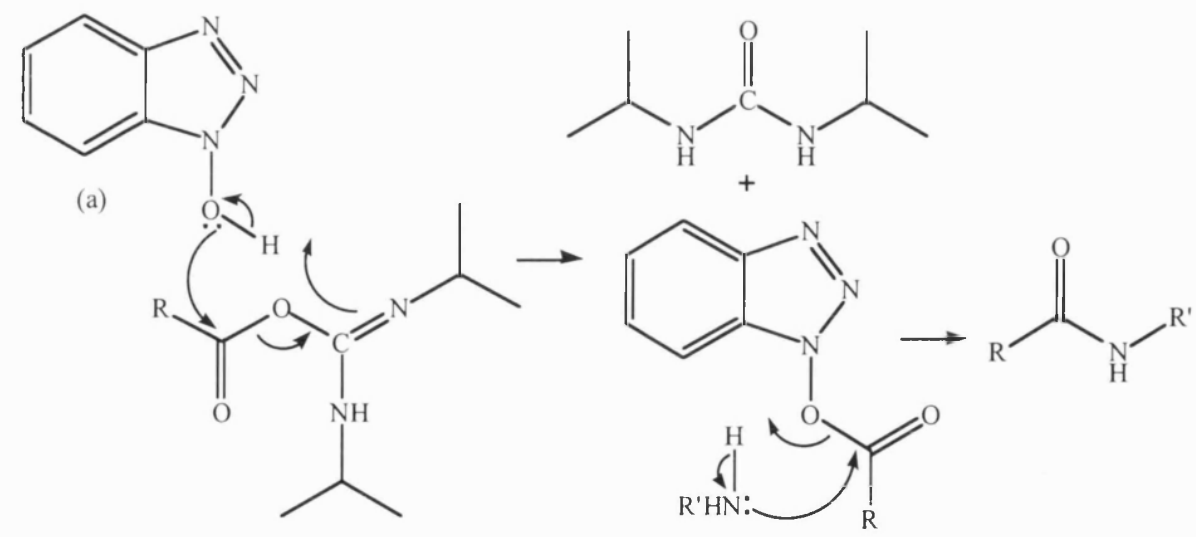

(b)

Figure 2.14. Formation of an amide bond via in situ activation of the $O$-acylisourea intermediate (b) with HOBt (a) 
HOBt also prevents the formation of $\mathrm{N}$-acylisourea via a competing intramolecular rearrangement of the $O$-acylisourea, which occurs when the latter is not immediately reacted with the nucleophilic amino group (Figure 2.15). ${ }^{104}$

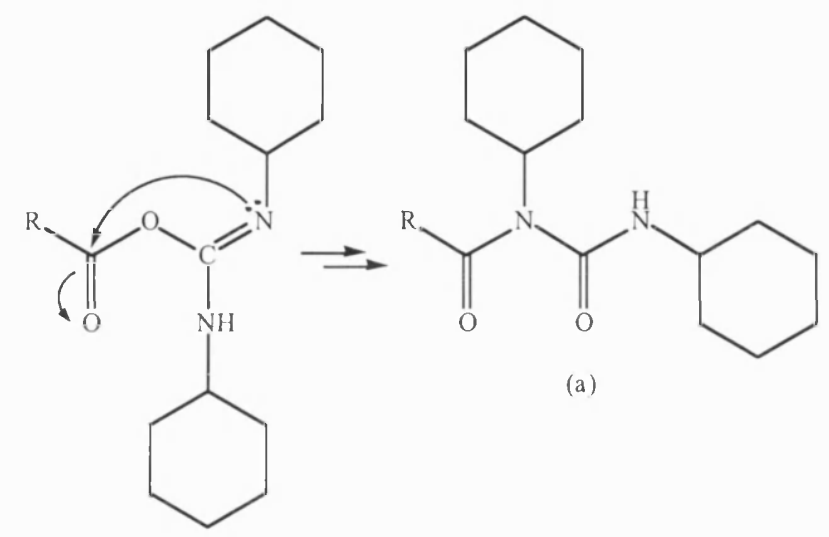

Figure 2.15. Formation of the unreactive $N$-acylisourea (a)

Phosphonium derivatives of $\mathrm{HOBt}$ - BOP reagents - were developed by Castro et al. and proved to be efficient coupling reagents for peptide synthesis (Figure 2.16). ${ }^{105 a} \mathrm{~A}$ major problem, however, is the formation of hexamethylphosphoric triamide (HMPA) by-product which is a carcinogen.

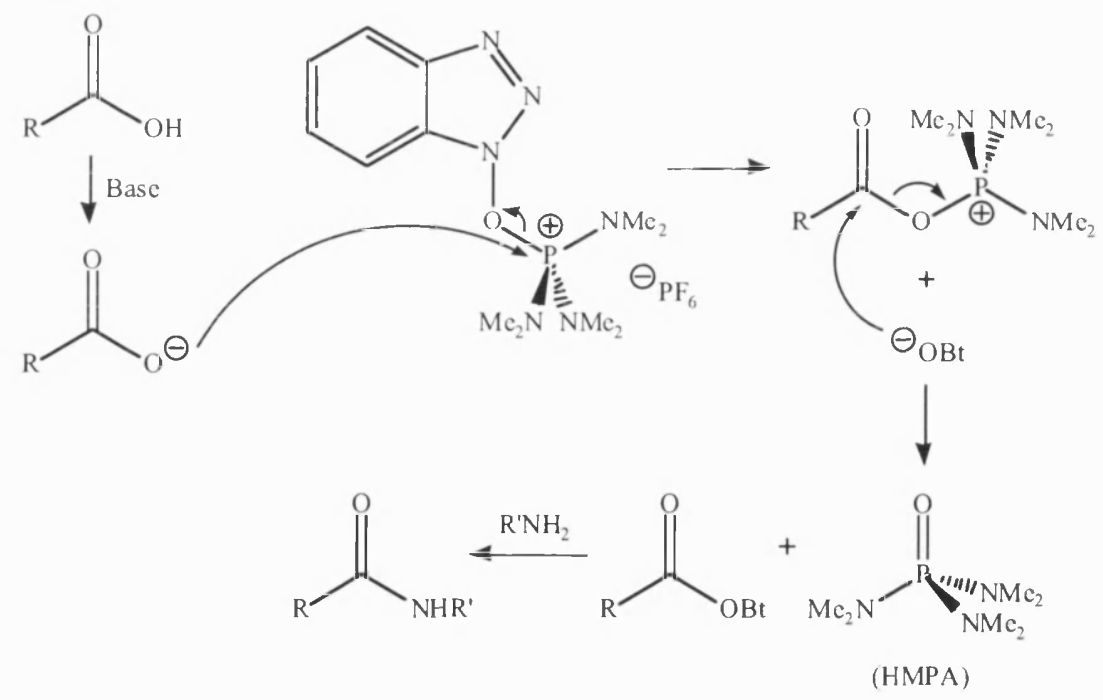

Figure 2.16. Formation of the carcinogenic HMPA during BOP activation

Over the years various non-toxic coupling reagents have been developed, including PyBOP ${ }^{105 b}$ and HBTU, ${ }^{105 c}$ mainly by varying the derivatives on the phosphorous (Figure 2.17). 


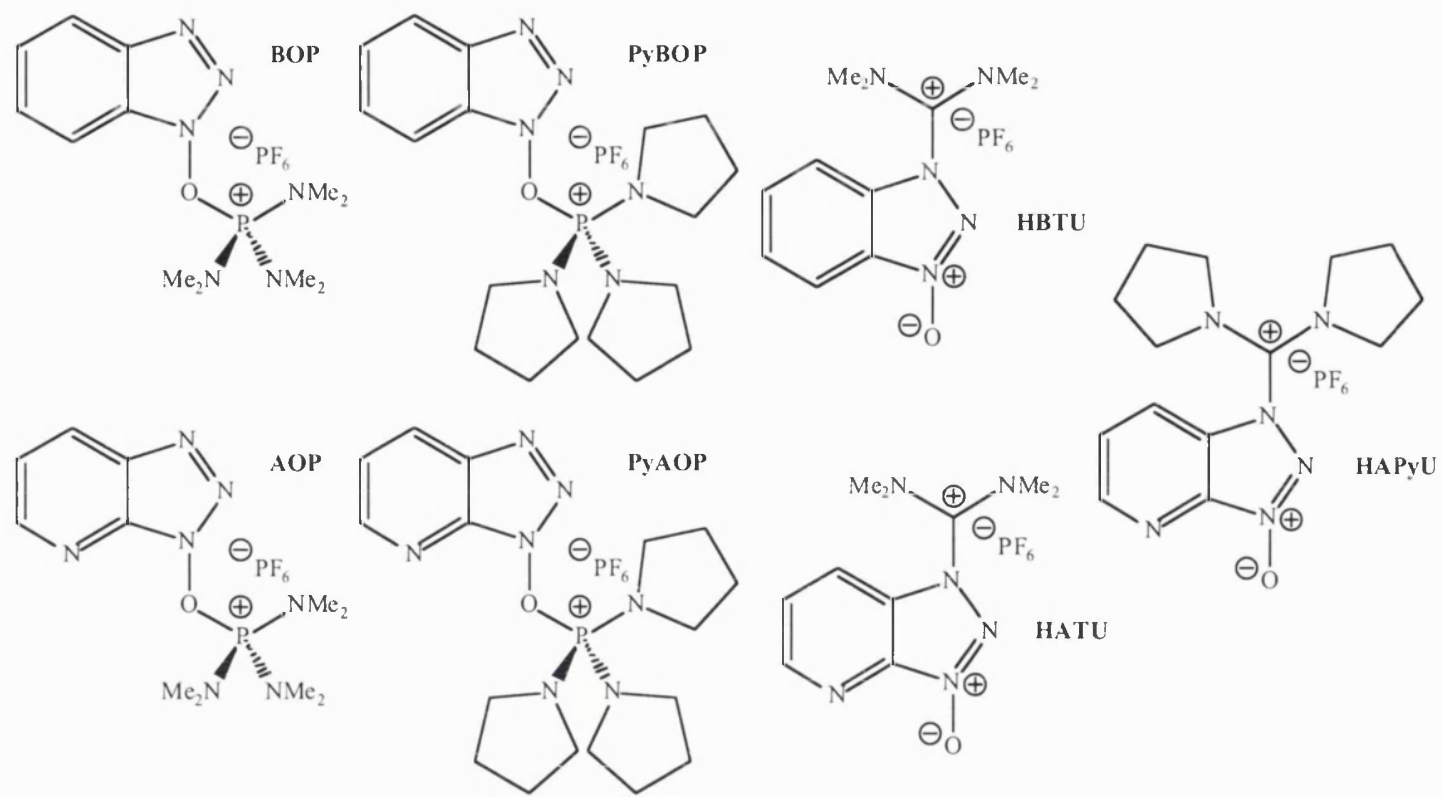

Figure 2.17. Various coupling and/or cyclisation reagents for SPPS

The 1-hydroxy-7-azabenzotriazole (HOAt) derived reagents have been shown to be superior to HOBt with increased coupling efficiencies and lower racemisation. ${ }^{106,107}$ These are due to the nitrogen on the 7-position, which stabilises the overall negative charge on the leaving group, as well as having a stabilising effect via a hydrogen bond formed with the amino group on the incoming amino acid (Figure 2.18). ${ }^{108}$

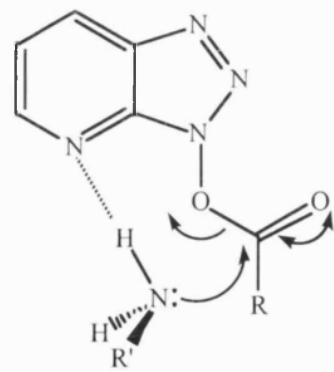

Figure 2.18. Stabilising effect of the pyridinium nitrogen on HOAt

In fact, the use of phosphonium and "so-called" uronium (aminium) salts of HOAt PyAOP and HATU/HAPyU, respectively - has been shown to be superior to coupling methods via carbodiimides and HOBt. Albericio et al. demonstrated that for a simple coupling of Z-Gly-Phe-OH and H-Pro- $\mathrm{NH}_{2}$, the use of PyAOP-HOAt and HAPyU-HOAt yielded negligible racemisation compared to the more traditional DCC-HOBt and BOP systems. ${ }^{109}$ Various other examples have been reported to 
highlight the lower racemisation ${ }^{110,111,112}$ and the fact that activation and coupling of amino acids are achieved much quicker. ${ }^{111}$ The superiority of the "so-called" uronium and phosphonium salts of HOAt has also been extended to the synthesis of cyclic peptides via lactam bridge formation. ${ }^{113,114,115}$

\subsection{Orthogonal protecting groups}

The use of different types of temporary and semi permanent protecting groups that are cleaved under different conditions plays an important role in SPPS. The orthogonality of the widely used $\mathrm{Fmoc} /{ }^{t} \mathrm{Bu}$ system has often been utilised in conjunction with other types of protecting groups. This section discusses two such protecting groups in terms of their orthogonality to the Fmoc methodology, incorporation onto amino acids and their deprotection conditions: the commonly used allyl and Aloc, and the relatively new dimedone-based protecting groups ivDde and Dmab.

\subsubsection{Allylic protecting groups}

The allyloxycarbonyl species (Aloc, Figure 2.19) was the first to have been used as an allylic protection for amino acids by Stevens and Watanabe in $1950 .^{116}$ Their report included the fact that there were no specific methods available for the cleavage of this group. After various attempts using platinum and palladium catalysts, phosphonium iodide in glacial acetic acid and metallic sodium in liquid ammonia, they came to the conclusion that deprotection was not straightforward. Competing hydrogenation of the allylic double bond also occurred, limiting the application of allylic protecting groups in synthetic organic chemistry. It was not until the early 1980 s that the development of mild methods for deprotection enabled the potential of allylic protecting groups in organic chemistry to be recognised. ${ }^{117}$ 


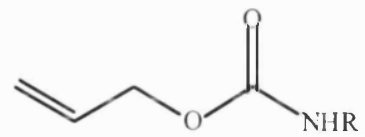

(a) $N$-Aloc protection<smiles>[R]OC(=O)OC/C=C\C</smiles>

(d) Allyl carbonate<smiles>[2H]C(=O)OCC=CC</smiles>

(b) Allyl ester

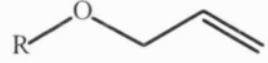

(c) Allyl ether

Figure 2.19. Allylic protection of various functional groups

In amino acid chemistry, the two main allylic groups (allyl and Aloc) are used for protecting carboxyl functions as allyl esters, alcohol functions as allyl ethers or allyl carbonates and amino functions as allyl carbamates. These have recently been extensively reviewed. ${ }^{118,119}$ Incorporation of the allyl group onto carboxyl functions is generally achieved by one of three means: acid catalysed esterification with allyl alcohol, activated carboxyl with allyl alcohol or nucleophilic displacement of allyl halides with carboxylates. Allyl carbonates and carbamates are generally generated from treatment of the alcohol and amine, respectively, with allyl chloroformate in the presence of base (Figure 2.20). There has also been a report on the use of diallyl dicarbonate for the synthesis of allyl carbamates. ${ }^{120}$<smiles>C=CCO</smiles><smiles>[2H][C@@H](N)C(=O)OCC=C</smiles>

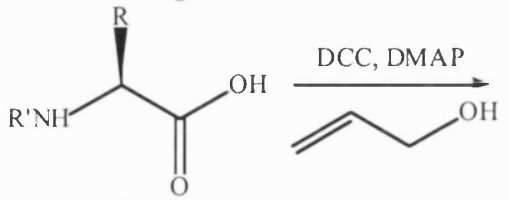<smiles>[B][C@@H]([18OH])C(=O)OCC=C</smiles>

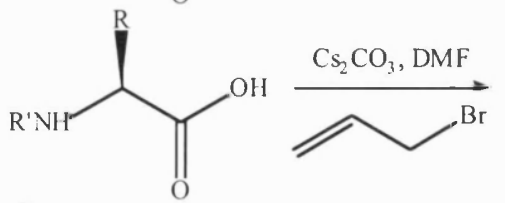<smiles>C=CCOC(=O)[C@H](C)N</smiles><smiles>[CH2][C@@H](N)C(=O)OCC=C</smiles><smiles>C=CCOC(=O)N[C@@H](C)C(=O)O</smiles>

Figure 2.20. Incorporation of allyl and Aloc onto alcohols, carboxyls and amines 
The main challenge in the use of allylic protecting groups for amino acids and peptides has been their deprotection. The well known and widespread method involves the cleavage of allyl esters or allyl carbamates using palladium(0) catalysts in the presence of nucleophiles under near-neutral conditions. The mechanism for allyl and Aloc deprotection is shown in Figure 2.21. The palladium( 0 ) catalyst is coordinated to the allylic double bond and is then oxidised, liberating the carboxyl group (or amino group and carbon dioxide for Aloc-protection) which results in a cationic palladium(II)-allyl species. A nucleophilic scavenger then attacks the allylic cation, resulting in a reductive elimination of the palladium(II).
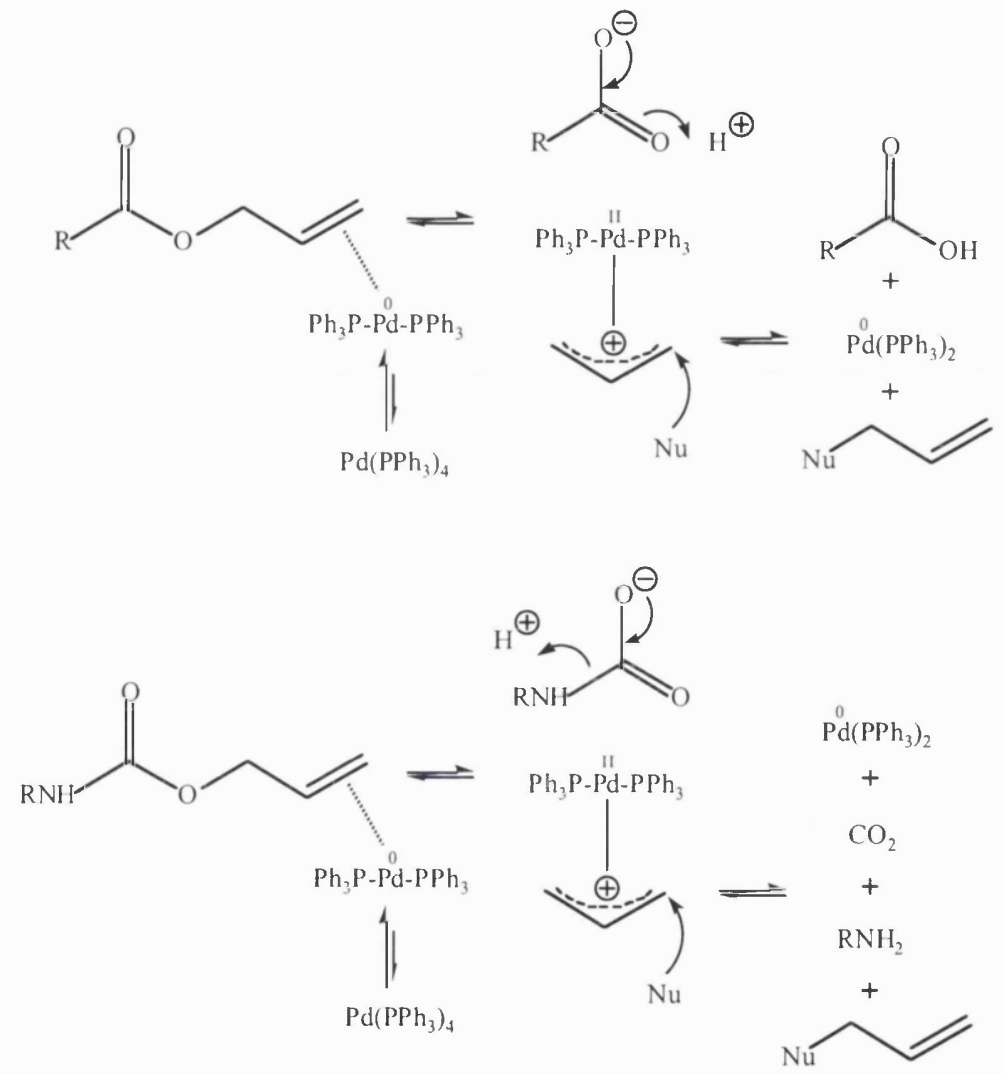

Figure 2.21. Mechanism for allylic deprotection

The problem often encountered in Aloc deprotection is the nucleophilicity of the liberated free amine towards the palladium(II)-allyl species, which can compete with the nucleophilicity of the allyl group scavenger (route (a) in Figure 2.22). This would result in the formation of a secondary allyl amine on an amino acid or peptide, which would put a halt to the synthesis. The use of an excess amount of scavenger would also be advantageous as it eliminates the formation of this secondary allyl amine via 
the routes (a) and (b) described in Figure 2.22. The use of a weakly basic nucleophile as a scavenger is also essential as it avoids Fmoc deprotection; this consolidates the orthogonality of the allyl group with the Fmoc/'Bu system.

(a)

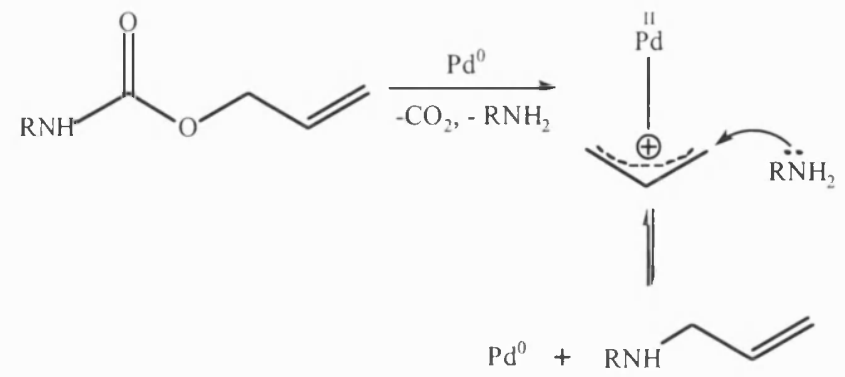

(b)

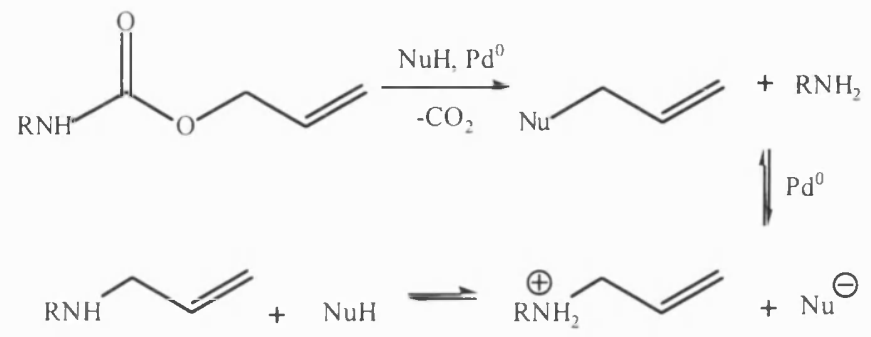

Figure 2.22. Competing allyl amine formation reaction via routes (a) and (b)

Scavengers that have been used include oxygen nucleophiles such as potassium 2ethylhexanoate, ${ }^{121}$ nitrogen nucleophiles such as morpholine, ${ }^{122,}{ }^{123} \mathrm{~N}$ methylaniline, ${ }^{124,}{ }^{125}$ diethylamine, ${ }^{126,127}$ and $N$-methylmorpholine ${ }^{128,129}$ carbon nucleophiles (acidic $\beta$-dicarbonyls) such as dimedone ${ }^{130}$ and $N, N$-dimethylbarbituric acid, ${ }^{119}$ sulfur nucleophiles such as 2-thiobenzoic acid, ${ }^{131}$ silylated derivatives of nitrogen nucleophiles such as trimethylsilylmorpholine ${ }^{132}$ and trimethylsilyldimethylamine ${ }^{132}$ and hydride donors such as formic acid, ${ }^{133}$ tributyltin hydride, ${ }^{134}$ borohydrides ${ }^{135}$ and silicon hydrides ${ }^{136,137}$ (Figure 2.23).

In SPPS, allylic groups were initially used as resin linkers. Kunz et al. were the pioneers in using 4-bromocrotonic acid (Figure 2.24), coupled to the aminomethyl polystyrene resin (DCC/HOBt) forming an allylic anchor. ${ }^{138}$ This and other allylic handles such as 4-trityloxybut-(Z)-2-enyloxyacetic $\operatorname{acid}^{139}$ and 4-(4'methoxytrityloxy)-but-2-enyloxyhexanoic acid ${ }^{140}$ are removed by palladium( 0 ) species in the presence of various nucleophiles. ${ }^{119,141}$ 
<smiles>CCCCC(CC)C(=O)[O-]</smiles><smiles>C1CCCCC1</smiles><smiles>CNc1ccccc1</smiles><smiles>CC1CC(=O)CC(=O)C1</smiles><smiles>CN1C(=O)CC(=O)N(C)C1=O</smiles><smiles>O=C(O)c1ccccc1S</smiles>

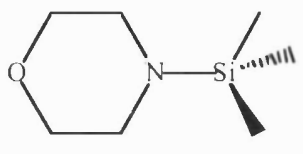<smiles>CN(C)[Si](C)(C)C</smiles><smiles>O=CO</smiles><smiles>[10BH][10BH]</smiles>

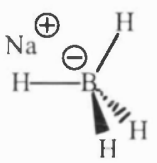<smiles>[IH2]</smiles>

Figure 2.23. Scavengers used for palladium-mediated allylic deprotection<smiles>O=C(O)/C=C\CBr</smiles>

(a)<smiles>CCOC/C=C\CCCC(=O)O</smiles><smiles>COc1ccc(C(OC/C=C\CCCCCCCC(=O)O)(c2ccccc2)c2ccccc2)cc1</smiles>

(c)

Figure 2.24. Structures of (a) 4-bromocrotonic acid, (b) 4-trityloxybut-(Z)-2enyloxyacetic acid and (c) 4-(4'-methoxytrityloxy)-but-2-enyloxyhexanoic acid

The high selectivity, mild and almost neutral conditions of the cleavage of allylic groups together with their compatibility with sulfur derivatives ${ }^{119}$ have made them an excellent choice for three-dimensional orthogonal protecting group strategy in SPPS. They have also been proven to be compatible with the traditional Fmoc/'Bu methodology. ${ }^{129,141,142,143,144,145,146}$

There have also been successful cases of simultaneous allyl and Aloc deprotection. Hayakawa et al. carried out the cleavage of the phosphate-bound allyl and amine- 
bound Aloc groups in the solution and solid phase synthesis of nucleic acids simultaneously, using tris(dibenzylideneacetone)dipalladium(0), $\mathrm{PPh}_{3}$, butylamine and formic acid in THF. ${ }^{147}$. Schmidt et al. have reported simultaneous cleavage of the amine-bound Aloc and the carboxyl-bound allyl groups of a linear peptide using $\mathrm{Pd}\left(\mathrm{PPh}_{3}\right)_{4} /$ morpholine/THF ${ }^{122}$ as have Arnould et al. who cleaved an allyl ester and a doubly Aloc protected amino function of a carbapenem antibiotic using $\mathrm{Pd}\left(\mathrm{PPh}_{3}\right)_{4} /$ dimedone/THF. ${ }^{148}$

\subsubsection{Dimedone-based protecting groups}

In 1993 Bycroft et al. introduced Dcm (N-4,4-dimethyl-2,6-dioxocyclohexylidenemethyl), the prototype of a series of novel temporary non-urethane based $\mathrm{N}$ protecting groups. ${ }^{149}$ Its precursor was formed by reacting dimedone with $\mathrm{N}, \mathrm{N}$ dimethylformamide dimethylacetal ${ }^{150}$ and the resulting 5,5-dimethyl-2(dimethylaminomethylene)cyclohexane-1,3-dione was treated with amino acids in ethanol at room temperature yielding stable crystalline derivatives (Figure 2.25).

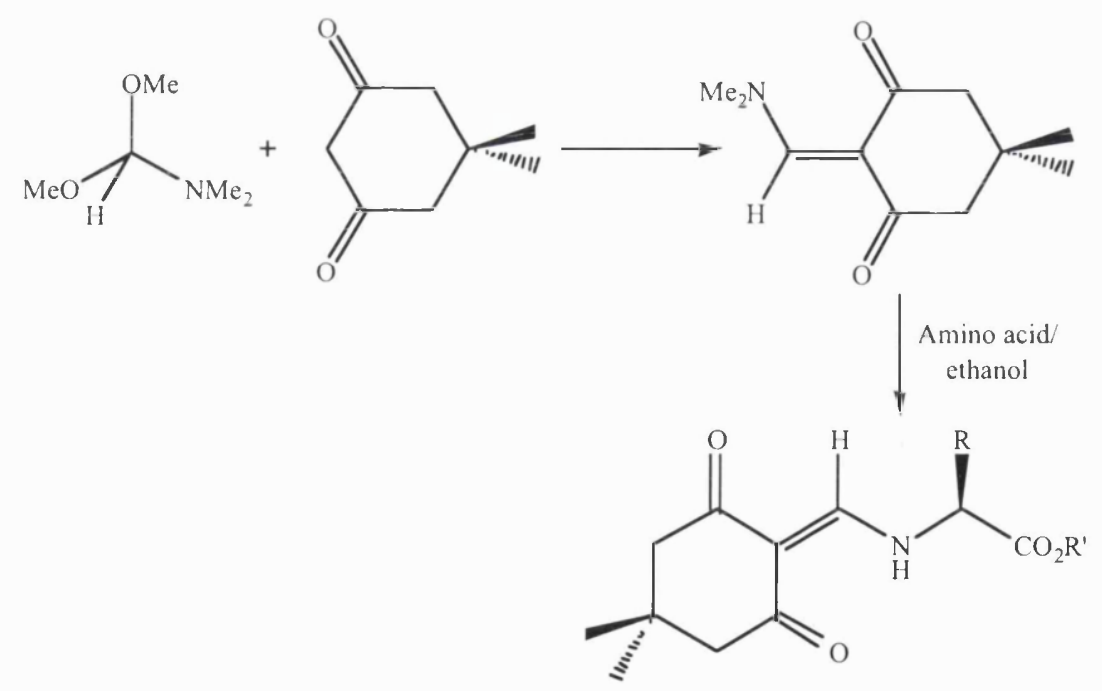

Figure 2.25. Synthesis of Dcm and its incorporation into amino acids

The removal of the Dcm protecting group was successfully carried out with $1 \%$ and $0.1 \%$ hydrazine hydrate solutions (v/v) in DMF over 10 and 20 minutes, respectively. The reaction is driven by the formation of the 1,2-diazole (Figure 2.26). It was discovered that Dcm could be removed under Fmoc deprotection conditions 
making it incompatible with Fmoc SPPS, although the piperidine-aided deprotection of Dcm required treatment for 20 minutes, which is longer than that required for the removal of Fnoc.
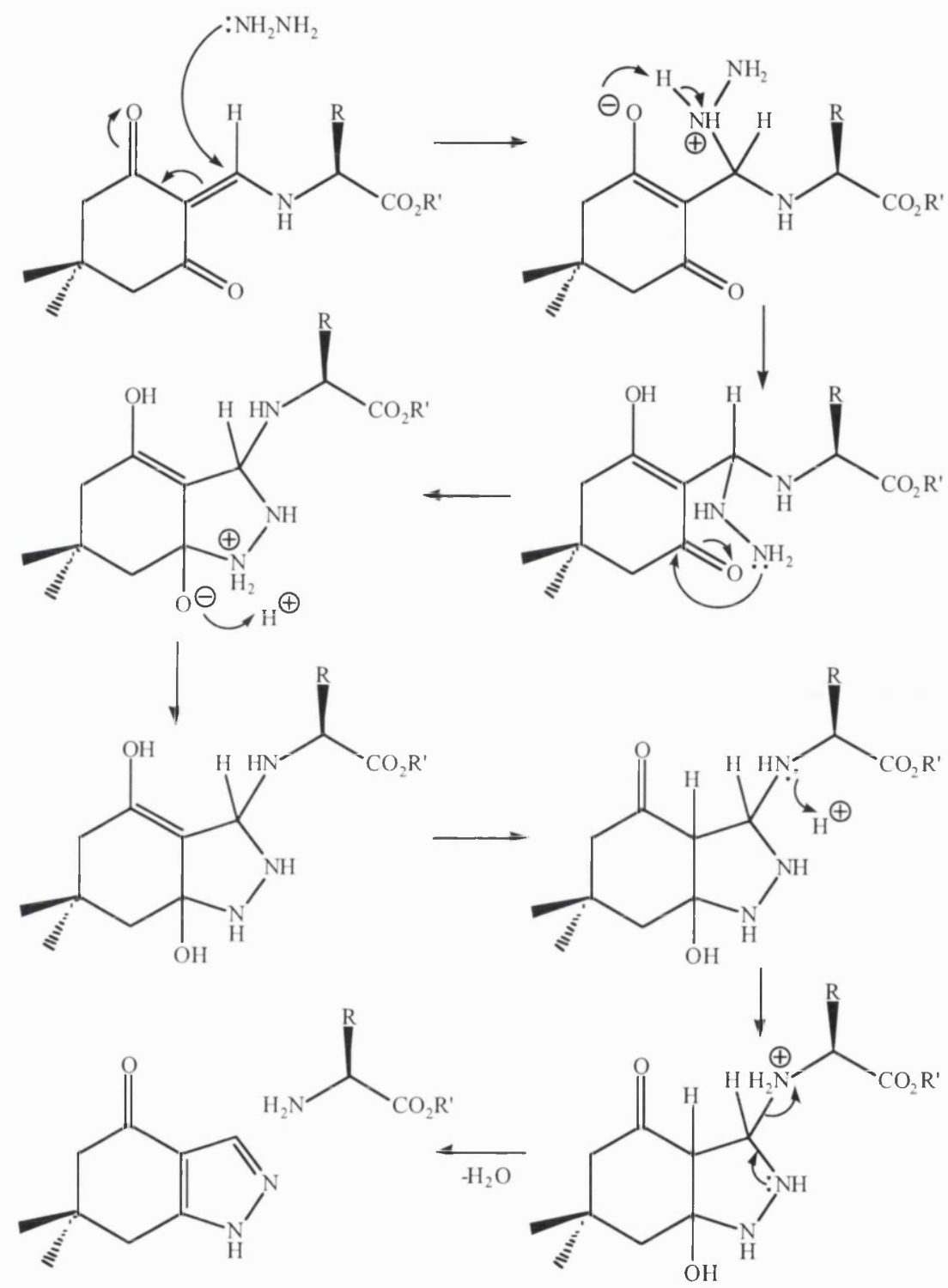

Figure 2.26. Mechanism for the deprotection of Dcm

Bycroft et al. also introduced the Dde group (N-1-(4,4-dimethyl-2.6dioxocyclohexylidene)ethyl), ${ }^{151}$ synthesised from dimedone and acetic acid with DCC and DMAP. ${ }^{152}$ Its reactivity with amino acids was readily shown by the side chain derivatisation of Fmoc-Lys-OH in ethanol. The deprotection of the Dde group was carried out by treatment with a $2 \%$ hydrazine hydrate solution in DMF under continuous flow conditions for 3 minutes (Figure 2.27). The base stability of the Dde 
group was found to be superior to that of its predecessor. A peptidyl-resin containing a Lys residue with its side chain protected with Dde was treated with a $20 \%$ piperidine solution in DMF for 2 and 4 hours and showed a mere $3 \%$ and $6.5 \%$ loss of the protecting group, respectively. It was argued that a total exposure time of 2 hours with $20 \%$ piperidine represents $17 \times 7$ minutes of Fmoc deprotection cycles on a peptide synthesiser; this would allow the synthesis of medium-sized peptides with insignificant loss of the Dde group.
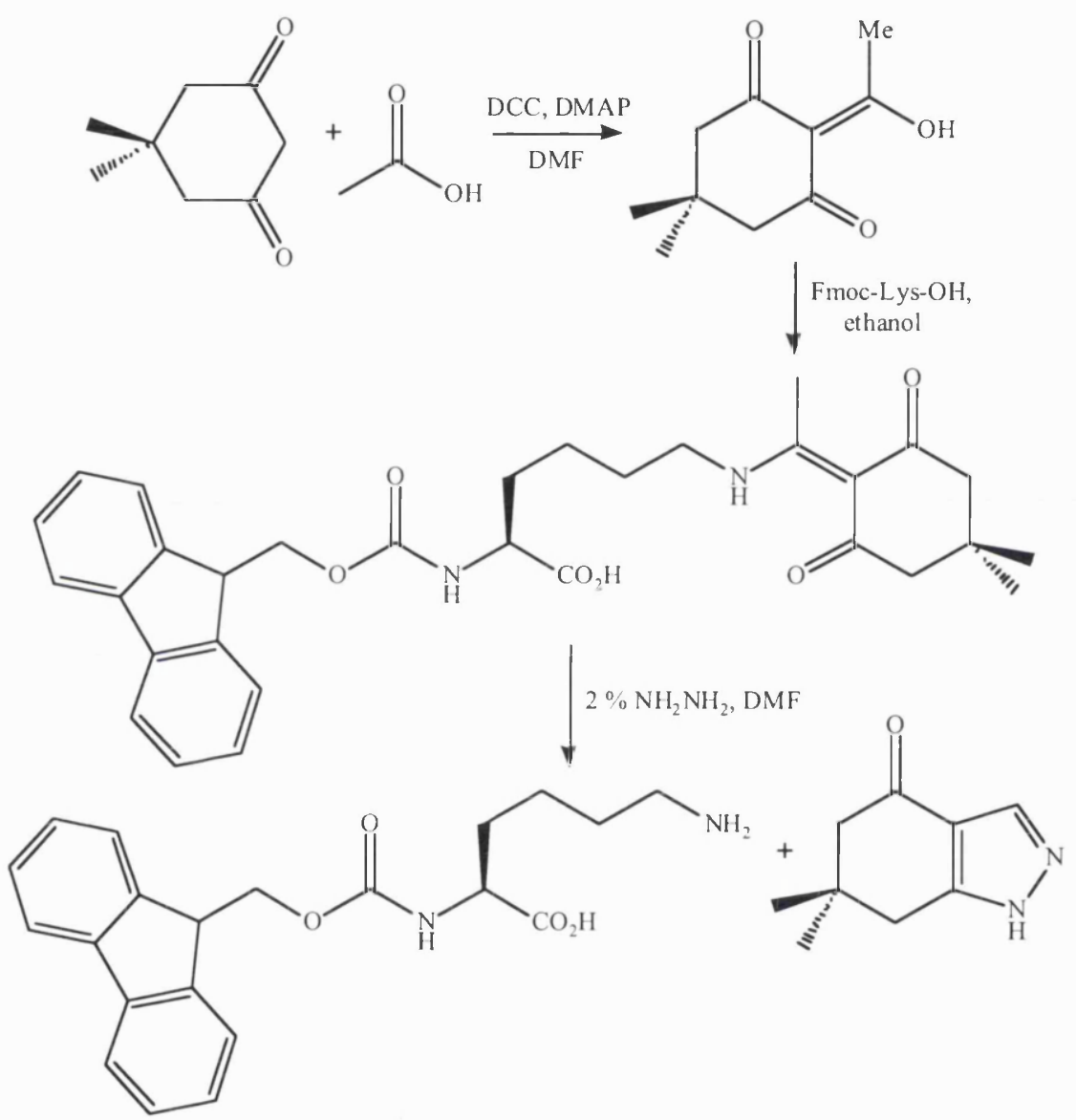

Figure 2.27. Synthesis of Dde, its incorporation onto Fmoc-Lys-OH and its deprotection

The isovaleryl derivative of Dde (N-1-(4,4-dimethyl-2,6-dioxocyclohexylidene) isovaleryl), previously known as Ddiv and currently known as ivDde (Figure 2.28), was developed mainly due to the recurring problem of Dde migration in peptides with unprotected Lys side chains during Fmoc deprotection. ${ }^{153}$ It was observed that neat DMF displaces the Dde group and the presence of piperidine during Fmoc deprotection creates an unstable piperidine-Dde adduct which in turn undergoes 
nucleophilic attack by an adjacent unprotected Lys side chain. Although the problem could be alleviated by using DBU for Fmoc removal, the introduction of the more hindered ivDde protecting group eliminates the problem almost completely. ${ }^{154}$

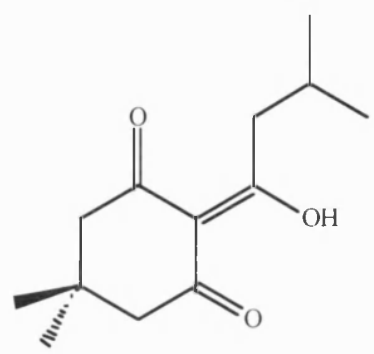

Figure 2.28. Structure of ivDde

Dcm, Dde and ivDde are primary amino protecting groups. The use of a pair of protecting groups in SPPS - one each for amino and carboxyl functions - could be useful for simultaneous deprotection, as seen for the allyl/Aloc, Bz/Z, Fm/Fmoc and ${ }^{t} \mathrm{Bu} /$ Boc pairs. The novel Dab ( $N$-Dde-4-aminobenzyl) carboxylic acid protecting group was therefore developed, based on the safety-catch principle, utilising the lability of the $N$-Dde and 4-aminobenzyl ester functions. ${ }^{155}$ It was synthesised from 4-aminobenzyl alcohol and Dde-OH, and the carboxylic acid moiety was subsequently esterified with the benzyl alcohol derivative via activation as the pivaloyl mixed anhydride in very good yields (Figure 2.29).

The deprotection of Dab is a two-step mechanism involving hydrazinolytic removal of the Dde moiety followed by spontaneous collapse of the 4-aminobenzyl ester group via a 1,6-elimination. ${ }^{155,156}$ This, combined with the knowledge that the Dde group is mildly unstable to $20 \%$ piperidine in DMF suggested that the Dab group, also, would be unstable to Fmoc deprotection condition. This was certainly the case as Bycroft et al. discovered a $13 \%$ loss of the Dab group after exposure to $20 \%$ piperidine for 1 hour. ${ }^{156}$ The Dmab (4-[N-\{1-(4,4-dimethyl-2,6-dioxocyclohex-1ylidene)-3-methylbutyl \}amino]benzyl) protecting group was developed mainly due to the relative instability of the Dab group to Fmoc deprotection conditions. ${ }^{156}$ Due to the superiority of the ivDde group compared to its Dde predecessor, the Dmab group was developed by synthesising 4-aminobenzyl alcohol with 2-(3methylbutyryl)dimedone in THF under reflux. Dmab protection of the carboxylic 
acid of Fmoc-Glu( $\left(\mathrm{O}^{t} \mathrm{Bu}\right)-\mathrm{OH}$ using the resulting benzyl alcohol derivative was successfully carried out with DCC, HOBt and DIPEA in DCM at room temperature for 18 hours. Its deprotection mechanism is identical to that of Dab (Figure 2.30).

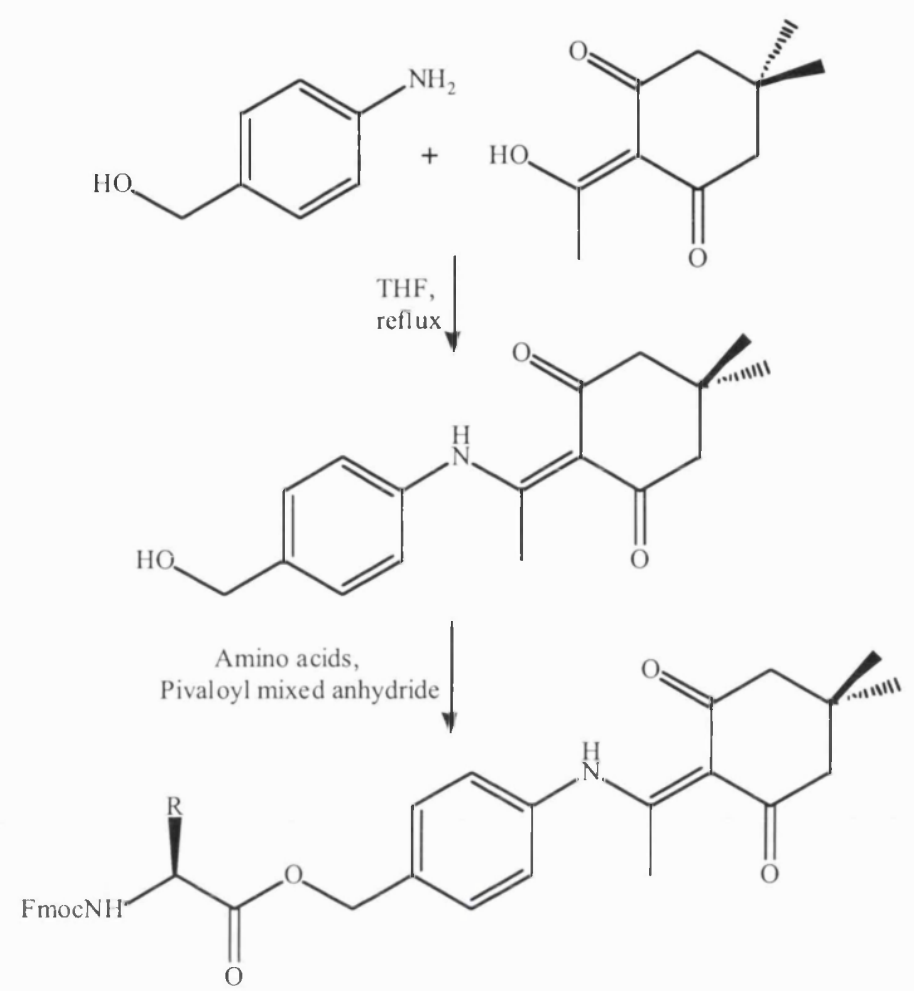

Figure 2.29. Synthesis of Dab and its incorporation onto amino acids

All the dimedone based protecting groups - Dcm, Dde, ivDde, Dab, Dmab - have been used in Fmoc SPPS as part of a triply orthogonal protecting group system,

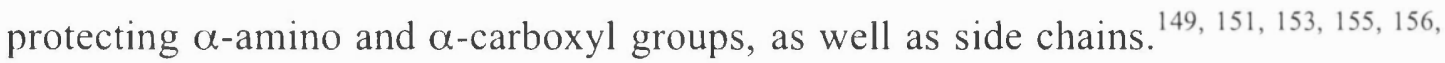
$157,158,159,160,161$

The use of Dmab in SPPS as a side chain protection to Glu is not without problems. Johnson et al. attempted to use it as an orthogonal side chain protecting group for the synthesis of side chain to side chain lactam bridged peptides. ${ }^{161}$ The use of Dmab resulted in the formation of the pyroglutamyl ring via an intramolecular nucleophilic attack of the secondary $N$-terminus to the activated $\gamma$-carboxyl of Glu in the presence of base (Figure 2.31). This shortens the peptide and contaminates the product, where the truncated by-product was found to be $15 \%$ of the final product. 


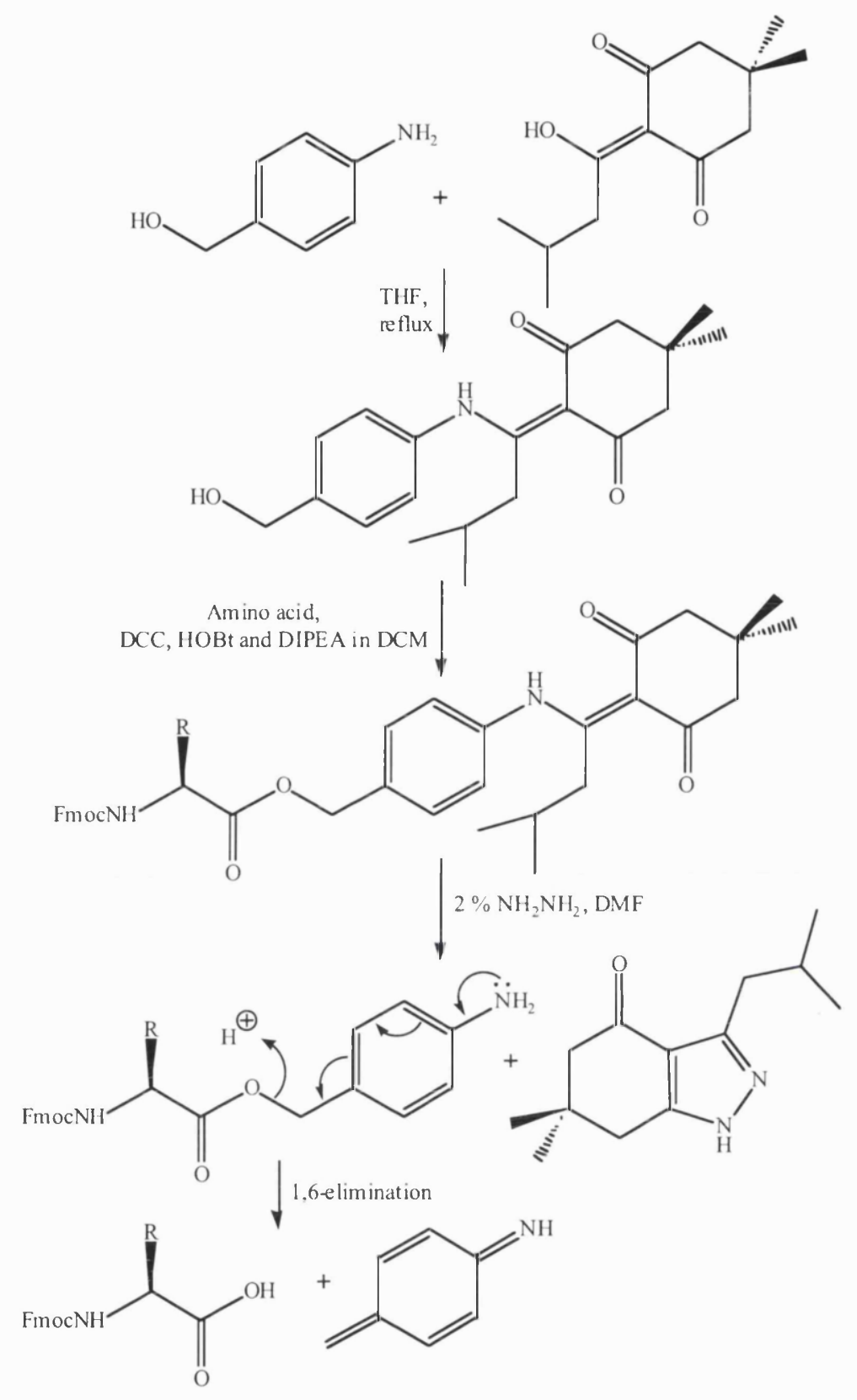

Figure 2.30. Synthesis of Dmab and its protection of and deprotection from amino acids

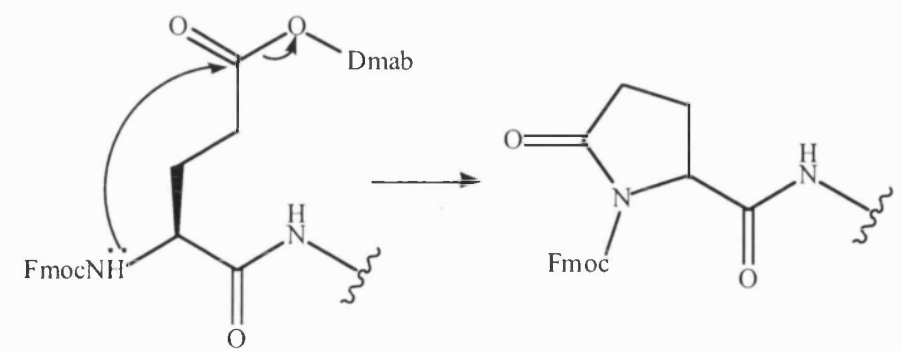

Figure 2.31. Formation of the pyroglutamyl ring on Glu 
There were also by-products that corresponded to the uncyclised peptide with an excess mass of 105. The limiting step for the Dmab deprotection is the disintegration of the 4-aminobenzyl ester group. Despite its ephemeral existence, the species was isolated by HPLC. This prevents the sufficient activation needed for the $\gamma$-carboxyl of Glu hence hindering cyclisation with the Lys side chain (Figure 2.32). ${ }^{161}$

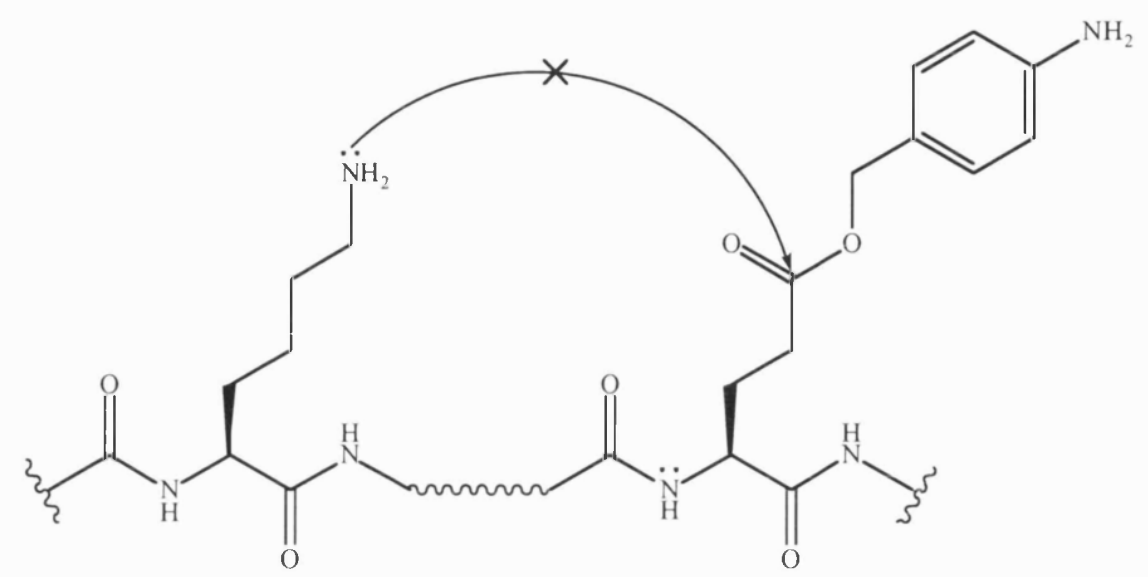

Figure 2.32. Unsuccessful cyclisation due to incomplete removal of Dmab

Another problem discovered with the use of dimedone based protecting groups was their partial incompatibility with allylic protecting groups. It was shown that the deprotection of Dde with hydrazine hydrate in the presence of Aloc, even for 5 minutes, led to some reduction of the Aloc double bond. ${ }^{159}$ This then, in turn, led to the failure of the subsequent Aloc deprotection step. It was thought that the cause of the problem was the presence of a small amount of diimide impurity in hydrazine hydrate, often formed in situ, arising from traces of oxidising agents or base. This problem, however, can be solved by means of degassing the solutions used and shortening deprotection times. However, the most advantageous method involved the use of 200 equivalents of allyl alcohol, acting as scavengers to the diimides. This made the dimedone based protecting groups completely orthogonal to the Fmoc ${ }^{t} \mathrm{Bu} /$ allyl system. 


\subsection{Synthesis of cyclic and polycyclic peptides}

Numerous advances and improvements have been made in SPPS over the last 3 decades since Merrifield first realised the use of insoluble resin beads for the assembly of amino acids to synthesise peptides. With the discovery of the lantibiotics, and the biological importance of conformationally restrained peptides, the synthesis of polycyclic peptides has been and remains a challenging undertaking for synthetic chemists in the peptide field. Nisin was synthesised via fragment condensation of individually prepared monocyclic structures in solution to produce the target peptide (see section 2.6.1). ${ }^{162}$ This is the more common method of synthesising polycyclic peptides.

The oxime resin ${ }^{163}$ has been widely used for the synthesis of monocyclic peptides. The nature of the utilisation of the oxime resin for the synthesis of cyclic peptides does not allow for the synthesis of polycyclic peptides. Its mechanism for an on-resin peptide cyclisation leads to a concomitant detachment of the peptide from the resin (Figure 2.33). The incorporation of additional cyclic structures would have to be carried out via segment condensation in solution.

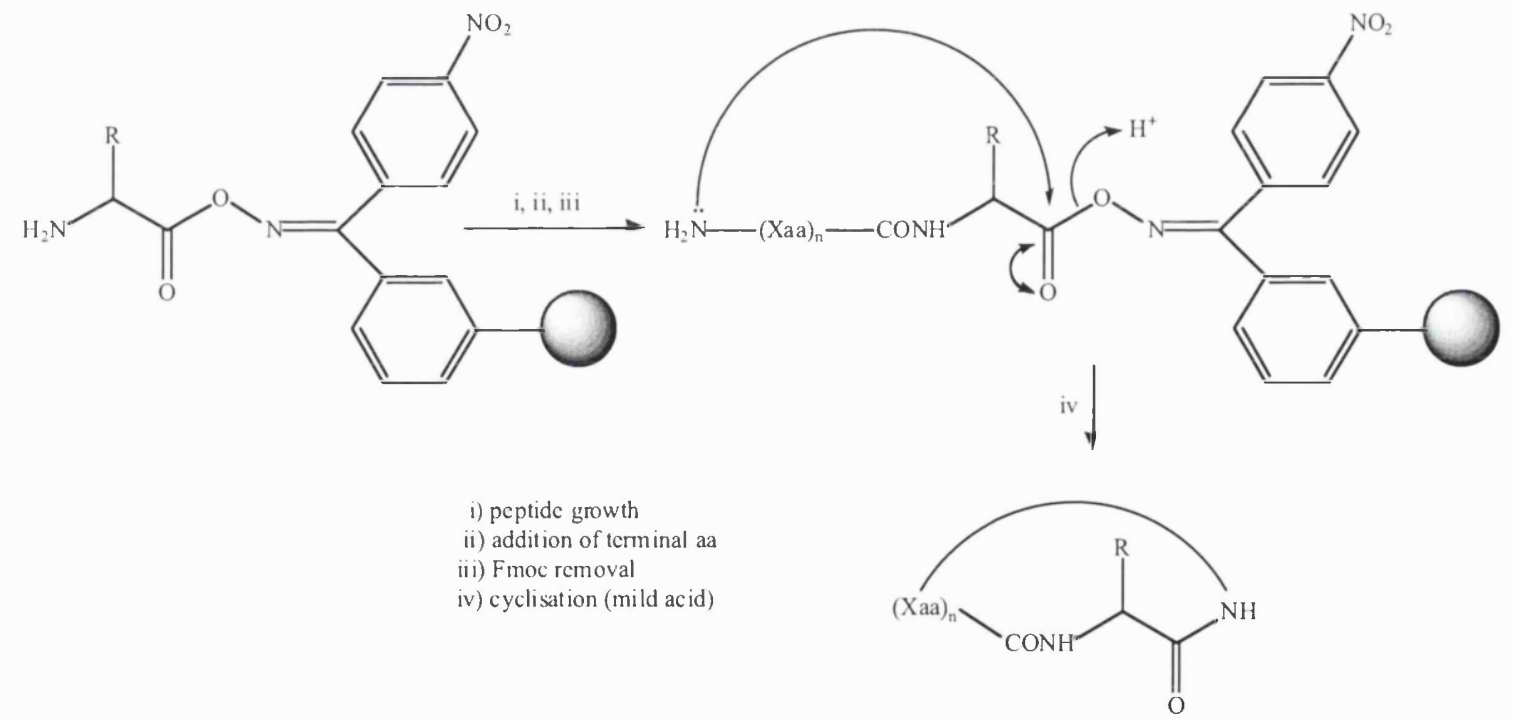

Figure 2.33. Peptide cyclisation mechanism on an oxime resin

Lee $^{164}$ synthesised an octadepsipeptide using the oxime resin in high yields. Although not eventually part of a bigger peptide structure, the synthesis of the 
peptide clearly highlights the limiting use of the oxime resin. In fact, Kaiser et al. ${ }^{163}$ and Ösapay and Taylor ${ }^{165 a}$ demonstrated that fully protected peptide fragments may be synthesised using the oxime resin, which are then combined via segment condensation to produce the final target peptides.

\subsubsection{Multiply-orthogonal protecting group strategies for SPPS}

An orthogonal protecting group system is one where the protecting groups may be removed independently of each other. The use of two orthogonal protecting groups is known as a two-dimensional orthogonal protecting group system and theoretically this can be as high as a six-dimensional orthogonal system with the wide availability of resins and linkers with varying degrees of sensitivities to acids, bases, metal catalysts, nucleophiles and U.V. radiation.

There have been numerous reports on the on-resin synthesis of mono- and polycyclic peptides utilising the orthogonality of various protecting groups. These cyclic peptides (Figure 2.34) are formed either with head-to-tail (a), tail-to-side chain (b), side chain-to-head (c) or side chain-to-side chain (d) cyclisation. These cyclisation strategies have recently been comprehensively reviewed ${ }^{165 b}$ and the following section therefore gives a brief overview of examples for each pathway.

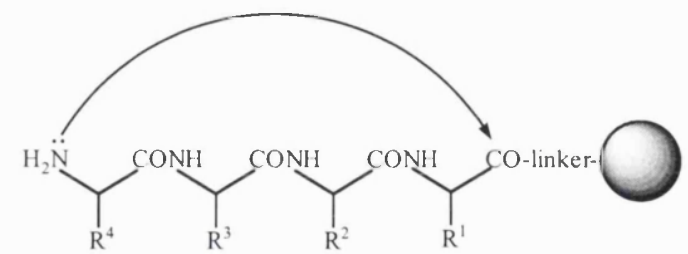

(a)

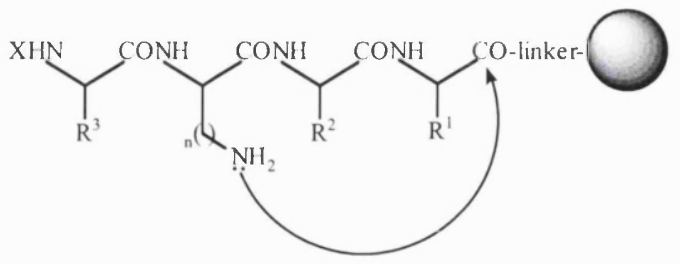

(c)

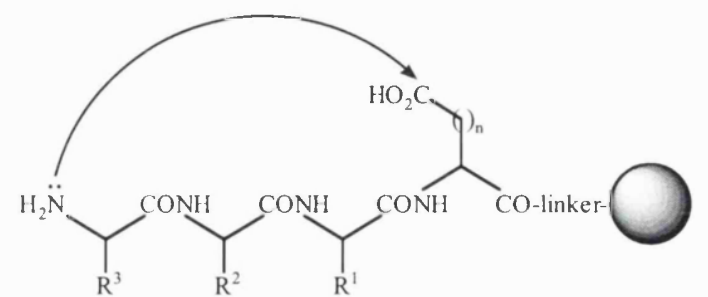

(b)

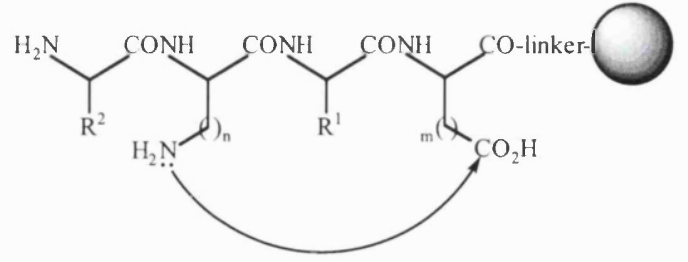

(d)

Figure 2.34. On-resin peptide cyclisation: (a) head-to-tail (b) tail-to-side chain (c) side chain-to-head and (d) side chain-to-side chain 
Albericio et al. reported the synthesis of monocyclic peptides utilising a threedimensional orthogonal protecting group strategy with side chain attachment to the resin and a head-to-tail cyclisation (Figure 2.35). ${ }^{142}$ Cyclisation was achieved using BOP in the presence of HOAt and a base. The use of an Fmoc/ $\mathrm{Bu} / \mathrm{allyl}$ system is perhaps the most commonly used three-dimensional orthogonal protecting group strategy.

Albericio and Carpino et al. synthesised a monocyclic peptide, again utilising the orthogonal properties of Fmoc ${ }^{t} \mathrm{Bu} / \mathrm{allyl}$ with head-to-tail cyclisation carried out using the superior coupling reagents PyAOP-HOAt (Figure 2.36). ${ }^{114}$
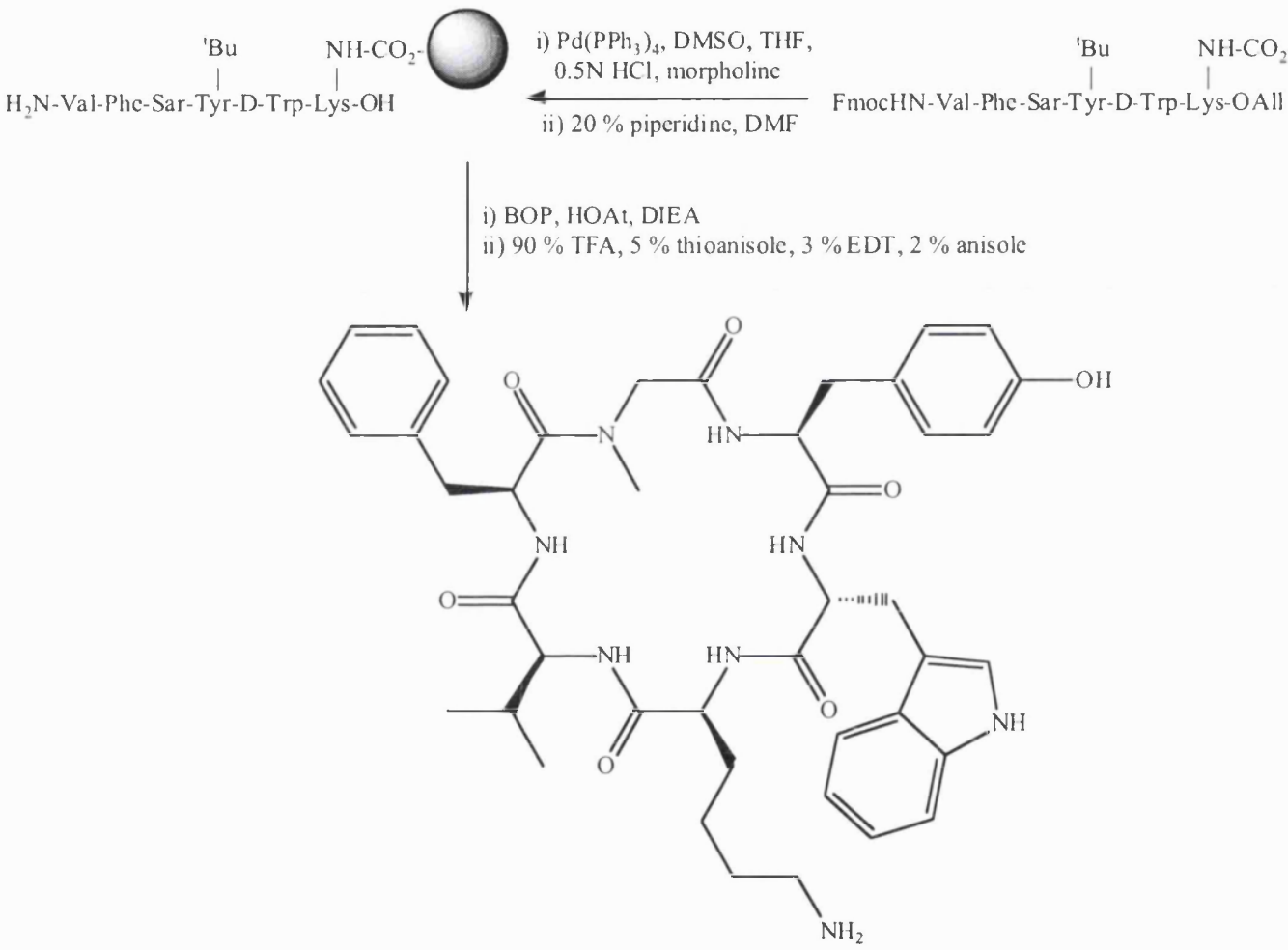

Figure 2.35. On-resin head-to-tail cyclisation via Lys side chain anchoring

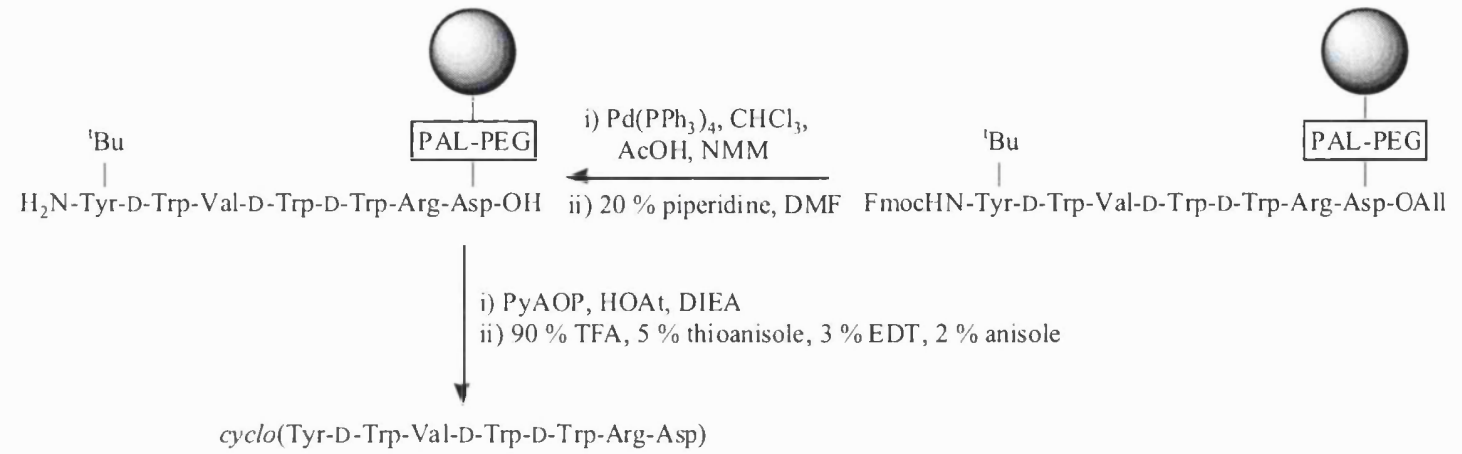

Figure 2.36. On-resin head-to-tail cyclisation via Asp side chain anchoring 
Sharma et al. also reported the synthesis of monocyclic peptides utilising a threedimensional orthogonal protecting group strategy, but with a side chain-to-head cyclisation (Figure 2.37). ${ }^{100}$ The synthesis was carried out utilising the orthogonality of the Fmoc, Boc $/{ }^{t} \mathrm{Bu}$ and Dde protecting groups.

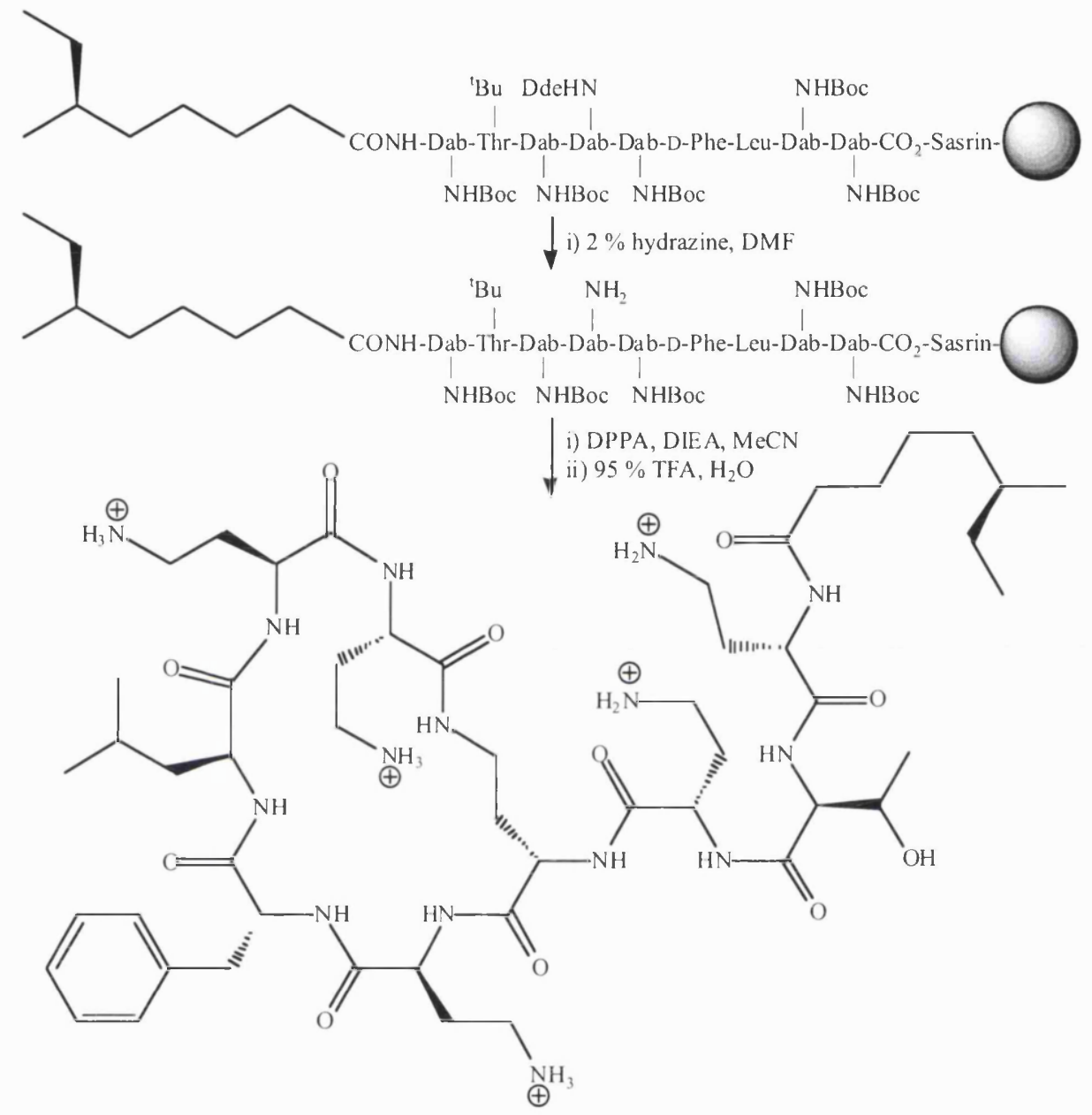

Figure 2.37. Solid phase synthesis of a monocyclic peptide

Bloomberg et al. reported the use of a four-dimensional protecting group strategy with a "tail-to-side chain" peptide cyclisation (Figure 2.38). ${ }^{129}$ The synthesis was carried out utilising the orthogonality of the Fmoc, Boc $/{ }^{t} \mathrm{Bu}$, allyl and Dde protecting groups and cyclisation was achieved with the use of HBTU, HOBt and base.

Taylor and Zhang reported the use of a four-dimensional protecting group strategy with double side chain-to-side chain cyclisation steps, creating a bi-cyclic peptide (Figure 2.39). ${ }^{166}$ The ingenious manipulation of protecting groups - Boc/allyl and 
Aloc/Fm and Fmoc/Bzl and $\mathrm{ClZ}$ - made way for the synthesis of this overlapping bicyclic peptide. Cyclisations via lactam bridge formation were carried out using BOP.

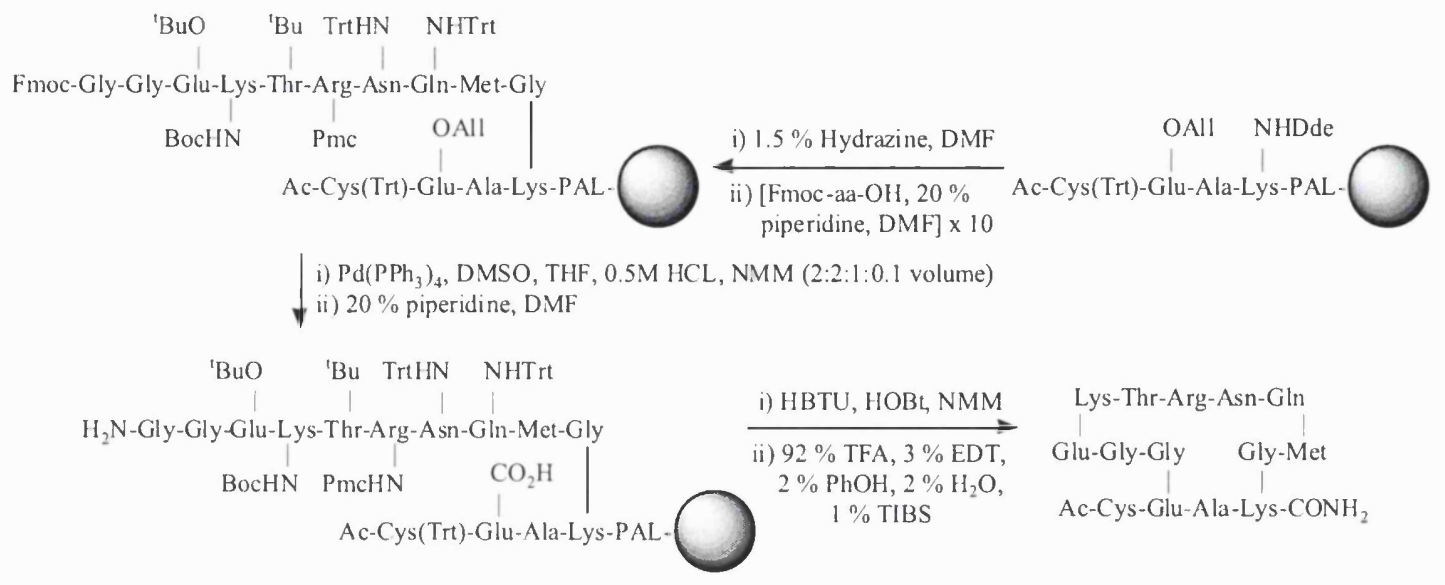

Figure 2.38. Synthesis of a branched cyclic peptide via a three-dimensional orthogonal protecting group strategy

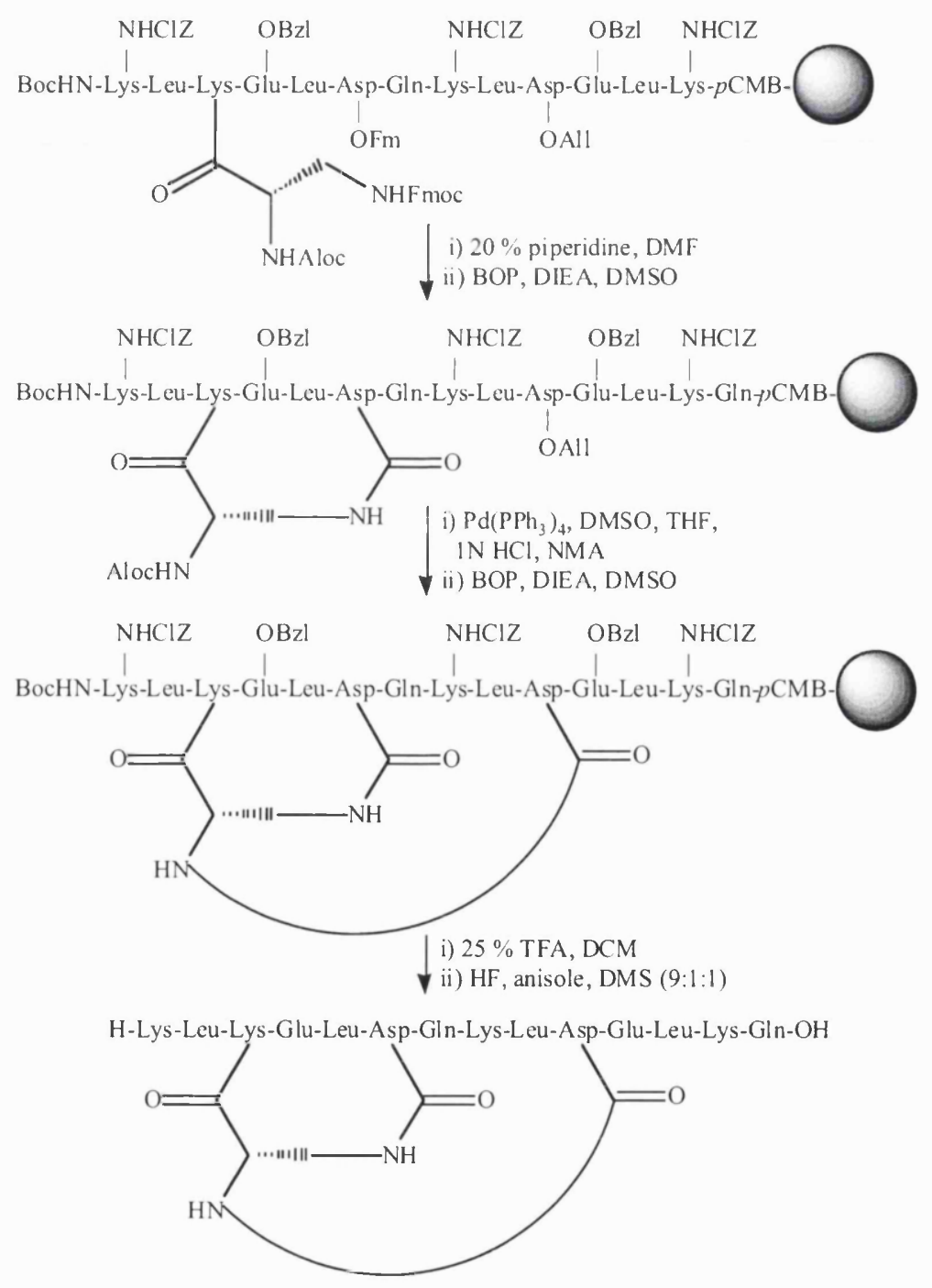

Figure 2.39. Synthesis of an overlapping bi-cyclic peptide 


\subsection{Synthesis of lanthionine-containing peptides}

There have been numerous reports on the synthesis of lanthionine-containing analogues of biologically active peptides, as described in section 1.4. and one report of the total synthesis of a complete lantibiotic, nisin.

\subsubsection{Sulfur extrusion}

This sulfur extrusion strategy was successfully employed by Shiba et al., ${ }^{162}$ in the presence of amino acids "under" the thioether bridge instead of the ethylene glycol tether in Figure 1.16, in their total synthesis of nisin. So far nisin is the only lantibiotic that has been successfully synthesised. All five ring structures of nisin were individually synthesised (with the exception of rings D and E which were synthesised as a bi-cyclic peptide) via solution peptide synthesis, employing Boc methodology, in the form of disulfide cyclic peptides. One sulfur atom from each cyclic structure was then extruded, using tris(diethylamino)phosphine, forming the desired thioether linkage and the rings were assembled in solution via segment condensation forming the target lantibiotic (Figure 2.40).

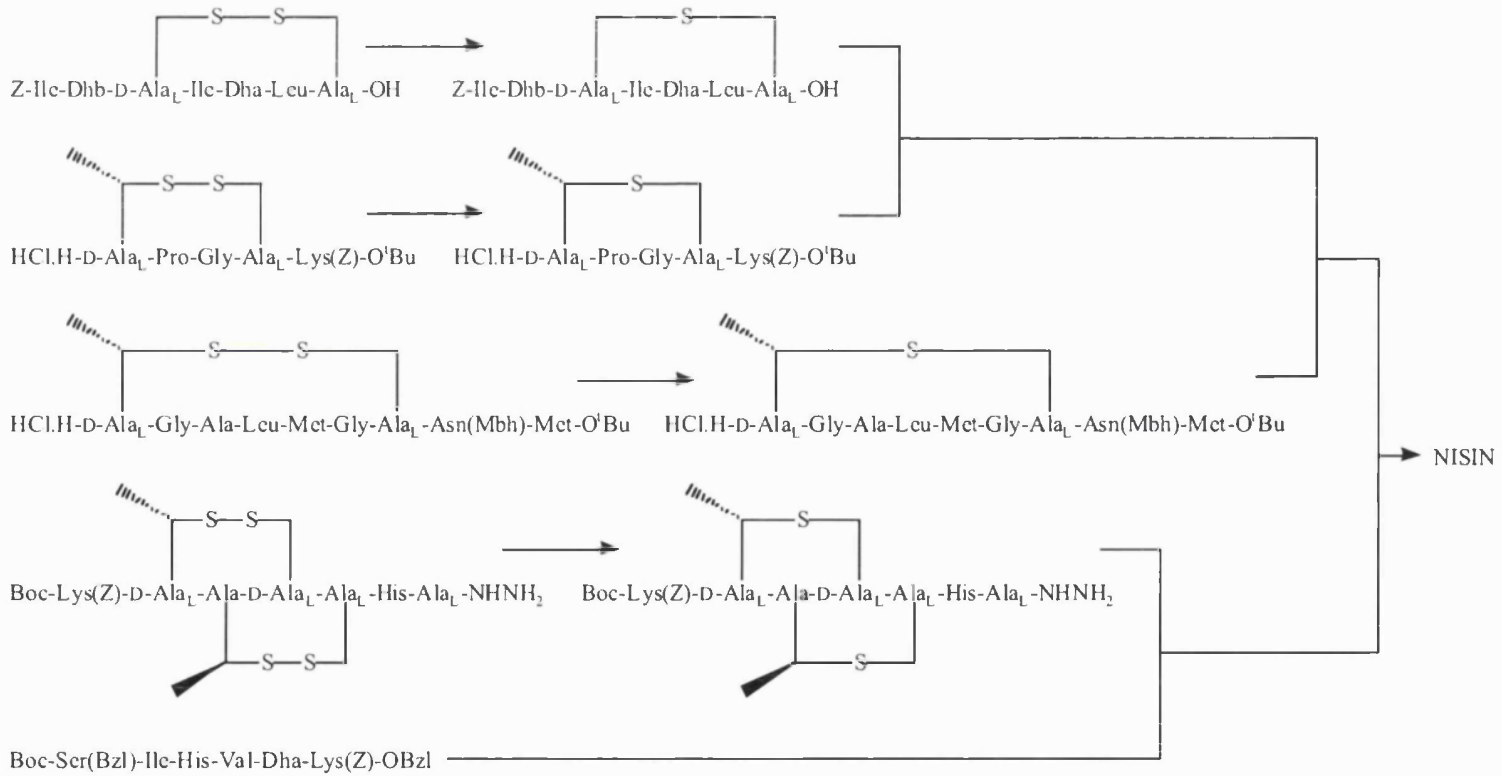

Figure 2.40. Schematics for the synthesis of nisin 


\subsubsection{Biomimetic approach}

The enkephalin opioid analogue shown in Figure 2.41 was synthesised on the solid phase with cyclisation achieved via side-chain Michael addition as described in section $1.5 .2^{50}$

Similarly, Bradley et al. applied the strategy on peptides (subtilin ring B) containing dehydroalanine and cysteine residues and achieved regio- and stereospecific cyclisation, mimicking the biosynthesis of lantibiotics (Figure 2.42) ${ }^{167}$ It seems that in a synthesis resulting in a constrained, cyclic environment the formation of the thioether bridge may favour the correct stereochemistry, forming the desired mesolanthionine moiety, which was also observed in the synthesis of ring $E$ of subtilin.

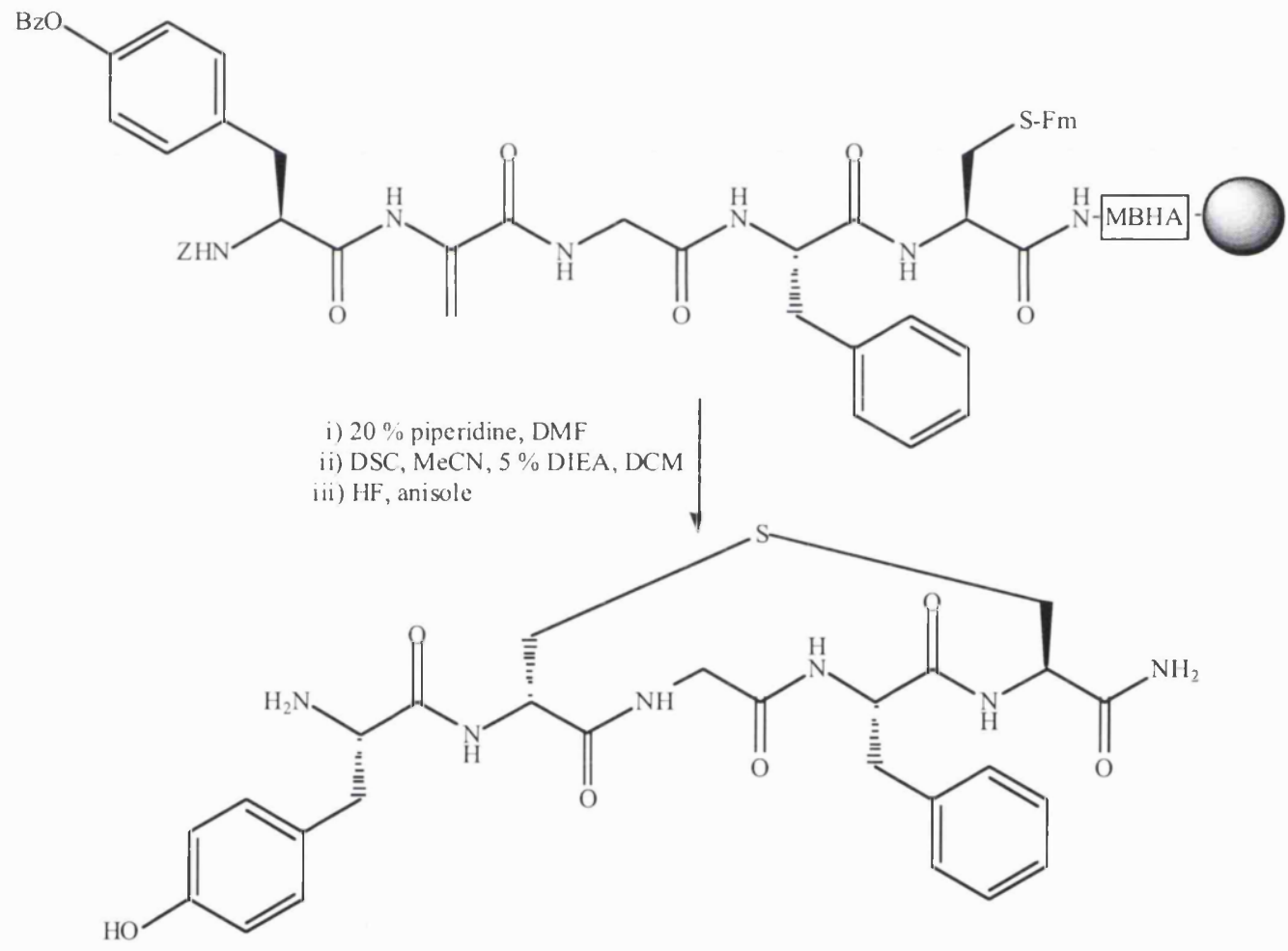

Figure 2.41. Synthesis of a lanthionine analogue of an enkephalin opioid peptide 


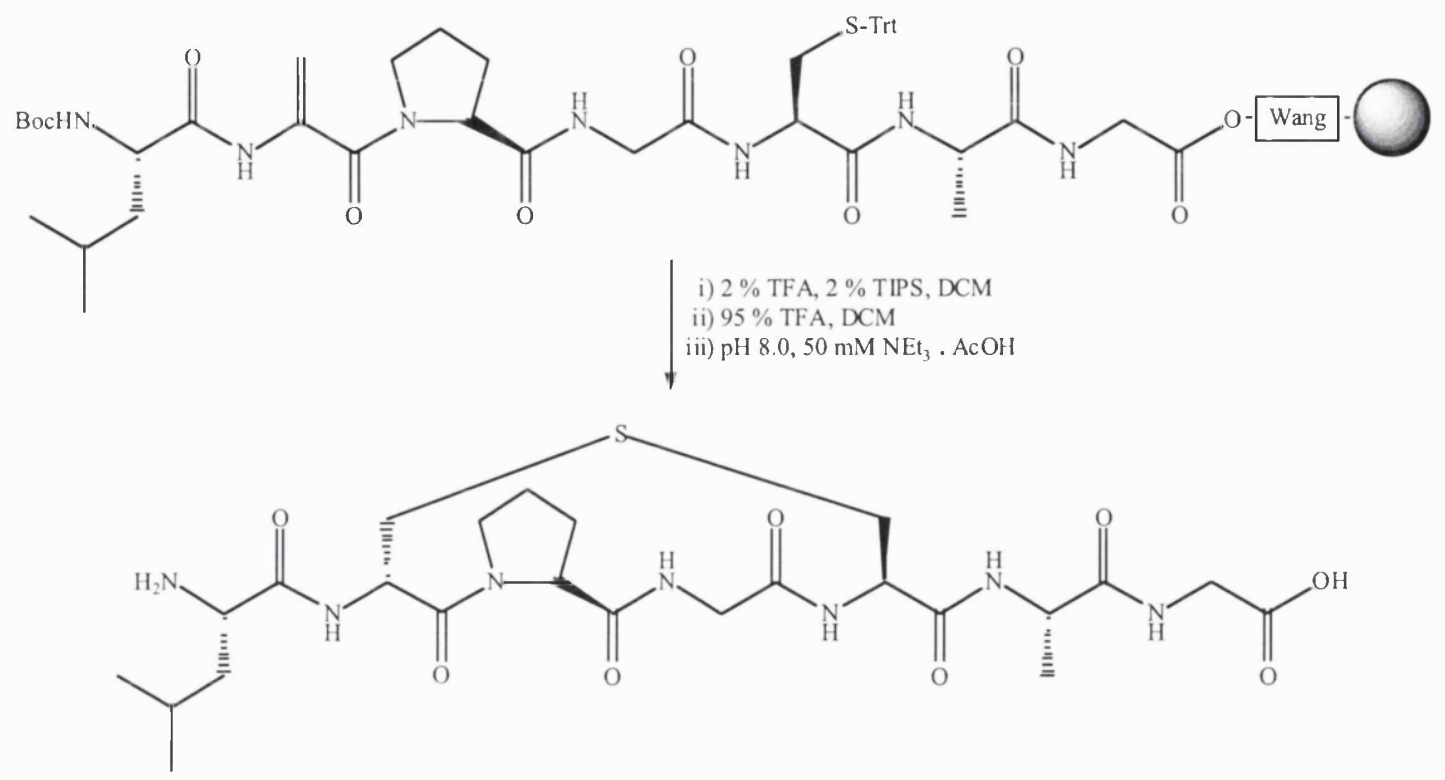

Figure 2.42. Formation of ring $\mathrm{B}$ of subtilin/nisin via a stereospecific Michael addition

However, the same methodology applied to the synthesis of ring A of subtilin produced a diastereoisomeric mixture of peptides, although the Michael addition was completely regiospecific in the presence of two dehydroalanine residues.

Despite the potential of this approach it is difficult to envisage its application to the synthesis of overlapping lanthionine- or 3-methyllanthionine-bridged peptides, which are widespread and prominent in most lantibiotics.

\subsubsection{Serine $\beta$-lactones}

The use of a lanthionine monomer, synthesised from serine- $\beta$-lactone derivatives, in the synthesis of cyclic peptides has also been reported. ${ }^{52,59,61,62}$ In each case the amino acid was synthesised along with its thioester by-product, as described in section 1.5.3. Lan-7 was synthesised in solution from lanthionine prepared via this method (Figure 2.43). ${ }^{59}$ 


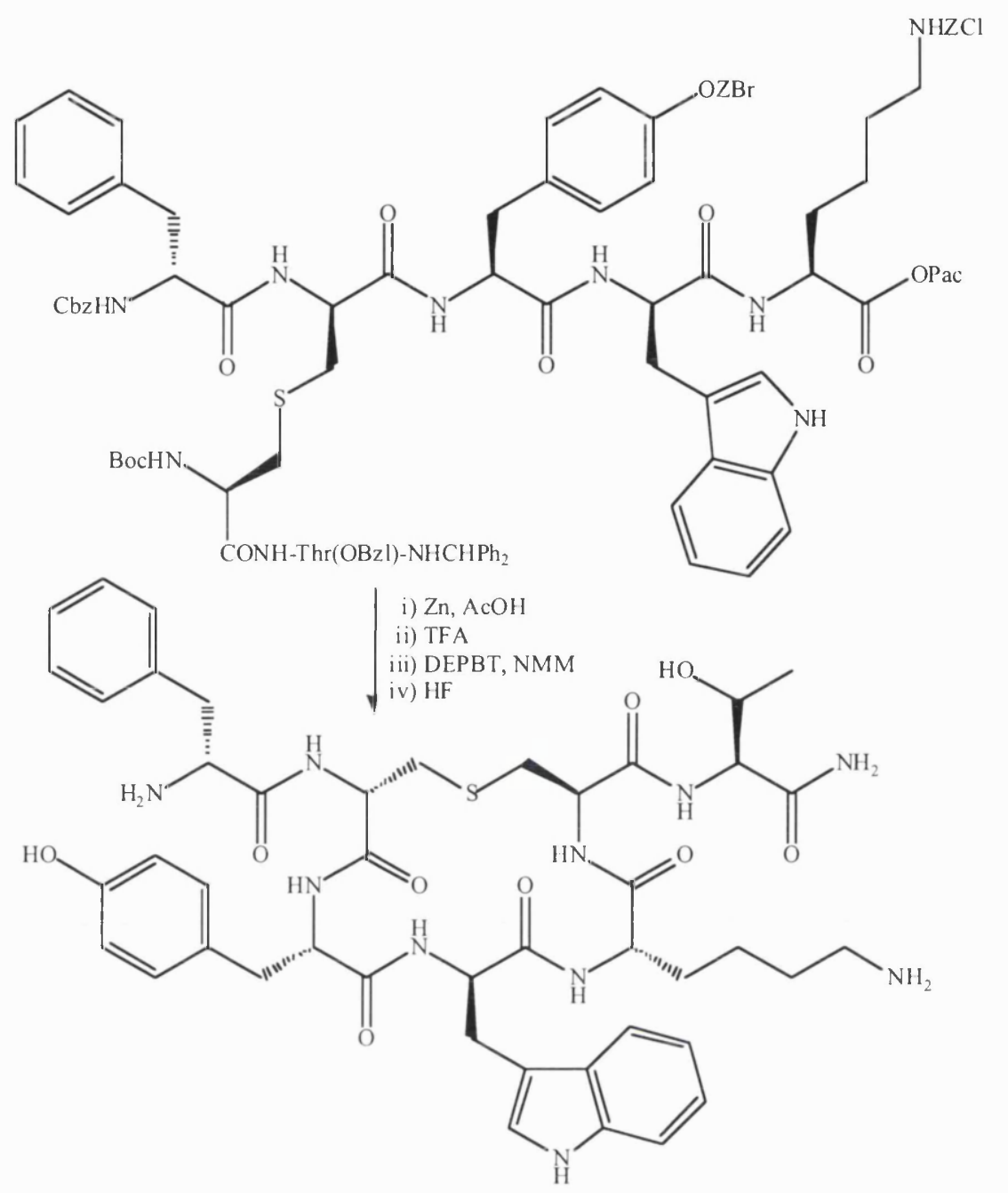

Figure 2.43. Synthesis of Lan-7 in solution

The lanthionine-somatostatin analogue in Figure 2.44 was also prepared from lanthionine synthesised from a serine- $\beta$-lactone derivative. ${ }^{6}$ 


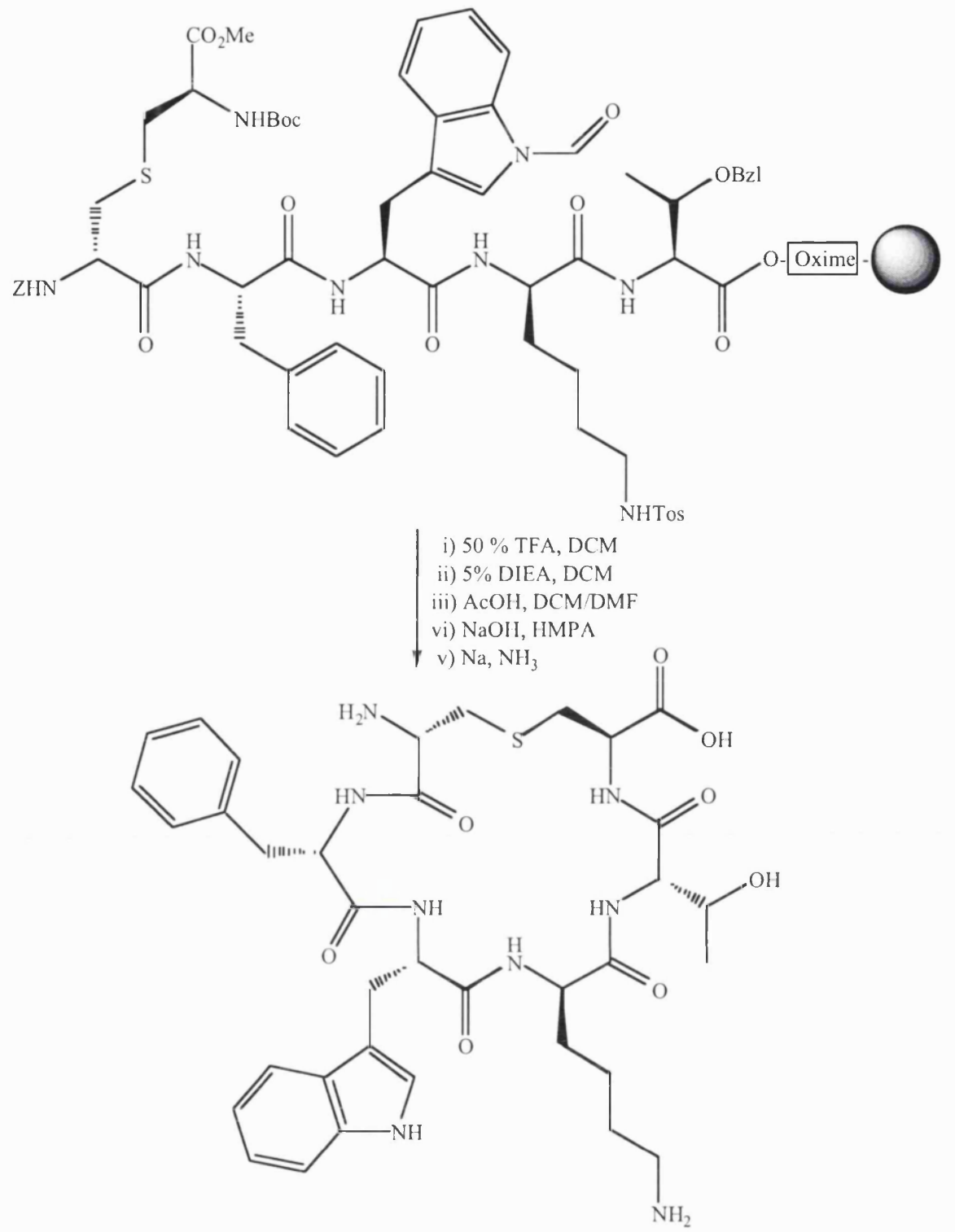

Figure 2.44. Synthesis of a lanthionine-somatostatin analogue

\subsection{4. $\beta$-Haloalanine derivatives}

The approach with the use of a cysteine-haloalanine coupling has also been used to synthesise lanthionine-containing cyclic peptides. In the synthesis of a $\beta, \beta$ dimethyllanthionine analogue of an opioid enkephalin, ${ }^{168}$ an iodoalanine derivative was treated with a peptide containing a $\beta, \beta$-dimethylcysteine in the presence of cesium carbonate to form a lanthionine analogue, within a short peptide chain. The cyclic structure was formed by cleavage of the peptide from the resin followed by cyclisation (Figure 2.45). There was no indication, however, on the presence of 
conformers at the iodoalanine derivative stage, which might have led to conformers in the peptide.

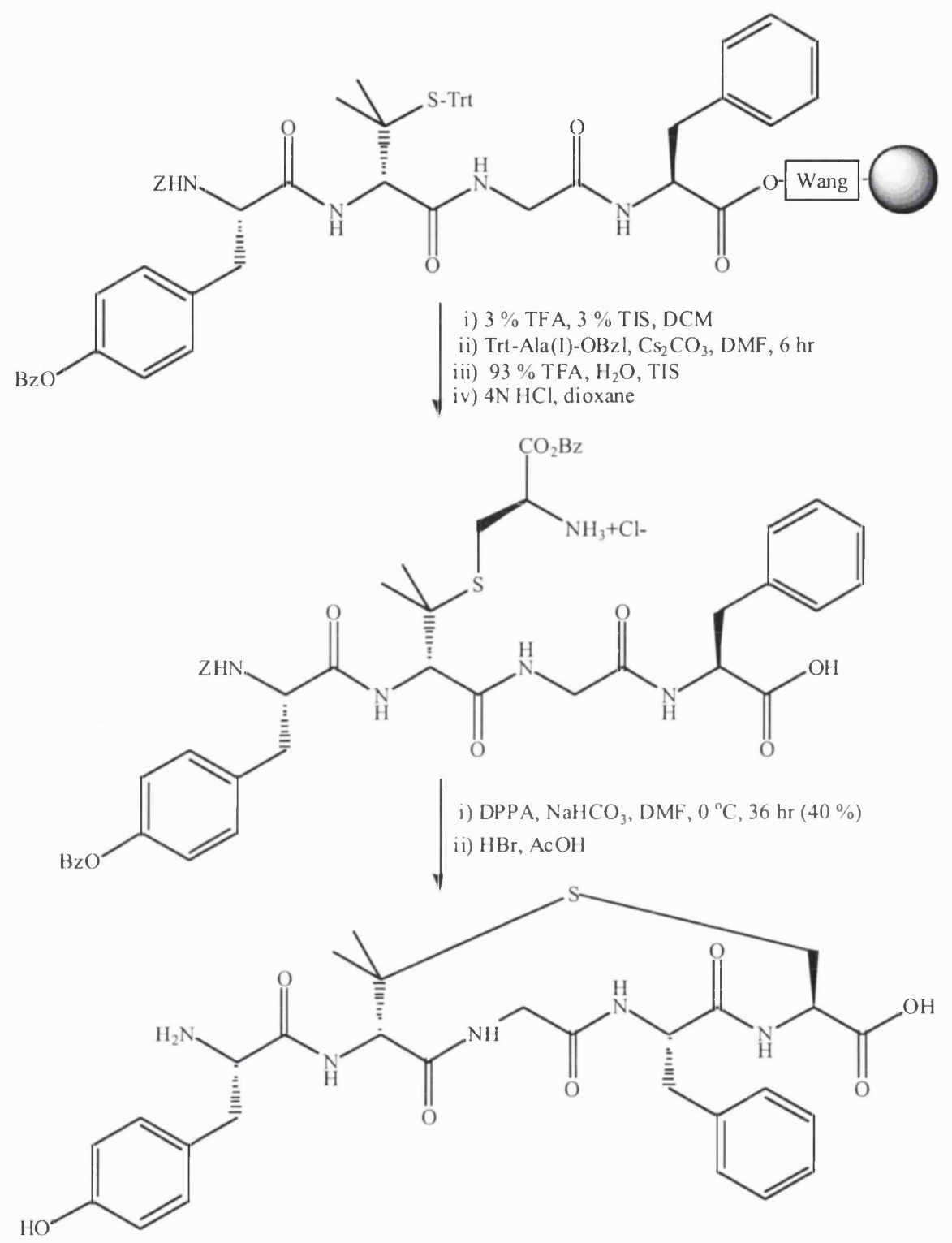

Figure 2.45. Synthesis of a $\beta, \beta$-dimethyllanthionine analogue of an enkephalin opioid

In theory, this particular route could be applied to synthesise polycyclic lanthioninecontaining peptides, especially ones that are more globular in conformation much like Type B lantibiotics, with some modifications to the choice of protecting groups.

In addition, there has also been a report of the use of a bromoalanine derivative in a peptidic on-resin macrocyclisation. ${ }^{53,54}$ Both enantiomers of serine were used in the 
assembly of the linear peptide with triphenylmethyl-protected cysteine residues. The serine residues were then converted to $\beta$-bromoalanine residues, allowing thiolate attack of the cysteine (Figure 2.46). The peptide is a cyclic analogue of an opioid enkephalin.

The use of both $(S)$ - and $(R)$-serine, resulting in $(R)$ - and $(S)$-bromoalanine, respectively, produced the same peptide with the correct lanthionine stereochemistry as seen in lantibiotics. It was concluded that the cyclisation via thioether formation went through a dehydroalanine intermediate resulting in a stereospecific Michael addition as reported by Bradley et al. ${ }^{167}$

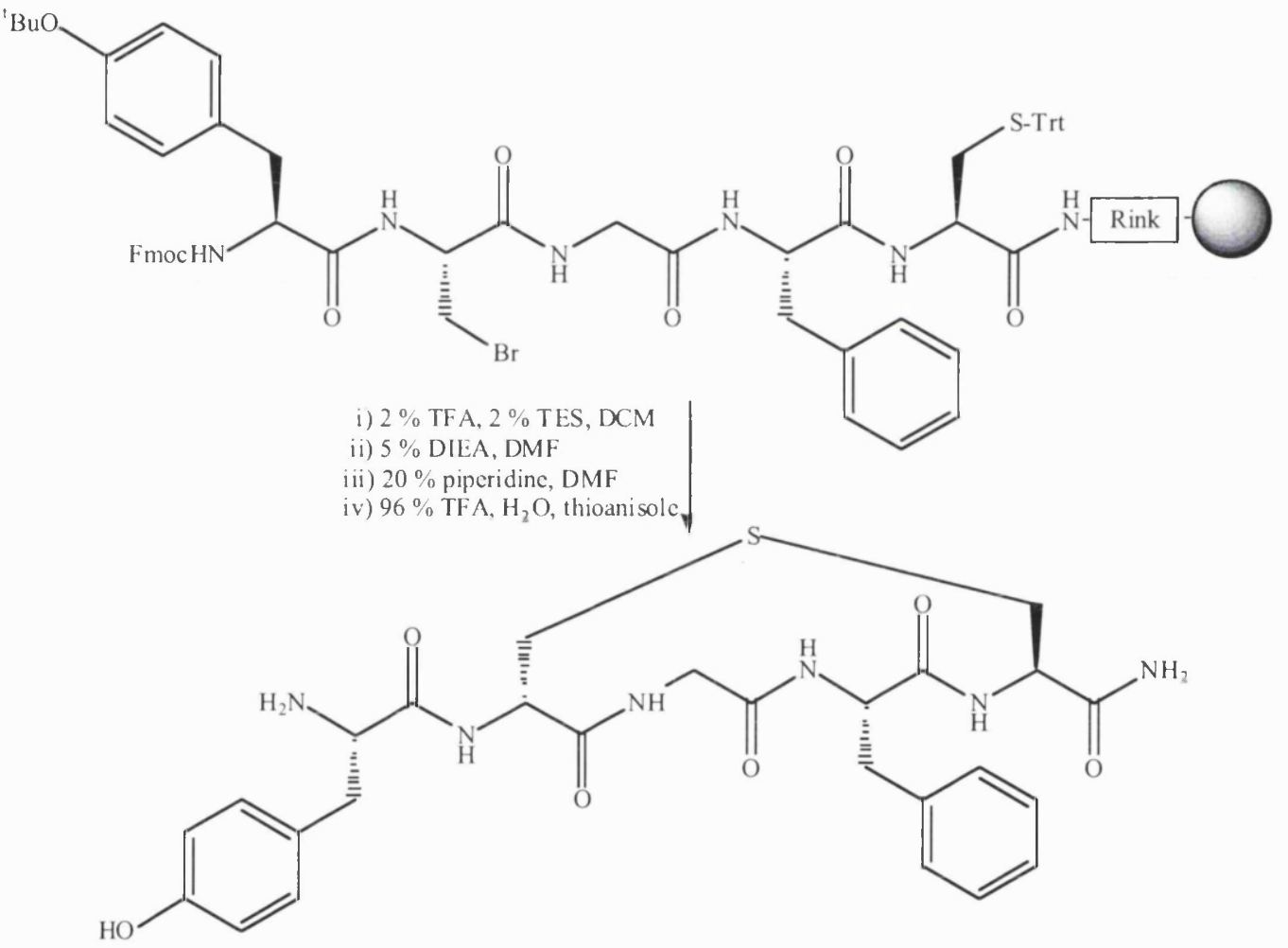

Figure 2.46. Lanthionine macrocyclisation by in situ activation of serine

The lanthionine-type cyclic peptide shown in Figure 1.12 was synthesised, on solid phase via Fmoc methodology, from a TBDMS-protected homoserine which was converted into a chloro-homoalanine with subsequent cyclisation with cysteine thiolate in the presence of base (Figure 2.47). ${ }^{65}$ 


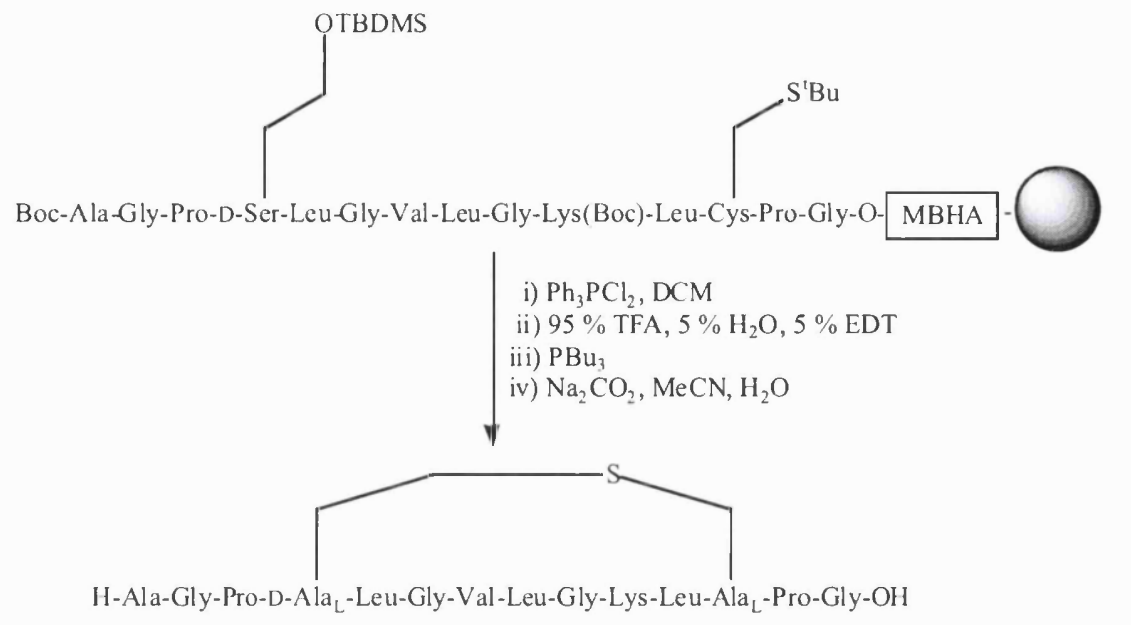

Figure 2.47. Synthesis of a thioether cyclic peptide 


\section{Chapter 3}

\section{Introduction III}

\subsection{Nucleophilic ring opening of aziridine-2-carboxylates}

Aziridine-2-carboxylates - either activated or not - can serve as key synthetic intermediates, to various unusual amino acids. This is due to their strained ring structure, analogous to other 3-membered cyclic ring structures such as cyclopropane and epoxides. Their susceptibility to various nucleophilic attacks has been reported.

Both soft and hard nucleophiles have been shown to ring-open aziridine-2carboxylates with varying results. The nucleophilic attack could occur at one of two 
positions on the ring $-C_{2}$ or $C_{3}$ - resulting in a $C_{2}-N$ or $C_{3}-N$ scission, respectively (Figure 3.1). Hard nucleophiles include amines, alcohols, azides and carbon nucleophiles and soft nucleophiles that have been used in the ring opening reactions of aziridine-2-carboxylates include thiols and halogens.

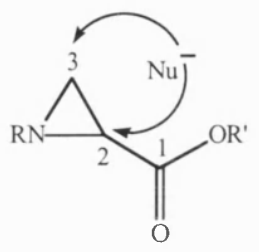

Figure 3.1. Nucleophilic ring opening at $C_{2}$ or $C_{3}$ of aziridine-2-carboxylates

One of the most common type of carbon nucleophiles is from organometallic reagents. ${ }^{169,170,171}$ Baldwin et al. demonstrated that $(R / S)$-1-benzyl-aziridine-2carboxylic acid tbutyl ester undergoes nucleophilic ring opening by the organocuprate reagent ${ }^{n} \mathrm{BuCu}(\mathrm{CN}) \mathrm{Li}_{2}$ in the presence of boron trifluoride etherate in modest yields (Figure 3.2). (S)-1-Tosyl-aziridine-2-carboxylic acid ${ }^{t}$ butyl ester can undergo ring opening by various Grignard reagents in the presence of a catalytic amount of $\mathrm{CuBr} . \mathrm{SMe}_{2}$. This suppressed the competing nucleophilic attack at the ester group on $C_{1} \cdot{ }^{170}$ Both types of reactions yielded a mixture of products from nucleophilic attack at $C_{2}\left(\alpha\right.$-amino acid) and $C_{3}(\beta$-amino acid) in moderate yields.

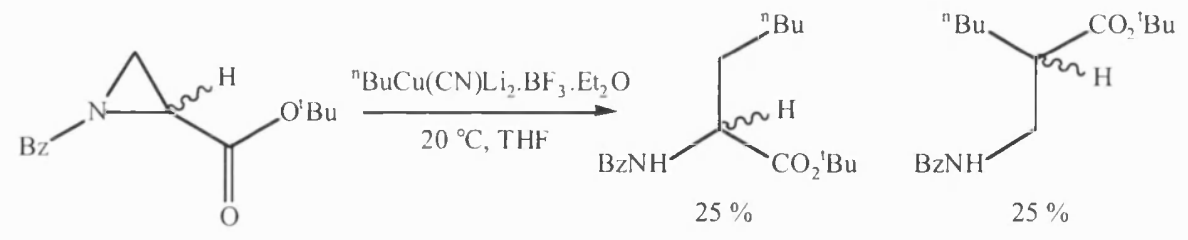

Figure 3.2. Reaction of an aziridine-2-carboxylate with ${ }^{n} \mathrm{BuCu}(\mathrm{CN}) \mathrm{Li}_{2}$

Young and Church, on the other hand, treated $(S)$-1-tosyl-aziridine-2-carboxylic acid with various organocuprate reagents in the absence of Lewis acids, yielding only the $\alpha$-amino acid products (Figure 3.3). ${ }^{169}$ 


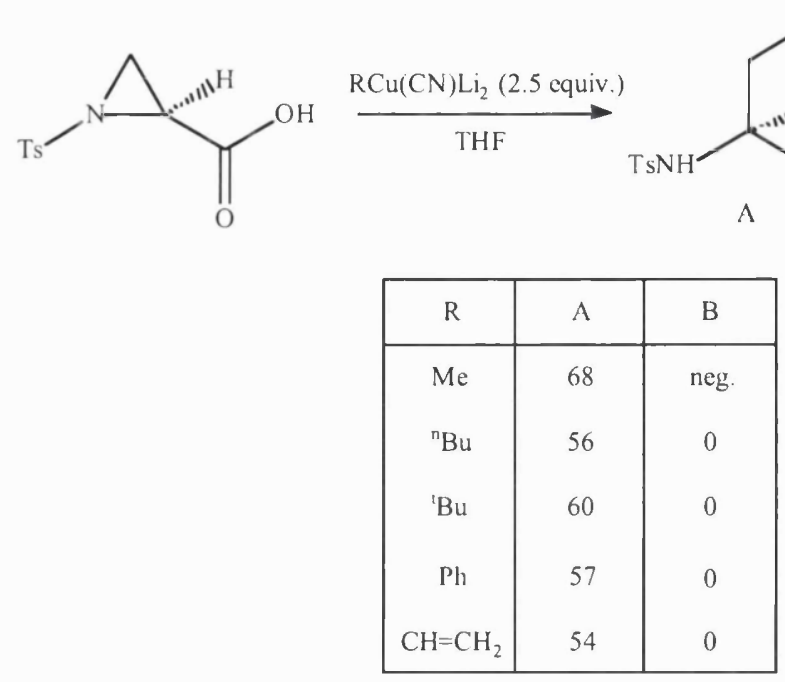

Figure 3.3. Reaction of an aziridine-2-carboxylic acid with various organocuprates

Baldwin et al. also demonstrated that the proportion of products from $C_{2}$ and $C_{3}$ attacks from $(S)$-1-tosyl-aziridine-2-carboxylic acid tbutyl ester varies with temperature and with the Grignard reagent (Figure 3.4). ${ }^{171}$ The bulky ${ }^{\mathrm{n}}$ butyl Grignard reagent prefers attack at $C_{3}$ whereas the methyl prefers attack at $C_{2}$, at $-25{ }^{\circ} \mathrm{C}$. It also seems that there is an optimal temperature for maximisation of yields.
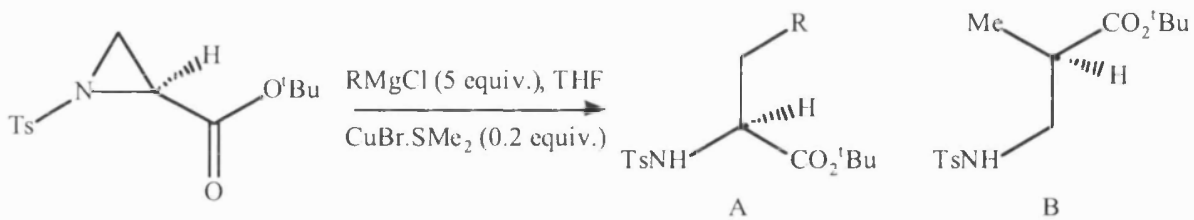

\begin{tabular}{|c|c|c|c|}
\hline $\mathrm{R}$ & $\mathrm{T} /{ }^{\circ} \mathrm{C}$ & $\mathrm{A}$ & $\mathrm{B}$ \\
\hline $\mathrm{Me}$ & -25 & 10 & 40 \\
$\mathrm{Me}$ & -20 & 30 & 55 \\
${ }^{\mathrm{n}} \mathrm{Bu}$ & -78 & 30 & 30 \\
${ }^{\mathrm{n}} \mathrm{Bu}$ & -25 & 50 & 20 \\
${ }^{\mathrm{n}} \mathrm{Bu}$ & -0 & 35 & 15 \\
${ }^{\mathrm{n}} \mathrm{Pr}$ & -16 & 42 & 21 \\
$\mathrm{Et}$ & -29 & 32 & 20 \\
\hline
\end{tabular}

Figure 3.4. Reaction of an aziridine-2-carboxylate with Grignard reagents in the presence of copper(I) bromide-dimethylsulfide 
Phosphonium ylides may also act as carbon nucleophiles. Baldwin et al. treated $(S)$ aziridine-2-carboxylic acid methyl ester derivatives with (triphenylphosphoranylidene)acetic acid ethyl ester in toluene and yielded ylide products from $C_{2}-N$ and $C_{3}-N$ scissions of the ring. ${ }^{172}$ It was also observed that $N$ amide $\left(\mathrm{COC}_{6} \mathrm{H}_{4} \mathrm{NO}_{2}-p\right)$ and $N$-sulphonyl $\left(\mathrm{SO}_{2} \mathrm{C}_{6} \mathrm{H}_{4} \mathrm{Me}-p\right)$ aziridine derivatives produced more of the $C_{3}-N$ cleavage product (yielding $\alpha$-amino acids) relative to the $\mathrm{C}_{2}-\mathrm{N}$ cleavage product and with higher combined yields, whilst $\mathrm{N}$-urethane $\left(\mathrm{CO}_{2} \mathrm{CH}_{2} \mathrm{C}_{6} \mathrm{H}_{4} \mathrm{NO}_{2}-p\right.$ and $\left.\mathrm{CO}_{2} \mathrm{CH}_{2} \mathrm{Ph}\right)$ aziridine derivatives yielded exclusively the $\mathrm{C}_{3}-\mathrm{N}$ cleavage product but with much lower yields (Figure 3.5). This is presumably due to the different electron withdrawing capacity of the two different types of protecting groups.

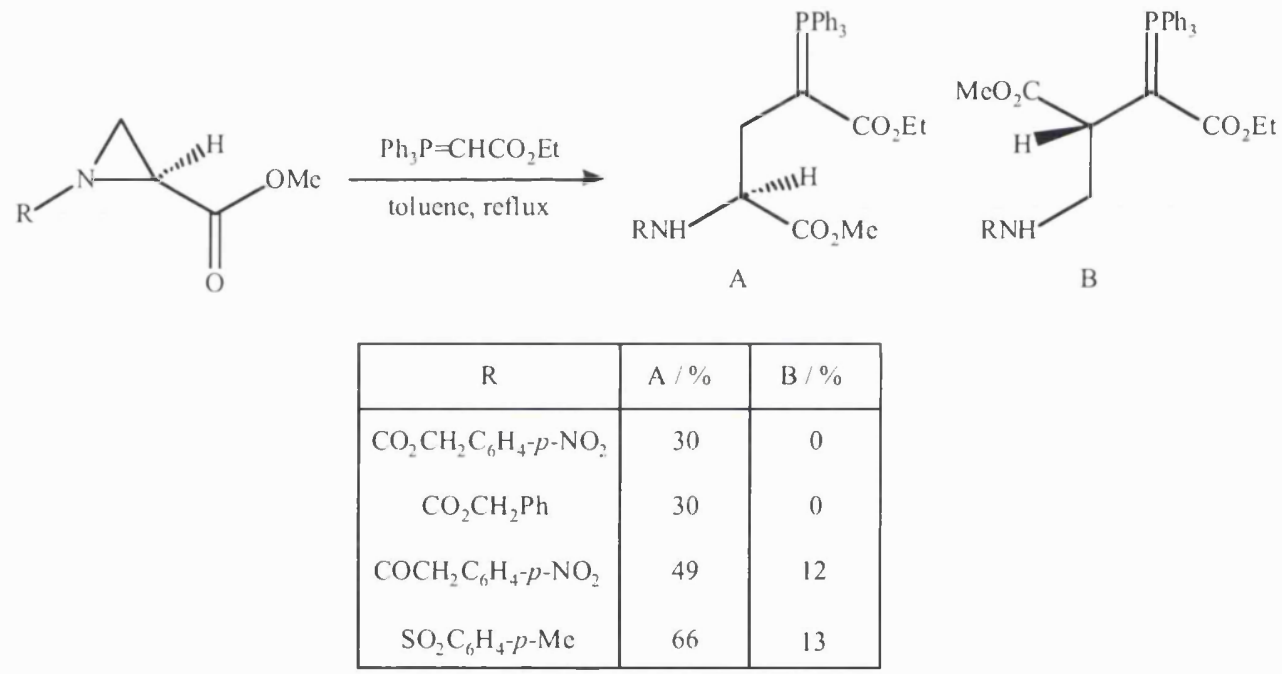

Figure 3.5. Reaction of an aziridine-2-carboxylates with a Wittig reagent

The ${ }^{11} \mathrm{C}$-labelled cyanide nucleophilic ring opening of $(R / S)-1$-'butoxycarbonylaziridine-2-carboxylic acid methyl ester has also been reported (Figure 3.6). ${ }^{173}$

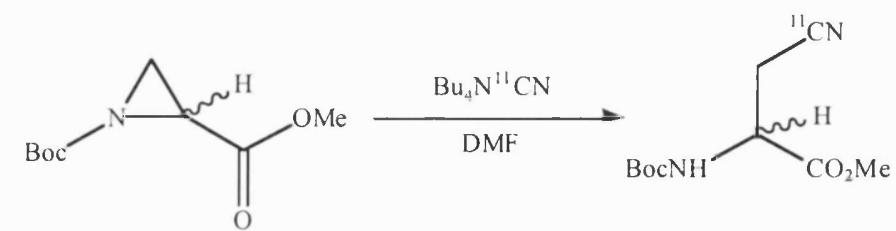

Figure 3.6. Aziridine-2-carboxylate reaction with tetrabutylammonium $\left[{ }^{11} \mathrm{C}\right]$ cyanide

Oxygen nucleophiles ( $\mathrm{R}-\mathrm{OH}$ or $\mathrm{H}_{2} \mathrm{O}$ ) have also been used to ring open aziridine-2carboxylates. Harada and Nakamura ${ }^{174,175}$ and Young et al. ${ }^{176}$ demonstrated that 
(2S,3S)-1-[(S)-1-phenylethyl]-aziridine-3-methyl-2-carboxylic acid methyl ester and (R)-1-triphenylmethyl-aziridine-2-carboxylic acid methyl ester, respectively, could be converted to $\beta$-hydroxy- $\alpha$-amino acids (Ser or Thr) by treatment with water in the presence of acid (Figure 3.7). In each case, the $\beta$-amino acids - resulting from nucleophilic attack at $C_{2}$ - were not observed.

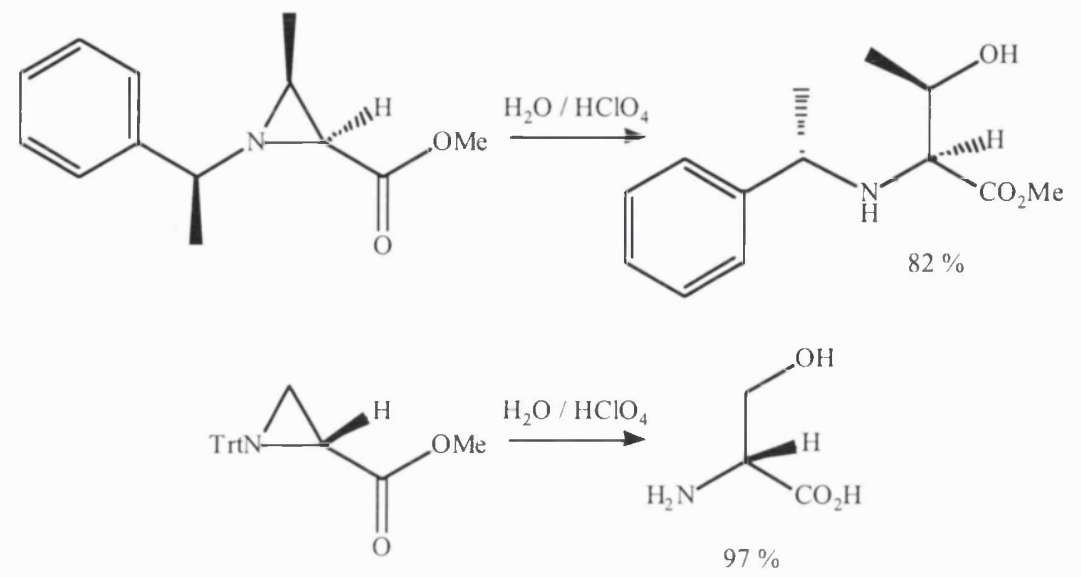

Figure 3.7. Hydration of aziridine-2-carboxylates

The nucleophilic ring opening of aziridine-2-carboxylates by alcohols is also highly regiospecific. Okawa et al. ${ }^{177}$ and Vessiere et al. ${ }^{178}$ have all reported on the attack of alcohols on aziridine-2-carboxylates - successful only in the presence of boron trifluoride etherate - leading exclusively to $C_{3}-N$ cleavage (Figure 3.8).

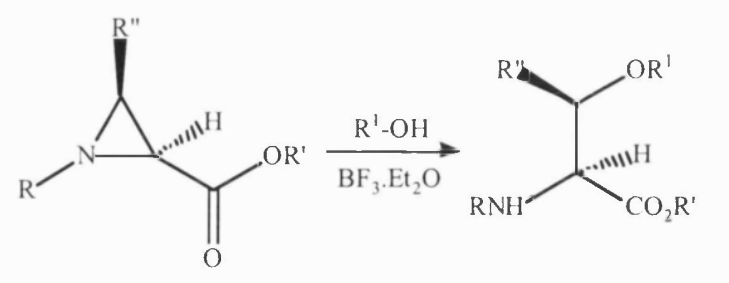

\begin{tabular}{|c|c|c|c|c|}
\hline $\mathrm{R}$ & $\mathrm{R}^{\prime}$ & $\mathrm{R}^{\prime \prime}$ & $\mathrm{R}^{\prime}$ & $\%$ \\
\hline $\mathrm{Z}$ & $\mathrm{Bz}$ & $\mathrm{H}$ & $\mathrm{Bz}$ & 100 \\
$\mathrm{Z}$ & $\mathrm{Me}$ & $\mathrm{Me}$ & $\mathrm{i} \mathrm{Pr}$ & 98 \\
$\mathrm{Z}$ & $\mathrm{Bz}$ & $\mathrm{H}$ & $\mathrm{i} \mathrm{Pr}$ & 95 \\
$\mathrm{Z}$ & $\mathrm{Me}$ & $\mathrm{Me}$ & $\mathrm{Me}$ & 98 \\
$\mathrm{i} \mathrm{Pr}$ & $\mathrm{Me}$ & $\mathrm{H}$ & $\mathrm{Me}$ & 88 \\
$\mathrm{i} \mathrm{Pr}$ & $\mathrm{Me}$ & $\mathrm{H}$ & $\mathrm{i} r$ & 87 \\
$\mathrm{H}$ & $\mathrm{i} \mathrm{Pr}$ & $\mathrm{H}$ & $\mathrm{Me}$ & 85 \\
$\mathrm{i} \mathrm{Pr}$ & $\mathrm{Me}$ & $\mathrm{H}$ & 2-MePrOH & 89 \\
\hline
\end{tabular}

Figure 3.8. Reaction of aziridine-2-carboxylates with various alcohols

Ben-Ishai et al. highlighted the same mode of nucleophilic attack of methanol towards several derivatives of $(R / S)$-aziridine-2-carboxylic acid methyl ester but in 
the presence of a catalytic amount of sulphuric acid instead of boron trifluoride etherate (Figure 3.9). ${ }^{179}$

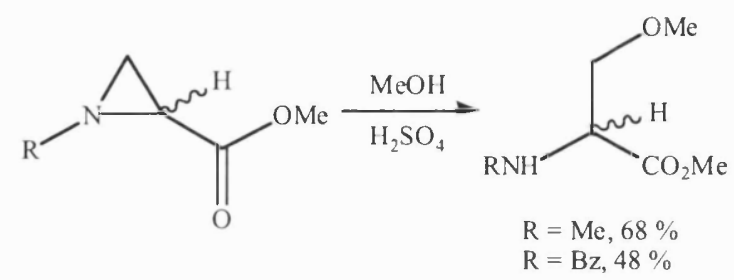

Figure 3.9. Reaction of aziridine-2-carboxylates with methanol

Nitrogen nucleophiles that have been used for the ring opening of aziridine-2carboxylates include amines and azides. Shiba et al. demonstrated the nucleophilic ring opening of (R)-1-tosyl-aziridine-3-( $N$-tbutoxycarbonyl)ethyleneamino-2carboxyl amide via ammonolysis only at $C_{3}$ on the ring (Figure 3.10). ${ }^{180,181}$

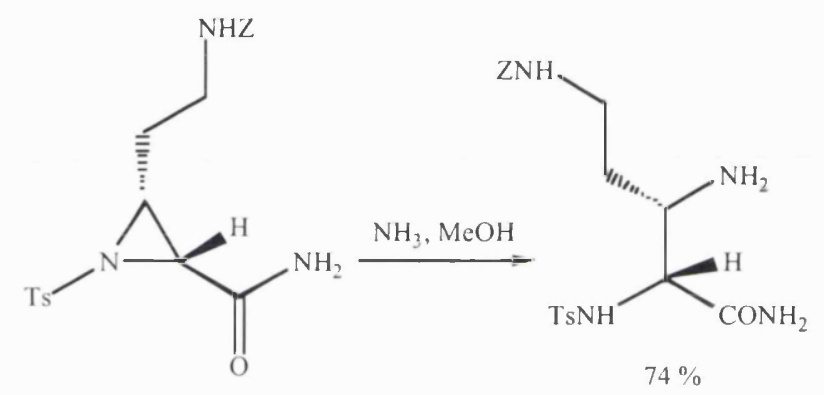

Figure 3.10. Reaction of a $C_{3}$-substituted aziridine-2-carboxyl amide with ammonia

Okawa et al. theorised that the concept could be extended to the reaction of primary amines (such as benzylamine) and secondary amines (such as diethylamine) on the peptide Z-Gly-Azi-Gly-OBz where Azi denotes an aziridine-2-carboxyl amide moiety. ${ }^{182}$ However only diethylamine reacted at the $C_{3}$ position to give the substituted peptide without any by-products (Figure 3.11).

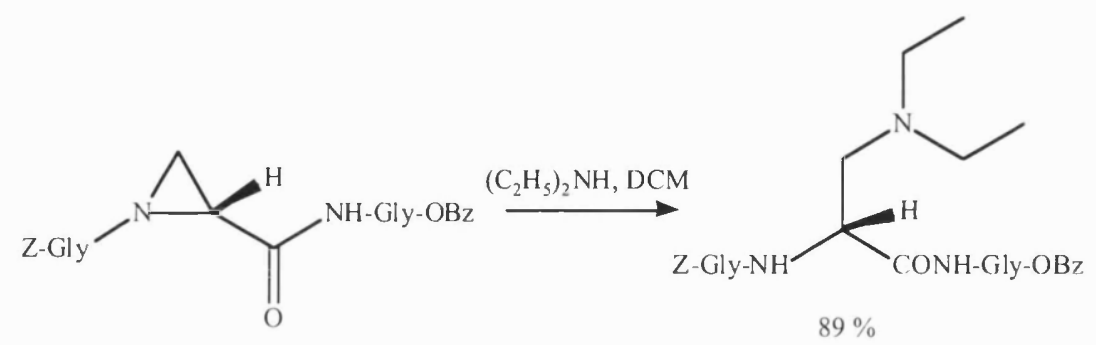

Figure 3.11. Reaction of an aziridine-containing peptide with diethylamine 
However, Hashimoto et al. successfully utilised the free amino group on L-His and reacted it with $(2 S, 3 S)$-1-tosyl-aziridine-3-methyl-2-carboxylic acid methyl ester yielding both $C_{2}$ and $C_{3}$ cleavage products (Figure 3.12). ${ }^{183}$
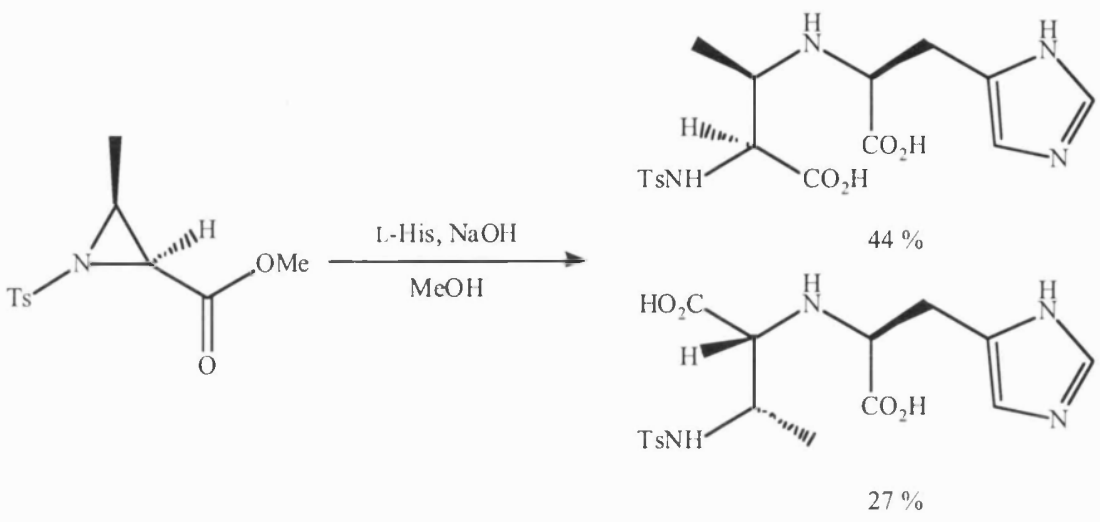

Figure 3.12. Reaction of an aziridine-2-carboxylate with the amino function of L-His

Ha et al. demonstrated that azides could also be used as nitrogen nucleophiles in ring opening reactions of $(2 S, 3 S)$-1-[(S)-1-phenylethyl]-aziridine-3-methyl-2-carboxylic acid methyl ester (Figure 3.13). ${ }^{184}$
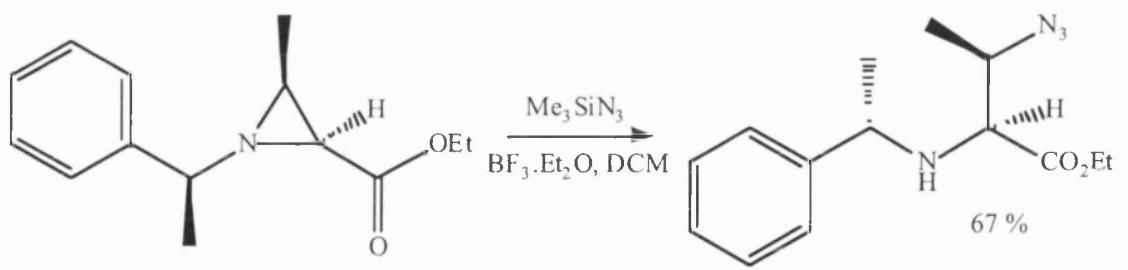

Figure 3.13. Reaction of an aziridine-2-carboxylate with trimethylsilyl azide

Soft nucleophiles that have been used in the ring opening reactions of aziridine-2carboxylates include thiols and halogens. $S$-methyl cysteine derivatives were synthesised by reaction of various $(R / S)$-aziridine-2-carboxylic acid methyl ester derivatives with methylmercaptan in the presence of boron trifluoride etherate exclusively via attack at $C_{3}$ of the aziridine ring (Figure 3.14). ${ }^{179}$

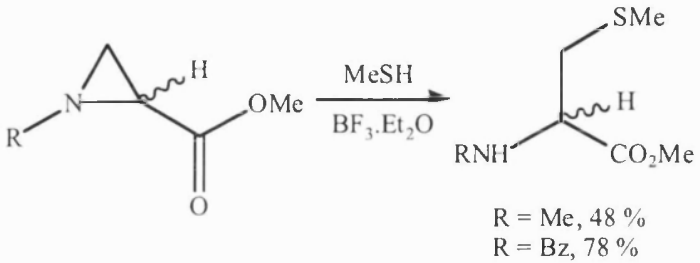

Figure 3.14. Reaction of an aziridine-2-carboxylate with methylmercaptan 
Okawa et al. have used various thiol nucleophiles for ring opening reactions of aziridine-2-carboxylates. The use of thiobenzoic acid in the presence of boron trifluoride etherate yielded only the $\alpha$-amino acid derivatives. ${ }^{80}$ The use of alkyl and aromatic thiols on Z-protected aziridine-2-carboxylic acid benzyl ester derivatives also produced the $S$-substituted cysteine derivatives in very good yields (Figure $3.15)^{185}$
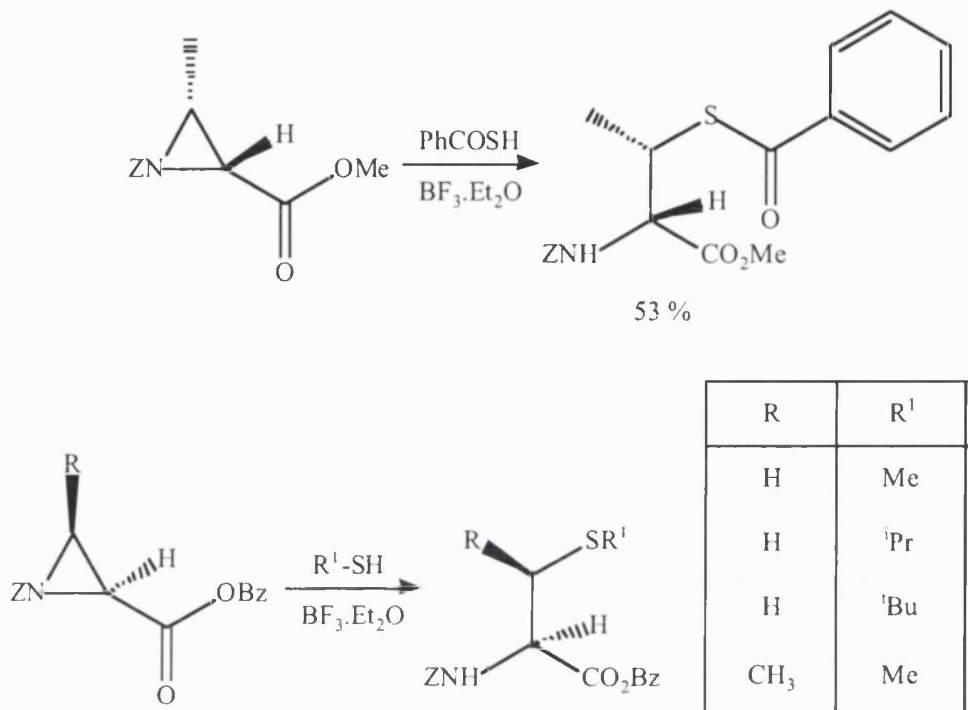

\begin{tabular}{|c|c|c|}
\hline $\mathrm{R}$ & $\mathrm{R}^{1}$ & $\%$ \\
\hline $\mathrm{H}$ & $\mathrm{Me}$ & 86 \\
$\mathrm{H}$ & ${ }^{\mathrm{Pr}}$ & 82 \\
$\mathrm{H}$ & ${ }^{\mathrm{t}} \mathrm{Bu}$ & 90 \\
$\mathrm{CH}_{3}$ & $\mathrm{Me}$ & 92 \\
$\mathrm{CH}_{3}$ & ${ }^{\mathrm{Pr}}$ & 73 \\
$\mathrm{CH}_{3}$ & ${ }^{\prime} \mathrm{Bu}$ & 54 \\
\hline
\end{tabular}

Figure 3.15. Reaction of aziridine-2-carboxylates with various thiols

Interestingly the use of $\mathrm{Z}-\mathrm{L}-\mathrm{Cys}-\mathrm{OBz}$ as nucleophile in the presence of boron trifluoride etherate was less successful - the lanthionine products were isolated in yields ranging from $12-37 \%$ only (Figure 3.16). There was no indication if the products resulting from $C_{2}$ thiol attack were isolated or even present.

Parry and Naidu ${ }^{186}$ and Baldwin et al. ${ }^{187}$ have all demonstrated that thiol nucleophilic attacks on aziridine-2-carboxylates yielded the $\alpha$-amino acid derivatives with no indication of the products for $C_{2}$ attack (Figure 3.17). 


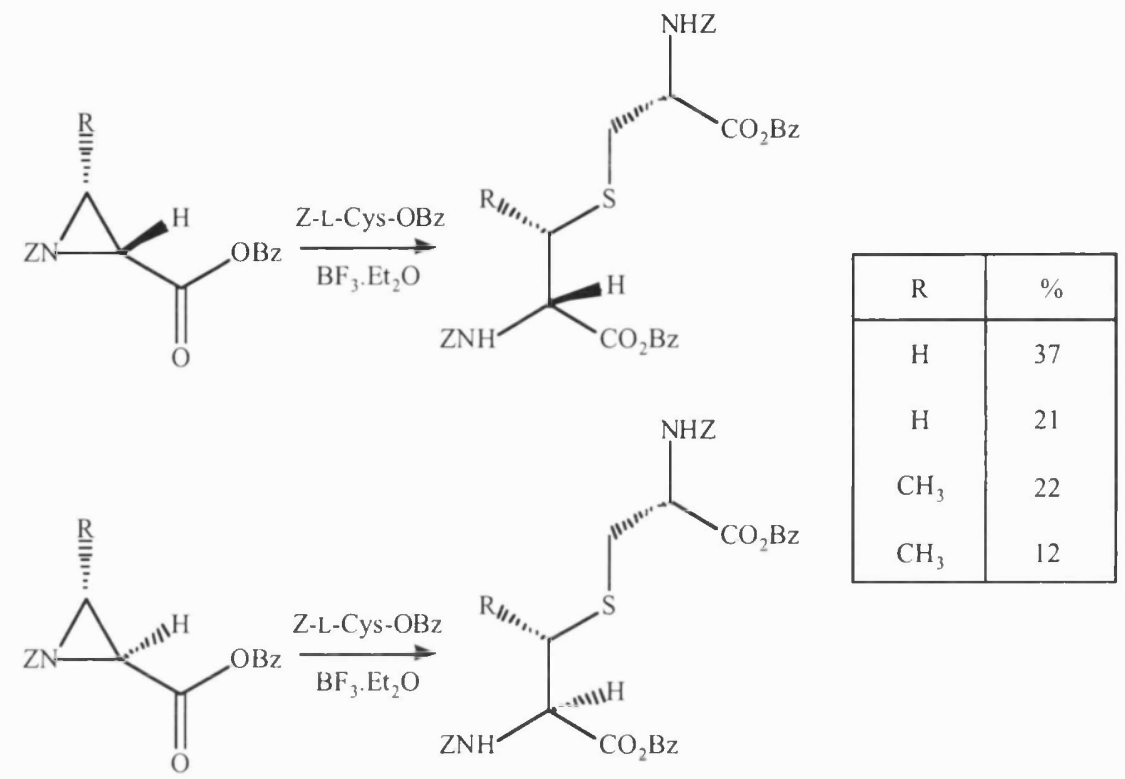

Figure 3.16. Formation of lanthionine and 3-methyllanthionine derivatives

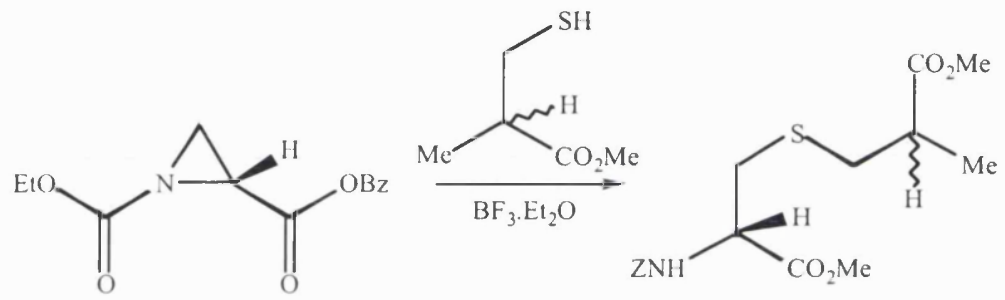

Figure 3.17. Reaction of an aziridine-2-carboxylate with a racemic thiol

Hata and Watanabe treated both $(R)$ - and $(S)$-aziridine-2-carboxylic acids and $(2 R, 3 R)$ - and $(2 S, 3 S)$-aziridine-3-methyl-2-carboxylic acids with thiol nucleophiles such as thiophenol and cysteine in a phosphate buffer and isolated both $\alpha$ - and $\beta$ amino acids with the latter being the predominant product. ${ }^{79}$ However, when the reactions with thiophenol were carried out in $95 \%$ acetonitrile in water and in $99 \%$ DMF in water the results were reversed, yielding 1.1:1 and 2:1 proportions, respectively, for the $(\alpha: \beta)$ amino acids. In the phosphate buffer, the lanthionine and 3-methyllanthionine derivatives produced were present in only between $20-29 \%$ of the total Cys-Azi coupling products (Figure 3.18). 


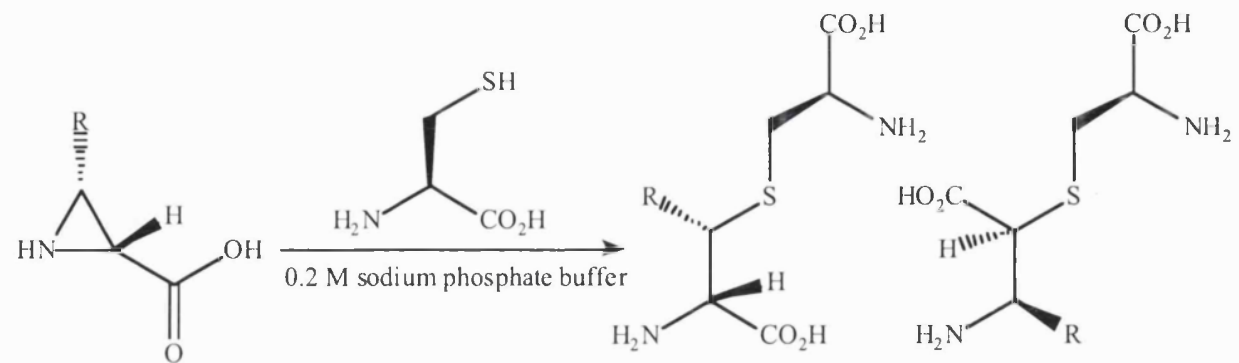

A

B

$\mathrm{R}=\mathrm{H}, \mathrm{A}: \mathrm{B}=2.5: 1,95 \%$

$\mathrm{R}=\mathrm{Me}, \mathrm{A}: \mathrm{B}=3.6: 1,80 \%$

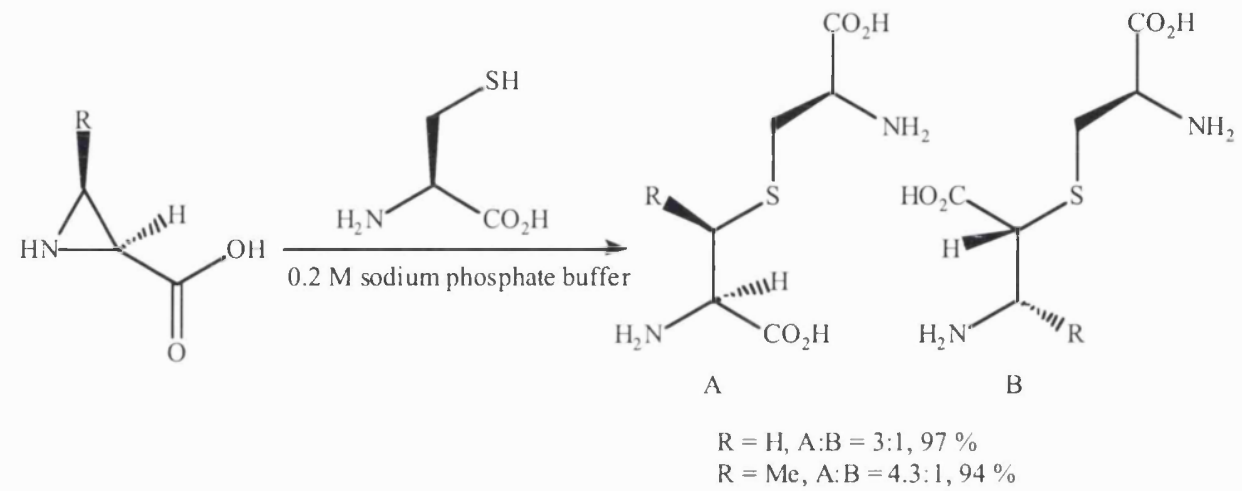

Figure 3.18. Formation of lanthionine and 3-methyllanthionine derivatives

Crousse et al. utilised a $C_{3}$-substituted (2S,3S)-1-benzyl-aziridine-3-trifluoromethyl2-carboxylic acid ethyl ester and various thiols in the presence of acid to produce exclusively the $\alpha$-thio $\beta$-amino acid derivatives (Figure 3.19). ${ }^{188}$

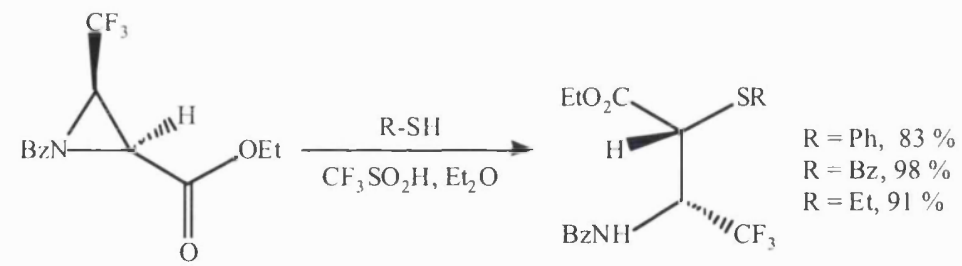

Figure 3.19. Reaction of a trifluoromethyl $C_{3}$-substituted aziridine-2-carboxylate with various thiols

Halogens have been used as nucleophiles in ring opening reactions of aziridine-2carboxylates. Wade et al. treated $(2 R / S, 3 R / S)$-aziridine-3-phenyl-2-carboxylic acid isopropyl ester and $(2 R / S, 3 R / S)$-aziridine-3-methyl-2-carboxylic acid 'butyl ester with hydrogen fluoride in the presence of base. This yielded the $\beta$-fluorinated alanine derivatives, with no indication of the presence of regioisomers (Figure 3.20). ${ }^{189,190}$ 


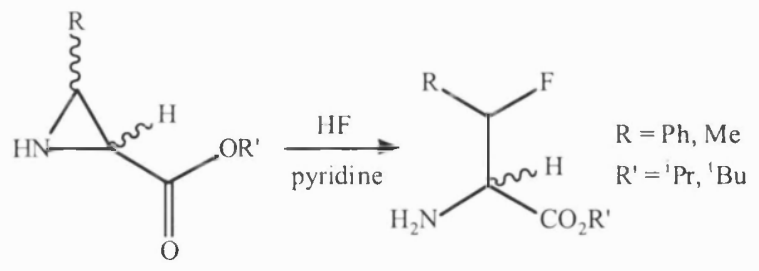

Figure 3.20. Reaction of an aziridine-2-carboxylate with hydrogen fluoride

Righi and D'Achille demonstrated that $C_{3}$-substituted $(2 R, 3 R)$-1-ethoxycarbonylaziridine-2-carboxylic acid methyl/ethyl esters undergo nucleophilic ring opening by metal halides at room temperature. ${ }^{191}$ Magnesium bromide was shown to ring open the rings at the $C_{3}$ position $\left(C_{3}: C_{2} 99: 1\right)$. Similar results were obtained with magnesium iodide; however, the use of sodium iodide and sodium bromide with Amberlyst 15 in acetone at $-40{ }^{\circ} \mathrm{C}$ gave a mixture of products, predominantly the $\beta$ amino acids, where up to $1: 1$ proportion of $C_{2}: C_{3}$ attack products were observed. Substitution of $C_{3}$ with bulky groups (isopropyl, cyclohexyl) yielded almost exclusively $\beta$-amino acids (Figure 3.21).

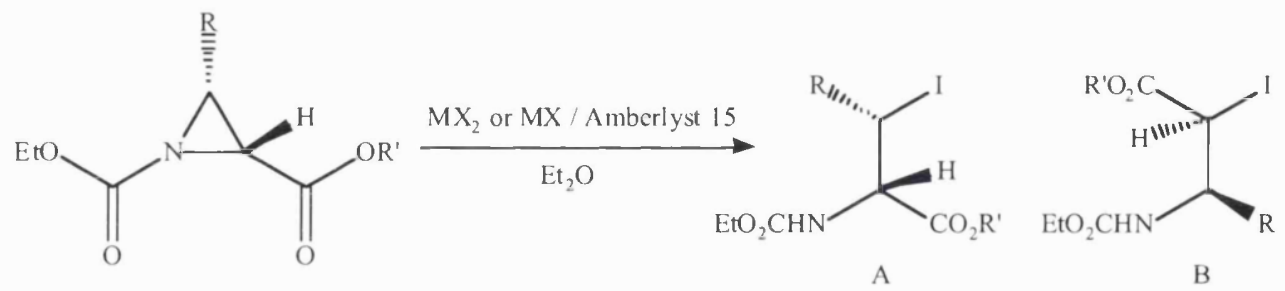

\begin{tabular}{|c|c|c|c|}
\hline $\mathrm{R}$ & $\mathrm{R}^{\prime}$ & $\mathrm{MX}_{\mathrm{n}}$ & $\mathrm{A}: \mathrm{B}$ \\
\hline${ }^{\mathrm{n}} \mathrm{Pr}$ & $\mathrm{Mc}$ & $\mathrm{MgBr}_{2}$ & $99: 1$ \\
$\mathrm{c}-\mathrm{Hcx}$ & $\mathrm{Mc}$ & $\mathrm{MgBr}_{2}$ & $99: 1$ \\
$\mathrm{Ph}$ & $\mathrm{Mc}$ & $\mathrm{MgBr}_{2}$ & $99: 1$ \\
$\mathrm{c}-\mathrm{Hex}$ & $\mathrm{Et}$ & $\mathrm{NaI}$ & $1: 99$ \\
${ }^{\mathrm{Pr}}$ & $\mathrm{Et}$ & $\mathrm{NaI}$ & $1: 99$ \\
${ }^{n} \mathrm{Pr}$ & $\mathrm{Et}$ & $\mathrm{NaI}$ & $1: 3$ \\
${ }^{n} \mathrm{Pr}$ & $\mathrm{Et}$ & $\mathrm{NaBr}$ & $22: 73$ \\
$\mathrm{Ph}$ & $\mathrm{Et}$ & $\mathrm{NaI}$ & $1: 1$ \\
\hline
\end{tabular}

Figure 3.21. Reaction of aziridine-2-carboxylates with metal halides 
Crousse et al. utilised trifluoromethyl $C_{3}$-substituted (2S,3S)-1-benzyl-aziridine-2carboxylic acid ethyl ester to produce the $\alpha$-chloro- $\beta$-amino acid derivative (Figure $3.22)^{188}$

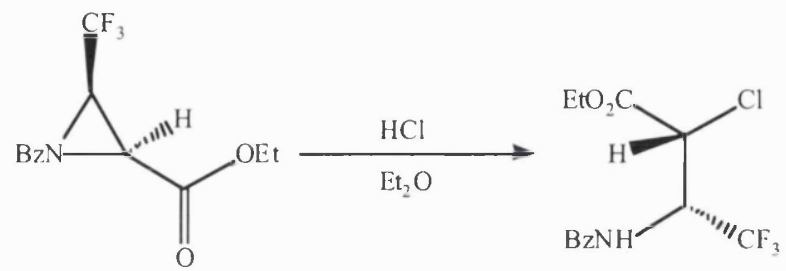

Figure 3.22. Reaction of an aziridine-2-carboxylate with hydrogen chloride

$C_{3}$-subsituted (ethyl/phenyl) and $C_{2}$-subsituted (methyl) (2R,3S)-1-tosyl-aziridine-2carboxylic acid methyl ester derivatives have also been subjected to nucleophilic ring opening as a subsequent reaction following reduction with lithium aluminium hydride. ${ }^{192}$ The products obtained were then oxidised to form the $\alpha, \beta$-substituted $\beta$ amino acids (Figure 3.23).

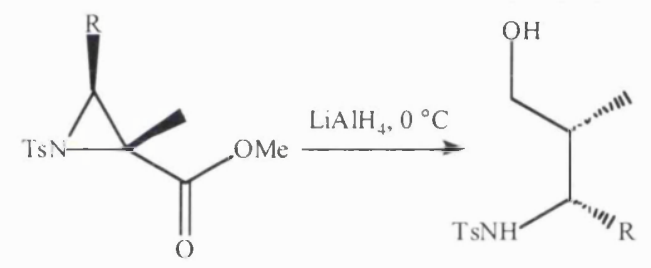

Figure 3.23. Reduction of an aziridine-2-carboxylate

In all of the examples given above retention of stereochemical integrity was observed, except for reactions using racemic mixtures of aziridine-2-carboxylates where retention or inversion would be difficult to predict.

From all the above examples it seemed that X (Figure 3.24) needs to be an electronwithdrawing group to avoid nucleophilic attack at $C_{1}$ (when $\mathrm{Y} \neq \mathrm{H}$ ) by making $C_{2}$ and $C_{3}$ more electrophilic. It also seemed that the electrophilicity of $C_{2}$ is decreased relative to $C_{3}$ when $\mathrm{Y}$ is a hydrogen (i.e. the acid rather than the ester). Bulky substituents on either $C_{2}$ or $C_{3}$ increase the susceptibility of $C_{3}$ or $C_{2}$, respectively, to nucleophiles. The different types of nucleophiles also contribute to the manner of ring opening reactions of aziridine-2-carboxylates. 


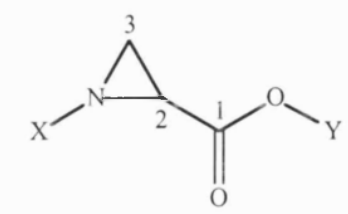

Figure 3.24. Aziridine-2-carboxylate system

The use of Lewis acids such as boron trifluoride etherate, $\mathrm{CuBr}$.SMe and perchloric acid promotes ring opening reactions, presumably via the mechanism illustrated in Figure 3.25 .

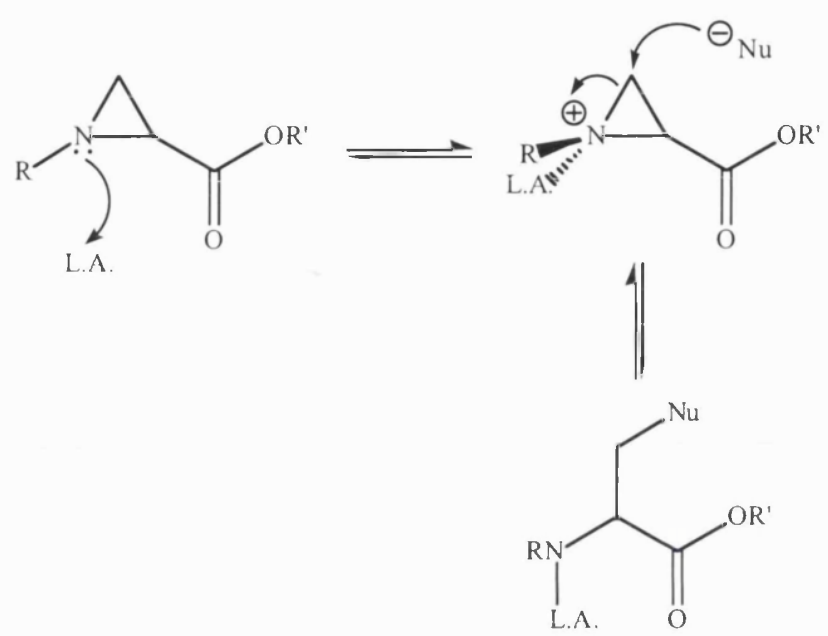

Figure 3.25. Proposed mechanism for Lewis acid-mediated $C_{3}$ ring opening of aziridine-2-carboxylates

\subsection{Aims}

The ultimate aim of this project was to lay the foundation for the synthesis of polycyclic lanthionine-containing peptides on the solid phase. With the dawn of methicillin-resistant staphylococcus aureus (MRSA) and staphylococcus epidermidis (MRSE) and vancomycin-resistant enterococci (VRE) the need is even greater, to find the next class of antibiotics for the future.

The total synthesis of nisin ${ }^{162}$ has not been optimised for the solid phase, although there have been reports of the synthesis of smaller lanthionine-containing analogues of biologically active peptides ${ }^{6,50,52,53,54,59,61,62,168}$ and monocyclic fragments of lantibiotics ${ }^{167}$ on solid phase. As discussed in Chapter 1, previous syntheses of 
lanthionine-containing peptides were carried out either via a lanthionine residue incorporation into a peptide, often with concomitant cleavage of peptide upon cyclisation or on-resin thioether bond formation between two side chains, some with inefficient stereochemical control.

This project aimed to synthesise differentially protected lanthionine residues for solid phase synthesis utilising Fmoc methodology and to incorporate them into cyclic and polycyclic lanthionine-containing peptides. It was also of interest to synthesise these peptides solely via continuous flow peptide synthesis. The orthogonality of Fmoc, ${ }^{t} \mathrm{Bu}$ or Boc and allylic protecting groups proved to be an attractive choice due to its precedence and widespread use in SPPS. ${ }^{129,141,142,143,144,145,146}$ In this project, its usage is highlighted in Figure 3.26 where target molecule 1 is used as a doubleheaded amino acid residue incorporated into peptides.

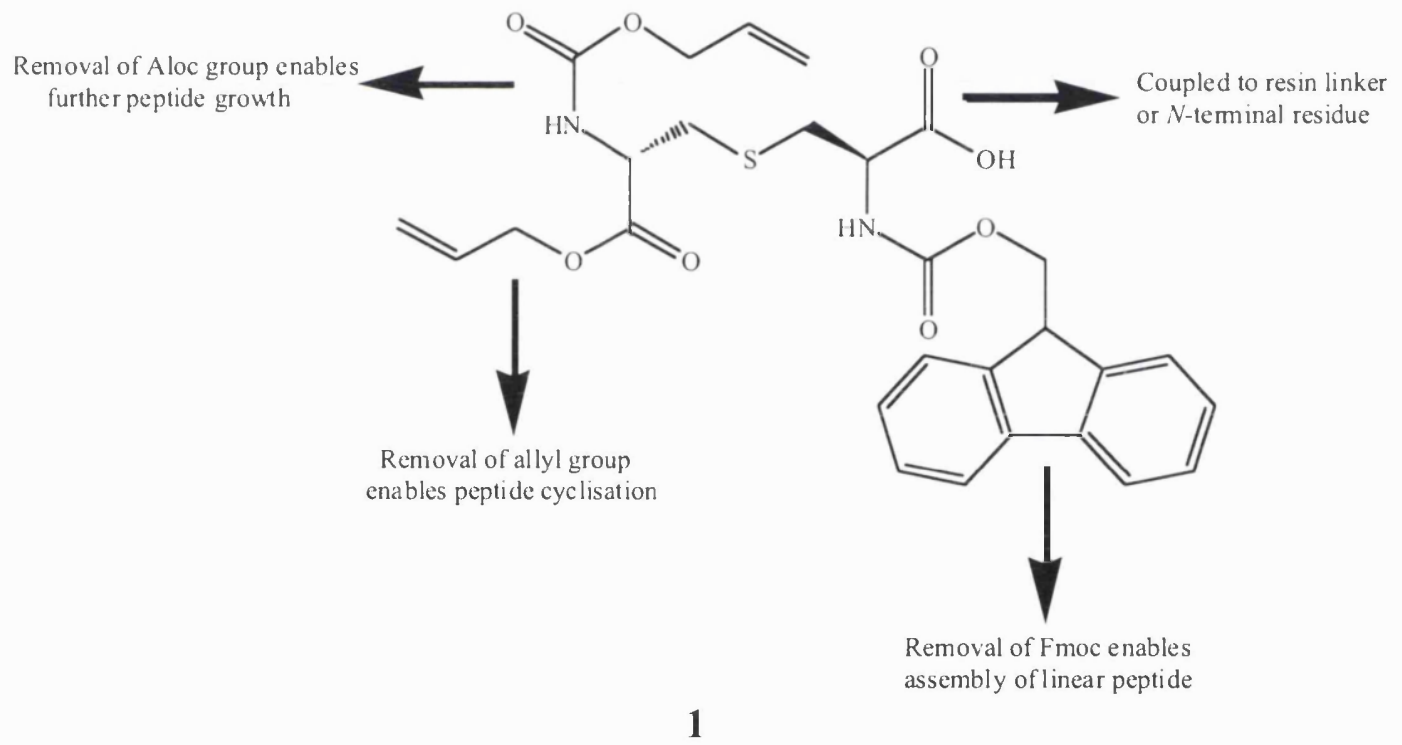

Figure 3.26. Target molecule 1 for SPPS

The proposed approach for synthesising lanthionine containing peptides on solid phase for this project is outlined in Figure 3.27. 


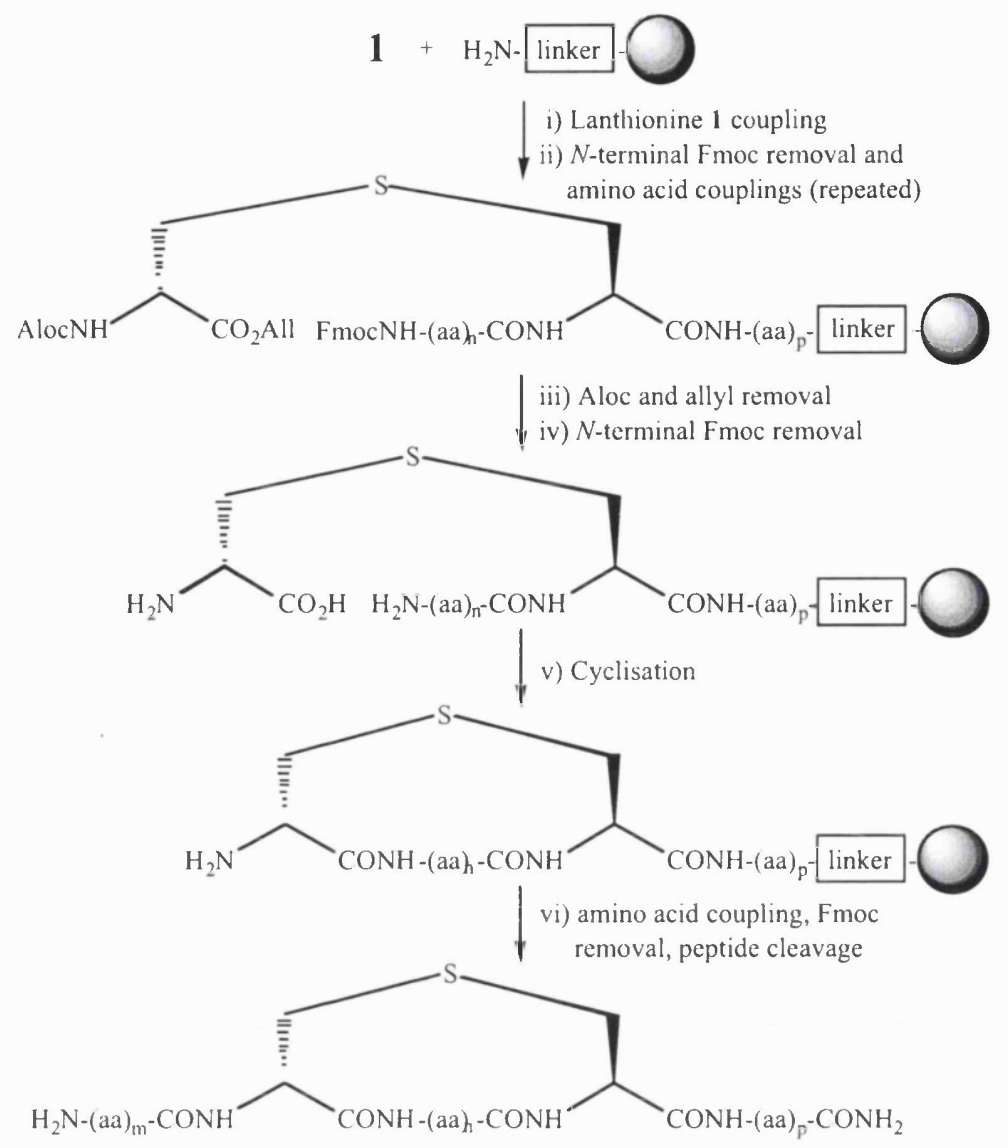

Figure 3.27. General scheme for proposed synthesis of lanthionine containing peptides, using a linker yielding peptide amides

The orthogonality of the allyl ester and Aloc groups to Fmoc and ${ }^{\mathrm{t}} \mathrm{Bu}$ allows their selective removal from the residue's second amino acid functionality. Due to the liberation of the $N$-terminal amine (Fmoc removal) and carboxyl function (allyl deprotection), cyclisation of the peptide could be attempted in the presence of a suitable coupling reagent. With the availability of the other $N$-terminal amine (Aloc removal), the peptide could be further extended. This novel methodology for lanthionine-containing peptides would allow for an unprecedented, solely on-resin synthesis of polycyclic lanthionine-containing peptides.

For the synthesis of overlapping bi-cyclic lanthionine/3-methyllanthionine structures encountered in lantibiotics (such as the rings D and E of nisin), a second, orthogonally protected lanthionine was required. It was envisaged that target molecule 2 (Figure 3.28) would be used as a second, differentially protected 
lanthionine structure to be incorporated into a linear peptide, prior to cyclisation of the first lanthionine (Figure 3.29).

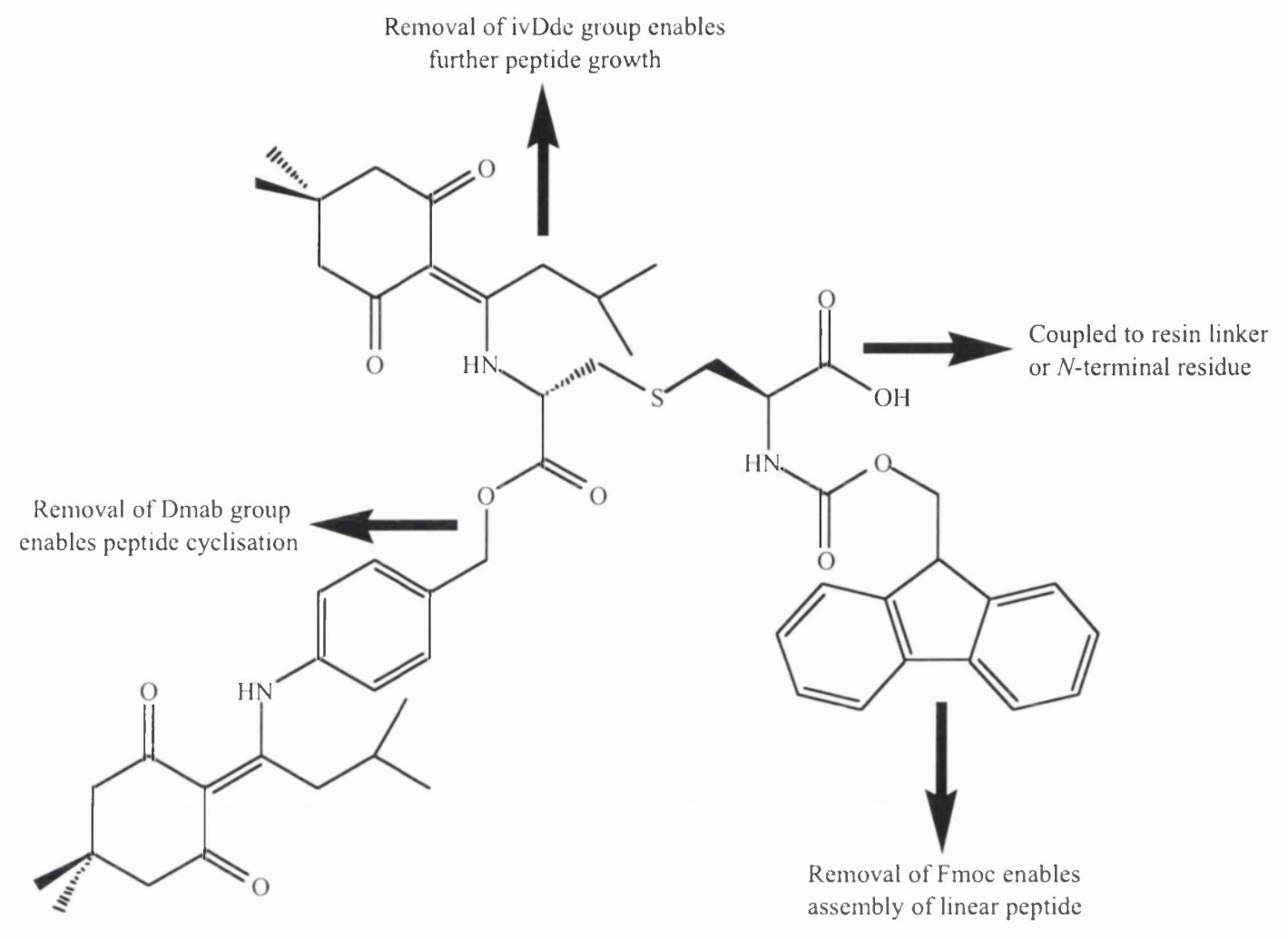

2

Figure 3.28. Target molecule 2 for SPPS

The unique combination of protecting groups for $\mathbf{1}$ and $\mathbf{2}$ would facilitate the synthesis of lanthionine containing peptides with overlapping thioether bridges, and hence lantibiotics. 


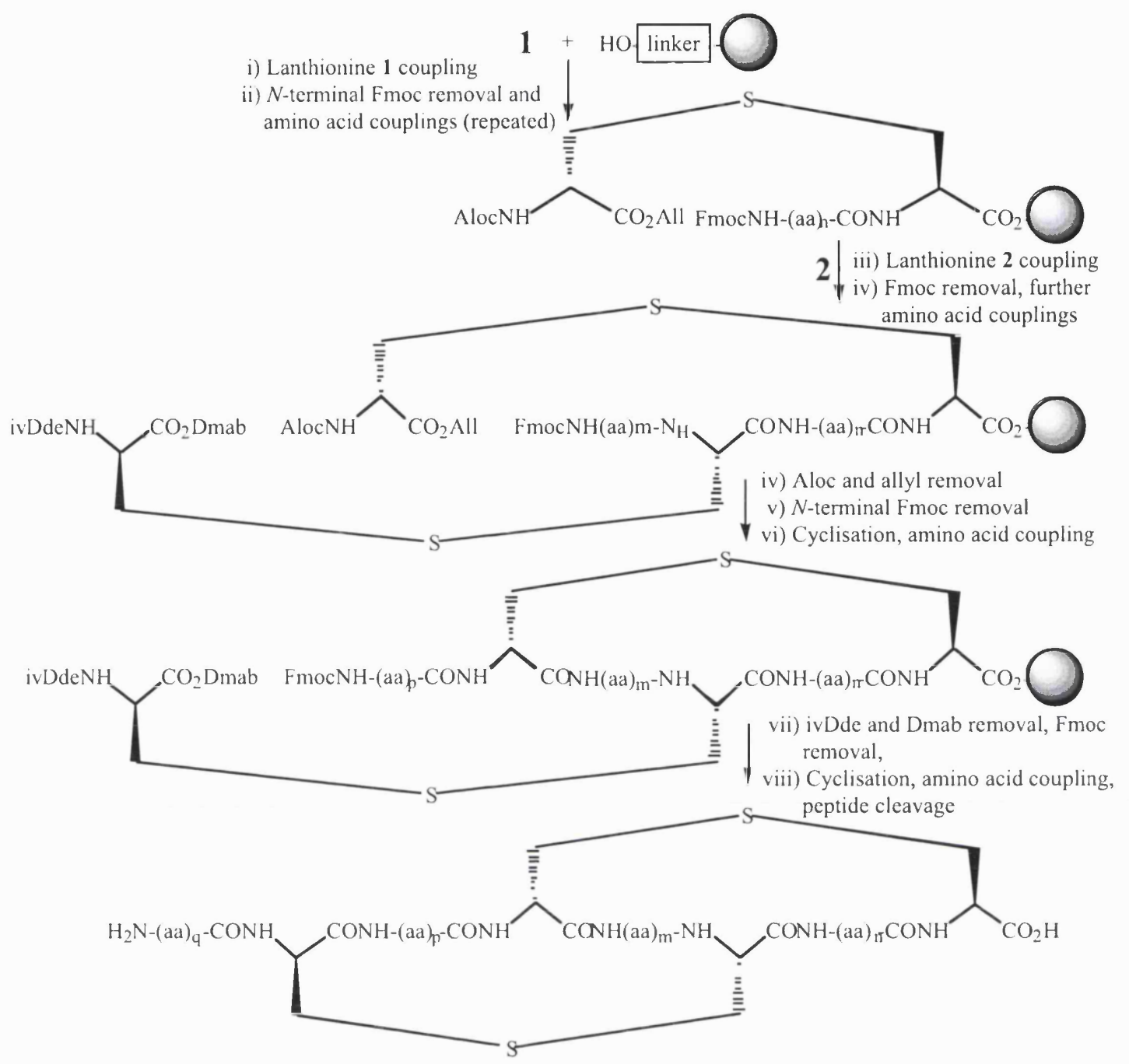

Figure 3.29. General scheme for the proposed synthesis of lanthionine containing peptides with overlapping thioether linkages, using a linker yielding peptide acids 


\section{Chapter 4}

\section{Results and Discussion I}

The aim of this project was to synthesise lanthionine-containing peptides, from orthogonally protected lanthionine residues, on the solid phase. The high yielding strategy of coupling an iodoalanine derivative with a cysteine derivative ${ }^{76}$ was chosen as the method to synthesise the orthogonally protected lanthionine. As discussed in Chapter 1, this approach appeared to be the most direct and reproducible, and had already been investigated with a range of protected iodoalanine derivatives. The observations that two conformers were present in a number of key compounds gave rise for concern, as it was far from clear why derivatives such as $N$-triphenylmethyl- $\beta$-iodoalanine methyl ester ${ }^{76}$ should exist in 
two locked conformations, despite the bulky triphenylmethyl group and the large iodine atom. However, in the original paper, extensive evidence supporting the chiral integrity of the lanthionine residues had been presented, as discussed in Chapter 1.

The original work of Dugave and Menez was followed closely initially, but with an allyl group on the iodoalanine, providing a lanthionine template for further protecting group manipulations. To compare structural and spectral properties, syntheses of both the unnatural and natural lanthionine derivatives $(\boldsymbol{R}, \boldsymbol{R})-\mathbf{1 0}$ and $(\boldsymbol{S}, \boldsymbol{R})$-10 were attempted. The general scheme for the synthesis is outlined in Figure 4.1.

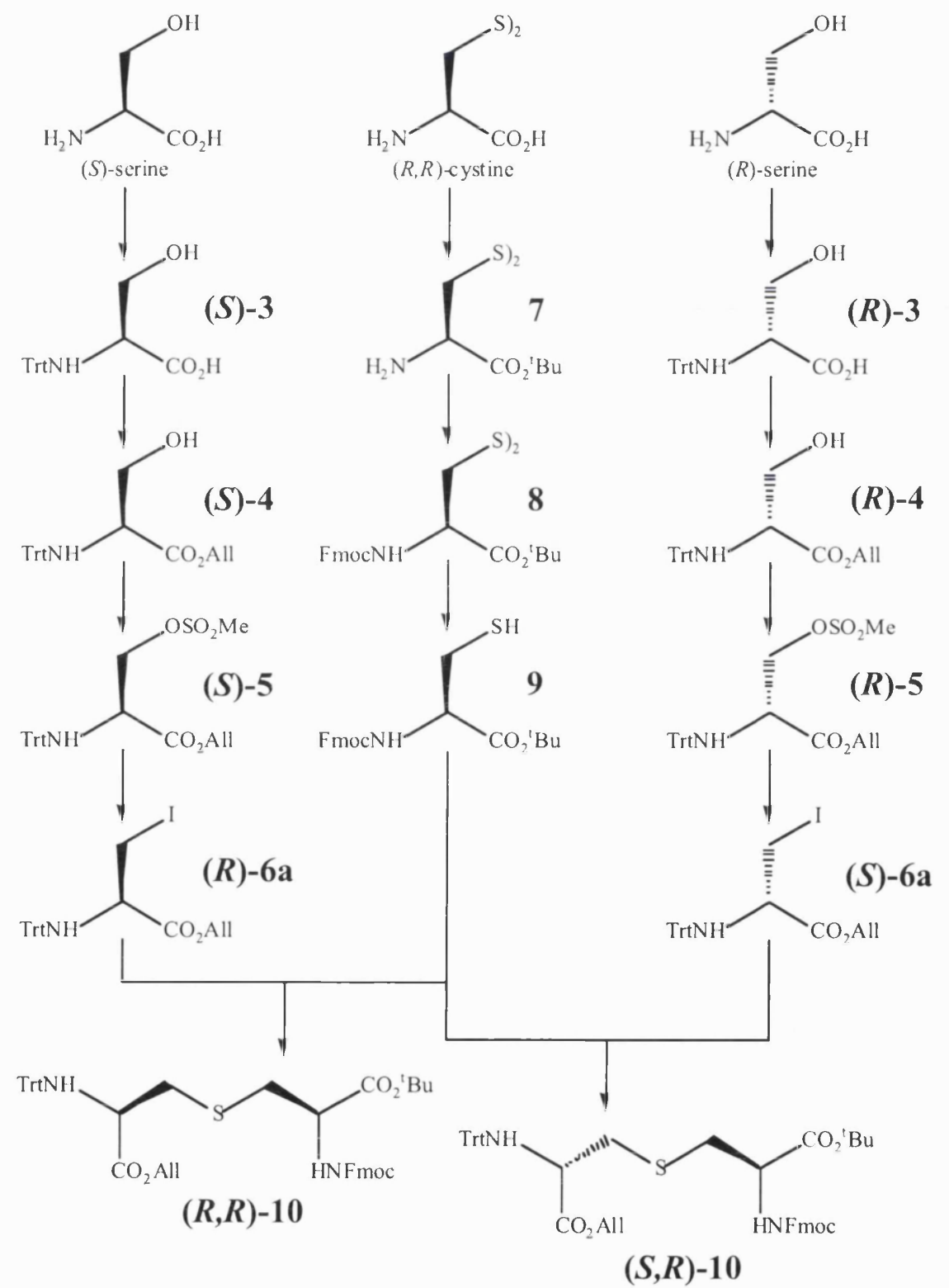

Figure 4.1. General scheme for the synthesis of lanthionine derivatives 
A critical discovery was made at later stages of the peptide synthesis, which completely invalidated the concept of locked conformations in the acyclic system of $N$-triphenylmethyl- $\beta$-iodoalanine derivatives reported by Dugave and Menez. Extensive NMR analyses have proven that the two conformers reported were in fact a mixture of the desired compound and its regioisomer $(\alpha-$-iodo- $\beta$-amino acid derivative, Figure 4.2) - present as a mixture of enantiomers - with the regioisomer being the predominant product. In this chapter the initial synthetic work is presented, with preliminary findings, thoughts and ideas. In Chapter 5, a complete analysis of the problem is discussed, with NMR and other analyses that led to the above conclusion.

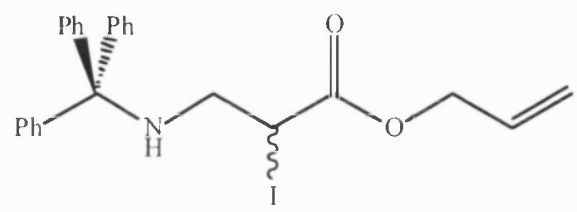

6b

Figure 4.2. Structure of $(R / S)$-2-iodo-3-(triphenylmethylamino)-propionic acid allyl ester, an $\alpha$-iodo- $\beta$-alanine derivative

\subsection{Synthesis of protected cysteine 9}

Protection of the carboxyl groups of cystine as their tbutyl esters (Figure 4.3) was first carried out following a literature procedure. ${ }^{193}$ Initial low yields of the desired product arose from the inefficiency of the reported work-up procedure, which was simplified to yield the doubly esterified compound, in excellent yields. Work carried out within the group indicated that only the use of the potentially explosive perchloric acid led to the solvation of cystine. ${ }^{78}$
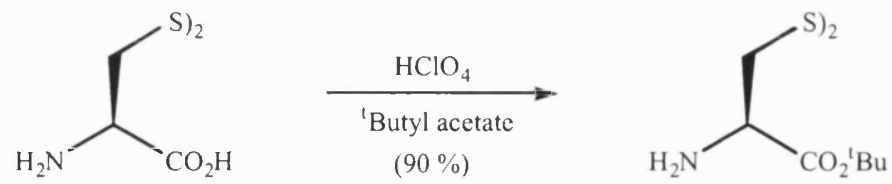

7

Figure 4.3. Trans-esterification of $(R, R)$-cystine with tbutyl acetate

The amino groups on 7 were then protected with the Fmoc group and the product purified by recrystallisation (Figure 4.4). ${ }^{194}$ The use of exactly two equivalents of 99 
Fmoc chloride was imperative as the Fm-OH by-product - which would have resulted from quenching of excess reagent - was precipitated together with the desired compound. This required an additional purification step by flash column chromatography. Additionally, the precautionary use of base in excess of two equivalents was also necessary to prevent the formation of $\mathrm{HCl}$, which would cleave the butyl group.

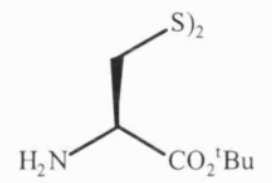

7

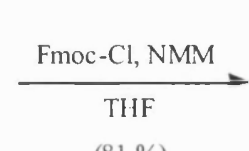

$(81 \%)$

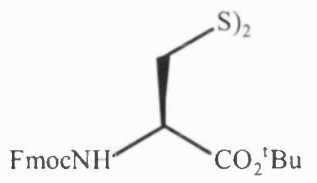

8

Figure 4.4. Fmoc protection of $(R, R)$-cystine bis- ${ }^{\mathrm{t}}$ butyl ester

The final step for preparing the first half of the lanthionine residues $(\boldsymbol{R}, \boldsymbol{R})-\mathbf{1 0}$ and $(\boldsymbol{S}, \boldsymbol{R})$-10 was the disulfide bond cleavage of $\mathbf{8}$ with tri-" ${ }^{\mathrm{n}}$ butylphosphine (Figure 4.5). Dugave and $\mathrm{Menez}^{76}$ reported the use of wet THF for this reaction. In our hands the use of at least one equivalent of water in this reaction resulted in the production of the desired compound in high yields. This is due to the fact that the water molecule is required to drive the reaction forward, forming the thermodynamically stable $\mathrm{P}=\mathrm{O}$ bond of the tri- ${ }^{\mathrm{n}}$ butylphosphine oxide by-product.
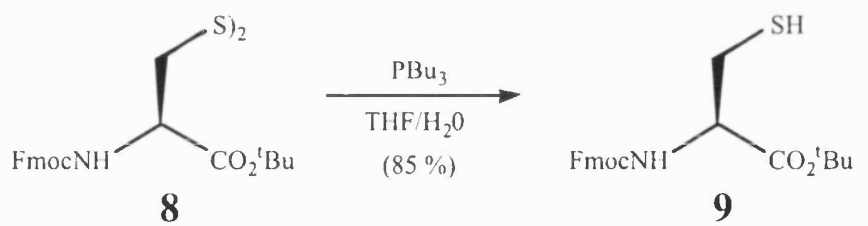

Figure 4.5. Disulfide reduction of $N, N^{\prime}$-bis(fluorenylmethoxycarbonyl)- $(R, R)$ cystine-bis- ${ }^{\mathrm{t}}$ butyl ester

The reactions described above were reproducible at larger scales of up to $21 \mathrm{mmol}$.

\subsection{Synthesis of protected (R)-iodoalanine $(R)-6 a$}

$(S)$-Serine provided the template for the second half of the lanthionine derivative $(\boldsymbol{R}, \boldsymbol{R})-10$. The $N$-triphenylmethylation of serine was carried out following a procedure described by Theodoropulous et al. ${ }^{195}$ where a one-pot synthesis was 
achieved (Figure 4.6). Silylation of all three functional groups of serine was followed by selective removal of the most labile trimethylsilyl protection with anhydrous methanol - at the amino function - thus enabling selective triphenylmethylation, forming $(\boldsymbol{S})$-3. The use of this temporary $N$-triphenylmethyl protection was to prevent abstraction of the $\alpha-H$ on the serine by a non-nucleophilic base, ${ }^{196 a, 196 b}$ which would lead to dehydration and hence the formation of a dehydroalanine derivative. Once the thioether linkage is formed the triphenylmethyl group is removed, as the risk of elimination by a base at this stage is almost non-existent.

The use of dry trimethylsilyl chloride was imperative as it affected the yield. In our hands, the use of exactly one equivalent of dry methanol in the selective removal of the amino-trimethylsilyl group instead of 1.5 equivalents as reported originally, increased the overall yield by over $5 \%$.

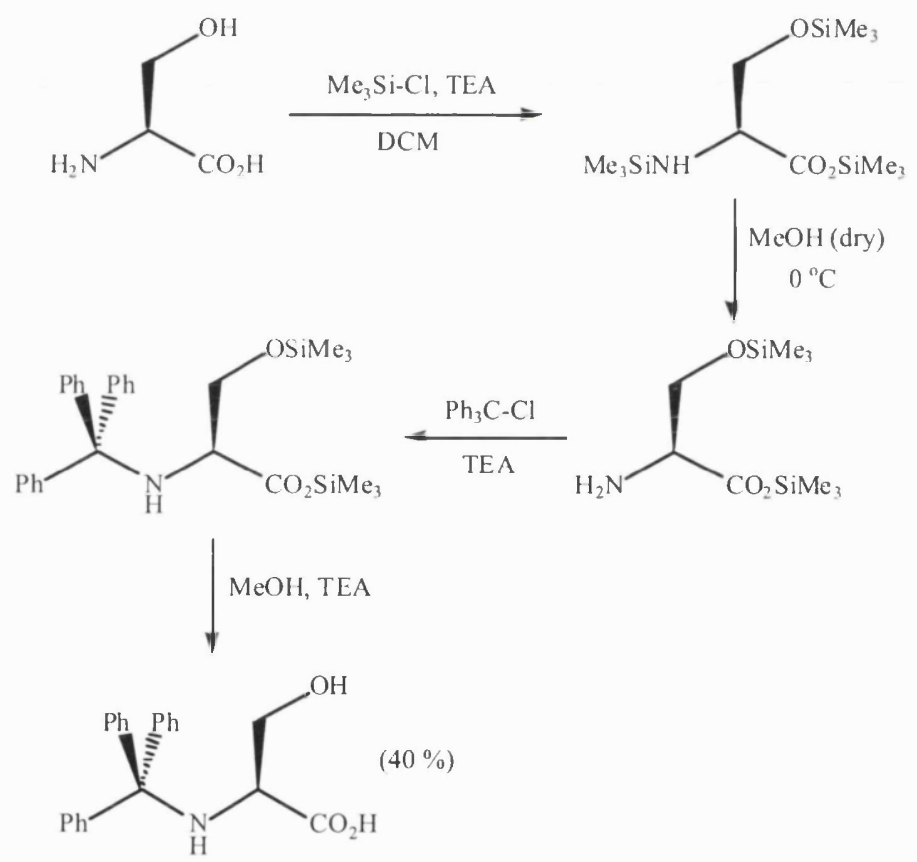

$(S)-3$

Figure 4.6. $N$-Triphenylmethylation of $(S)$-serine

The carboxyl function of the serine was then protected with an allyl group. The first attempt at allylation was carried out in dichloromethane with triethylamine as the base. This however produced the desired compound in a maximum $50 \%$ yield. 
Another method was then attempted, using phase-transfer catalysis with Aliquat 336, sodium hydrogen carbonate and allyl bromide in dichloromethane. ${ }^{197}$ The reaction was quantitative by t.l.c., however the crude material proved to be impossible to purify.

Using a known allylation procedure, ${ }^{198}$ involving initial treatment with caesium carbonate to form the carboxylate salt, followed by addition of allyl bromide, carboxyl protection as an allyl ester was achieved (Figure 4.7).

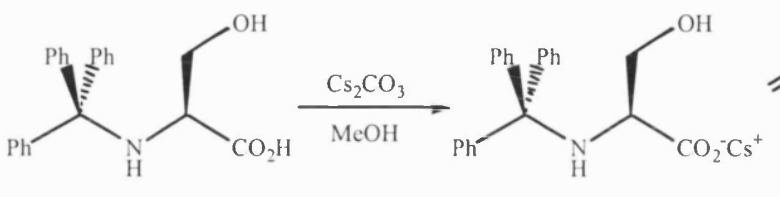

$(S)-3$

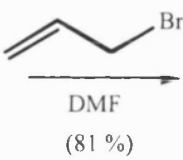

$(81 \%)$<smiles>C=CCOC(=O)[C@H](CO)NC([18OH])[18OH]</smiles>

$(S)-4$

Figure 4.7. Allylation of $N$-triphenylmethyl-( $S)$-serine

With the successful allylation reaction, it was imperative that the use of caesium carbonate be kept to 0.5 equivalents and the allyl bromide to one equivalent relative to the amino acid, or else the doubly allyl-protected species could be formed, even with a mere 0.51 equivalents of the base and 1.1 equivalents of the bromide.

Treatment of the $N$-triphenylmethyl-(S)-serine allyl ester $(S)-4$ with methanesulfonyl chloride under standard conditions ${ }^{76}$ (Figure 4.8) afforded the mesylated derivative $(S)-5$.

Compound $(S)-5$ was then treated with 10 equivalents of sodium iodide (Figure 4.9) to displace the methanesulfonyl group giving the iodoalanine derivative $(\boldsymbol{R})-\mathbf{6 a}$.

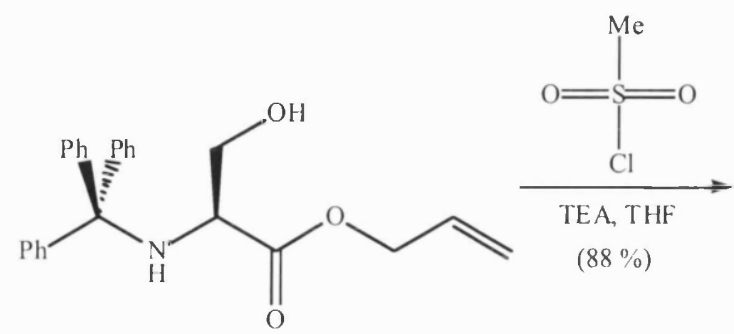

$(S)-4$<smiles>C=CCOC(=O)[C@H](COS(C)(=O)=O)NC(c1ccccc1)c1ccccc1</smiles>

$(S)-5$

Figure 4.8. Mesylation of $N$-triphenylmethyl-(S)-serine allyl ester 
<smiles>C=CCOC(=O)[C@H](COS(C)(=O)=O)NC(c1ccccc1)c1ccccc1</smiles>

$(S)-5$

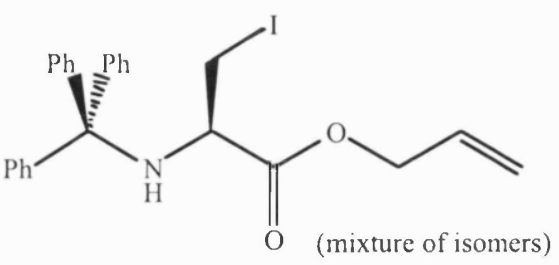

$(R)-6 a$

Figure 4.9. Iodination of $N$-triphenylmethyl-( $S)$-serine( $O$-methanesulfonyl) allyl ester

The results obtained, by ${ }^{1} \mathrm{H}$ and ${ }^{1} \mathrm{H}$-COSY NMR, concurred with the observations made by Dugave and Menez ${ }^{76}$ with their $N$-triphenylmethyl- $\beta$-iodoalanine benzyl and methyl esters, where two isomers and an aziridine-2-carboxylate by-product (approximately $10 \%$ ) were present (Appendix A). It was noticed that the proportion of these two isomers were dependent upon the temperature at which the iodination reaction was carried out. At $15{ }^{\circ} \mathrm{C}$ over 3 days the proportion - isomers $(\boldsymbol{R})-6 \mathrm{a}$ and 6b - was $3: 2(60: 40)$ compared to $1: 2(33: 67)$ at $25^{\circ} \mathrm{C}$.

The aziridine-2-carboxylate derivative $(S)$-11 (Figure 4.10) has an almost identical $\mathrm{R}_{\mathrm{f}}$ to iodoalanine $(\boldsymbol{R})-\mathbf{6 a}$ and its regioisomer $\mathbf{6 b}$ by t.l.c., which led to difficulties in purification. After chromatographic purification to remove traces of starting material, the products - including the aziridine $(\boldsymbol{S}) \mathbf{- 1 1}$ - were dissolved in warm dichloromethane then methanol (1:5) followed by gradual cooling to room temperature, then to $4{ }^{\circ} \mathrm{C}$ and finally $-20^{\circ} \mathrm{C}$ when the viscous oily mixture precipitated out. The ${ }^{1} \mathrm{H}$ NMR spectrum of the precipitated product confirmed the absence of the aziridine by-product. However, the proportion of the isomers had changed from 1:2 to approximately $1: 4((\boldsymbol{R}) \mathbf{- 6 a}: \mathbf{6 b})$.

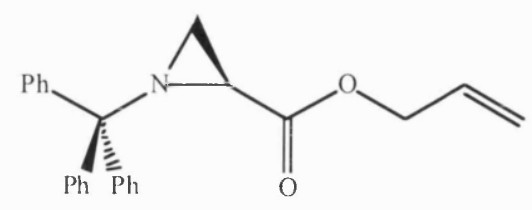

$(S)-11$

Figure 4.10. (S)-1-Triphenylmethyl-aziridine-2-carboxylic acid allyl ester 
${ }^{1} \mathrm{H}$ NMR revealed that the filtrate consisted of the two isomers, $(\boldsymbol{R})-\mathbf{6} \mathbf{a}$ and $\mathbf{6} \mathbf{b}$, and the aziridine derivative $(S)-11$ in a 2:1:1 mixture. Further precipitation attempts failed. Attempts were also made to separate out the two isomers via flash column chromatography with very slow gradients but these were unsuccessful.

A hypothesis was previously proposed, ${ }^{78}$ that restricted rotation about the $C_{\alpha}-C_{\beta}$ bond led to the formation of the two spectroscopically distinct rotational isomers. Therefore, although three different conformations were possible (Figure 4.11), two were thought to appear similar by NMR leading to the observation of only two apparent sets of signals. The same hypothesis was initially applied to $(\boldsymbol{R})-\mathbf{6 a}$ and $\mathbf{6} \mathbf{b}$.

The formation of two locked conformers in this reaction, although precedented in the work of Dugave and Menez, appeared unlikely: apart from this work, restricted rotation in acyclic compounds of such a size has not, to our knowledge, been widely reported elsewhere. Careful analysis of the purified products, however, did not clarify the situation; microanalysis, high resolution mass spectrometry, ${ }^{13} \mathrm{C}$ and ${ }^{1} \mathrm{H}$ COSY NMR and HPLC experiments proved to be insufficient. The hypothesis that the second isomer might have arisen from triphenylmethyl cleavage by acidic deuterated chloroform in the NMR tube was also rejected, as the ${ }^{1} \mathrm{H}$ NMR spectrum of the sample recorded with the use of alumina-treated deuterated chloroform produced identical results.
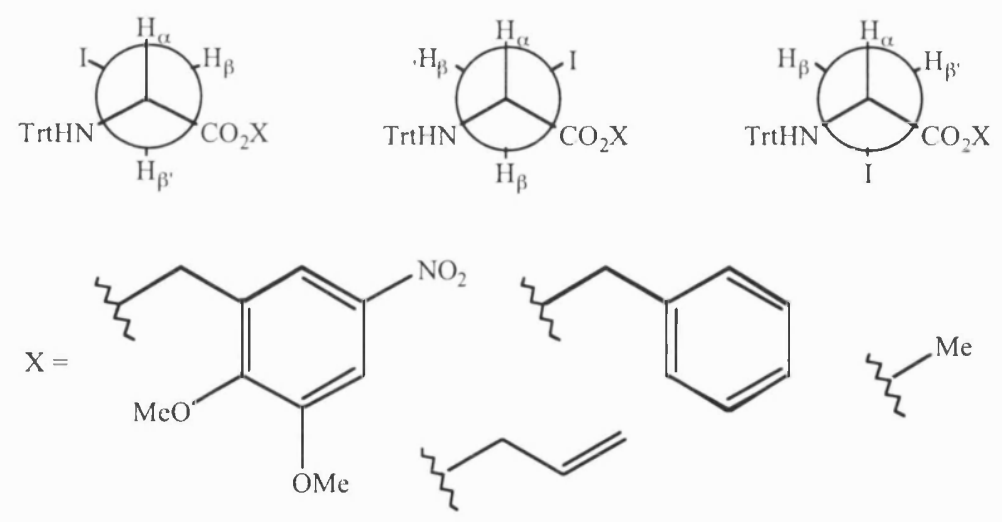

Figure 4.11. Fisher projections displaying three conformations of $N$ triphenylmethyl-3-iodo- $(R)$-alanine 6-nitroveratryl, ${ }^{78}$ benzyl, ${ }^{76}$ methyl ${ }^{76}$ and allyl ester 
Both the crude iodoalanine derivative (in the presence of the aziridine-2-carboxylate) and the precipitated product were used in the next coupling step with cysteine derivative 9.

The reactions described above were reproducible at larger scales of up to $42 \mathrm{mmol}$.

\subsection{Synthesis of protected lanthionine $(R, R)-10$}

With the apparent success of the synthetic route to the $N$-triphenylmethyl- $\beta$-iodo- $(R)$ alanine allyl ester $(\boldsymbol{R})$-6a and $N$-fluorenylmethoxycarbonyl-(R)-cysteine tbutyl ester 9, the two compounds were treated with caesium carbonate in an attempt to form the lanthionine derivative $(\boldsymbol{R}, \boldsymbol{R})$-10 (Figure 4.12).

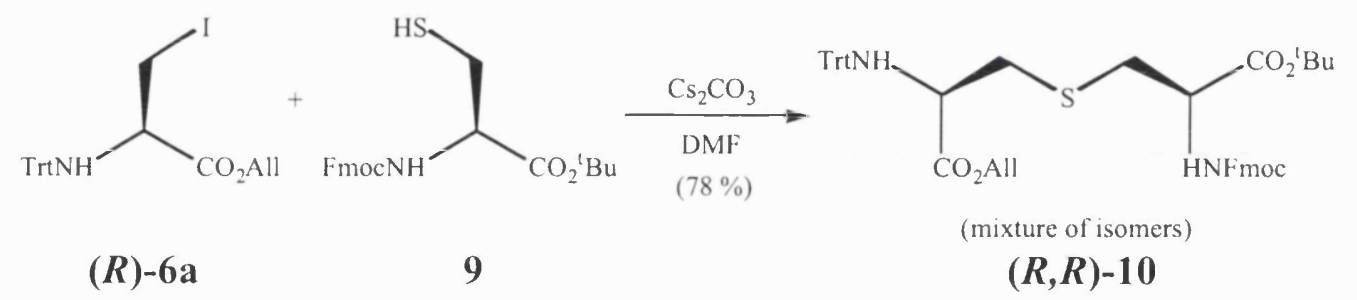

Figure 4.12. Formation of the triphenylmethyl-derived $(R, R)$-lanthionine

The order in which the reagents were added was significant to the success of the reaction. Although caesium carbonate was needed to promote the formation of the cysteine thiolate anion, it could also, to a certain extent, remove the Fmoc group if left solely in solution with the cysteine derivative for as little as 5 minutes. It was therefore imperative to have both residues in solution before adding the caesium carbonate.

The reaction seemed to produce the desired lanthionine in respectable yields as what appeared to be two rotational isomers as determined by ${ }^{1} \mathrm{H}$ NMR. The proportion of the isomers was identical $(1: 2)$ to that observed for the crude iodoalanine starting material. Again, the products were carefully analysed by microanalysis, high resolution mass spectrometry, ${ }^{13} \mathrm{C}$ and ${ }^{1} \mathrm{H}-\mathrm{COSY}$ NMR and HPLC experiments to clarify the issue of conformational isomers, but without success. 
In previous work, Goodman et al. ${ }^{199}$ produced all four diastereoisomeric lanthionine residues (via serine- $\beta$-lactone route), albeit with different protecting groups, and concluded that the difference in chemical shifts between them were small but detectable by $1-\mathrm{D}{ }^{1} \mathrm{H}$ NMR $(500 \mathrm{MHz}, 300 \mathrm{~K})$. The fact that the proportion of isomers in the reaction above was retained from the proportion of the iodoalanine isomers, and that there was an absence of any additional ${ }^{1} \mathrm{H}$ signals adjacent to the expected signals, would suggest retention of stereochemistry at both stereogenic centres as suggested by Dugave and Menez. ${ }^{76}$ However, when the precipitated iodoalanine derivative with isomer ratio of $1: 4((\boldsymbol{R})-\mathbf{6 a}: \mathbf{6 b})$ was treated with the cysteine derivative 9 the reaction yielded the major isomer as the sole product, enabling the ${ }^{1} \mathrm{H}$ NMR spectrum of the previous 1:2 mixture of isomers to be unambiguously assigned (Appendix B). The reason for this observation is discussed in Chapter 5, section 5.1.2.

The protecting groups on $(\boldsymbol{R}, \boldsymbol{R})$-10 were then adjusted, to afford the target molecule. The bulky triphenylmethyl group was cleaved with $5 \%$ trifluoroacetic acid in DCM liberating the free amine, which was not isolated but was immediately treated with allyloxycarbonyl chloride to give $(\boldsymbol{R}, \boldsymbol{R})-\mathbf{1 2}$ (Figure 4.13). The low acid concentration of the first step was not strong enough to cleave the tbutyl group.

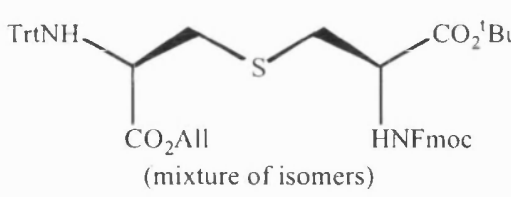

$(R, R)-10$

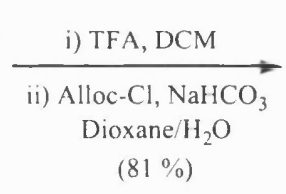

$(81 \%)$

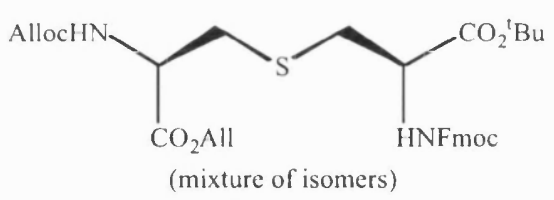

$(R, R)-12$

Figure 4.13. Replacement of triphenylmethyl with allyloxycarbonyl on lanthionine $(R, R)-10$

Initial analysis of the ${ }^{1} \mathrm{H}$ NMR of the compound appeared to indicate that there were no longer two different isomers present, as expected from the removal of the triphenylmethyl group, which was originally thought to be responsible for the restricted bond rotations resulting in two conformers. However, careful observation revealed that the integrations for the ${ }^{1} \mathrm{H}$ NMR did not add up to the correct number of protons and in some cases gave integrations of less than one ${ }^{1} \mathrm{H}$. Further analyses were carried out, discussed in Chapter 5, which clearly support the evidence 
suggesting that the iodoalanine $(\boldsymbol{R})-\mathbf{6}$ in fact consisted of a mixture of regioisomers, $(R)-6 \mathrm{a}$ and $(R / S)-6 \mathrm{~b}$.

The reactions described above were reproducible at larger scales of up to $2 \mathrm{mmol}$.

Believing that the lanthionine derivative $(\boldsymbol{R}, \boldsymbol{R}) \mathbf{- 1 2}$ had been synthesised, efforts then turned to producing the protected meso-lanthionine $(\boldsymbol{S}, \boldsymbol{R})$-10.

\subsection{Synthesis of protected (S)-iodoalanine (S)-6a}

The synthesis of the lanthionine derivative $(\boldsymbol{S}, \boldsymbol{R})$-10 was then attempted, starting from $(R)$-serine. The $N$-triphenylmethylation of serine was carried out following the method described above. This was then followed by esterification of the carboxyl functionality with an allyl group, followed by the mesylation step as described above with the reaction carried out in THF. The next step was the iodination reaction, which produced similar results to $(\boldsymbol{R})$-6a (Figure 4.14$)$.

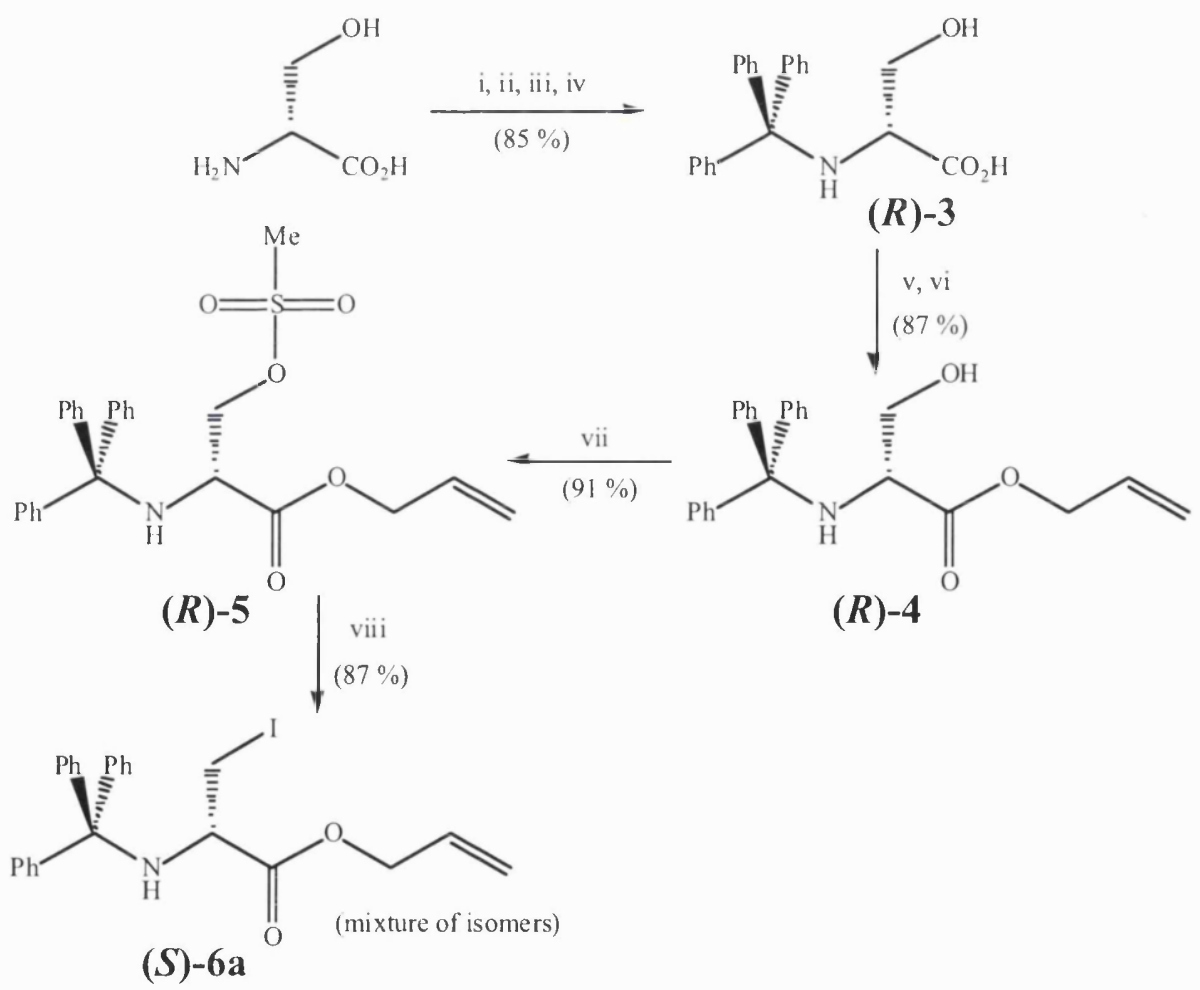

i) $\mathrm{Me}_{3} \mathrm{Si}-\mathrm{Cl}$, TEA, DCM, ii) $\mathrm{MeOH}$ (dry), $0{ }^{\circ} \mathrm{C}$, iii) $\mathrm{Ph}_{3} \mathrm{C}-\mathrm{Cl}$, TEA, iv MeOH, TEA, v) $\mathrm{Cs}_{2} \mathrm{CO}_{3}, \mathrm{MeOH}$,

vi) Allyl bromide, DMF, viii) Methanesulfonyl chloride, TEA, THF, viii) Nal, acetone

Figure 4.14. Preparation of $(S)$-iodoalanine derivative $(S)$-6a 
The proportion of the isomers, $(\boldsymbol{S}) \mathbf{- 6} \mathbf{a}$ and $\mathbf{6} \mathbf{b}$, prior to precipitation was 1:3 (with approximately $10 \%$ aziridine derivative $(\boldsymbol{R})$-11 by-product, (Figure 4.15 )) and 1:5 after precipitation (Appendix C).

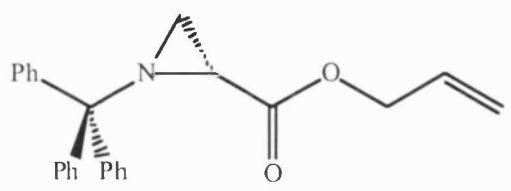

(R)-11

Figure 4.15. (R)-1-Triphenylmethyl-aziridine-2-carboxylic acid allyl ester

In an attempt to clarify the isomer issue, optical rotation experiments were carried out on both $(R)$ - and $(S)$-iodoalanine derivatives (Table 4):

\begin{tabular}{|c|c|c|}
\hline & $(R)-6$ & $(S)-6$ \\
\hline$|\alpha|_{\mathrm{D}}{ }^{\mathrm{T}} /{ }^{\circ}$ & $+8.9\left(15^{\circ} \mathrm{C}\right), 1: 4$ isomers & $-3.3\left(17^{\circ} \mathrm{C}\right), 1: 5$ isomers \\
\hline
\end{tabular}

Table 4. Optical rotation values for $(R)-6$ and $(S)-6$ as mixtures of isomers

It should be noted that even though the two enantiomers deflect the plane of polarised light oppositely, two conformationally locked conformers would have different optical rotation values. It was therefore not surprising that the modulus of the optical rotation values $\left(\left|[\alpha]_{\mathrm{D}}^{\mathrm{T}}\right|\right)$ for the two enantiomers were not identical, initially thought to be due to the difference in conformer proportions for each enantiomer. However, evidence discussed in Chapter 5 clearly demonstrates that the difference in optical rotation values between $(R)-6$ and $(S)-6$ (both as mixtures of isomers) was caused by a mixture of regioisomers for each enantiomer.

\subsection{Synthesis of protected lanthionine $(S, R)-10$}

With the synthetic route to the fully protected lanthionine $(\boldsymbol{R}, \boldsymbol{R})-\mathbf{1 0}$ apparently resolved, the same methodology was applied in an attempt to synthesise the corresponding derivative of meso-lanthionine. With the same conditions as above the reaction proceeded in high yields (Figure 4.16). 


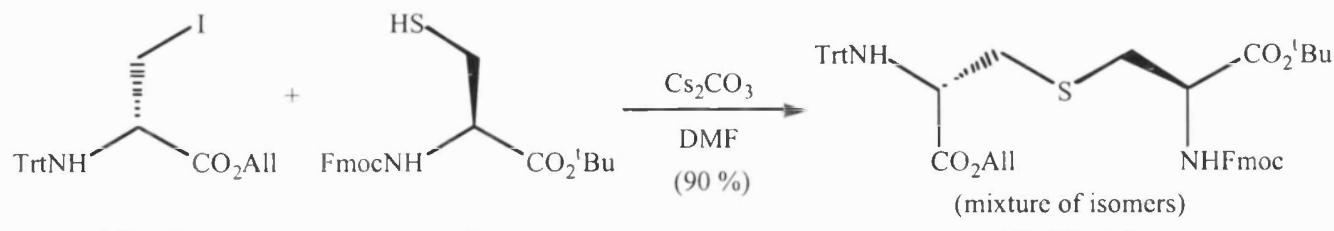

(S)-6a

9

$(S, R)-10$

Figure 4.16. Formation of the lanthionine derivative $(S, R)-10$

Again, the proportion of isomers in $(\boldsymbol{S}, \boldsymbol{R})$-10 was retained (1:3) from the crude iodoalanine (S)-6. Interestingly, the proportion of the isomers in $(S, R)-\mathbf{1 0}$ was also retained (1:5) from the precipitated iodoalanine derivative $(\boldsymbol{S})$-6. Small differences were observed in the ${ }^{1} \mathrm{H}$ NMR chemical shifts of this derivative (Appendix D) compared to its diastereoisomer $(\boldsymbol{R}, \boldsymbol{R})-\mathbf{1 0}$, which would suggest that the two are diastereoisomers, confirming the observations made by Goodman et al. concerning their very similar spectra of diastereoisomeric lanthionine derivatives differing by only one stereogenic centre. ${ }^{199}$

With the difference in isomer proportions it was difficult to establish what the optical rotation for one diastereoisomer should be compared to the other (Table 5). Goodman et al. ${ }^{199}$ reported that two identical derivatives of lanthionine diastereoisomers, which differ by only one chiral centre, possessed optical rotation values differing only in magnitude but not necessarily in sign. However, the difference in protecting groups used here would probably account for this. Further discussion in Chapter 5 examines the nature of the isomerism of the two species in each diastereoisomer.

Optical rotations were also carried out on both residues (Table 5):

\begin{tabular}{|c|c|c|}
\hline & $(\boldsymbol{R}, \boldsymbol{R})-\mathbf{1 0}$ & $(\boldsymbol{S}, \boldsymbol{R})-\mathbf{1 0}$ \\
\hline$\left[\left.\boldsymbol{\alpha}\right|_{\mathrm{D}}{ }^{\mathrm{T}} /^{\circ}\right.$ & $+12.1\left(16^{\circ} \mathrm{C}\right), 1: 2$ isomers & $-4.1\left(18^{\circ} \mathrm{C}\right), 1: 3$ isomers \\
\hline
\end{tabular}

Table 5. Optical rotation values for $(R, R)-10$ and $(S, R)-10$

Replacement of the triphenylmethyl group with an allyloxycarbonyl protecting group then followed, as before (Figure 4.17). The 'H NMR for this compound was virtually 
identical to that of the $(R, R)$-diastereoisomer $(\boldsymbol{R}, \boldsymbol{R})$-12. Further discussion follows, in Chapter 5.

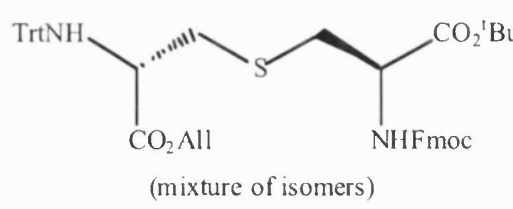

$(S, R)-10$

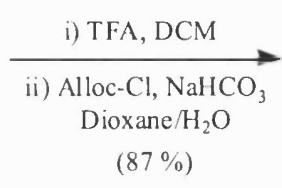

$(87 \%)$

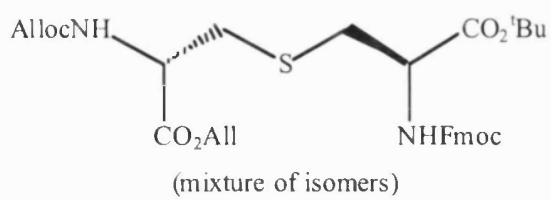

$(S, R)-12$

Figure 4.17. Replacement of triphenylmethyl with allyloxycarbonyl on lanthionine $(S, R)-10$

The final step was deprotection of the tbutyl protecting group with trifluoroacetic acid (Figure 4.18).

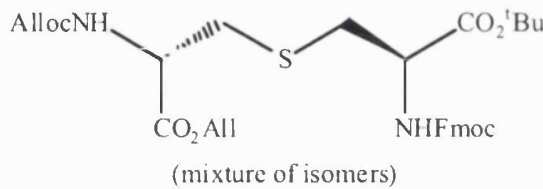

$(S, R)-12$

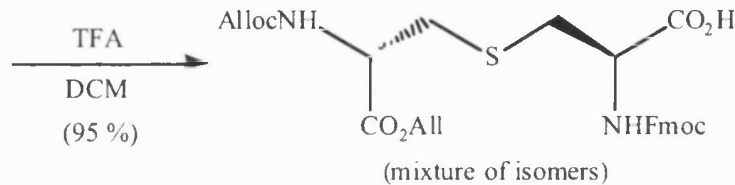

1

Figure 4.18. Removal of tbutyl from allyloxycarbonyl-derived lanthionine $(S, R)-12$

The ${ }^{1} \mathrm{H}$ NMR of this final residue, which was ready for incorporation onto solid phase resin, produced very broad signals in deuterated chloroform and relatively broad signals in methanol- $d_{4}$ due to the free carboxyl group. It was therefore difficult to assign (Appendix E). However, high-resolution mass spectrometry and elemental analysis apparently demonstrated that the desired compound was present in high purity.

\subsection{Synthesis of ivDde/Dmab protected (S,R)-lanthionine 2}

With the ultimate aim of this research being the synthesis of peptides with overlapping thioether bridges, as found in lantibiotics such as nisin, ${ }^{7}$ subtilin $^{8}$ and plantaricin $\mathrm{W},{ }^{37}$ the synthesis of $(S, R)$-lanthionine having protecting groups orthogonal to Fmoc, ${ }^{t} \mathrm{Bu} / \mathrm{Boc}$ and allyl/Aloc was required. Due to the proven orthogonality of the dimedone-based protecting groups to $\mathrm{Fmoc},{ }^{t} \mathrm{Bu} / \mathrm{Boc}$ and allyl/Aloc systems (Chapter 2, section 2.4.2.), the synthesis of the ivDde/Dmabprotected $(S, R)$-lanthionine 2 (Figure 4.19$)$ was therefore attempted. 


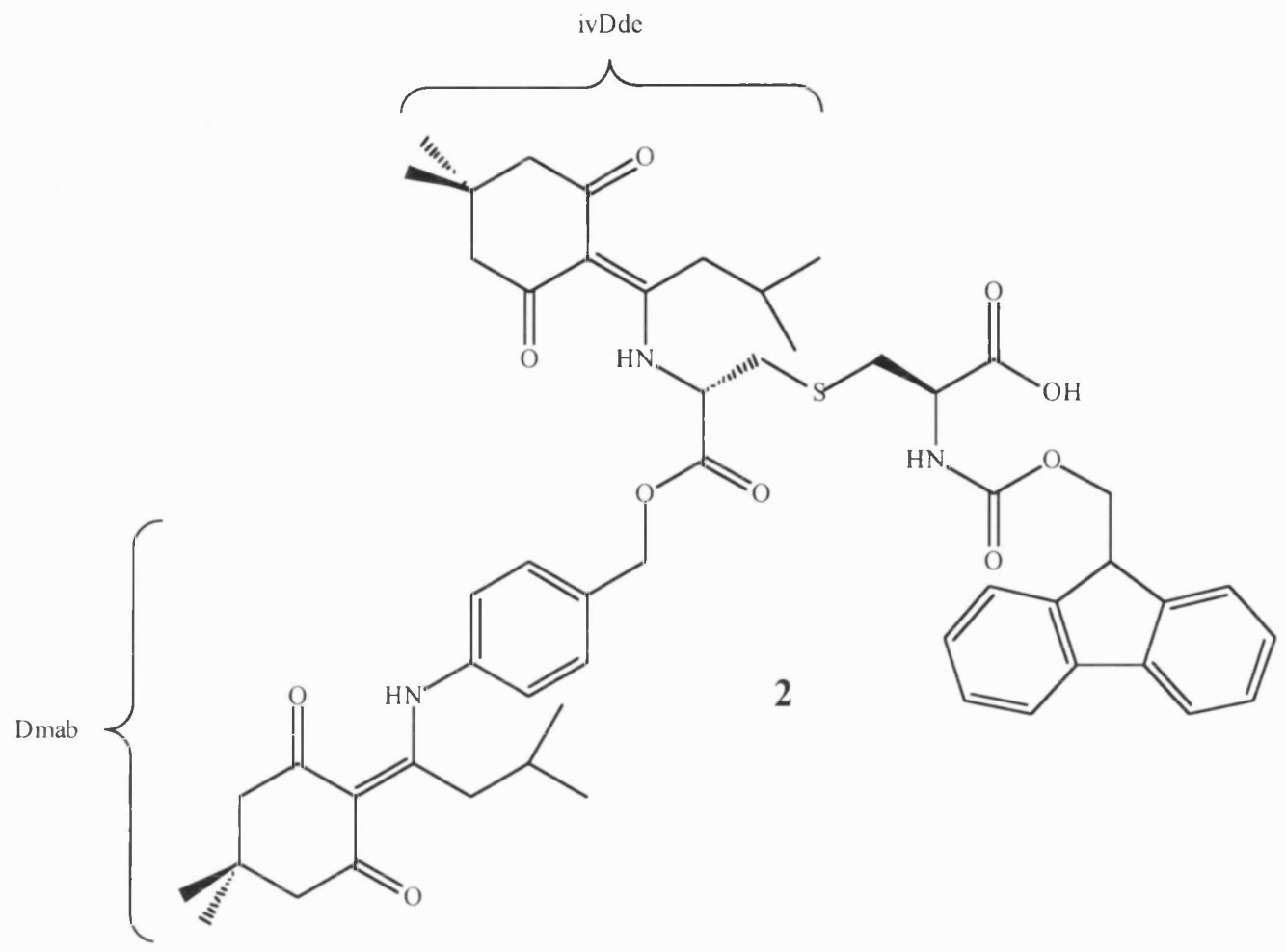

Figure 4.19. ivDde/Dmab derivative of $(S, R)$-lanthionine

The proposed strategy for synthesising ivDde/Dmab $(S, R)$-lanthionine initially involved incorporation of the Dmab group at the $N$-triphenylmethyl- $(R)$-serine $(\boldsymbol{R})-\mathbf{3}$ stage to give 13. The first attempt at esterification, with diisopropylcarbodiimide, 1hydroxybenzotriazole and DIPEA in dichloromethane, was carried out using the method described by Chan et al. ${ }^{156}$ (Figure 4.20). The attempt was unsuccessful, with recovery of the Dmab-OH and an unidentified triphenylmethyl-containing compound. It was noted that 1-hydroxybenzotriazole did not dissolve in dichloromethane. Hence a second attempt was carried out omitting this reagent, and using dicyclohexyl - instead of diisopropyl - carbodiimide where the urea by-product could now be easily removed through filtration. This attempt also proved to be unsuccessful. A third attempt was carried out using 4-dimethylaminopyridine, which, apart from being a base, is also a powerful acylation catalyst, but this also failed. It seemed that all efforts were futile. In each case only the Dmab-OH starting material was isolated (Figure 4.20). 


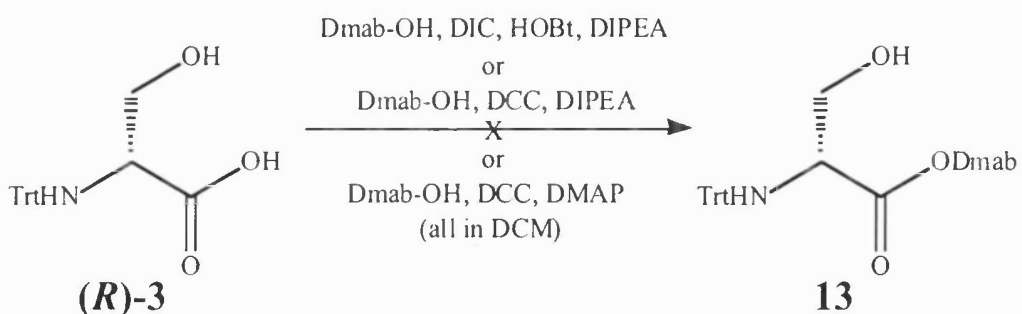

Figure 4.20. Unsuccessful attempts at esterification of $N$-triphenylmethyl-(R)-serine (R)-3 with Dmab-OH

The reason for the lack of success of the coupling of the serine with the Dmab-OH was thought to be due to the availability of the $\beta-\mathrm{OH}$ on serine. This could have led to intra- and/or intermolecular $\beta$-hydroxyl displacement of the -ODCC group. However, there is no hard evidence to explain why these reactions failed.

As an alternative, the $\beta-\mathrm{OH}$ was protected as the tbutyldimethylsilyl ether $\mathbf{1 4}$ (Figure 4.21). Esterification of $\mathbf{1 4}$ with Dmab-OH was then attempted using various conditions but without success.

Conversion of the Dmab-OH to the bromo analogue $\mathbf{1 5}$, to allow a similar reaction with the caesium salt of $N$-triphenylmethyl- $(R)$-serine $(\boldsymbol{R})-3$ to that used in the synthesis of $N$-triphenylmethyl- $(R)$-serine allyl ester $(\boldsymbol{R}) \mathbf{- 4}$, was then attempted (Figure 4.22). Although the reaction produced the desired Dmab-Br, as confirmed by $\left[\mathrm{M}+{ }^{79} \mathrm{Br}\right]^{+}$and $\left[\mathrm{M}+{ }^{81} \mathrm{Br}\right]^{+}$in mass spectrometry, purification of the compound proved to be extremely problematic. After numerous flash column chromatographic attempts, this approach was abandoned.

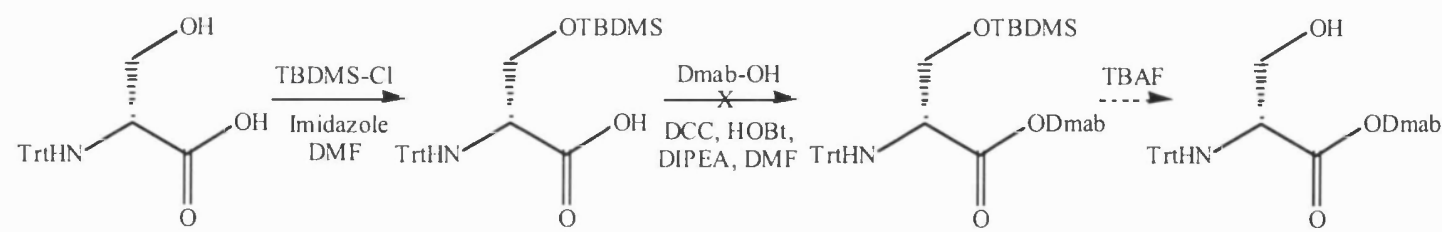
$(R)-3$

14

Figure 4.21. Scheme for carboxyl protection of $N$-triphenylmethyl- $(R)$-serine $(\boldsymbol{R})-\mathbf{3}$ with Dmab-OH via TBDMS $\beta-\mathrm{OH}$ protection 


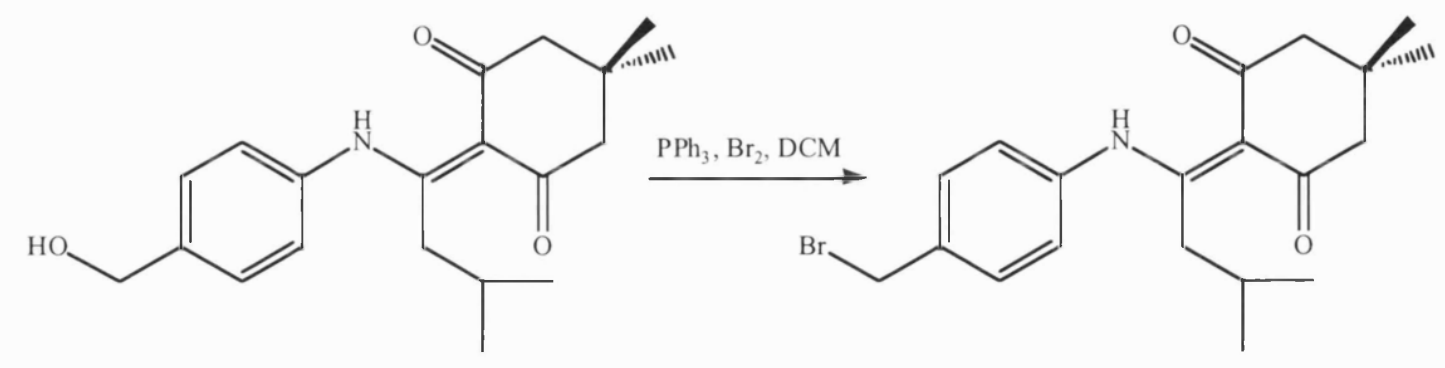

15

Figure 4.22. Bromination of Dmab-OH

A different approach, in which the protecting groups were manipulated once at the lanthionine stage, was therefore attempted. This has the added advantage of using common intermediates up to the thioether bridge formation step.

Compound $(\boldsymbol{S}, \boldsymbol{R})-\mathbf{1 0}$ (as a mixture of isomers - see Chapter 5) was treated with $5 \%$ trifluoroacetic acid in DCM to selectively cleave the triphenylmethyl group, liberating the free amine. The resulting product was then treated, without further purification, with a solution of ivDde-OH in ethanol at $40{ }^{\circ} \mathrm{C}$ (Figure 4.23). ${ }^{154}$

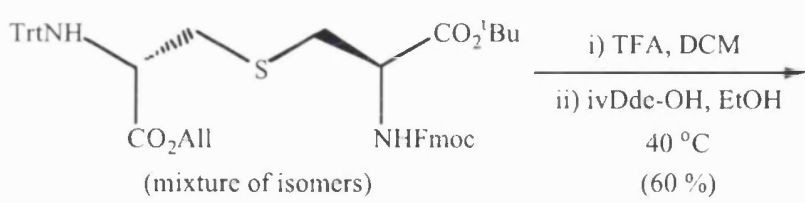

$(S, R)-10$<smiles>NC(CSC[C@H](N[N+](=O)[O-])C(=O)O)C(=O)O</smiles>

16

Figure 4.23. Replacement of triphenylmethyl protecting group with ivDde on compound $(S, R)-10$

The ${ }^{1} H$ NMR of the product initially suggested the presence of one single isomer as a result of the removal of the triphenylmethyl group. However, repeated attempts at this reaction produced the desired compound in a maximum yield of $60 \%$ only, for a batch of starting material containing isomers in a 1:3 ratio. This suggested that only one isomer had reacted with the ivDde-OH.

The search for the other isomer, either already protected with the ivDde group or not, led to the discovery of a polar species that was eluted last from the flash column. It was thought to be the other isomer present with some impurities, but without the ivDde group attached. Mass spectrometry and the colourimetric ninhydrin test 
confirmed this. This crude material was then treated with the ivDde-OH as before, however the amino acid did not dissolve in the solvent used. This resulted in the failure of the ivDde-OH to couple with the free amine. Various solvents were tested for solubility properties, including water, but none dissolved the other conformer. It did, however, dissolve in deuterated dimethylsulfoxide and this confirmed, by ${ }^{1} \mathrm{H}$ NMR, that the crude material did contain the other isomer, de-triphenylmethylated, along with other unidentified impurities which were not separable via flash column chromatography. The peculiarity of one isomer reacting with the ivDde-OH but not the other was difficult to explain at the time. However, it was decided that the available ivDde-protected species should be pushed forward, prior to dealing with the solubility issue of the other isomer. Further discussion, explaining these observations, follows in Chapter 5.

With one isomer of the ivDde-protected species in hand, the allyl group was cleaved with tetrakis(triphenylphosphine) palladium(0) using the published procedure (Figure 4.24). ${ }^{128}$ The resulting product was carried through to the next step without purification, and reacted with Dmab-OH to apparently give the desired ester (Figure 4.24).

The reaction conditions for coupling the Dmab group onto the de-allylated species 16 were identical to earlier trial reactions which had been carried out with $\mathrm{N}$ triphenylmethyl- $(R)$-serine( $O$-'butyldimethylsilyl) but had failed. The reason for this was unclear. Compound 17 was carried through to the next step without purification as several attempts to purify via normal phase flash column chromatography proved unsuccessful. This may be due to the difficulty of separating tetrakis(triphenylphosphine) palladium $(0)$ debris.

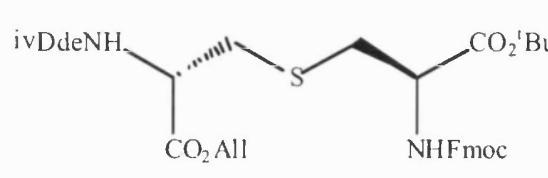

16

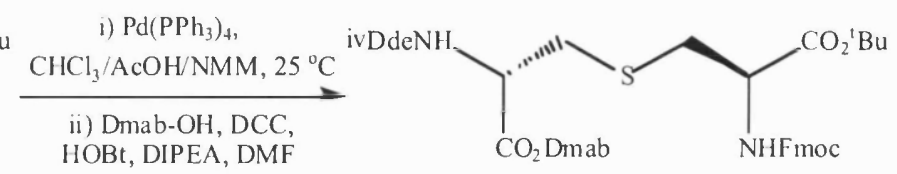

17

Figure 4.24. Replacement of allyl with Dmab protecting group on ivDde-derived $(S, R)$-lanthionine 16 
With the ivDde/Dmab product 17 thought to be in hand, the crude material was treated with $50 \%$ trifluoroacetic acid in DCM to cleave the tbutyl group (Figure 4.25).

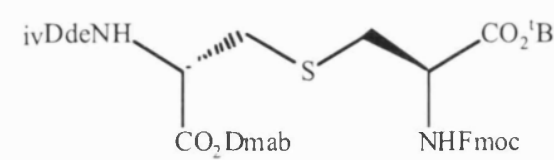

17
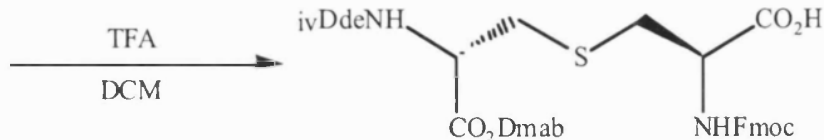

2

Figure 4.25. Removal of ${ }^{t}$ butyl from ivDde-derived $(S, R)$-lanthionine 17

A small amount of $\mathbf{2}$ was purified via preparative reverse phase HPLC for full characterisation and ready for solid phase peptide synthesis. 


\section{Chapter 5}

\section{Results and Discussion II}

\subsection{Investigation of the conformational isomer issue}

The anomalies observed by NMR for the iodoalanine derivatives $(R)-6$ and $(S)-6$ and the thioether amino acid derivatives $(R, R)-10,(S, R)-10,(R, R)-12,(S, R)-12,16$, and 17 (Figure 5.1) raised concerns with regard to the stereochemical integrity of these molecules, despite the apparently proven hypothesis that these anomalies are caused by sterically locked conformational isomers. ${ }^{76}$ With the apparent success of the synthesis of the target lanthionine derivatives $\mathbf{1}$ and $\mathbf{2}$, an in-depth investigation on the stereochemistry of these and related compounds commenced. HPLC analyses, 
further NMR experiments, test reactions and attempts at recrystallisation for x-ray structural determination were carried out, yielding unexpected results.

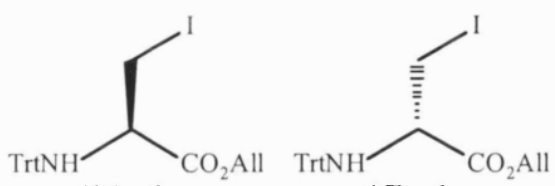

$$
(R)-6 a \quad(S)-6 a
$$
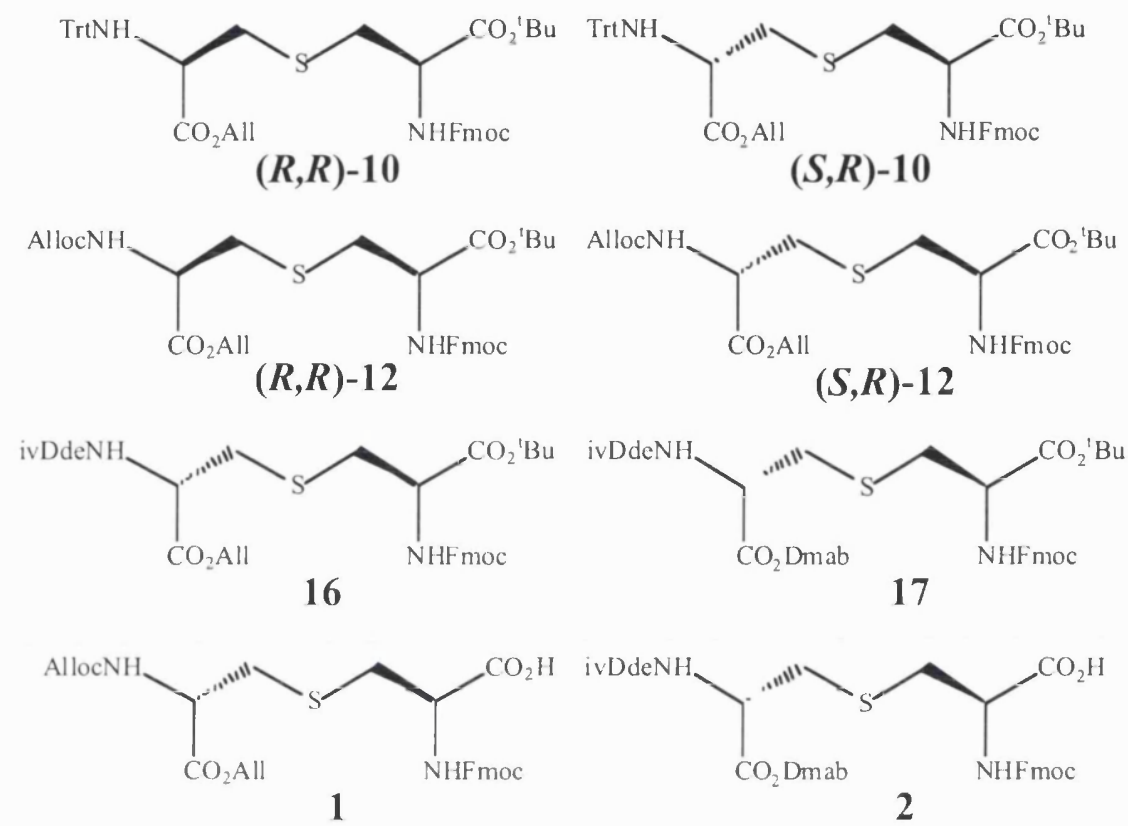

Figure 5.1. Iodoalanine and lanthionine targets

\subsubsection{HPLC analyses}

The fact that the two isomers of each iodoalanine enantiomer $(S)-6$ and $(R)-6$ were partially separable via precipitation led to HPLC studies of these compounds. Preliminary analytical normal phase HPLC suggested that separation of the two isomers of $(S)-6$ and $(R)-6$ could be achieved. Separation of the isomers of $(S)-6$ by preparative normal phase HPLC was attempted. A gradient of $2-3 \%$ ethyl acetate in hexane over $40 \mathrm{~min}$ gave only partial separation [samples contained approximately $9: 1$ of $(S)-\mathbf{6 a}: \mathbf{6 b}$ and $\mathbf{6 b}:(S)-\mathbf{6 a}$ ] of the two species (Appendix F, Appendix G). It was noticed that the sample containing predominantly the major isomer was a solid and the one containing predominantly the minor isomer was a liquid. 
Recrystallisation of the sample containing predominantly the major isomer was unsuccessful, preventing structure determination by x-ray crystallography.

Further analyses were carried out on the precipitated (major isomer enriched) $(\boldsymbol{S})-\mathbf{6}$. Elemental analysis indicated a high accuracy to the calculated theoretical mass and mass spectrometry indicated the presence of only one species where $\mathrm{M}^{+}=497$, with all the expected fragmentations. Although these data indicated the absence of an impurity of a different elemental constitution or mass, they did not rule out an impurity of the same mass, i.e. some form of isomer.

Compound $(\boldsymbol{S}, \boldsymbol{R}) \mathbf{- 1 0}$ was also analysed by HPLC. Analytical reverse phase HPLC under a range of conditions showed only a single peak. Under the same conditions, a single peak was also observed for compound $(\boldsymbol{R}, \boldsymbol{R}) \mathbf{- 1 0}$, with a very marginal difference in the retention times. Each analytical normal phase HPLC of $(\boldsymbol{R}, \boldsymbol{R})$-10 and $(\boldsymbol{S}, \boldsymbol{R})-\mathbf{1 0}$, on the other hand, produced a broad peak.

Recrystallisation of $(\boldsymbol{R}, \boldsymbol{R})-\mathbf{1 0}$ and $(\boldsymbol{S}, \boldsymbol{R})$-10 (Figure 5.2) was also unsuccessful, again preventing structure determination by x-ray crystallography.

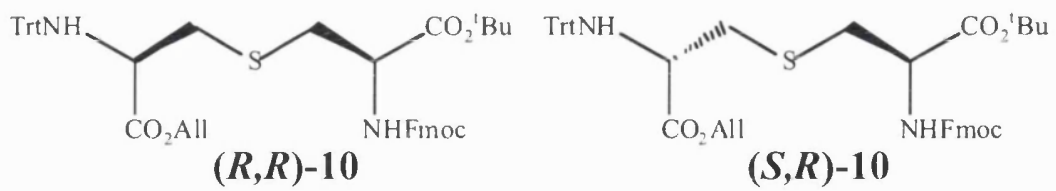

Figure 5.2. Structures of $(R, R)-10$ and $(S, R)-10$

Analytical normal phase HPLC analysis of compound $(\boldsymbol{S}, \boldsymbol{R}) \mathbf{- 1 2}$ revealed that it contained two separable components. The two components were successfully separated by preparative normal phase HPLC (Appendix H). It was thought that ${ }^{1} \mathrm{H}$ NMR indicated that the two components were the major and minor isomers. The spectra of the isolated isomers were more readily interpretable (Appendix I) than that of the mixture (with a marked difference in chemical shift values between their corresponding $\alpha-\mathrm{H}$ signals near the Aloc moiety), enabling complete interpretation of the initially ambiguous integrations described in Chapter 4. 
These results made the conformational isomer hypothesis increasingly unlikely and therefore detailed NMR analyses were carried out.

\subsubsection{NMR analyses}

${ }^{1} \mathrm{H}-\mathrm{COSY}$ NMR analysis of both enantiomers of the iodoalanine $(\boldsymbol{R})-\mathbf{6}$ and $(\boldsymbol{S})-\mathbf{6}$ confirmed that there was no overlap between the off-diagonal cross peaks of the major and minor isomers. This observation was also true for compounds $(\boldsymbol{R}, \boldsymbol{R})-\mathbf{1 0}$ and $(S, R)-10$, and for compounds $(R, R)-12$ and $(S, R)-12$. Each isomer's COSY pattern was independent of that of the other.

If the two species observed by ${ }^{1} \mathrm{H}$ NMR for iodoalanine and supposed lanthionine derivatives were conformers as originally reported, ${ }^{76}$ a VT experiment should confirm this by coalescence of the signals at higher temperatures. As a general example, the two singlets observed by ${ }^{1} \mathrm{H}$ NMR for the $N$-methyl groups of DMF are caused by restricted rotation about the $\mathrm{N}-\mathrm{C}(\mathrm{O})$ bond. On warming, the two lines broaden and coalesce with a coalescence temperature of $64^{\circ} \mathrm{C}(60 \mathrm{MHz})$, as the molecules are given sufficient energy to overcome the barrier to rotation about that bond, indicating that the time averaged environment of the two methyl groups is now identical. $^{200}$ This concept was applied to compound $(\boldsymbol{R})-\mathbf{6}$, as it had been hypothesised that it existed as two spectroscopically different conformers. ${ }^{76} \mathrm{VT}^{1} \mathrm{H}$ NMR experiments were carried out with $\mathrm{T}_{\max }=50^{\circ} \mathrm{C}$. Unfortunately the precipitated iodoalanine derivative $(\boldsymbol{R})-6$ started to degrade at higher temperatures in solution. It was also noticed that once $(\boldsymbol{R})-6$ was dissolved in deuterated DMSO, the aziridine derivative $(S)$-11 immediately started to form (the precipitated iodoalanine derivative $(R)-6$ did not show the presence of the aziridine by-product in deuterated chloroform). As the temperature was increased the amount of the aziridine-2carboxylate also increased (Figure 5.3), along with other unidentified degradation product(s) (Appendix J). In fact, Kato et al. conveniently prepared optically active $(S)$-1-triphenylmethyl-aziridine-2-carboxylates from $\beta$-halo amino acids with heating, in the presence of potassium hydrogen sulfate. ${ }^{201}$ It was therefore not 
possible to verify that $(\boldsymbol{R}) \mathbf{- 6} \mathbf{a}$ and $\mathbf{6 b}$ were conformationally restricted conformers using this technique.

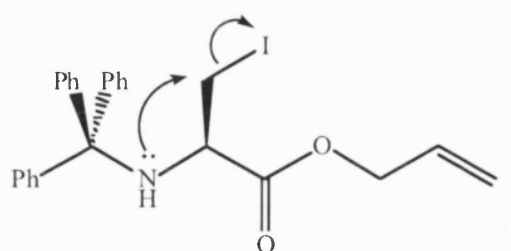

$(R)-6 a$

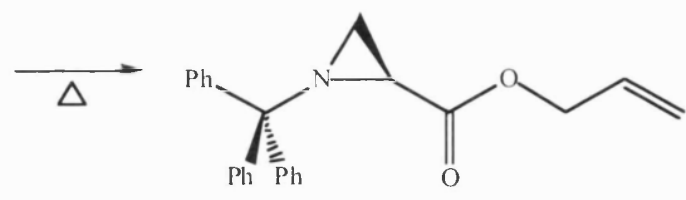

$(S)-11$

Figure 5.3. Mechanism for the formation of aziridine $(S)-11$ from $N$ triphenylmethyl- $\alpha$-iodo- $(R)$-alanine allyl ester $(\boldsymbol{R})$-6a

VT 'H NMR experiments were also carried out on the presumed lanthionine derivative $(\boldsymbol{R}, \boldsymbol{R})-\mathbf{1 0}$. However, even at temperatures over $100^{\circ} \mathrm{C}$, no coalescence of peaks was observed. Again, only unidentified degradation products were observed (Appendix K).

Assignment of the ${ }^{13} \mathrm{C}$ spectra for the iodoalanine and lanthionine derivatives proved to be difficult due to their being mixtures. The isomers of the iodoalanine $(\boldsymbol{S})-\mathbf{6}$ (as well as ( $R$ )-6) each gave a ${ }^{13} \mathrm{C}$ signal between $9-21 \mathrm{ppm}$ and another between $48-$ $56 \mathrm{ppm}$, with the former being the expected upfield signals for a carbon atom attached to iodine (shielding effect) and the latter representing carbon atoms attached to nitrogen; they had been assumed to be the $\beta$ - and $\alpha-\mathrm{C}$, respectively. $\mathrm{A}{ }^{13} \mathrm{C}$ DEPT experiment confirmed that, for the minor product, the peak at $9.6 \mathrm{ppm}$ was a $\beta-\mathrm{CH}_{2}-\mathrm{I}$ signal and the peak at $55.8 \mathrm{ppm}$ was an $\alpha-\mathrm{CH}-\mathrm{N}$ signal, which is consistent with the desired product. However, the DEPT experiment also clearly indicated that, for the major product, the peak at $20.1 \mathrm{ppm}$ was a $\mathrm{CH}$ instead of the expected $\mathrm{H}_{2} \mathrm{C}$-I signal and the peak at $48.2 \mathrm{ppm}$ was a $\mathrm{CH}_{2}$ signal. The implication was that, for the major isomer, the iodine was attached to a tertiary carbon instead of a secondary one, with the structure proposed in Figure 5.4. It was therefore decided that ${ }^{13} \mathrm{C}-{ }^{1} \mathrm{H}$ correlation experiments (HSQC/HMQC/HMBC) should be carried out in order to clarify the assignments and to further investigate the structures of the isomers. 


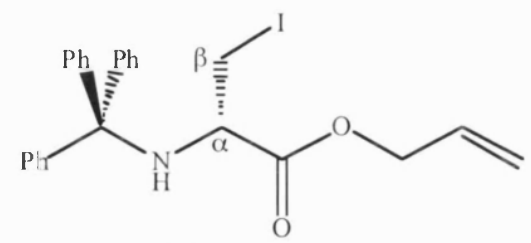

$(S)-6 a$

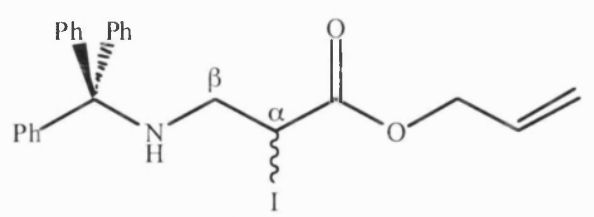

6b

Figure 5.4. ( $S)$-6a and the regioisomeric iodination product, $(R / S)$-2-iodo-3(triphenylmethylamino)-propionic acid allyl ester, an $\alpha$-substituted $\beta$-alanine derivative

A ${ }^{13} \mathrm{C}-{ }^{1} \mathrm{H}$ HSQC experiment confirmed the observation made above (Appendix L). As expected for iodoalanine $(\boldsymbol{S})-\mathbf{6 a}$, the $\beta$-Hs on the minor isomer correlated to the ${ }^{13} \mathrm{C}$ signal at $9.6 \mathrm{ppm}$ and the $\alpha-\mathrm{H}$ correlated to the ${ }^{13} \mathrm{C}$ signal at $55.8 \mathrm{ppm}$. As for the major isomer $\mathbf{6 b}$, however, the $\beta$-Hs correlated to the ${ }^{13} \mathrm{C}$ signal at $48.2 \mathrm{ppm}$ and the $\alpha-\mathrm{H}$ correlated to the ${ }^{13} \mathrm{C}$ signal at $20.1 \mathrm{ppm}$. This is consistent with the structure proposed in Figure 5.4 - an upfield ${ }^{13} \mathrm{C}$ chemical shift for a carbon attached to iodine. $\mathrm{A}^{13} \mathrm{C}-{ }^{1} \mathrm{H}$ HMBC experiment consolidated the theory, indicating an absence of a ${ }^{3} J$ correlation between the NH proton and the carbonyl carbon for the major isomer. It was extrapolated that the regioisomeric product then reacted with cysteine 9 to afford regioisomers of lanthionine. This indicates that not only had the wrong compound been predominantly synthesised at the iodoalanine and all subsequent lanthionine stages, but also that Dugave and $\mathrm{Menez}^{76}$ had wrongly characterised their iodoalanine and hence lanthionine derivatives. As discussed in Chapter 1, Dugave and Menez probed the chiral integrity of lanthionine derivatives synthesised via the iodoalanine route. Only the FLEC $^{\circledR}$ derivatives of L-alanine were detected for a $(R, R)$-lanthionine derivative, and only traces of $\mathrm{D}$-alanine derivatives were found, indicating negligible racemisation during the thioether bridge formation. Since the chiral HPLC results are compared against chromatographic traces for true samples of FLEC $^{\circledR}$-L-alanine and FLEC ${ }^{\circledR}$-D-alanine, any other peak in the chromatogram would have been considered impurities, including a $\operatorname{FLEC}^{\circledR}$ - $\beta$-alanine derivative peak. Work carried out within the group ${ }^{202}$ showed that the $N$-triphenylmethyl- $\beta$ iodoalanine benzyl ester claimed by Dugave and Menez to be present as a mixture of locked conformers, produced under identical conditions, undoubtedly gave similar ${ }^{13} \mathrm{C}-{ }^{1} \mathrm{H}$ correlation patterns in an HSQC experiment. 
The regioisomer $\mathbf{6 b}$ of the iodoalanine derivative $(\boldsymbol{S})-\mathbf{6 a}$ was thought to have been formed via the ring opening of the aziridine-2-carboxylate derivative $(\boldsymbol{R}) \mathbf{- 1 1}$ by iodide (which was present in abundance during the iodination reaction of $(\boldsymbol{R})-5$ ), at the more hindered, but perhaps more electronically favoured, $\alpha$-position (Figure 5.5).
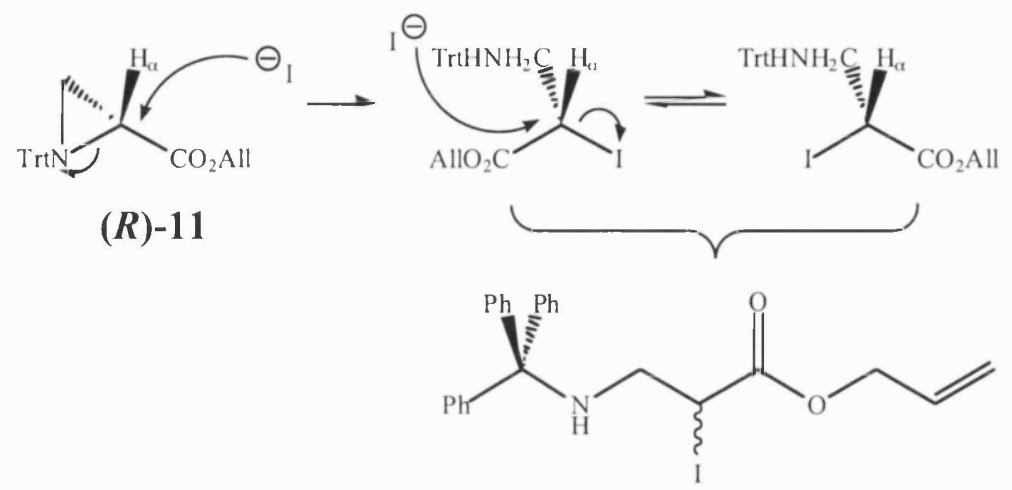

6b

Figure 5.5. Proposed mechanism for the formation of $\mathbf{6 b}$ via the aziridine-2carboxylate $(\boldsymbol{R})-\mathbf{1 1}$

As discussed in Chapter 3, aziridine-2-carboxylate ring opening by a nucleophile has been reported to take place at either $\alpha$ - or $\beta$ - or both positions depending on various different factors. Nucleophilic attack by iodide at the $\beta$-position of $(\boldsymbol{R}) \mathbf{- 1 1}$, formed from $(S)-6 a$, would straightforwardly return the original starting material. However, attack by an iodide at the $\alpha$-position of $(\boldsymbol{R})-\mathbf{1 1}$ would be more complicated. Due to the chirality at the $\alpha-C$, an $\mathrm{S}_{\mathrm{N}} 2$ attack by iodide would initially result in stereoinversion; indeed, there have been reports of this after nucleophilic ring opening reactions at $C_{2}$ of aziridine-2-carboxylates. ${ }^{170,171,183,188}$ However, due to the lability of the iodine substituent and the abundance of sodium iodide (10 equivalents) in the reaction, a second inversion by another iodide would then be possible. The result would be a mixture of enantiomers: $(R)-\mathbf{6 b}$ and $(S)-\mathbf{6 b}$ as a mixture (Figure 5.5), represented as $\mathbf{6 b}$. Notably, this potential loss of stereochemical integrity was not apparently significant in the work of Righi and D'Achille who produced predominantly $\alpha$-iodo- $\beta$-amino acid derivatives by reacting different derivatives of aziridine-2-carboxylates with sodium iodide, presumably due partly to the fact that only one equivalent of sodium iodide was used. ${ }^{191}$ 
In light of these results, NMR studies were carried out on the compounds $(\boldsymbol{R}, \boldsymbol{R})-\mathbf{1 0}$ and $(\boldsymbol{S}, \boldsymbol{R})-\mathbf{1 0}$. The HSQC results indicated a big difference in ${ }^{13} \mathrm{C}$ chemical shifts for the $\alpha_{1}$-Cs of the two species present in each sample, as well as the $\beta_{1}$-Cs (Figure $5.6)$.

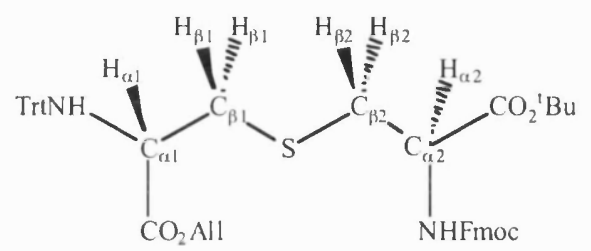

Figure 5.6. Indication of $\alpha_{1}-\mathrm{C}, \alpha_{2}-\mathrm{C}, \beta_{1}-\mathrm{C}$ and $\beta_{2}-\mathrm{C}$ for $(\boldsymbol{R}, \boldsymbol{R})-10$ and $(\boldsymbol{S}, \boldsymbol{R})-10$

The $\alpha_{1}-\mathrm{C}$ on the major product has a lower chemical shift by $9 \mathrm{ppm}$ compared to the corresponding ${ }^{13} \mathrm{C}$ signal for the minor product. The $\beta_{1}-\mathrm{C}$ has a 6 -ppm higher chemical shift compared to the corresponding ${ }^{13} \mathrm{C}$ signal for the minor product. Such large differences in chemical shifts could not be caused by a difference in conformation.

An HMBC experiment was carried out on $(\boldsymbol{S}, \boldsymbol{R})-\mathbf{1 0}$ to explore the connectivity of these molecules. In the experiment, $\beta_{2}$-Hs of the lanthionine should have a ${ }^{3} J$ correlation with the $\beta_{1}-\mathrm{C}$, across the thioether linkage (Figure 5.7). The same pattern should be observed between $\beta_{1}$-Hs and the $\beta_{2}-\mathrm{C}$ (Figure 5.7).

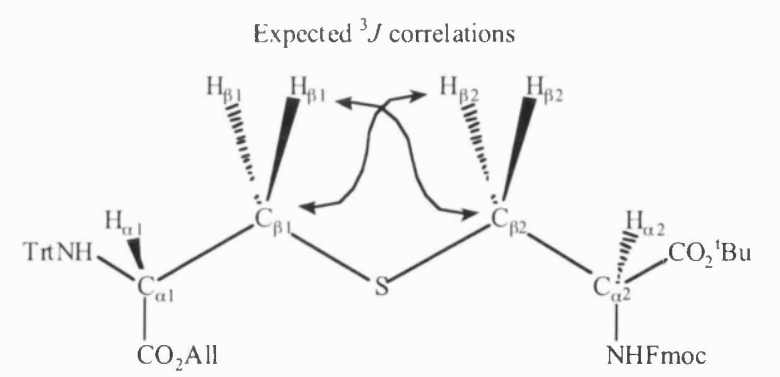

$(S, R)-10$

Figure 5.7. Expected HMBC ${ }^{3} J$ correlations for $(\boldsymbol{S}, \boldsymbol{R})$-10 across the thioether linkage

However, according to the $\mathrm{HMBC}$ experiment carried out, this is not the case for the major product. The $\beta_{2}$-Hs have a ${ }^{3} J$ correlation with the $\alpha_{1}-\mathrm{C}$ instead, which is unlikely for lanthionines, due to the fact that the $\alpha_{1}-\mathrm{C}$ is four bonds away. The same observation was made with the $\alpha_{1}-\mathrm{H}$, which had a ${ }^{3} J$ correlation with the $\beta_{2}-\mathrm{C}$ 
(Appendix M). This is consistent with the proposed regioisomeric structure $\mathbf{1 8}$, henceforth referred to as nor-lanthionine (nor-Lan), not that of the desired lanthionine (Figure 5.8). As for the minor component, which was thought to be the correct lanthionine derivative, the ${ }^{13} \mathrm{C}$ signals were relatively weak compared to the major component (1:5 isomer proportion). Therefore, the HMBC correlations were not strong enough to unambiguously deduce the structure of the minor isomer.

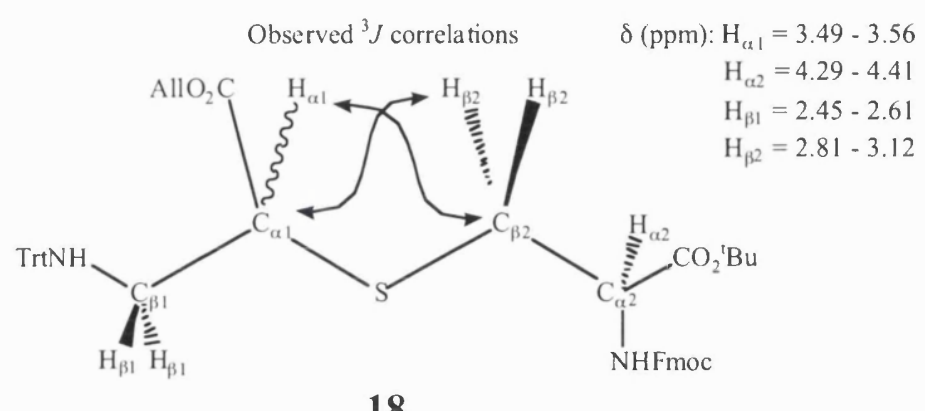

18

Figure 5.8. Structure of nor-lanthionine 18, as proposed by NMR

NMR studies were also carried out on the lanthionine $(\boldsymbol{S}, \boldsymbol{R}) \mathbf{- 1 2}$, as a mixture of two species. Apart from the large difference in ${ }^{1} H$ chemical shifts for the $\alpha_{1}$-Hs between the two species, it was noticed that the ${ }^{13} \mathrm{C}$ signal for the $\alpha_{1}-\mathrm{C}$ for the major component was too different (according to the HMQC experiment) to be a conformer of the minor component. It is now thought that the major component is the allyloxycarbonyl-derived nor-lanthionine 19 (Figure 5.9). Due to the low proportion of the minor component relative to the major one, its ${ }^{13} \mathrm{C}-{ }^{1} \mathrm{H}$ correlation was weak. It was thought that, based on the HSQC experiments carried out on iodoalanine (S)-6, the minor component was the desired lanthionine derivative (Figure 5.9).

NMR studies were then carried out on compound 1, which had been thought to contain a mixture of the correct lanthionine and its regioisomer, nor-lanthionine $\mathbf{2 0}$ (Figure 5.10). HSQC experiments clearly indicated a similar pattern for $\alpha_{1}$-C and $\beta_{1}$ $\mathrm{C}$ and ${ }^{1} \mathrm{H}$ chemical shifts to that observed for $\mathbf{1 8}$ and 19. The correct lanthionine, with the correct thioether connectivity, was not detected. It was either absent from the sample, or in such small quantity it was undetected by the HSQC experiment. 
<smiles>C=CCOC(=O)[C@H](CI)NC(c1ccccc1)c1ccccc1</smiles>

(S)-6a<smiles>CNC(CSC[C@H](N)C(=O)O)C(=O)OCc1ccccc1</smiles><smiles>N[C@@H](CSC[C@H](N)C(=O)O)C(=O)O</smiles>

$(S, R)-12$<smiles>C=CCOC(=O)[C@H](CI)NC(c1ccccc1)c1ccccc1</smiles>

(R)-6a<smiles>N[C@H](CSC[C@H](N)C(=O)O)C(=O)O</smiles><smiles>N[C@@H](CSC[C@H](N)C(=O)O)C(=O)O</smiles>

$(R, R)-12$<smiles>C=CCOC(=O)C(I)CNC(c1ccccc1)c1ccccc1</smiles><smiles>NCC(OC(=O)O)SC[C@H](N)C(=O)O</smiles>

18<smiles>CNC(=O)OCC(N)C(=O)O</smiles>

19<smiles>C=CCOC(=O)C(I)CN[C@@H](N)c1ccccc1</smiles>

6b<smiles>NCC(N)C(=O)OCC(=O)OCc1ccccc1</smiles>

18

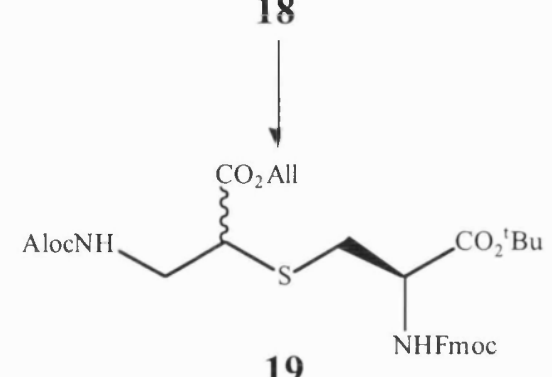

19

Figure 5.9. Scheme indicating how $(S)-6$ gave $(S, R)-10$ and hence $(S, R)-12$; and $\mathbf{6 b}$ gave 18 and hence 19. Similarly, $(R)-6$ gave $(R, R)-10$ and hence $(R, R)-12$; and $\mathbf{6 b}$ gave 18 and hence 19. The loss of stereochemical integrity is discussed later in this chapter and in Chapter 6. 
<smiles>CCCCCC(CSC[C@H](NC(=O)O)C(=O)O)C(=O)O</smiles>

1

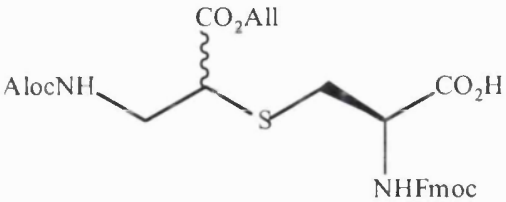

20

Figure 5.10. Structure of nor-lanthionine 20, a regioisomer of $\mathbf{1}$

NMR studies were also carried out on the supposed ivDde-derivative $16 .{ }^{13} \mathrm{C}-{ }^{1} \mathrm{H}$ correlation experiments identified compound $\mathbf{2 1}$, a regioisomer of lanthionine, as the only product (Figure 5.11). This explained the observation, discussed in Chapter 4, that only the major isomer reacted with the ivDde-OH. This does not mean that the correct lanthionine will not react at all to form its ivDde derivative. There could simply be competition between the correct lanthionine, which has a primary amine attached to a tertiary carbon, and its regioisomer, which has a primary amine connected to a less sterically hindered secondary carbon. Related to this, it has been reported that Dde can migrate between unprotected lysine side chains. However, no Dde migration onto an unprotected $\alpha-\mathrm{NH}_{2}$ was detected. ${ }^{153}$ Unfortunately further studies could not be carried out due to the poor solubility of the triphenylmethylcleaved lanthionine. The use of the ivDde and Dmab protecting groups, as part of an orthogonal protecting group system should therefore not be dismissed until further studies can be carried out using a genuine lanthionine derivative with ivDde-OH.

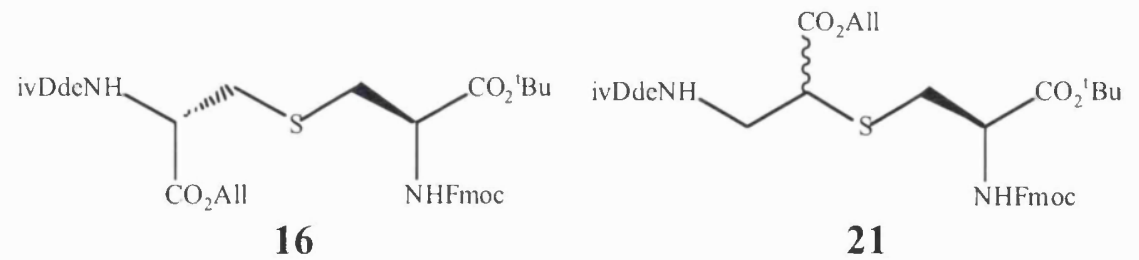

Figure 5.11. Structure of nor-lanthionine 21, a regioisomer of $\mathbf{1 6}$

As a result of the above, the lanthionine derivatives $\mathbf{1 7}$ and $\mathbf{2}$ were not present. Their regioisomers $\mathbf{2 2}$ and $\mathbf{2 3}$ (Figure 5.12) had instead been synthesised by the method described in Chapter 4. 


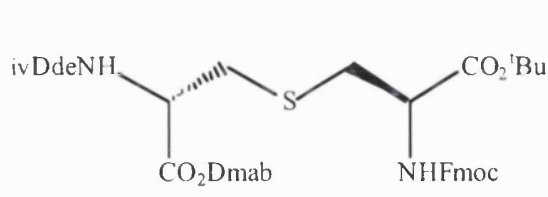

17

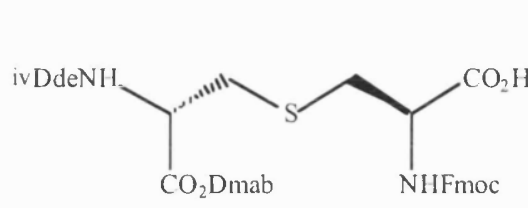

2

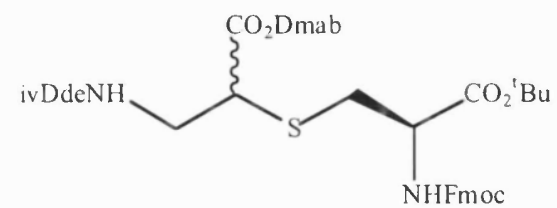

22

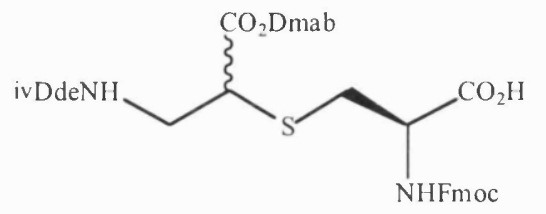

23

Figure 5.12. Lanthionine derivatives 17 and $\mathbf{2}$ and their regioisomers 22 and $\mathbf{2 3}$, respectively

The fact that the ${ }^{13} \mathrm{C}$ NMR spectrum for compound $(\boldsymbol{R}, \boldsymbol{R})-\mathbf{1 0}$ (in the presence of its regioisomer 18) produced three peaks for many of the carbon environments indicated the presence of three species. One carbon signal was for the desired lanthionine $(\boldsymbol{R}, \boldsymbol{R})-10$ and two for the diastereoisomeric nor-lanthionine 18. This hypothesis was further supported by the ${ }^{13} \mathrm{C}$ NMR spectrum of $\mathbf{1 8}$ prepared free of $(\boldsymbol{R}, \boldsymbol{R})$-10 from the precipitated iodoalanine (1:4 for $(\boldsymbol{R})-\mathbf{6 a} \mathbf{6} \mathbf{6 b}$, see Chapter 4 , section 4.3$)$ : two signals were present for many of the carbon environments with the experiment carried out at $125 \mathrm{MHz}$. Although enantiomers cannot be distinguished by ${ }^{13} \mathrm{C} \mathrm{NMR}$, two diastereoisomers can. It was therefore hypothesised that compounds $\mathbf{1 8}$ and $\mathbf{1 9}$

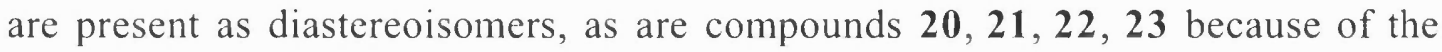
partial racemisation of $\mathbf{6} \mathbf{b}$ (Figure 5.13). It was thought that $\mathbf{6 b}$ was an unequal mixture of enantiomers and not completely racemised mixture because the intensities of ${ }^{13} \mathrm{C}$ signals for the diastereoisomeric nor-lanthionine derivatives were not equal. This is further supported by the outcome of one of the peptide syntheses, discussed in Chapter 6, sections 6.2 and 6.4.

With regards to the exclusive formation of $\mathbf{1 8}$ when a precipitated batch of iododalanine (1:4 for $(\boldsymbol{R}) \mathbf{- 6 a} \mathbf{6} \mathbf{6}$ ) was used in an attempt at forming $(\boldsymbol{R}, \boldsymbol{R}) \mathbf{- 1 0}$ (Chapter 4, section 4.3), it was thought that this was due to the cysteine 9 being contaminated with the oxidised product (cystine 8 ) hence resulting in a reduced amount of thiol species, with thiolate attack favouring $\mathbf{6 b}$ over $(\boldsymbol{R})-\mathbf{6 a}$. 


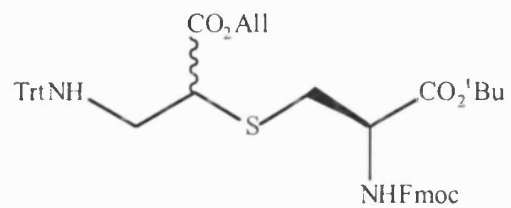

18<smiles>NC[C@H](CS[C@H](CNC(=O)O)C(=O)O)C(=O)O</smiles>

20

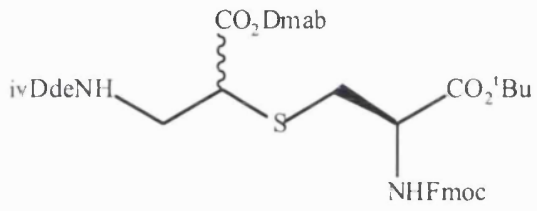

22<smiles>CCN[C@@H](CS[C@@H](CN[N+](=O)[O-])C(=O)O)C(=O)O</smiles>

19<smiles>[R6]NCC(SC[C@H](NCCCC)C(=O)O)C(=O)O</smiles>

21<smiles>[R6]NCC(SCC(C(=O)O)C(=O)O)C(=O)O</smiles>

23

Figure 5.13. Diastereoisomeric nor-lanthionine derivatives inadvertently synthesised

\subsubsection{Test reactions}

Several tests were carried out to determine the mechanism of ring opening of aziridine-2-carboxylate $(\boldsymbol{R}) \mathbf{- 1 1}$ by nucleophiles; sodium iodide and cysteine derivative 9 were used.

The hypothesis for the mechanism of formation of the incorrect iodoalanine regioisomer was tested. The aziridine-2-carboxylate $(\boldsymbol{R})$-11 was isolated by treating $N$-triphenylmethyl- $(R)$-serine $(O$-methanesulfonyl) allyl ester $(\boldsymbol{R})-\mathbf{5}$ with base at room temperature for $18 \mathrm{hr}$ (Figure 5.14).<smiles>C=CCOC(=O)[C@H](COS(C)(=O)=O)NC(c1ccccc1)c1ccccc1</smiles>

$(R)-5$

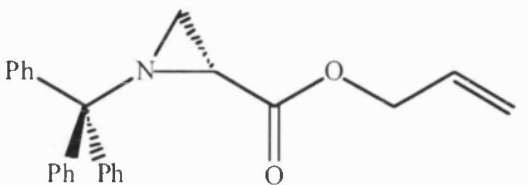

$(R)-11$

Figure 5.14. Partial conversion of $N$-triphenylmethyl- $(R)$-serine $(O$-methanesulfonyl $)$ allyl ester $(\boldsymbol{R})-\mathbf{5}$ into aziridine $(\boldsymbol{R})-\mathbf{1 1}$ 
Under these conditions the reaction was not complete, however the aziridine-2carboxylate could be separated out from the mesylate $(\boldsymbol{R})-\mathbf{5}$ and purified via flash column chromatography. Aziridine-2-carboxylate $(\boldsymbol{R})-\mathbf{1 1}$ was then treated with 10 equivalents of sodium iodide for 3 days at $20{ }^{\circ} \mathrm{C}$ (Figure 5.15).

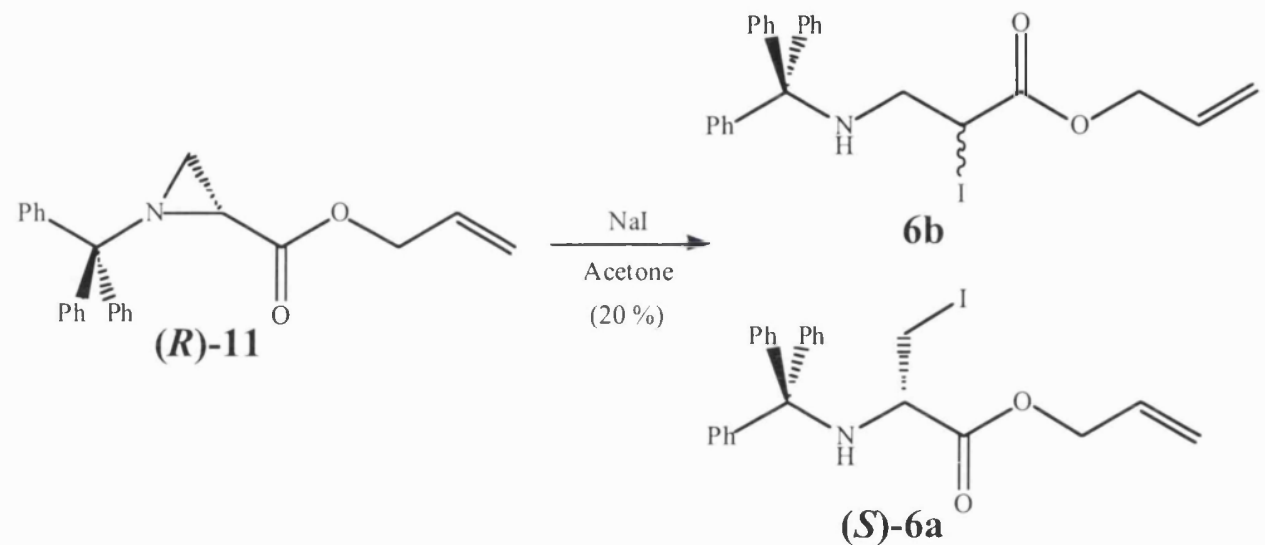

Figure 5.15. Ring opening of aziridine $(R)-11$ by sodium iodide

The ${ }^{1} \mathrm{H}$ NMR of the crude material clearly indicated the presence of the regioisomer $\mathbf{6 b}$ in a $\underline{3: 1}$ ratio to the desired $\beta$-iodoalanine $(S)-6 a$ (despite still containing $75 \%$ of the starting aziridine $(\boldsymbol{R})-\mathbf{1 1})$ whereas the iodination of mesylate $(\boldsymbol{R})-\mathbf{5}$ at $20{ }^{\circ} \mathrm{C}$ yielded the iodoalanine isomers in a ratio of $2: 1$. The increase in the proportion of $(S)$-6a, from the reaction of $(R)-\mathbf{1 1} \rightarrow(S)$-6 over the reaction of $(R)-5 \rightarrow(S)-6$ suggested that the formation of the correct $\beta$-iodoalanine $(S)$-6a goes via both an $\mathrm{S}_{\mathrm{N}} 2$ mechanism (displacement of the methanesulfonyl group by an iodide) and via the ring opening of $(\boldsymbol{R})-\mathbf{1 1}$. It was thought that iodoalanine regioisomer $\mathbf{6} \mathbf{b}$ is in fact present as a mixture of enantiomers due to stereo-inversion of an $\mathrm{S}_{\mathrm{N}} 2$ nucleophilic attack (see Figure 5.5). Also, there is probably an equilibrium between $(\boldsymbol{S})$-6a and $(R)$-11 during the iodination reaction (Figure 5.16). The same hypothesis could be made for the corresponding compounds originating from $(R)$-serine. 


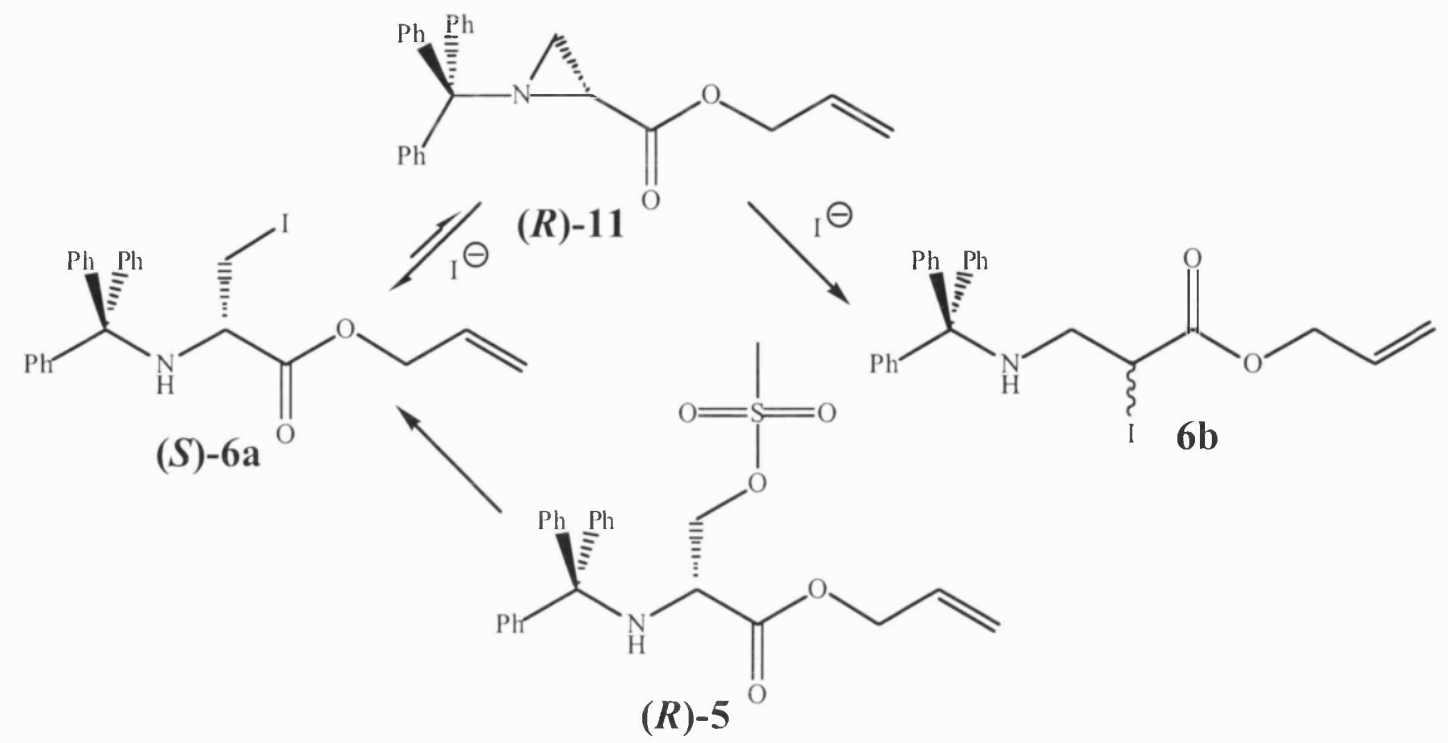

Figure 5.16. Equilibrium between the correct iodoalanine $(S)-6 a$ and its regioisomer 6b via $(\boldsymbol{R})-11$

Since the formation of the aziridine derivative was also seen during the thioether bridge formation step, another test reaction was carried out. The ring opening of the aziridine derivative $(\boldsymbol{R}) \mathbf{- 1} \mathbf{1}$ by the cysteine 9 was investigated. Dugave and Menez ${ }^{76}$ reported that a coupling between cysteine 9 and serine mesylates in the presence of caesium carbonate did not produce the desired lanthionine. Instead, starting materials and the aziridine derivative were isolated, implying that no ring opening took place by the cysteine. The test reaction, where the aziridine-2-carboxylate $(\boldsymbol{R})-\mathbf{1 1}$ was treated with the cysteine derivative 9 in DMF with caesium carbonate for $4 \mathrm{hr}$ at room temperature, was therefore carried out (Figure 5.17). Neither the desired lanthionine derivative $(\boldsymbol{S}, \boldsymbol{R})$-10 nor its diastereoisomeric nor-lanthionine regioisomer 18 was formed under these conditions. Since the role of the caesium carbonate was to generate thiolate anions but the reaction did not work, its presence led to the basic cleavage of the Fmoc group on cysteine instead (Figure 5.17). However, Hata and Watanabe $^{79}$ succeeded in ring opening unprotected aziridine-2-carboxylates with unprotected cysteines, without the addition of any Lewis acid, which is often required for aziridine-2-carboxylate ring opening reactions, in the presence of a phosphate buffer. This difference in observations remains unclear. Okawa et al. observed that boron trifluoride etherate was necessary for coupling a cysteine derivative with aziridine-2-carboxylates to yield lanthionines. ${ }^{185}$ Other contributing 
factors could be the ineffectiveness of the triphenylmethyl group on the nitrogen as an electron-withdrawing group, to increase the susceptibility of $C_{2}$ and $C_{3}$ on the aziridine ring to thiolate nucleophilic attack, and the bulkiness of the triphenylmethyl group. It has been shown on numerous occasions that the triphenylmethyl group is often used in the synthesis of aziridine-2-carboxylates, but it is more often than not removed and/or substituted during aziridine-2-carboxylate ring opening reactions. ${ }^{80,}$ $171,176,172,203$

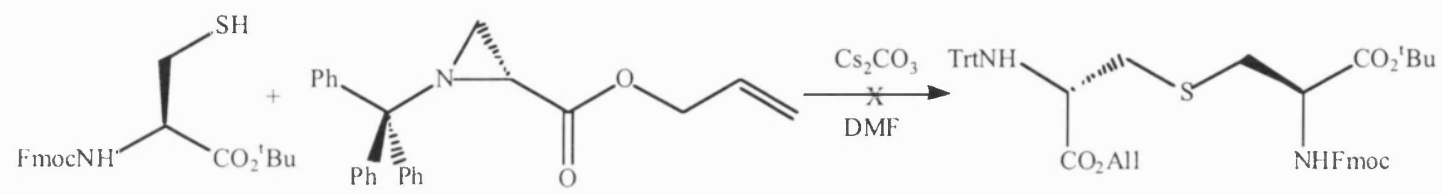

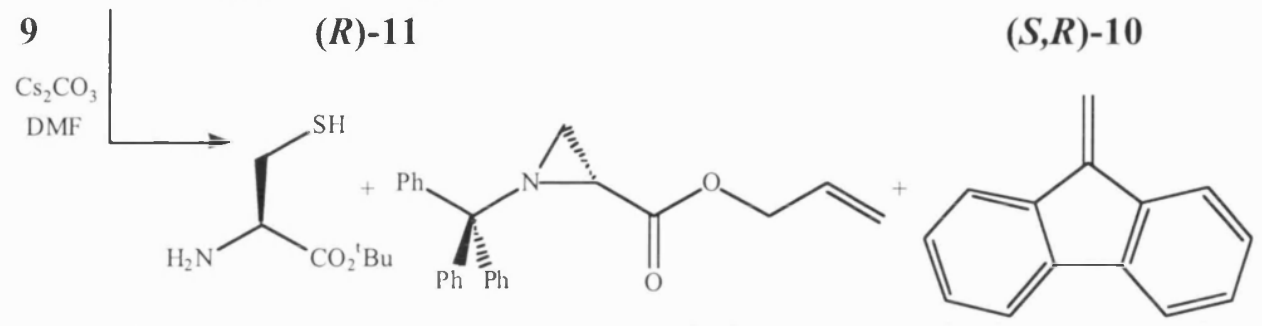

$(R)-11$

Figure 5.17. Unsuccessful ring-opening reaction of $(R)-11$ with cysteine derivative 9

The formation of the aziridine-2-carboxylate during the thioether bridge formation step was caused by intramolecular iodide displacement by the nitrogen on the iodoalanine derivatives, presumably assisted by the presence of a polar solvent: DMF. Since the cysteine 9 does not ring open the aziridine, this was thought to be the reason for the presence of the aziridine-2-carboxylates $(S)-11$ and $(R)-11$ as byproducts in the thioether bridge formation reactions.

\subsubsection{Synthesis of $(\mathrm{R}, \mathrm{R})$ and ( $\mathrm{S}, \mathrm{R})$-lanthionine derivatives 14 and 15 (via the} Mitsunobu reaction)

Work carried out within the group ${ }^{204}$ indicated that lanthionine derivatives could be directly synthesised via a modified version of the Mitsunobu reaction, in which the hydroxyl groups on serine derivatives $(S)-4$ and $(R)-4$ are activated with trimethylphosphine and 1,1'-(azodicarbonyl)dipiperidine (ADDP) $)^{205 a, 205 b}$ and this 
mixture was treated with the cysteine derivative 9 in the presence of a $\operatorname{zinc}(\mathrm{II})^{205 \mathrm{c}}$ catalyst (Figure 5.18).

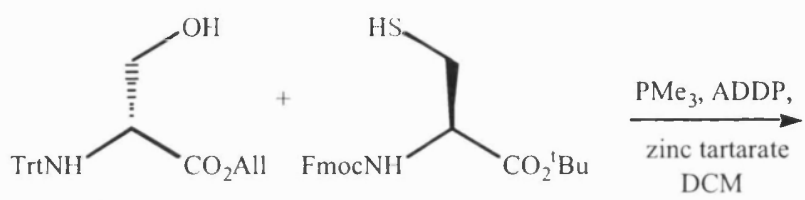

$(R)-4$

9<smiles>N[C@@H](CSC[C@H](N)C(=O)O)C(=O)O</smiles>

11

Figure 5.18. Formation of lanthionine via Mitsunobu reaction conditions

Although work is in progress to improve the mass recovery and yield of this reaction, coupling $(\boldsymbol{S}) \mathbf{- 4}$ with $\mathbf{9}$ yielded only one isomer. The use of the serine derivative $(\boldsymbol{R}) \mathbf{- 4}$ also yielded only one isomer, with small differences in the ${ }^{1} \mathrm{H}$ NMR chemical shift values (Appendix N). HSQC experiments carried out on both diastereoisomers confirmed the correct thioether bridge connectivity with similar ${ }^{13} \mathrm{C}$ chemical shift values between the $\alpha$ - and $\beta$-Hs, and $\alpha$ - and $\beta$-Cs on both sides of the thioether bridge. HMBC experiments confirmed this with the presence of ${ }^{3} J$ couplings between $\beta$-Hs and $\beta$-Cs across the bridge (see Figure 5.7). Not surprisingly, each diastereoisomer produced via this method had identical ${ }^{1} \mathrm{H}$ and ${ }^{13} \mathrm{C}$ chemical shifts and coupling patterns to the minor component of the corresponding lanthionine derivatives $(\boldsymbol{R}, \boldsymbol{R})$-10 and $(\boldsymbol{S}, \boldsymbol{R})$-10 produced via the iodoalanine route. 


\section{Chapter 6}

\section{Results and Discussion III}

\subsection{Synthesis of a lanthionine analogue of an enkephalin opioid peptide}

With orthogonally protected nor-lanthionine derivatives in hand, an investigation of the SPPS of cyclic peptides containing thioether bridges using the proposed methodology commenced. During the course of these investigations the NMR studies described in Chapter 5 proved that only compounds $\mathbf{2 0}$ and $\mathbf{2 3}$ had been synthesised, instead of the desired $\mathbf{1}$ and $\mathbf{2}$, respectively (Figure 6.1). However, the SPPS studies were continued, in order to test the methodology. It was also suspected that the resulting novel cyclic peptides could possess unusual conformational properties, as the nor-lanthionine bridge was previously unknown. 
<smiles>CNC(CSC(CSC(=O)O)C(=O)O)C(=O)O</smiles>

20

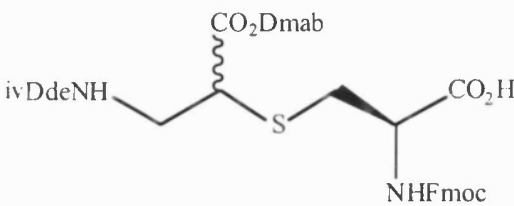

23

Figure 6.1. nor-Lanthionine derivatives $\mathbf{2 0}$ and $\mathbf{2 3}$ for SPPS

Although the lanthionine analogue of the enkephalin opioid peptide (Figure 6.2) is no longer of interest for further biological studies, ${ }^{50}$ it was chosen as the first target for the peptide synthesis due to the small size of the peptide and hence short synthesis. A number of attempts at the synthesis were made; in each case the synthesis was carried out either manually in a Merrifield bubbler (Appendix O) or in an automated fashion (continuous flow) on a peptide synthesiser.<smiles>NC(=O)[C@H](Cc1ccccc1)NC(=O)[C@H](Cc1ccccc1)NC(=O)CNC(=O)[C@H](CSC[C@H](NC(=O)[C@H](N)Cc1ccccc1)C(N)=O)NC(=O)[C@H](N)Cc1ccc(O)cc1</smiles>

(a)<smiles>NC(=O)[C@H](Cc1ccccc1)NC(=O)[C@H](Cc1ccccc1)NC(=O)CNC(=O)C(CNC(=O)[C@H](N)Cc1ccc(O)cc1)SC[C@H](NC(=O)C[C@H](N)Cc1ccccc1)C(N)=O</smiles>

24

Figure 6.2. Lanthionine opioid enkephalin peptide analogue (a) and the analogue synthesised 24

\section{First attempt}

The resin used for the synthesis was the highly acid labile Sieber amide resin ${ }^{87}$ (Figure 6.3) with a loading of $0.47 \mathrm{mmol} / \mathrm{g}$ and the synthesis is outlined in Figure 6.4. The Fmoc protecting group at each stage of the peptide synthesis was removed by a $20 \%$ piperidine solution in DMF. A small amount of resin was washed after each Fmoc deprotection step and treated with the Kaiser test solution. ${ }^{83}$ 


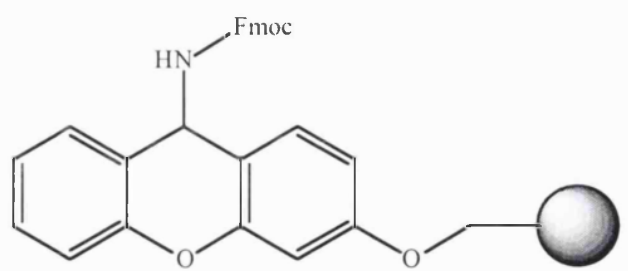

Figure 6.3. Sieber amide resin

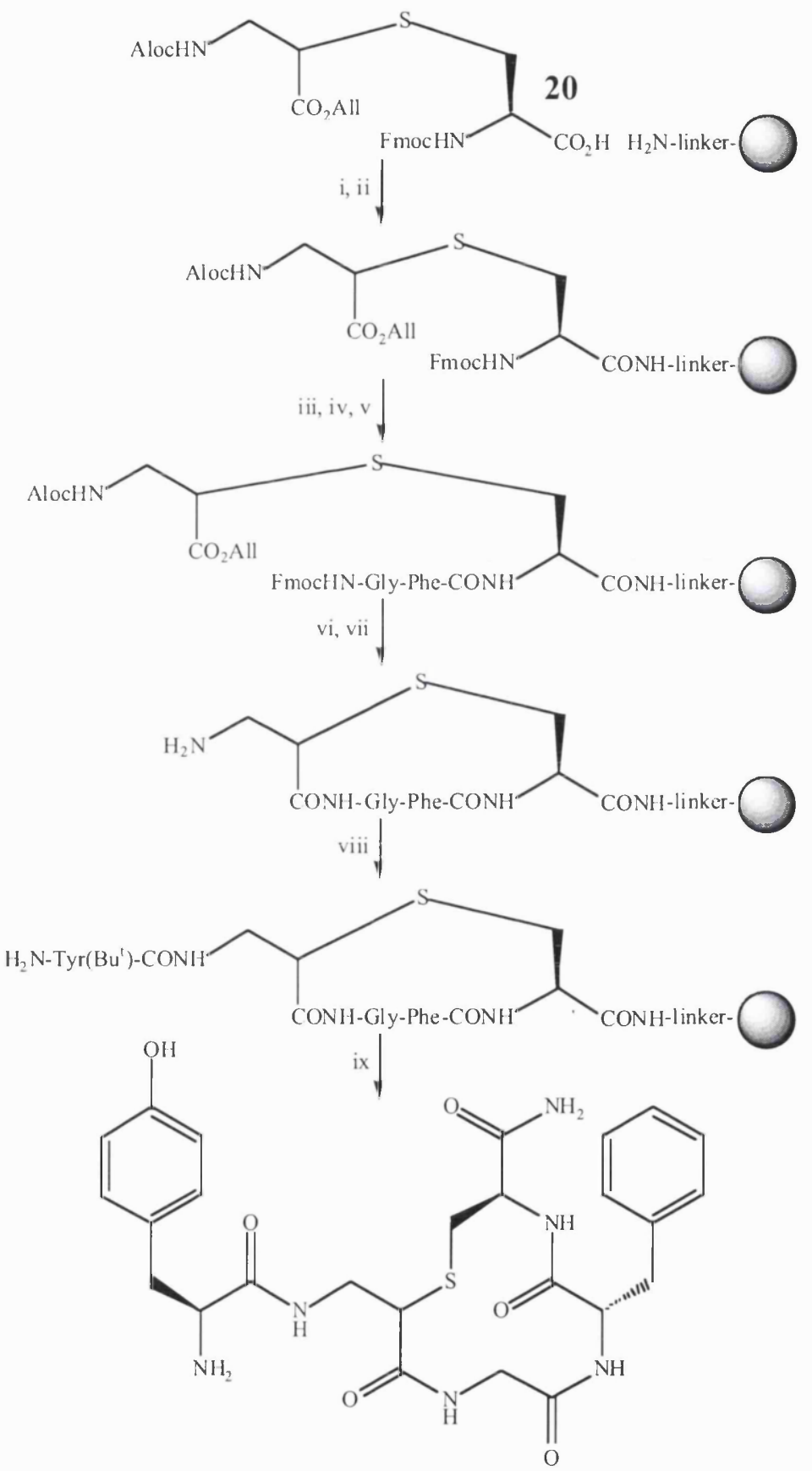

24

i) $\mathrm{HOB} / \mathrm{DMF}$, DIC/Dioxane, ii) $\mathrm{Ac}_{2} \mathrm{O}, \mathrm{HOBt}$, DIPEA, DMF, iii) Piperidine/DMF

iv) Fmoc-Phe, i,ii, iii, v) Fmoc-Gly, i,ii, vi) $\mathrm{Pd}\left(\mathrm{PPh}_{3}\right)_{4}, \mathrm{CHCl}_{3} / \mathrm{AcOH} / \mathrm{NMM}$, iii,

vii) PyAOP,HOAvDIPEA/DMF, viii) Fmoc-Tyr, i, ii,iii, ix) TFA/H ${ }_{2} \mathrm{O}, \mathrm{Et}_{3} \mathrm{SiH}$

Figure 6.4. Synthesis of a thioether-bridged opioid enkephalin peptide analogue 24 
Incorporation of the amino acids (nor-Lan, Phe, Gly) was carried out by preactivation of these residues with $N, N^{\prime}$-diisopropylcarbodiimide (DIC) and 1hydroxybenzotriazole (HOBt) for 15-20 minutes before being added to the resin. Each coupling step was carried out twice and the resin subsequently washed in preparation for the colorimetric Kaiser test. No problems were encountered during incorporation of the first residue, nor-Lan 20, onto the resin as confirmed by Kaiser test.

Each amino acid coupling step was also followed by a capping wash. The capping wash consisted of an acetic anhydride solution in DMF. Its role was to ensure that all unreacted amino groups are capped, leading to a truncated sequence. This is to prevent the formation of peptides with sequences lacking one or more (random) residues, which are more difficult to remove in the purification step than shorter, truncated sequences.

After assembling the linear peptide, the allylic protecting groups on the norlanthionine were removed with tetrakis(triphenylphosphine) palladium(0) in a mixture of $\mathrm{CHCl}_{3} / \mathrm{AcOH} / \mathrm{NMM}(37: 2: 1)^{128}$ or $\mathrm{DMF} / \mathrm{CHCl}_{3} / \mathrm{AcOH} / \mathrm{NMM}$ $(18.5: 18.5: 2: 1)$. Following that were several washes with solutions of diisopropylethylamine (DIPEA) and sodium diethyldithiocarbamate - to remove traces of acetic acid and organometallic by-products - and the Fmoc deprotection step. The results from the Kaiser test after the allylic deprotection was a positive blue and an intense purple was observed after the Fmoc deprotection step. This was due to the presence of firstly one then two amino groups on the resin. A small amount of the resin was taken at the allylic deprotection and Fmoc deprotection stages and treated with a $1 \%$ solution of TFA in dichloromethane and the expected peptide ion searched for by electrospray mass spectrometry. Each of the two stages in the synthesis above gave dubious parent ion peaks at very low relative abundances. This is perhaps not surprising as the presence of trifluoroacetic acid, or its salts, tends to suppress signals in mass spectrometry. 
Cyclisation of the peptide was carried out using the highly reactive mixture of PyAOP, HOAt and DIPEA. ${ }^{109}$ This mixture has been shown to be a potent peptide coupling reagent, especially for cyclisation of peptides. The Kaiser test following the cyclisation step was a positive blue (i.e. not purple), which suggested that the amine intended for cyclisation was no longer present, hence, the success of the cyclisation step was indicated. A small amount of the resin was again set aside for cleavage and examination by mass spectrometry. The result was similar to the previous test where the parent ion, 18 mass units less than the previous stage due to loss of $\mathrm{H}_{2} \mathrm{O}$ was observed but gave a very low relative abundance.

As a test of the methodology, the final amino acid on the sequence, Tyr, was coupled to the remaining amino group and its Fmoc group then removed. Removal of the peptide from the resin would then release a $C$-terminal peptide amide. The peptide was repeatedly washed with dichloromethane to remove traces of DMF, which could hinder the cleavage reaction ${ }^{206}$ and treated with an $85 \%$ trifluoroacetic acid solution, plus water $(10 \%)$ and triethylsilane (TES, $5 \%$ ) portion-wise on the bubbler with collection of the aliquots in a round-bottom flask. The high concentration of the acid was to ensure that the tbutyl side chain protection on Tyr was completely removed. After stirring for 4 hours the mixture was concentrated in vacuo and the peptide was precipitated with cold ether. Removal of the solvent followed by lyophilisation from acetic acid solution yielded the peptide in crude form.

The mass spectrum of the crude peptide gave the expected parent ion as the base peak (554, Appendix P), despite the fact that the crude weight was $240 \mathrm{mg}$ when the maximum possible yield was only $55 \mathrm{mg}$. Analytical HPLC, however, showed that the peptide contained a host of peaks especially at higher concentrations of acetonitrile (Appendix Q).

Purification via preparative HPLC proved difficult due to impurities with retention times close to that of the peptide. Eventually isolation of the peptide was achieved but in a disappointing $0.8 \%$ yield. Two HPLC peaks adjacent to the desired peptide displayed, among others, the parent ion but at less than $30 \%$ relative abundance, and 
consist mainly of unidentified contaminants. Insufficient peptide was isolated to obtain full ${ }^{1} \mathrm{H}$ NMR characterisation.

\section{Second attempt}

A second attempt at the synthesis was then carried out. This time, the linear peptide was synthesised via continuous flow solid phase peptide synthesis (MilliGen 9050 peptide synthesiser) since it was part of the aim of this project to apply the methodology to continuous flow solid phase peptide synthesis eventually, with the automation of the allyl deprotection and cyclisation steps as described by Albericio et al. ${ }^{128}$

A PEG version of the Sieber amide resin was used $\left(\text { NovaSyn }{ }^{\circledR} \text { TG Sieber resin) }\right)^{87}$ (Figure 6.5) for continuous flow synthesis with a lower loading of $0.16 \mathrm{mmol} / \mathrm{g}$, to avoid inter-chain coupling at the cyclisation stage.

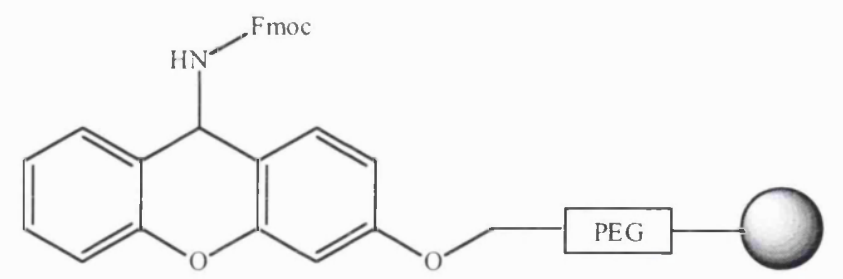

Figure 6.5. NovaSyn ${ }^{\circledR}$ TG Sieber resin

Each Fmoc deprotection step and amino acid coupling was monitored by U.V. at 365 $\mathrm{nm}$. The U.V. traces were integrated to indicate the degree and comparative success of each Fmoc deprotection and amino acid coupling step (Appendix R). All the reagents used were the same as in the batch synthesis described above with the exception of 1 -acetylimidazole, which was used as the capping reagent.

The Fmoc deprotection step for the incorporated nor-lanthionine was inefficient and thought to be caused by a dilution factor in the sequence of the synthesiser protocol. This resulted in poor Phe coupling, inefficient Fmoc deprotection and low Gly coupling. Despite all these, it was decided to continue the synthesis offline, with the 
allylic deprotection, Fmoc deprotection on Gly, cyclisation and Tyr coupling as described above. The Kaiser tests at these stages were ambiguous.

The cleavage of the peptide was carried out as before. It was perhaps not surprising that the parent ion was absent by mass spectrometry, considering the results of the U.V. integrations. Analytical HPLC showed a peptide that was at least $70 \%$ pure with a different $\mathrm{R}_{\mathrm{T}}$ compared to the first peptide synthesised. There was still a host of peaks present on the HPLC trace at higher acetonitrile concentrations.

Isolation of the peptide gave a mass of 222 as the base peak and ${ }^{1} \mathrm{H}$ NMR suggested that the peptide that was synthesised was in fact a $\mathrm{H}_{2} \mathrm{~N}-\mathrm{Tyr}-\mathrm{Phe}-\mathrm{CONH}_{2}$ dipeptide. This in turn suggested that the incorporation of nor-lanthionine onto the resin was not as successful as it should have been, which explained the weak U.V. trace and integration for the following Fmoc deprotection step (Appendix R). It was then decided that the concentration of piperidine needed to be increased to $30 \%$ due to the dilution factor in the sequence of the synthesiser protocol to maximise Fmoc deprotection. However, it could not be explained how the capping step immediately after the incorporation of nor-lanthionine in the synthesis failed to prevent the formation of this dipeptide side product.

\section{Third attempt}

The third attempt to synthesise peptide 24 was carried out in a similar manner to the second attempt with the linear peptide synthesised on the MilliGen 9050. However, the nor-lanthionine residue was incorporated onto the resin offline, as a precautionary measure. A Kaiser test gave a negative result indicating a successful coupling of the first residue and the synthesis was continued as before, online, followed by offline allylic deprotection, cyclisation and Tyr coupling as described above.

The cleavage of the peptide from the resin and precipitation were carried out as before. The analytical HPLC trace indicated one major product, apart from the 
cluster of peaks at higher acetonitrile concentrations, but the retention time was again different from the first attempt under identical HPLC conditions. The crude mass spectrum revealed a peak at $100 \%$ relative abundance for a mass of 586 . As the desired mass should have been 554, this implied double oxidation of the thioether bridge (Figure 6.6). This was thought to be caused by prolonged storage of the peptidyl-resin in DMF at the linear stage prior to allylic deprotection and cyclisation, despite care being taken to keep the resin at temperatures below $4{ }^{\circ} \mathrm{C}$ under nitrogen. Purification of the peptide was carried out via preparative HPLC and an MS-MS experiment was carried out. The results appeared to show that the peptide contained the right sequence of amino acids with the corresponding daughter ion peaks for the peptide.

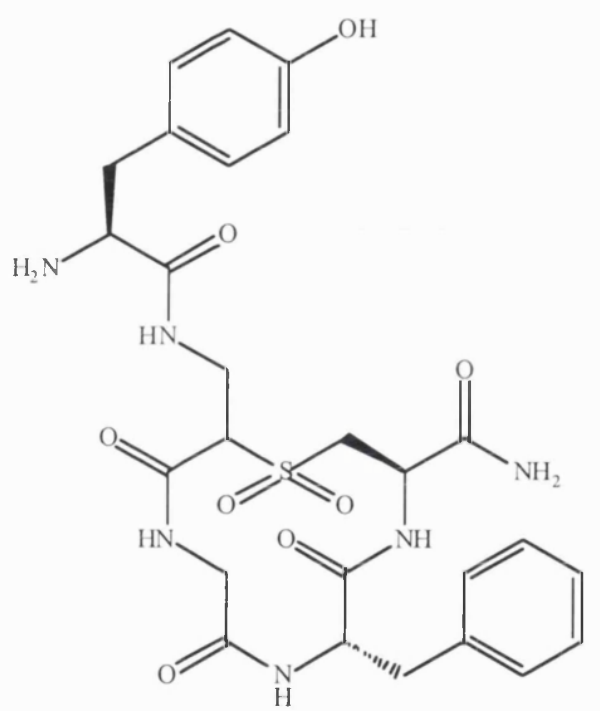

Figure 6.6. Structure of the doubly oxidised peptide $\mathbf{2 4 a}$

With the yield of the peptide being just under $10 \mathrm{mg}$ (indicating a $17 \%$ yield based on the doubly oxidised peptide) the ${ }^{1} \mathrm{H}$ NMR of the peptide was now more interpretable. The ${ }^{1} \mathrm{H}$ spectrum initially seemed to suggest that the right peptide had been synthesised, except that it had been oxidised, although the aromatic ${ }^{1} \mathrm{H}$ integrations appeared to be greater than expected. However, careful investigation of the ${ }^{1} \mathrm{H}$-COSY spectrum indicated that the structure proposed for $\mathbf{2 4 a}$ was incorrect. Although mass spectrometry and HPLC indicated the presence of predominantly one species with a molecular mass of 586, and MS/MS experiments indicated the correct fragmentations for the doubly oxidised peptide, it was thought that only a small 
amount of this was present in the sample. Data from the ${ }^{1} \mathrm{H}$-COSY experiment suggested that the major product was a $\mathrm{H}_{2} \mathrm{~N}-\mathrm{Tyr}-\mathrm{Gly}-\mathrm{Gly}-\mathrm{Phe}-\mathrm{Phe}-\mathrm{CONH}_{2}$ pentapeptide, which coincidentally has a molecular mass identical to that of the doubly-oxidised 24a. Again it could not be explained how the capping steps in the synthesis failed to prevent the formation of this predominant peptide by-product.

The repeating trend observed by HPLC, where a cluster of peaks was observed at higher concentrations of acetonitrile, gave rise to concern. The hypothesis was advanced that the conditions for the peptide cleavage were so highly acidic that they led to degradation of the linker. It was therefore thought that a two-step cleavage procedure could improve product purity. The first step involved a mild acid cleavage in the bubbler with repeated washes of a $5 \%$ TFA, $2.5 \% \mathrm{H}_{2} \mathrm{O}$ and $2.5 \%$ TES (as the scavenger for tbutyl or trityl species) solution in DCM, followed by a methanol wash and collection of the filtrate in a flask containing trifluoroacetic acid. Stirring the mixture for 2 - 3 hours then followed.

\section{Fourth attempt}

The next attempt at synthesising peptide 24 was carried out solely on the peptide synthesiser. This involved some manual intervention with changes in solvents and reagents required for the allylic deprotection and cyclisation steps. The original settings subsequently needed to be restored before the Tyr was coupled to the peptide.

In order to monitor the progress of the first few steps of the synthesis, a small amount of resin was removed from the column following Fmoc deprotection, and also following nor-lanthionine coupling. Each batch of resin was checked by I.R. and U.V. The I.R. spectrum of the resin following Fmoc deprotection showed the absence of a carbamate $\mathrm{C}=\mathrm{O}$ stretch: this was visible again, although more weakly, after nor-lanthionine coupling. U.V. measurements were carried out to determine the amount of the Fmoc group present at each of these two stages. This was carried out by taking a known quantity of the resin and cleaving the Fmoc group with a $30 \%$ 
piperidine solution on DMF and measuring the absorbance of the aliquot at $300 \mathrm{~nm}$, which is the absorbance of the piperidine-dibenzofulvene adduct (Appendix S).

The absorbance for the resin after Fmoc removal gave a near-zero value, which was expected. The loading was quantitatively assigned by using the absorbance value for the nor-lanthionine-incorporated resin in the calculation in Appendix $S(0.16$ $\mathrm{mmol} / \mathrm{g}, 0.16 \mathrm{mmol} / \mathrm{g}$ claimed by manufacturer).

The synthesis was then carried through to the end, despite the apparent lack of increase in carbamate $\mathrm{C}=\mathrm{O}$ stretch intensity in I.R. as the synthesis progressed, and low integrations on the peptide synthesiser.

Once the synthesis was completed, the peptide was cleaved from the resin following the two-step method and precipitated as previously described to give $70 \mathrm{mg}$ of crude material. Mass spectrometry indicated that the material contained the desired peptide. The analytical HPLC trace showed that the impurity peaks previously detected by U.V. at higher concentrations of acetonitrile were no longer present, confirming the hypothesis that the Sieber amide linker was not stable to the highly acidic cleavage conditions previously employed.

Purification of the peptide was carried out via preparative HPLC. As previously observed the parent ion of the peptide was the base peak. Two other adjacent peaks gave the parent ion with much smaller relative abundances. These were thought to be impurities that contained an extremely small amount of the peptide. A very poor yield $(2.7 \%)$ was obtained.

12-membered cyclic tetrapeptides are known to be difficult to synthesise via lactam bridge formation. This is mainly due to the elongated conformation of the linear precursor, maximising the distance between the two termini, which affects the degree of success during cyclisation. ${ }^{207,208}$ Cyclisation is favoured by the presence of Pro or Gly or D-amino acids ${ }^{209}$ and the absence of these leads to slow cyclisation resulting in dimerisation and racemisation. ${ }^{210}$ These factors perhaps account for the difficulty 
in obtaining a reasonable yield from the synthesis of peptide $\mathbf{2 4}$, which is a $\mathbf{1 2}$ membered cyclic tetrapeptide formed via ring closure to give an amide bond, containing a sulfur bridge and a $\beta$-amino acid.

To test the above explanation for a poor yield of cyclic tetrapeptide, the synthesis of a bigger cyclic peptide was considered. The best candidate was thought to be an analogue of ring $\mathrm{C}$ of nisin, which is a 21 -membered ring with no dehydro-amino acids (Figure 6.7).

\subsection{Synthesis of a nor-lanthionine analogue of ring $C$ of nisin}

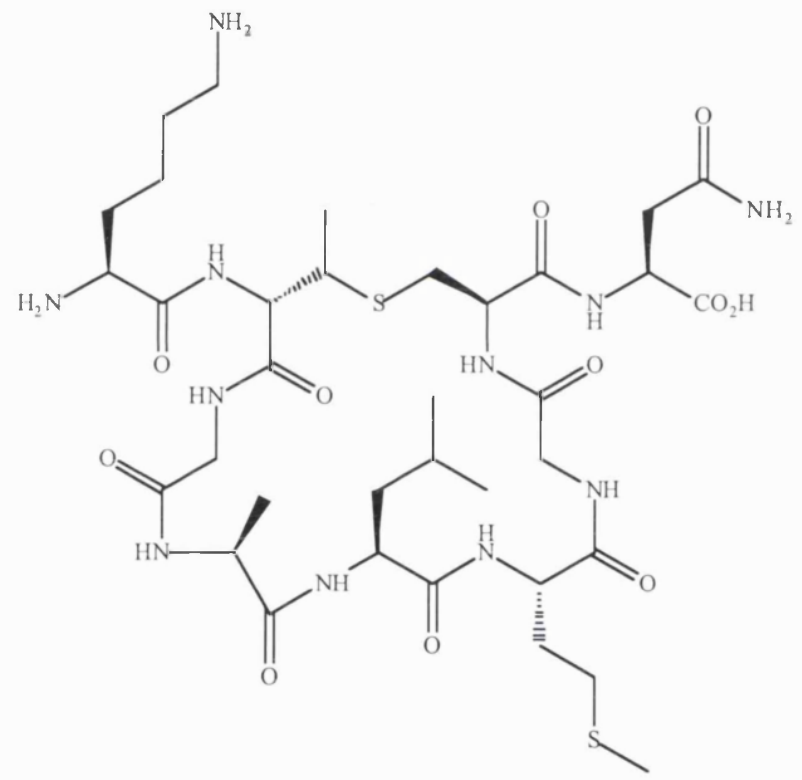

(a)<smiles>CSCC[C@H](C)NC(=O)[C@H](CCSC)NC(=O)C(CC(C)C)NC(=O)[C@H](C)NC(=O)CNC(=O)C(CNC(=O)[C@H](N)CCCCN)SC[C@H](NC(=O)CNC(=O)[C@H](C)CCCN)C(=O)N[C@@H](CC(N)=O)C(=O)O</smiles>

25

Figure 6.7. (a) Ring $\mathrm{C}$ of nisin and an analogue of ring $\mathrm{C}$ of nisin 25

Since the $C$-terminus of the peptide is an acid, the resin used for the synthesis was the highly acid labile NovaSyn ${ }^{\circledR}$ TGT alcohol resin pre-loaded with Asn(Trt) (Figure $6.8)^{90,91,211}$

The whole synthesis was carried out offline on the Merrifield bubbler. The Fmoc deprotection steps, amino acid couplings, capping washes, allylic deprotection, 
cyclisation and Kaiser tests were all carried out as described above. The loading was quantitatively assigned to be $0.21 \mathrm{mmol} / \mathrm{g}(0.20 \mathrm{mmol} / \mathrm{g}$ determined by the manufacturer) by U.V. measurements (Appendix T).

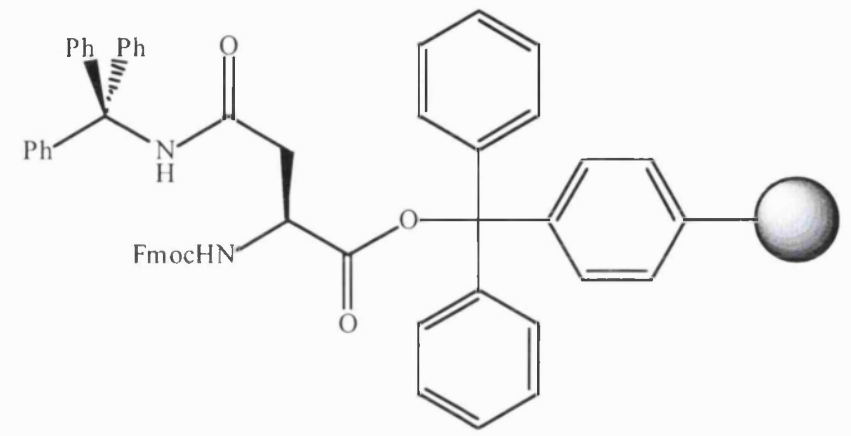

Figure 6.8. NovaSyn ${ }^{\circledR}$ TGT alcohol resin pre-loaded with Asn(Trt)

The synthesis (Figure 6.9) proceeded well, as monitored by Kaiser test, and the peptide cleavage was carried out via the two-step procedure described above. Precipitation of the peptide with cold ether followed by lyophilisation with aqueous acetic acid yielded the crude material as a pale yellow powder $(85 \mathrm{mg})$.

The crude material gave a mass spectrum with the parent ion as a $50 \%$ relative abundance peak and $[\mathrm{M}+2]^{2+}$ as the base peak. Analytical LCMS showed that there were two peaks on the HPLC trace, which gave identical mass spectra - the parent ion of the peptide and $[M+2]^{2+}$ (Appendix U). Purification of the peptide by preparative HPLC confirmed the presence of two peptidic species, 25a and $\mathbf{2 5} \mathbf{b}$, with the same mass with a ratio of approximately $3: 1$, giving a total yield of $55 \%$. This implied an average coupling efficiency of $94 \%$ over 10 steps, assuming the Fmoc deprotection steps proceeded quantitatively. The ratio of the two peptidic species was the same as the proportion of what was originally thought to be lanthionine conformers $(\boldsymbol{S}, \boldsymbol{R})$-10 (Figure 6.10). However, due to the disproval of the conformer theory no correlation could be made. Further purification was carried out under a different gradient by HPLC. It was observed that there was a small shoulder on the broad HPLC trace of the major product, which did not separate out even with two other gradient systems on HPLC. 


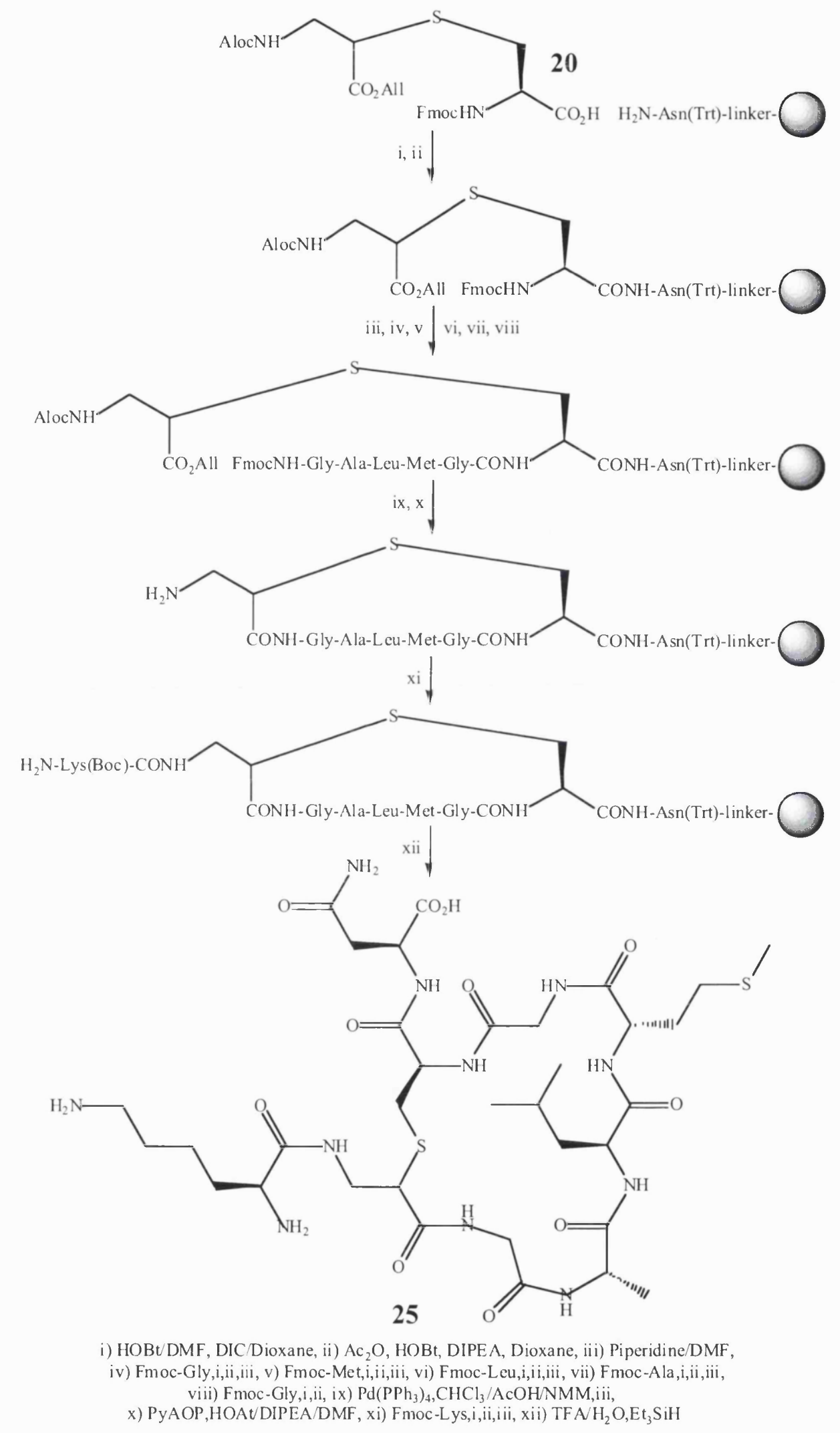

Figure 6.9. Synthesis of a lanthionine analogue of ring $\mathrm{C}$ of nisin $\mathbf{2 5}$ 


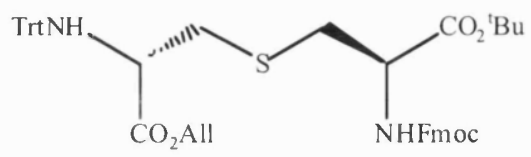

$(S, R)-10$

Figure 6.10. Lanthionine derivative $(S, R)-10$

The ${ }^{1} \mathrm{H}$ NMR of the two peptides in deuterated methanol at $283 \mathrm{~K}$ confirmed that only the minor product was pure. The major product produced a ${ }^{1} \mathrm{H}$ spectrum with doubling of the methyl peaks for Met, Ala and Leu at approximately 2.0, 1.4 and 0.9 ppm respectively and increased breadth of some aliphatic signals for Lys, Leu and Met. At this stage the impurity in the major product was thought to be a deletion sequence with common residues such as Lys, Leu and Met. In the meantime another peptide was synthesised, an analogue of rings $\mathrm{A}$ and $\mathrm{B}$ of nisin, a sequential bi-cyclic thioether-bridged peptide.

\subsection{Synthesis of an analogue of rings $A$ and $B$ of nisin}

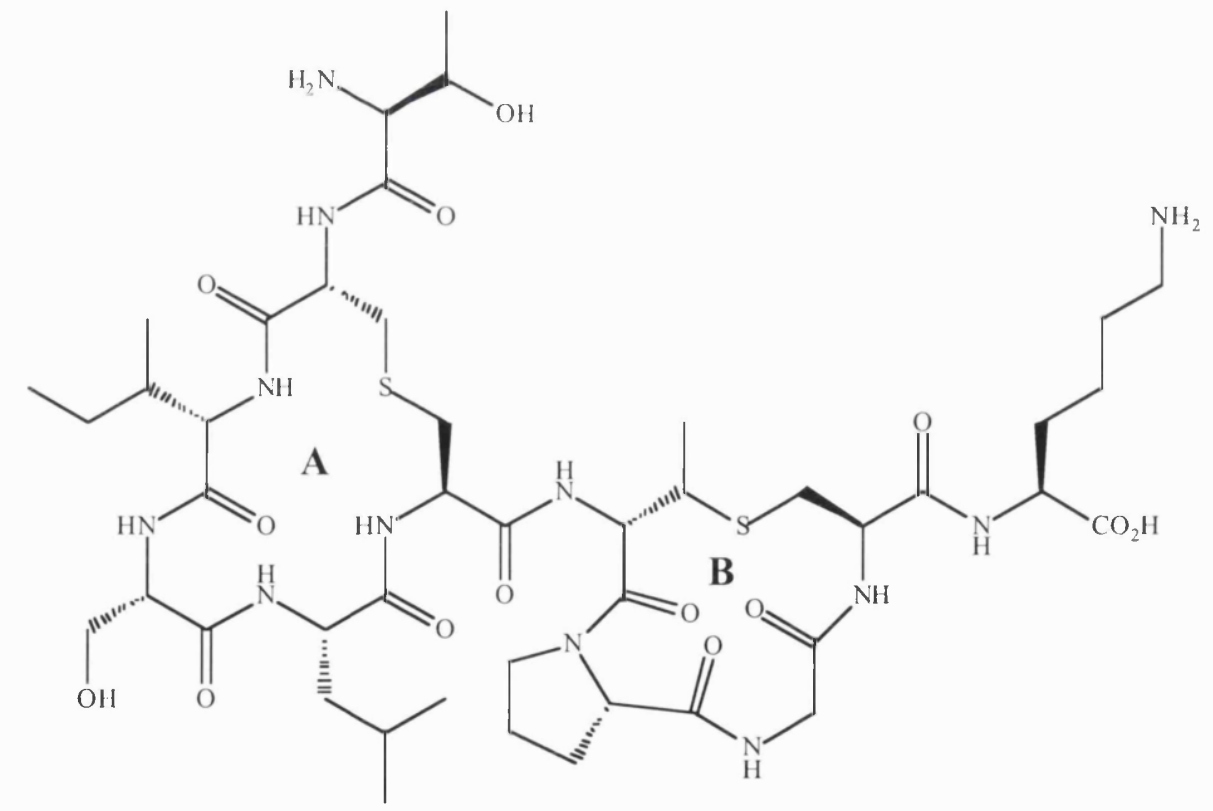

Figure 6.11. Rings $\mathrm{A}$ and $\mathrm{B}$ of nisin

One of the advantageous features of this method for the synthesis of cyclic sidechain bridged peptides is the possibility of making several successive rings on resin. It was decided that synthesising an analogue of rings $\mathrm{A}$ and $\mathrm{B}$ of nisin, the non- 
overlapping bi-cyclic peptide $\mathbf{2 6}$, should test the strategy. In this case, the synthesis was carried out entirely on the Merrifield bubbler and all reagents used were identical to the synthesis of ring $\mathrm{C}$ of nisin. The loading was determined by U.V. measurements as previously described $(0.22 \mathrm{mmol} / \mathrm{g}, 0.20 \mathrm{mmol} / \mathrm{g}$ specified by manufacturer) (Appendix V).

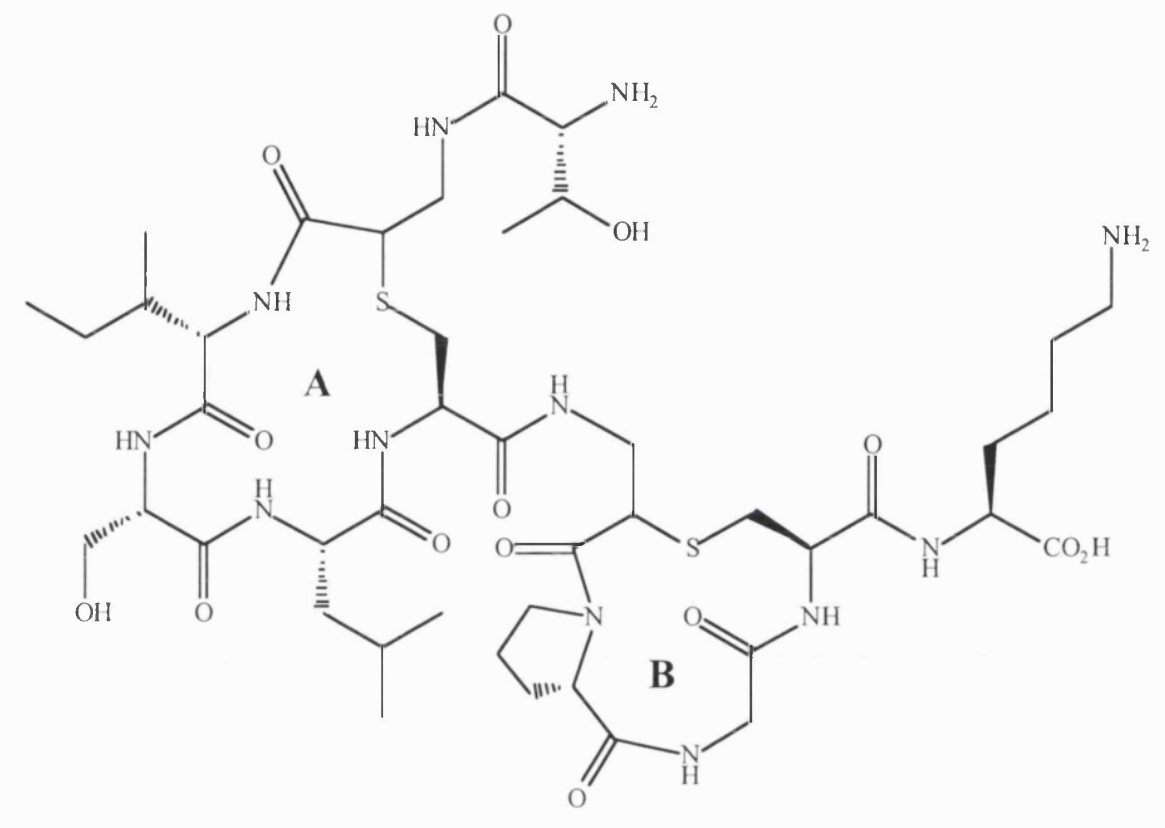

26

Figure 6.12. nor-Lanthionine analogue of rings $\mathrm{A}$ and $\mathrm{B}$ of nisin 26

Despite the fact that the analogue of ring $\mathrm{B}$ is a 12 -membered ring, it was envisaged that the proline residue would encourage cyclisation by twisting the linear peptide. A small amount of the resin was therefore treated with a $5 \%$ trifluoroacetic acid solution in dichloromethane after the cyclisation step and checked by mass spectrometry. Indeed, the crude material gave a relative abundance of $83 \%$ for the parent ion for the ring $\mathrm{B}$ analogue.

With this information the synthesis was carried through, with the incorporation of a second nor-lanthionine and subsequent residues, to form the second ring (ring A of nisin, Figure 6.13).

Cleavage of the peptide from the resin was carried out as previously described. Mass spectrometric analysis of the crude product gave the parent ion peak with a relative 
abundance of $18 \%$ and $[\mathrm{M}+2]^{2+}$ as the base peak. Purification via preparative HPLC however indicated that only a small amount of the peptide with the correct mass was present $(<1 \%)$. The presence of proline in the sequence was thought not to have increased the yield significantly during cyclisation of the analogue of ring B of nisin.

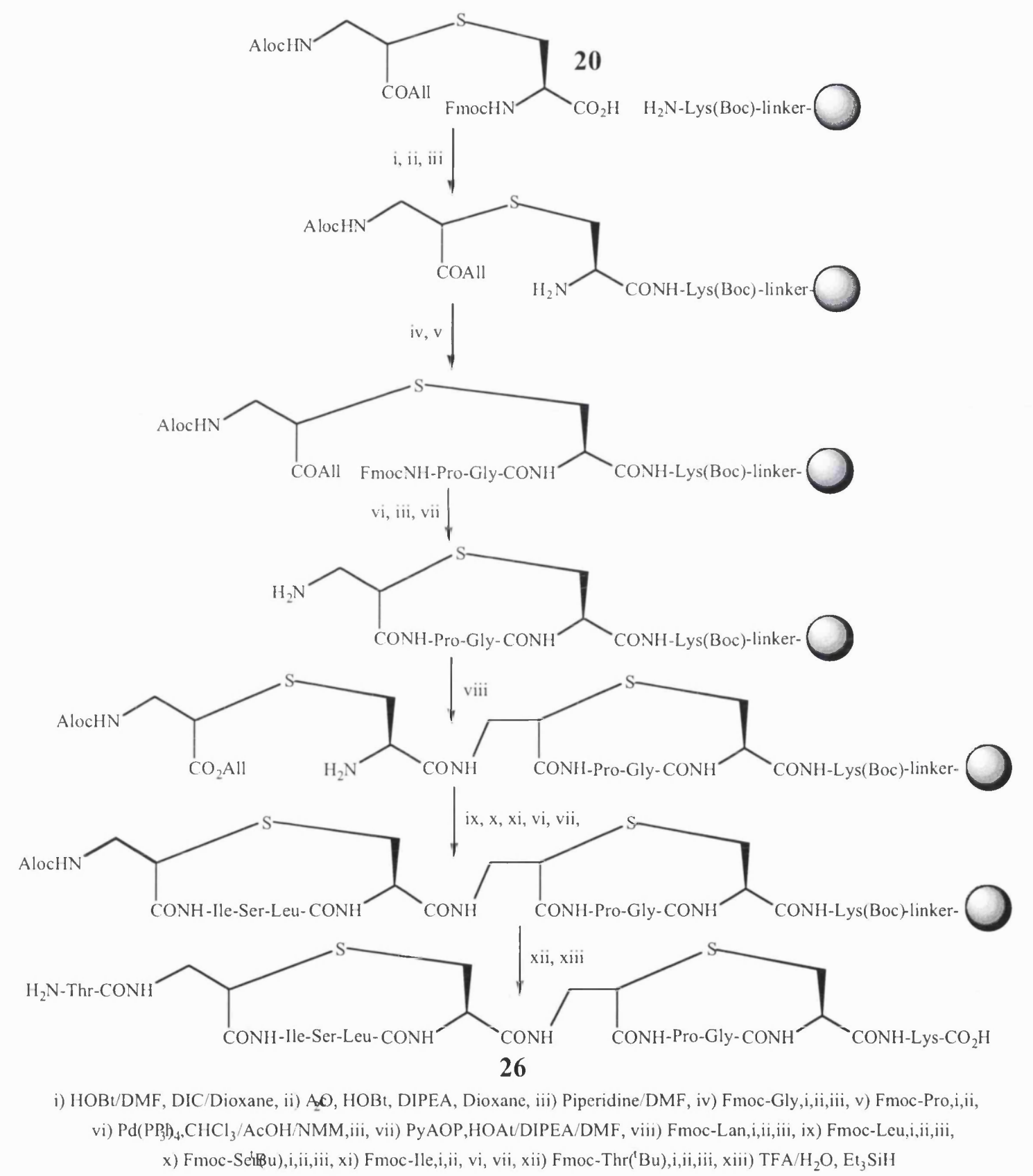

Figure 6.13. Synthesis of an analogue of rings A and B of nisin 26 


\subsection{Further NMR analyses of peptide 25}

Although all other contaminants seemed to have been removed, the ${ }^{1} \mathrm{H}$ NMR for the minor product of 25a was difficult to assign despite the availability of ${ }^{1} \mathrm{H}$ COSY and TOCSY spectra. The major product, $\mathbf{2 5} \mathbf{b}$, on the other hand, produced signals at lower fields in the $\alpha-\mathrm{H}$ region with integrations less than that for one proton and which did not correlate to any other signals in the TOCSY spectrum. In light of these observations and following the high yielding synthesis of peptide $\mathbf{2 5}$, extensive NMR studies were carried out in collaboration with Dr. Richard Harris (Department of Biochemistry, UCL).

${ }^{1} \mathrm{H}-{ }^{13} \mathrm{C}$ HSQC (Appendix W, Appendix X) and HMBC (Appendix X-a) correlation experiments were first carried out on the two peptides isolated in the synthesis of $\mathbf{2 5}$. The results obtained confirmed earlier findings: both peptidic species showed what would have been unlikely ${ }^{3} J$ correlations in a lanthionine moiety between $\beta_{2}$-Hs and the $\alpha_{1}-\mathrm{C}$ across the sulfur bridge, hence giving a structure illustrated in Figure 6.14. The same observation was made with the $\alpha_{1}-\mathrm{H}$, which had a ${ }^{3} J$ correlation with the $\beta_{2}-\mathrm{C}$ on the across the thioether bridge. At the time of these findings the nature of the isomerism of the lanthionine precursors was not known. The determination of the thioether connectivity at the peptide stage provided further proof to the conclusion made in Chapter 5.

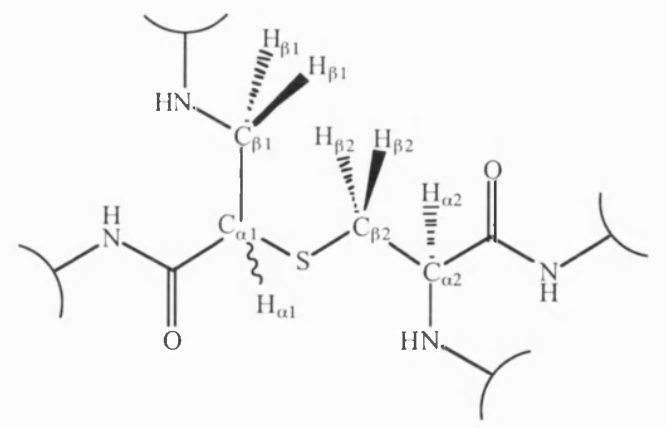

Figure 6.14. Structure of the nor-Lan analogue of ring $C$ of nisin 25 across the thioether linkage as proposed by NMR

Structural determination was carried out by Dr. Richard Harris, involving NOESY experiments, where structure calculations were carried out using the 
PARALLHDGv5.1 (parameter all hydrogens version 5.1) parameter, with nonbonded energy function of PROLSQ, within the CNS (Crystallography and NMR System) program. 175 and 63 inter- ${ }^{1} \mathrm{H}$ distance restraints were applied in restrained molecular dynamics calculations for $\mathbf{2 5 a}$ and $\mathbf{2 5 b}$, respectively. Each calculation was run with both $R$ - and $S$-stereochemistry at the $\alpha$-C position of the $\beta$-amino acid moiety of the peptide. The superimposition of the 20 lowest energy structures for the calculations indicated that the minor isomer 25a displayed a distinct conformation for the $R$-diastereoisomer (Appendix Y). This strongly suggests that 25a is the $R$ diastereoisomer. However, the superimposition of the 20 lowest energy structures calculated for an $S$-diastereoisomer was less defined (Appendix Y). Both $S$ - and $R$ models for the major component 25b were vague (Appendix $Z$ ) due to the low number of inter- ${ }^{1} \mathrm{H}$ distance restraints obtained from NOESY, as well as ROESY experiments. This could be due to the fact that $\mathbf{2 5 b}$ is inherently more flexible, therefore giving rise to fewer NOEs. Since it has been proven that the major product does not contain a lanthionine moiety but a nor-lanthionine one instead, and its mass and ${ }^{1} \mathrm{H}$ NMR spectra are almost identical to those of $\mathbf{2 5 a}$, it could be deduced that it is in fact the $S$-diastereoisomer $\mathbf{2 5 b}$ (Figure 6.15).

Although the hypothesis was made, that partial racemisation occurred at the iodoalanine stage (for regioisomer 6b), it could not be confirmed due to the inefficiency of separation throughout the synthesis. The peptide synthesis, finally provided concrete evidence that the stereochemistry of the $\alpha$-iodo- $\beta$-alanine derivative $6 \mathbf{b}$ was compromised during the ring-opening reaction of aziridine derivatives $(S)-11$ and $(R)-11$. This resulted in a mixture of diastereoisomers for the subsequent nor-lanthionine derivatives 18, 19, 20, 21, 22 and 23 as discussed in Chapter 5, section 5.1.3. 


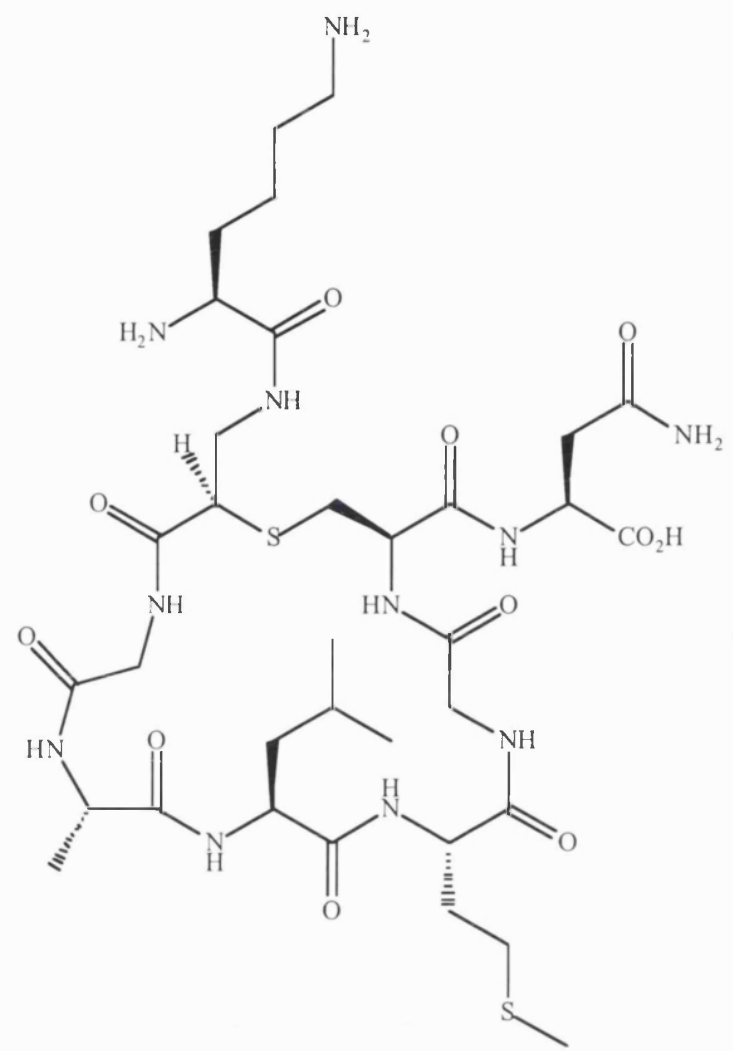

$25 a$

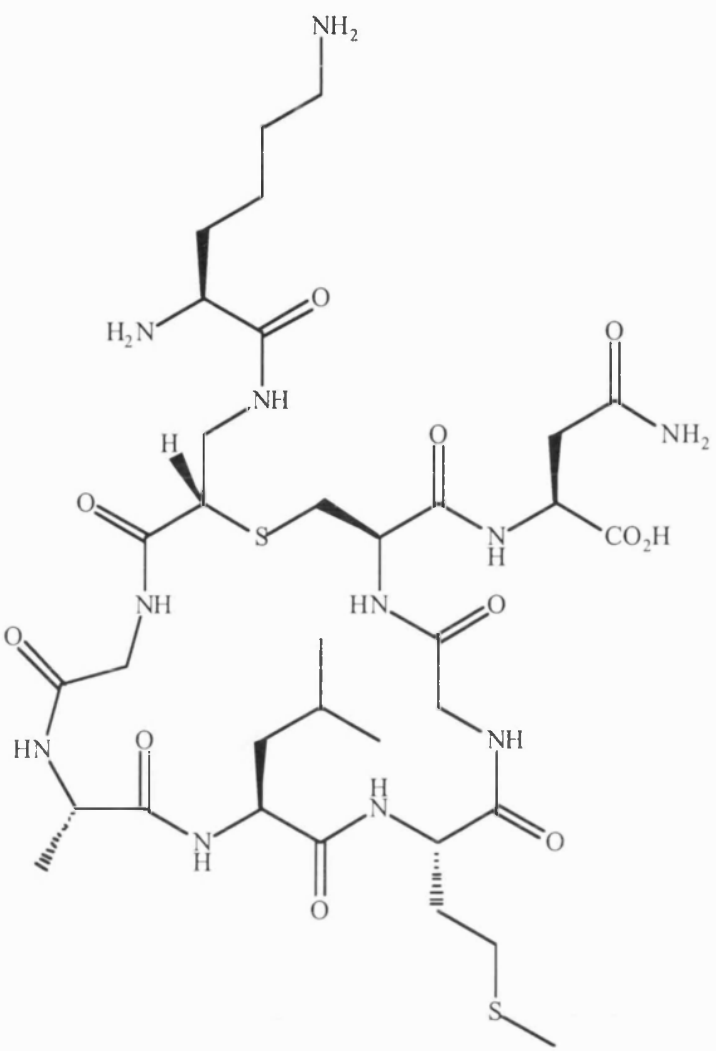

$25 b$

Figure 6.15. Structures of $25 \mathrm{a}$ and $25 \mathrm{~b}$, indicating the stereochemistry at the $\beta$ amino acid moiety

\subsection{Future work}

The use of a double-headed amino acid in the synthesis of cyclic peptides on solid phase has now been proven. ${ }^{212 a, 212 b}$ Although the wrong regioisomer of lanthionine was used, forming an isomeric cyclic peptide, it is highly probable that the methodology will work on the correct lanthionine residue. Future work will need to be concentrated on the synthesis of suitably protected lanthionines.

As mentioned in Chapter 5 (section 5.1.4), both lanthionine derivatives $(\boldsymbol{R}, \boldsymbol{R})-\mathbf{1 0}$ and $(S, R)-10$ could be synthesised utilising new Mitsunobu reaction conditions (Figure 6.16). It was discovered that the use of trimethylphosphine with ADDP $^{205 a, 205 b}$ is more powerful than the original Mitsunobu reactants triphenylphosphine and diethylazodicarboxylate. ${ }^{213}$ Recent reports have also indicated that the use of $\mathrm{Zn}$ 
catalysts could be employed in Mitsunobu reactions involving nitrogen nucleophiles. ${ }^{205 \mathrm{c}}$ The use of trimethylphosphine and ADDP in the presence of zinc(II) tartarate produced the desired lanthionine successfully if in only moderate yields. ${ }^{202,204}$ Attempts to optimise the yield are still continuing.

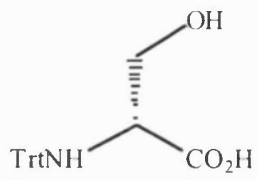

$(R)-4$

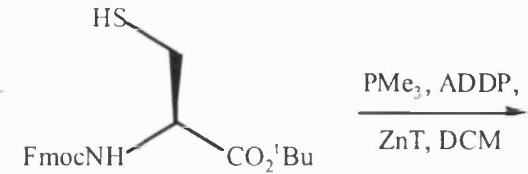

9<smiles>N=C(N)C(=O)OC[C@H](N)C(=O)O</smiles>

$(S, R)-10$

Figure 6.16. Formation of lanthionine via Mitsunobu reaction conditions

These preliminary findings provided invaluable information with regards to the ${ }^{1} \mathrm{H}$ NMR of the two diastereoisomeric lanthionine residues. Crucially, this produced even further confirmation of the regioisomer theory, which was proved by ${ }^{1} \mathrm{H}-{ }^{13} \mathrm{C}$ NMR correlation studies.

Once the synthesis of the lanthionine residue has been optimised, the use of ivDde as part of an orthogonal protecting group strategy must be carried out, as discussed in Chapter 5, section 5.1.2.

Effort will also need to be focused on the synthesis of 3-methyllanthionine, whose occurrence is widespread in lantibiotics. Theoretically the Mitsunobu conditions could also be applied in this instance (Figure 6.17).<smiles>CC(S)C(N)C(=O)O</smiles><smiles>N[C@@H](CO)C(=O)O</smiles>

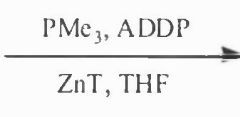<smiles>CC(SC[C@H](N)C(=O)O)C(N)C(=O)O</smiles>

Figure 6.17. Application of Mitsunobu conditions to the synthesis of MeLan

Work will also need to be carried out on introducing dehydro-amino acids into the synthesis, either during the on-resin synthesis or after peptide-resin cleavage. 


\section{Chapter 7}

\section{Experimental}

Unless otherwise indicated, all reagents were obtained from commercial suppliers and used without further purification. THF was distilled using sodium and benzophenone. TEA was distilled over $\mathrm{CaH}_{2}$ and DCM was distilled over $\mathrm{P}_{2} \mathrm{O}_{5}$. $\mathrm{MeOH}$ was distilled using magnesium and iodine, and stored over $4 \AA$ molecular sieves. DMF, NMM, piperidine, acetic anhydride and dioxane were distilled from $\mathrm{CaH}_{2}$ and DIEA was distilled from KOH: all were stored over $4 \AA$ molecular sieves. Hexane is described as the fraction boiling between $67-70{ }^{\circ} \mathrm{C}$. Ether refers to diethyl ether and brine refers to a saturated solution of sodium chloride. 
NMR spectra were recorded using Bruker AC 300, Varian VXR400, AMX400, AVANCE500 and Varian UNITYplus 500 and $600 \mathrm{MHz}$ machines and all samples were dissolved in deuterated chloroform, unless otherwise stated. The chemical shifts (ס) are given in units ppm relative to tetramethylsilane. Coupling constants $(J)$ are measured in Hertz (Hz). Multiplicities for ${ }^{1} \mathrm{H}$ are shown as s (singlet), $\mathrm{d}$ (doublet), $\mathrm{t}$ (triplet), dd (doublet of doublets), dt (doublet of triplets), m (complex multiplet), bs (broad singlet), bd (broad doublet), bt (broad triplet) and bm (broad multiplet). ${ }^{13} \mathrm{C}$ chemical shifts are listed where characterisation of peaks were carried out from experiments described in parentheses following the " $\delta_{C}$ " sign. The term " $c a$." in front of a ${ }^{13} \mathrm{C}$ chemical shift indicates an approximate value for two or three signals for the same carbon that are indistinguishable (regioisomers and/or diastereoisomers) and ${ }^{13} \mathrm{C}$ chemical shifts expressed as $(\mathrm{X}+\mathrm{Y})$ indicate two distinct diastereoisomeric carbon signals.

Mass spectra were recorded using a VG ZAB 2SE mass spectrometer for FAB and Micromass Quattro for ES and APCI (MassLynx software). High resolution mass spectra were recorded using a FAB VG ZAB SE machine.

I.R. spectra were recorded on Perkin-Elmer 1600 FTIR or Shimadzu FTIR-8700 spectrophotometers for solutions in chloroform (with $\mathrm{KBr}$ cell) unless otherwise stated, and Nicolet Protégé for solid I.R.

U.V. spectra were recorded on Shimadzu UV-2401C and are measured against absolute ethanol.

Optical rotations were recorded on Optical Activity:polAAR2000 polarimeter in alumina-treated chloroform solutions (unless otherwise indicated) quoted in $10^{-1} \mathrm{deg}$ $\mathrm{cm}^{2} \mathrm{~g}^{-1}$ with concentration (c.) in $\mathrm{g} / 100 \mathrm{ml}$.

Melting points were determined using a Gallenkemp instrument and are uncorrected. 
Microanalyses were determined, where possible, using a Perkin Elmer 2400 Elemental Analyser (CHN) and Perkin Elmer 240 Elemental Analyser (S, halogen). However a number of compounds prepared in this work were extremely viscous liquids and their purity was determined by accurate mass spectrometry and ${ }^{1} \mathrm{H}$ and ${ }^{13}$ C NMR spectroscopy.

Thin layer chromatography (t.l.c.) was performed on pre-coated aluminium/glassbacked Merck $60 \mathrm{~F}_{254}$ silica plates with $0.25-\mathrm{mm}$ thickness. Visualisation was done by absorption of U.V. light and by dipping with permanganate solution or bromocresol green solution where applicable. Normal phase flash column chromatography was carried out using silica gel with particle size $40-63 \mu \mathrm{m}$ and reverse phase flash column chromatography was carried out using silicagel 60 silanized $(63-200 \mu \mathrm{m})$, both supplied by Merck.

Analytical reverse phase HPLC was carried out using a Waters 600E quaternary gradient system pump, Rheodyne 7125 valve injector, Gilson 115 variablewavelength U.V. detector and Hewlett-Packard HP3396A integrator with a Vydac ${ }^{\text {TM }}$ $\mathrm{C}_{18}$ column, $4.6 \mathrm{~mm} \times 25 \mathrm{~cm}$. Preparative reverse phase HPLC was carried out using a Waters $600 \mathrm{E}$ quaternary gradient system pump, Rheodyne 7125 valve injector, Gilson Holochrome variable-wavelength U.V. detector and Waters 745B Data Module with a $\mathrm{Vydac}^{\mathrm{TM}} \mathrm{C}_{18}$ column, $21.4 \mathrm{~mm} \times 25 \mathrm{~cm}$. Analytical normal phase HPLC was carried out using a Gilson binary gradient system pump, Rheodyne 7725 valve injector, spectroMonitor 3320 Holochrome variable-wavelength U.V. detector and Hewlett-Packard HP3390A integrator with an Alphasil5SILICA column, $4.6 \mathrm{~mm}$ x $25 \mathrm{~cm}$. Preparative normal phase HPLC was carried out using a Waters $600 \mathrm{E}$ quaternary gradient system pump, Rheodyne 7125 valve injector, Gilson Holochrome variable-wavelength U.V. detector and Waters 745B Data Module with a Techsil5SILICA column, $22 \mathrm{~mm} \times 25 \mathrm{~cm}$. LC-MS was carried out using a HewlettPackard HP1100 for HPLC with a Phenomenex ${ }^{\circledR}$ LUNA $3 \mu$ C18(2) column, 2 x 50 $\mathrm{mm}$ and Micromass Quattro LC (MassLynx software) or Finnigan A Q A (XCALIBUR software) for mass spectrometry. Retention times are given in min. 
Kaiser tests were carried out with a small amount of peptidyl-resin using 4 drops each of ethanolic solutions of phenol $(40 \mathrm{~g} / 10 \mathrm{ml})$, ninhydrin $(0.5 \mathrm{~g} / 10 \mathrm{ml})$ and a pyridine $(49 \mathrm{ml})$ solution of $1 \mathrm{mM}$ aqueous potassium cyanide $(1 \mathrm{ml})$ and the combined solution was heated at $\mathrm{T} \sim 100{ }^{\circ} \mathrm{C}$ for approximately $4 \mathrm{~min}$ ). Solutions containing potassium cyanide were treated with a $1 \mathrm{M}$ solution of iron (II) sulfate before being disposed off in the normal fashion for organic materials.

\section{1. ${\underline{(R, R)-C y s t i n e ~ b i s-{ }^{t} \text { butyl ester }}}^{193}$}

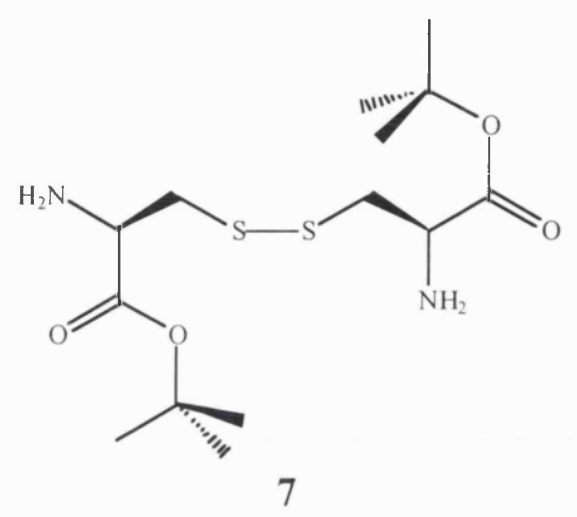

${ }^{t}$ Butyl acetate $(50 \mathrm{ml})$ was added to a stirred solution of L-cystine $(5.0 \mathrm{~g}, 21 \mathrm{mmol})$ in perchloric acid (70\% aq. v/v, $7.8 \mathrm{ml}, 84 \mathrm{mmol}, 4$ equiv.) at room temperature resulting in two immiscible layers, which dispersed on stirring after approximately 3 hr. The reaction mixture was left to stir for a further $72 \mathrm{hr}$ after which it was cooled in an ice bath for $30 \mathrm{~min}$. Sodium hydroxide (2M aq.) was slowly added until the solution reached basic $\mathrm{pH}(11)$. The product was extracted with ether $(6 \mathrm{x} 100 \mathrm{ml})$ and ethyl acetate $(6 \times 100 \mathrm{ml})$ and the combined organic layers were dried over anhydrous sodium sulfate. Removal of the solvents in vacuo yielded the product as a yellow liquid.

Yield: $6.6 \mathrm{~g}, 19 \mathrm{mmol}, 90 \%$ (lit. ${ }^{193} 67 \%$ )

NMR: $\boldsymbol{\delta}_{\mathbf{H}}(400 \mathrm{MHz}) 3.63\left(2 \mathrm{H}, \mathrm{dd}, J\right.$ 7.9, 4.5, 2 x $\left.\mathrm{CHCH}_{2} \mathrm{~S}\right), 3.08(2 \mathrm{H}, \mathrm{dd}, J$ 13.4, 4.6, $2 \times \mathrm{CHCHHS}), 2.82(2 \mathrm{H}, \mathrm{dd}, J 13.4,7.9,2 \times \mathrm{CHCH} H \mathrm{~S}), 1.42$ (18H, s, 2x $\left.\mathrm{CO}_{2} \mathrm{C}\left(\mathrm{CH}_{3}\right)_{3}\right)$

Mass spectrum: m/z (APCI+) $353\left([\mathrm{M}+\mathrm{H}]^{+}, 100 \%\right), 375\left([\mathrm{M}+\mathrm{Na}]^{+}, 0.4 \%\right), 297$ $\left(\left[\mathrm{M}-{ }^{\mathrm{t}} \mathrm{Bu}+2\right]^{+}, 39 \%\right), 241\left(\left[\mathrm{M}-2^{\mathrm{t}} \mathrm{Bu}+3\right]^{+}, 18 \%\right)$ 


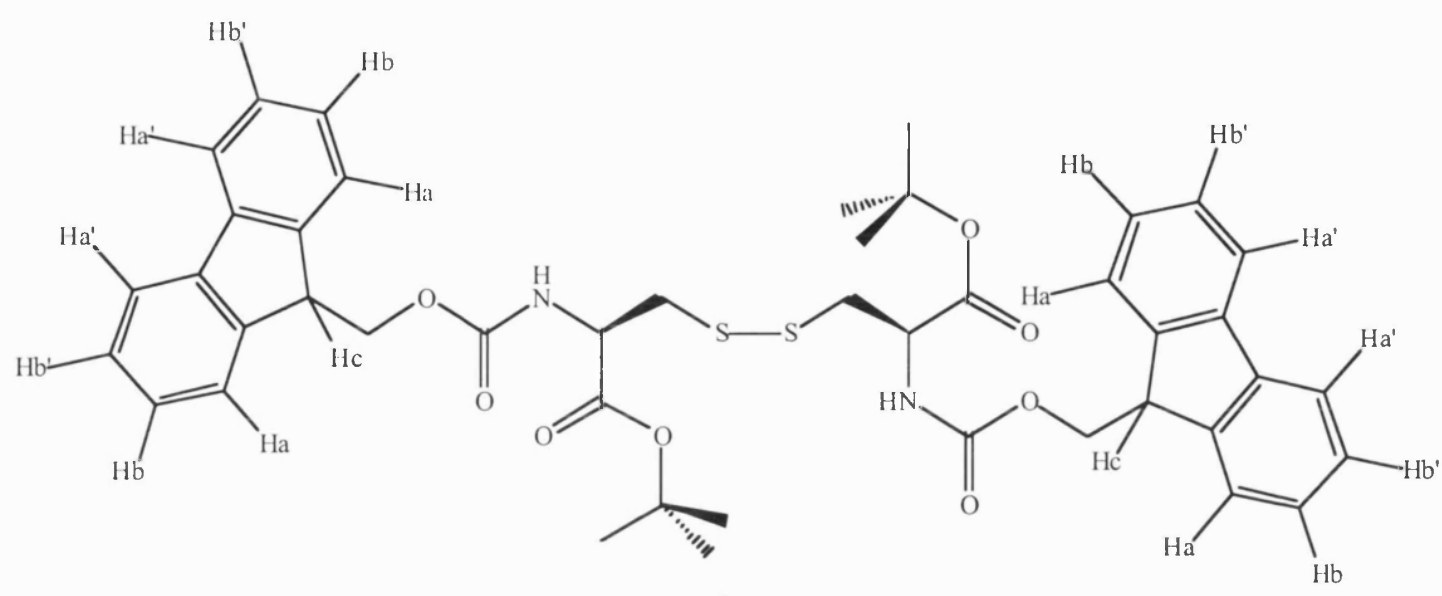

8

$(R, R)$-Cystine bis-'butyl ester $7(6.6 \mathrm{~g}, 18 \mathrm{mmol})$ and fluorenylmethoxycarbonyl chloride ( $10 \mathrm{~g}, 36 \mathrm{mmol}, 2$ equiv.) were dissolved in THF under inert conditions. The reaction was cooled in an ice bath and NMM ( $4.5 \mathrm{ml}, 36 \mathrm{mmol}, 2$ equiv.) was slowly added to the solution and the resulting mixture was stirred for $3 \mathrm{hr}$. Ethyl acetate $(200 \mathrm{ml})$ was added to the mixture, which was then washed with potassium hydrogen sulfate $(5 \%$ aq. w/v, $4 \times 50 \mathrm{ml})$ and water $(3 \times 50 \mathrm{ml})$. The organic layer was dried over anhydrous sodium sulfate and the solvent was removed in vacuo yielding the crude product as a yellow liquid. The product was precipitated with DCM:MeOH, $1: 5$ at $-20{ }^{\circ} \mathrm{C} .{ }^{13} \mathrm{C}$ and ${ }^{1} \mathrm{H}$ NMR data were consistent with literature. ${ }^{194,215,216}$

Yield: $12 \mathrm{~g}, 15 \mathrm{mmol}, 81 \%$

$\mathbf{R}_{\mathbf{f}}: 0.25$ (hexane:ether, $2: 1$ )

M.p.: $146-148^{\circ} \mathrm{C}\left(\right.$ lit. $\left.^{216} 151.5-152{ }^{\circ} \mathrm{C}\right)$

Elemental analysis: Found $\mathrm{C}, 66.59 ; \mathrm{H}, 6.06 ; \mathrm{N}, 3.38 ; \mathrm{S}, 7.97 \% . \mathrm{C}_{44} \mathrm{H}_{48} \mathrm{~N}_{2} \mathrm{O}_{8} \mathrm{~S}_{2}$ requires $\mathrm{C}, 66.31 ; \mathrm{H}, 6.07 ; \mathrm{N}, 3.51 ; \mathrm{S}, 8.05 \%$.

NMR: $\delta_{\mathbf{H}}(400 \mathrm{MHz}) 7.78\left(4 \mathrm{H}, \mathrm{bd}, J 7.5, H_{\mathrm{a}^{\prime}}\right), 7.62\left(4 \mathrm{H}, \mathrm{bd}, J 7.4, H_{\mathrm{a}}\right), 7.41(4 \mathrm{H}, \mathrm{bt}$, $\left.J 7.2, H_{\mathrm{b}^{\prime}}\right), 7.33\left(4 \mathrm{H}, \mathrm{bt}, J 7.3, H_{\mathrm{b}}\right), 5.74(2 \mathrm{H}, \mathrm{d}, J 7.0, \mathrm{NH}), 4.56\left(2 \mathrm{H}, \mathrm{bm}, \mathrm{CHCH}_{2} \mathrm{~S}\right)$, $4.41\left(4 \mathrm{H}, \mathrm{bm}, \mathrm{CH}_{\mathrm{c}} \mathrm{CH}_{2} \mathrm{O}\right), 4.23\left(2 \mathrm{H}, \mathrm{bm}, \mathrm{H}_{\mathrm{c}}\right), 2.99\left(4 \mathrm{H}, \mathrm{bm}, \mathrm{CHCH}_{2} \mathrm{~S}\right), 1.51(18 \mathrm{H}, \mathrm{s}$, $\left.\mathrm{CO}_{2} \mathrm{C}\left(\mathrm{CH}_{3}\right)_{3}\right) ; \delta_{\mathrm{C}}(75.4 \mathrm{MHz}$, DEPT 135) 169.7, 156.0, 144.3, 144.2, 141.7, 128.1, $127.5,125.5,120.3,83.5,67.6,54.6,47.5,28.4$

Mass spectrum: $m / z$ (FAB) $819.2750\left([\mathrm{M}+\mathrm{Na}]^{+} . \mathrm{C}_{44} \mathrm{H}_{48} \mathrm{~N}_{2} \mathrm{O}_{8} \mathrm{~S}_{2} \mathrm{Na}\right.$ requires 819.2725), $797\left(\mathrm{M}^{+}, 30 \%\right), 685\left(\left[\mathrm{M}-2^{\mathrm{t}} \mathrm{Bu}+3\right]^{+}, 100 \%\right), 463\left(\left[\mathrm{M}-2^{t} \mathrm{Bu}-\mathrm{Fmoc}+\right.\right.$ $\left.4]^{+}, 54 \%\right), 237\left(\left[\mathrm{M}-2^{t} \mathrm{Bu}-2 \mathrm{Fmoc}+5\right]^{+}, 49 \%\right)$ 


\section{3. $\mathbf{N - 9 - F l u o r e n y l m e t h o x y c a r b o n y l - ( R ) - c y s t e i n e ~}{ }^{t}$ butvl ester $^{76}$}

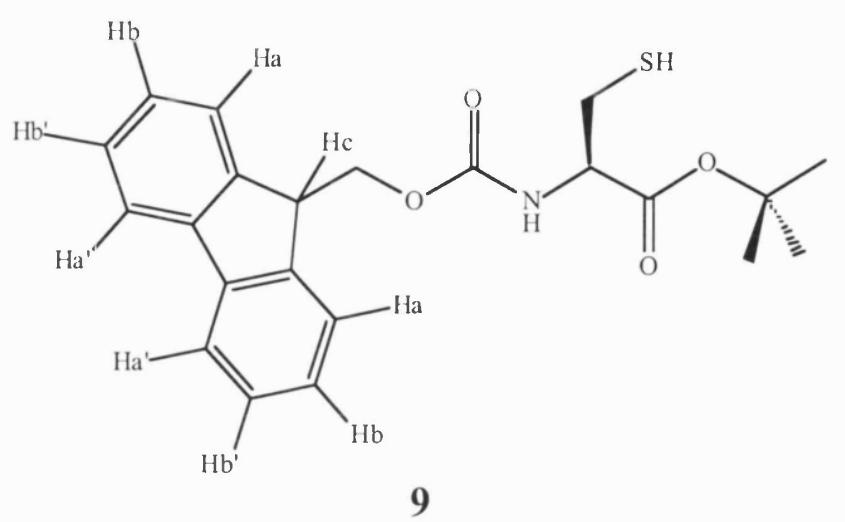

A solution of $N, N$ '-bis(fluorenylmethoxycarbonyl)-( $R, R)$-cystine-bis- 'butyl ester 8 $(7.5 \mathrm{~g}, 9.4 \mathrm{mmol})$ in dry THF $(50 \mathrm{ml})$ was treated with tributylphosphine $(2.4 \mathrm{ml}, 9.4$ $\mathrm{mmol}$ ). The mixture was stirred for 2 minutes after which water (distilled, $0.94 \mathrm{ml}$, $10 \%$ of THF volume, 5 equiv.) was added and the reaction was stirred for a further 2 hr. The mixture was then concentrated in vacuo and dissolved in ethyl acetate (200 $\mathrm{ml})$. The solution was then washed with citric acid $(10 \% \mathrm{aq} . \mathrm{w} / \mathrm{v}, 150 \mathrm{ml})$ then with brine $(100 \mathrm{ml})$. The organic layer was dried over anhydrous sodium sulfate and the solvent was removed under reduced pressure yielding a pale yellow liquid. Purification via flash column chromatography (hexane:ether $2: 1,6 \mathrm{~cm}$ width, $15 \mathrm{~cm}$ height) yielded the product as an extremely viscous colourless liquid.

Yield: $6.8 \mathrm{~g}, 17 \mathrm{mmol}, 85 \%$

$\mathbf{R}_{\mathbf{f}}: 0.30$ (hexane:ether, $2: 1$ )

Elemental analysis: Found $\mathrm{C}, 65.96 ; \mathrm{H}, 6.40 ; \mathrm{N}, 3.42 ; \mathrm{S}, 7.83 \% . \mathrm{C}_{22} \mathrm{H}_{25} \mathrm{NO}_{4} \mathrm{~S}$ requires $\mathrm{C}, 66.14 ; \mathrm{H}, 6.31 ; \mathrm{N}, 3.51 ; \mathrm{S}, 8.02 \%$.

NMR: $\delta_{\mathrm{H}}(400 \mathrm{MHz}) 7.78\left(2 \mathrm{H}, \mathrm{d}, J 7.5, H_{\mathrm{a}}\right), 7.62\left(2 \mathrm{H}, \mathrm{d}, \mathrm{J} 7.4, H_{\mathrm{a}}\right), 7.41(2 \mathrm{H}, \mathrm{t}, J$ 7.4, $\left.H_{\mathrm{b}}\right), 7.33\left(2 \mathrm{H}, \mathrm{dt}, J 7.5,1.2, H_{\mathrm{b}}\right), 5.74(1 \mathrm{H}, \mathrm{d}, J 7.0, \mathrm{NH}), 4.56(1 \mathrm{H}, \mathrm{m}$, $\left.\mathrm{CHCH}_{2} \mathrm{SH}\right), 4.41\left(2 \mathrm{H}, \mathrm{m}, \mathrm{CH}_{\mathrm{c}} \mathrm{CH}_{2} \mathrm{O}\right), 4.23\left(1 \mathrm{H}, \mathrm{m}, \mathrm{H}_{\mathrm{c}}\right), 3.00\left(2 \mathrm{H}, \mathrm{m}, \mathrm{CHCH}_{2} \mathrm{SH}\right)$, $1.51\left(9 \mathrm{H}, \mathrm{s}, \mathrm{CO}_{2} \mathrm{C}\left(\mathrm{CH}_{3}\right)_{3}\right) ; \delta_{\mathrm{C}}(75.4 \mathrm{MHz}, \mathrm{DEPT} 135)$ 169.4, 156.1, 144.1, 141.7, $128.2,127.5,125.5,120.4,83.5,67.5,55.8,47.6,28.4,27.8$

Mass spectrum: $m / z(\mathrm{FAB}) 422.1402\left([\mathrm{M}+\mathrm{Na}]^{+} \cdot \mathrm{C}_{22} \mathrm{H}_{25} \mathrm{NO}_{4} \mathrm{SNa}\right.$ requires $422.1391), 400\left([\mathrm{M}+\mathrm{H}]^{+}, 0.3 \%\right), 344\left(\left[\mathrm{M}-{ }^{\mathrm{t}} \mathrm{Bu}\right]^{+}, 3.8 \%\right), 220\left([\mathrm{M}-\mathrm{Fmoc}]^{+}, 0.2\right.$ $\%), 179\left(\mathrm{Fmoc}^{+}, 100 \%\right), 163\left(\left[\mathrm{M}-\mathrm{Fmoc}-{ }^{\mathrm{t}} \mathrm{Bu}\right]^{+}, 6.9 \%\right)$ 


\section{4. $\underline{N \text {-Triphenvlmethyl-(S)-serine }}{ }^{195}$}

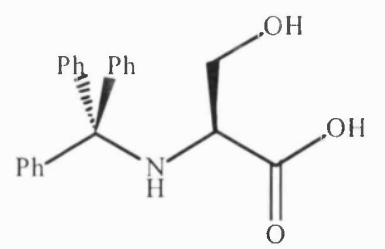

$(S)-3$

To a suspension of $(S)$-serine $(4.4 \mathrm{~g}, 42 \mathrm{mmol})$ in DCM $(65 \mathrm{ml})$ under nitrogen was added trimethylsilyl chloride ( $17 \mathrm{ml}, 0.13 \mathrm{~mol}, 3.1$ equiv.). The mixture was allowed to reflux for 20 minutes. After cooling the mixture to room temperature, triethylamine (18 ml, $130 \mathrm{mmol}, 3.1$ equiv.) in DCM $(20 \mathrm{ml})$ was added and the reaction was heated under reflux for a further 45 minutes. After cooling in an ice bath anhydrous $\mathrm{MeOH}(2.5 \mathrm{ml}, 42 \mathrm{mmol})$ was added dropwise to the mixture, which was then allowed to warm to room temperature. Triethylamine $(6 \mathrm{ml}, 42 \mathrm{mmol})$ was added followed by triphenylmethyl chloride ( $12 \mathrm{~g}, 42 \mathrm{mmol})$ and the mixture was stirred for $20 \mathrm{hr}$. An excess of triethylamine $(20 \mathrm{ml})$ and $\mathrm{MeOH}(200 \mathrm{ml})$ was then added until the white solids present in the mixture dissolved. The solvents were removed in vacuo yielding a mixture of yellow and white solids, which was partitioned between ethyl acetate $(200 \mathrm{ml})$ and citric acid pre-cooled to $4{ }^{\circ} \mathrm{C}(5 \%$ aq. $\mathrm{w} / \mathrm{v}, 150 \mathrm{ml}$ ). The organic layer was washed with a solution of sodium hydroxide $(1 \mathrm{M}, 2 \times 50 \mathrm{ml})$ followed by water $(2 \times 30 \mathrm{ml})$. The aqueous layers were combined, washed with ethyl acetate $(50 \mathrm{ml})$ and neutralised with glacial acetic acid at $0{ }^{\circ} \mathrm{C}$. The precipitated product was extracted with ethyl acetate $(3 \times 50 \mathrm{ml})$ and the organic layer was washed with water $(2 \times 20 \mathrm{ml})$. Removal of the solvent under reduced pressure produced a white solid.

Yield: $5.8 \mathrm{~g}, 17 \mathrm{mmol}, 40 \%$

R: 0.33 (DCM:MeOH, 9:1)

M.p.: $107-108^{\circ} \mathrm{C}$ (dec.) (lit. ${ }^{214} 111-112^{\circ} \mathrm{C}$, racemic)

Elemental analysis: Found $\mathrm{C}, 75.93 ; \mathrm{H}, 6.21 ; \mathrm{N}, 3.29 \% . \mathrm{C}_{22} \mathrm{H}_{21} \mathrm{NO}_{3}$ requires $\mathrm{C}$, $76.06 ; \mathrm{H}, 6.09 ; \mathrm{N}, 4.03 \%$.

NMR: $\delta_{\mathrm{H}}(400 \mathrm{MHz}) 7.41(6 \mathrm{H}, \mathrm{m}, \mathrm{Trt}), 7.24(9 \mathrm{H}, \mathrm{m}, \mathrm{Trt}), 3.71(1 \mathrm{H}, \mathrm{dd}, J 11.2,3.1$, $\left.\mathrm{CHCH}_{2} \mathrm{OH}\right), 3.49\left(1 \mathrm{H}, \mathrm{dd}, J 4.2,3.5, \mathrm{CHCH}_{2} \mathrm{OH}\right), 2.88(1 \mathrm{H}, \mathrm{dd}, J 11.2,4.5$, $\mathrm{CHCH}_{2} \mathrm{OH}$ ) 
Mass spectrum: $m / z(\mathrm{FAB}) 370.1419\left([\mathrm{M}+\mathrm{Na}]^{+} . \mathrm{C}_{22} \mathrm{H}_{21} \mathrm{NO}_{3} \mathrm{Na}\right.$ requires 370.1430), $370\left([\mathrm{M}+\mathrm{Na}]^{+}, 1.5 \%\right), 348\left([\mathrm{M}+\mathrm{H}]^{+}, 0.6 \%\right), 243(\operatorname{Trt}, 100 \%) ; \mathrm{m} / z(\mathrm{APCI}+) 243$ $\left(\mathrm{Trt}^{+}, 100 \%\right), 106\left([\mathrm{M}-\mathrm{Trt}+2]^{+}, 0.7 \%\right)$

\section{5. $\underline{N \text {-Triphenvlmethvl- }(R)-\text { serine }^{214}}$}

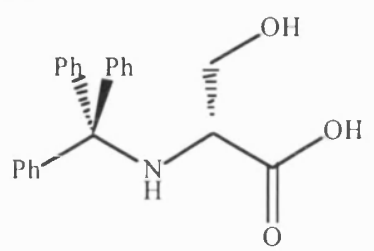

$(R)-3$

To a suspension of $(R)$-serine $(15 \mathrm{~g}, 0.14 \mathrm{~mol})$ in DCM $(200 \mathrm{ml})$ under nitrogen was added trimethylsilyl chloride ( $56 \mathrm{ml}, 0.43 \mathrm{~mol}, 3.1$ equiv.). The mixture was allowed to reflux for 20 minutes. After cooling the mixture to room temperature, triethylamine ( $62 \mathrm{ml}, 0.43 \mathrm{~mol}, 3.1$ equiv.) in DCM $(200 \mathrm{ml})$ was added and the reaction was heated under reflux for a further 45 minutes. After cooling in an ice bath anhydrous $\mathrm{MeOH}(7 \mathrm{ml}, 0.14 \mathrm{~mol})$ was added dropwise to the mixture, which was then allowed to warm to room temperature. Triethylamine $(20 \mathrm{ml}, 0.14 \mathrm{~mol})$ was added followed by triphenylmethyl chloride $(30 \mathrm{~g}, 0.14 \mathrm{~mol})$ and the mixture was stirred for $20 \mathrm{hr}$. An excess of triethylamine $(100 \mathrm{ml})$ and $\mathrm{MeOH}(700 \mathrm{ml})$ was then added until the white solids present in the mixture dissolved. The solvents were removed in vacuo yielding a mixture of yellow and white solids, which was partitioned between ethyl acetate $(500 \mathrm{ml})$ and citric acid pre-cooled to $4{ }^{\circ} \mathrm{C}(5 \%$ aq. $\mathrm{w} / \mathrm{v}, 300 \mathrm{ml}$ ). The organic layer was washed with a solution of sodium hydroxide $(2 \mathrm{M}, 2 \times 150 \mathrm{ml})$ followed by water $(3 \times 100 \mathrm{ml})$. The aqueous layers were combined, washed with ethyl acetate $(100 \mathrm{ml})$ and neutralised with glacial acetic acid at $0{ }^{\circ} \mathrm{C}$. The precipitated product was extracted with ethyl acetate $(6 \times 200 \mathrm{ml})$ and the organic layer was dried over anhydrous sodium sulfate. Removal of the solvent under reduced pressure yielded the product as a white solid.

Yield: $7.2 \mathrm{~g}, 21 \mathrm{mmol}, 85 \%$

$\mathbf{R}_{\mathbf{f}}: 0.33$ (DCM:MeOH, 9:1)

M.p.: $109-110^{\circ} \mathrm{C}$ (dec.) (lit. ${ }^{214} 111-112^{\circ} \mathrm{C}$, racemic) 
NMR: $\delta_{\mathbf{H}}(500 \mathrm{MHz}) 7.45(6 \mathrm{H}, \mathrm{m}, \mathrm{Trt}), 7.28(9 \mathrm{H}, \mathrm{m}, \mathrm{Trt}), 3.72(1 \mathrm{H}, \mathrm{dd}, J 11.3,3.0$, $\left.\mathrm{CHCH}_{2} \mathrm{OH}\right), 3.52\left(1 \mathrm{H}, \mathrm{m}, \mathrm{CHCH} \mathrm{H}_{2} \mathrm{OH}\right), 2.88\left(1 \mathrm{H}, \mathrm{dd}, J 11.3,4.3, \mathrm{CHCH}_{2} \mathrm{OH}\right) ; \boldsymbol{\delta}_{\mathrm{C}}$ (75.4 MHz, DEPT135) 175.9, 144.4, 128.7 128.2, 127.1, 72.3, 62.9, 59.3

Mass spectrum: $m / z(F A B) 370.1419\left([\mathrm{M}+\mathrm{Na}]^{+} . \mathrm{C}_{22} \mathrm{H}_{21} \mathrm{NO}_{3} \mathrm{Na}\right.$ requires 370.1430), $370\left([\mathrm{M}+\mathrm{Na}]^{+}, 4 \%\right), 243\left(\mathrm{Trt}^{+}, 100 \%\right)$

\section{6. $\underline{\mathbf{N}-T r i p h e n y l m e t h y l-(\mathbf{S}) \text {-serine allyl ester }}$}

A suspension of $N$-triphenylmethyl- $(S)$-serine $(S)-3(0.51 \mathrm{~g}, 1.5 \mathrm{mmol})$ in a solution of sodium hydrogen carbonate $(0.13 \mathrm{~g}, 1.6 \mathrm{mmol})$ in water $(5 \mathrm{ml})$ was treated with a solution of Aliquat $336(0.70 \mathrm{ml}, 1.5 \mathrm{mmol})$ and allyl bromide $(0.14 \mathrm{ml}, 1.6 \mathrm{mmol})$ in DCM (2.2 ml). After $10-15$ seconds of vigorous stirring the starting compound dissolved and the mixture was left stirring for $95 \mathrm{hr}$. The mixture was extracted with $\operatorname{DCM}(3 \times 15 \mathrm{ml})$ and the combined organic layers were dried over anhydrous sodium sulfate. Removal of the solvent in vacuo yielded a yellow liquid whose NMR spectrum contained the desired product and the Aliquat 336. This proved impossible to purify by chromatography.

\section{7. $\underline{\text { N-Triphenylmethyl-(S)-serine allyl ester }}$}

A solution of $N$-triphenylmethyl-(S)-serine $(S)-3(0.31 \mathrm{~g}, 0.89 \mathrm{mmol})$ in DCM (2 ml) was prepared under argon and treated with triethylamine $(0.18 \mathrm{ml}, 1.3 \mathrm{mmol}, 1.5$ equiv.). A solution of allyl bromide ( $0.08 \mathrm{ml}, 0.92 \mathrm{mmol}, 1.04$ equiv.) in DCM was added to the mixture and the reaction was stirred for $42 \mathrm{hr}$. The mixture was treated with citric acid (5\% aq. w/v, $2 \mathrm{ml})$ and extraction with $\operatorname{DCM}(3 \times 10 \mathrm{ml})$ was performed. The combined organic layers were washed with water $(3 \times 10 \mathrm{ml})$ and dried over anhydrous sodium sulfate. A yellow liquid was produced and purified via flash column chromatography (DCM:MeOH, 9:1, $5 \mathrm{~cm}$ width, $8 \mathrm{~cm}$ height) yielding the desired product as a viscous pale yellow liquid, and the starting material

Yield: $0.17 \mathrm{~g}, 0.44 \mathrm{mmol}, 50 \%$

$\mathbf{R}_{\mathbf{f}}: 0.64$ (DCM:MeOH, 9:1) 


\section{N-Triphenvlmethvl-(S)-serine allvl ester}

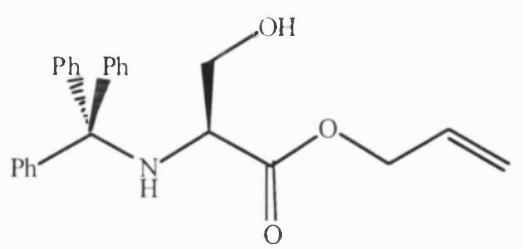

$(S)-4$

$N$-triphenylmethyl-(S)-serine $(S)-3(2.0 \mathrm{~g}, 5.7 \mathrm{mmol})$ was converted to its cesium salt by dissolving in dry $\mathrm{MeOH}(40 \mathrm{ml})$ with cesium carbonate $(1.0 \mathrm{~g}, 2.9 \mathrm{mmol}, 0.51$ equiv.) under argon. After removing the solvent in vacuo, the salt produced was taken up in DMF $(40 \mathrm{ml})$ and the resulting mixture was treated with allyl bromide ( $0.50 \mathrm{ml}, 5.8 \mathrm{mmol}, 1.01$ equiv.). The mixture was left stirring for a further $18 \mathrm{hr}$, after which it was partitioned between ethyl acetate $(200 \mathrm{ml})$ and citric acid $(5 \%$ aq. $\mathrm{w} / \mathrm{v}, 50 \mathrm{ml})$. The aqueous layer was washed with further ethyl acetate $(2 \times 50 \mathrm{ml})$ and the organic layers were combined, washed with water $(15 \times 50 \mathrm{ml})$ and then dried over anhydrous sodium sulfate. Removal of the solvent in vacuo yielded a very pale yellow (almost colourless) liquid, which was purified by flash columnchromatography (DCM:MeOH, 40:1, $5 \mathrm{~cm}$ width) to give an extremely viscous colourless liquid.

Yield: $1.8 \mathrm{~g}, 4.6 \mathrm{mmol}, 81 \%$

$\mathbf{R}_{\mathbf{f}}: 0.33$ (DCM:MeOH, 40:1)

$\left[\left.\alpha\right|_{D}{ }^{19}:+3.7\right.$ (c 0.98$)$

I.R.: $v_{\max }\left(\mathrm{cm}^{-1}\right) 3581(\mathrm{OH}), 3325(\mathrm{NH}), 1732(\mathrm{C}=\mathrm{O}$ ester $), 1672(\mathrm{C}=\mathrm{C}$ Ar $), 1596$ $(\mathrm{C}=\mathrm{C} \mathrm{Ar}$ ). Absence of i) a broad $\mathrm{H}$-bonded $\mathrm{OH}$ (acid) signal and ii) $\mathrm{C}=\mathrm{O}$ (acid) signal between $1700-1725 \mathrm{~cm}^{-1}$

NMR: $\boldsymbol{\delta}_{\mathbf{H}}(400 \mathrm{MHz}) 7.48(6 \mathrm{H}, \mathrm{m}, \mathrm{Trt}), 7.22(9 \mathrm{H}, \mathrm{m}, \mathrm{Trt}), 5.70\left(1 \mathrm{H}, \mathrm{m}, \mathrm{CH}=\mathrm{CH}_{2}\right)$, $5.17\left(2 \mathrm{H}, \mathrm{m}, \mathrm{CH}=\mathrm{CH}_{2}\right), 4.20\left(1 \mathrm{H}, \mathrm{dd}, J 13.1,5.8, \mathrm{OCH}_{2} \mathrm{CH}\right), 4.08(1 \mathrm{H}, \mathrm{dd}, J 13.1$, 5.8, $\left.\mathrm{OCH}_{2} \mathrm{CH}\right), 3.70\left(1 \mathrm{H}, \mathrm{m}, \mathrm{HNCHCO}_{2}\right), 3.56\left(2 \mathrm{H}, \mathrm{m}, \mathrm{CHCH}_{2} \mathrm{OH}\right) ; \delta_{\mathrm{C}}(75.4 \mathrm{MHz}$, DEPT135) 173.1, 145.6, 131.6, 128.5, 127.8, 126.5, 118.3, 70.9, 65.6, 64.9, 57.9

Mass spectrum: $m / z(\mathrm{FAB}) 410.1732\left([\mathrm{M}+\mathrm{Na}]^{+} . \mathrm{C}_{22} \mathrm{H}_{25} \mathrm{NO}_{3} \mathrm{Na}\right.$ requires 410.1750), $410\left([\mathrm{M}+\mathrm{Na}]^{+}, 0.3 \%\right), 388\left([\mathrm{M}+\mathrm{H}]^{+}, 0.1 \%\right), 387\left(\mathrm{M}^{+}, 0.3 \%\right), 243\left(\operatorname{Trt}^{+}, 100 \%\right)$, $146\left([\mathrm{M}-\mathrm{Trt}+2]^{+}, 1.8 \%\right)$ 


\section{9. $\mathbf{N}$-Triphenvlmethvl-(R)-serine allvl ester}

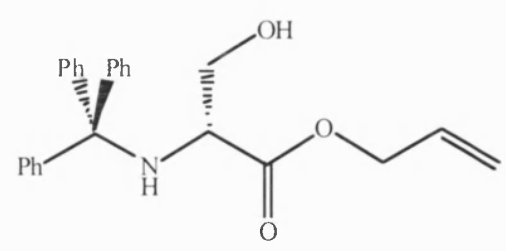

$(R)-4$

$N$-triphenylmethyl-(R)-serine $(\boldsymbol{R})-\mathbf{3}(20 \mathrm{~g}, 58 \mathrm{mmol})$ was converted to its cesium salt by dissolving in dry $\mathrm{MeOH}(200 \mathrm{ml})$ with cesium carbonate $(1.0 \mathrm{~g}, 2.9 \mathrm{mmol}, 0.51$ equiv.) under argon. After removing the solvent in vacuo, the salt produced was treated with DMF $(40 \mathrm{ml})$ and the resulting mixture was treated with allyl bromide (0.52 ml, $6.0 \mathrm{mmol}, 1.01$ equiv.). The mixture was left stirring for a further $18 \mathrm{hr}$, after which the DMF was removed under high vacuum. The mixture was then redissolved in ethyl acetate $(200 \mathrm{ml})$ and washed with citric acid $(5 \%$ aq. w/v, $50 \mathrm{ml})$. The aqueous layer was washed with further ethyl acetate $(2 \times 50 \mathrm{ml})$ and the organic layers were combined, washed with water $(12 \times 50 \mathrm{ml})$ and then dried over anhydrous sodium sulfate. Removal of the solvent in vacuo yielded the product as an extremely viscous colourless liquid.

Yield: $19 \mathrm{~g}, 50 \mathrm{mmol}, 87 \%$

$\mathbf{R}_{\mathbf{f}}: 0.33$ (DCM:MeOH, 40:1)

$|\alpha|_{\mathrm{D}}{ }^{19}:-2.0(\mathrm{c} 0.75)$

I.R.: $v_{\max }\left(\mathrm{cm}^{-1}\right) 3580(\mathrm{OH}), 3320(\mathrm{NH}), 1732(\mathrm{C}=\mathrm{O}$ ester $), 1493(\mathrm{C}-\mathrm{C}$ Ar). Absence of i) a broad $\mathrm{H}$-bonded $\mathrm{OH}$ (acid) signal and ii) $\mathrm{C}=\mathrm{O}$ (acid) signal between 1700$1725 \mathrm{~cm}^{-1}$

Elemental analysis: Found $\mathrm{C}, 75.65 ; \mathrm{H}, 6.41 ; \mathrm{N}, 3.49 \% . \mathrm{C}_{25} \mathrm{H}_{25} \mathrm{NO}_{3}$ requires $\mathrm{C}$, $77.49 ; \mathrm{H}, 6.50 ; \mathrm{N}, 3.61 \%$.

NMR: $\boldsymbol{\delta}_{\mathbf{H}}(500 \mathrm{MHz}) 7.51(6 \mathrm{H}, \mathrm{m}, \mathrm{Trt}), 7.25(9 \mathrm{H}, \mathrm{m}, \mathrm{Trt}), 5.71\left(1 \mathrm{H}, \mathrm{m}, \mathrm{CH}=\mathrm{CH}_{2}\right)$, $5.17\left(2 \mathrm{H}, \mathrm{m}, \mathrm{CH}=\mathrm{CH}_{2}\right), 4.22\left(1 \mathrm{H}, \mathrm{dd}, J 13.1,5.8, \mathrm{OCH}_{2} \mathrm{CH}\right), 4.11(1 \mathrm{H}, \mathrm{dd}, J 13.1$, 5.8, $\left.\mathrm{OCH}_{2} \mathrm{CH}\right), 3.72\left(1 \mathrm{H}, \mathrm{m}, \mathrm{HNCHCO}_{2}\right), 3.58\left(2 \mathrm{H}, \mathrm{m}, \mathrm{CHCH}_{2} \mathrm{OH}\right) ; \boldsymbol{\delta}_{\mathrm{C}}(75.4 \mathrm{MHz}$, DEPT 135) 173.6, 146.1 132.1, 129.2, 128.3, 127.0, 118.8, 71.5, 66.1, 65.3, 58.3

Mass spectrum: $m / z(F A B) 410.1732\left([\mathrm{M}+\mathrm{Na}]^{+} . \mathrm{C}_{22} \mathrm{H}_{25} \mathrm{NO}_{3} \mathrm{Na}\right.$ requires 410.1750), $388\left([\mathrm{M}+\mathrm{H}]^{+}, 3 \%\right), 387\left(\mathrm{M}^{+}, 0.7 \%\right), 243\left(\mathrm{Trt}^{+}, 100 \%\right), 146\left([\mathrm{M}-\mathrm{Trt}+2]^{+}, 2 \%\right)$ 


\section{0. $\mathbf{N}$-Triphenvlmethvl-(S)-serine(O-methanesulfonvl) allvl ester}

A solution of $N$-triphenylmethyl- $(S)$-serine allyl ester $(S)-4(0.092 \mathrm{~g}, 0.23 \mathrm{mmol})$ in DCM $(3 \mathrm{ml})$ was treated with triethylamine $(0.10 \mathrm{ml}, 0.70 \mathrm{mmol}, 3$ equiv. $)$ and methanesulfonyl chloride $(0.04 \mathrm{ml}, 0.47 \mathrm{mmol}, 2$ equiv. $)$ at $0{ }^{\circ} \mathrm{C}$. The reaction mixture was stirred for $2 \mathrm{hr}$ at room temperature after which it was diluted with DCM $(10 \mathrm{ml})$. The mixture was washed with a saturated solution of ammonium chloride $(8 \mathrm{ml})$ and then with a saturated solution of sodium hydrogen carbonate $(2 \mathrm{x}$ $10 \mathrm{ml}$ ). The organic layer was dried over anhydrous sodium sulfate and removal of the solvent in vacuo gave a yellow-orange liquid. Flash column chromatography was used to purify the crude compound (hexane:ethyl acetate, $2: 1,1 \mathrm{~cm}$ width, $15 \mathrm{~cm}$ height) yielding a colourless liquid, which crystallised slowly. 1-D and 2-D ${ }^{1} \mathrm{H}$ NMR confirmed the correct compound, however a better procedure - described below was followed.

Yield: $0.09 \mathrm{~g}, 0.18 \mathrm{mmol}, 80 \%$

$\mathbf{R}_{\mathbf{f}}: 0.3$ (hexane:ethyl acetate, $2: 1$ )

\section{1. $\underline{\text { N-Triphenvlmethvl-(S)-serine(O-methanesulfonvl) allvl ester }}$}

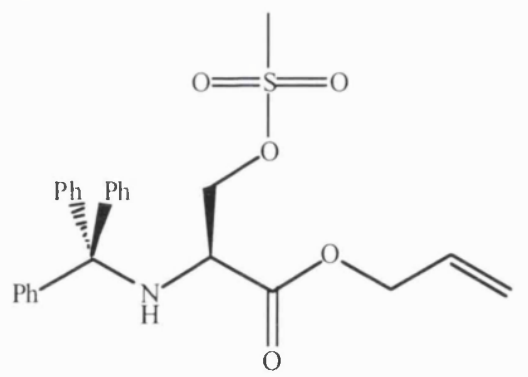

$(S)-5$

$N$-Triphenylmethyl-(S)-serine allyl ester $(S)-4(1.8 \mathrm{~g}, 4.5 \mathrm{mmol})$ was dissolved in dry THF $(22 \mathrm{ml})$ under argon and the solution was cooled to $0{ }^{\circ} \mathrm{C}$. Triethylamine $(0.66$ $\mathrm{ml}, 4.5 \mathrm{mmol})$ and methanesulfonyl chloride $(0.70 \mathrm{ml}, 9.0 \mathrm{mmol}, 2$ equiv.) were added, the ice bath removed and the mixture stirred for $4 \mathrm{hr}$. The reaction mixture was diluted with ether $(150 \mathrm{ml})$ and washed with ice-cold water $(5 \times 50 \mathrm{ml})$ and then brine $(3 \times 40 \mathrm{ml})$. The organic layer was dried over anhydrous sodium sulfate and removal of the solvent yielded a pale yellow liquid that crystallised slowly. This material was recrystallised from DCM and $\mathrm{MeOH}(1: 4)$ yielding white crystals. 
Yield: $1.9 \mathrm{~g}, 4.0 \mathrm{mmol}, 88 \%$

$\mathbf{R}_{\mathrm{f}}: 0.3$ (hexane:ethyl acetate, $2: 1$ )

M.p.: $115-118^{\circ} \mathrm{C}$ (dec.)

$[\alpha]_{D}{ }^{15}:+27.2(\mathrm{c} 1.11)$

I.R.: $v_{\max }\left(\mathrm{cm}^{-1}\right) 3018\left(\mathrm{CH}_{3}\right), 1736(\mathrm{C}=\mathrm{O}), 1544(\mathrm{C}=\mathrm{C}$ Ar$), 1346+1178\left(\mathrm{SO}_{2}-\mathrm{O}\right)$

Elemental analysis: Found $\mathrm{C}, 66.74 ; \mathrm{H}, 5.76 ; \mathrm{N}, 2.91 ; \mathrm{S}, 6.55 \% . \mathrm{C}_{26} \mathrm{H}_{27} \mathrm{NO}_{5} \mathrm{~S}$ requires $\mathrm{C}, 67.08 ; \mathrm{H}, 5.85 ; \mathrm{N}, 3.01 ; \mathrm{S}, 6.89 \%$.

NMR: $\boldsymbol{\delta}_{\mathrm{H}}(400 \mathrm{MHz}) 7.49(6 \mathrm{H}, \mathrm{m}, \mathrm{Trt}), 7.27(6 \mathrm{H}, \mathrm{m}, \mathrm{Trt}), 7.20$ (3H, m, Trt), 5.72 $\left(1 \mathrm{H}, \mathrm{m}, \mathrm{CH}=\mathrm{CH}_{2}\right), 5.21\left(2 \mathrm{H}, \mathrm{m}, \mathrm{CH}=\mathrm{CH}_{2}\right), 4.46\left(1 \mathrm{H}, \mathrm{dd}, J 10.1,4.2, \mathrm{CHCH}_{2} \mathrm{OMs}\right)$, $4.28\left(1 \mathrm{H}, \mathrm{dd}, J 10.1,6.1, \mathrm{CHCH}_{2} \mathrm{OMs}\right), 4.25$ (1H, dd, $\left.J 13.0,5.9, \mathrm{OCH}_{2} \mathrm{CH}\right), 4.08$ $\left(1 \mathrm{H}, \mathrm{dd}, J 13.0,5.9, \mathrm{OCH}_{2} \mathrm{CH}\right), 3.73\left(1 \mathrm{H}, \mathrm{m}, \mathrm{HNCHCO}_{2}\right), 2.97\left(3 \mathrm{H}, \mathrm{s}, \mathrm{OS}(\mathrm{O})_{2} \mathrm{CH}_{3}\right)$; $\delta_{\mathrm{C}}(75.4 \mathrm{MHz}$, DEPT 135) 171.3, 145.3, 131.4, 128.6, 128.1, 126.7, 118.8, 71.1, 70.7, $66.1,55.7,37.7$

Mass spectrum: $m / z(F A B) 466.1688\left([\mathrm{M}+\mathrm{H}]^{+} . \mathrm{C}_{26} \mathrm{H}_{28} \mathrm{NO}_{5} \mathrm{~S}\right.$ requires 466.1664), $488\left([\mathrm{M}+\mathrm{Na}]^{+}, 0.4 \%\right), 243\left(\mathrm{Trt}^{+}, 100 \%\right), 224\left([\mathrm{M}-\mathrm{Trt}+2]^{+}, 0.2 \%\right)$

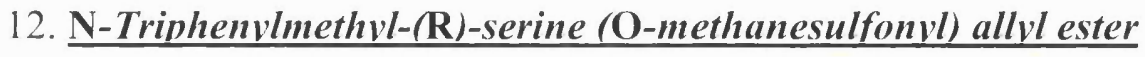

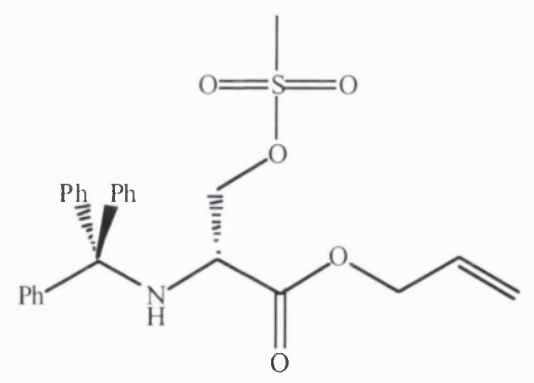

$(R)-5$

$N$-Triphenylmethyl-(R)-serine allyl ester $(\boldsymbol{R})-4(19 \mathrm{~g}, 49 \mathrm{mmol})$ was dissolved in dry THF $(200 \mathrm{ml})$ under argon and the solution was cooled to $0{ }^{\circ} \mathrm{C}$. Triethylamine $(7.0$ $\mathrm{ml}, 49 \mathrm{mmol})$ and methanesulfonyl chloride $(8.3 \mathrm{ml}, 98 \mathrm{mmol}, 2$ equiv.) were added, the ice bath removed and the mixture stirred for $4 \mathrm{hr}$. The reaction mixture was diluted with ether $(400 \mathrm{ml})$ and washed with ice-cold water $(6 \times 100 \mathrm{ml})$ and then brine $(4 \times 100 \mathrm{ml})$. The organic layer was dried over anhydrous sodium sulfate and removal of the solvent yielded a pale yellow liquid. Recrystallisation of the crude product was carried out in DCM and MeOH (1:4) yielding white crystals.

Yield: $21 \mathrm{~g}, 44 \mathrm{mmol}, 91 \%$ 
$\mathbf{R}_{\mathbf{f}}: 0.3$ (hexane:ethyl acetate, $2: 1$ )

M.p.: $116-118^{\circ} \mathrm{C}$ (dec.)

$[\alpha]_{\mathrm{D}}^{20}:-32.7(\mathrm{c} 0.46)$

I.R.: $v_{\max }\left(\mathrm{cm}^{-1}\right) 1736(\mathrm{C}=\mathrm{O}), 1489(\mathrm{C}=\mathrm{C} \mathrm{Ar}), 1346+1177\left(\mathrm{SO}_{2}-\mathrm{O}\right)$

NMR: $\boldsymbol{\delta}_{\mathbf{H}}(400 \mathrm{MHz}) 7.50(6 \mathrm{H}, \mathrm{m}, \mathrm{Trt}), 7.29(6 \mathrm{H}, \mathrm{m}, \mathrm{Trt}), 7.21(3 \mathrm{H}, \mathrm{m}, \mathrm{Trt}), 5.70$

$\left(1 \mathrm{H}, \mathrm{m}, \mathrm{CH}=\mathrm{CH}_{2}\right), 5.20\left(2 \mathrm{H}, \mathrm{m}, \mathrm{CH}=\mathrm{CH}_{2}\right), 4.45\left(1 \mathrm{H}, \mathrm{dd}, J 10.0,4.2, \mathrm{CHCH}_{2} \mathrm{OMs}\right)$, 4.25 (1 H, dd, $J 10.0,6.1, \mathrm{CHCH}_{2} \mathrm{OMs}$ ), 4.21 ( $\left.1 \mathrm{H}, \mathrm{dd}, J 13.1,6.0, \mathrm{OCH}_{2} \mathrm{CH}\right), 4.05$ $\left(1 \mathrm{H}, \mathrm{dd}, J 13.1,5.9, \mathrm{OCH}_{2} \mathrm{CH}\right), 3.69(1 \mathrm{H}, \mathrm{m}, \mathrm{HNCHCO} 2), 2.99\left(3 \mathrm{H}, \mathrm{s}, \mathrm{OS}(\mathrm{O})_{2} \mathrm{CH}_{3}\right)$; $\delta_{\mathrm{C}}(75.4 \mathrm{MHz}$, DEPT135) 171.3, 145.3, 131.5, 128.6, 128.1, 126.7, 118.8, 71.2, 70.7, $66.1,55.8,37.7$

Mass spectrum: $m / z(F A B) 488.1508\left([\mathrm{M}+\mathrm{Na}]^{+} \cdot \mathrm{C}_{26} \mathrm{H}_{27} \mathrm{NO}_{5} \mathrm{SNa}\right.$ requires $488.1520), 488\left([\mathrm{M}+\mathrm{Na}]^{+}, 1 \%\right), 243\left(\operatorname{Trt}^{+}, 100 \%\right)$

\section{3. $\mathbf{N}$-Triphenv/methvl-B-iodo-(R)-alanine allvl ester}

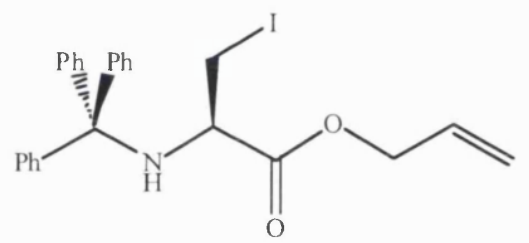

(R)-6a

A solution of $N$-triphenylmethyl-( $S)$-serine( $O$-methanesulfonyl) allyl ester $(\boldsymbol{S})-5$ (1.1 g, $2.5 \mathrm{mmol})$ in acetone $(6 \mathrm{ml})$ was added to a solution of sodium iodide $(4.0 \mathrm{~g}, 29$ mmol, 11 equiv.) in dry acetone $(6 \mathrm{ml})$ under argon. The solution was stirred for 96 hr at $25^{\circ} \mathrm{C}$, and the resulting yellow-brown slurry was then concentrated in vacuo. Ether $(100 \mathrm{ml})$ then sodium thiosulfate (10\% aq. w/v, $4 \mathrm{ml}$ approx.) was then slowly added, dissolving the insoluble solids and substantially decolourising the organic layer to a pale yellow solution. The organic layer was separated and dried over anhydrous sodium sulfate and the solvent was removed in vacuo. The crude product was separated from the starting material (flash column chromatography, hexane:ethyl acetate, 2:1, $4 \mathrm{~cm}$ width) giving a pale yellow liquid. Further purification by precipitation (DCM:MeOH, 1:4) afforded the desired product, along with the regioisomeric by-product, $(R / S)$-2-iodo-3-(triphenylmethylamino)-propionic acid allyl ester, in 1:4 proportion as an inseparable mixture. 
Yield: $1.02 \mathrm{~g}, 2.0 \mathrm{mmol}, 82 \%$ (overall); $0.20 \mathrm{~g}, 0.41 \mathrm{mmol}, 16 \%$ (desired product, based on NMR)

$\mathbf{R}_{\mathbf{f}}: 0.60$ (hexane:ethyl acetate. $2: 1$ )

I.R.: $v_{\max }\left(\mathrm{cm}^{-1}\right) 1730(\mathrm{C}=\mathrm{O}), 1489(\mathrm{C}=\mathrm{C}$ Ar $)$. Absence of signals at $1346\left(\mathrm{SO}_{2}-\mathrm{O}\right)$ and $1177\left(\mathrm{SO}_{2}-\mathrm{O}\right) \mathrm{cm}^{-1}$

Elemental analysis: Found $\mathrm{C}, 60.15 ; \mathrm{H}, 4.77 ; \mathrm{N}, 2.72 ; \mathrm{I}, 25.59 \%$ on mixture. $\mathrm{C}_{25} \mathrm{H}_{24} \mathrm{NO}_{2} \mathrm{I}$ requires $\mathrm{C}, 60.37 ; \mathrm{H}, 4.86 ; \mathrm{N}, 2.82 ; \mathrm{I}, 25.51 \%$.

NMR: $\delta_{\mathbf{H}}(400 \mathrm{MHz}) 7.47(6 \mathrm{H}, \mathrm{m}, \mathrm{Trt}), 7.30$ (6H, m, Trt), 7.21 (3H, m, Trt), 5.74 $\left(1 \mathrm{H}, \mathrm{m}, \mathrm{CH}=\mathrm{CH}_{2}\right), 5.24\left(1 \mathrm{H}, \mathrm{m}, \mathrm{CH}=\mathrm{CH}_{2}\right), 5.21\left(1 \mathrm{H}, \mathrm{m}, \mathrm{CH}=\mathrm{CH}_{2}\right), 4.25(1 \mathrm{H}, \mathrm{dd}, J$ $\left.13.0,5.8, \mathrm{OCH}_{2} \mathrm{CH}\right), 4.10\left(1 \mathrm{H}, \mathrm{dd}, J 13.0,5.7, \mathrm{OCH}_{2} \mathrm{CH}\right), 3.52\left(1 \mathrm{H}, \mathrm{m}, \mathrm{HNCHCO}_{2}\right)$, $3.33\left(1 \mathrm{H}, \mathrm{dd}, J 9.7,3.1, \mathrm{CHCH}_{2} \mathrm{I}\right), 3.22\left(1 \mathrm{H}, \mathrm{dd}, J 9.5,7.0, \mathrm{CHCH}_{2} \mathrm{I}\right), 2.91(1 \mathrm{H}, \mathrm{d}, J$ 9.6, $\operatorname{Tr} \mathrm{N} H \mathrm{CH}) ; \delta_{\mathrm{C}}(75.4 \mathrm{MHz}, \mathrm{DEPT} 135)$ 172.0, 145.6, 131.2, 128.6, 128.0, 126.5, $118.8,70.8,66.0,56.4,9.7$

(R/S)-2-iodo-3-(triphenylmethylamino)-propionic acid allyl ester (mixture of enantiomers): $\delta_{\mathbf{H}}(400 \mathrm{MHz}) 7.52(6 \mathrm{H}, \mathrm{m}, \mathrm{Trt}), 7.30(6 \mathrm{H}, \mathrm{m}, \mathrm{Trt}), 7.21(3 \mathrm{H}, \mathrm{m}, \mathrm{Trt})$, $5.94\left(1 \mathrm{H}, \mathrm{m}, \mathrm{CH}=\mathrm{CH}_{2}\right), 5.40\left(1 \mathrm{H}, \mathrm{dd}, J 17.2,1.4, \mathrm{CH}=\mathrm{CH}_{2}\right), 5.30(1 \mathrm{H}, \mathrm{dd}, J 10.5$, 1.1, $\left.\mathrm{CH}=\mathrm{CH}_{2}\right), 4.66\left(2 \mathrm{H}, \mathrm{d}, J 5.7, \mathrm{OCH}_{2} \mathrm{CH}\right), 4.42(1 \mathrm{H}, \mathrm{m}, \mathrm{CHICO}), 2.74(1 \mathrm{H}, \mathrm{dd}, J$ 12.8, 8.6, $\left.\mathrm{HNCH}_{2} \mathrm{CHI}\right), 2.57$ ( $\left.1 \mathrm{H}, \mathrm{dd}, J 12.9,5.8, \mathrm{HNCH}_{2} \mathrm{CHI}\right), 2.27\left(\mathrm{TrtN} H \mathrm{CH}_{2}\right)$; $\boldsymbol{\delta}_{\mathrm{C}}(75.4 \mathrm{MHz}, \mathrm{DEPT} 135)$ 170.4, 145.4, 131.1, 128.3, 128.0, 126.5, 118.8, 70.8, 66.1, $48.2,20.1$

Mass spectrum: $m / z(\mathrm{FAB}) 498.0930\left([\mathrm{M}+\mathrm{H}]^{+} . \mathrm{C}_{25} \mathrm{H}_{25} \mathrm{NO}_{2} \mathrm{I}\right.$ requires 498.0954), $520\left([\mathrm{M}+\mathrm{Na}]^{+}, 3.7 \%\right), 498\left([\mathrm{M}+\mathrm{H}]^{+}, 2.7 \%\right), 497\left(\mathrm{M}^{+}, 0.4 \%\right) ; \mathrm{m} / z(\mathrm{APCI}+) 520$ $\left([\mathrm{M}+\mathrm{Na}]^{+},<0.1 \%\right), 243\left(\mathrm{Trt}^{+}, 100 \%\right), 256\left([\mathrm{M}-\mathrm{Trt}+2]^{+}, 6 \%\right)$

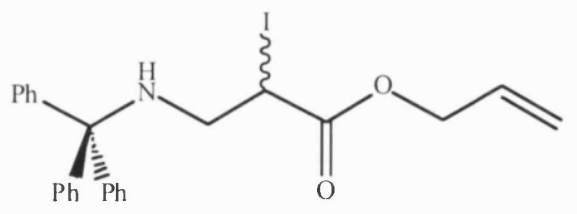

6b

Figure 7.1. Major product: $(R / S)$-2-iodo-3-(triphenylmethylamino)-propionic acid allyl ester 


\section{N-Triphenvlmethvl-B-iodo-(S)-alanine allvl ester}

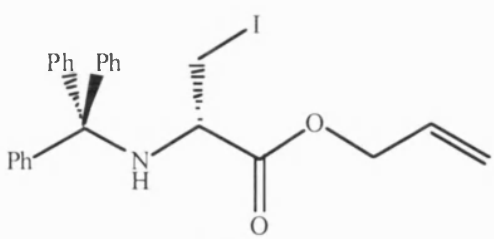

$(S)-6 a$

A solution of $N$-triphenylmethyl-( $R)$-serine( $O$-methanesulfonyl) allyl ester $(\boldsymbol{R})-5$ (22 $\mathrm{g}, 46 \mathrm{mmol})$ in acetone $(80 \mathrm{ml})$ was added to a solution of sodium iodide ( $70 \mathrm{~g}, 0.46$ mol, 10 equiv.) in dry acetone ( $100 \mathrm{ml})$ under argon. The mixture was stirred for 72 hr at $25{ }^{\circ} \mathrm{C}$ and the resulting yellow-brown slurry was then concentrated in vacuo. Ether $(400 \mathrm{ml})$ was added and sodium thiosulfate ( $10 \%$ aq. w/v, $25 \mathrm{ml}$ approx.) was then slowly added, dissolving the insoluble solids and substantially decolourising the organic layer to a pale yellow solution. The organic layer was separated and dried over anhydrous sodium sulfate and the solvent was removed in vacuo. The crude product was purified (flash column chromatography, hexane:ethyl acetate, 2:1, $5 \mathrm{~cm}$ width) to remove a small amount of starting material, giving a pale yellow liquid. Further purification by precipitation (DCM:MeOH, 1:5) afforded the desired product, along with the regioisomeric by-product, $(R / S)$-2-iodo-3(triphenylmethylamino)-propionic acid allyl ester in a ratio of 1:5. These two products were not separable by HPLC, however, incomplete separation of the two species was attempted using preparative normal phase HPLC ( 2 - $3 \%$ ethyl acetate in hexane over 10 minutes, $15 \mathrm{ml} / \mathrm{min}$ with $\mathrm{DCM}$ loading, $\mathrm{R}_{\mathrm{T}} 12.1,12.8$ (regioisomer)).

Yield: $19.9 \mathrm{~g}, 40 \mathrm{mmol}, 87 \%$ (overall); $3.3 \mathrm{~g}, 6.7 \mathrm{mmol}, 15 \%$ (desired product, based on NMR)

$\mathbf{R}_{\mathbf{f}}: 0.60$ (hexane:ethyl acetate. $2: 1$ )

I.R.: $v_{\max }\left(\mathrm{cm}^{-1}\right) 1730(\mathrm{C}=\mathrm{O}), 1489(\mathrm{C}=\mathrm{C}$ Ar $)$. Absence of signals at $1346\left(\mathrm{SO}_{2}-\mathrm{O}\right)$ and $1177\left(\mathrm{SO}_{2}-\mathrm{O}\right) \mathrm{cm}^{-1}$

NMR: $\delta_{\mathbf{H}}(600 \mathrm{MHz}) 7.51(6 \mathrm{H}, \mathrm{m}, \mathrm{Trt}), 7.29(6 \mathrm{H}, \mathrm{m}, \mathrm{Trt}), 7.20(3 \mathrm{H}, \mathrm{m}, \mathrm{Trt}), 5.73$

$\left(1 \mathrm{H}, \mathrm{m}, \mathrm{CH}=\mathrm{CH}_{2}\right), 5.22\left(1 \mathrm{H}, \mathrm{dd}, J 17.3,1.4, \mathrm{CH}=\mathrm{CH}_{2}\right), 5.19(1 \mathrm{H}, \mathrm{dd}, J 10.2,1.1$, $\left.\mathrm{CH}=\mathrm{CH}_{2}\right), 4.22\left(1 \mathrm{H}, \mathrm{dd}, J 13.1,6.1, \mathrm{OCH}_{2} \mathrm{CH}\right), 4.09\left(1 \mathrm{H}, \mathrm{dd}, J 13.1,6.1, \mathrm{OCH}_{2} \mathrm{CH}\right)$, $3.50(1 \mathrm{H}, \mathrm{m}, \mathrm{HNCHCO} 2), 3.30\left(1 \mathrm{H}, \mathrm{dd}, J 9.8,3.4, \mathrm{CHCH}_{2} \mathrm{I}\right), 3.23(1 \mathrm{H}, \mathrm{dd}, J 9.8$, 
6.9, $\left.\mathrm{CHCH}_{2} \mathrm{I}\right), 2.88(1 \mathrm{H}, \mathrm{d}, J$ 9.8, TrtNHCH$) ; \boldsymbol{\delta}_{\mathrm{C}}(150.8 \mathrm{MHz}$, DEPT 135, HSQC, HМBC) $171.9,145.6,131.3,128.4,127.9,126.4,118.6,70.9,65.8,55.8,9.55$

(R/S)-2-iodo-3-(triphenylmethylamino)-propionic acid allyl ester (mixture of enantiomers): $\delta_{\mathrm{H}}(600 \mathrm{MHz}) 7.52(6 \mathrm{H}, \mathrm{m}, \mathrm{Trt}), 7.30(6 \mathrm{H}, \mathrm{m}, \mathrm{Trt}), 7.21(3 \mathrm{H}, \mathrm{m}, \mathrm{Trt})$, $5.94\left(1 \mathrm{H}, \mathrm{m}, \mathrm{CH}=\mathrm{CH}_{2}\right), 5.40\left(1 \mathrm{H}, \mathrm{dd}, J 17.2,1.4, \mathrm{CH}=\mathrm{CH}_{2}\right), 5.30(1 \mathrm{H}, \mathrm{dd}, J 10.5$, 1.1, $\left.\mathrm{CH}=\mathrm{CH}_{2}\right), 4.67\left(2 \mathrm{H}, \mathrm{m}, \mathrm{OCH}_{2} \mathrm{CH}\right), 4.42\left(1 \mathrm{H}, \mathrm{m}, \mathrm{CHICO}_{2}\right), 2.73(1 \mathrm{H}, \mathrm{dd}, J$ 12.9, 8.5, $\left.\mathrm{HNCH}_{2} \mathrm{CHI}\right), 2.57\left(1 \mathrm{H}, \mathrm{dd}, J 12.9,5.9, \mathrm{HNCH}_{2} \mathrm{CHI}\right), 2.27\left(\operatorname{TrtN} \mathrm{HCH}_{2}\right) ; \boldsymbol{\delta}_{\mathrm{C}}$ (150.8 MHz, DEPT135, HSQC, HMBC) 170.4, 145.4, 131.1, 128.3, 128.0, 126.5, $118.8,70.8,66.1,48.2,20.1$

Mass spectrum: $m / z$ (FAB) $520.0750\left([\mathrm{M}+\mathrm{Na}]^{+} \cdot \mathrm{C}_{25} \mathrm{H}_{24} \mathrm{NO}_{2} \mathrm{INa}\right.$ requires $520.0732), 520\left([\mathrm{M}+\mathrm{Na}]^{+}, 0.2 \%\right), 243\left(\mathrm{Trt}^{+}, 100 \%\right) ; \mathrm{m} / z(\mathrm{APCI}+) 498\left([\mathrm{M}+\mathrm{H}]^{+}\right.$, $2 \%), 243\left(\mathrm{Trt}^{+}, 100 \%\right), 256\left([\mathrm{M}-\mathrm{Trt}+2]^{+}, 2 \%\right)$<smiles>C=CCOC(=O)C(I)CNC(c1ccccc1)c1ccccc1</smiles>

6b

Figure 7.2. Major product: $(R / S)$-2-iodo-3-(triphenylmethylamino)-propionic acid allyl ester

15. 3-I(R)-2-'Butoxvcarbonvl-2-(fluoren-9-vlmethoxvcarbonvlamino)ethvlsulfanvll-(R)-2-(triphenvlmethylamino)-propionic acid allvl ester

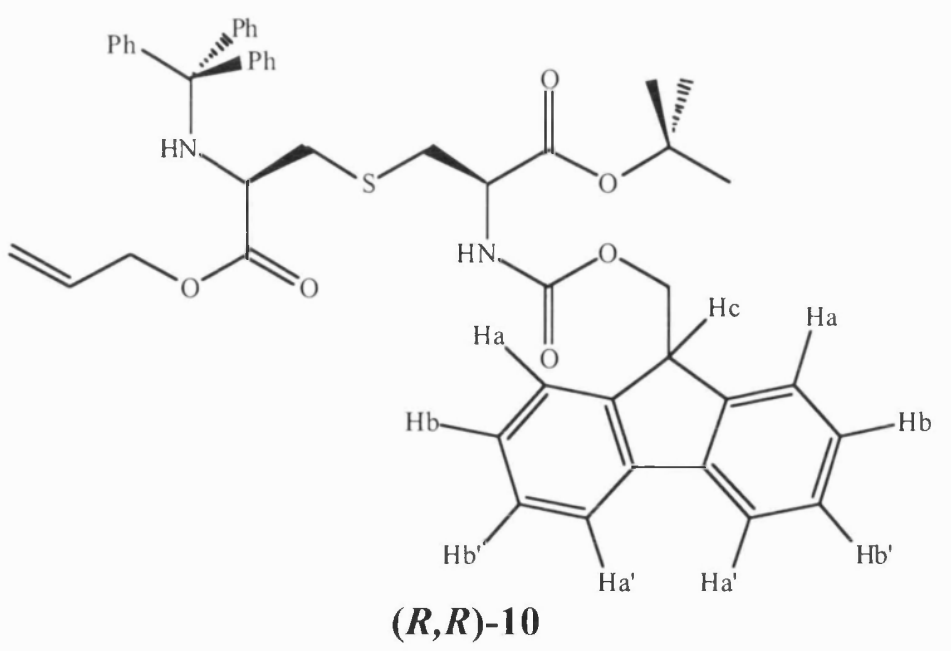

$N$-9-Fluorenylmethoxycarbonyl-( $R)$-cysteine tbutyl ester $9(0.29 \mathrm{~g}, 0.73 \mathrm{mmol})$ was dissolved in DMF $(3.2 \mathrm{ml})$ under inert conditions. To it a solution of $N$ - 
triphenylmethyl-3-iodo- $(R)$-alanine allyl ester $(\boldsymbol{R})-\mathbf{6 a}$ (mixture with regioisomer, $0.41 \mathrm{~g}, 0.83 \mathrm{mmol}, 1.1$ equiv.) in DMF $(3.2 \mathrm{ml})$ was added. Cesium carbonate $(0.24$ $\mathrm{g}, 0.73 \mathrm{mmol}$ ) was then added and the mixture was stirred for $4 \mathrm{hr}$ at $25^{\circ} \mathrm{C}$. The solvent was removed in vacuo and the residue was partly dissolved in ethyl acetate $(75 \mathrm{ml})$. The organic layer was washed with citric acid (5\% aq. w/v, $25 \mathrm{ml})$, dissolving the remainder of the residue. The organic layer was then washed with water $(10 \times 30 \mathrm{ml})$ and dried over anhydrous sodium sulfate. Removal of the solvent in vacuo yielded a pale yellow oil which was purified via flash column chromatography (hexane:ethyl acetate, $4: 1,5.5 \mathrm{~cm}$ width) and produced the desired product along with its regioisomer in diastereoisomeric mixture $(1: 2$ product:regioisomers), as a very pale yellow oil which foamed and solidified under highly reduced pressure.

Yield: $0.44 \mathrm{~g}, 0.57 \mathrm{mmol}, 78 \%$ (overall); $0.15 \mathrm{~g}, 0.19 \mathrm{mmol}, 26 \%$ (desired product, based on NMR)

$\mathbf{R}_{\mathbf{f}}: 0.28$ (hexane:ethyl acetate, $4: 1$ )

I.R.: $v_{\max }\left(\mathrm{cm}^{-1}\right) 1724(\mathrm{C}=\mathrm{O}), 1506(\mathrm{C}=\mathrm{C}$ Ar $), 1450(\mathrm{C}=\mathrm{C}$ Ar), $1215+1155(\mathrm{C}-\mathrm{S}-\mathrm{C})$

Found: $\mathrm{C}, 72.92 ; \mathrm{H}, 6.45 ; \mathrm{N}, 3.60 ; \mathrm{S}, 3.98 \% . \mathrm{C}_{47} \mathrm{H}_{48} \mathrm{~N}_{2} \mathrm{O}_{6} \mathrm{~S}$ requires $\mathrm{C}, 73.41 ; \mathrm{H}$, $6.29 ; \mathrm{N}, 3.64 ; \mathrm{S}, 4.17 \%$.

NMR: $\delta_{\mathbf{H}}(400 \mathrm{MHz}) 7.73\left(2 \mathrm{H}, \mathrm{m}, H_{\mathrm{a}^{\prime}}\right), 7.64\left(2 \mathrm{H}, \mathrm{m}, H_{\mathrm{a}}\right), 7.53(6 \mathrm{H}, \mathrm{m}, \mathrm{Trt}), 7.36$ $\left(2 \mathrm{H}, \mathrm{m}, H_{\mathrm{b}}\right), 7.20-7.31\left(2 \mathrm{H}, \mathrm{m}, H_{\mathrm{b}}+6 \mathrm{H}, \mathrm{m}, \mathrm{Trt}\right), 7.16(3 \mathrm{H}, \mathrm{m}, \mathrm{Fmoc}(\mathrm{Ar})), 5.68$ $\left(1 \mathrm{H}, \mathrm{m}, \mathrm{CH}=\mathrm{CH}_{2}\right), 5.62(1 \mathrm{H}, \mathrm{bd}, \mathrm{FmocN} H), 5.22\left(1 \mathrm{H}, \mathrm{m}, \mathrm{CH}=\mathrm{CH}_{2}\right), 5.13(1 \mathrm{H}, \mathrm{m}$, $\left.\mathrm{CH}=\mathrm{CH}_{2}\right), 4.47(1 \mathrm{H}, \mathrm{m}, \mathrm{FmocNHCH}), 4.35\left(2 \mathrm{H}, \mathrm{m}, \mathrm{CH}_{2} \mathrm{OCONH}\right), 4.20\left(1 \mathrm{H}, \mathrm{m}, H_{\mathrm{c}}\right)$, $4.05\left(2 \mathrm{H}, \mathrm{m}, \mathrm{OCH}_{2} \mathrm{CH}=\mathrm{CH}_{2}\right), 3.58(1 \mathrm{H}, \mathrm{m}, \mathrm{TrtNHCH}), 2.98\left(2 \mathrm{H}, \mathrm{m}, \mathrm{CHCH}_{2} \mathrm{~S}\right), 2.72$ $-2.90\left(2 \mathrm{H}, \mathrm{m}, \operatorname{TrtNHCHCH}{ }_{2}\right), 2.20(1 \mathrm{H}, \mathrm{bs}, \operatorname{TrtNH}), 1.49\left(9 \mathrm{H}, \mathrm{s}, \mathrm{C}\left(\mathrm{CH}_{3}\right)_{3}\right) ; \boldsymbol{\delta}_{\mathrm{C}}(75.4$ MHz, DEPT135) 173.4, ca. 170, 156.1, ca. 146, 144.1, 141.6, ca. 132, ca. 129, ca. $128,128.1,127.5$, ca. 127, 125.5, 120.3, ca. 119, ca. 83, ca. 71, 67.6, ca. 66, 56.9, ca. $54,47.5,38.6,36.3,28.3$

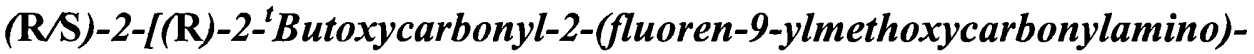

ethylsulfanyl]-3-(triphenylmethylamino)-propionic acid allyl ester (diastereoisomeric mixture): $\delta_{\mathrm{H}}(400 \mathrm{MHz}) 7.73\left(2 \mathrm{H}, \mathrm{m}, H_{\mathrm{a}^{\prime}}\right), 7.56\left(2 \mathrm{H}, \mathrm{m}, H_{\mathrm{a}}\right), 7.43$ $(6 \mathrm{H}, \mathrm{m}, \mathrm{Trt}), 7.36\left(2 \mathrm{H}, \mathrm{m}, H_{\mathrm{b}^{\prime}}\right), 7.20-7.31\left(2 \mathrm{H}, \mathrm{m}, H_{\mathrm{b}}+6 \mathrm{H}, \mathrm{m}, \mathrm{Trt}\right), 7.16(3 \mathrm{H}, \mathrm{m}$, Fmoc (Ar)), $5.91\left(1 \mathrm{H}, \mathrm{m}, \mathrm{CH}=\mathrm{CH}_{2}\right), 5.58+5.69(1 \mathrm{H}, \mathrm{bd}, \mathrm{FmocN} H), 5.33(1 \mathrm{H}, \mathrm{m}$, 
$\left.\mathrm{CH}=\mathrm{CH}_{2}\right), 5.23\left(1 \mathrm{H}, \mathrm{m}, \mathrm{CH}=\mathrm{CH}_{2}\right), 4.66\left(2 \mathrm{H}, \mathrm{m}, \mathrm{OCH}_{2} \mathrm{CH}=\mathrm{CH}_{2}\right), 4.50(1 \mathrm{H}, \mathrm{m}$, FmocNHCH $), 4.29-4.41\left(2 \mathrm{H}, \mathrm{m}, \mathrm{CH}_{2} \mathrm{OCONH}\right), 4.18-4.25\left(1 \mathrm{H}, \mathrm{m}, H_{\mathrm{c}}\right), 3.49+$ $3.56\left(1 \mathrm{H}, \mathrm{t}, J 6.4+6.7, \mathrm{TrtNHCH}_{2} \mathrm{CH}\right), 2.81-3.12\left(2 \mathrm{H}, \mathrm{m}, \mathrm{CHCH}_{2} \mathrm{~S}\right), 2.61(1 \mathrm{H}, \mathrm{m}$, $\left.\operatorname{TrtNHCH} H_{2}\right), 2.45\left(1 \mathrm{H}, \mathrm{m}, \operatorname{TrtNHCH} H_{2}\right), 1.48-1.49\left(9 \mathrm{H}, \mathrm{s}, \mathrm{C}\left(\mathrm{CH}_{3}\right)_{3}\right) ; \delta_{\mathrm{C}}(75.4 \mathrm{MHz}$, DEPT135) 171.8, ca. 169, 156.1, ca. 146, 144.1, 141.6, ca. 132, ca. 129, ca. 128, $128.1,127.5, c a .127,125.5,120.3, c a .119, c a .83, c a .71,67.6, c a .66, c a .54,(48.8$ $+48.0), 47.5,(44.9+44.6),(34.8+34.3), 28.3$

Mass spectrum: $m / z(F A B) 769.3311\left([\mathrm{M}+\mathrm{H}]^{+} . \mathrm{C}_{47} \mathrm{H}_{49} \mathrm{~N}_{2} \mathrm{O}_{6} \mathrm{~S}\right.$ requires 769.3326), $769\left(\mathrm{M}^{+}, 4.8 \%\right), 527\left([\mathrm{M}-\mathrm{Trt}+1]^{+}, 1.9 \%\right), 471\left(\left[\mathrm{M}-\mathrm{Trt}-{ }^{t} \mathrm{Bu}+2\right]^{+}, 0.2 \%\right), 243$ $\left(\mathrm{Trt}^{+}, 100 \%\right), 208\left(\left[\mathrm{M}-\mathrm{Trt}-\mathrm{Fmoc}-{ }^{\mathrm{t}} \mathrm{Bu}-\mathrm{All}+3\right]^{+}, 0.3 \%\right)$

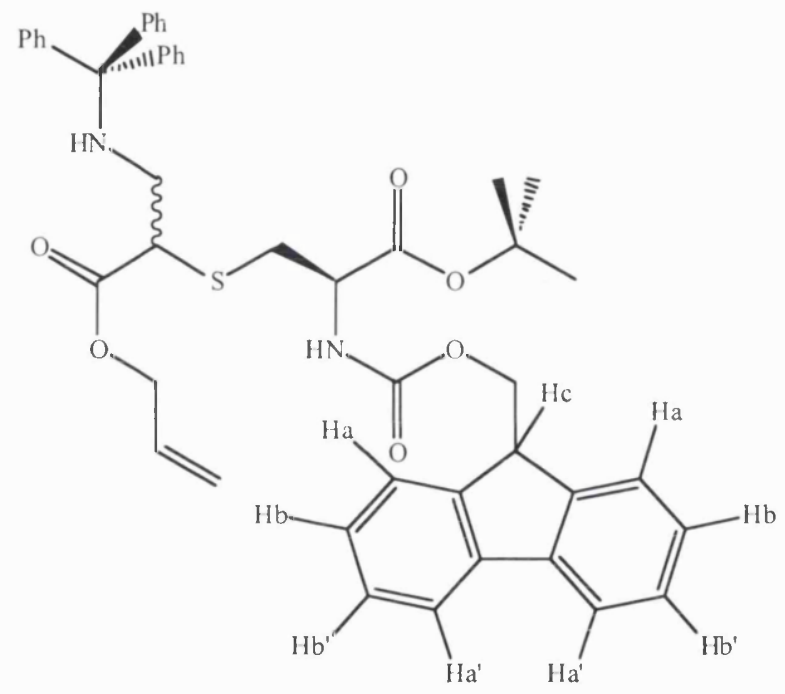

18

Figure 7.3. Major product: (R/S)-2-[(R)-2-'butoxycarbonyl-2-(fluoren-9ylmethoxycarbonylamino)-ethylsulfanyl]-3-(triphenylmethylamino)-propionic acid allyl ester 


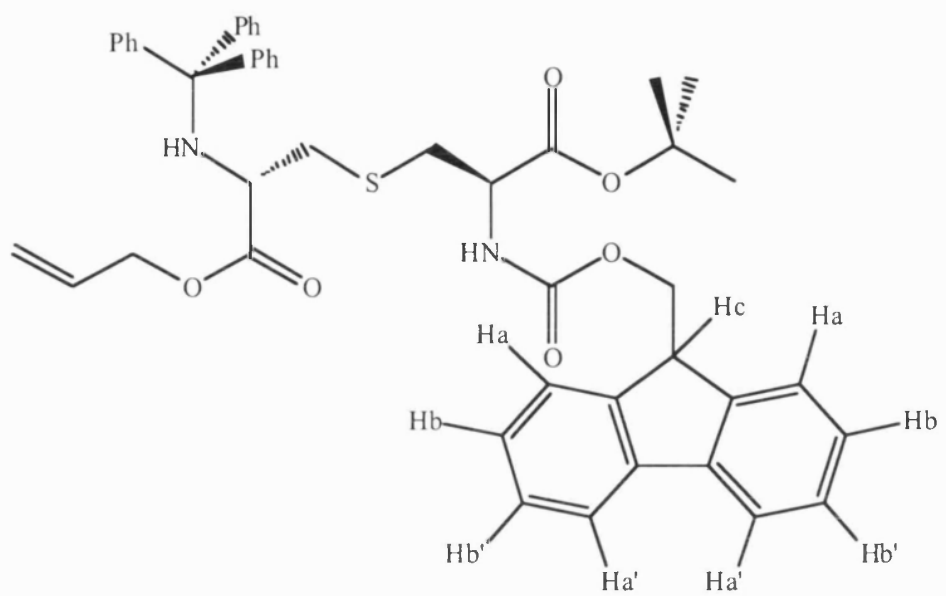

$(S, R)-10$

$N$-9-Fluorenylmethoxycarbonyl- $(R)$-cysteine tbutyl ester 9 (5.6 g, $14 \mathrm{mmol})$ was dissolved in dry DMF $(75 \mathrm{ml})$ under inert conditions. To it a solution of $\mathrm{N}$ triphenylmethyl-3-iodo-( $S$ )-alanine allyl ester $(\boldsymbol{S})$-6a (mixture with regioisomer, 7.7 g, 16 mmol, 1.1 equiv) in DMF ( $75 \mathrm{ml}$ ) was added. Cesium carbonate $(4.6 \mathrm{~g}, 14$ mmol) was then added and the mixture was stirred for $4 \mathrm{hr}$ at $25{ }^{\circ} \mathrm{C}$. The solvent was removed in vacuo and the residue was partly dissolved in ethyl acetate $(250 \mathrm{ml})$. The organic layer was washed with citric acid $(5 \%$ aq. w/v, $100 \mathrm{ml})$, dissolving the remainder of the residue. The organic layer was then washed with water $(8 \times 100 \mathrm{ml})$ and dried over anhydrous sodium sulfate. Removal of the solvent in vacuo yielded a pale yellow oil which was purified via flash column chromatography (hexane:ethyl acetate, 4:1, $9 \mathrm{~cm}$ width) produced the desired product along with its regioisomer in diastereoisomeric mixture (1:5 product:regioisomers), as a very pale yellow oil which foamed and solidified under highly reduced pressure.

Yield: $9.7 \mathrm{~g}, 12.6 \mathrm{mmol}, 90 \%$ (overall); $1.6 \mathrm{~g}, 2.1 \mathrm{mmol}, 15 \%$ (desired product, based on NMR)

$\mathbf{R}_{\mathbf{f}}: 0.28$ (hexane:ethyl acetate, $4: 1$ )

I.R.: $v_{\max }\left(\mathrm{cm}^{-1}\right) 1724(\mathrm{C}=\mathrm{O}), 1504(\mathrm{C}=\mathrm{C}$ Ar), $1450(\mathrm{C}=\mathrm{C}$ Ar), $1215+1153(\mathrm{C}-\mathrm{S}-\mathrm{C})$ NMR: $\boldsymbol{\delta}_{\mathbf{H}}(500 \mathrm{MHz}) 7.74(2 \mathrm{H}, \mathrm{m}, \mathrm{Fmoc}(\mathrm{Ar})), 7.60(2 \mathrm{H}, \mathrm{m}, \mathrm{Fmoc}(\mathrm{Ar})), 7.49$ (6H, m, Trt), 7.38 (2H, m, Fmoc (Ar)), $7.20-7.31$ (2H, m, Fmoc (Ar) +6H, m, Trt), 7.16 $(3 \mathrm{H}, \mathrm{m}, \mathrm{Fmoc}(\mathrm{Ar})), 5.69\left(1 \mathrm{H}, \mathrm{m}, \mathrm{CH}=\mathrm{CH}_{2}\right), 5.63(1 \mathrm{H}, \mathrm{bd}, \mathrm{FmocN} H), 5.18(1 \mathrm{H}, \mathrm{m}$, $\left.\mathrm{CH}=\mathrm{CH}_{2}\right), 5.13\left(1 \mathrm{H}, \mathrm{m}, \mathrm{CH}=\mathrm{CH}_{2}\right), 4.49(1 \mathrm{H}, \mathrm{m}, \mathrm{FmocNHCH}), 4.34(2 \mathrm{H}, \mathrm{m}$, 
$\left.\mathrm{CH}_{2} \mathrm{OCONH}\right), 4.18\left(1 \mathrm{H}, \mathrm{m}, \mathrm{CHCH} \mathrm{CHCNH}_{2} \mathrm{O}, 4.04\left(2 \mathrm{H}, \mathrm{m}, \mathrm{OCH}_{2} \mathrm{CH}=\mathrm{CH}_{2}\right), 3.56\right.$ $(1 \mathrm{H}, \mathrm{m}, \operatorname{TrtNHCH}), 2.89-3.03\left(2 \mathrm{H}, \mathrm{m}, \mathrm{CHCH}_{2} \mathrm{~S}\right), 2.70-2.89(2 \mathrm{H}, \mathrm{m}$, TrtNHCHCH $\left.H_{2}\right), 2.20(1 \mathrm{H}$, bs, $\operatorname{TrtN} H), 1.50\left(9 \mathrm{H}, \mathrm{s}, \mathrm{C}\left(\mathrm{CH}_{3}\right)_{3}\right) ; \boldsymbol{\delta}_{\mathrm{c}}(125.8 \mathrm{MHz}$, DEPT135) 171.9, 169.7, 156.1, 146.0, 144.2, 141.7, 132.1, 129.2, 128.4, 128.1, $127.0,127.5,125.6,120.4,119.2,83.4,71.2,67.6,66.3,56.2$, ca. 54.3, 47.5, 28.4 (both $\beta$-Cs were too weak to be detected)

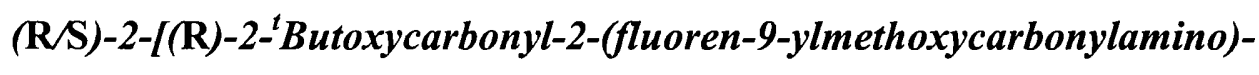
ethylsulfanyl]-3-(triphenylmethylamino)-propionic acid allyl ester (diastereoisomeric mixture): $\boldsymbol{\delta}_{\mathbf{H}}(400 \mathrm{MHz}) 7.73\left(2 \mathrm{H}, \mathrm{m}, H_{\mathrm{a}^{\prime}}\right), 7.56\left(2 \mathrm{H}, \mathrm{m}, H_{\mathrm{a}}\right), 7.43$ (6H, m, Trt), $7.36\left(2 \mathrm{H}, \mathrm{m}, H_{\mathrm{b}}\right), 7.20-7.31\left(2 \mathrm{H}, \mathrm{m}, H_{\mathrm{b}}+6 \mathrm{H}, \mathrm{m}, \mathrm{Trt}\right), 7.16(3 \mathrm{H}, \mathrm{m}$, Fmoc (Ar)), $5.91\left(1 \mathrm{H}, \mathrm{m}, \mathrm{CH}=\mathrm{CH}_{2}\right), 5.58+5.69(1 \mathrm{H}, \mathrm{bd}, \mathrm{FmocN} H), 5.33(1 \mathrm{H}, \mathrm{m}$, $\left.\mathrm{CH}=\mathrm{CH}_{2}\right), 5.23\left(1 \mathrm{H}, \mathrm{m}, \mathrm{CH}=\mathrm{CH}_{2}\right), 4.66\left(2 \mathrm{H}, \mathrm{m}, \mathrm{OCH}_{2} \mathrm{CH}=\mathrm{CH}_{2}\right), 4.50(1 \mathrm{H}, \mathrm{m}$, FmocNHCH), $4.29-4.41\left(2 \mathrm{H}, \mathrm{m}, \mathrm{CH}_{2} \mathrm{OCONH}\right), 4.18-4.25\left(1 \mathrm{H}, \mathrm{m}, H_{\mathrm{c}}\right), 3.49+$ $3.56\left(1 \mathrm{H}, \mathrm{t}, J 6.4+6.7, \mathrm{TrtNHCH}_{2} \mathrm{CH}\right), 2.81-3.12\left(2 \mathrm{H}, \mathrm{m}, \mathrm{CHCH}_{2} \mathrm{~S}\right), 2.61(1 \mathrm{H}, \mathrm{m}$, TrtNHCH$\left.\left.H_{2}\right), 2.45(1 \mathrm{H}, \mathrm{m}, \operatorname{TrtNHCH})_{2}\right), 1.48-1.49\left(9 \mathrm{H}, \mathrm{s}, \mathrm{C}\left(\mathrm{CH}_{3}\right)_{3}\right) ; \delta_{\mathrm{C}}(75.4 \mathrm{MHz}$, DEPT135) 171.8, ca. 169, 156.1, ca. 146, 144.1, 141.6, ca. 132, ca. 129, ca. 128, $128.1,127.5, c a .127,125.5,120.3, c a .119, c a .83, c a .71,67.6, c a .66, c a .54,(48.8$ + 48.0), 47.5, $(44.9+44.6),(34.8+34.3), 28.3$

Mass spectrum: $m / z$ (FAB) $901.2287\left([\mathrm{M}+\mathrm{Cs}]^{+} . \mathrm{C}_{47} \mathrm{H}_{48} \mathrm{~N}_{2} \mathrm{O}_{6} \mathrm{SCs}\right.$ requires 901.2264), $527\left([\mathrm{M}-\mathrm{Trt}+1]^{+}, 0.4 \%\right), 471\left(\left[\mathrm{M}-\mathrm{Trt}-{ }^{\mathrm{t}} \mathrm{Bu}+2\right]^{+}, 0.3 \%\right), 243\left(\mathrm{Trt}^{+}\right.$, $100 \%)$ 


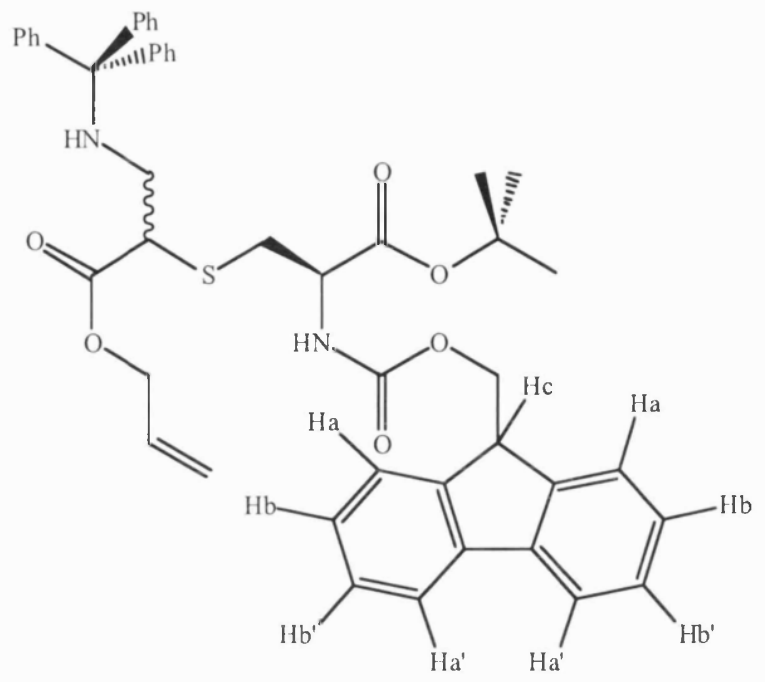

18

Figure 7.4. Major product: ( $R / S)$-2-[(R)-2-'butoxycarbonyl-2-(fluoren-9ylmethoxycarbonylamino)-ethylsulfanyl]-3-(triphenylmethylamino)-propionic acid allyl ester

\section{3-I(R)-2- ${ }^{t}$ Butoxvcarbonvl-2-(fluoren-9-vlmethoxvcarbonvlamino)-}

\section{ethvlsulfanvll-(R)-2-(allvloxvcarbonvlamino)-propionic acid allvl ester}

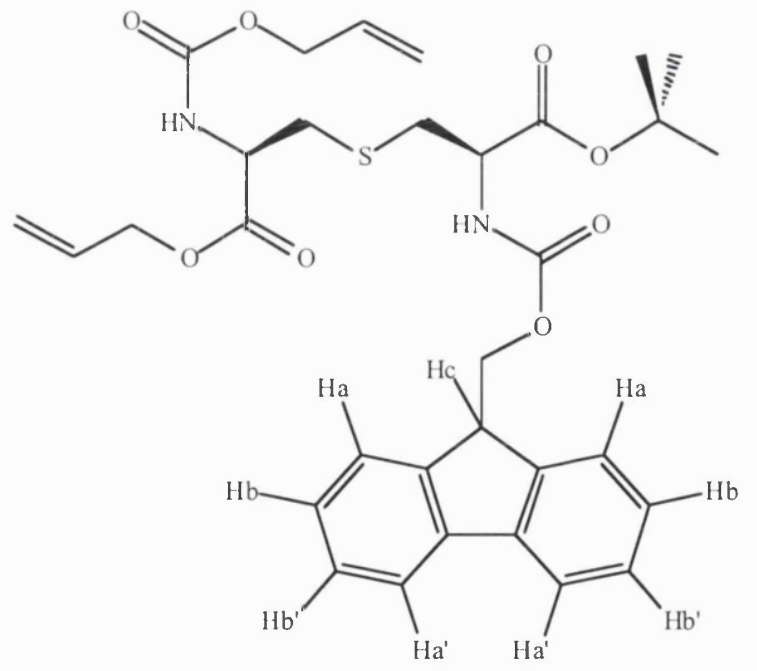

$(R, R)-12$

3-[(R)-2- ${ }^{t}$ Butoxycarbonyl-2-(fluoren-9-ylmethoxycarbonylamino)-ethylsulfanyl]-

$(R)$-2-(triphenylmethylamino)-propionic acid allyl ester $(\boldsymbol{R}, \boldsymbol{R})$-10 (mixture with regioisomer, $0.69 \mathrm{~g}, 0.90 \mathrm{mmol}$ ) was treated with a $5 \%$ solution of trifluoroacetic acid $(0.30 \mathrm{ml}, 3.8 \mathrm{mmol}, 4.2$ equiv. $)$ in DCM $(6.4 \mathrm{ml})$ under inert conditions for $4 \mathrm{~h}$. The resulting solution was diluted with DCM $(50 \mathrm{ml})$ and washed with sodium 
hydrogen carbonate $(5 \%$ aq. w/v, $2 \times 10 \mathrm{ml})$ and water $(2 \times 10 \mathrm{ml})$. The solvent was removed in vacuo. DCM $(20 \mathrm{ml})$ and $\mathrm{MeOH}(20 \mathrm{ml})$ were then added and reevaporation yielded a pale yellow liquid, which was then treated with sodium hydrogen carbonate $(0.30 \mathrm{~g}, 3.6 \mathrm{mmol})$ in water $(7.5 \mathrm{ml})$. The mixture was then cooled to $0{ }^{\circ} \mathrm{C}$ and a solution of allyl chloroformate $(0.19 \mathrm{ml}, 1.8 \mathrm{mmol})$ in dioxane $(7.5 \mathrm{ml})$ was added. The resulting mixture was stirred at a temperature $<5{ }^{\circ} \mathrm{C}$ for 18 $\mathrm{hr}$. The solvents were then removed in vacuo and the residue was re-dissolved in ethyl acetate $(100 \mathrm{ml})$. The organic layer was washed with water $(10 \times 40 \mathrm{ml})$, dried over anhydrous sodium sulfate and concentrated yielding a viscous yellow liquid. Purification was carried out via flash column chromatography (hexane:ethyl acetate, $4: 1$ then $2: 1,4 \mathrm{~cm}$ width) yielding the title compound as a mixture of regioisomers and a yellow oil.

Yield: $0.44 \mathrm{~g}, 0.73 \mathrm{mmol}, 81 \%$ (overall); $0.15 \mathrm{~g}, 0.24 \mathrm{mmol}, 27 \%$ (desired product, based on starting material)

$\mathbf{R}_{\mathbf{f}}: 0.29$ (hexane:ethyl acetate, $2: 1$ )

I.R.: $v_{\max }\left(\mathrm{cm}^{-1}\right) 1720(\mathrm{C}=\mathrm{O}), 1508(\mathrm{C}=\mathrm{C}$ Ar), 1450 (C=C Ar), $1223+1153$ (C-S-C) NMR: $\delta_{\mathbf{H}}(400 \mathrm{MHz}) 7.77\left(2 \mathrm{H}, \mathrm{m}, H_{\mathrm{a}^{\prime}}\right), 7.62\left(2 \mathrm{H}, \mathrm{m}, H_{\mathrm{a}}\right), 7.40\left(2 \mathrm{H}, \mathrm{m}, H_{\mathrm{b}}\right), 7.31$ $\left(2 \mathrm{H}, \mathrm{m}, H_{\mathrm{b}}\right), 5.80-5.96\left(2 \mathrm{H}, \mathrm{m}, \mathrm{CH}=\mathrm{CH}_{2}\right), 5.75(1 \mathrm{H}, \mathrm{m}, \mathrm{FmocN} H), 5.66(1 \mathrm{H}, \mathrm{m}$, AlocNH), $5.17-5.35\left(4 \mathrm{H}, \mathrm{m}, \mathrm{CH}=\mathrm{CH}_{2}\right), 4.56-4.65\left(4 \mathrm{H}, \mathrm{m}, \mathrm{OCH}_{2} \mathrm{CH}=\mathrm{CH}_{2}\right), 4.61$ (1H, m, AlocNHCH), 4.50 (1H, m, FmocNHCH), 4.39 (2H, m, $\left.\mathrm{CH}_{2} \mathrm{OCONH}\right), 4.25$ $\left(1 \mathrm{H}, \mathrm{m}, H_{\mathrm{C}}\right), 2.94-3.10\left(4 \mathrm{H}, \mathrm{m}, \mathrm{CH}_{2} \mathrm{SCH}_{2}\right), 1.50\left(9 \mathrm{H}, \mathrm{s}, \mathrm{C}\left(\mathrm{CH}_{3}\right)_{3}\right) ; \boldsymbol{\delta}_{\mathrm{C}}(125.8 \mathrm{MHz}$, DEPT135) 170.2, ca. 169.2, ca. 156.1, ca. 155.7, ca. 143.8, 141.3, ca. 132.5, ca. 131.3, ca. 127.8, 127.1, 125.1, 120.0, ca. 119.1, ca. 117.8, ca. 83.1, ca. 67.2, 66.4, ca. 66.1, ca. $54.0(3 \mathrm{C}), c a .47 .1, c a .35 .5(2 \mathrm{C}), 28.0\left(\delta_{\mathrm{H}}\right.$ values were extrapolated from the separated samples of 3-[(R)-2-'butoxycarbonyl-2-(fluoren-9ylmethoxycarbonylamino)-ethylsulfanyl]-(S)-2- (allyloxycarbonylamino)-propionic acid allyl ester $(\boldsymbol{S}, \boldsymbol{R})-\mathbf{1 2})$

(RS)-2-[(R)-2-'Butoxycarbonyl-2-(fluoren-9-ylmethoxycarbonylamino)ethylsulfanyl]-3-(allyloxycarbonylamino)-propionic acid allyl ester (diastereoisomeric mixture): $\delta_{\mathrm{H}}(400 \mathrm{MHz}) 7.76\left(2 \mathrm{H}, \mathrm{m}, H_{\mathrm{a}^{\prime}}\right), 7.62\left(2 \mathrm{H}, \mathrm{m}, H_{\mathrm{a}}\right), 7.40$ $\left(2 \mathrm{H}, \mathrm{m}, H_{\mathrm{b}}\right), 7.32\left(2 \mathrm{H}, \mathrm{m}, H_{\mathrm{b}}\right), 5.90\left(2 \mathrm{H}, \mathrm{m}, \mathrm{CH}=\mathrm{CH}_{2}\right), 5.76(1 \mathrm{H}, \mathrm{bm}, \mathrm{FmocN} H)$, $5.14-5.36\left(4 \mathrm{H}, \mathrm{m}, \mathrm{CH}=\mathrm{CH}_{2}\right), 5.33(1 \mathrm{H}, \mathrm{m}, \mathrm{AlocN} H), 4.50-4.70(4 \mathrm{H}, \mathrm{m}$, 
$\left.\mathrm{OCH}_{2} \mathrm{CH}=\mathrm{CH}_{2}\right), 4.54(1 \mathrm{H}, \mathrm{m}, \mathrm{FmocNHCH}), 4.40\left(2 \mathrm{H}, \mathrm{m}, \mathrm{CH}_{2} \mathrm{OCONH}\right), 4.25(1 \mathrm{H}$, $\left.\mathrm{m}, H_{\mathrm{c}}\right), 3.45-3.66\left(2 \mathrm{H}, \mathrm{m}, \mathrm{SCHCH} \mathrm{H}_{2}\right), 3.52(1 \mathrm{H}, \mathrm{m}, \mathrm{AlocNHCH}), 2.97-3.25(2 \mathrm{H}$, $\left.\mathrm{m}, \mathrm{SCH} \mathrm{H}_{2}\right), 1.50\left(9 \mathrm{H}, \mathrm{s}, \mathrm{C}\left(\mathrm{CH}_{3}\right)_{3}\right) ; \boldsymbol{\delta}_{\mathrm{C}}(125.8 \mathrm{MHz}$, DEPT135) 170.7, (169.2+ 169.1), ca. 156, ca. 157, 143.7, 141.2, 132.6, (131.4 + 131.3), 127.7, 127.0, 125.1, 119.9, $(119.0+118.9),(117.72+117.65)$, ca. 83.1, $(67.24+67.16),(66.1+66.0), 65.7$, $(54.3+53.9), 47.0,(46.7+46.0),(41.5+41.4),(34.34+34.26), 27.9$

Mass spectrum: $m / z(F A B) 611.2427\left([\mathrm{M}+\mathrm{H}]^{+} . \mathrm{C}_{32} \mathrm{H}_{39} \mathrm{~N}_{2} \mathrm{O}_{8} \mathrm{~S}\right.$ requires 611.2440), $633\left([\mathrm{M}+\mathrm{Na}]^{+}, 19 \%\right), 61 \mathrm{l}\left([\mathrm{M}+\mathrm{H}]^{+}, 20 \%\right), 555\left(\left[\mathrm{M}-{ }^{\prime} \mathrm{Bu}+2\right]^{+}, 55 \%\right), 333([\mathrm{M}$ $\left.\left.-{ }^{\mathrm{B}} \mathrm{Bu}-\mathrm{Fmoc}+3\right]^{+}, 35 \%\right) ; m / z(\mathrm{APCI}+) 633\left([\mathrm{M}+\mathrm{Na}]^{+}, 4.5 \%\right), 611\left([\mathrm{M}+\mathrm{H}]^{+}, 13\right.$ $\%), 555\left(\left[\mathrm{M}-{ }^{\mathrm{t}} \mathrm{Bu}+2\right]^{+}, 100 \%\right), 333\left(\left[\mathrm{M}-{ }^{\mathrm{t}} \mathrm{Bu}-\mathrm{Fmoc}+3\right]^{+}, 38 \%\right)$

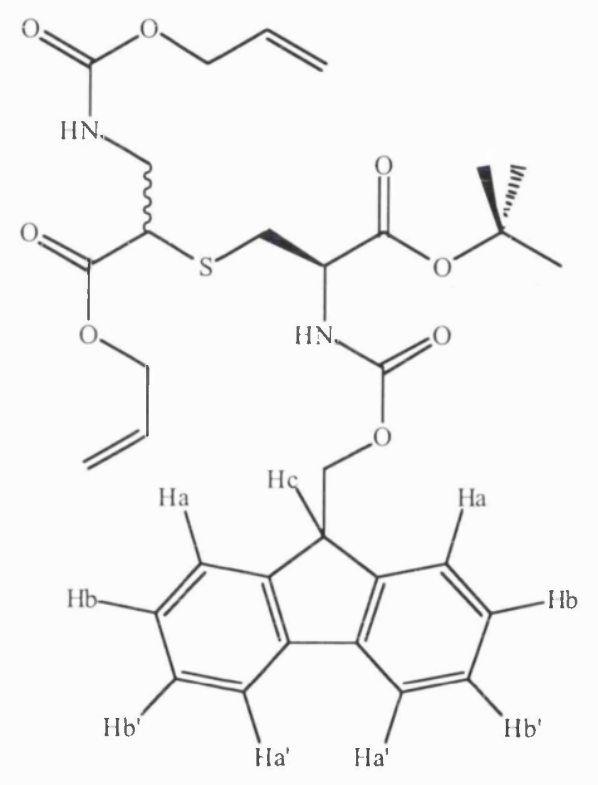

19

Figure 7.5. Major product: ( $R / S)-2[(R)-2$-butoxycarbonyl-2-(fluoren-9ylmethoxycarbonylamino)-ethylsulfanyl]-3-(allyloxycarbonylamino)-propionic acid allyl ester 


\section{3-I(R)-2- ${ }^{t}$ Butoxvcarbonvl-2-(fluoren-9-vlmethoxvcarbonvlamino)-}

\section{ethvlsulfanvll-(S)-2-(allvloxvcarbonvlamino)-propionic acid allvl ester}

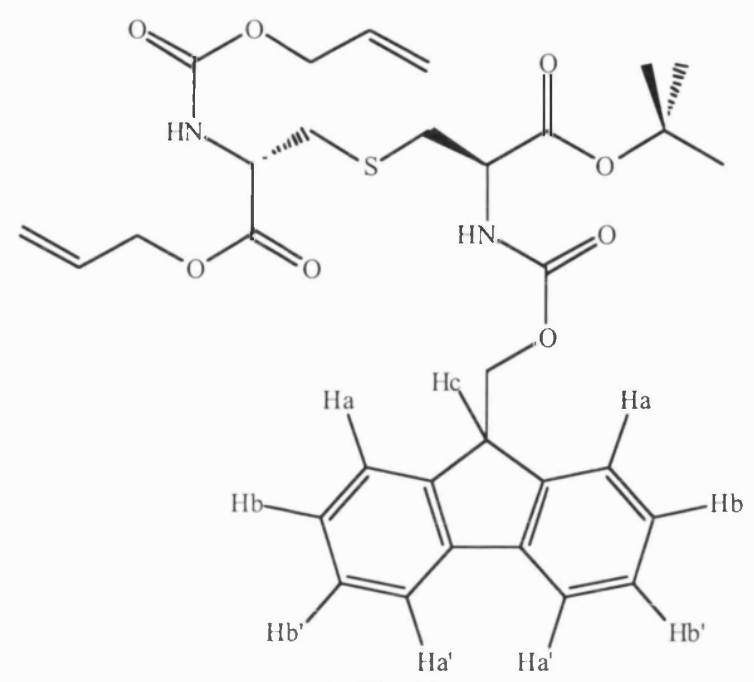

$(S, R)-12$

3-[(R)-2- ${ }^{\mathrm{t}}$ Butoxycarbonyl-2-(fluoren-9-ylmethoxycarbonylamino)-ethylsulfanyl $]-(S)$ 2-(triphenylmethylamino)-propionic acid allyl ester $(\boldsymbol{S}, \boldsymbol{R})$-10 (mixture with regioisomer, $3.5 \mathrm{~g}, 4.6 \mathrm{mmol}$ ) was treated with a $5 \%$ solution of trifluoroacetic acid $(1.4 \mathrm{ml}, 18 \mathrm{mmol}, 4$ equiv.) in DCM $(27 \mathrm{ml})$ under inert conditions for $4 \mathrm{hr}$. The resulting solution was diluted with DCM $(200 \mathrm{ml})$ and washed with sodium hydrogen carbonate $(5 \%$ aq. w/v, $2 \times 75 \mathrm{ml})$ and water $(2 \times 50 \mathrm{ml})$. The solvent was removed in vacuo. DCM $(20 \mathrm{ml})$ and $\mathrm{MeOH}(20 \mathrm{ml})$ were then added and reevaporation yielded a pale yellow liquid, which was then treated with sodium hydrogen carbonate $(1.5 \mathrm{~g}, 18 \mathrm{mmol}, 4$ equiv. $)$ in water $(30 \mathrm{ml})$. The mixture was then cooled to $0{ }^{\circ} \mathrm{C}$ and a solution of allyl chloroformate $(0.80 \mathrm{ml}, 9.2 \mathrm{mmol}, 2$ equiv.) in dioxane $(30 \mathrm{ml})$ was added. The resulting mixture was stirred at a temperature $<5{ }^{\circ} \mathrm{C}$ for $18 \mathrm{hr}$. The solvents were then removed in vacuo and the residue was re-dissolved in ethyl acetate $(250 \mathrm{ml})$. The organic layer was washed with water $(8 \times 50 \mathrm{ml})$, dried over anhydrous sodium sulfate and concentrated yielding a yellow liquid. Purification was carried out via flash column chromatography (hexane:ethyl acetate, $4: 1$ then $2: 1,7.5 \mathrm{~cm}$ width) yielding the title compound as a viscous yellow oil. Separation of the regioisomers was carried out via preparative normal phase HPLC (20-23\% ethyl acetate in hexane over 45 minutes, $15 \mathrm{ml} / \mathrm{min}$, DCM loading, $\mathrm{R}_{\mathrm{T}} 16.6,20.1$ (regioisomer)) yielding both products as viscous, pale yellow liquids. 
Yield: $2.4 \mathrm{~g}, 4.0 \mathrm{mmol}, 87 \%$ (overall); $0.41 \mathrm{~g}, 0.67 \mathrm{mmol}, 14 \%$ (desired product, based on starting material)

$\mathbf{R}_{\mathbf{f}}: 0.29$ (hexane:ethyl acetate, $2: 1$ )

I.R.: $v_{\max }\left(\mathrm{cm}^{-1}\right) 3429(\mathrm{NH}), 1724(\mathrm{C}=\mathrm{O}), 1512(\mathrm{C}=\mathrm{C}$ Ar$), 1450(\mathrm{C}=\mathrm{C} \mathrm{Ar}), 1226+$ $1153(\mathrm{C}-\mathrm{S}-\mathrm{C})$

NMR: $\delta_{\mathrm{H}}(400 \mathrm{MHz}) 7.78\left(2 \mathrm{H}, \mathrm{m}, H_{\mathrm{a}}{ }^{\prime}\right), 7.63\left(2 \mathrm{H}, \mathrm{m}, H_{\mathrm{a}}\right), 7.42\left(2 \mathrm{H}, \mathrm{m}, H_{\mathrm{b}^{\prime}}\right), 7.33$ $\left(2 \mathrm{H}, \mathrm{m}, H_{\mathrm{b}}\right), 5.80-5.96\left(2 \mathrm{H}, \mathrm{m}, \mathrm{CH}=\mathrm{CH}_{2}\right), 5.75(1 \mathrm{H}, \mathrm{m}, \mathrm{FmocN} H), 5.66(1 \mathrm{H}, \mathrm{m}$, AlocNH), $5.17-5.35\left(4 \mathrm{H}, \mathrm{m}, \mathrm{CH}=\mathrm{CH}_{2}\right), 4.56-4.65\left(4 \mathrm{H}, \mathrm{m}, \mathrm{OCH}_{2} \mathrm{CH}=\mathrm{CH}_{2}\right), 4.61$ $(1 \mathrm{H}, \mathrm{m}, \mathrm{AlocNHCH}), 4.50$ (1H, m, FmocNHCH), 4.39 (2H, m, $\left.\mathrm{CH}_{2} \mathrm{OCONH}\right), 4.25$ $\left(1 \mathrm{H}, \mathrm{m}, \mathrm{H}_{\mathrm{c}}\right), 2.94-3.10\left(4 \mathrm{H}, \mathrm{m}, \mathrm{CH}_{2} \mathrm{SCH}\right), 1.50\left(9 \mathrm{H}, \mathrm{s}, \mathrm{C}\left(\mathrm{CH}_{3}\right)_{3}\right) ; \boldsymbol{\delta}_{\mathrm{C}}(125.8 \mathrm{MHz}$, DEPT135) 170.2, ca. 169.2, ca. 156.1, ca. 155.7, 143.8, 141.3, ca. 132.5, ca. 131.3, 127.7, 127.1, 125.1, 120.0, ca. 119.1, ca. 117.7, ca. 83.1, ca. 67.2, 66.4, ca. 66.1, ca. $54.0(3 \mathrm{C}), c a .47 .1, c a .35 .5(2 \mathrm{C}), 28.0$

(RS)-2-I(R)-2-'Butoxycarbonyl-2-(fluoren-9-ylmethoxycarbonylamino)ethylsulfanyl]-3-(allyloxycarbonylamino)-propionic acid allyl ester (diastereoisomeric mixture): $\boldsymbol{\delta}_{\mathbf{H}}(400 \mathrm{MHz}) 7.76\left(2 \mathrm{H}, \mathrm{m}, H_{\mathrm{a}^{\prime}}\right), 7.62\left(2 \mathrm{H}, \mathrm{m}, H_{\mathrm{a}}\right), 7.40$ $\left(2 \mathrm{H}, \mathrm{m}, H_{\mathrm{b}}\right), 7.32\left(2 \mathrm{H}, \mathrm{m}, H_{\mathrm{b}}\right), 5.90\left(2 \mathrm{H}, \mathrm{m}, \mathrm{CH}=\mathrm{CH}_{2}\right), 5.76(1 \mathrm{H}, \mathrm{bm}, \mathrm{FmocN} H)$, $5.14-5.36\left(4 \mathrm{H}, \mathrm{m}, \mathrm{CH}=\mathrm{CH}_{2}\right), 5.31(1 \mathrm{H}, \mathrm{m}, \operatorname{AlocN} H), 4.50-4.70(4 \mathrm{H}, \mathrm{m}$, $\left.\mathrm{OCH}_{2} \mathrm{CH}=\mathrm{CH}_{2}\right), 4.54(1 \mathrm{H}, \mathrm{m}, \mathrm{FmocNHCH}), 4.40\left(2 \mathrm{H}, \mathrm{m}, \mathrm{CH}_{2} \mathrm{OCONH}\right), 4.25(1 \mathrm{H}$, $\left.\mathrm{m}, H_{\mathrm{c}}\right), 3.45-3.66(2 \mathrm{H}, \mathrm{m}, \mathrm{SCHCH})_{2}, 3.52(1 \mathrm{H}, \mathrm{m}, \mathrm{AlocNHCH}), 2.97-3.25(2 \mathrm{H}$, $\left.\mathrm{m}, \mathrm{SCH} \mathrm{H}_{2}\right), 1.50\left(9 \mathrm{H}, \mathrm{s}, \mathrm{C}\left(\mathrm{CH}_{3}\right)_{3}\right) ; \boldsymbol{\delta}_{\mathrm{C}}(125.8 \mathrm{MHz}, \mathrm{DEPT} 135) 170.7,(169.2+169.1)$, ca. 156, ca. 157, 143.7, 141.2, 132.6, (131.4 + 131.3), 127.7, 127.0, 125.1, 119.9, $(119.0+118.9),(117.72+117.65), c a .83 .1,(67.24+67.16),(66.1+66.0), 65.7$, $(54.3+53.9), 47.0,(46.7+46.0),(41.5+41.4),(34.34+34.26), 27.9$

Mass spectrum: $m / z(\mathrm{FAB}) 633.2247\left([\mathrm{M}+\mathrm{Na}]^{+} \cdot \mathrm{C}_{32} \mathrm{H}_{38} \mathrm{~N}_{2} \mathrm{O}_{8} \mathrm{SNa}\right.$ requires 633.2265), $633\left([\mathrm{M}+\mathrm{Na}]^{+}, 31 \%\right), 611\left([\mathrm{M}+\mathrm{H}]^{+}, 38 \%\right), 555\left(\left[\mathrm{M}-{ }^{\mathrm{t}} \mathrm{Bu}+2\right]^{+}, 56\right.$ $\%), 333\left(\left[\mathrm{M}-{ }^{\mathrm{t}} \mathrm{Bu}-\mathrm{Fmoc}+3\right]^{+}, 100 \%\right)$ 


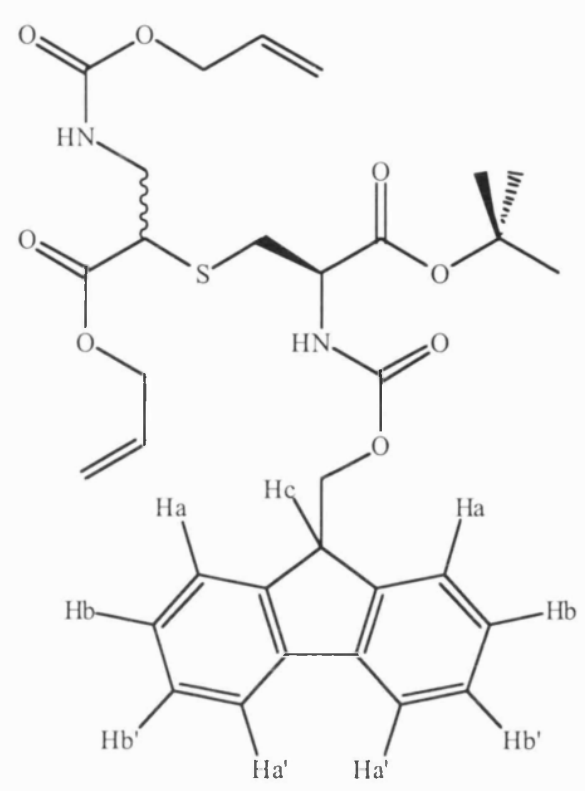

19

Figure 7.6. Major product: ( $R / S)$-2-[(R)-2-'butoxycarbonyl-2-(fluoren-9ylmethoxycarbonylamino)-ethylsulfanyl]-3-(allyloxycarbonylamino)-propionic acid allyl ester

19. 3-I(R/S)-1-Allvloxvcarbonvl-2-(allvloxvcarbonvlamino)-ethvlsulfanvll-(R)-2(fluoren-9-vlmethoxvcarbonvlamino)-propionic acid<smiles>C=CCOC(=O)NC[C@@H](SC[C@H](NC(=O)OCC1(C)c2c(C)c(Br)c(Br)c(Br)c2-c2c(C)c(Br)c(Br)c(Br)c21)C(=O)O)C(=O)OCC=C</smiles>

20

3-[(R)-2- ${ }^{\mathrm{B}}$ Butoxycarbonyl-2-(fluoren-9-ylmethoxycarbonylamino)-ethylsulfanyl]-(S)2-(allyloxycarbonylamino)-propionic acid allyl ester $(\boldsymbol{S}, \boldsymbol{R}) \mathbf{- 1 2}$ (mixture with regioisomer, $2.1 \mathrm{~g}, 3.4 \mathrm{mmol})$ was treated with a solution of TFA ( $5 \mathrm{ml}$, excess) in DCM $(5 \mathrm{ml})$ and the mixture was stirred for $4 \mathrm{hr}$. The mixture was concentrated in vacuo and re-dissolved in DCM $(100 \mathrm{ml})$ and $\mathrm{MeOH}(100 \mathrm{ml})$. The solvents were 
then removed. The crude material was purified via reverse phase flash column chromatography $\left(0-35 \% \mathrm{MeCN}, 20 \%\right.$ saturated $\left.\mathrm{NaHCO}_{3}, \mathrm{H}_{2} \mathrm{O}\right)$ yielding a colourless liquid, which foamed and solidified under highly reduced pressure at -50 ${ }^{\circ} \mathrm{C}$. Only the regioisomer, as a mixture of diastereoisomers, was isolated.

Yield: $1.4 \mathrm{~g}, 2.6 \mathrm{mmol}, 76 \%$

$\mathbf{R}_{\mathbf{f}}: 0.31\left(\mathrm{MeCN}: \mathrm{H}_{2} \mathrm{O}:\right.$ sat. $\left.\mathrm{NaHCO}_{3}, 4: 5: 1\right)$

I.R.: $v_{\max }\left(\mathrm{cm}^{-1}\right) 3433(\mathrm{OH}$, negligible H-bonding), $3337(\mathrm{NH}), 1720(\mathrm{C}=\mathrm{O}), 1512$ $(\mathrm{C}=\mathrm{C}$ Ar $), 1450(\mathrm{C}=\mathrm{C}$ Ar $), 1223+1150(\mathrm{C}-\mathrm{S}-\mathrm{C})$

NMR: $\boldsymbol{\delta}_{\mathbf{H}}\left(500 \mathrm{MHz}, \mathrm{CD}_{3} \mathrm{OD}\right)$ Diastereoisomeric $7.76\left(2 \mathrm{H}, \mathrm{m}, \mathrm{H}_{\mathrm{a}}\right), 7.62(2 \mathrm{H}, \mathrm{m}$, $\left.H_{\mathrm{a}}\right), 7.35\left(2 \mathrm{H}, \mathrm{m}, H_{\mathrm{b}}\right), 7.27\left(2 \mathrm{H}, \mathrm{m}, H_{\mathrm{b}}\right), 5.80-5.96\left(2 \mathrm{H}, \mathrm{m}, \mathrm{CH}=\mathrm{CH}_{2} \mathrm{All}+\mathrm{Aloc}\right)$, $5.10-5.35\left(4 \mathrm{H}, \mathrm{m}, \mathrm{CH}=\mathrm{CH}_{2} \mathrm{All}+\mathrm{Aloc}\right), 4.60\left(2 \mathrm{H}, \mathrm{m}, \mathrm{OCH}_{2} \mathrm{CH}=\mathrm{CH}_{2}\right), 4.48(2 \mathrm{H}$, $\left.\mathrm{m}, \mathrm{OCH}_{2} \mathrm{CH}=\mathrm{CH}_{2}\right), 4.38(1 \mathrm{H}, \mathrm{m}, \mathrm{FmocNHCH}), 4.28-4.41\left(2 \mathrm{H}, \mathrm{m}, \mathrm{CH}_{2} \mathrm{OCONH}\right)$, $\left.4.22\left(1 \mathrm{H}, \mathrm{m}, H_{\mathrm{c}}\right), 3.64\left(1 \mathrm{H}, \mathrm{m}, \mathrm{AlocNHCH}_{2} \mathrm{CH}\right), 3.44(2 \mathrm{H}, \mathrm{m}, \mathrm{AlocNHCH})_{2}\right), 2.88-$ $3.24\left(2 \mathrm{H}, \mathrm{m}, \mathrm{CHCH}_{2} \mathrm{~S}\right) ; \boldsymbol{\delta}_{\mathrm{C}}(125.8 \mathrm{MHz}, \mathrm{HSQC})(172.5+172.4), 172.0, \mathrm{ca} .158$ $(2 \mathrm{C}), c a .145 .2,142.5,(134.2+134.1),(133.2+133.0), 129.2,(128.7+128.6)$, $126.3,120.6,118.8,(117.7+117.6),(68.23+68.18),(66.99+66.96),(66.6+66.5)$, $55.6,48.3,47.2,(43.1+42.8),(34.7+34.2)$

Elemental analysis: Found $\mathrm{C}, 61.06 ; \mathrm{H}, 6.02 ; \mathrm{N}, 4.68 ; \mathrm{S}, 5.22 \% . \mathrm{C}_{44} \mathrm{H}_{48} \mathrm{~N}_{2} \mathrm{O}_{8} \mathrm{~S}_{2}$ requires $\mathrm{C}, 60.64 ; \mathrm{H}, 5.45 ; \mathrm{N}, 5.05 ; \mathrm{S}, 5.78 \%$.

Mass spectrum: $m / z(\mathrm{ES}+) 577\left([\mathrm{M}+\mathrm{Na}]^{+}, 100 \%\right), 555\left([\mathrm{M}+\mathrm{H}]^{+}, 4 \%\right), 333([\mathrm{M}-$ $\mathrm{Fmoc}+2]^{+}, 4 \%$ )

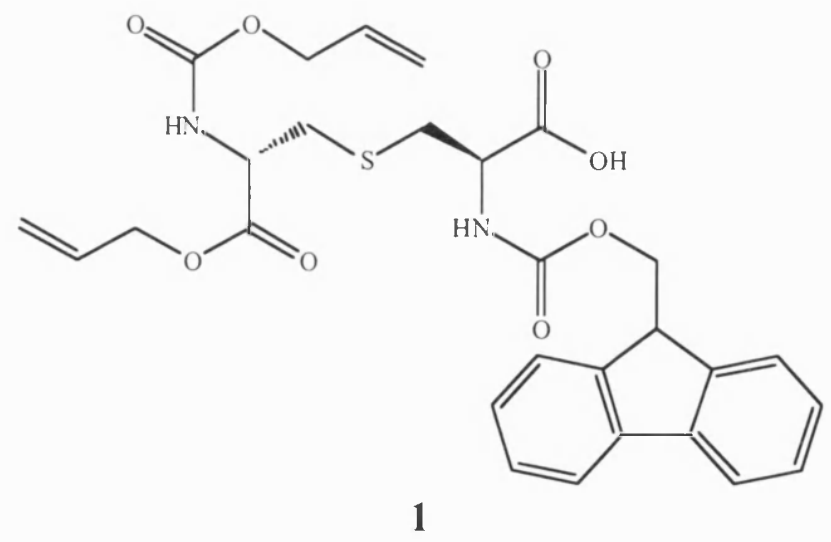

Figure 7.7. Intended product: 3-[(S)-2-allyloxycarbonyl-2-(allyloxycarbonylamino)ethylsulfanyl]-(R)-2-(fluoren-9-ylmethoxycarbonylamino)-propionic acid 


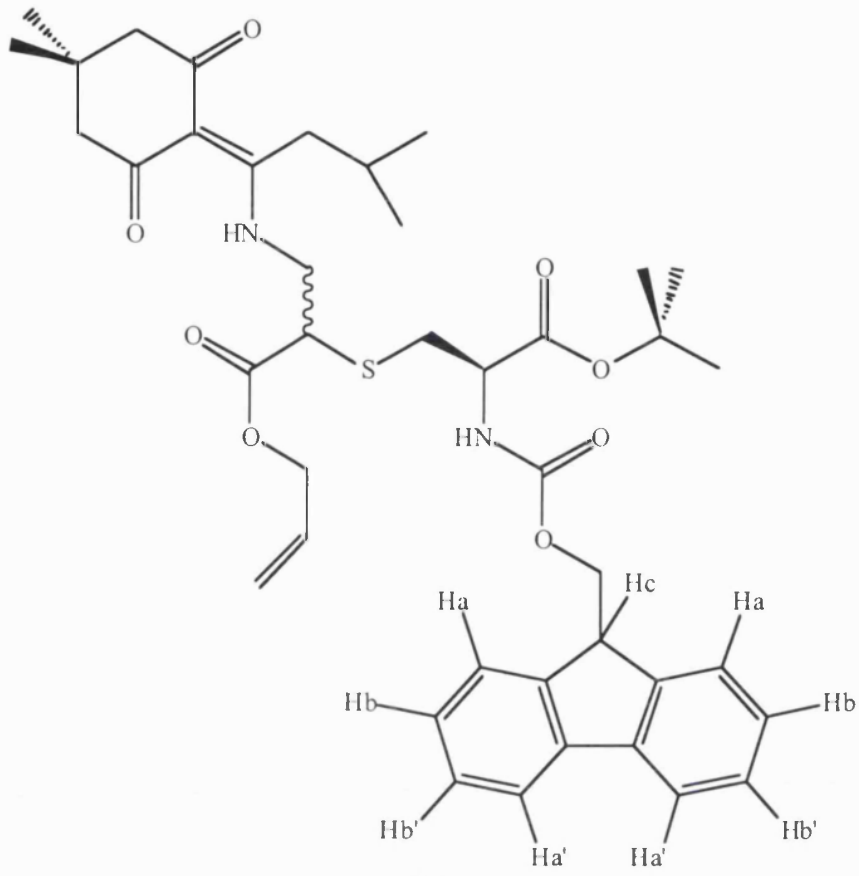

21

3-[(R)-2-'Butoxycarbonyl-2-(fluoren-9-ylmethoxycarbonylamino)-ethylsulfanyl $]-(S)$ 2-(triphenylmethylamino)-propionic acid allyl ester $(\boldsymbol{S}, \boldsymbol{R})-10$ (mixture with regioisomer, $3.5 \mathrm{~g}, 4.6 \mathrm{mmol}$ ) was treated with a $5 \%$ solution of trifluoroacetic acid ( $1.4 \mathrm{ml}, 18 \mathrm{mmol}, 4$ equiv.) in DCM $(27 \mathrm{ml})$ under inert conditions for $4 \mathrm{hr}$. The resulting solution was diluted with DCM $(100 \mathrm{ml})$ and washed with sodium hydrogen carbonate $(5 \%$ aq. w/v, $2 \times 75 \mathrm{ml})$ and water $(2 \times 50 \mathrm{ml})$. The solvent was removed in vacuo. DCM $(20 \mathrm{ml})$ and $\mathrm{MeOH}(20 \mathrm{ml})$ were then added and reevaporation yielded a pale yellow liquid, which was then dissolved in ethanol (abs., $30 \mathrm{ml})$. ivDde-OH ( $0.99 \mathrm{ml}, 4.6 \mathrm{mmol})$ was then added and the reaction mixture was stirred for $18 \mathrm{hr}$ at $35^{\circ} \mathrm{C}$. The solvent was removed in vacuo and the resulting oil was re-dissolved in ethyl acetate $(250 \mathrm{ml})$. The organic layer was washed with citric acid $(5 \%$ aq. w/v, $100 \mathrm{ml})$ and water $(3 \times 50 \mathrm{ml})$ and dried over anhydrous sodium sulfate. Removal of the solvent yielded a pale yellow liquid, which was purified via flash column chromatography (hexane:ethyl acetate, $2: 1,7.5 \mathrm{~cm}$ width). The product, which contained only the regioisomer as a mixture of diastereoisomers, was an extremely viscous colourless liquid. 
Yield: $2.0 \mathrm{~g}, 2.7 \mathrm{mmol}, 60 \%$

$\mathbf{R}_{\mathbf{f}}: 0.35$ (cyclohexane:ethyl acetate, $2: 1$ )

I.R.: $v_{\max }\left(\mathrm{cm}^{-1}\right) 1728(\mathrm{C}=\mathrm{O}), 1566(\mathrm{C}=\mathrm{C}$ Ar $), 1450(\mathrm{C}=\mathrm{C}$ Ar $), 1224+1151(\mathrm{C}-\mathrm{S}-\mathrm{C})$ NMR: $\boldsymbol{\delta}_{\mathrm{H}}(500 \mathrm{MHz})$ Diastereoisomeric $7.77\left(2 \mathrm{H}, \mathrm{m}, H_{\mathrm{a}}{ }^{\prime}\right), 7.63\left(2 \mathrm{H}, \mathrm{m}, H_{\mathrm{a}}\right), 7.40$ $\left(2 \mathrm{H}, \mathrm{m}, H_{\mathrm{b}}\right), 7.30\left(2 \mathrm{H}, \mathrm{m}, H_{\mathrm{b}}\right), 6.15+6.24(1 \mathrm{H}, \mathrm{bd}, \mathrm{FmocNH}), 5.94(1 \mathrm{H}, \mathrm{m}$, $\left.\mathrm{CH}=\mathrm{CH}_{2}\right), 5.36\left(1 \mathrm{H}, \mathrm{dd}, J 18.4,1.3, \mathrm{CH}=\mathrm{CH}_{2}\right), 5.21\left(1 \mathrm{H}, \mathrm{dd}, J 10.4,1.1, \mathrm{CH}=\mathrm{CH}_{2}\right)$, $4.70\left(2 \mathrm{H}, \mathrm{m}, \mathrm{OCH}_{2} \mathrm{CH}\right), 4.44-4.52(1 \mathrm{H}, \mathrm{m}, \mathrm{FmocNHCH}), 4.35-4.45(2 \mathrm{H}, \mathrm{m}$, $\left.\mathrm{CH}_{2} \mathrm{OCONH}\right), 4.24\left(1 \mathrm{H}, \mathrm{m}, \mathrm{H}_{\mathrm{c}}\right), 3.61-3.95\left(2 \mathrm{H}, \mathrm{m}, \mathrm{NCH}_{2}\right), 3.60(1 \mathrm{H}, \mathrm{m}$, $\left.\mathrm{NCH}_{2} \mathrm{CH}\right), 3.03-3.23\left(2 \mathrm{H}, \mathrm{m}, \mathrm{CH}_{2} \mathrm{~S}\right), 3.02\left(2 \mathrm{H}, \mathrm{m}, \mathrm{C}=\mathrm{CCH}_{2}\right), 2.30-2.45(4 \mathrm{H}, \mathrm{m}$, $\left.\mathrm{CH}_{2} \mathrm{C}=\mathrm{O}\right), 1.94\left(1 \mathrm{H}, \mathrm{m}, \mathrm{CH}\left(\mathrm{CH}_{3}\right)_{2}\right), 1.50\left(9 \mathrm{H}, \mathrm{s}, \mathrm{C}\left(\mathrm{CH}_{3}\right)_{3}\right), 0.96-1.05(12 \mathrm{H}, \mathrm{m}$, $\left.\left.\left(\mathrm{CH}_{3}\right)_{2} \mathrm{CCH}_{2},\left(\mathrm{CH}_{3}\right)_{2} \mathrm{CH}\right)\right) ; \boldsymbol{\delta}(125.8 \mathrm{MHz}, \mathrm{DEPT} 135$, HMQC) $(199.7+196.1)$, $(176.5+176.3),(169.9+169.8),(169.0+168.9),(155.9+155.8),(143.74+$ $143.67),(141.18+141.17),(131.2+131.1), 127.6,126.9,(125.1+125.0), 119.9$, $(119.3+119.1),(107.5+107.4),(83.04+82.98),(67.1+67.0),(66.5+66.4),(54.3$ $+54.0),(53.7+52.2), 47.0,(46.4+45.9),(43.9+43.7),(37.1+37.0),(34.7+34.6)$, $(29.74+29.71),(29.03+29.00),(28.1+28.0),(27.85+27.83),(22.42+22.37)$

Mass spectrum: $m / z(F A B) 733.3521\left([\mathrm{M}+\mathrm{H}]^{+} . \mathrm{C}_{41} \mathrm{H}_{53} \mathrm{~N}_{2} \mathrm{O}_{8} \mathrm{~S}\right.$ requires 733.3583), $m / z(\mathrm{ES}+) 755\left([\mathrm{M}+\mathrm{Na}]^{+}, 38 \%\right), 733\left([\mathrm{M}+\mathrm{H}]^{+}, 51 \%\right)$

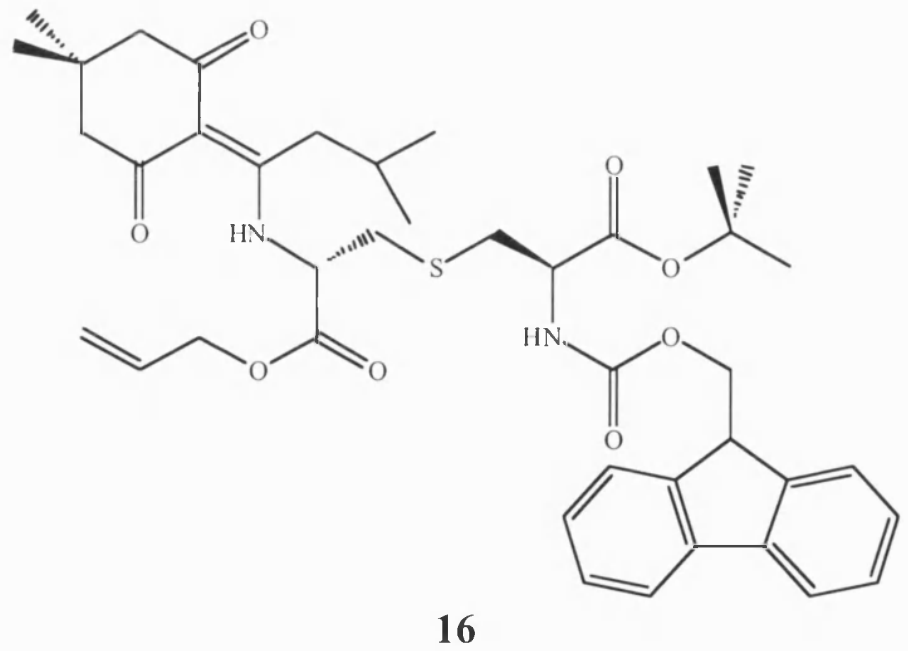

Figure 7.8. Intended product: 3-[( $R$ )-2-tbutoxycarbonyl-2-(fluoren-9ylmethoxycarbonylamino)-ethylsulfanyl]-(S)-2-\{1-(4,4-dimethyl-2,6-dioxocyclohex1-ylidene)isovalerylamino -propionic acid allyl ester 


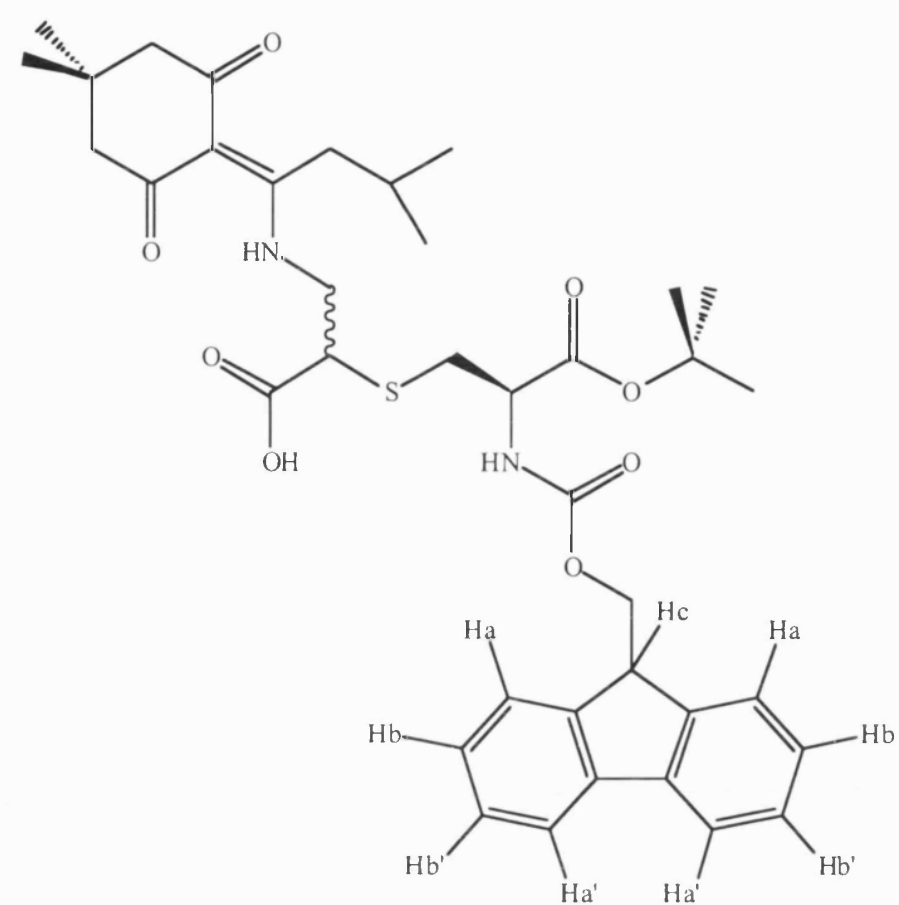

27

$(R / S)-2-\left[(R)-2-{ }^{\prime}\right.$ Butoxycarbonyl-2-(fluoren-9-ylmethoxycarbonylamino)-

ethylsulfanyl]-3-\{1-(4,4-dimethyl-2,6-dioxocyclohex-1-ylidene)isovalerylamino $\}$ propionic acid allyl ester 21 (1.7 g, $2.4 \mathrm{mmol})$ was treated with tetrakis(triphenylphosphine) palladium(0) (1.2 g, $1.0 \mathrm{mmol}, 0.4$ equiv.) in a cocktail solution of DMF:CHCl $: A c O H: N M M(18.5: 18.5: 2: 1 \mathrm{v} / \mathrm{v} / \mathrm{v} / \mathrm{v}, 9.3: 9.3: 1: 0.5 \mathrm{ml})$. The solution was stirred at $25{ }^{\circ} \mathrm{C}$ for $3 \mathrm{hr}$. The mixture was then diluted with DCM (250 $\mathrm{ml})$, washed with water $(5 \times 50 \mathrm{ml})$ and dried over anhydrous sodium sulfate. Removal of the solvent yielded a yellow liquid, which was used without further purification, in crude form. A small amount of the crude material was purified via preparative reverse phase HPLC for analyses $(40-60 \% \mathrm{MeCN}$ in water over 30 $\min , 15 \mathrm{ml} / \mathrm{min}, \mathrm{R}_{\mathrm{T}}$ 13.2).

Yield: $100 \%$ (crude)

R: 0.1 (DCM:MeOH, 19:1)

I.R.: $v_{\max }\left(\mathrm{cm}^{-1}\right) 3300(\mathrm{OH}$, broad $), 1717(\mathrm{C}=\mathrm{O}), 1570(\mathrm{C}=\mathrm{C}$ Ar $), 1450(\mathrm{C}=\mathrm{C}$ Ar $)$, $1223+1153(\mathrm{C}-\mathrm{S}-\mathrm{C})$ 
NMR: $\boldsymbol{\delta}_{\mathbf{H}}(500 \mathrm{MHz}, 323 \mathrm{~K})$ Diastereoisomeric $7.68\left(2 \mathrm{H}, \mathrm{bm}, H_{\mathrm{a}^{\circ}}\right), 7.54(2 \mathrm{H}, \mathrm{bm}$, $\left.H_{\mathrm{a}}\right), 7.30\left(2 \mathrm{H}, \mathrm{bm}, H_{\mathrm{b}}\right), 7.20\left(2 \mathrm{H}, \mathrm{bm}, H_{\mathrm{b}}\right), 6.30+6.13(1 \mathrm{H}, \mathrm{bm}, \mathrm{FmocN} H), 4.34-$ $4.59(1 \mathrm{H}, \mathrm{bm}, \mathrm{FmocNHCH}), 4.30\left(2 \mathrm{H}, \mathrm{bm}, \mathrm{CH}_{2} \mathrm{OCONH}\right), 4.15\left(1 \mathrm{H}, \mathrm{bm}, H_{\mathrm{c}}\right), 3.73$ $\left(2 \mathrm{H}, \mathrm{bm}, \mathrm{NCH}_{2}\right), 3.57\left(1 \mathrm{H}, \mathrm{bm}, \mathrm{NCH}_{2} \mathrm{CH}\right), 2.90-3.27\left(2 \mathrm{H}, \mathrm{bm}, \mathrm{CH}_{2} \mathrm{~S}\right), 2.95(2 \mathrm{H}$, bm, $\left.\mathrm{C}=\mathrm{CCH}_{2}\right), 2.26\left(4 \mathrm{H}, \mathrm{bs}, \mathrm{CH}_{2} \mathrm{C}=\mathrm{O}\right), 1.87\left(1 \mathrm{H}, \mathrm{bm}, \mathrm{CH}\left(\mathrm{CH}_{3}\right)_{2}\right), 1.40(9 \mathrm{H}, \mathrm{bs}$, $\left.\left.\mathrm{C}\left(\mathrm{CH}_{3}\right)_{3}\right), 0.91\left(12 \mathrm{H}, \mathrm{bm},\left(\mathrm{CH}_{3}\right)_{2} \mathrm{CCH}_{2},\left(\mathrm{CH}_{3}\right)_{2} \mathrm{CH}\right)\right) ; \boldsymbol{c}(125.8 \mathrm{MHz}$, DEPT 135, HMQC, 323 K) 197.0, 176.3, 169.7, (169.0 + 168.9), 157.2, 156.2, (143.9 + 143.8), $141.3,(128.6+128.5),(128.0+127.9) 125.2,119.9,107.7,82.8,,(67.7+67.5)$, $(55.1+54.9), 52.9,47.9,(47.12+47.08), 45.6,37.0,34.0,29.7,29.0,28.1,28.0$, 22.5

Mass spectrum: $m / z(\mathrm{FAB}) \quad 825.2186\left([\mathrm{M}+\mathrm{Cs}]^{+} \cdot \mathrm{C}_{38} \mathrm{H}_{48} \mathrm{~N}_{2} \mathrm{O}_{8} \mathrm{SCs}\right.$ requires 825.2204), $m / z(\mathrm{ES}+) 693\left([\mathrm{M}+\mathrm{H}]^{+}, 72 \%\right), 715\left([\mathrm{M}+\mathrm{Na}]^{+}, 4 \%\right)$

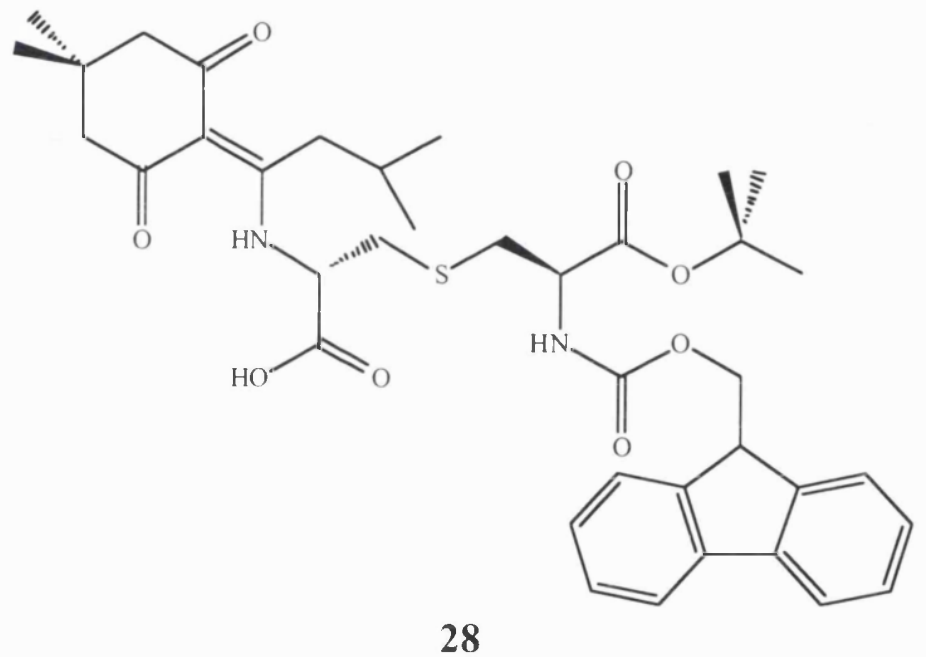

Figure 7.9. Intended product: 3-[( $R)$-2 -'butoxycarbonyl-2-(fluoren-9ylmethoxycarbonylamino)-ethylsulfanyl]-(S)-2-\{1-(4,4-dimethyl-2,6-dioxocyclohex1-ylidene)isovalerylamino\}-propionic acid 
22. ( $\mathrm{R} / \mathrm{S}$ )-2-/(R)-2- ${ }^{t}$ Butoxvcarbonvl-2-(fluoren-9-vlmethoxvcarbonvlamino)ethylsulfanvll-3-\{1-(4,4-dimethyl-2,6-dioxocvclohex-1-vlidene)isovalervlamino\}propionic acid 4-\{N-/1-(4,4-Dimethvl-2,6-dioxocvclohex-1vlidene)-3methvlbutvl/amino?benzvl ester

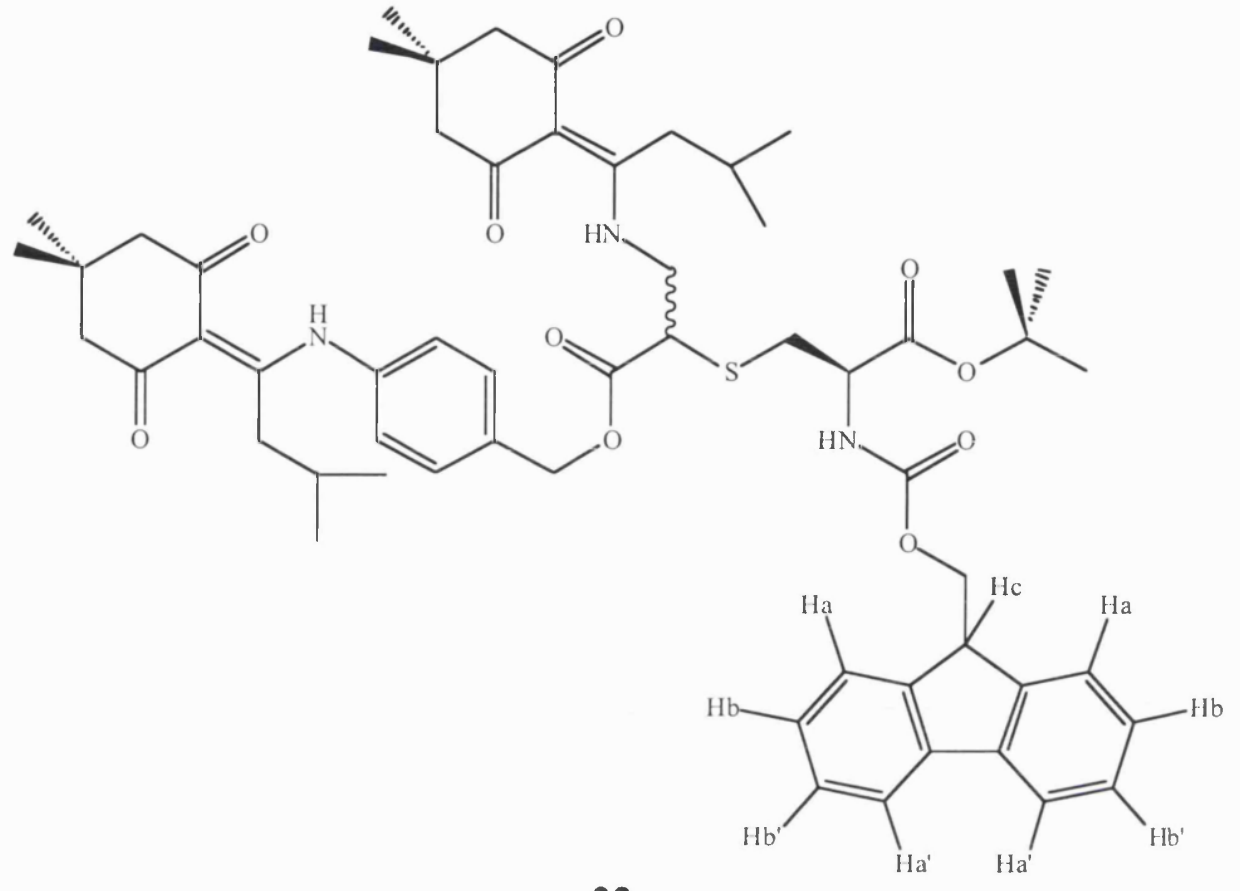

22

To a solution of crude $(R / S)-2-[(R)$ - 2 -'butoxycarbonyl-2-(fluoren-9ylmethoxycarbonylamino)-ethylsulfanyl\}-3-\{1-(4,4-dimethyl-2,6-dioxocyclohex-1ylidene)isovaleryl-amino -propionic acid 27 (1.5 g, crude) in DMF (20 ml) was added Dmab-OH (0.66 g, $2.0 \mathrm{mmol})$, dicyclohexylcarbodiimide $(0.4 \mathrm{~g}, 2.0 \mathrm{mmol}), 1$ hydroxybenzotriazole $(0.27 \mathrm{~g}, 2.0 \mathrm{mmol})$ and 4-dimethylaminopyridine $(0.24 \mathrm{~g}, 2.0$ $\mathrm{mmol}$ ). The mixture was stirred for $18 \mathrm{hr}$. The precipitated urea by-product was filtered off and the mixture was diluted with ethyl acetate $(200 \mathrm{ml})$. The organic layer was washed with citric acid $(5 \%$ aq. w/v, $50 \mathrm{ml})$ and water $(6 \times 50 \mathrm{ml})$ and dried over anhydrous sodium sulfate. Removal of the solvent yielded a yellow liquid, which foamed and solidified under highly reduced pressure at $-50{ }^{\circ} \mathrm{C}$ and used without further purification, in crude form. A small amount of the crude material was purified via preparative reverse phase HPLC for analyses $(70-90 \% \mathrm{MeCN}$ in water over $30 \mathrm{~min}, 15 \mathrm{ml} / \mathrm{min}, \mathrm{R}_{\mathrm{T}} 22.1$ ).

Yield: $100 \%$ (crude)

$\mathbf{R}_{\mathbf{f}}: 0.45$ (hexane:ethyl acetate, $1: 1$ ) 
I.R.: $v_{\max }\left(\mathrm{cm}^{-1}\right) 1728(\mathrm{C}=\mathrm{O}), 1566(\mathrm{C}=\mathrm{C}$ Ar $), 1450(\mathrm{C}=\mathrm{C}$ Ar $), 1215+1152(\mathrm{C}-\mathrm{S}-\mathrm{C})$ NMR: $\boldsymbol{\delta}_{\mathrm{H}}(500 \mathrm{MHz})$ Diastereoisomeric $7.74\left(2 \mathrm{H}, \mathrm{m}, H_{\mathrm{a}}\right), 7.58\left(2 \mathrm{H}, \mathrm{m}, H_{\mathrm{a}}\right), 7.33$ $7.40\left(2 \mathrm{H}, \mathrm{m}, H_{\mathrm{b}},+2 \mathrm{H}, \mathrm{m}, \mathrm{NHC}=\mathrm{CH}(\mathrm{Ar})\right), 7.24\left(2 \mathrm{H}, \mathrm{m}, H_{\mathrm{b}}\right), 7.06(2 \mathrm{H}, \mathrm{m}$, $\left.\mathrm{OCH}_{2} \mathrm{C}=\mathrm{CH}(\mathrm{Ar})\right), 5.22\left(2 \mathrm{H}, \mathrm{m}, \mathrm{ArOCH}_{2}\right), 4.55(1 \mathrm{H}, \mathrm{m}, \mathrm{FmocNHCH}), 4.33-4.44$ $\left(2 \mathrm{H}, \mathrm{m}, \mathrm{CH}_{2} \mathrm{OCONH}\right), 4.22\left(1 \mathrm{H}, \mathrm{m}, H_{\mathrm{c}}\right), 3.63-3.95\left(2 \mathrm{H}, \mathrm{m}, \mathrm{NCH}_{2}\right), 3.60(1 \mathrm{H}, \mathrm{m}$, $\left.\mathrm{NCH}_{2} \mathrm{CH}\right), 3.03-3.29\left(2 \mathrm{H}, \mathrm{m}, \mathrm{CH}_{2} \mathrm{~S}\right), 2.99\left(4 \mathrm{H}, \mathrm{bm}, \mathrm{C}=\mathrm{CCH}_{2}\right), 2.31-2.49(8 \mathrm{H}$, bm, $\left.\mathrm{CH}_{2} \mathrm{C}=\mathrm{O}\right), 1.92\left(1 \mathrm{H}, \mathrm{m}, \mathrm{CH}\left(\mathrm{CH}_{3}\right)_{2}\right), 1.81\left(1 \mathrm{H}, \mathrm{m}, \mathrm{CH}\left(\mathrm{CH}_{3}\right)_{2}\right), 1.45(9 \mathrm{H}, \mathrm{s}$, $\left.\mathrm{C}\left(\mathrm{CH}_{3}\right)_{3}\right), 1.07\left(6 \mathrm{H}, \mathrm{s},\left(\mathrm{CH}_{3}\right)_{2} \mathrm{CCH}_{2}\right), 0.96-0.99\left(12 \mathrm{H}, \mathrm{m},\left(\mathrm{CH}_{3}\right)_{2} \mathrm{CCH}_{2},\left(\mathrm{CH}_{3}\right)_{2} \mathrm{CH}\right)$, $0.75\left(6 \mathrm{H}, \mathrm{d}, J 6.7,\left(\mathrm{CH}_{3}\right)_{2} \mathrm{CH}\right) ; \boldsymbol{\delta}_{\mathrm{C}}(125.8 \mathrm{MHz}$, DEPT 135, HMQC) $(200.2+199.9)$, 196.3, ca.176.5, (170.1 + 169.9), (169.0 + 168.8), (155.9 + 155.8), (143.7 + 143.6), $(141.27+141.25),(137.12+137.10),(134.6+134.5), 129.2,127.7,127.0,126.7$, $(125.1+125.0), 120.0,107.6,107.5,(83.23+83.16), 67.1,(66.8+66.7),(54.2+$ $54.1), 53.7,52.3,47.1,(46.5+46.0),(44.1+43.8), 38.3,(34.8+34.7), 30.0,(29.83$ $+29.81), 29.5,(29.12+29.09), 28.22,28.17,(27.92+27.91), c a .22 .5$

Mass spectrum: $m / z(F A B) 1004.5095\left([\mathrm{M}+\mathrm{H}]^{+} \cdot \mathrm{C}_{58} \mathrm{H}_{74} \mathrm{~N}_{3} \mathrm{O}_{10} \mathrm{~S}\right.$ requires 1004.5020), $m / z(\mathrm{ES}+) 1026\left([\mathrm{M}+\mathrm{Na}]^{+}, 17 \%\right), 1004\left([\mathrm{M}+\mathrm{H}]^{+}, 9 \%\right)$

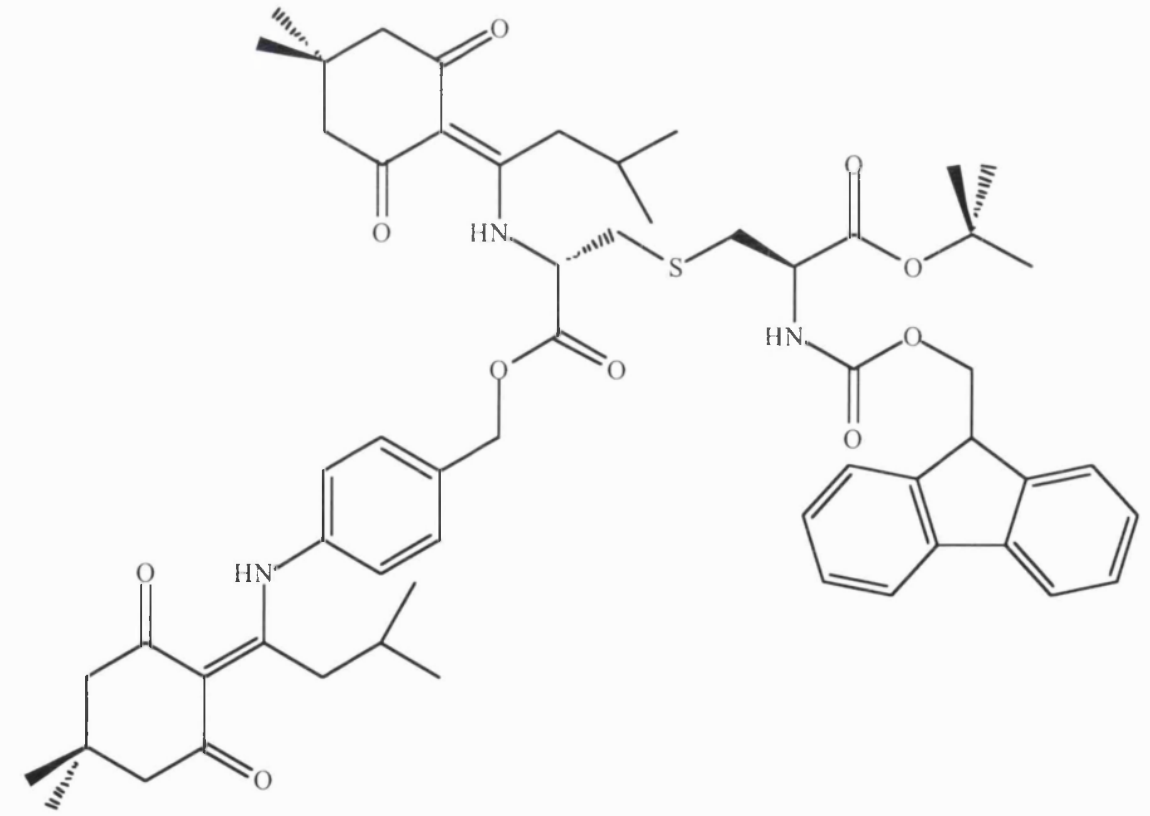

17

Figure 7.10. Intended product: 3-[(R)-2-'butoxycarbonyl-2-(fluoren-9ylmethoxycarbonylamino)-ethylsulfanyl]-(S)-2-\{1-(4,4-dimethyl-2,6-dioxocyclohex1 -ylidene)isovalerylamino $\}$-propionic acid 4-\{N-[1-(4,4-dimethyl-2,6dioxocyclohex-1ylidene)-3-methylbutyl]amino benzyl ester 


\section{methvlbutvl)aminolbenzvloxvcarbonvl\}-2-\{1-(4,4-dimethvl-2,6-dioxocvclohex-1-}

vlidene)isovalervlamino\}-ethvlsulfanvll-(R)-2-(fluoren-9-

vlmethoxvcarbonvlamino)-propionic acid

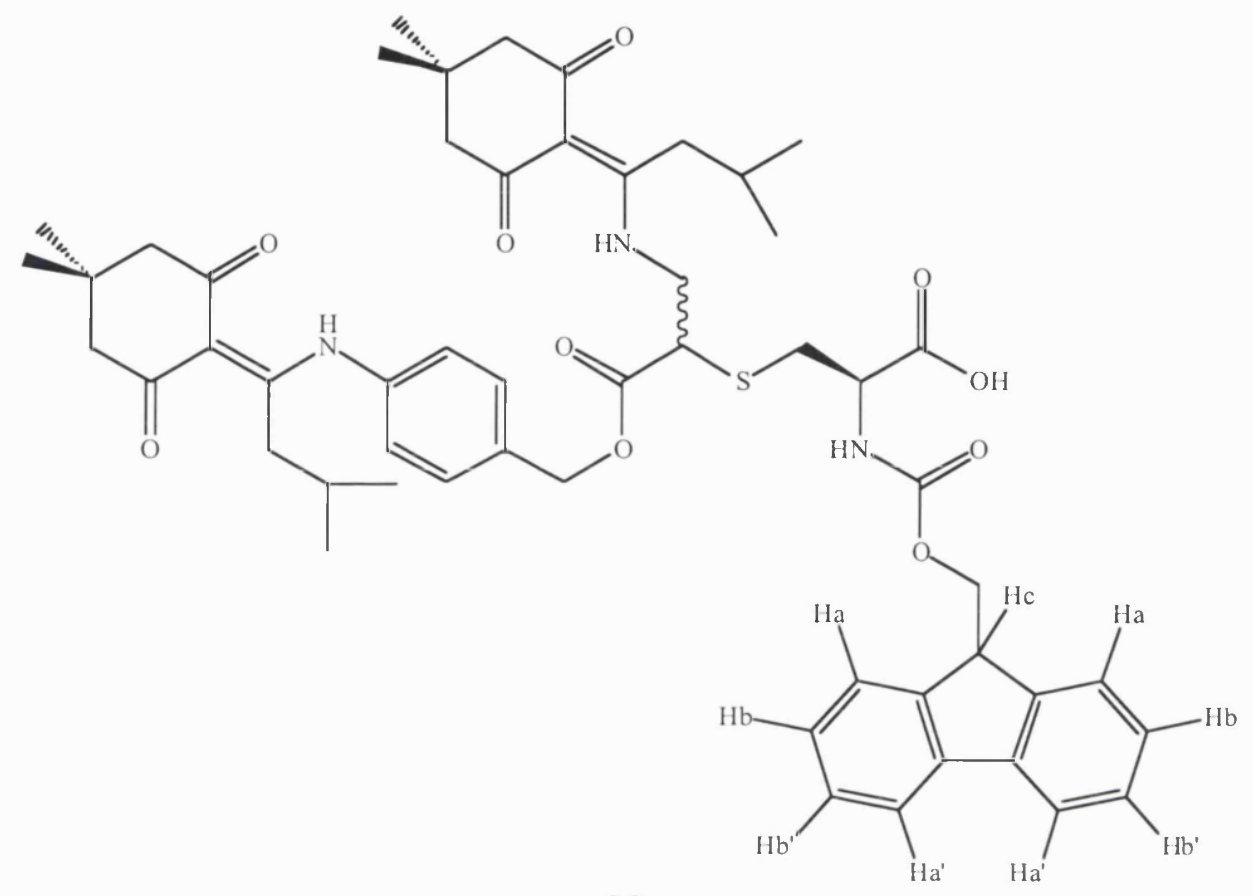

23

$(R / S)$-2-[(R)-2-'Butoxycarbonyl-2-(fluoren-9-ylmethoxycarbonylamino)-

ethylsulfanyl]-3-\{1-(4,4-dimethyl-2,6-dioxocyclohex-1-ylidene)isovaleryl-amino $\}$ -

propionic acid 4-\{N-[1-(4,4-Dimethyl-2,6-dioxocyclohex-1ylidene)-3methylbutyl]amino benzyl ester 22 ( $1.4 \mathrm{~g}$, crude) was treated with a solution of TFA ( $5 \mathrm{ml}$, excess) in DCM $(5 \mathrm{ml})$ and the mixture was stirred for $4 \mathrm{hr}$. The mixture was concentrated in vacuo and re-dissolved in DCM (100 ml) and MeOH (100 ml). The solvents were then removed yielding a yellow liquid, which foamed and solidified in vacuo. A small amount of the sample was purified via preparative reverse phase HPLC (40-60\% MeCN in water over $30 \mathrm{~min}, 15 \mathrm{ml} / \mathrm{min}, \mathrm{R}_{\mathrm{T}} 12.6$ ).

Yield: $100 \%$ (crude)

$\mathbf{R}_{\mathbf{f}}: 0.20$ (DCM:MeOH, 19:1)

I.R.: $v_{\max }\left(\mathrm{cm}^{-1}\right) 3400(\mathrm{OH}$, broad $), 1717(\mathrm{C}=\mathrm{O}), 1562(\mathrm{C}=\mathrm{C} \mathrm{Ar}), 1450(\mathrm{C}=\mathrm{C}$ Ar$)$, $1211+1151(\mathrm{C}-\mathrm{S}-\mathrm{C})$

NMR: $\boldsymbol{\delta}_{\mathbf{H}}(500 \mathrm{MHz})$ Diastereoisomeric $7.63\left(2 \mathrm{H}, \mathrm{m}, H_{\mathrm{a}^{\prime}}\right), 7.48\left(2 \mathrm{H}, \mathrm{m}, H_{\mathrm{a}}\right), 7.23$ $\left(2 \mathrm{H}, \mathrm{m}, H_{\mathrm{b}},+2 \mathrm{H}, \mathrm{m}, \mathrm{NHC}=\mathrm{CH}(\mathrm{Ar})\right), 7.11\left(2 \mathrm{H}, \mathrm{m}, H_{\mathrm{b}}\right), 6.92\left(2 \mathrm{H}, \mathrm{m}, \mathrm{OCH}_{2} \mathrm{C}=\mathrm{CH}\right.$ 
(Ar)), $5.07\left(2 \mathrm{H}, \mathrm{bm}, \operatorname{ArOCH}_{2}\right), 4.36(1 \mathrm{H}, \mathrm{bm}, \mathrm{FmocNHCH}), 4.20(2 \mathrm{H}, \mathrm{bm}$, $\left.\mathrm{CH}_{2} \mathrm{OCONH}\right), 4.09\left(1 \mathrm{H}, \mathrm{bm}, H_{\mathrm{c}}\right), 3.60-3.90\left(3 \mathrm{H}, \mathrm{bm}, \mathrm{NCH}_{2}, \mathrm{NCH}_{2} \mathrm{CH}\right), 2.98-$ $3.33\left(2 \mathrm{H}, \mathrm{bm}, \mathrm{CH}_{2} \mathrm{~S}\right), 2.91\left(4 \mathrm{H}, \mathrm{bm}, \mathrm{C}=\mathrm{CCH}_{2}\right), 2.12-2.49\left(8 \mathrm{H}, \mathrm{bm}, \mathrm{CH}_{2} \mathrm{C}=\mathrm{O}\right), 1.71$ - $1.88\left(2 \mathrm{H}, \mathrm{bm}, \mathrm{CH}\left(\mathrm{CH}_{3}\right)_{2}\right), 1.04\left(6 \mathrm{H}, \mathrm{s},\left(\mathrm{CH}_{3}\right)_{2} \mathrm{CCH}_{2}\right), 0.75-0.93(12 \mathrm{H}, \mathrm{bm}$, $\left.\left(\mathrm{CH}_{3}\right)_{2} \mathrm{CCH}_{2},\left(\mathrm{CH}_{3}\right)_{2} \mathrm{CH}\right), 0.68\left(6 \mathrm{H}, \mathrm{bm},\left(\mathrm{CH}_{3}\right)_{2} \mathrm{CH}\right) ; \delta_{\mathrm{C}}(125.8 \mathrm{MHz}$, DEPT 135 , HMQC) $200.0,196.2,176.8,176.2,170.3,156.6$, $(143.7+143.5), 141.0,137.0$, $134.2,129.0,127.7,127.0,126.5,125.0,119.9,107.7,67.2,66.8,55.0,53.6,52.1$, $46.9,45.9,43.9,38.2,34.3,30.0,29.9,29.7,29.6,29.4$, ca. 28.1, 22. 6, 22.5

Mass spectrum: $m / z(F A B) 970.4288\left([\mathrm{M}+\mathrm{H}]^{+} \cdot \mathrm{C}_{54} \mathrm{H}_{66} \mathrm{~N}_{3} \mathrm{O}_{10} \mathrm{Na}\right.$ requires $970.4310), m / z(E S+) 970\left([\mathrm{M}+\mathrm{Na}]^{+}, 10 \%\right), 949\left([\mathrm{M}+\mathrm{H}]^{+}, 92 \%\right),\left([\mathrm{Dmab}+\mathrm{H}]^{+}\right.$, $100 \%$ )

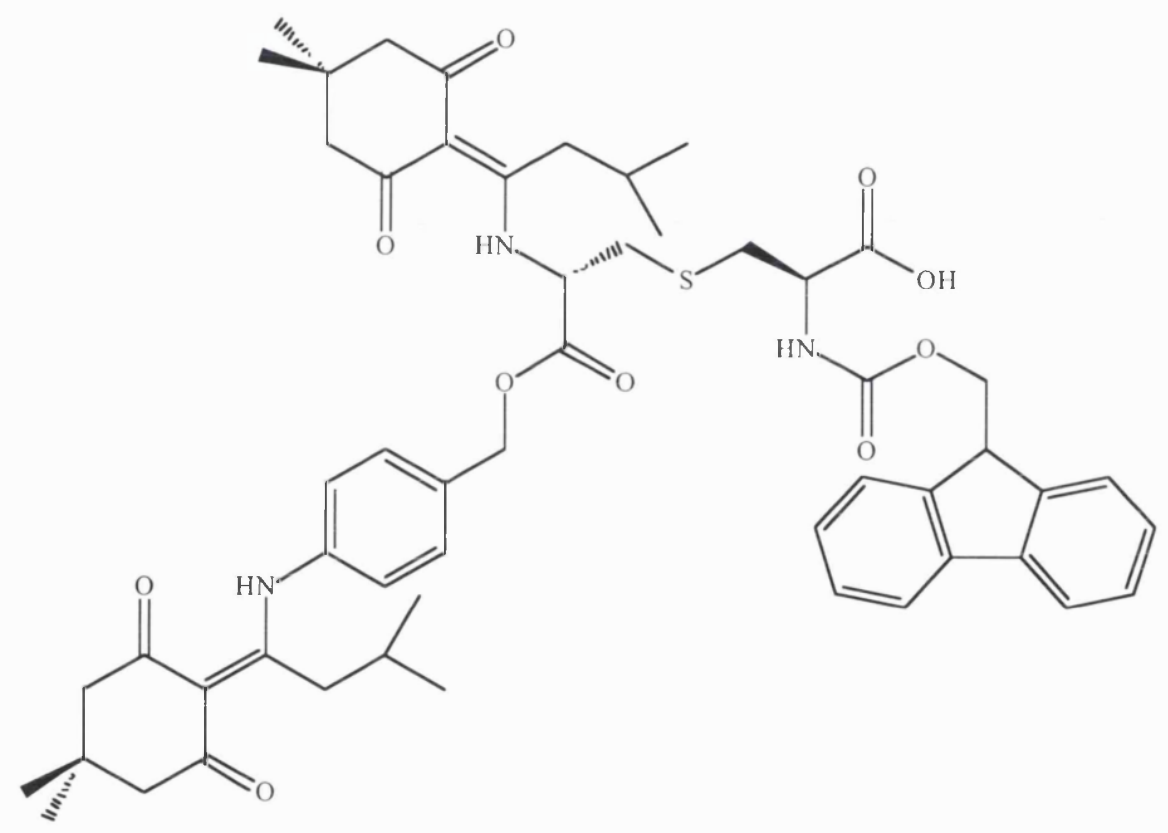

2

Figure 7.11. Intended product: $3-[(S)-2-\{4-[N-(1-(4,4-d i m e t h y l-2,6-d i o x o c y c l o h e x-$ 1-ylidene)-3-methylbutyl)amino]benzyloxycarbonyl $\}-2-\{1-(4,4-d i m e t h y l-2,6-$ dioxocyclohex-1-ylidene)isovalerylamino - -ethylsulfanyl]-( $R$ )-2-(fluoren-9ylmethoxy carbonylamino)-propionic acid 


\section{2-(1-Hydroxy-3-methyl-butvlidene)-5.5-dimethyl-cyclohexane-1,3-dione ${ }^{154}$}

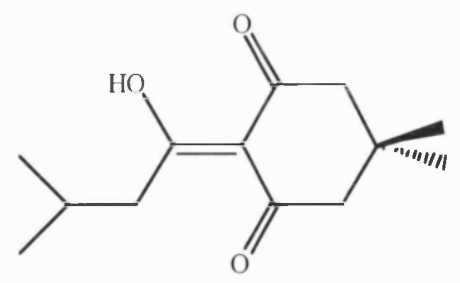

29

To a stirred solution of isovaleric acid $(0.11 \mathrm{ml}, 1.0 \mathrm{mmol})$ in DMF $(5 \mathrm{ml})$ was added dimedone $(0.14 \mathrm{~g}, 1.0 \mathrm{mmol})$, DCC $(0.40 \mathrm{~g}, 1.0 \mathrm{mmol})$ and DMAP $(0.12 \mathrm{~g}, 1.0$ mmol). The mixture was stirred for $48 \mathrm{hr}$, during which the urea by-product was precipitated. The solvent was removed in vacuo and re-dissolved in ethyl acetate (50 $\mathrm{ml}$ ). The solid urea was filtered off and the organic layer was washed with $\mathrm{KHSO}_{4}$ solution (1 M, $20 \mathrm{ml})$. Extraction was carried out with $\mathrm{NaOH}(5 \%$ aq., 3 x $50 \mathrm{ml})$ and the aqueous layers were combined and neutralised with acetic acid. Further extraction was carried out using DCM $(5 \times 50 \mathrm{ml})$. The organic layer was dried with anhydrous sodium sulfate and removal of the solvent yielded the product as a yellow liquid.

Yield: $0.18 \mathrm{~g}, 0.8 \mathrm{mmol}, 80 \%$

NMR: $\delta_{\mathbf{H}}(300 \mathrm{MHz}) 2.92\left(2 \mathrm{H}, \mathrm{d}, \mathrm{Me}_{2} \mathrm{CHCH}_{2} \mathrm{COH}\right), 2.45\left(4 \mathrm{H}, \mathrm{m}, \mathrm{CH}_{2} \mathrm{CMe}_{2} \mathrm{CH}_{2}\right)$, $2.13\left(1 \mathrm{H}, \mathrm{m}, \mathrm{Me}_{2} \mathrm{CHCH}_{2} \mathrm{COH}\right), 1.08\left(6 \mathrm{H}, \mathrm{s}, \mathrm{CH}_{2} \mathrm{CMe}_{2} \mathrm{CH}_{2}\right), 0.97(6 \mathrm{H}, \mathrm{d}$, $\left.\mathrm{Me}_{2} \mathrm{CHCH}_{2} \mathrm{COH}\right)$

Mass spectrum: $m / z(F A B) 225\left([\mathrm{M}+\mathrm{H}]^{+}, 100 \%\right)$

\section{Attempted synthesis of $N$-Triphenylmethyl-(R)-serine 4-IN-\{1-(4,4-dimethyl-}

\section{$\underline{\text { 2,6-dioxocvclohex-1-vlidene)-3-methylbutvl\}aminolbenzyl ester }}$}

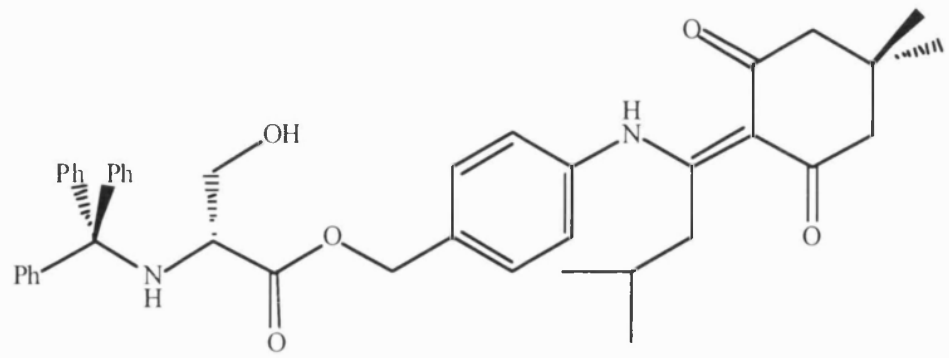

13

$N$-triphenylmethyl- $(R)$-serine $(\boldsymbol{R})-3(0.10 \mathrm{~g}, 0.29 \mathrm{mmol})$ was treated with Dmab-OH (0.099g, $0.29 \mathrm{mmol})$, diisopropylcarbodiimide $(0.045 \mathrm{ml}, 0.59 \mathrm{mmol}, 2$ equiv.), 1 - 
hydroxybenzotriazole $(0.040 \mathrm{~g}, 0.29 \mathrm{mmol})$ and diisopropylethylamine $(0.035 \mathrm{ml}$, $0.20 \mathrm{mmol}, 0.7$ equiv.) in dichloromethane. The mixture was stirred for 18 hours and then diluted with dichloromethane $(20 \mathrm{ml})$ and washed with citric acid $(5 \%$, aq., $10 \mathrm{ml})$ and water $(2 \times 10 \mathrm{ml})$. The organic layer was dried over anhydrous sodium sulfate. The product was not formed, as indicated by mass spectrometry (ES+), t.l.c. and ${ }^{1} \mathrm{H}$ NMR.

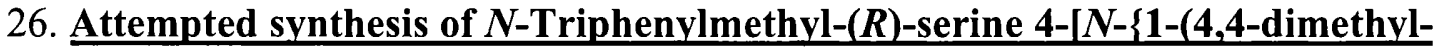

\section{2,6-dioxocyclohex-1-ylidene)-3-methylbutyl\}aminolbenzyl ester}

$N$-triphenylmethyl- $(R)$-serine $(\boldsymbol{R})-3(0.050 \mathrm{~g}, 0.14 \mathrm{mmol})$ was treated with Dmab-OH $(0.047 \mathrm{~g}, 0.14 \mathrm{mmol})$, dicyclohexylcarbodiimide $(0.059 \mathrm{~g}, 0.29 \mathrm{mmol}, 2$ equiv.) and diisopropylethylamine $(0.035 \mathrm{ml}, 0.20 \mathrm{mmol}, 0.7$ equiv. $)$ in dichloromethane. The mixture was stirred for 18 hours. The urea by-product was filtered and the mixture was then diluted with dichloromethane $(10 \mathrm{ml})$ and washed with citric acid (5\%, aq., $5 \mathrm{ml})$ and water $(2 \times 10 \mathrm{ml})$. The organic layer was dried over anhydrous sodium sulfate. The product was not formed, as indicated by mass spectrometry (ES+), t.l.c. and ${ }^{1} \mathrm{H}$ NMR.

\section{Attempted synthesis of $N$-Triphenylmethyl-(R)-serine 4-[N-\{1-(4,4-dimethyl-}

\section{2,6-dioxocyclohex-1-ylidene)-3-methylbutyl\}aminolbenzyl ester}

$N$-triphenylmethyl- $(R)$-serine $(R)-3(0.050 \mathrm{~g}, 0.14 \mathrm{mmol})$ was treated with Dmab-OH $(0.047 \mathrm{~g}, 0.14 \mathrm{mmol})$, dicyclohexylcarbodiimide $(0.059 \mathrm{~g}, 0.59 \mathrm{mmol}, 2$ equiv.) and 4-dimethylaminopyridine $(0.018 \mathrm{~g}, 0.14 \mathrm{mmol})$ in dichloromethane. The mixture was stirred for 18 hours. The urea by-product was filtered and then diluted with dichloromethane $(20 \mathrm{ml})$ and washed with citric acid $(5 \%$, aq., $10 \mathrm{ml})$ and water $(2 \mathrm{x}$ $10 \mathrm{ml})$. The organic layer was dried over anhydrous sodium sulfate. The product was not formed, as indicated by mass spectrometry (ES+), t.l.c. and ${ }^{1} \mathrm{H}$ NMR. 


\section{8. (R)-2-triphenvlmethvlamino-3-'butvldimethvlsilvloxv-propionic acid}

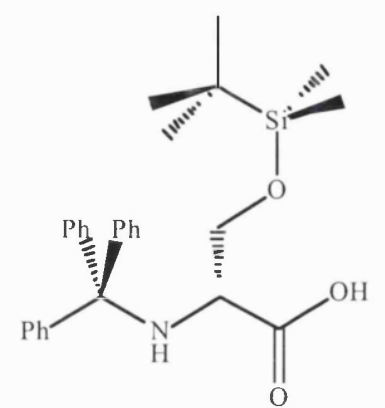

14

To a stirred solution of $N$-triphenylmethyl- $(R)$-serine $(\boldsymbol{R})-3(0.10 \mathrm{~g}, 0.29 \mathrm{mmol})$ in DMF $(0.2 \mathrm{ml})$ was added tert-butyldimethylsilyl chloride $(0.052 \mathrm{~g}, 0.35 \mathrm{mmol}, 1.2$ equiv.) and imidazole ( $0.049 \mathrm{~g}, 0.72 \mathrm{mmol}, 2.5$ equiv.) and the mixture was stirred for 10 hours. The mixture was diluted with ethyl and then washed with water $(3 \times 10$ $\mathrm{ml})$ and brine $(10 \mathrm{ml})$. The organic layer was then dried over anhydrous sodium sulfate and removal of the solvent yielded a viscous, colourless liquid. The compound was used without further purification in the presence of the tertbutyldimethylsilyl alcohol by-product.

Yield: $100 \%$ (crude)

$\mathbf{R}_{\mathbf{f}}: 0.55$ (hexane: ethyl acetate, $2: 1$ )

NMR: $\boldsymbol{\delta}_{\mathbf{H}}(400 \mathrm{MHz}) 7.21-7.46(15 \mathrm{H}, \mathrm{m}, \mathrm{Trt}(\mathrm{Ar})), 3.68\left(1 \mathrm{H}, \mathrm{m}, \mathrm{CH}_{2} \mathrm{OSi}\right), 3.42$ $(1 \mathrm{H}, \mathrm{m}, \mathrm{TrtNHCH}), 2.67\left(1 \mathrm{H}, \mathrm{m}, \mathrm{CH}_{2} \mathrm{OSi}\right), 0.93\left(9 \mathrm{H}, \mathrm{s}, \mathrm{Si}\left(\mathrm{CCH}_{3}\right)_{3}\right), 0.11(6 \mathrm{H}, \mathrm{s}$, $\left.\mathrm{Si}\left(\mathrm{CH}_{3}\right)_{2}\right)$

Mass spectrum: $m / z(E S+) 466\left([\mathrm{M}+\mathrm{H}]^{+}, 0.1 \%\right), 223\left([\mathrm{M}-\mathrm{Trt}+\mathrm{H}]^{+}, 0.2 \%\right), 243$ $\left(\mathrm{Trt}^{+}, 100 \%\right)$ 


\section{1vlidene)-3-methylbutyl\} aminolbenzyl ester}<smiles>CC(C)CC(Nc1ccc(COC(=O)[C@H](CO[Si](C)(C)C(C)(C)C)NC(c2ccccc2)c2ccccc2)cc1)=C1C(=O)CC(C)CC1=O</smiles>

30

2-triphenylmethylamino-3-'butyldimethylsilyloxy-propionic acid 37 (0.033g, 80 $\mu \mathrm{mol})$ in DMF $(1 \mathrm{ml})$ was treated with Dmab-OH $(0.025 \mathrm{~g}, 80 \mu \mathrm{mol})$, dicyclohexylcarbodiimide $(0.016 \mathrm{~g}, 80 \mu \mathrm{mol}), 1$-hydroxybenzotriazole $(0.010 \mathrm{~g}, 80$ $\mu \mathrm{mol})$ and 4-dimethylaminopyridine $(0.0093 \mathrm{~g}, 80 \mu \mathrm{mol})$ under inert conditions at room temperature. The reaction mixture was stirred for 18 hours. The mixture was then diluted with ethyl acetate $(20 \mathrm{ml})$ and the urea by-product was filtered off. The organic layer was then washed with citric acid (5\%, aq., $10 \mathrm{ml})$ and water $(2 \times 10$ $\mathrm{ml}$ ). The organic layer was dried over anhydrous sodium sulfate. Removal of the solvent yielded a mixture of viscous liquid and solids. T.l.c. and mass spectrometry indicated that the reaction had not worked, isolating starting materials only.

30. 4 - /N-(1-\{4.4-Dimethvl-2.6-dioxocvclohex-1vlidene)-3-

\section{methvlbutvllaminolbenzvl bromide}<smiles>CC(C)CC(Nc1ccc(CBr)cc1)=C1C(=O)CC(C)(C)CC1=O</smiles>

15

Bromine $(0.01 \mathrm{ml}, 0.2 \mathrm{mmol}, 1.3$ equiv.) was added to a solution of triphenylphosphine $(0.040 \mathrm{~g}, 0.15 \mathrm{mmol})$ in dichloromethane $(2.5 \mathrm{ml})$ at $0{ }^{\circ} \mathrm{C}$. Upon warming to room temperature Dmab-OH $(0.050 \mathrm{~g}, 0.15 \mathrm{mmol})$ in dichloromethane 
$(2.5 \mathrm{ml})$ was added and the reaction mixture was left stirring for 5 hours. The mixture was then diluted with dichloromethane $(50 \mathrm{ml})$ and washed with sodium hydrogen carbonate $(5 \%$, aq., $2 \times 25 \mathrm{ml})$, water $(2 \times 25 \mathrm{ml})$ and finally brine $(2 \times 25$ $\mathrm{ml}$ ). Removal of the solvent yielded a viscous yellow-brown liquid. Purification attempts were unsuccessful.

Mass spectrum: $m / z(\mathrm{ES}+) 435\left(\left[\mathrm{M}+{ }^{81} \mathrm{Br}+\mathrm{K}\right]^{+}, 20 \%\right), 433\left(\left[\mathrm{M}+{ }^{81} \mathrm{Br}+\mathrm{K}\right]^{+}, 19\right.$ $\left.\%), 394\left(\left[\mathrm{M}+{ }^{81} \mathrm{Br}\right]^{+}, 100 \%\right), 392\left(\left[\mathrm{M}+{ }^{79} \mathrm{Br}\right]\right]^{+}, 98 \%\right)$

\section{1. (R)-1-Triphenvlmethvl-aziridine-2-carboxvlic acid allvl ester}

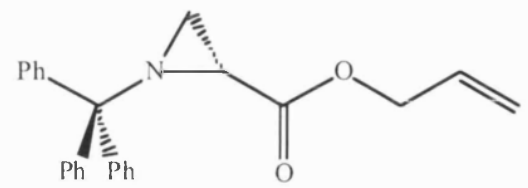

(R)-11

$N$-Triphenylmethyl- $(R)$-serine( $O$-methanesulfonyl) allyl ester $(\boldsymbol{R})-5(0.10 \mathrm{~g}, 0.21$ mmol) was dissolved in acetonitrile $(2.5 \mathrm{ml})$ and treated with $N, N$ diisopropylethylamine $(0.04 \mathrm{ml}, 0.43 \mathrm{mmol}, 2$ equiv. $)$. The reaction was stirred for 18 hours at room temperature. The reaction mixture was diluted with ethyl acetate $(20 \mathrm{ml})$ and washed with citric acid $(5 \%$ aq., $10 \mathrm{ml})$ and water $(2 \times 10 \mathrm{ml})$ and dried over anhydrous sodium sulfate. Removal of the solvent yielded a mixture of colourless liquid and white solid. The aziridine derivative was purified via normal phase preparative HPLC (0-4\% ethyl acetate in hexane over 40 min, DCM loading, $15 \mathrm{ml} / \mathrm{min}, \mathrm{R}_{\mathrm{t}} 37.0$ ) to give a colourless liquid.

Yield: $0.0096 \mathrm{~g}, 0.026 \mathrm{mmol}, 12 \%$

$\mathbf{R}_{\mathbf{f}}: 0.60$ (hexane:ethyl acetate. $2: 1$ )

NMR: $\delta_{\mathbf{H}}(300 \mathrm{MHz}) 7.43(6 \mathrm{H}, \mathrm{m}, \mathrm{Trt}), 7.17(9 \mathrm{H}, \mathrm{m}, \mathrm{Trt}), 5.87\left(1 \mathrm{H}, \mathrm{m}, \mathrm{CH}=\mathrm{CH}_{2}\right)$, $5.25\left(1 \mathrm{H}, \mathrm{dd}, J 17.2,1.3, \mathrm{CH}=\mathrm{CH}_{2}\right), 5.20\left(1 \mathrm{H}, \mathrm{dd}, J 10.4,1.4, \mathrm{CH}=\mathrm{CH}_{2}\right), 4.61(1 \mathrm{H}$, $\left.\mathrm{d}, J 4.5, \mathrm{OCH}_{2} \mathrm{CH}\right), 4.60\left(1 \mathrm{H}, \mathrm{d}, J 4.5, \mathrm{OCH}_{2} \mathrm{CH}\right), 2.20(1 \mathrm{H}, \mathrm{dd}, J 2.6,1.6$, $\left.\mathrm{NCHCO}_{2}\right), 1.84\left(1 \mathrm{H}, \mathrm{dd}, J 6.2,2.6, \mathrm{NCH}_{2} \mathrm{CH}\right), 1.34\left(1 \mathrm{H}, \mathrm{dd}, J 6.2,1.5, \mathrm{NCH}_{2} \mathrm{CH}\right)$; $\delta_{\mathrm{C}}(75.4 \mathrm{MHz}, \mathrm{DEPT} 135) 171.5,144.0,132.4,129.7,128.0,127.3,118.9,65.9,32.2$, 29.1 
Mass spectrum: $m / z(F A B) 392.1626\left([\mathrm{M}+\mathrm{Na}]^{+} . \mathrm{C}_{25} \mathrm{H}_{23} \mathrm{NO}_{2} \mathrm{Na}\right.$ requires 392.1645), $m / z(\mathrm{ES}+) 370\left([\mathrm{M}+\mathrm{H}]^{+}, 9 \%\right), 243\left(\mathrm{Trt}^{+}, 100 \%\right) ;(\mathrm{FAB}) 393\left([\mathrm{M}+\mathrm{Na}+\mathrm{H}]^{+}, 13\right.$ $\%), 243\left(\mathrm{Trt}^{+}, 100 \%\right)$

32. N-Triphenvlmethvl-3-iodo-(S)-alanine allvl ester (via aziridine ring-opening)

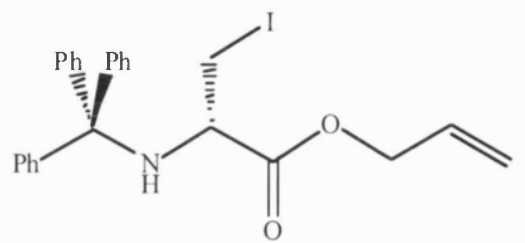

$(S)-6 a$

(R)-N-triphenylmethyl-aziridine-2-carboxylic acid allyl ester $(\boldsymbol{R})-11(0.012 \mathrm{~g}, 33$ $\mu \mathrm{mol})$ was treated with sodium iodide $(0.049 \mathrm{~g}, 0.33 \mathrm{mmol}, 10$ equiv. $)$ in acetone $(0.5 \mathrm{ml})$ under argon and the mixture was stirred for $72 \mathrm{hr}$ at $25^{\circ} \mathrm{C}$. The solvent was then removed in vacuo. Ether $(10 \mathrm{ml})$ was added and sodium thiosulfate $(10 \% \mathrm{aq}$. $\mathrm{w} / \mathrm{v}, 1 \mathrm{ml}$ approx.) was then slowly added, decolourising the organic layer to a pale yellow solution. The organic layer was separated and dried over anhydrous sodium sulfate and the solvent was removed in vacuo. The crude product was purified by HPLC, however, incomplete separation of the product and its regioisomer $(1: 4$ approx., respectively) was attempted using preparative normal phase HPLC $(2-3 \%$ ethyl acetate in hexane over 60 minutes, $15 \mathrm{ml} / \mathrm{min}$ with DCM loading, $\mathrm{R}_{\mathrm{T}} 46.0,47.1$ (regioisomer)). The purification isolated the starting material at $\mathrm{R}_{T} 53.6$ as the predominant species.

Yield: $0.0044 \mathrm{~g}, 8.8 \mu \mathrm{mol}, 27 \%$ (overall); $0.0010 \mathrm{~g}, 1.9 \mu \mathrm{mol}, 6 \%$ (desired product, based on NMR)

$\mathbf{R}_{\mathbf{f}}: 0.60$ (hexane:ethyl acetate. $2: 1$ )

NMR: $\boldsymbol{\delta}_{\mathbf{H}}(600 \mathrm{MHz}) 7.51(6 \mathrm{H}, \mathrm{m}, \mathrm{Trt}), 7.29(6 \mathrm{H}, \mathrm{m}, \mathrm{Trt}), 7.20(3 \mathrm{H}, \mathrm{m}, \mathrm{Trt}), 5.73$ $\left(1 \mathrm{H}, \mathrm{m}, \mathrm{CH}=\mathrm{CH}_{2}\right), 5.22\left(1 \mathrm{H}, \mathrm{dd}, J 17.3,1.4, \mathrm{CH}=\mathrm{CH}_{2}\right), 5.19(1 \mathrm{H}, \mathrm{dd}, J 10.2,1.1$, $\left.\mathrm{CH}=\mathrm{CH}_{2}\right), 4.22\left(1 \mathrm{H}, \mathrm{dd}, J 13.1,6.1, \mathrm{OCH}_{2} \mathrm{CH}\right), 4.09\left(1 \mathrm{H}, \mathrm{dd}, J 13.1,6.1, \mathrm{OCH}_{2} \mathrm{CH}\right)$, $3.50\left(1 \mathrm{H}, \mathrm{m}, \mathrm{HNCHCO}_{2}\right), 3.30\left(1 \mathrm{H}, \mathrm{dd}, J 9.8,3.4, \mathrm{CHCH}_{2} \mathrm{I}\right), 3.23(1 \mathrm{H}, \mathrm{dd}, J 9.8$, 6.9, $\left.\mathrm{CHCH}_{2} \mathrm{I}\right), 2.88(1 \mathrm{H}, \mathrm{d}, J 9.8, \operatorname{TrtNHCH})$

(R/S)-2-iodo-3-(triphenylmethylamino) propionic acid allyl ester (mixture of enantiomers): $\delta_{\mathrm{H}}(600 \mathrm{MHz}) 7.52(6 \mathrm{H}, \mathrm{m}, \mathrm{Trt}), 7.30(6 \mathrm{H}, \mathrm{m}, \mathrm{Trt}), 7.21(3 \mathrm{H}, \mathrm{m}, \mathrm{Trt})$, $5.94\left(1 \mathrm{H}, \mathrm{m}, \mathrm{CH}=\mathrm{CH}_{2}\right), 5.40\left(1 \mathrm{H}, \mathrm{dd}, J 17.2,1.4, \mathrm{CH}=\mathrm{CH}_{2}\right), 5.30(1 \mathrm{H}, \mathrm{dd}, J 10.5$, 
1.1, $\left.\mathrm{CH}=\mathrm{CH}_{2}\right), 4.67\left(2 \mathrm{H}, \mathrm{m}, \mathrm{OCH}_{2} \mathrm{CH}\right), 4.42\left(1 \mathrm{H}, \mathrm{m}, \mathrm{CHICO}_{2}\right), 2.73(1 \mathrm{H}, \mathrm{dd}, J 12.9$, 8.5, $\left.\mathrm{HNCH}_{2} \mathrm{CHI}\right), 2.57$ (1 H, dd, $\left.J 12.9,5.9, \mathrm{HNCH}_{2} \mathrm{CHI}\right), 2.27\left(\mathrm{TrtNHCH}_{2}\right)$

Mass spectrum: $m / z(\mathrm{ES}+) 520\left([\mathrm{M}+\mathrm{Na}]^{+}, 7 \%\right), 243\left(\mathrm{Trt}^{+}, 100 \%\right)$<smiles>C=CCOC(=O)C(I)CNC(c1ccccc1)c1ccccc1</smiles>

6b

Figure 7.12. Major product: $(R / S)$-2-iodo-3-(triphenylmethylamino) propionic acid allyl ester

33. Attempted synthesis of 3-I(R)-2-'Butoxycarbonyl-2-(fluoren-9-vlmethoxvcarbonylamino)-ethylsulfanvll-(S)-2-(triphenylmethvlamino)-propionic acid allyl ester (via aziridine ring-opening)

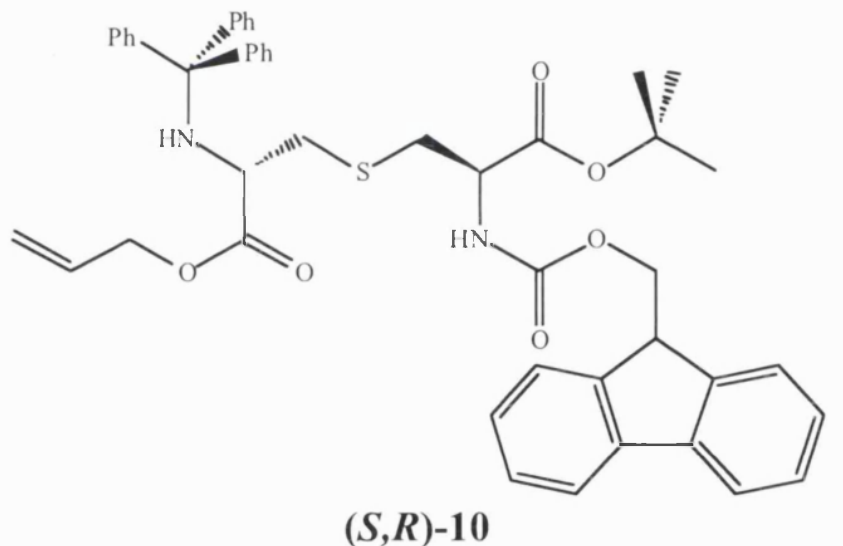

$N$-9-Fluorenylmethoxycarbonyl-( $R$ )-cysteine tbutyl ester 9 (0.0088 g, $22 \mu \mathrm{mol})$ was dissolved in dry DMF $(0.15 \mathrm{ml})$ under inert conditions. To it a solution of $(R)-N$ triphenylmethyl-aziridine-2-carboxylic acid allyl ester $(\boldsymbol{R})-11(0.0081 \mathrm{~g}, 22 \mu \mathrm{mol})$ in DMF $(0.15 \mathrm{ml})$ was added. Cesium carbonate $(0.0072 \mathrm{~g}, 22 \mu \mathrm{mol})$ was then added and the mixture was stirred for $4 \mathrm{hr}$ at $25^{\circ} \mathrm{C}$. The solvent was removed in vacuo and the residue was partly dissolved in ethyl acetate $(10 \mathrm{ml})$. The organic layer was washed with citric acid ( $5 \%$ aq. w/v, $1 \mathrm{ml})$, dissolving the remainder of the residue. The organic layer was then washed with water $(4 \times 5 \mathrm{ml})$ and dried over anhydrous sodium sulfate. Removal of the solvent in vacuo yielded a mixture of pale yellow oil and white solids. 'H NMR of the crude material revealed that the reaction had not taken place, producing signals for the aziridine derivative and dibenzofulvene only. 
General procedure for solid phase peptide synthesis with double-headed amino acids

\section{Batch synthesis}

All glassware is placed in the oven one day prior to synthesis. The resin used is suspended in DMF ( $5 \mathrm{ml}$ ) for $20 \mathrm{~min}$ in a vial and then transferred to the bubbler.

A. Removal of the solvent was followed by Fmoc deprotection of the resin, or, the pre-loaded amino acid on the resin using a piperidine solution in DMF $(20 \%, 4 \mathrm{ml})$ with vigorous argon bubbling for 20 minutes. The resin is then thoroughly washed with DMF (6 x $10 \mathrm{ml})$ after which the qualitative Kaiser test, to check the presence of free amines, is performed on a small amount of the resin with an anticipated positive result, yielding a blue-purple colouration.

B. The resin is then washed with DCM $(3 \times 10 \mathrm{ml})$. A small amount of the resin was taken (10 $\pm 0.1 \mathrm{mg}$ approx.), dried, treated with a solution of piperidine in DMF (30 $\%, 0.5 \mathrm{ml}$ ) and left to stand for $30 \mathrm{~min}$. Ethanol (abs., $19.5 \mathrm{ml}$ ) is then added and the mixture is left to settle. The aliquot is transferred into a U.V. cell and checked for a negative result for piperidyl-dibenzofulvene adduct $(\lambda 300 \mathrm{~nm})$.

C. 3-[(R/S)-1-Allyloxycarbonyl-2-(allyloxycarbonylamino)-ethylsulfanyl $]-(R)-2-$ (fluoren-9-ylmethoxycarbonylamino)-propionic acid 20 (0.13 g, $0.25 \mathrm{mmol}, 2.5$ equiv.) is activated under inert conditions, in a separate vial, with a HOBt solution in DMF (0.033 g, 0.25 mmol, 2.5 equiv., $0.25 \mathrm{M}, 1 \mathrm{ml}$ ) and a DIC solution in DMF $(0.039 \mathrm{ml}, 0.25 \mathrm{mmol}, 2.5$ equiv., $0.25 \mathrm{M}, 1 \mathrm{ml})$ for $20 \mathrm{~min}$. The activated amino acid solution is then added to the resin with bubbling for $30 \mathrm{~min}$. After $15 \mathrm{~min}$ of the first coupling, another batch of 3-[(R/S)-1-allyloxycarbonyl-2-(allyloxycarbonylamino)-ethylsulfanyl]-(R)-2-(fluoren-9-ylmethoxycarbonylamino)-propionic acid 20 $(0.13 \mathrm{~g}, 0.25 \mathrm{mmol}, 2.5$ equiv. $)$ is activated in exactly the same manner. Removal of the first coupling solution is followed by addition of the second, with the same coupling duration. The resin is then washed with DMF $(6 \times 10 \mathrm{ml})$ after removal of 
the second coupling solution. A Kaiser test is performed with an anticipated negative result.

D. The resin is then capped with a solution of 1-acetylimidazole in DMF $(0.16 \mathrm{~g}, 1.5$ mmol, $0.3 \mathrm{M}, 4 \mathrm{ml}$ ). Steps A, B, C and D are repeated for the subsequent amino acid in the sequence and steps $\mathrm{A}, \mathrm{C}$ and $\mathrm{D}$ only are repeated thereafter.

E. Simultaneous allylic deprotection is performed using tetrakistriphenylphosphine palladium(0) ( $0.23 \mathrm{~g}, 0.20 \mathrm{mmol}, 2$ equiv. $)$, dissolved by gentle heating in a solution of $\mathrm{DMF} / \mathrm{CHCl}_{3} / \mathrm{AcOH} / \mathrm{NMM}$ (18.5:18.5:2:1 volume, $1.85 / 1.85 / 0.2 / 0.1 \mathrm{ml}$, where the concentration of palladium $(0)$ catalyst in the cocktail solution $=0.050 \mathrm{M})$. The mixture is bubbled for $2 \mathrm{hr}$ in the dark. The resin is then washed with $0.5 \% \mathrm{v} / \mathrm{v}$ DIEA in DMF $\left(\begin{array}{llll}4 & \mathrm{x} & 5 \mathrm{ml}\end{array}\right)$, DMF $\left(\begin{array}{lllllll}6 & \mathrm{x} & 10 & \mathrm{ml}\end{array}\right), 0.5 \% \mathrm{w} / \mathrm{v}$ sodium diethyldithiocarbamate trihydrate in DMF $(4 \times 5 \mathrm{ml})$ and more DMF $(6 \times 10 \mathrm{ml})$.

A Kaiser test is performed at this stage. This is followed by Fmoc deprotection as before (Kaiser test should yield a richer purple coloration, due to the presence of 2 free amines) and a $\mathrm{HOBt} / \mathrm{DMF}$ acidic wash $(0.4 \% \mathrm{w} / \mathrm{v}, 5 \mathrm{ml})$ - to prevent any unwanted piperidyl-amide formation at the free carboxy end ${ }^{217}-$ This is followed by further DMF washes $(2 \times 10 \mathrm{ml})$.

The cyclisation reagents are prepared during the Fmoc deprotection. PyAOP $(0.26 \mathrm{~g}$, 0.5 mmol, 5 equiv. $)$ is dissolved in DMF $(1 \mathrm{ml})$ and mixed with a solution of HOAt ( $0.068 \mathrm{~g}, 0.5 \mathrm{mmol}, 5$ equiv.) in DMF $(1 \mathrm{ml})$. The mixture is transferred to the bubbler and a solution of DIEA $(0.18 \mathrm{ml}, 1.0 \mathrm{mmol}, 10$ equiv.) in DMF $(0.5 \mathrm{ml})$ is added 1 min later. The mixture is bubbled for $1 \mathrm{hr}$ followed by DMF washings $(6 \mathrm{x}$ $10 \mathrm{ml}$ ). Steps $\mathrm{A}, \mathrm{C}$ and $\mathrm{D}$ are repeated for the appropriate amino acid.

F. Removal of the Fmoc group on the terminal residue is followed by DMF washes $(6 \times 10 \mathrm{ml})$ and DCM washes $(6 \times 10 \mathrm{ml})$. The cleavage of the peptide is carried out by treating the resin with a $5 \%$ TFA, $2.5 \% \mathrm{H}_{2} \mathrm{O}, 2.5 \%$ TES solution in DCM $(5 \mathrm{x}$ $10 \mathrm{ml}, 1 \mathrm{~min}$ each portion), collected in a flask containing TFA $(50 \mathrm{ml})$ with 
vigorous stirring for $3 \mathrm{hr}$. The mixture is then concentrated in vacuo to approximately $1 \mathrm{ml}$, after which cold ether is added $(100 \mathrm{ml})$. The product is precipitated in the fridge overnight. The precipitate is collected by filtration, dissolved in a solution of $20 \% \mathrm{AcOH} / \mathrm{H}_{2} \mathrm{O}$ and lyophilised. Purification is carried out via preparative reverse phase HPLC and removal of the solvents followed by lyophilisation yielded the peptide as white solid.

\section{Continuous flow synthesis}

The peptide synthesiser used is a MilliGen 9050 synthesiser. The "notebook" is initially prepared, programming in detail the sequences, duration and reagents to be used for the whole synthesis, or parts of it.

The resin used is suspended in DMF ( $5 \mathrm{ml}$ ) for $20 \mathrm{~min}$ in a vial and then transferred to the synthesiser column (10 mm width). Fmoc deprotection is carried out using a 20 \% piperidine solution in DMF for 5 minutes for standard Fmoc-protected amino acids and 10 minutes for the resin and the lanthionine residue.

The amino acid cycles included a 20 bubble-cycle activation of the residues with 0.6 M HOBt and 0.6 M DIC solutions, both in DMF, followed by 15-minute coupling cycles for standard amino acids which was carried out twice and a 30-minute coupling cycle for the lanthionine, also performed twice. Both the Fmoc deprotection and amino acid couplings were monitored quantitatively by measuring the U.V. absorbance $(\lambda 365 \mathrm{~nm})$. Each double-cycle was followed by a capping cycle using a $0.3 \mathrm{M}$ solution of 1-acetylimidazole in DMF.

3-[(R/S)-1-Allyloxycarbonyl-2-(allyloxycarbonylamino)-ethylsulfanyl]-(R)-2(fluoren-9-ylmethoxycarbonylamino)-propionic acid 20 (0.22 g, $0.4 \mathrm{mmol}, 4$ equiv.) is activated with the DIC solution $(1.6 \mathrm{ml})$ and the HOBt solution $(1.5 \mathrm{ml})$ and delivered to the column on the synthesiser for the coupling cycle. DMF washes, a capping cycle, further washes and the Fmoc deprotection of the newly attached residue follow the second cycle. Subsequent amino acid couplings then followed. 
The simultaneous allylic deprotection, cyclisation and final amino acid coupling steps are then carried out manually on the Merrifield bubbler, as described previously (step E). Cleavage of the peptide from the resin and purification as described above in step F yielded the peptide as white solid.

34.

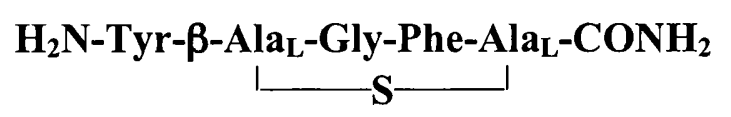

24

Batch synthesis:

Resin: Sieber amide resin ( $0.47 \mathrm{mmol} / \mathrm{g}$ loading - manufacturer determination)

Yield: $0.5 \mathrm{mg}, 0.8 \mu \mathrm{mol}, 0.8 \%$

Mass spectrum: $m / z(\mathrm{ES}+) 579\left([\mathrm{M}+\mathrm{Na}]^{+}, 100 \%\right), 557\left([\mathrm{M}+\mathrm{H}]^{+}, 37 \%\right)$

HPLC: $\mathrm{R}_{\mathrm{T}} 20.2$ (analytical, $0-50 \% \mathrm{MeCN}$ in $30 \mathrm{~min}, 50-90 \% \mathrm{MeCN}$ in $1 \mathrm{~min}$, $90-90 \% \mathrm{MeCN}$ in $4 \mathrm{~min}, 90-0 \% \mathrm{MeCN}$ in $1 \mathrm{~min}$ )

Continuous flow:

Resin: NovaSyn ${ }^{\circledR}$ TG Sieber resin $(0.16 \mathrm{mmol} / \mathrm{g})$

Yield: $1.1 \mathrm{mg}, 2.0 \mu \mathrm{mol}, 2.0 \%$

Mass spectrum: $m / z(\mathrm{ES}+) 758\left([\mathrm{M}+\mathrm{H}]^{+}, 20 \%\right), 780\left([\mathrm{M}+\mathrm{Na}]^{+}, 100 \%\right)$ for the linear tripeptide; $579\left([\mathrm{M}+\mathrm{Na}]^{+}, 37 \%\right), 557\left([\mathrm{M}+\mathrm{H}]^{+}, 100 \%\right)$

HPLC: $\mathrm{R}_{\mathrm{T}} 20.2$ (analytical, $0-50 \% \mathrm{MeCN}$ in $30 \mathrm{~min}, 50-90 \% \mathrm{MeCN}$ in $1 \mathrm{~min}$, $90-90 \% \mathrm{MeCN}$ in $4 \mathrm{~min}, 90-0 \% \mathrm{MeCN}$ in $1 \mathrm{~min}$ )

35.

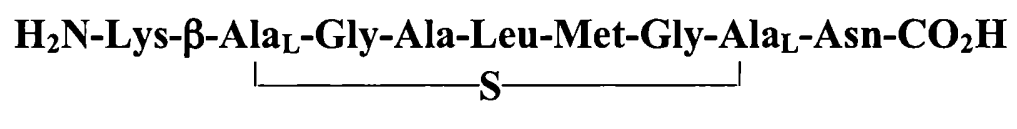

25

Batch synthesis:

Resin: NovaSyn ${ }^{\circledR}$ TGT resin, pre-loaded with Fmoc-Asn(Trt) $(0.21 \mathrm{mmol} / \mathrm{g}$ calculated loading, $0.20 \mathrm{mmol} / \mathrm{g}$ manufacturer determination)

Yield: Diastereoisomer 25a 14.3 mg, 16.6 mmol, 16.6 \%, Diastereoisomer 25b 33.1 $\mathrm{mg}, 38.4 \mathrm{mmol}, 38.4 \%$, $55 \%$ overall

NMR: $\boldsymbol{\delta}_{\mathbf{H}}(600 \mathrm{MHz})$ see Table 6 and Table 7. 
Mass spectrum: $m / z(\mathrm{ES}+)$ Diastereoisomer 25a $863\left([\mathrm{M}]^{+}, 63 \%\right), 432\left([\mathrm{M}+2]^{2+}\right.$, $100 \%)$; Diastereoisomer 25b $863\left([\mathrm{M}]^{+}, 100 \%\right), 432\left([\mathrm{M}+2]^{2+}, 51 \%\right)$

Diastereoisomer $\mathbf{2 5 \mathbf { a } ^ { 2 1 8 }}$

\begin{tabular}{|c|c|c|c|}
\hline Residue & Atom & $\delta_{\mathrm{H}}(\mathrm{ppm})$ & $\delta_{C}(\mathrm{ppm})$ \\
\hline \multirow[t]{5}{*}{ Lys } & $\alpha$ & 3.88 & 53.8 \\
\hline & $\bar{\beta}$ & 1.88 & 31.7 \\
\hline & $\gamma$ & 1.48 & 22.4 \\
\hline & $\delta$ & 1.69 & 27.9 \\
\hline & $\varepsilon$ & 2.95 & 40.1 \\
\hline \multirow[t]{2}{*}{$\beta-\mathrm{Ala}_{\mathrm{L}}$} & $\alpha$ & 3.94 & 47.7 \\
\hline & $\beta$ & $3.61,3.37$ & 41.1 \\
\hline Gly & $\alpha$ & 3.89 & 43.7 \\
\hline \multirow[t]{2}{*}{ Ala } & $\alpha$ & 4.24 & 50.9 \\
\hline & $\beta$ & 1.38 & 16.8 \\
\hline \multirow[t]{5}{*}{ Leu } & $\alpha$ & 4.27 & 53.5 \\
\hline & $\beta$ & $2.08,1.55$ & 40.2 \\
\hline & $\gamma$ & 1.87 & 25.6 \\
\hline & $\delta 1$ & 1.00 & 23.5 \\
\hline & $\delta 2$ & 0.94 & 20.9 \\
\hline \multirow[t]{4}{*}{ Met } & $\alpha$ & 4.22 & 55.8 \\
\hline & $\bar{\beta}$ & 2.04 & 31.5 \\
\hline & $\gamma$ & $2.63,2.55$ & 30.5 \\
\hline & $\varepsilon$ & 2.10 & 14.9 \\
\hline Gly & $\alpha$ & $3.89,3.85$ & 44.2 \\
\hline \multirow[t]{2}{*}{$\mathrm{Ala}_{\mathrm{L}}$} & $\alpha$ & 4.58 & 55.0 \\
\hline & $\beta$ & 3.03 & 31.8 \\
\hline \multirow[t]{2}{*}{ Asn } & $\alpha$ & 4.82 & 50.4 \\
\hline & $\beta$ & $2.76,2.67$ & 38.6 \\
\hline
\end{tabular}

Table 6. ${ }^{1} \mathrm{H}$ and ${ }^{13} \mathrm{C}$ chemical shifts for peptide 25a 
Diastereoisomer $\mathbf{2 5} \mathbf{b}^{218}$

\begin{tabular}{|c|c|c|c|}
\hline Residue & Atom & $\delta_{\mathrm{H}}(\mathrm{ppm})$ & $\delta_{C}(\mathrm{ppm})$ \\
\hline \multirow[t]{5}{*}{ Lys } & $\alpha$ & 3.89 & 53.7 \\
\hline & $\beta$ & 1.90 & 31.6 \\
\hline & $\gamma$ & 1.49 & 22.4 \\
\hline & $\delta$ & 1.69 & 27.9 \\
\hline & $\varepsilon$ & 2.91 & 40.1 \\
\hline \multirow[t]{2}{*}{$\beta-\mathrm{Ala}_{\mathrm{L}}$} & $\alpha$ & 3.75 & 46.5 \\
\hline & $\beta$ & $3.78,3.56$ & 41.1 \\
\hline Gly & $\alpha$ & $4.18,3.81$ & 43.1 \\
\hline \multirow[t]{2}{*}{ Ala } & $\alpha$ & 4.13 & 52.0 \\
\hline & $\beta$ & 1.40 & 17.0 \\
\hline \multirow[t]{5}{*}{ Leu } & $\alpha$ & 4.30 & 54.4 \\
\hline & $\beta$ & $1.91,1.60$ & 40.4 \\
\hline & $\gamma$ & 1.68 & 25.8 \\
\hline & $\delta 1$ & 0.96 & 23.1 \\
\hline & $\delta 2$ & 0.91 & 21.1 \\
\hline \multirow[t]{4}{*}{ Met } & $\alpha$ & 4.45 & 543. \\
\hline & $\beta$ & $2.13,2.05$ & 31.8 \\
\hline & $\gamma$ & $2.62,2.49$ & 30.6 \\
\hline & $\varepsilon$ & 2.08 & 14.8 \\
\hline Gly & $\alpha$ & $4.13,3.85$ & 43.2 \\
\hline \multirow[t]{2}{*}{$\mathrm{Ala}_{\mathrm{L}}$} & $\alpha$ & 4.81 & 52.2 \\
\hline & $\beta$ & $3.47,2.70$ & 34.1 \\
\hline \multirow[t]{2}{*}{ Asn } & $\alpha$ & 4.77 & 50.0 \\
\hline & $\beta$ & $2.84,2.80$ & 37.4 \\
\hline
\end{tabular}

Table 7. ${ }^{1} \mathrm{H}$ and ${ }^{13} \mathrm{C}$ chemical shifts for peptide $\mathbf{2 5 b}$

HPLC: Preliminary preparative HPLC of the crude material: $10-20 \% \mathrm{MeCN}$ in 30 $\min , 20-90 \% \mathrm{MeCN}$ in $15 \mathrm{~min}, 90-90 \% \mathrm{MeCN}$ in $5 \mathrm{~min}, 90-10 \%$ in $1 \mathrm{~min}$ (25a $\mathrm{R}_{\mathrm{T}} 17.0$, 25b $\mathrm{R}_{\mathrm{T}}$ 20.1). Diastereoisomer 25a $\mathrm{R}_{\mathrm{T}} 8.9(15-18 \% \mathrm{MeCN}$ in 30 $\min , 18-50 \% \mathrm{MeCN}$ in $5 \mathrm{~min}, 50-90 \% \mathrm{MeCN}$ in $1 \mathrm{~min}, 90-90 \% \mathrm{MeCN}$ in 4 
$\min , 90-15 \% \mathrm{MeCN}$ in $1 \mathrm{~min})$; Diastereoisomer $25 \mathrm{~b} \mathrm{R}_{\mathrm{T}} 11.7$ (15-18\% $\mathrm{MeCN}$ in $30 \mathrm{~min}, 18-90 \% \mathrm{MeCN}$ in $5 \mathrm{~min}, 90-90 \% \mathrm{MeCN}$ in $5 \mathrm{~min}, 90-15 \% \mathrm{MeCN}$ in $1 \mathrm{~min})$

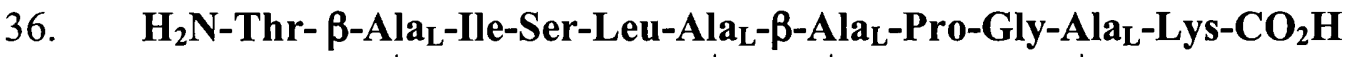

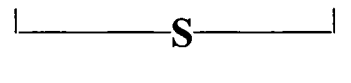 \\ 26}

Batch synthesis:

Resin: NovaSyn ${ }^{\circledR}$ TGT resin, pre-loaded with Fmoc-Lys(Boc) (0.22 mmol/g loading, $0.20 \mathrm{mmol} / \mathrm{g}$ manufacturer determination)

Yield: Negligible

Mass spectrum: $m / z(\mathrm{ES}+) 473\left([\mathrm{M}+1]^{+}, 83 \%\right)$ for ring B only; $1060\left([\mathrm{M}+1]^{+}, 18\right.$ $\%), 530\left([\mathrm{M}+2]^{2+}, 100 \%\right)$ 
Appendix A
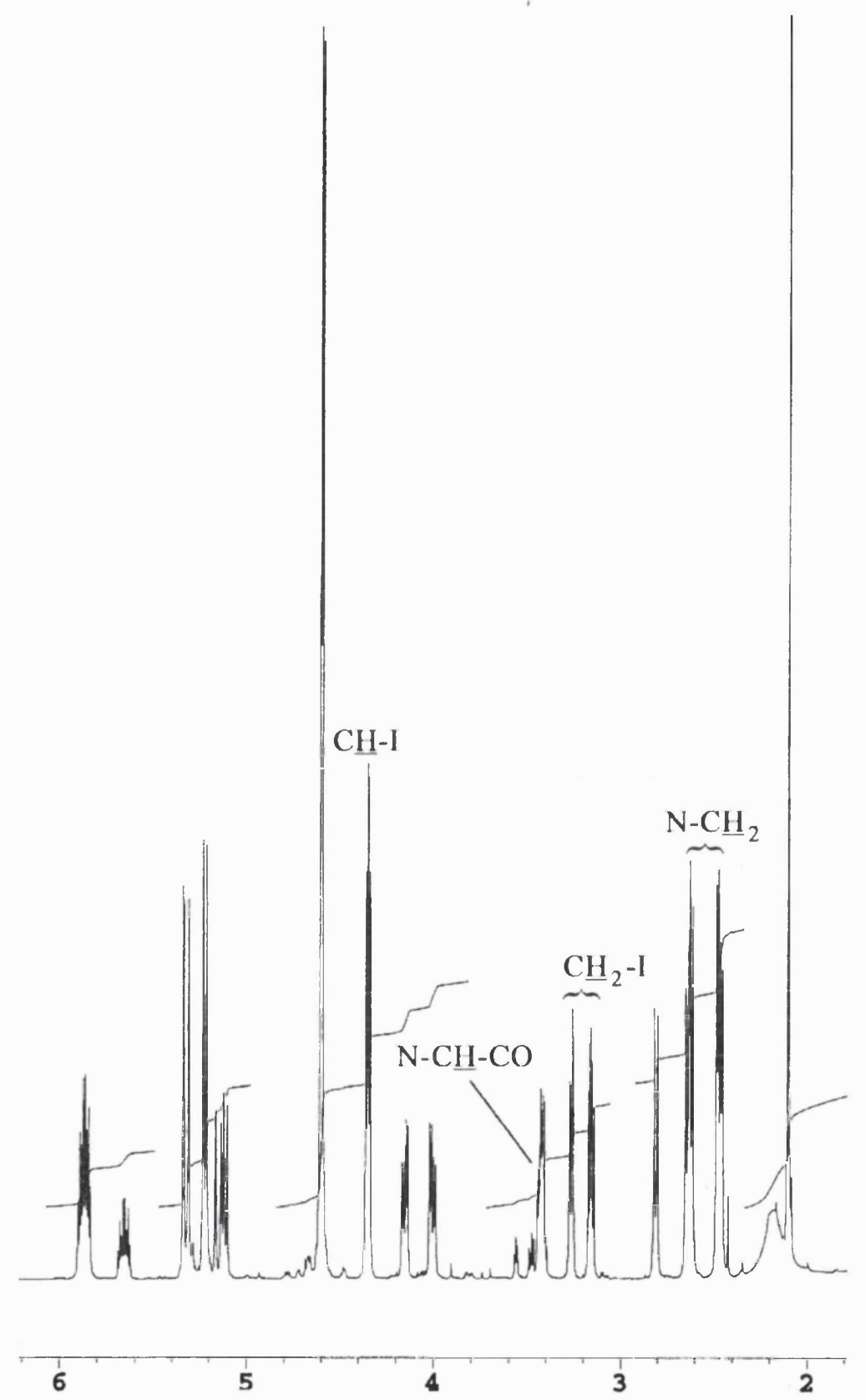<smiles></smiles>

$(R)-6 a$<smiles>C=CCOC(=O)C(I)CNC(c1ccccc1)c1ccccc1</smiles>

$6 b$

${ }^{1}$ H NMR spectrum of $(\boldsymbol{R})-\mathbf{6 a}$ and $\mathbf{6} \mathbf{b}$ 


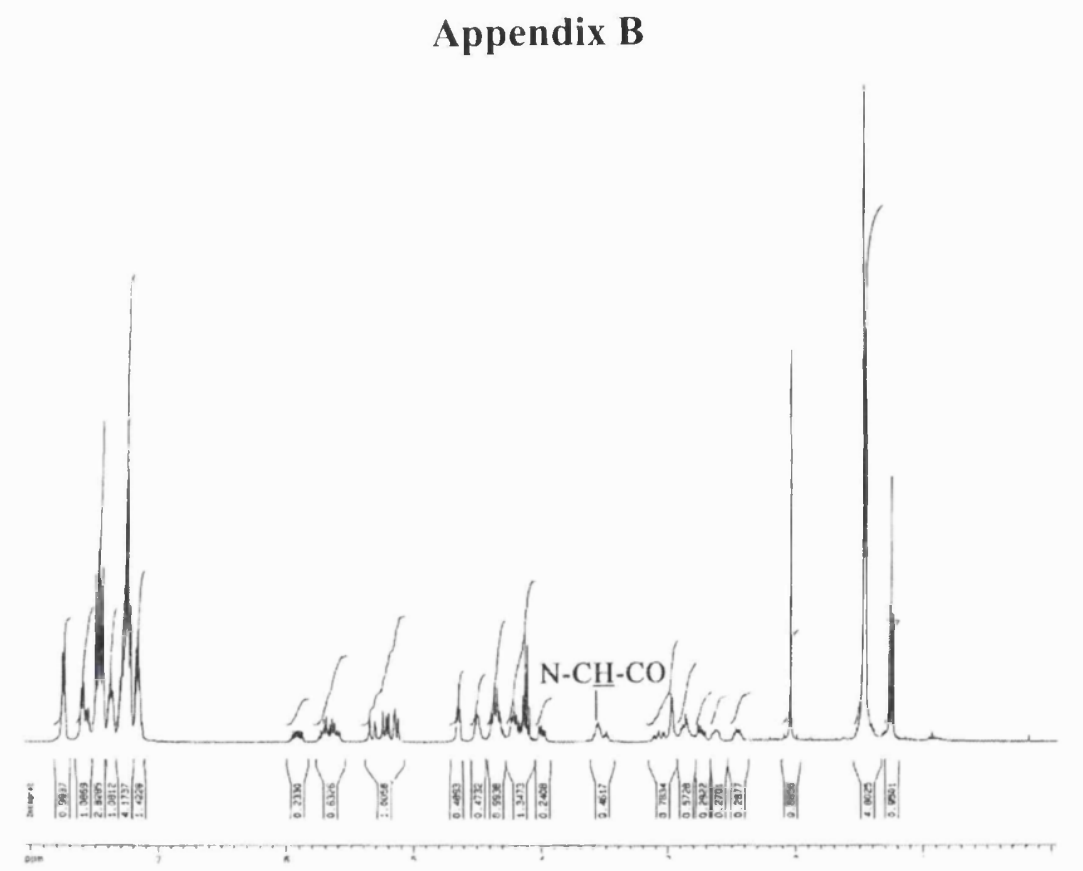

(a)

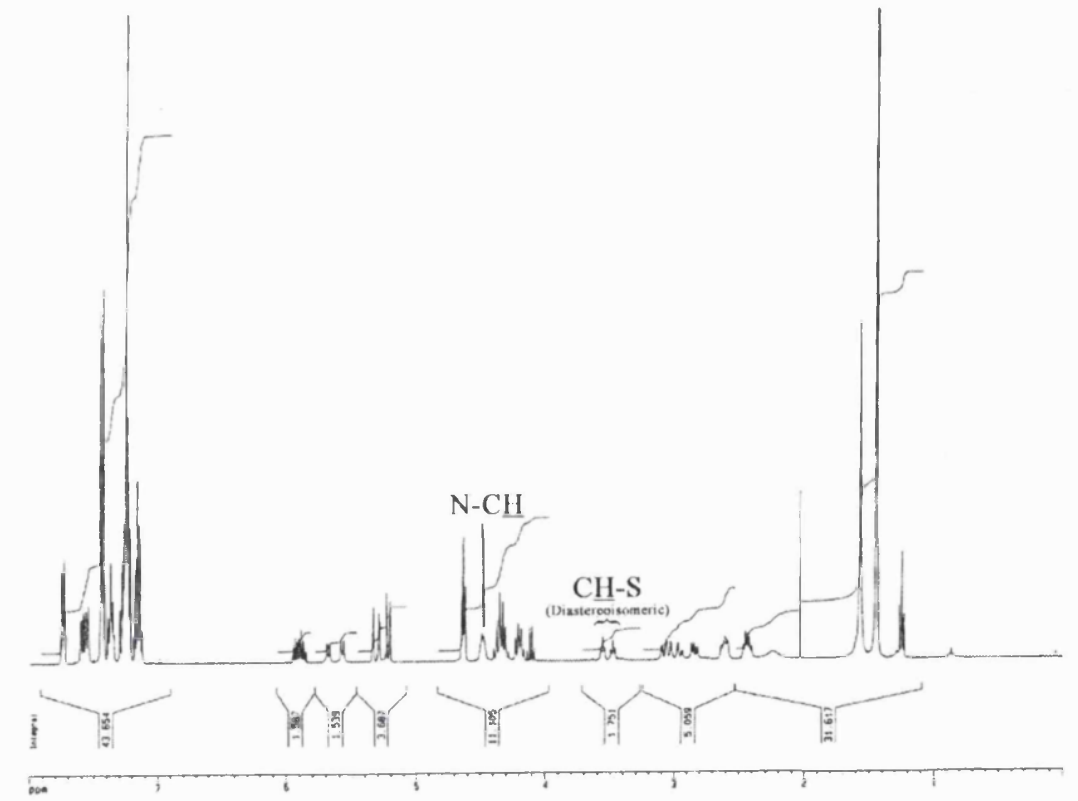

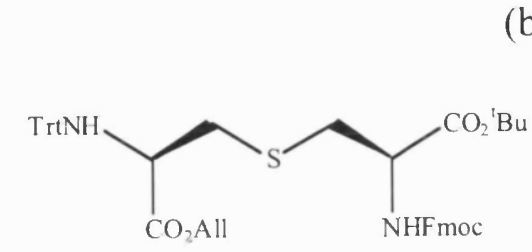

(a)

(b)

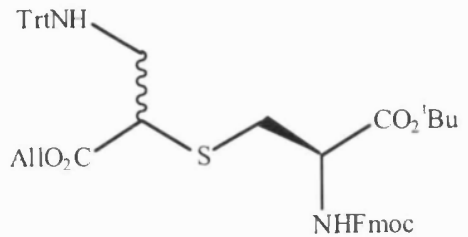

(b)

H NMR spectra for (a) $(\boldsymbol{R}, \boldsymbol{R})$-10 with 18 and (b) 18 
Appendix C
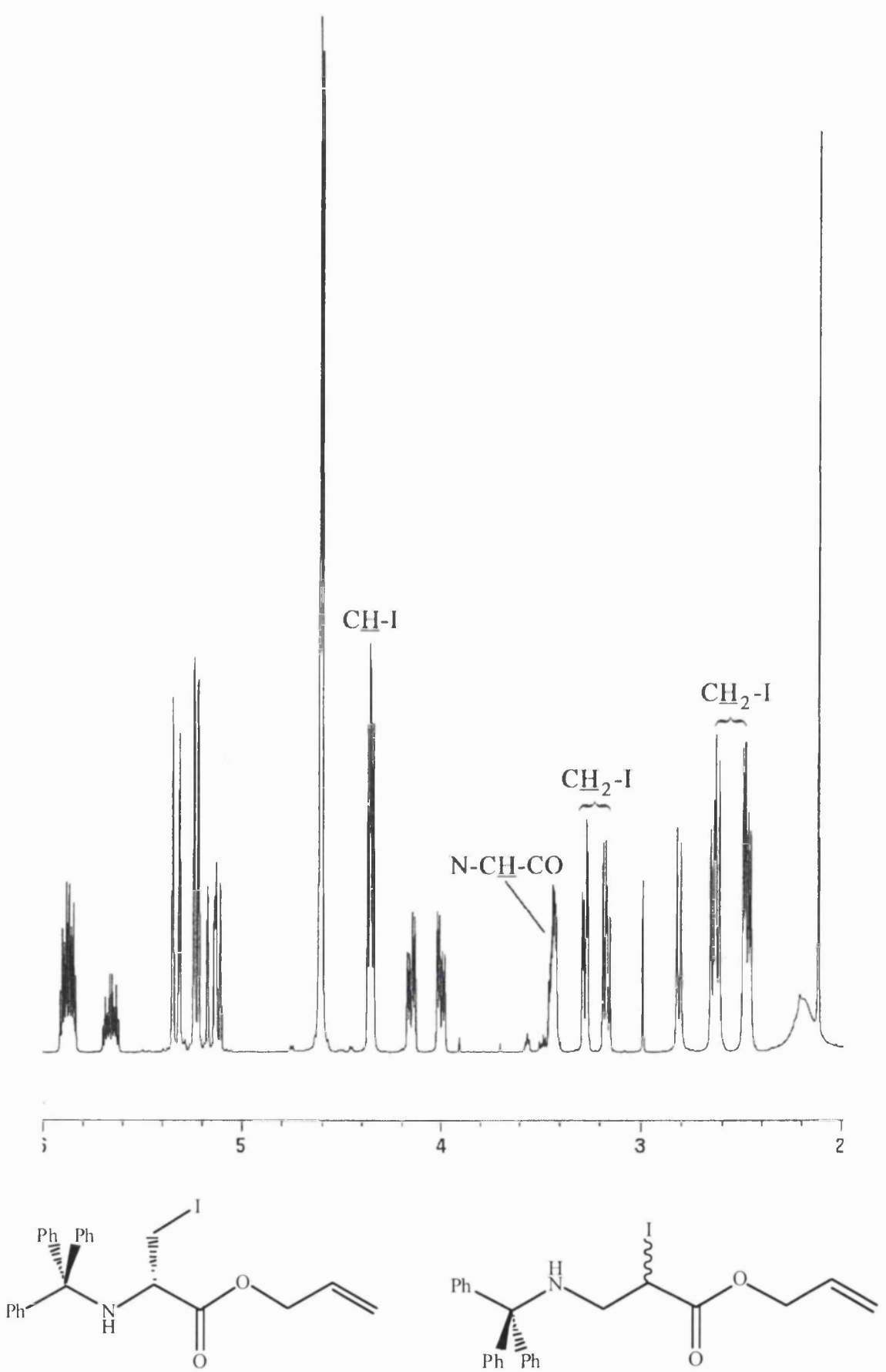

(S)-6a

$6 \mathrm{~b}$

${ }^{1}$ H NMR spectrum of (S)-6a and $\mathbf{6 b}$ 


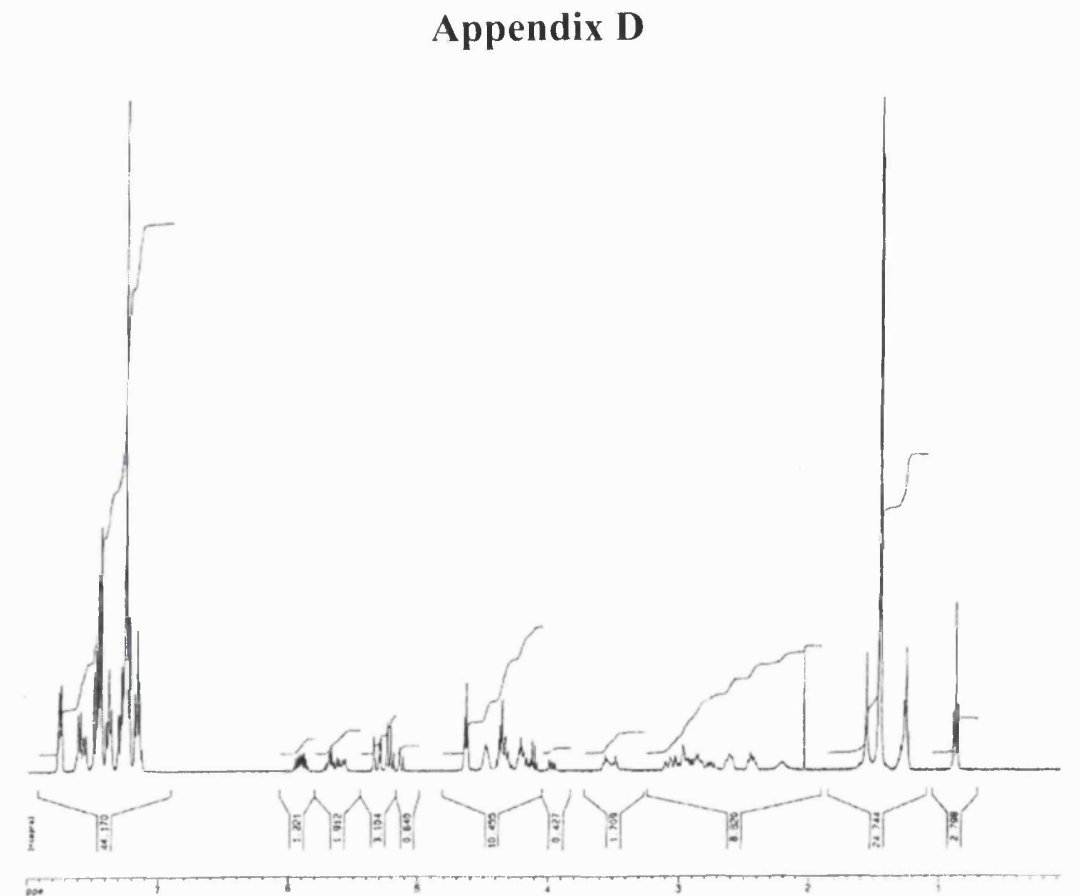

(a)

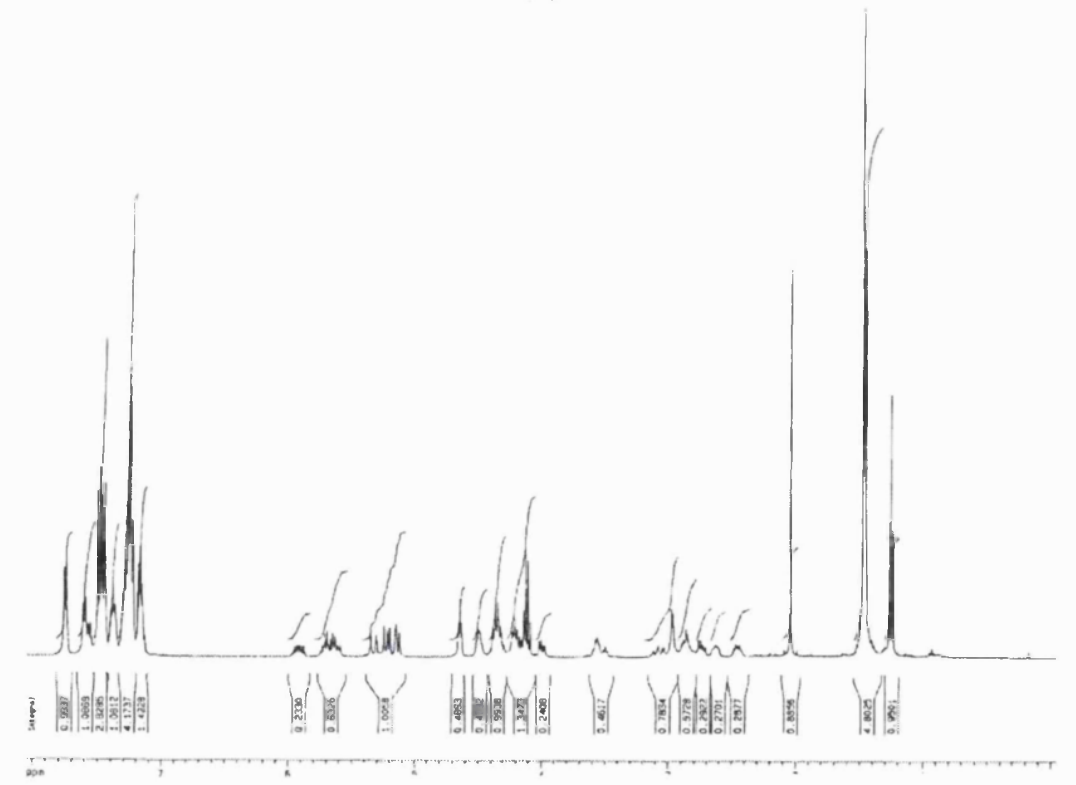

(b)

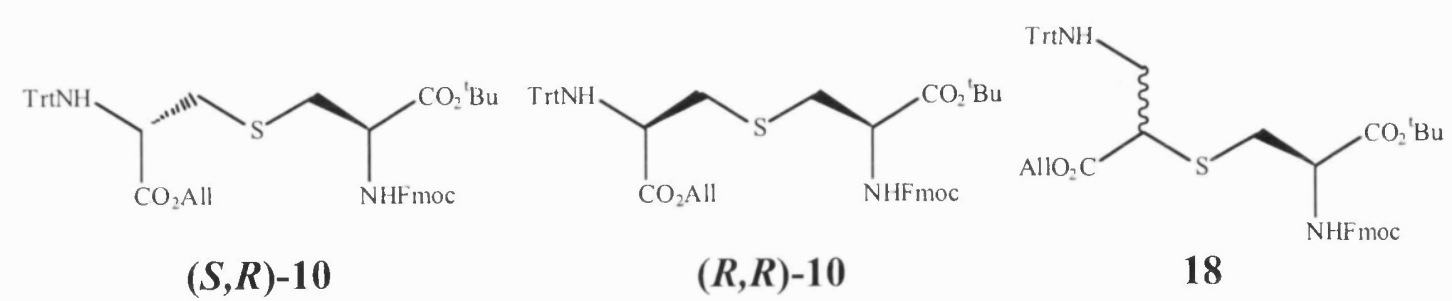

${ }^{1}$ H NMR spectra for (a) $(\boldsymbol{S}, \boldsymbol{R})$-10 with $\mathbf{1 8}$ and (b) $(\boldsymbol{R}, \boldsymbol{R})$-10 with $\mathbf{1 8}$ 


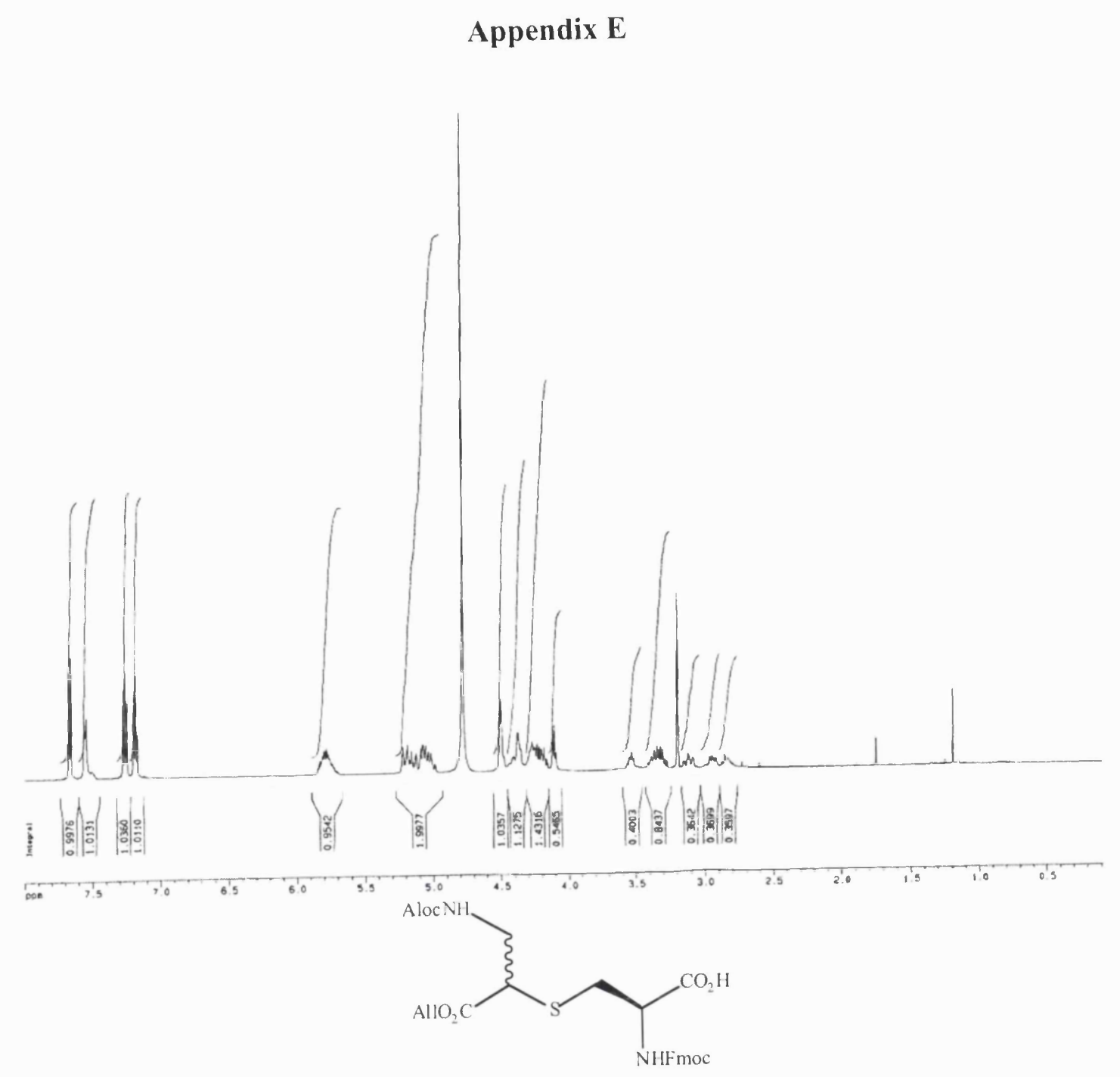

${ }^{1} \mathrm{H}$ NMR spectrum for $\mathbf{2 0}$ 

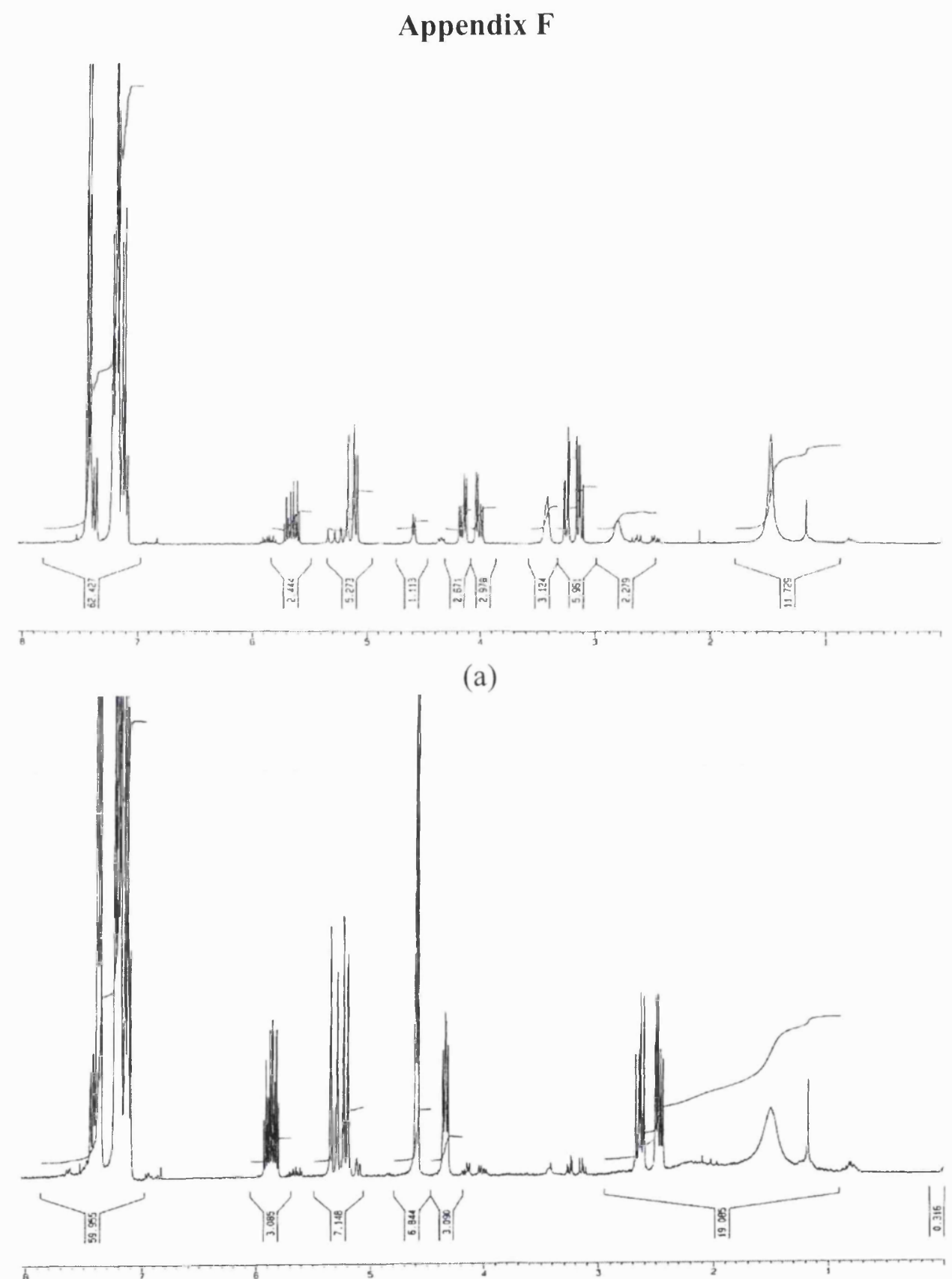

(b)<smiles>C=CCOC(=O)[C@H](CI)N[C@@H]([15NH])c1ccccc1</smiles>

(S)-6a<smiles>C=CCOC(=O)C(I)CNC(c1ccccc1)c1ccccc1</smiles>

6b

${ }^{1}$ H NMR of partially separated (a) (S)-6a (minor component) and (b) $6 \mathbf{b}$ (major component) 


\section{Appendix G}

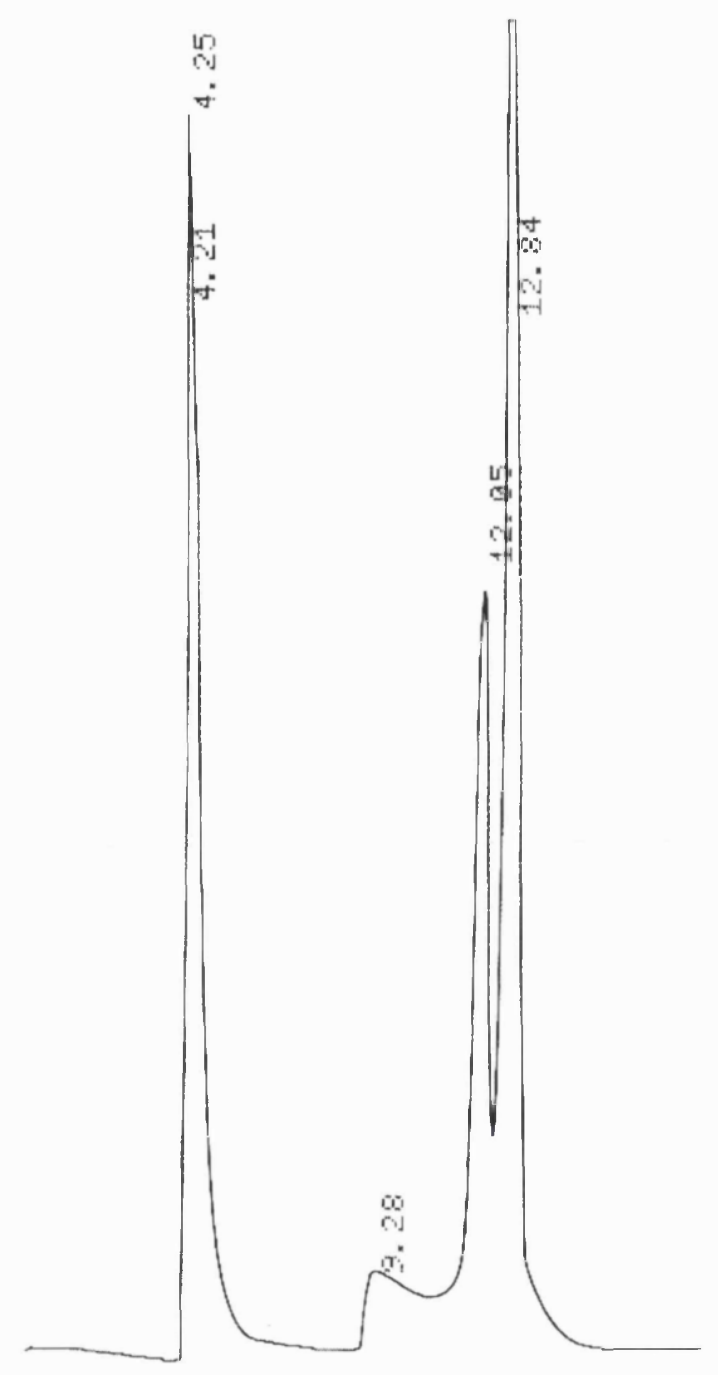<smiles>C=CCOC(=O)[C@H](CI)NC(c1ccccc1)c1ccccc1</smiles><smiles>C=CCOC(=O)[C@H](I)CNC(c1ccccc1)c1ccccc1</smiles>

Partial normal phase HPLC separation of $(\boldsymbol{S})$-6a (minor component) and $\mathbf{6 b}$ (major component) 


\section{Appendix H}

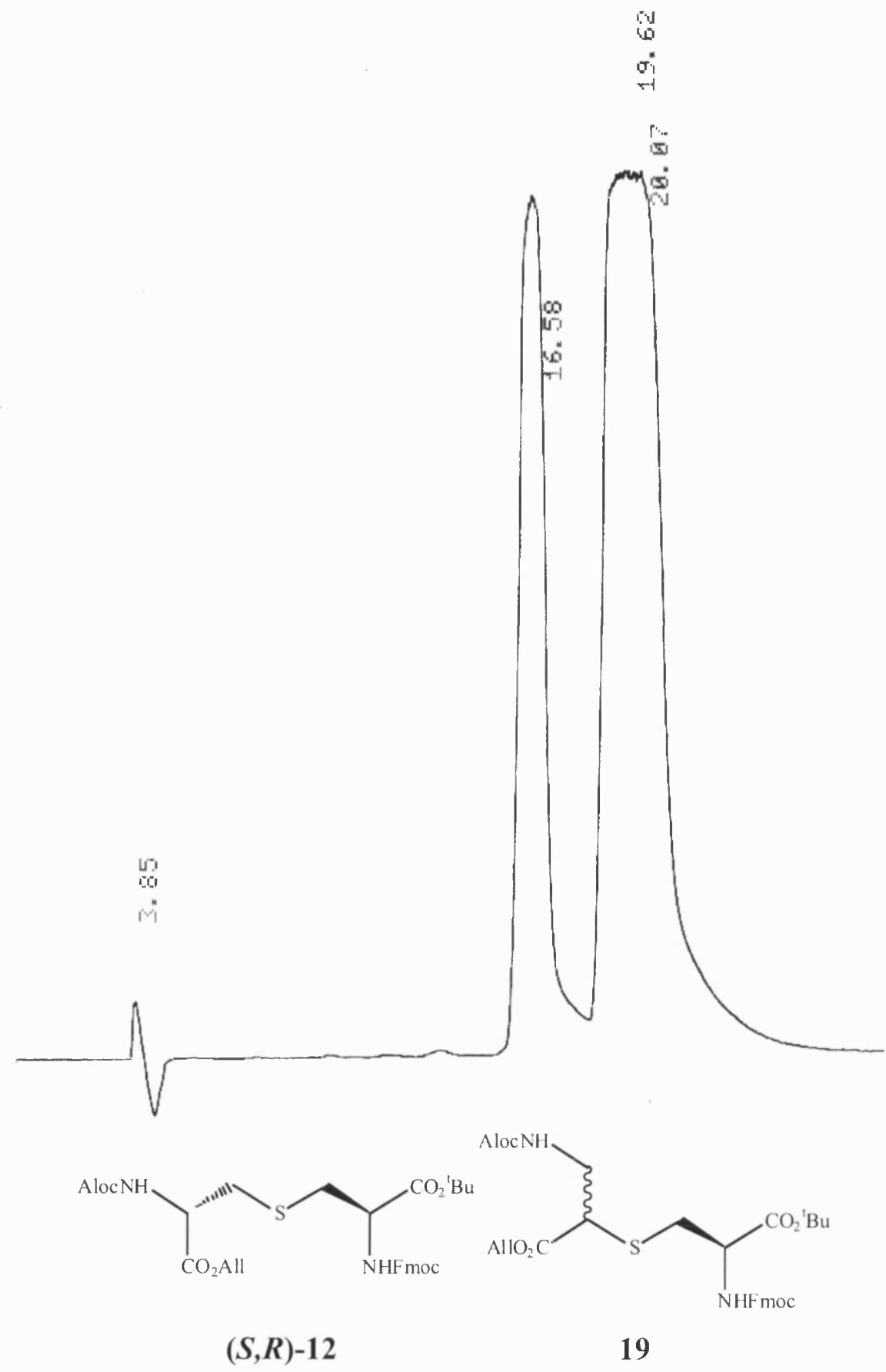

Normal phase HPLC separation of $(\boldsymbol{S}, \boldsymbol{R})-\mathbf{1 2}$ (minor component) and $\mathbf{1 9}$ (major component) 


\section{Appendix I}

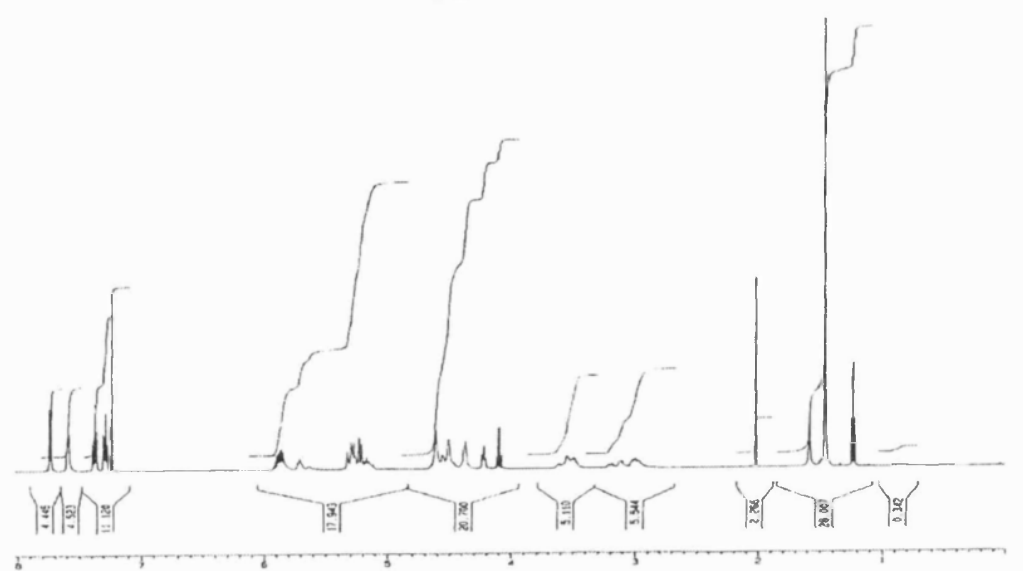

(a)

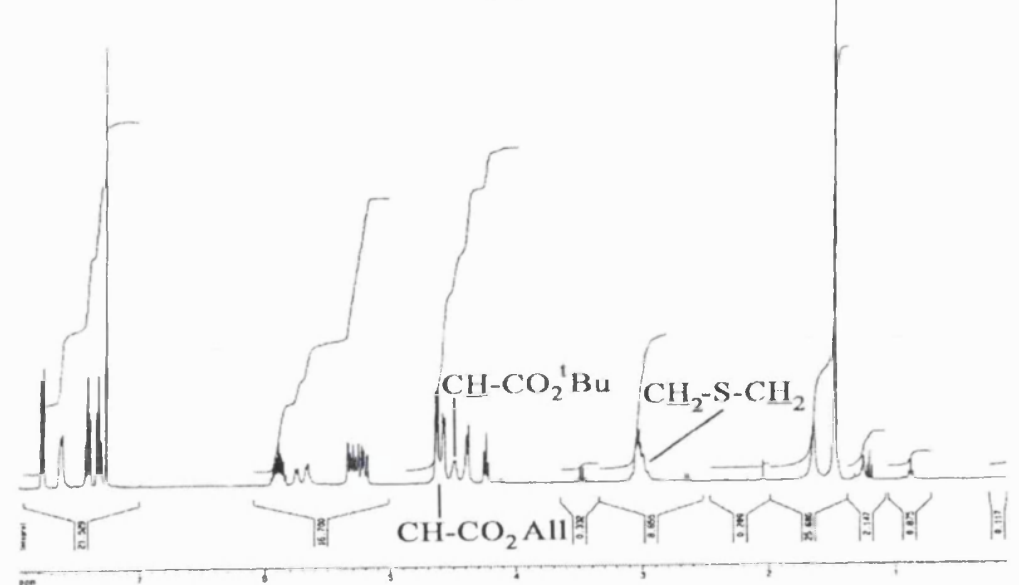

(b)

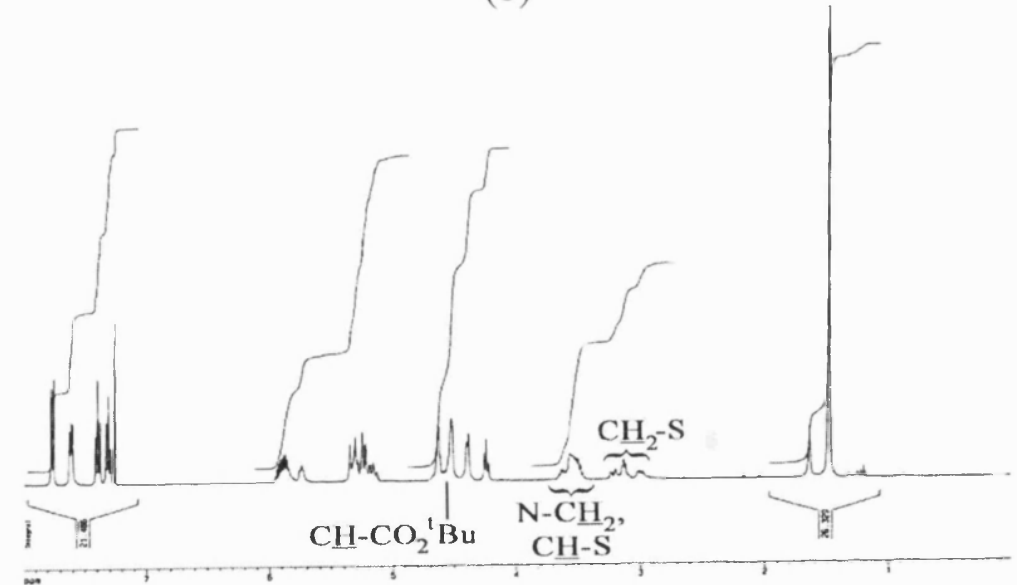

(c)

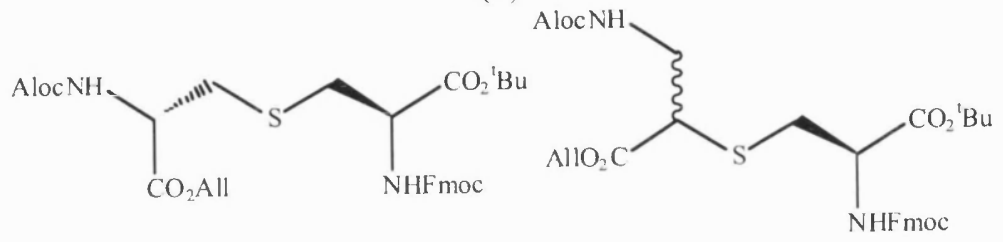

$(S, R)-12$

19

${ }^{1}$ H NMR of (a) $(S, R)-12$ as a mixture and separated (b) $(S, R)-12$ and (c) 19 


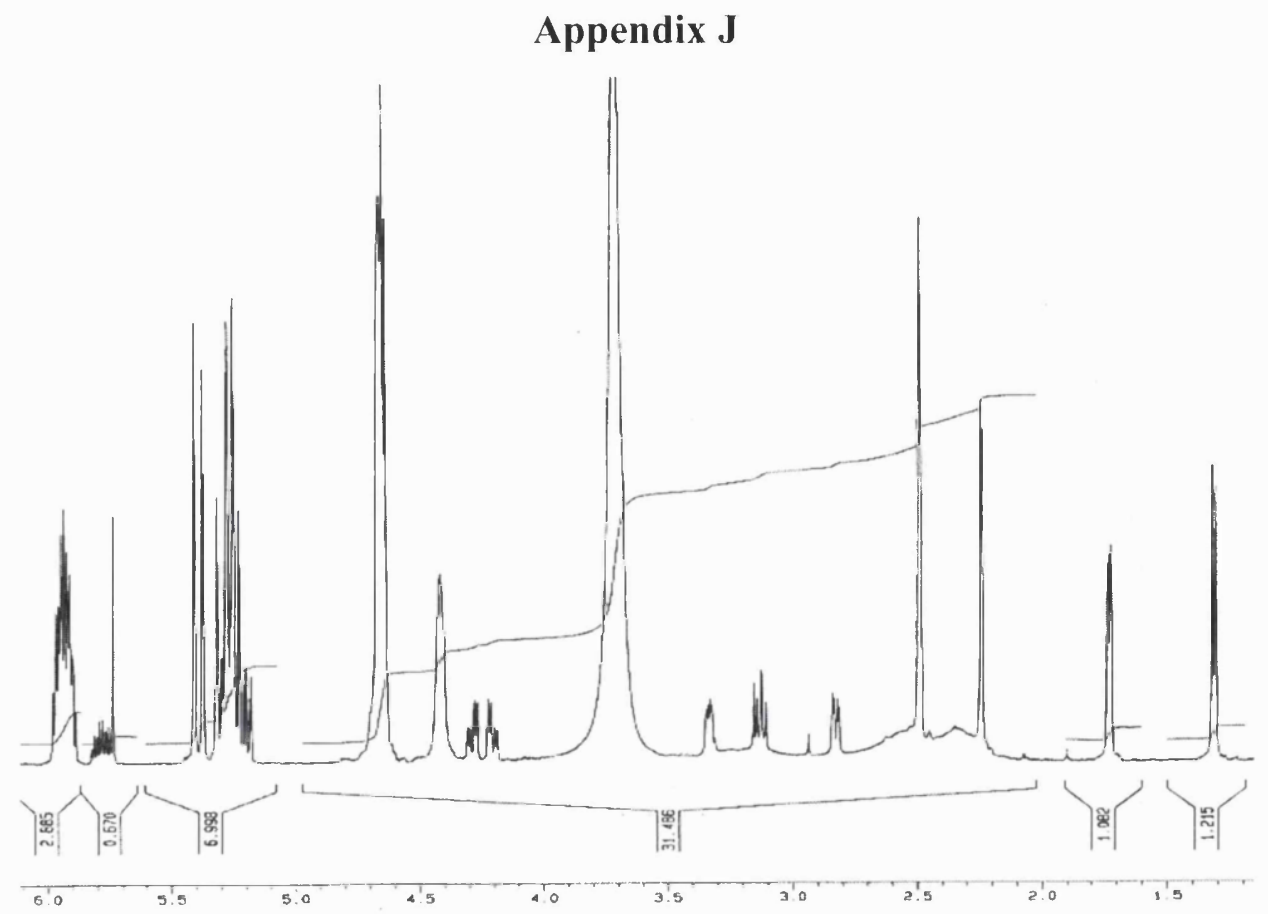

(a)

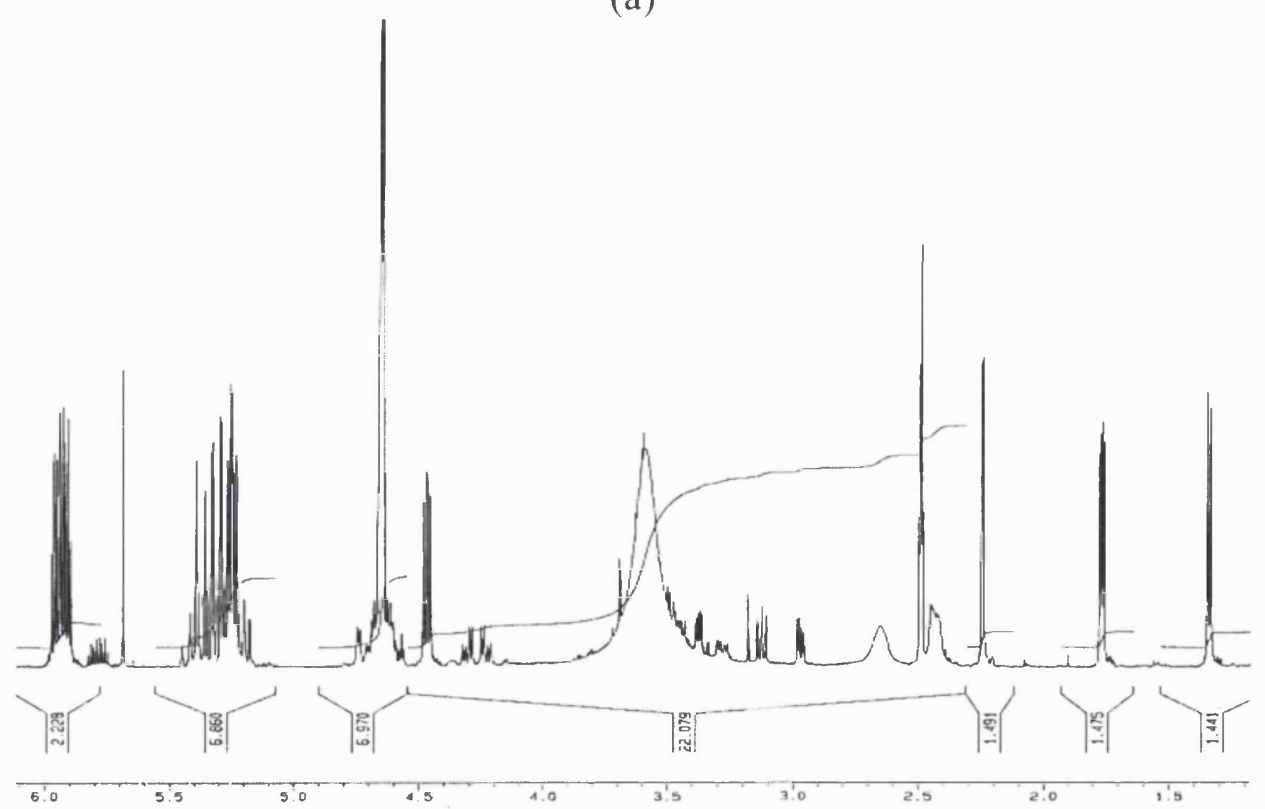

(b)

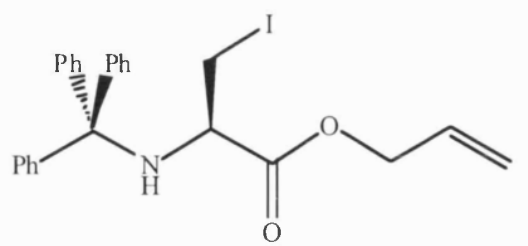

(R)-6a

Unsuccessful 'H NMR VT experiment of what was originally thought to be rotameric (R)-6a: (a) $25{ }^{\circ} \mathrm{C} \mathrm{K}$ (b) $50{ }^{\circ} \mathrm{C}$ 
Appendix K

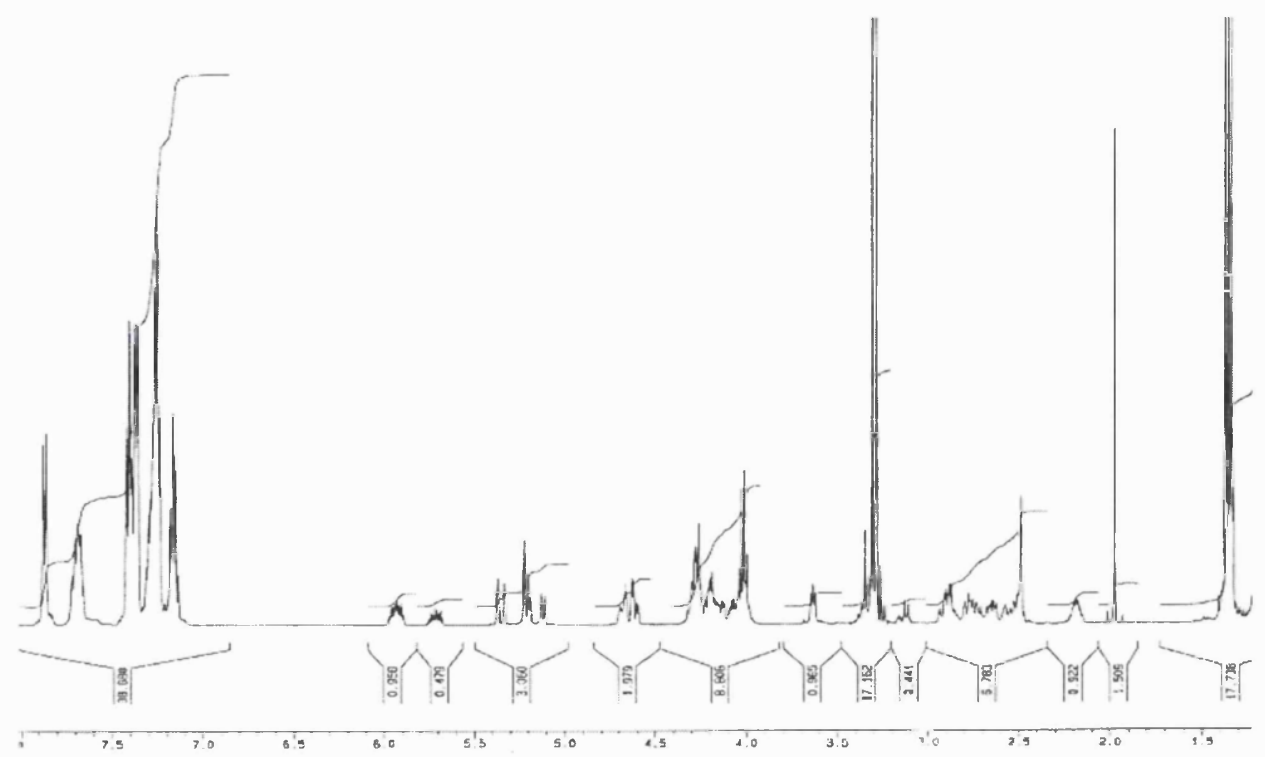

(a)

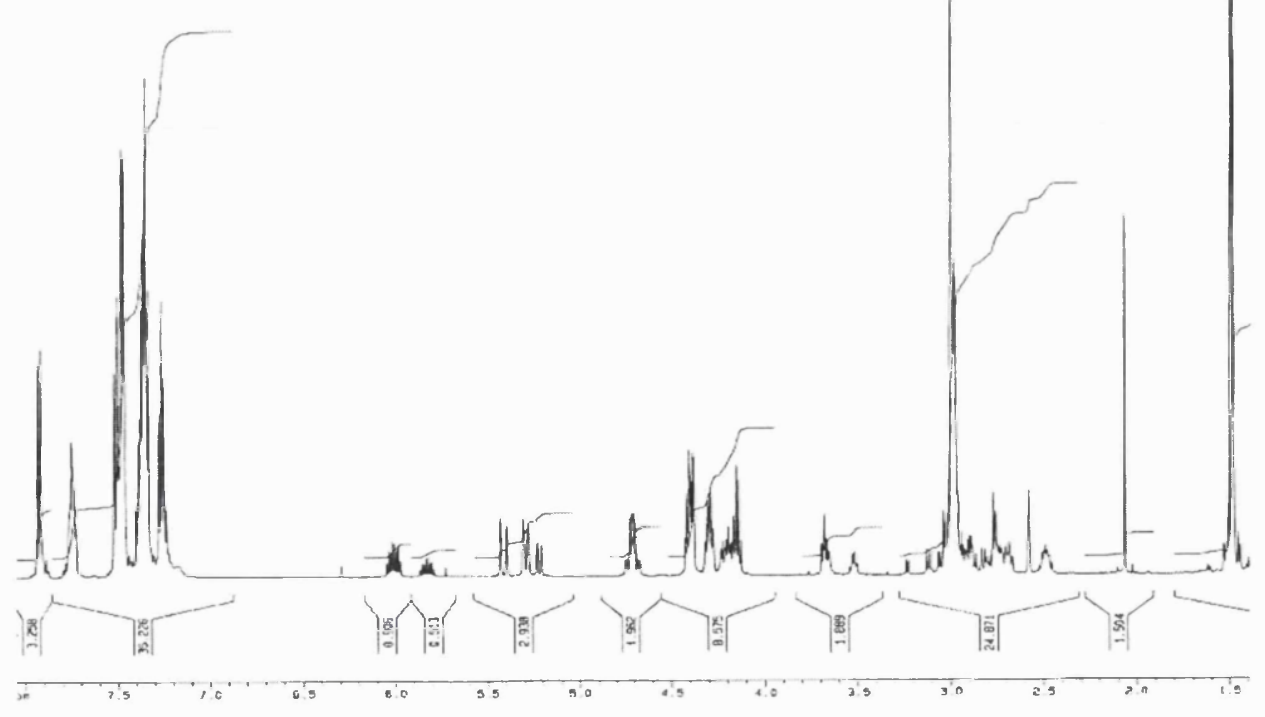

(b)

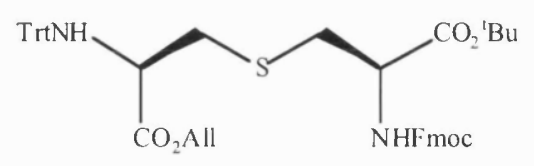

$(R, R)-10$

Unsuccessful ${ }^{1} \mathrm{H}$ NMR VT experiment of what was originally thought to be rotameric $(\boldsymbol{R}, \boldsymbol{R})-\mathbf{1 0}$ : (a) $25{ }^{\circ} \mathrm{C} \mathrm{K}$ (b) $100{ }^{\circ} \mathrm{C}$ 
Appendix L

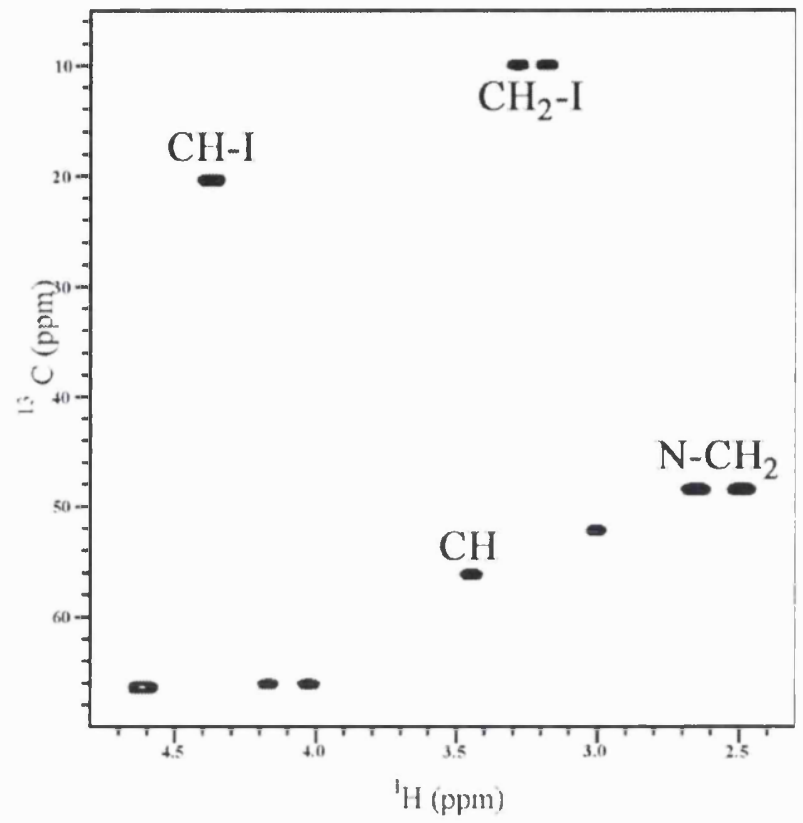<smiles>C=CCOC(=O)[C@H](CI)N[C@@H]([15NH])c1ccccc1</smiles>

(R)-6a<smiles>C=CCOC(=O)C(I)CNC(c1ccccc1)c1ccccc1</smiles>

6b

${ }^{1} \mathrm{H}-{ }^{13} \mathrm{C}$ HSQC NMR spectrum of $(\boldsymbol{S})-\mathbf{6 a}$ and $\mathbf{6 b}$ 
Appendix M

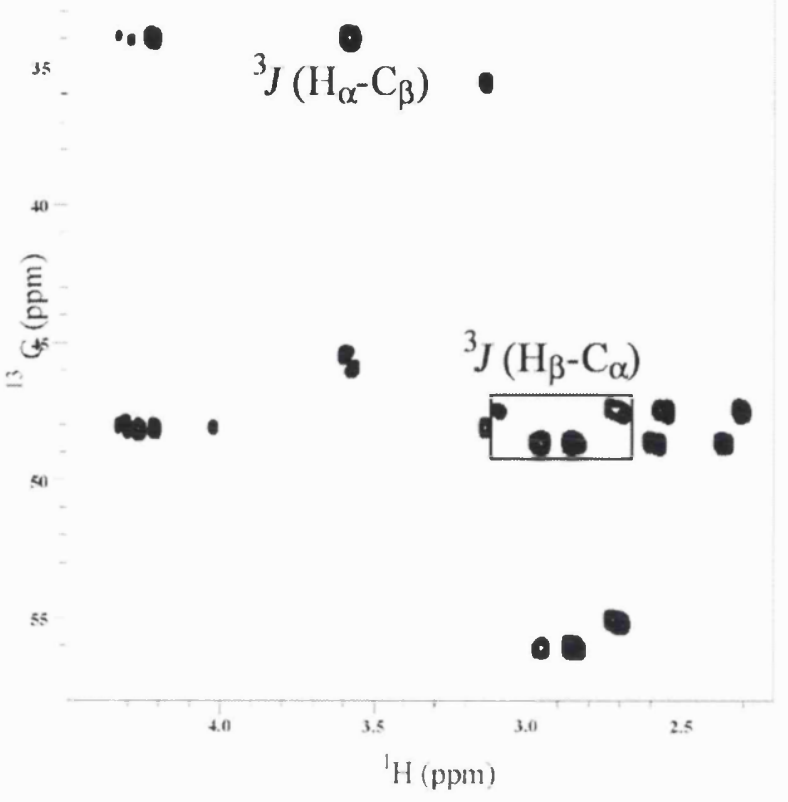

${ }^{1} \mathrm{H}-{ }^{13} \mathrm{C}$ HMBC NMR spectrum of $(\boldsymbol{S}, \boldsymbol{R})-\mathbf{1 0}$ 


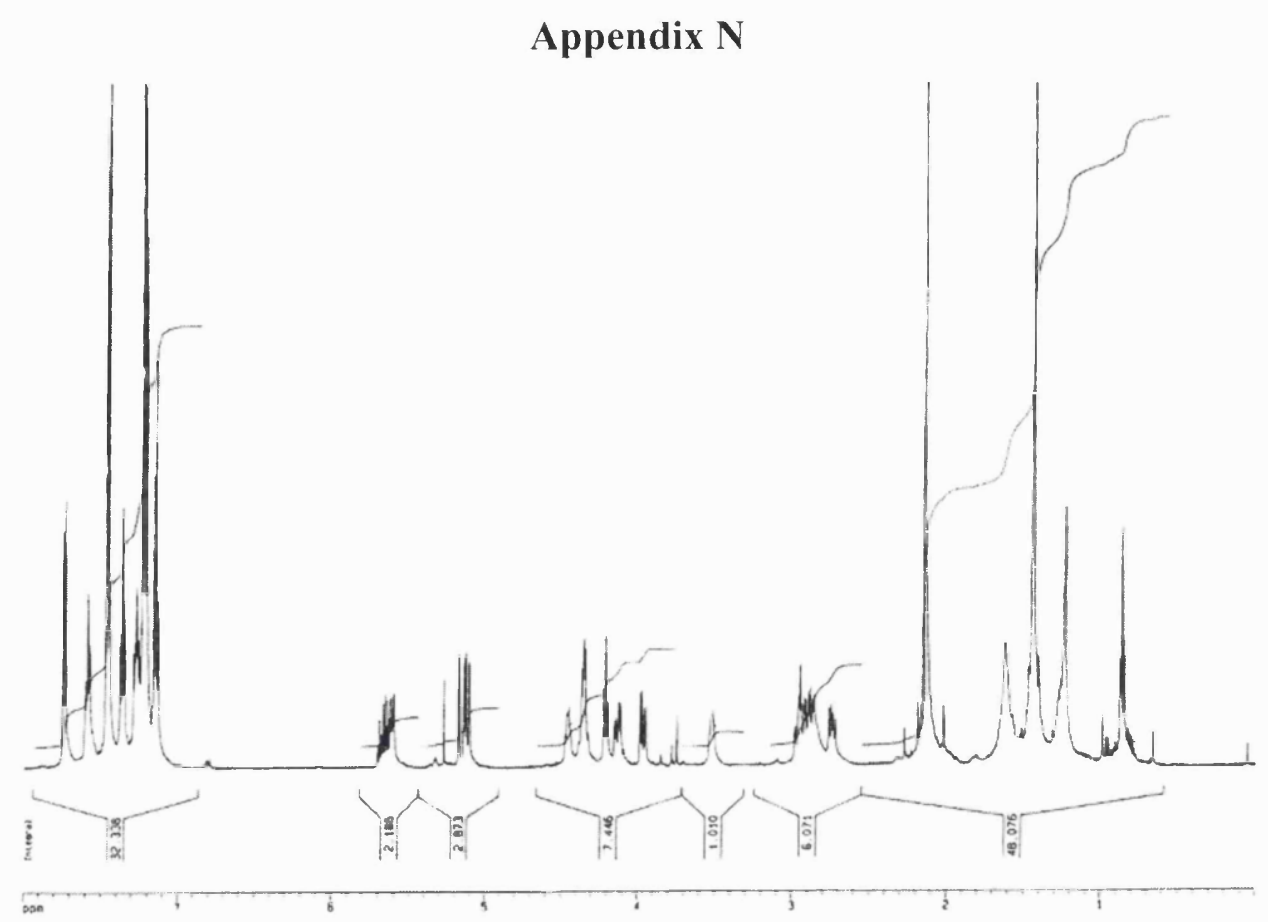

(a)

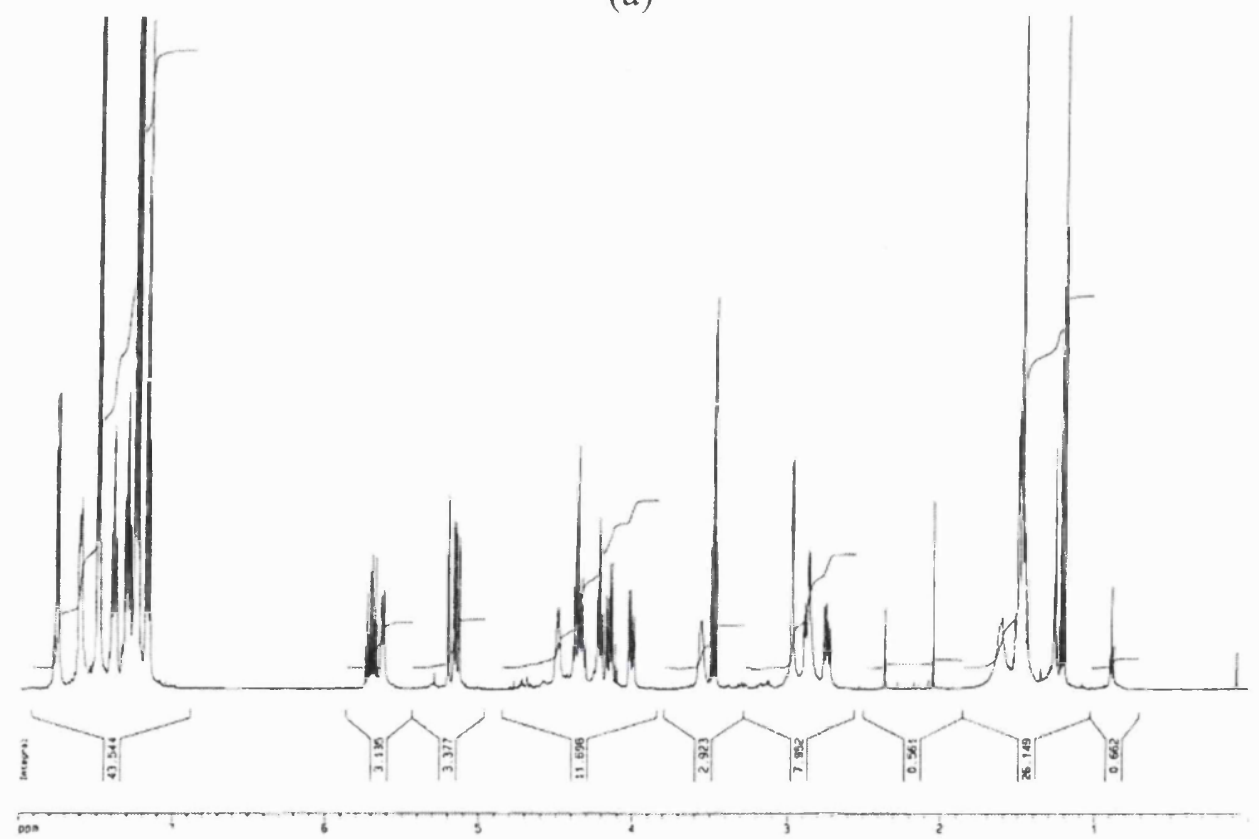

(b)

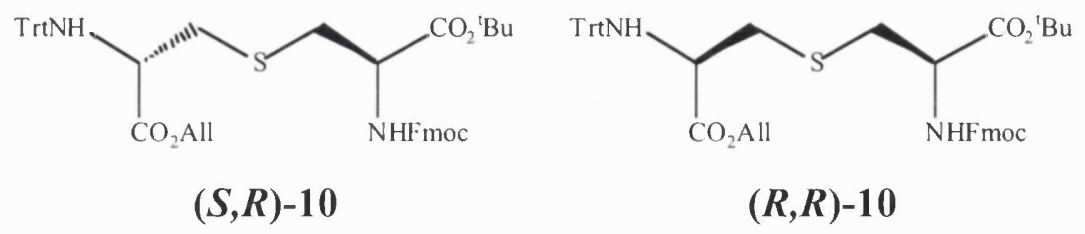

${ }^{1}$ H NMR spectra for (a) $(\boldsymbol{S}, \boldsymbol{R})-\mathbf{1 0}$ and (b) $(\boldsymbol{R}, \boldsymbol{R})$-10, both synthesised as a single isomer via the Mitsunobu route 
Appendix O

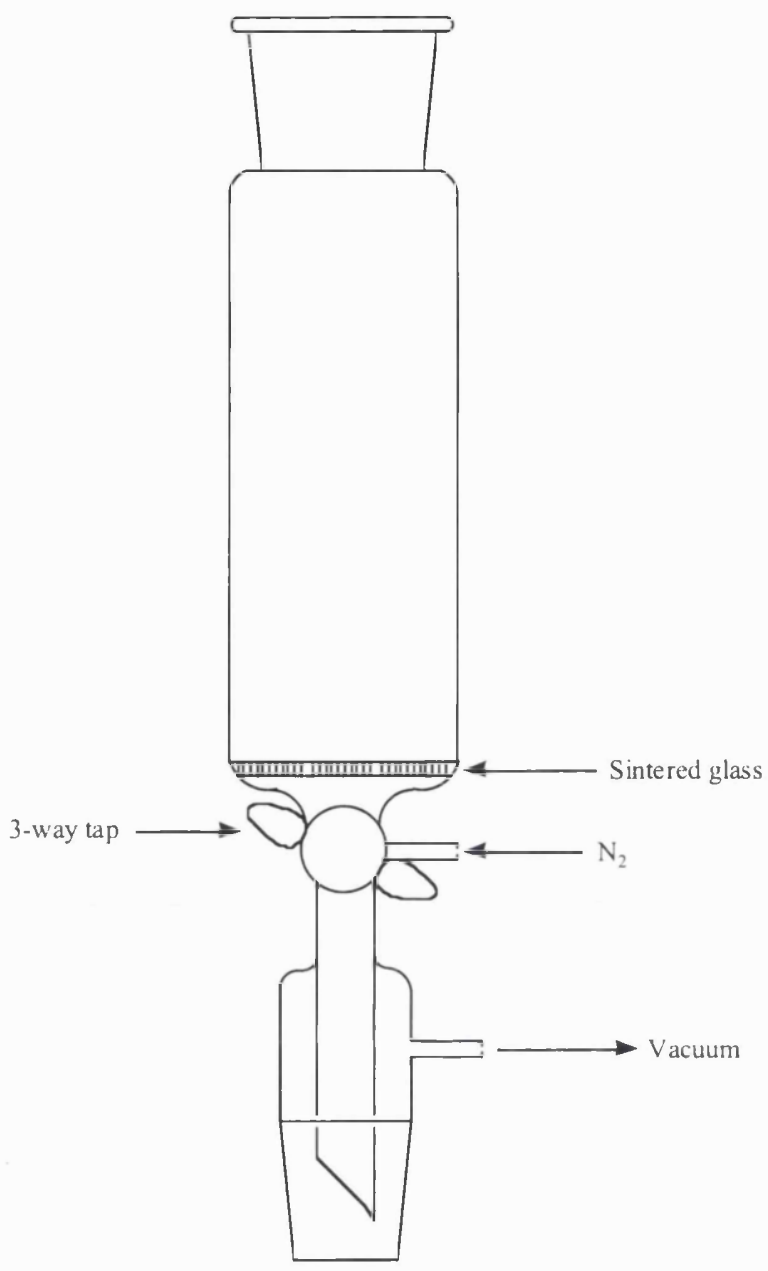

Diagram of Merrifield bubbler 


\section{Appendix $\mathbf{P}$}
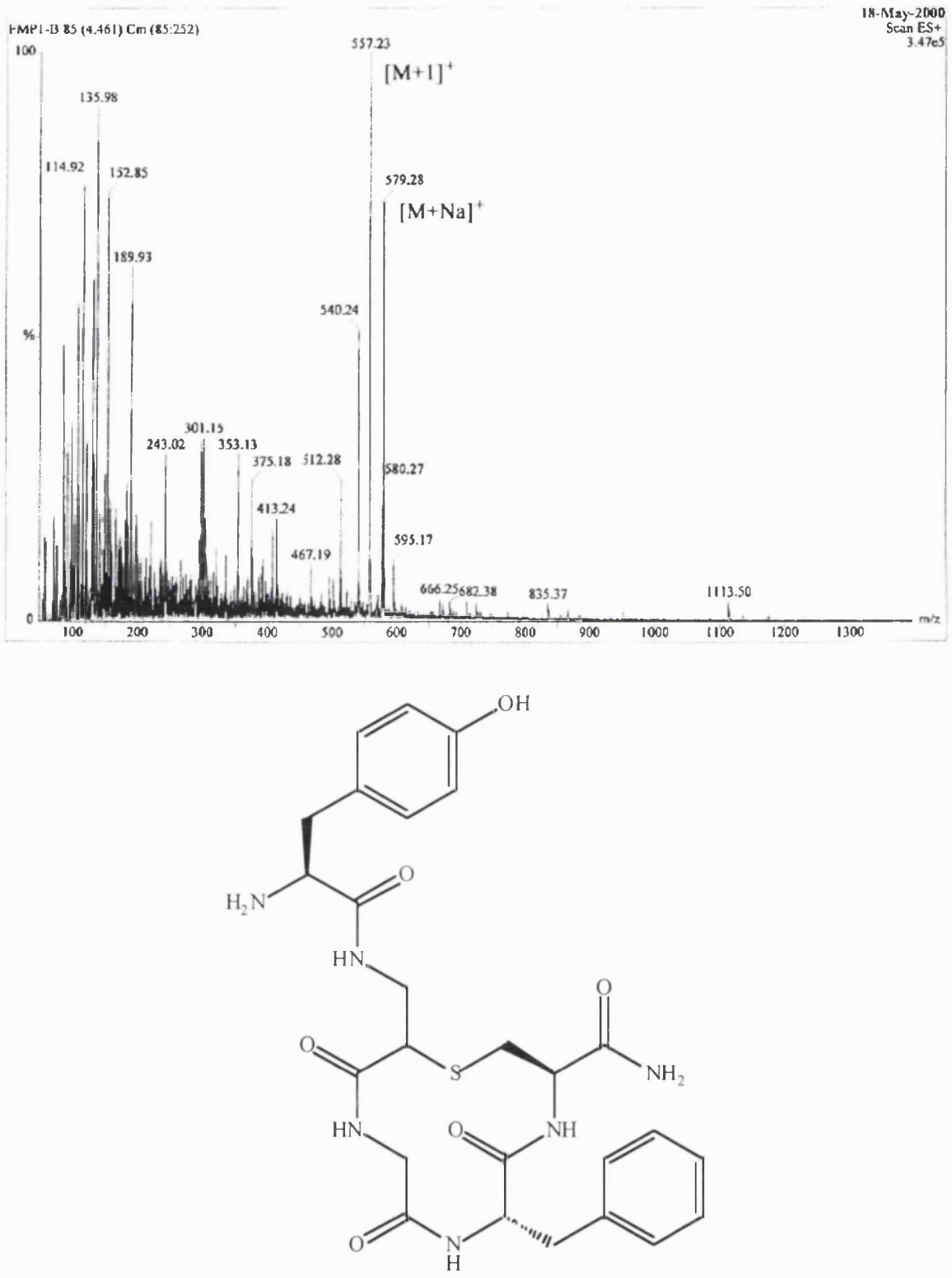

24

Crude mass spectrum of peptide $\mathbf{2 4}$, clearly indicating the parent ion as the baseline peak 


\section{Appendix Q}

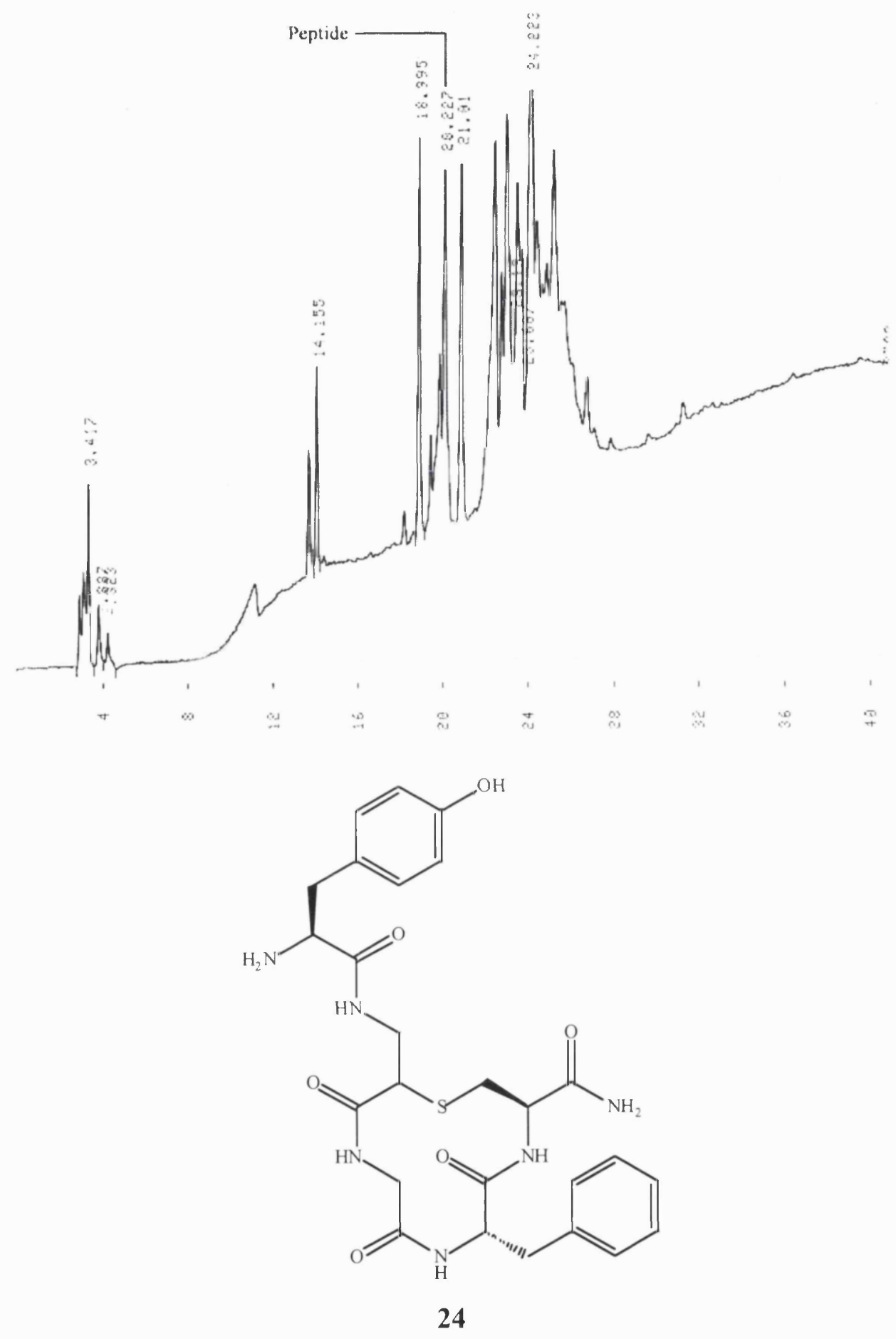

Crude analytical reverse phase HPLC trace of peptide 24 

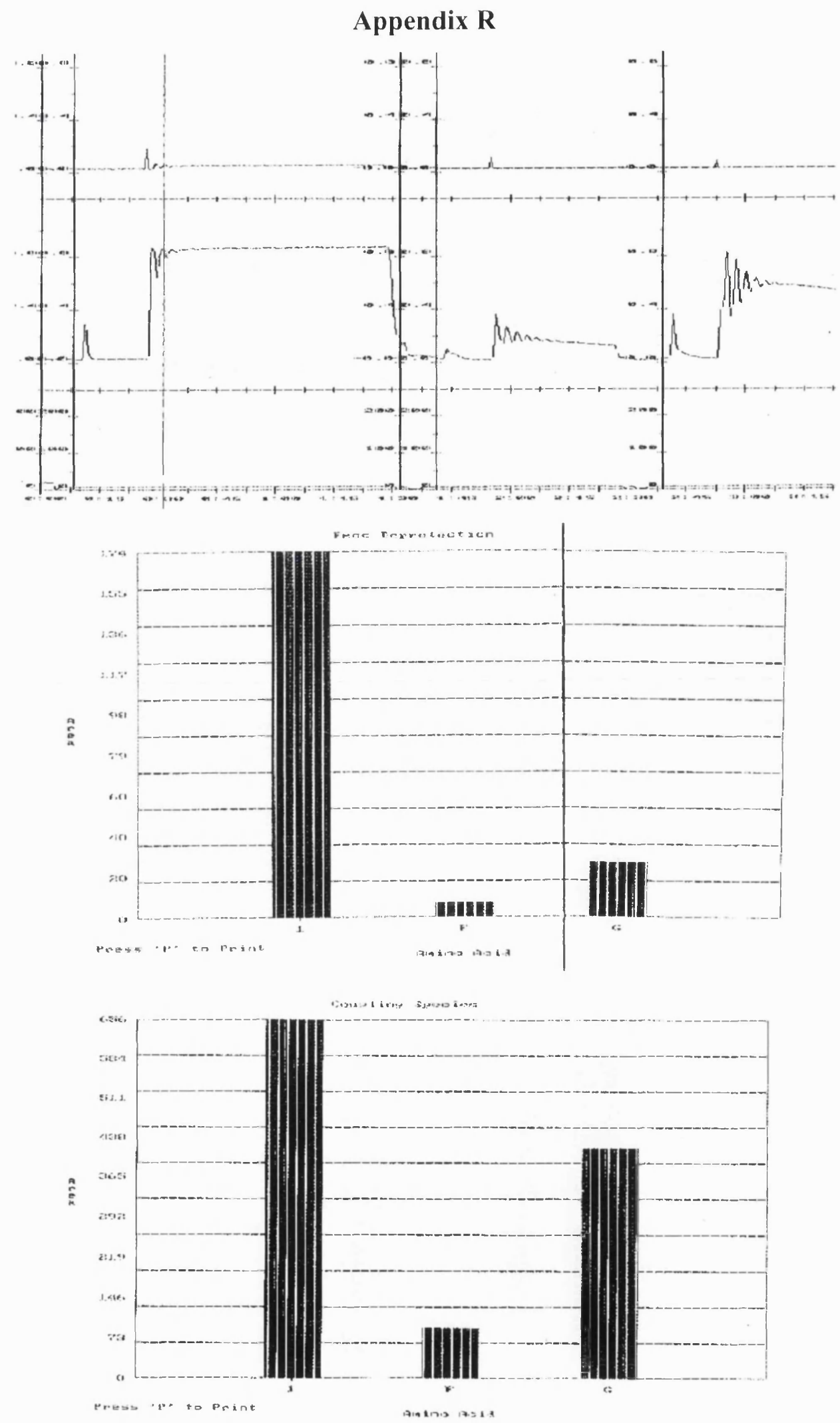

U.V. traces and integrations for Fmoc deprotection and amino acid coupling steps for continuous flow peptide synthesis of $\mathbf{2 4}$ 


\section{Appendix S}

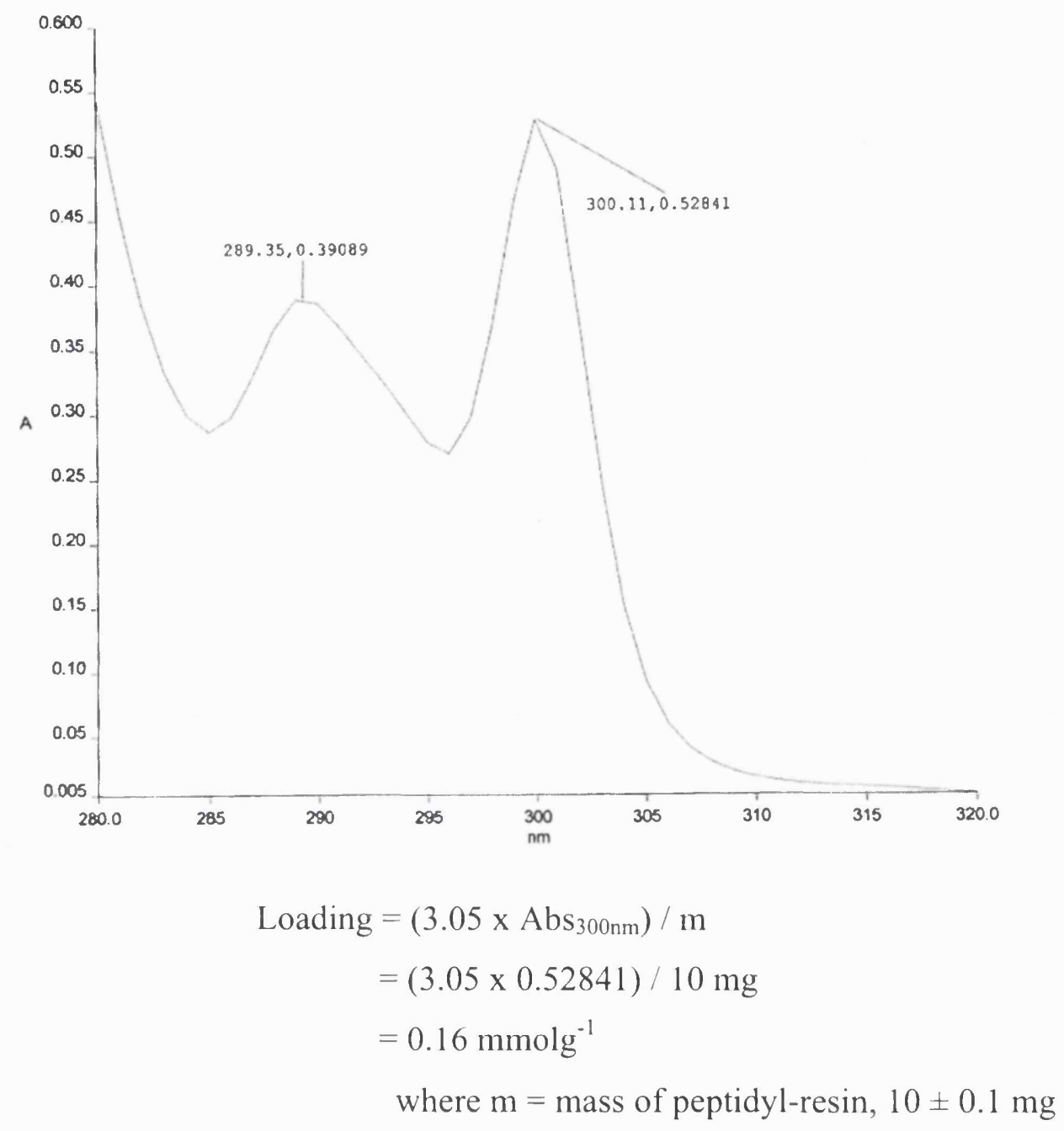

UV spectrum for Fmoc deprotection of nor-lanthionine incorporated onto Sieber TG resin in the synthesis of $\mathbf{2 4}$, for the quantitative determination of resin loading 


\section{Appendix T}

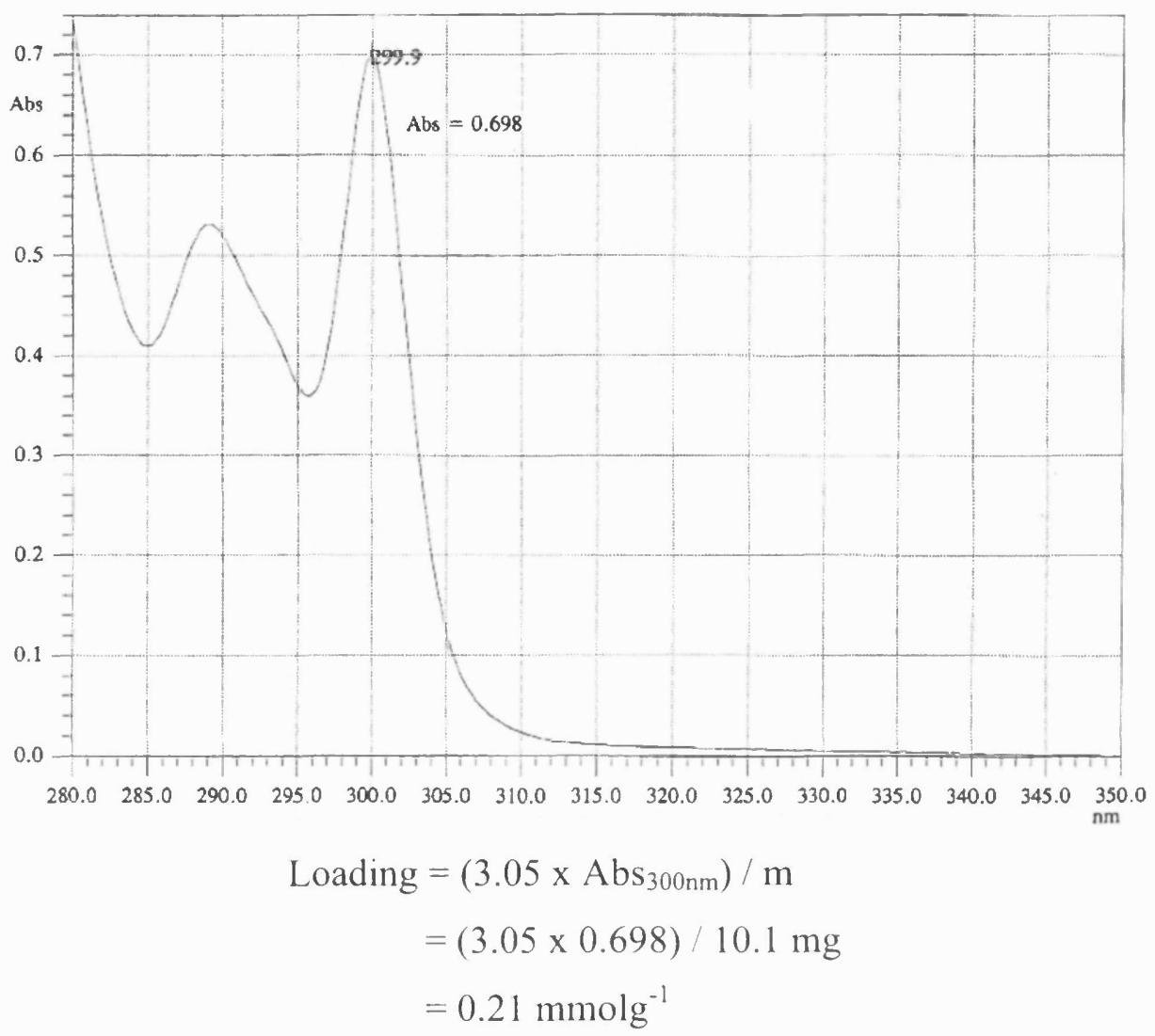

where $\mathrm{m}=$ mass of peptidyl-resin, $10 \pm 0.1 \mathrm{mg}$

UV spectrum for Fmoc deprotection of nor-lanthionine incorporated onto Sieber TG resin in the synthesis of $\mathbf{2 5}$, for the quantitative determination of resin loading 


\section{Appendix U}

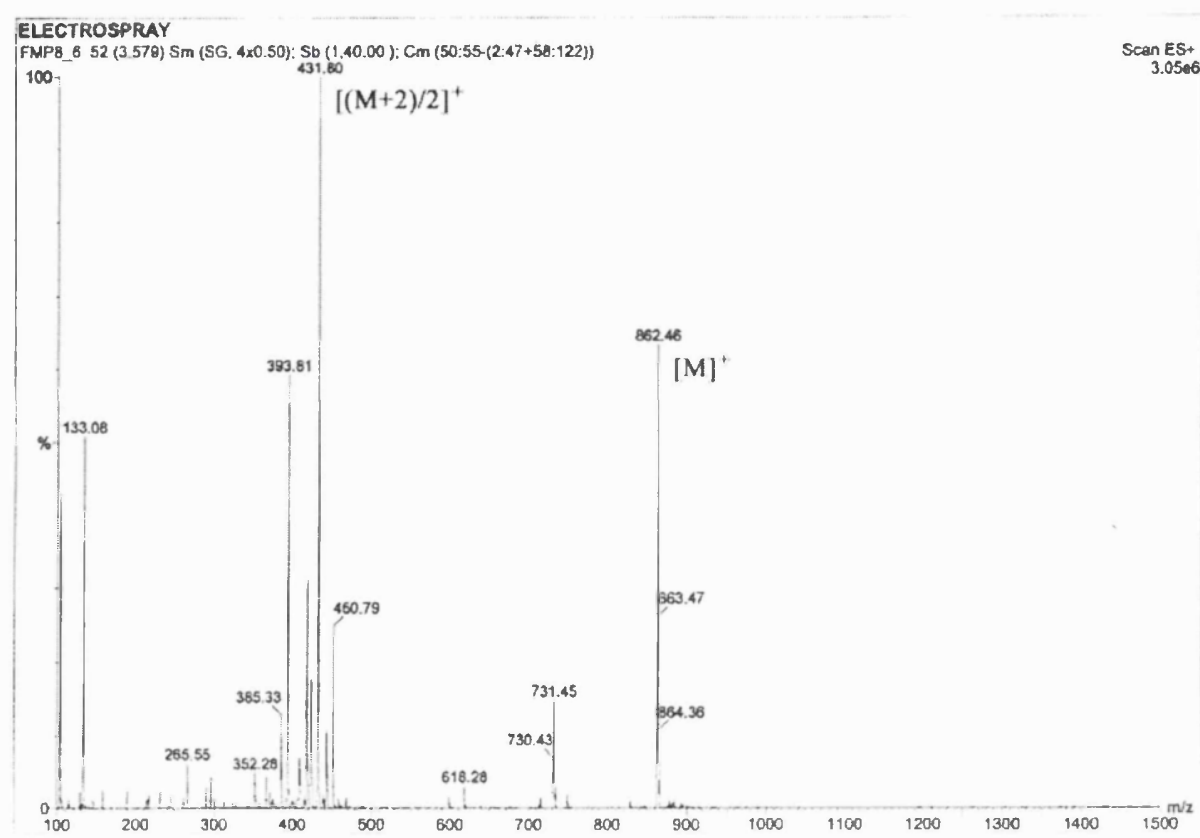

(a)

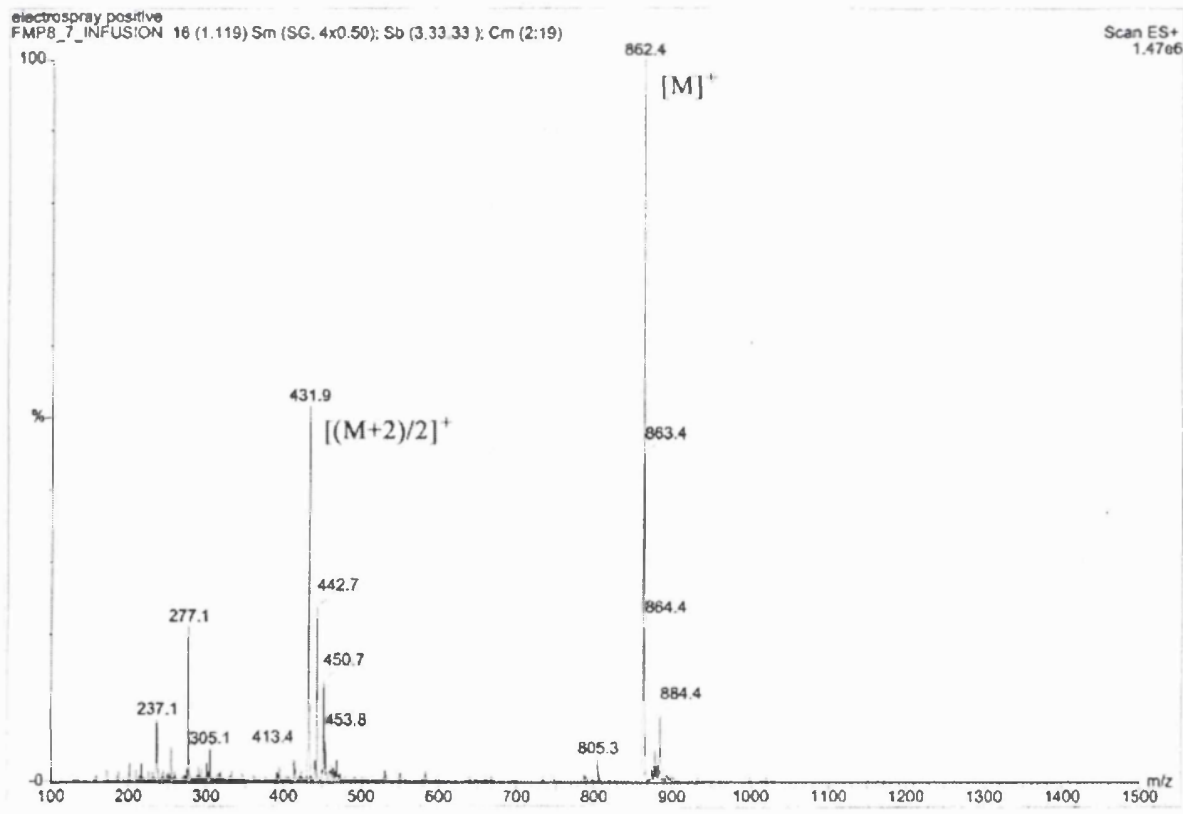

(b)

Mass spectra for (a) minor diastereoisomer and (b) major diastereoisomer of $\mathbf{2 5}$ 


\section{Appendix V}



where $\mathrm{m}=$ mass of peptidyl-resin, $10 \pm 0.1 \mathrm{mg}$

$*$ after baseline correction

UV spectrum for Fmoc deprotection of nor-lanthionine incorporated onto Sieber TG resin in the synthesis of $\mathbf{2 6}$, for the quantitative determination of resin loading 


\section{Appendix W}

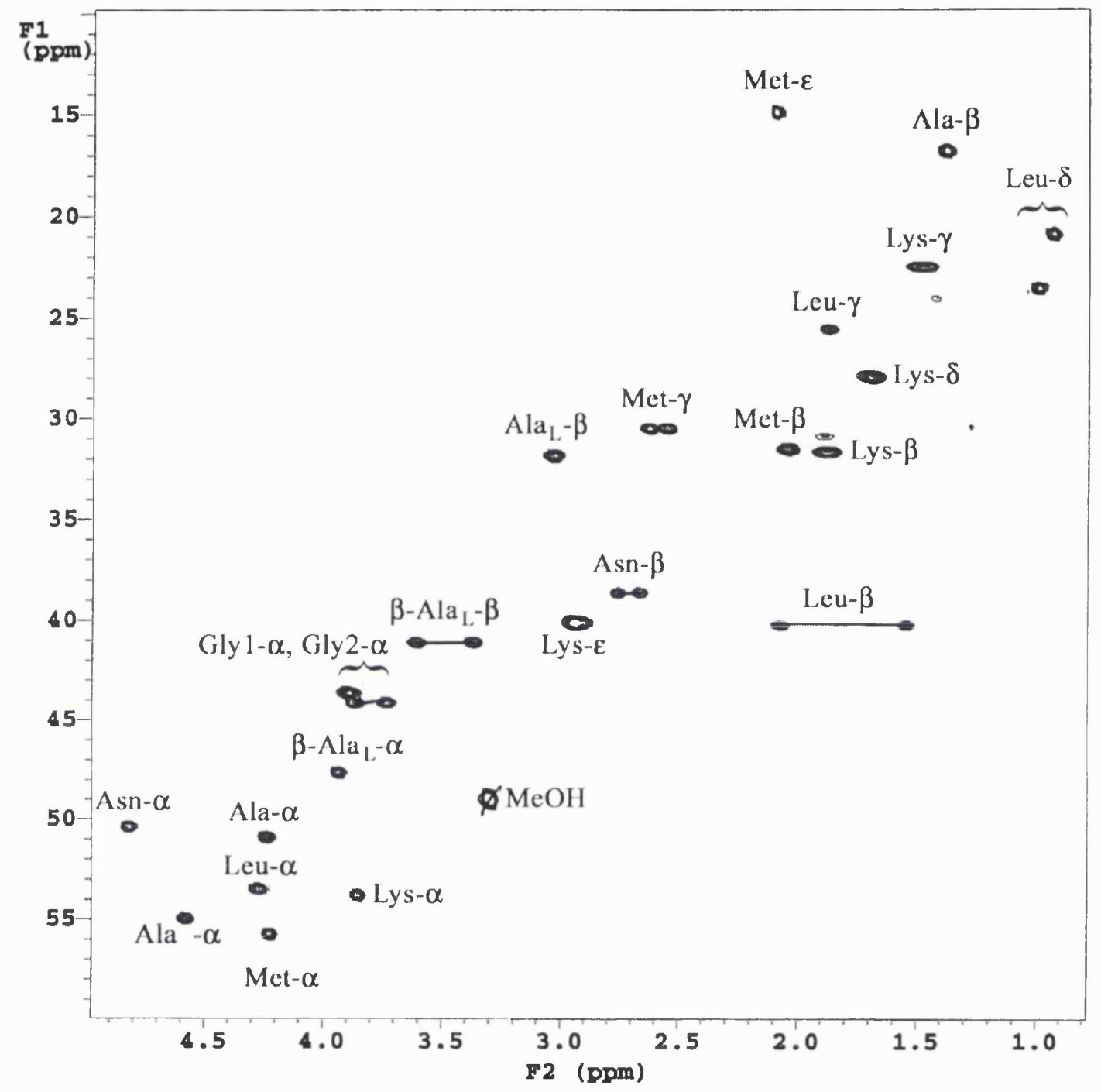

${ }^{1} \mathrm{H}-{ }^{13} \mathrm{C}$ HSQC spectrum of the minor diastereoisomer of $\mathbf{2 5 a}$, clearly indicating the difference in ${ }^{13} \mathrm{C}$ chemical shifts between the $\alpha$-Cs on both sides of the thioether bridge 


\section{Appendix X}

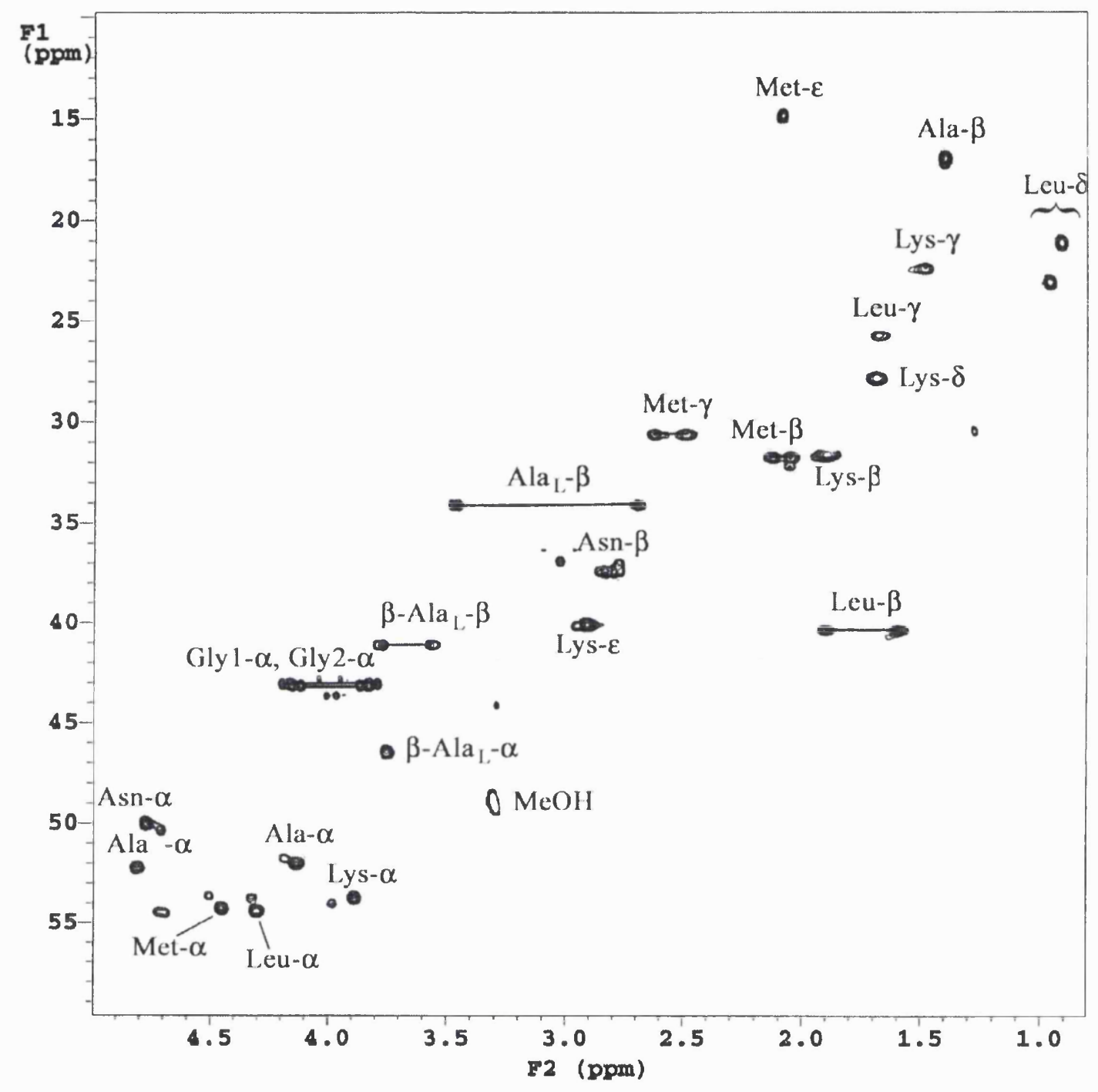

${ }^{1} \mathrm{H}-{ }^{13} \mathrm{C}$ HSQC spectrum of the major diastereoisomer of 25 , clearly indicating the difference in ${ }^{13} \mathrm{C}$ chemical shifts between the $\alpha$-Cs on both sides of the thioether bridge. It also shows the presence of a small amount of unidentified impurities. 


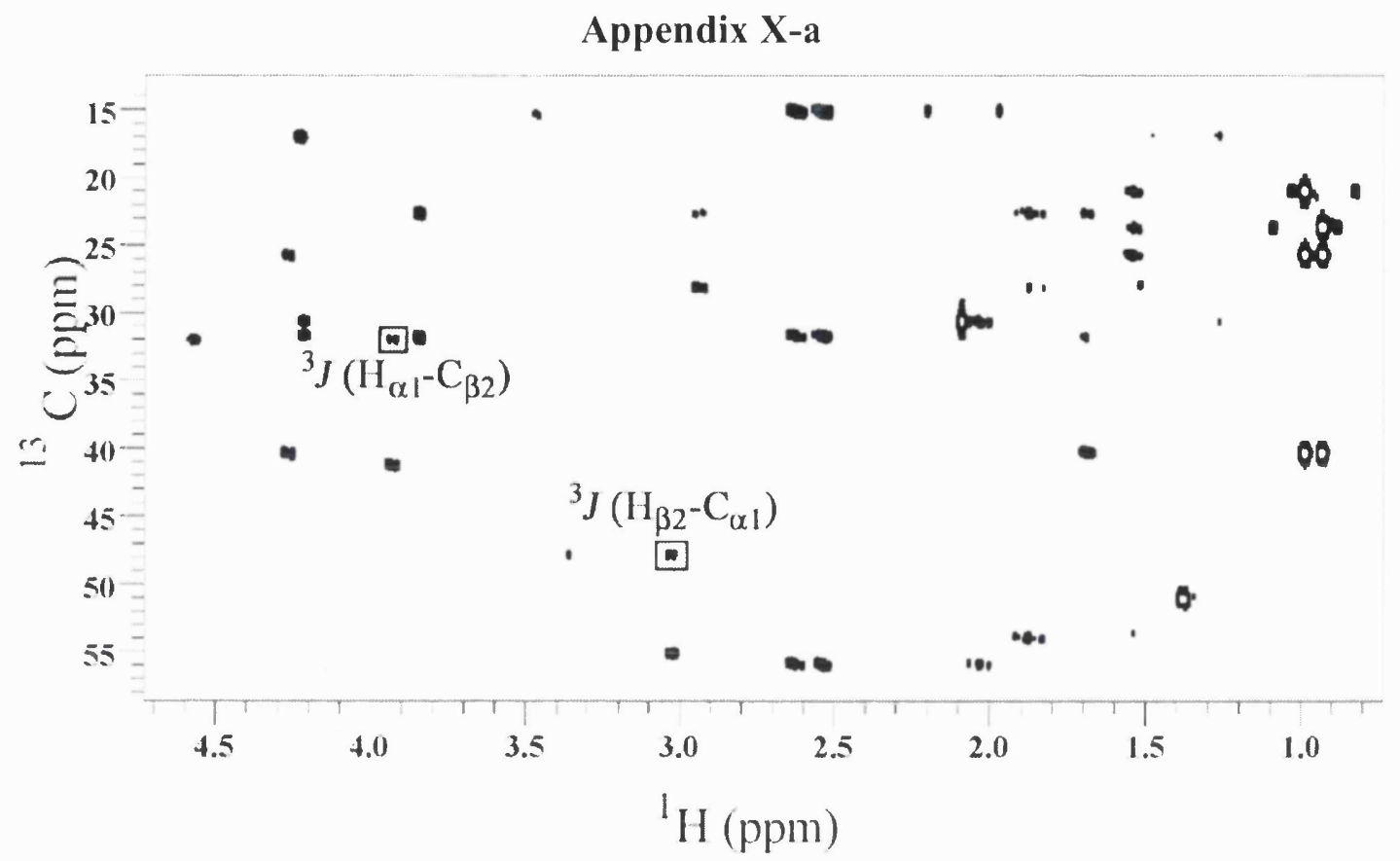

(a)

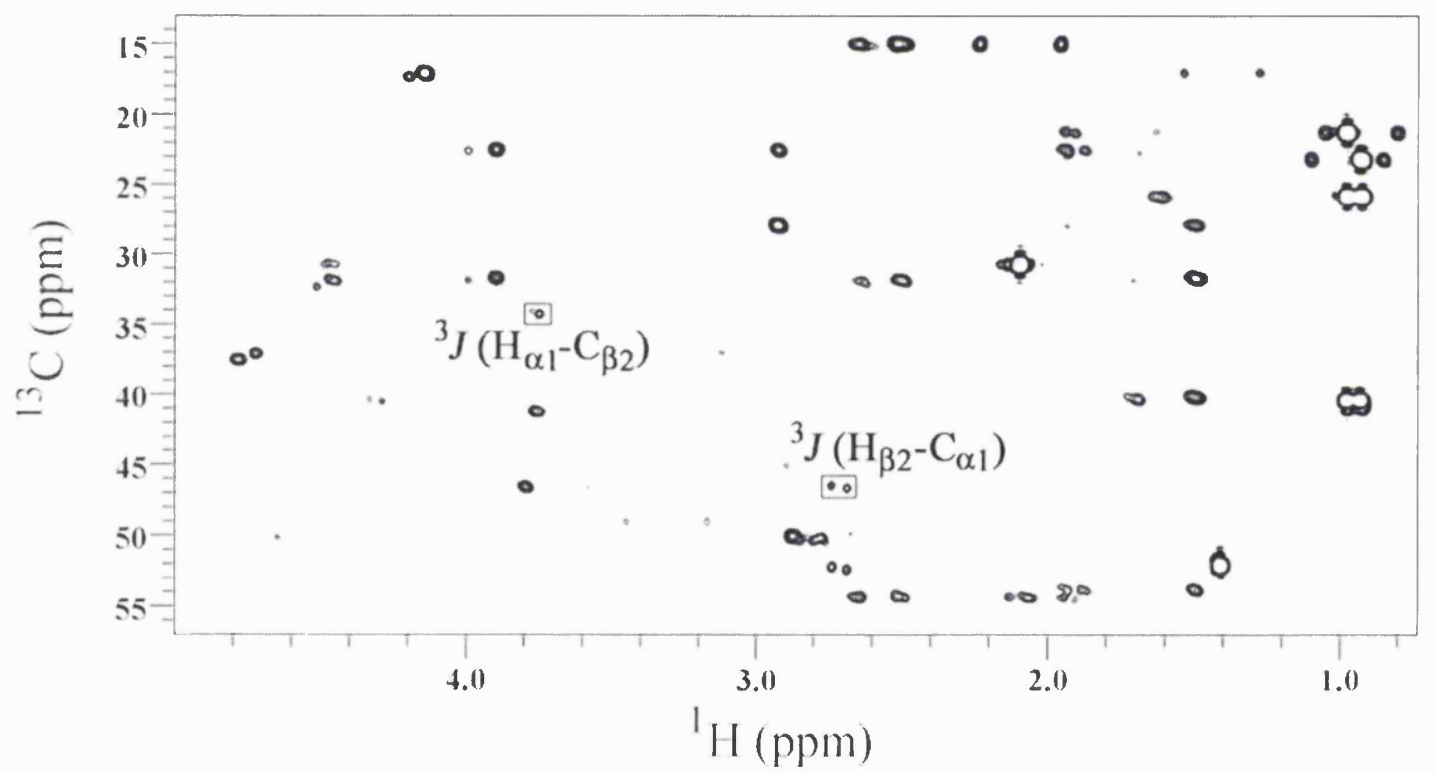

(b)

${ }^{1} \mathrm{H}-{ }^{13} \mathrm{C}$ HMBC spectrum of (a) the minor diastereoisomer of $\mathbf{2 5 a}$ and (b) the major diastereoisomer of $\mathbf{2 5} \mathbf{b}$, indicating the connectivity across the thioether bridge 


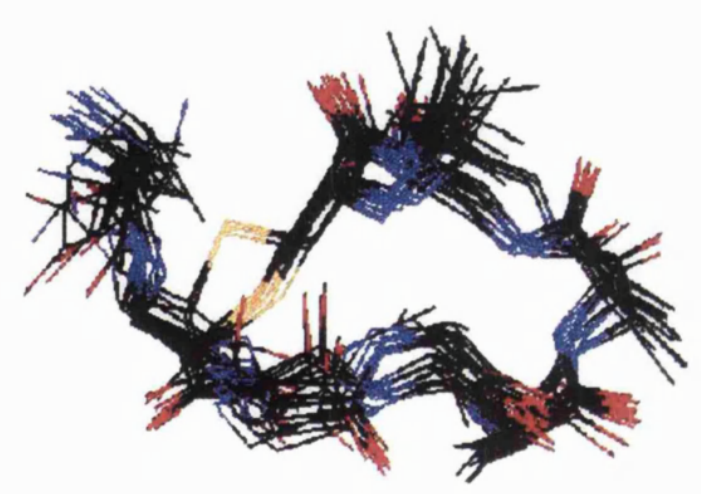

(a)

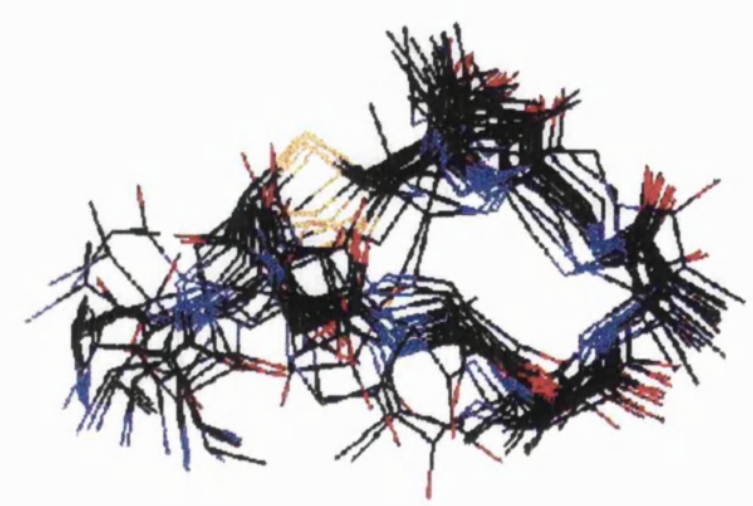

(b)

20 energy-minimised conformations for the minor diastereoisomer of peptide 25 in $\mathrm{CD}_{3} \mathrm{OD}$, with (a) $R$-stereochemistry at the $\beta$-amino acid moiety and (b) $S$-stereochemistry at the $\beta$-amino acid moiety 


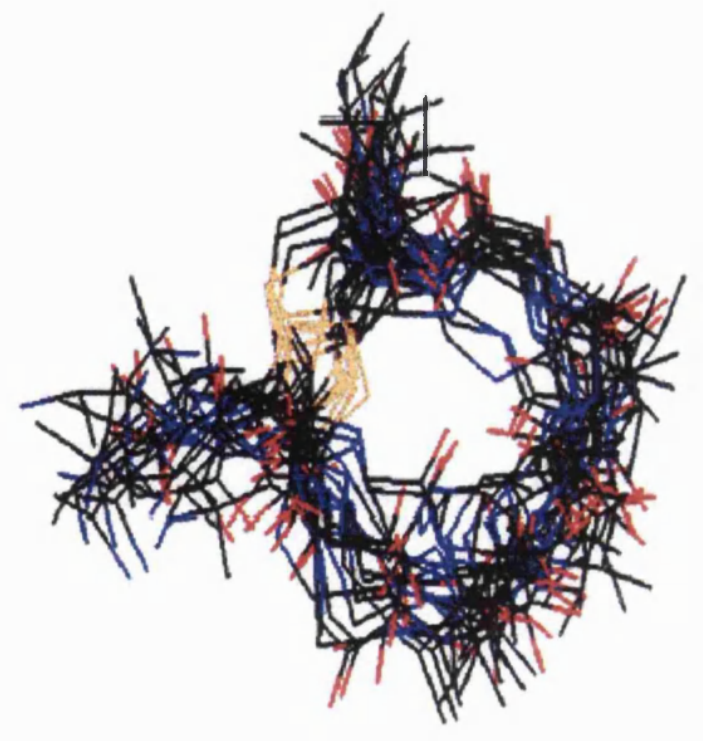

(a)

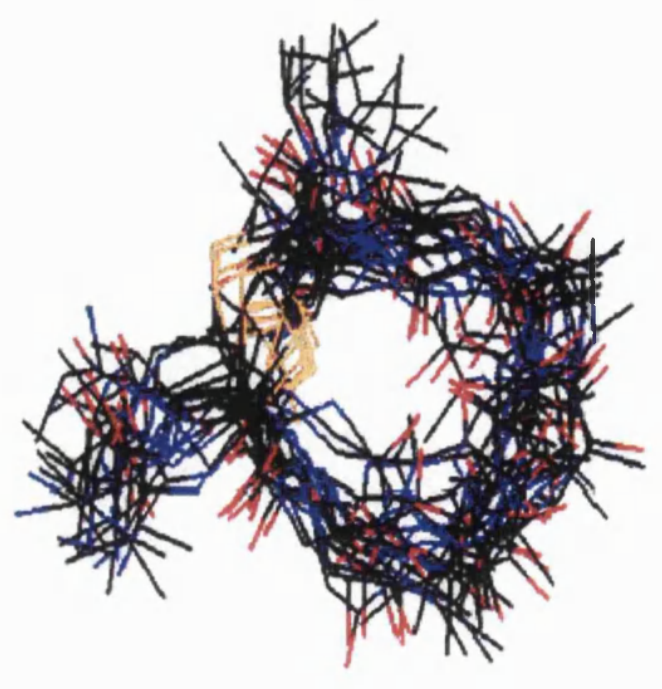

(b)

20 energy-minimised conformations for the major diastereoisomer of peptide 25 in $\mathrm{CD}_{3} \mathrm{OD}$, with (a) $R$-stereochemistry at the $\beta$-amino acid moiety and (b) $S$-stereochemistry at the $\beta$-amino acid moiety 


\section{References}

1. Horn, M. J., Jones, D. B., J. Biol. Chem., 1941, 138, 141-149

2. Horn, M. J., Jones, D. B., J. Biol. Chem., 1941, 139, 473

3. M. J. Horn, D. B. Jones, S. J. Ringel, J. Biol. Chem., 1942, 144, 87-91

4. T. Shiba, T. Wakamiya, K. Fukase, A. Sano, K. Shimbo, Y. Ueki, Biopolymers, 1986, 25, S11- S19

5. H. Shao, S. H. H. Wang, C.-W. Lee, G. Ösapay, M. Goodman, J. Org. Chem., 1995, 60, 2956-2957

6. G. Ösapay, L. Prokai, H.-S. Kim, K. F. Medzihradszky, D. H. Coy, G. Liapakis, T. Reisine, G. Melacini, Q. Zhu, S. H.-H. Wang, R.-H. Mattern, M. Goodman, J. Med. Chem., 1997, 40, 2241-2251

7. E. Gross, J. L. Morell, J. Am. Chem. Soc., 1971, 93, 4634-4635

8. G. Alderton, H. L. Fevold, J. Am. Chem. Soc, 1951, 73, 463-464 
9. H. Allgaier, G. Jung, R. G. Werner, U. Schneider, H. Zähner, Angew. Chem. Int. Ed. Engl., 1985, 24, 1051-1053

10. H. Allgaier, G. Jung, R. G. Werner, U. Schneider, H. Zähner, Eur. J. Biochem., 1986, 160, 9-22

11. N. Schnell, K.-D. Entian, U. Schneider, F. Götz, H. Zähner, R. Kellner, G. Jung, Nature, 1988, 333, 276-278

12. R. Kellner, G. Jung, T, Hörner, H. Zähner, N. Schnell, K.-D. Entian, F. Götz, Eur. J. Biochem., 1988, 177, 53-59

13. Y. Kido, T. Hamakado, T. Yoshida, M. Anno, Y. Motoki, J. Antibiotics (Tokyo), 1983, 36, 1295-1299

14. G. Jung, Angew. Chem. Int. Ed. Engl., 1991, 30, 1051-1192

15. N. Zimmermann, S. Freund, A. Fredenhagen, G. Jung, Eur. J. Biochem., $1993,216,419-428$

16. C. Kaletta, K. -D. Entian, G. Jung, Eur. J. Biochem., 1991, 199, 411-415

17. G. Bierbaum, C. Szekat, M. Josten, C. Heidrich, C. Kempter, G. Jung, H. -G. Sahl, Appl. Environ. Microbiol., 1996, 62, 385-392

18. N. Zimmermann, J. W. Metzger, G. Jung, Eur J. Biochem., 1995, 228, 786797

19. W. -W. Niu, H. C. Neu, Antimicrob. Agents and Chemother., 1991, 35, 9981000

20. R. W. Jack, A. Carne, J. Metzger, S. Stefanovic, H. -G. Sahl, G. Jung, J. Tagg, Eur. J. Biochem., 1994, 220, 455-462

21. M. Van De Kamp, H. W. Van Den Hooven, R. N. H. Konings, G. Bierbaum, H. -G. Sahl, O. P. Kuipers, R. J. Siezen, W. M. De Vos, C. W. Hilbers, F. J. M. Van De Ven. Eur. J. Biochem., 1995, 230, 587-600

22. C. I. Mørtvedt, J. Nissen-Meyer, K. Sletten, I. F. Nes, Appl. Environ. Microbiol., 1991, 57, 1829-1834

23. K. F. Ross, C. W. Ronson, J. R. Tagg, Appl. Environ. Microbiol., 1993, 59, 2014-2021

24. D. Pridmore, N. Rekhil, A.-C. Pittet, B. Suri, B. Mollet, Appl. Environ. Microbiol., 1996, 62, 1799-1802 
25. J.-C. Piard, O. P. Kuipers, H. S. Rollema, M. J. Desmazeaud, W. M. De Vos, J. Biol. Chem., 1993, 268, 16361-16368

26. H. Kimura, H. Matsusaki, T. Sashihara, K. Sonomoto, A. Ishizaki, Biosci. Biotechnol. Biochem., 1998, 62, 2341-2345

27. D. L. Turner, L. Brennan, H. E. Meyer, C. Lohaus, C. Siethoff, H. S. Costa, B. Gonzalez, H. Santos, J. E. Suárez, Eur. J. Biochem., 1999, 264, 833-839

28. B. Furmanek, T. Kaczorowski, R. Bugalski, K. Bielawski, J. Bogdanowicz, A. J. Podhajska, J. Appl. Microbiol., 1999, 87, 856-866

29. W. A. Woodruff, J. Novak, P. W. Caufield, Gene, 1998, 206, 37-43

30. S. H. Paik, A. Chakicherla, J. M. Hansen, J. Biol. Chem., 1998, 273, 2313423142

31. H. -G. Sahl, R. W. Jack, G. Bierbaum, Eur. J. Biochem., 1995, 230, 827-853

32. A. Guder, I. Wiedermann, H. -G. Sahl, Biopolymers, 2000, 55, 62-73

33. H. E. Meyer, M. Heber, B. Eisermann, H. Korte, J. W. Metzger, G. Jung, Anal. Biochem., 1994, 223, 185-190

34. T. B. Walk, R. Süßmuth, C. Kempter, V. Gnau, R. W. Jack, G. Jung, Biopolymers, 1999, 49, 329-340

35. H. -G. Sahl, H. Brandis, FEMS Microbiol. Lett., 1983, 16, 75-79

36. H. Brötz, M. Josten, I. Wiedemann, U. Schneider, F. Götz, G. Bierbaum, H. -G. Sahl, Mol. Microbiol., 1998, 30, 317-327

37. H. Holo, Z, Jeknic, M. Daeschel, S. Stevanovic, I. F. Nes, Microbiology, 2001, 147, 643-651

38. O. McAuliffe, M. P. Ryan, R. P. Ross, C. Hill, P. Breeuwer, T. Abee, Appl. Environ. Microbiol., 1998, 64, 439-445

39. M. C. Booth, C. P. Bogie, H. -G. Sahl, R. J. Siezen, K. L. Hatter, M. S. Gilmore, Mol. Microbiol., 1996, 21, 1175-1184

40. M. A. D. B. Navaratna, H. -G. Sahl, J. R. Tagg, Appl. Environ. Microbiol., 1998, 64, 4803-4808

41. L. A. Rogers, E. O. Whittier, J. Bacteriol., 1928, 16, 211-229

42. L. A. Rogers, E. O. Whittier, J. Bacteriol., 1928, 16, 321-325

43. A. T. R. Mattick, A. Hirsch, Lancett, 1947, 2, 5-8

44. A. Hirsch, Nature, 1951, 167, 1031-1032 
45. A. Hirsch, J. Gen. Microbiol., 1951, 5, 208-221

46. A. A. Anderson, H. D. Michener, H. S. Olcott, Antibiotics Chemother., 1953, 3,521

47. E. Gross, J. L. Morell, J. Am. Chem. Soc., 1967, 89, 2791-2792

48. K. Fukase, M. Kitazawa, A. Sano, K. Shimbo, S. Horimoto, H. Fujita, A. Kubo, T. Wakamiya, T. Shiba, Bull. Chem. Soc. Jpn., 1992, 65, 2227-2240

49. H. S. Rollema, O. P. Kuipers, P. Both, W. M. De Vos, R. J. Siezen, Appl. Environ. Microbiol., 1995, 61, 2873/2878

50. A. Polinsky, M. G. Cooney, A. Toy-Palmer, G. Ösapay, M. Goodman, J. Med. Chem., 1992, 35, 4185-4194

51. G. A. Olson, R. D. Olson, A. J. Kastin, Peptides, 1996, 17, 1421-1466 (review)

52. G. Ösapay, M. Goodman, J. Chem. Soc., Chem. Commun., 1993, 1599-1600

53. J. P. Mayer, J. Zhang, S. Gröger, C. F. Liu, Peptides: Proceed. $15^{\text {th }} \mathrm{Am}$. Peptide Symp., 1999, 291-292

54. J. P. Mayer, J. Zhang, S. Gröger, C. F. Liu, M. A. Jarosinski, J. Peptide Res., 1998, 51, 432-436

55. P. Brazeau, W. Vale, R. Burgus, N. Ling, M. Butcher, J. Rivier, R. Guillemin, Science, 1973, 179, 77-79

56. W. Bauer, U. Briner, W. Doepfner, R. Haller, R. Huguenin, P. Merbach, T. J. Petcher, J. Pless, Life Sci., 1982, 31, 1133-1140

57. C. Wynants, G. Van Binst, H. R. Loosli, Int. J. Peptide Protein Res., 1985, $25,622-627$

58. G. Melacini, Q. Zhu, G. Ösapay, M. Goodman, J. Med. Chem., 1997, 40, 2252-2258

59. H. Li, X. Jiang, S. B. Howell, M. Goodman, J. Peptide Sci., 2000, 6, 26-35

60. H. Zheng, D. Fink, X, Jiang, S. Aebi, P. Law, M. Goodman, S. B. Howell, Clinical Cancer Res., 1997, 3, 1323-1330

61. H. Li, X. Jiang, M. Goodman, J. Peptide Sci., 2001, 7, 82-91

62. H. Li, X. Jiang, M. Goodman, Peptides: Proceed. $15^{\text {th }}$ Am. Peptide Symp., 1999, 455-456

63. R. O. Hynes, Cell, 1987, 48, 549-554 
64. D. M. Nowlin, F. Gorcsan, M. Moscinski, S. -L. Chiang, T. J. Lobl, P. M. Cardarelli, J. Biol. Chem., 1993, 268, 20352-20359

65. L. Yiu, Y. Lai, J. V. Wade, S. M. Coutts, Tetrahedron Lett., 1998, 39, 66336636

66. D. N. Harp, J. G. Gleason, J. Org. Chem., 1971, 36, 73-80

67. D. N. Harp, J. G. Gleason, J. Org. Chem., 1970, 35, 3259-3263

68. D. N. Harp, J. G. Gleason, J. Am. Chem. Soc., 1971, 93, 2437-2445

69. F. Cavelier-Frontin, J. Daunis, R. Jacquier, Tetrahedron: Asymmetry, 1992, 3, $85-94$

70. J. M. Probert, D. Rennex, M. Bradley, Tetrahedron Lett., 1996, 37, 11011104

71. L. S. Monteiro, P. M. T. Ferreira, H. L. S. Maria, J. Sacramento, Peptides: Proceed. $17^{\text {th }}$ Am. Peptide Symp., 2001, 44-45

72. L. D. Arnold, J. C. G. Drover, J. C. Vederas, J. Am. Chem. Soc., 1987, 109, 4649-4659

73. L. D. Arnold, T. H. Kalantar, J. C. Vederas, J. Am. Chem. Soc., 1985, 107, 7105-7109

74. E. S. Ratemi, J. C. Vederas, Tetrahedron Lett., 1994, 35, 7605-7608

75. M. Sakai, K. Hashimoto, H. Shirahama, Heterocycles, 1997, 44, 319-324

76. C. Dugave, A. Menez, Tetrahedron: Asymmetry, 1997, 8, 1453-1465

77. J. -K. Son, D. Kalvin, R. W. Woodard, Tetrahedron Lett., 1988, 29, 40454048

78. M. F. A. Groussier, "A new orthogonal protecting group strategy for lanthionine-containing peptides", Ph.D. Thesis, University College London, 2000

79. Y. Hata, M. Watanabe, Tetrahedron, 1987, 43, 3881-3888

80. T. Wakamiya, K. Shimbo, T. Shiba, K. Nakajima, M. Neya, K. Okawa, Bull. Chem. Soc. Jpn., 1982, 55, 3878-3881

81. R. B. Merrifield, J. Am. Chem. Soc., 1963, 85, 2149-2154

82. a) R. C. Sheppard, Peptides 1971: Proceed. $11^{\text {th }}$ Eur. Peptide Symp., 1973, 111 ; b) E. Atherton, D. L. J. Clive, R. C. Sheppard, J. Am. Chem. Soc., 1975, $97,6584-6585$ 
83. E. Kaiser, R. L. Colescott, C, D. Bossinger, P. I. Cook, Anal. Biochem., 1970, $84,595-598$

84. E. Bayer, W. Rapp, Chemistry of Peptides and Proteins (W. Voelter, E. Bayer, Y. A. Ovchinnikov, V. T. Ivanov, eds), W. de Gruyter, Berlin, 1986, $3,3-8$

85. E. Bayer, Angew. Chem. Int. Ed. Engl., 1991, 30, 113-129

86. H. Rink, Tetrahedron Lett., 1987, 28, 3787-3790

87. P. Sieber, Tetrahedron Lett., 1987, 28, 2107-2110

88. K. Barlos, D. Gatos, J. Kallitsis, G. Papaphotiu, P. Sotiriu, Y. Wenging, W. Schäffer, Tetrahedron Lett., 1989, 30, 3943-3946

89. S. -S. Wang, J. Am. Chem. Soc., 1973, 95, 1328-1333

90. E. Bayer, N. Clausen, C. Goldammer, B. Henkel, W. Rapp, L. Zhang, Peptides: Proceed. $13^{\text {th }}$ Am. Peptide Symp., 1994, 156-158

91. J. M. J. Frechet, K. E. Hague, Tetrahedron Lett., 1975, 14, 3055-3056

92. M. S. Bernatowicz, S. B. Daniels, H. Küster, Tetrahedron Lett., 1989, 30, $4645-4648$

93. R. C. Sheppard, B. J. Williams, Int. J. Peptide Protein Res., 1982, 20, 451

94. M. Mergler, R. Nyfeler, R. Tanner, J. Gosteli, P. Grogg, Tetrahedron Lett., 1988, 29, 4009-4012

95. A. Florsheimer, B. Riniker, Peptides 1990: Proceed. $21^{\text {st }}$ Eur. Peptide Symp., $131-133$

96. E. Atherton, C. J. Logan, R. C. Sheppard, J. Chem. Soc., Perkin Trans. I, 1981, 538-546

97. C. P. Holmes, D. G. Jones, J. Org. Chem., 1995, 60, 2318-2319

98. T. Curtius, Chem. Ber., 1902, 35, 3226-3228

99. T. Shioiri, K. Ninomiya, S. -I. Yamada, J. Am. Chem. Soc., 1972, 94, 62036205

100. S. K. Sharma, A. D. Wu, N. Chandramouli, C. Fotsch, G. Kardash, K. W. Bair, J. Peptide Res., 1999, 53, 501-506

101. E. Atherton, J. L. Holder, M. Meldal, R. C. Sheppard, R. M. Valerio, J. Chem. Soc., Perkin Trans. 1, 1988, 2887 
102. D. Sarantakis, J. Teichman, E. L. Lien, R. L. Fenichel, Biochem. Biophys. Res. Commun., 1976, 73, 336-342

103. W. Konig, R. Geiger, Chem. Ber., 1970, 103, 788-798

104. C. L. Stevens, M. E. Munk, J. Am. Chem. Soc., 1958, 80, 4065-4071

105. a) B. Castro, J. R. Dormoy, G. Evin, C. Selve, Tetrahedron Lett., 1975, 14, 1219-1222; b) J. Coste, J. Le-Nguyen, B. Castro, Tetrahedron Lett., 1990, 31, 205-508; c) R. Knorr, A. Trzeciak, W. Bannwarth, D. Gillessen, Tetrahedron Lett., 1989, 30, 1927-1930

106. L. A. Carpino, A. El-Faham, C. A. Minor, F. Albericio, J. Chem. Soc., Chem. Commun., 1994, 201-203

107. L. A. Carpino, A. El-Faham, F. Albericio, Tetrahedron Lett., 1994, 35, 2279-2282

108. L. A. Carpino, J. Am. Chem. Soc., 1993, 115, 4397-4398

109. F. Albericio, I. Abdelmoty, J. M. Bofill, L. A. Carpino, A. El-Faham, B. M. Foxman, M. Gairi, E. Giralt, G. W. Griffin, S. A. Kates, P. Lloyd-Williams, C. A. Minor, L. M. Scarmoutzos, H. N. Shroff, S. Triolo, H. Wenschuh, Peptides 1995: Proceed. 23 ${ }^{\text {rd }}$ Eur. Peptide Symp., 23-25

110. S. A. Kates, S. Triolo, E. Diekman, L. A. Carpino, A. El-Faham, D. Ionescu, F. Albericio, Peptides: Proceed. $14^{\text {th }}$ Am. Peptide Symp., 1998, 115-116

111. F. Albericio, J. M. Bofill, A. El-Faham, S. A. Kates, J. Org. Chem., 1998, $63,9678-9683$

112. J. Alsina, G. Barany, F. Albericio, S. A. Kates, Lett. Peptide Sci., 1999, 6, 243-245

113. A. Ehrlich, H. -U. Heyne, R. Winter, M. Beyermann, H. Haber, L. A. Carpino, M. Bienert, J. Org. Chem., 1996, 61, 8831-8838

114. F. Albericio, M. Cases, J. Alsina, S. Triolo, L. A. Carpino, S. A. Kates, Tetrahedron Lett., 1997, 38, 4853-4856

115. P. Lloyd-Williams, G. Jou, I. González, J. M. Caba, F. Albericio, E. Giralt, Peptides 1996: Proceed. 24 ${ }^{\text {th }}$ Eur. Peptide Symp., 589-590

116. C. M. Stevens, R. Watanabe, J. Am. Chem. Soc., 1950, 72, 725-727

117. B. M. Trost, Acc. Chem. Res., 1980, 11, 385-393

118. F. Guibè, Tetrahedron, 1997, 53, 13509-13556 
119. F. Guibè, Tetrahedron, 1998, 54, 2967-3042

120. G. Senneyey, G. Barcelo, J. -P. Senet, Tetrahedron Lett., 1987, 28, 58095810

121. P. D. Jeffrey, S. W. McCombie, J. Org. Chem., 1982, 47, 587-590

122. U. Schmidt, B. Riedl, J. Chem. Soc., Chem Commun., 1992, 1186-1187

123. H. Kunz, H. Waldmann, Angew. Chem. Int. Ed. Engl., 1984, 23, 71-72

124. H. Mastalerz, V. Vinet, J. Chem. Soc., Chem. Commun., 1987, 1283-1284

125. M. Ciommer, H. Kunz, Synlett, 1991, 593-595

126. J. P. Genêt, E Blart, M Savignac, S. Lemeune, J.-M. Paris, Tetrahedron Lett., 1993, 34, 4189-4192

127. J. P. Genêt, E Blart, M Savignac, S. Lemaire-Audoire, G. Pourcelot, , J.-M. Bernard, Tetrahedron Lett., 1994, 35, 8783-8786

128. S. A. Kates, B. G. de la Torre, R. Eritja, F. Albericio, Tetrahedron Lett., 1994, 35, 1033-1034

129. G. B. Bloomberg, D. Askin, A. R. Gargaro, M. J. A. Tanner, Tetrahedron Lett., 1993, 34, 4709-4712

130. H. Kunz, C. Unverzagt, Angew. Chem. Int. Ed. Engl., 1984, 23, 436-437

131. J. P. Genêt, E Blart, M Savignac, S. Lemeune, S. Lemaire-Audoire, J.-M. Bernard, Synlett, 1993, 680-682

132. A. Merzouk, G. Guibé, Tetrahedron Lett., 1992, 33, 477-480

133. H. Hey, H. -J. Arpe, Angew. Chem. Int. Ed. Engl., 1973, 12, 928-929

134. O. Dangles, F. Guibé, G. Balavoine, S. Lavielle, A. J. Marquet, J. Org. Chem., 1987, 52, 4984-4993

135. R. O. Hutchins, K. Learn, R. P. Fulton, Tetrahedron Lett., 1980, 21, 27-30

136. M. Dessolin, M. -G, Guillerez, N. Thieriet, F. Guibé, A. Loffet, Tetrahedron Lett., 1995, 36, 5741-5744

137. N. Thieriet, J. Alsina, E. Giralt, F. Guibé, F. Albericio, Tetrahedron Lett., 1997, 38, 7275-7278

138. H. Kunz, B. Dombo, Angew. Chem. Int. Ed. Engl., 1988, 27, 711-713

139. F. Guibé, O Dangles, G. Balavoine, A Loffet, Tetrahedron Lett., 1989, 30, 2641-2644

140. B. Blankemeyer-Menge, R. Frank, Tetrahedron Lett., 1988, 29, 5871-5874 
141. S. A. Kates, S. B. Daniels, F. Albericio, Anal. Biochem., 1993, 212, 303-310

142. J. Alsina, F. Rabanal, E. Giralt, F. Albericio, Tetrahedron Lett., 1994, 35, 9633-9636

143. I. R. Marsh, M. Bradley, J. Org. Chem., 1997, 62, 6199-6203

144. S. A. Kates, N. A. Solé, C. R. Johnson, D. Hudson, G. Barany, F. Albericio, Tetrahedron Lett., 1993, 34, 1549-1552

145. P. Lloyd-Williams, A. Merzouk, F. Guibè, F. Albericio, E. Giralt, Tetrahedron Lett., 1994, 35, 4437-4440

146. D. Delforge, M. Dieu, E. Delaive, M. Art, B. Gillon, D. Devreese, M. Raes, J. Van Beeuman, J. Remacle, Lett. Peptide Sci., 1996, 3, 89-97

147. Y. Hayakawa, S. Wakabayashi, H. Kato, R. Noyori, J. Am. Chem. Soc., 1990, $112,1691-1696$

148. J. C. Arnould, F. Landier, M. J. Pasquet, Tetrahedron Lett., 1992, 33, 71337136

149. B. W. Bycroft, W. C. Chan, S. R. Chhabra, P. H. Teesdale-Spittle, P. M. Hardy, J. Chem. Soc., Chem. Commun., 1993, 776-777

150. P. Schenone, L Mosti, G. Menozzi, J. Heterocyclic Chem., 1982, 19, $1355-$ 1361

151. B. W. Bycroft, W. C. Chan, S. R. Chhabra, N. D. Hone, J. Chem. Soc., Chem. Commun., 1993, 778-779

152. B. Kellam, W. C. Chan, S. R. Chhabra, B. W. Bycroft, Tetrahedron Lett., 1997, 38, 5391-5394

153. K. Augustyns, W. Kraas, G. Jung, J. Peptide Res., 1998, 51, 127-133

154. S. R. Chhabra, B. Hothi, D. J. Evans, P. D. White, B. W. Bycroft, W. C. Chan, Tetrahedron Lett., 1998, 39, 1603-1606

155. W. C. Chan, B. W. Bycroft, D. J. Evans, P. D. White, Peptides 1994: Proceed. 23 ${ }^{\text {rd }}$ Eur. Peptide Symp., 153-154

156. W. C. Chan, B. W. Bycroft, D. J. Evans, P. D. White, J. Chem. Soc., Chem. Commun., 1995, 2209-2210

157. D. Lelièvre, D. Daguet, A. Brack, Tetrahedron Lett., 1995, 36, 9317-9320

158. I. A. Nash, B. W. Bycroft, W. C. Chan, Tetrahedron Lett., 1996, 37, 26252628 
159. B. Rohwedder, Y. Mutti, P. Dumy, M. Mutter, Tetrahedron Lett., 1998, 39, $1175-1178$

160. G. Greiner, L. Seyfarth, S. Reissmann, Peptides 1996: Proceed. $24^{\text {th }}$ Eur. Peptide Symp., 435-436

161. T. Johnson, M. Liley, T. J. Cheeseright, F. Begum, J. Chem. Soc., Perkin Trans. I, 2000, 2811-2820

162. K. Fukase, M. Kitazawa, A. Sano, K. Shimbo, S. Horimoto, H. Fujita, T. Wakamiya, T. Shiba, Tetrahedron Lett., 1988, 29, 795-798

163. W. F. DeGrado, E. T. Kaiser, J. Org. Chem., 1980, 45, 1295-1300

164. B. H. Lee, Tetrahedron Lett., 1997, 38, 757-760

165. (a) G. Ösapay, J. W. Taylor, J. Am. Chem. Soc., 1990, 112, 6046-6051; (b)

C. Blackburn, S. A. Kates, Methods in Enzymology, 1997, 289, 175-198

166. W. Zhang, J. W. Taylor, Tetrahedron Lett., 1996, 37, 2173-2179

167. S. Burrage, T. Raynham, G. Williams, J. W. Essex, C. Allen, M. Cardno, V. Swali, M. Bradley, Chem. Eur. J., 2000, 8, 1455-1466

168. T. J. Baker, Y. Rew, M. Goodman, Pure Appl. Chem., 2000, 72, 347-354

169. N. J. Church, D. W. Young, Tetrahedron Lett., 1995, 36, 151-154

170. J. E. Baldwin, R. M. Adlington, I. A. O’Neil, C. J. Schofield, A. C. Spivey,

J. B. Sweeney, J. Chem. Soc., Chem. Commun., 1989, 1852-1854

171. J. E. Baldwin, C. J. Schofield, A. C. Spivey, J. B. Sweeney, Tetrahedron, 1993, 49, 6309-6330

172. J. E. Baldwin, R. M. Adlington, N. G. Robinson, J. Chem. Soc., Chem. Commun., 1987, 153-155

173. N. M. Gillings, A. D. Gee, J. Labelled Cpd. Radiopharm., 2001, 44, 909920

174. I. Nakamura, K. Harada, Chem. Lett., 1979, 313-314

175. K. Harada, I. Nakamura, J. Chem. Soc., Chem. Commun., 1978, 522-523

176. B. S. Axelsson, K. J. O'Toole, P. A. Spencer, D. W. Young, J. Chem. Soc., Perkin Trans. I, 1994, 807-815

177. K. Nakajima, M. Neya, S. Yamada, K. Okawa, Bull. Chem. Soc. Jpn., 1982, $55,3049-3050$

178. J. Bodenan, J. Chanet-Ray, R. Vessiere, Synthesis, 1992, 288-292 
179. Z. Bernstein, D. Ben-Ishai, Tetrahedron, 1977, 33, 881-883

180. T. Shiba, T. Ukita, K. Mizuno, T. Teshima, T. Wakamiya, Tetrahedron Lett., 1977, 18, 2681-2684

181. T. Shiba, T. Ukita, K. Mizuno, T. Teshima, T. Wakamiya, Bull. Chem. Soc. Jpn., 1978, 51, 850-854

182. K. Nakajima, T. Tanaka, K. Morita, K. Okawa, Bull. Chem. Soc. Jpn., 1980, $53,283-284$

183. N. Shigematsu, H. Setoi, I. Uchida, T. Shibata, H. Terano, M Hashimoto, Tetrahedron Lett., 1988, 29, 5147-5150

184. K. -D. Lee, J. -M. Suh, J. -H. Park, H. -J. Ha, H. G. Choi, C. S. Park, J. W. Chang, W. K. Lee, Y. Dong, H. Yun, Tetrahedron, 2001, 57, 8267-8276

185. K. Nakajima, H. Oda, K. Okawa, Bull. Chem. Soc. Jpn., 1983, 56, 520-522

186. R. J. Parry, M. V. Naidu, Tetrahedron Lett., 1983, 24, 1133-1134

187. J. E. Baldwin, R. M. Adlington, N. Moss, N. Robinson, J. Chem. Soc., Chem. Commun., 1987, 1664-1666

188. B. Crousse, S. Narizuka, D. Bonnet-Delpon, J. -P. Bégué, Synlett, 2001, 679-681

189. T. N. Wade, F. Gaymard, R. Guedj, Tetrahedron Lett., 1979, 20, 2681-2682

190. T. N. Wade, R. Kheribet, J. Chem. Res., 1980, 210-211

191. G. Righi, R. D’Achille, Tetrahedron Lett., 1996, 37, 6893-6896

192. F. A. Davis, G. V. Reddy, C. -H. Liang, Tetrahedron Lett., 1997, 38, 51395142

193. J. W. Metzger, K.-H. Wiesmüller, G. Jung, Int. J. Peptide Protein Res., 1991, 38, 545-554

194. J. S. A. Amaral, A. Macedo, I. A. Oliveira, J. Chem. Soc., Perkin Trans. 1, $1977,205-206$

195. K. Barlos, D. Papaioannou, D. Theodoropoulos, J. Org. Chem., 1982, 47, 1324-1326

196. C. Dugave, P. Kessler, C. Colas, C. Hirth, Tetrahedron Lett., 1994, 35, 9199-9202

197. S. Friedrich-Bochnitschek, H. Waldman, H. Kunz, J. Org. Chem., 1989, 54, $751-756$ 
198. H. Kunz, H. Waldman, C. Unverzagt, Int. J. Peptide Protein Res., 1985, 26, 493-497

199. G. Ösapay, Q. Ziu, H. Shao, R.K. Chadha, M. Goodman, Int. J. Peptide Protein Res., 1995, 46, 290-301

200. D. H. Williams, I. Flemming, Spectroscopic Methods in Organic Chemistry, $5^{\text {th }}$ Ed., 1995, McGraw-Hill Book Company Europe

201. Y. Kato, K. Fukumoto, Chem. Commun., 2000, 245-246

202. N. Bulic-Subanovic, A. B. Tabor, Department of Chemistry, University College London, unpublished work

203. B. S. Axelsson, K. J. O'Toole, P. A. Spencer, D. W. Young, J. Chem. Soc., Chem. Commun., 1991, 1085-1086

204. S. L. Elliot, "Synthesis of Orthogonally Protected Lanthionine via Mitsunobu Conditions", M.Sci. Thesis, University College London, 2000, P. R. J. Gaffney, A. B. Tabor, Department of Chemistry, University College London, personal communication

205. a) J. R. Flack, J. -Y. Lai, S. -D. Cho, J. Yu, Tetrahedron Lett., 1999, 40, 2903-2906; b) R. A. Falconer, I. Jablonkai, I. Toth, Tetrahedron Lett., 1999, 40, 8663-8666; c) S. B. Kang, E. J. Ahn, Y. Kim, Tetrahedron Lett., 1996, $37,9317-932088$

206. Residual DMF, which is basic, would have an inhibitory effect on the cleavage of peptides from acid labile linkers.

207.F. Cavelier-Frontin, S. Achmad, J. Verducci, R. Jacquier, G. Pépe, J. Mol. Structure, 1993, 286, 125-130

208. W. D. F. Meutermans, S. W. Golding, G. T. Bourne, L. P Miranda, M. J. Dooley, P. F. Alewood, M. L. Smythe, J. Am. Chem. Soc., 1999, 121, 97909796

209. H. Kessler, B. Haas, Int. J. Peptide Protein Res., 1992, 39, 36-40

210. U. Schmidt, J. Langner, J Peptide Res., 1997, 49, 67-73

211. European Patent 0,394,194 and US Patent 5,324,833 granted to Calbiochem-Novabiochem AG

212. a) L. M. Alexander McNamara, "Synthesis of Stable $\alpha$-Helical Peptides", Ph.D. Thesis, University College London, 2000 ; b) L. M. Alexander 
McNamara, M. J. I. Andrews, F. Mietzel, G. Siligardi, A. B. Tabor, J. Org. Chem., 2001, 66, 4585-4594

213. O. Mitsunobu, Synthesis, 1981, 1-28

214. G. Amiard, R. Heymes, L. Velluz, Mémoires Présentés a la Société Chimiqué, 1954, 191-193

215. J. W. Metzger, K. -H. Wiesmüller, G. Jung, Int. J. Peptide Protein Res., $1991,38,545-554$

216. T. Hida, K. Hayashi, K. Yukishige, S. Tanida, N. Kawamura, J. Antibiotics, 1995, 48, 589-603

217. J. S. McMurray, C. A. Lewis, N. U. Obeyeskere, Peptide Research, 1994, 7, 195-206

218. R. Harris, Department of Biochemistry and Molecular Biology, University College London, personal communication 